\title{
Nanolithography Toolbox
}

Krishna C. Balram, Daron A. Westly, Marcelo Davanco, Karen E. Grutter, Qing Li, Thomas Michels, Christopher H. Ray, Liya Yu, Richard J. Kasica,

Christopher B. Wallin, Ian J. Gilbert, Brian A. Bryce, Gregory Simelgor, Juraj Topolancik, Nicolae Lobontiu, Yuxiang Liu, Pavel Neuzil, Vojtech Svatos, Kristen A. Dill, Neal A. Bertrand, Meredith G. Metzler, Gerald Lopez, David A. Czaplewski, Leonidas Ocola, Kartik A. Srinivasan, Samuel M. Stavis, Vladimir A. Aksyuk, J. Alexander Liddle, Slava Krylov, and B. Robert Ilic

This publication is available free of charge from:

http://dx.doi.org/10.6028/NIST.HB.160 


\section{NIST Handbook 160}

\section{Nanolithography Toolbox}

Krishna C. Balram, Daron A. Westly, Marcelo

Davanco, Karen E. Grutter, Qing Li, Thomas

Michels, Christopher H. Ray, Liya Yu, Richard J. Kasica, Christopher B. Wallin, Ian J. Gilbert, Kristen

A. Dill, Neal A. Bertrand, Kartik A. Srinivasan, Samuel M. Stavis, Vladimir A. Aksyuk, J. Alexander

Liddle, Slava Krylov, and B. Robert Ilic Center for Nanoscale Science and Technology

Brian A. Bryce

Harvey Mudd College, Claremont, CA 91711 USA

Gregory Simelgor, Edico Genome, La Jolla, CA 92037 USA

Juraj Topolancik

Roche Sequencing Solutions, Pleasanton, CA 94588

Nicolae Lobontiu University of Alaska, Mechanical Engineering, Anchorage, AK 99508 USA
Pavel Neuzil

Brno University of Technology (BUT), Central European Institute of Technology (CEITEC), Technicka 3058/10, CZ-616 00 Brno, Czech Republic Department of Microsystems, Northwestern Polytechnical University, Xi'an, P.R. China

Vojtech Svatos Brno University of Technology (BUT), Central

European Institute of Technology (CEITEC), Technicka 3058/10, CZ-616 00 Brno, Czech Republic

Meredith G. Metzler and Gerald Lopez Quattrone Nanofabrication Facility, University of Pennsylvania, Philadelphia, PA 19104 USA

David A. Czaplewski and Leonidas Ocola Center for Nanoscale Materials, Argonne National Laboratory, Lemont, IL 60439 USA

Slava Krylov

Tel Aviv University, School of Mechanical Engineering, Ramat Aviv 69978 Tel Aviv, Israel

This publication is available free of charge from: http://dx.doi.org/10.6028/NIST.HB.160

October 2016

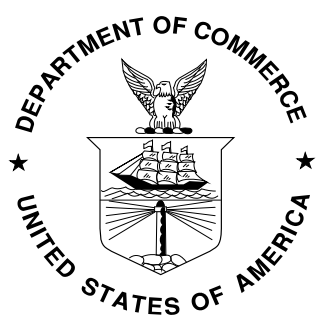

U.S. Department of Commerce Penny Pritzker, Secretary

National Institute of Standards and Technology Willie May, Under Secretary of Commerce for Standards and Technology and Director 


\section{Nanolithography Toolbox

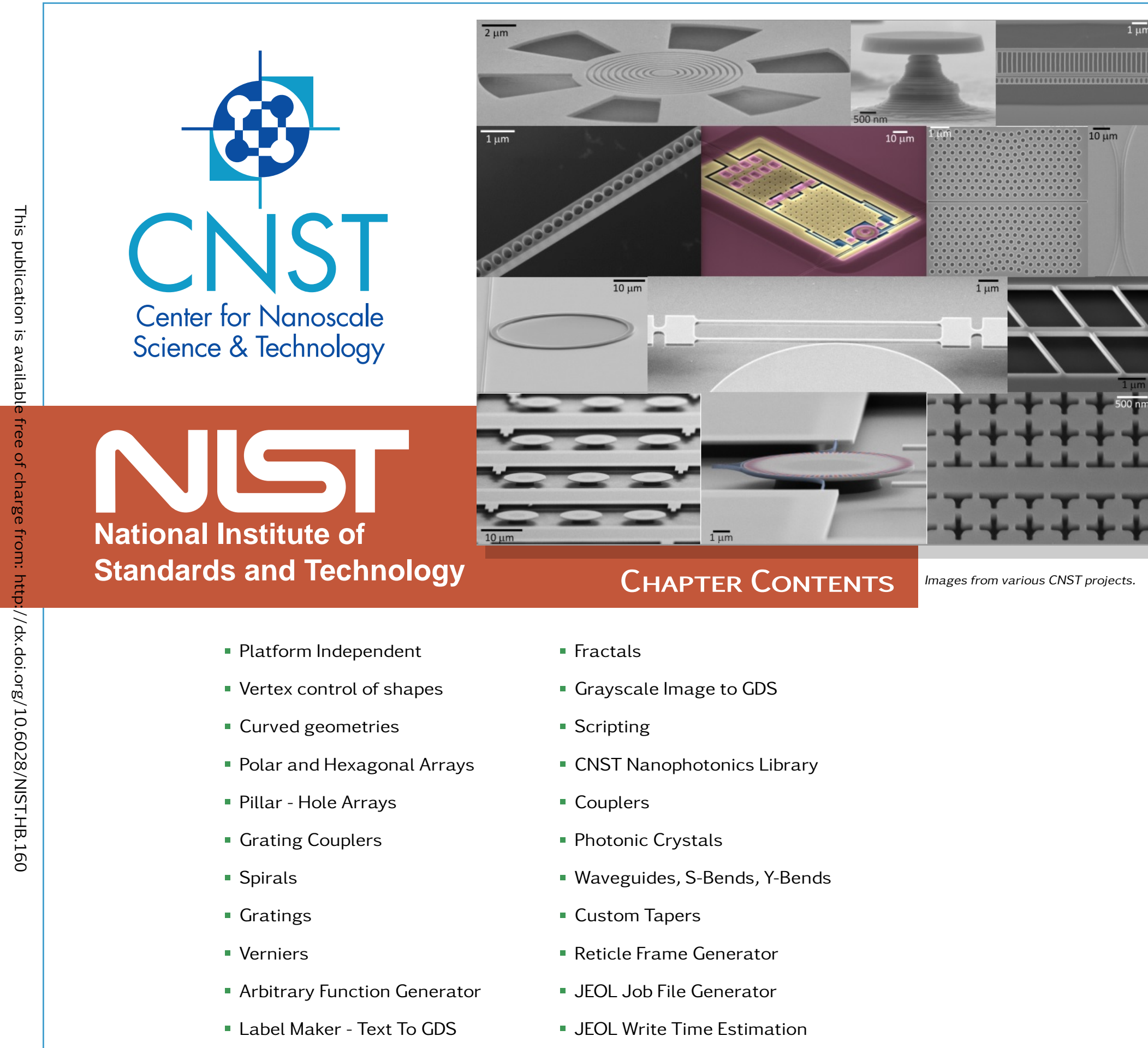


1 Nanolithography Toolbox $\quad 1$

Nanolithography Toolbox 8

1.1 Overview ..................... 8

1.1 .1 Terms Of Use . . . . . . . . . . . . . . . . 10

$1.1 .2 \quad$ Disclaimer . . . . . . . . . . . . . . . . 10

1.1.3 Acknowledgements ... . . . . . . . . . . 10

1.1.4 Development Team . . . . . . . . . . . . . . . . 10

1.1 .5 Future Developments . . . . . . . . . . . . . 11

1.1.6 Java 8 Requirements . . . . . . . . . . . . . 12

1.1 .7 Distribution Package . . . . . . . . . . . . . . . 12

1.1.8 Modes of Operation . . . . . . . . . . . . . . 12

1.1.8.1 CNST Scripting . . . . . . . . . . . . . . . . 12

1.1.8.2 Graphical User Interface . . . . . . . . . . . 15

$\begin{array}{ll}\text { GDS Scripting } & 16\end{array}$

2.1 Setup, Execution and Examples . . . . . . . . . . . . . . 16

2.1 .1 Quick Start Setup . . . . . . . . . . . . . . . . . 16

2.1.2 Setup - CNSTdefaultValues.xml open and save directories 17

2.1.3 Graphical User Interface Execution . . . . . . . . . . 18

2.1.4 Running CNST Scripts From a Terminal Command Prompt 19

2.1.5 Running Multiple CNST Scripts - Batch File Mode . . . . 19

2.1.6 Running CNST Scripts Within Matlab . . . . . . . . . 20

2.1.7 Python Script Generation . . . . . . . . . . . . . . . . 21

2.1.8 NotePad++ Syntax Coloring . . . . . . . . . . . . 22

2.1 .9 Scripting Examples . . . . . . . . . . . . 23

2.1.9.1 Basic Scripting Example . . . . . . . . . . . . 23

2.1.9.2 Scripting Example - Labeled Calibration Ruler . . 24

2.1.9.3 Labeled Electrodes . . . . . . . . . . . . . . 25

2.1.9.4 MEMS Comb Drive Flexures . . . . . . . . . . . 26

2.1.9.5 MEMS Radial Comb Drive Circular Hub . . . . . . 27

2.1.9.6 Scripting Example Files Description . . . . . . . . 28

2.2 Interface Functions . . . . . . . . . . . . . . . . . 32

2.2 .1 Comments . . . . . . . . . . . . . . . 32

2.2.2 Creating Structures (Cells) . . . . . . . . . . . . . 32

2.2 .3 Layer . . . . . . . . . . . . . . . . . . 32

2.2 .4 Data Type . . . . . . . . . . . . . . . . . 32

2.2.5 GDS Rendering Resolution . . . . . . . . . . . . . 32

2.2.6 Shape Resolution . . . . . . . . . . . . . . . . 33 


\section{Chapter 1 CONTENTS}

2.2.7 Font Outline Width . . . . . . . . . . . . . . . 33

2.2.8 Calls To Multiple CNST Script Files . . . . . . . . . . . . . 34

2.2.9 Log File Time Date Stamp . . . . . . . . . . . . . . . 34

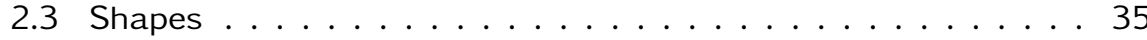

2.3.1 Circles and Ellipses . . . . . . . . . . . . 35

2.3.1.1 Primitive Ellipse . . . . . . . . . . . . . . 35

2.3.1.2 Vectorized Ellipse . . . . . . . . . . . . . . . 35

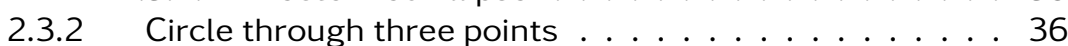

2.3.3 Circle With a Wave Boundary . . . . . . . . . . . . . 37

2.3.4 Cross ..................... . . 38

$2.3 .5 \quad$ L-Shape . . . . . . . . . . . . . . . 39

2.3.6 Pie Shaped Arc . . . . . . . . . . . . . . . . . . . . 40

2.3.7 Pie Shaped Arc - Vector . . . . . . . . . . . . . . 40

2.3 .8 Polygon . . . . . . . . . . . . . . . 41

2.3 .9 Rectangle . . . . . . . . . . . . . . . . . . 42

2.3.10 Rounded Rectangle . . . . . . . . . . . . . . . . 43

2.3.11 Rectangular SU-Shape . . . . . . . . . . . . . . 44

2.3.12 Rectangle With a Linear Taper . . . . . . . . . . . . . . . 45

2.3 .13 Star .................... 46

2.3.14 Torus - Arc . . . . . . . . . . . . . . . . . . . . 47

2.3.15 Torus - Vector . . . . . . . . . . . . . . . . . . . . . . 47

2.3.16 Torus With a Wave boundary . . . . . . . . . . . . . . . 48

2.4 Arrays and Instances . . . . . . . . . . . . . . . . . . . . . 49

2.4.1 Rectangular Arrays . . . . . . . . . . . . . . 49

2.4 .2 Hexagonal Arrays . . . . . . . . . . . . . . 50

2.4 .3 Polar Arrays . . . . . . . . . . . . . . . 51

2.4.4 Instancing GDS Structures . . . . . . . . . . 52

2.4 .5 Points To Instance . . . . . . . . . . . . . 53

2.4.6 Pillar-Hole Hexagonal and Square Arrays . . . . . . . . . 54

2.5 General Area Operations . . . . . . . . . . . . . . . . . 56

2.5.1 Boolean Operations . . . . . . . . . . . . . . . 56

2.5.2 General Area Copies, Shape Bias and Affine Transfor-

mations . . . . . . . . . . . . . . 58

2.6 Text Labels, PostScript and Logos . . . . . . . . . . . . . . . 60

$2.6 .1 \quad$ Text. . . . . . . . . . . . . . . 6 60

2.6 .2 Text Outline . . . . . . . . . . . . . . . . 61

2.6 .3 Label Maker . . . . . . . . . . . . . . . . . 62

2.6.4 Label Maker - Outline Text . . . . . . . . . . . . 64

2.6 .5 PostScript to GDS . . . . . . . . . . . . . . 66

2.6.5.1 PostScript Pixel Value . . . . . . . . . . . 66

2.6.5.2 PostScript Fracturing . . . . . . . . . . . 666

2.6.5.3 Defining Postscript Shapes . . . . . . . . . . . 66

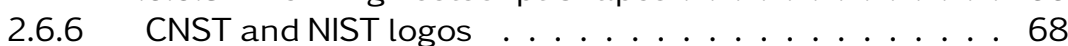

2.7 Objects . . . . . . . . . . . . . . . . . . 69

2.7.1 Arc (Torus-Circle) bounded square-hex arrays . . . . . . 69

2.7.2 Bezier Curve . . . . . . . . . . . . . . . . 70 


\section{Chapter 1 CONTENTS}

2.7 .3 Fractals . . . . . . . . . . . . . . 74

2.7.4 Function Plot . . . . . . . . . . . . . . . . . 75

2.7 .5 Grayscale . . . . . . . . . . . . . . 77

2.7.5.1 Polygons . . . . . . . . . . . . . . 77

2.7 .5 .2 Ramp . . . . . . . . . . . . . . . 79

2.7.5.3 Spiral Staircase . . . . . . . . . . . . . . . 81

2.7.6 Interdigitated Electrodes . . . . . . . . . . . 83

2.7 .7 Junctions . . . . . . . . . . . . . . . . . . . 86

2.7.7.1 T Junction . . . . . . . . . . . . . . . 86

2.7.7.2 H Junction . . . . . . . . . . . . . . . . 87

2.7.7.3 Arrow Junction . . . . . . . . . . . . . . . . . . . . . . . . . . . . . . .

2.7 .8 Meander Channel . . . . . . . . . . . . . . 89

2.7.9 Points To Shape . . . . . . . . . . . . . . . . . . . . . 92

2.7.10 Polygon Along a Path . . . . . . . . . . . . . 93

2.7.11 Random Polygons . . . . . . . . . . . . . . . . 94

2.7.12 Random Ellipses and Vectorized Ellipses . . . . . . . . 95

2.7.13 Resolution Test Pattern . . . . . . . . . . . . . . 96

2.7 .14 Spirals . . . . . . . . . . . . . . . . 99

2.7.15 Spiral-Rectangular . . . . . . . . . . . . . 100

2.8 Alignment and Reticle Elements . . . . . . . . . . . . . . . . . . . 101

2.8.1 Alignment Marks - Predefined . . . . . . . . . . . . . 101

2.8.2 Alignment Marks - Custom . . . . . . . . . . . . . 105

2.8.3 Reticle Barcode and Label Frames . . . . . . . . . . 107

2.8 .4 Vernier . . . . . . . . . . . . . . . . . . . . 108

2.8 .5 Vernier With Labels . . . . . . . . . . . . . . . . . . . . 109

2.8 .6 Arrows . . . . . . . . . . . . . . . . 110

2.9 CNST Nanophotonics Library . . . . . . . . . . . . . . . . . 111

2.9.1 Waveguides . . . . . . . . . . . . . 112

2.9.1.1 Waveguide . . . . . . . . . . . 112

2.9.1.2 Waveguide Slot . . . . . . . . . . . . . . 113

2.9.1.3 Waveguide Inverse . . . . . . . . . . . . 114

2.9.1.4 Waveguide Inverse Slot . . . . . . . . . . . . 115

2.9.1.5 Waveguide Expander . . . . . . . . . . . 116

2.9 .2 Tapers . . . . . . . . . . . . . . . . . . 117

2.9.2.1 Linear Taper . . . . . . . . . . . . . . . . 117

2.9.2.2 Linear Taper Slot . . . . . . . . . . . . . . . 118

2.9.2.3 Linear Taper Inverse Slot . . . . . . . . . . . . . . 119

2.9.2.4 Exponential Taper . . . . . . . . . . . . . . . . 120

2.9.2.5 Exponential Taper Inverse . . . . . . . . . . . . 121

2.9.2.6 Exponential Taper Inverse Slot . . . . . . . . . . . 122

2.9.2.7 Custom Taper . . . . . . . . . . . . . . . . . . 123

2.9 .3 Couplers . . . . . . . . . . . . . . . . . . . . 124

2.9.3.1 Directional Couplers . . . . . . . . . . . . . 124

2.9.3.2 S-Bend Taper . . . . . . . . . . . . . . . . . . . . 128

2.9.3.3 S-Bend Funnel . . . . . . . . . . . . . . . . . . . 129

2.9.3.4 S-Bend . . . . . . . . . . . . . . . 130 


\section{Chapter 1 CONTENTS}

2.9.3.5 S-Bend Inverse . . . . . . . . . . . . . . . . . . 131

2.9.3.6 S-Bend Inverse Slot . . . . . . . . . . . . . . . . . 132

2.9.3.7 Y-Bend . . . . . . . . . . . . . . . . 133

2.9.3.8 Y-Bend Inverse . . . . . . . . . . . . . . . . . . . . 134

2.9.3.9 Y-Bend Inverse Slot . . . . . . . . . . . . . . . . 135

2.9.3.10 Y-Bend - 90 degree . . . . . . . . . . . . . 136

2.9.3.11 Y-Bend Inverse - 90 degree . . . . . . . . . . . . 137

2.9.3.12 Y-Bend Inverse Slot - 90 degree . . . . . . . . . 138

2.9.3.13 90 Degree Bend . . . . . . . . . . . . . . . . . 139

2.9.3.14 90 Degree Bend Inverse . . . . . . . . . . . . 140

2.9.3.1590 Degree Bend Inverse Slot . . . . . . . . . . . 141

2.9.3.16 180 Degree Bend . . . . . . . . . . . . . . . . . 142

2.9.3.17 180 Degree Bend Inverse . . . . . . . . . . . . . . 143

2.9.3.18 180 Degree Bend Inverse Slot . . . . . . . . . . . 144

2.9 .4 Racetrack . . . . . . . . . . . . . . . . . . 145

2.9 .5 Spiral Delay Line . . . . . . . . . . . . . . . . . . 146

2.9.6 Inverse Spiral Delay Line . . . . . . . . . . . . . . 148

2.9 .7 Gratings . . . . . . . . . . . . . . . . . . . . 150

2.9.7.1 Grating . . . . . . . . . . . . . . . . . . . . . . . . . . . . . . . . . . . . . . . . . . .

2.9.7.2 Apodized Grating . . . . . . . . . . . . . . 151

2.9.7.3 Grating Coupler . . . . . . . . . . . . . . . . 152

2.9.7.4 Grating Couplers With Waveguides . . . . . . . . 153

2.9.8 Photonic Crystals and Hexagonal Arrays . . . . . . . . 156

2.9.9 Disc-Ring Architectures . . . . . . . . . . . . . . . . 157

2.9.9.1 Disc-Ring - Bezier Curves, Arcs and Endcaps . 157

2.9.9.2 Disc-Ring Infinite . . . . . . . . . . . . . . 159

2.9.9.3 Disc-Ring Infinite Inverse . . . . . . . . . . . . 161

2.9.9.4 Disc-Ring Infinite Inverse Positive Tone . . . . . . 163

2.9.9.5 Disc-Ring Symmetric Bezier . . . . . . . . . . 165

2.9.9.6 Disc-Ring Symmetric Arc . . . . . . . . . . . . 175

2.9.9.7 Disc-Ring Symmetric Inverse Bezier . . . . . . 185

2.9.9.8 Disc-Ring Symmetric Inverse Arc . . . . . . . . 195

2.9.9.9 Disc-Ring Symmetric Inverse Positive Tone Bezier 205

2.9.9.10 Disc-Ring Symmetric Inverse Positive Tone Arc . 215

2.9.9.11 Disc-Ring Pulley Bezier . . . . . . . . . . . . . 225

2.9.9.12 Disc-Ring Pulley Arc . . . . . . . . . . . . . . . 235

2.9.9.13 Disc-Ring Pulley Inverse Bezier . . . . . . . . . 245

2.9.9.14 Disc-Ring Pulley Inverse Arc . . . . . . . . . . 255

2.9.9.15 Disc-Ring Pulley Inverse Positive Tone Bezier . . 265

2.9.9.16 Disc-Ring Pulley Inverse Positive Tone Arc . . . . 275

2.9.10 Waveguide Inverse Photonic Crystals (Ellipse) . . . . . . 285

2.9.11 Waveguide Photonic Crystals (Ellipse) . . . . . . . . . 286

2.9.12 Waveguide Inverse Photonic Crystals (Rectangle) . . . 287

2.9.13 Waveguide Photonic Crystals (Rectangle) . . . . . . . . 288

2.9.14 Waveguide Photonic Crystals (Flush Rectangle) . . . . . 289

2.9.15 Various Waveguide-Disc-Tip Coupled Structures . . . . . 290 


\section{Chapter 1 CONTENTS}

2.10 MEMS - NEMS Library . . . . . . . . . . . . . . . . . . . . . . 298

2.10 .1 Actuators . . . . . . . . . . . . . . . . . 298

2.10.1.1 Bent Beams . . . . . . . . . . . . . . 298

2.10.1.2 Bi-Morph Thermal Actuator . . . . . . . . . . 299

2.10.1.3 Combs and Drive Elements . . . . . . . . . . . . 300

2.10.1.4 Folded Springs . . . . . . . . . . . . . . . . . 302

2.10 .2 Bolometers . . . . . . . . . . . . . . . . 311

2.10 .3 Gears . . . . . . . . . . . . . . . 313

2.10.3.1 Hub With Straight and Circular Springs . . . . 314

2.10.3.2 Radial Comb Drive . . . . . . . . . . . . . . . 317

2.10 .4 Anchored Flexures . . . . . . . . . . . . . . . . . . 319

2.10 .5 Cantilevers . . . . . . . . . . . . . . . 329

2.10 .6 Doubly Clamped Beams . . . . . . . . . . . . . . . . 340

2.10 .7 Interacting Arrays . . . . . . . . . . . . . . 347

2.10.8 Stress, Strain Measurement Structures . . . . . . . . . 357

2.10.8.1 Guckel Rings . . . . . . . . . . . . . . . . . . . . . . . . . . . . . . . . . . .

2.10.8.2 Diamond Ring . . . . . . . . . . . . . . 358

2.10 .9 Suspended Fluid Cell . . . . . . . . . . . . . . . . . . 359

Graphical User Interface $\quad 360$

3.1 Basic Shapes . . . . . . . . . . . . . . . . . . . . . 360

3.1.1 Pillar-Hole (Square/Hex) Array . . . . . . . . . . . . 360

3.1 .2 Torus . . . . . . . . . . . . . . . 360

3.1.3 Grating Coupler - Bulls Eye . . . . . . . . . . . 360

3.1 .4 Spirals . . . . . . . . . . . . . . 361

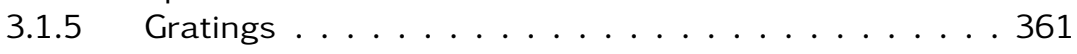

3.2 Lithography Machine Resources . . . . . . . . . . . . . 363

3.2.1 CNST Reticle Frame Generator . . . . . . . . . . . . . . 363

3.2.2 Generic Reticle Frames . . . . . . . . . . . . . . . 363

3.2.3 Ebeam Lithography - Job and Schedule File Generator . 363

3.2.3.1 Default Values Initialization File . . . . . . . . . 363

3.2 .3 .2 Main . . . . . . . . . . . . . . 363

3.2.3.3 Align . . . . . . . . . . . . . . . . 364

3.2.3.4 Pattern . . . . . . . . . . . . . . 364

3.2.3.5 Dose Matrix . . . . . . . . . . . . . . . 365

3.2.4 EBL Alignment Offset . . . . . . . . . . . . 368

3.2 .5 EBL Max Clock . . . . . . . . . . . . . . . 368

3.2.6 EBL Write Time Estimation . . . . . . . . . . . 368

3.3 Advanced CAD Resources . . . . . . . . . . . . . . . . . 370

3.3.1 Label Maker . . . . . . . . . . . . . . . . . . . 370

3.3 .2 Text To GDS . . . . . . . . . . . . . . . . . 370

3.3.3 Arbitrary Function Generator . . . . . . . . . . . . . . . 370

3.3.4 Binary Zone Plate . . . . . . . . . . . . . . . . . . . . 371

3.3.5 Photonic Crystals . . . . . . . . . . . . . . . . . . 372

3.3.6 Random Polygons . . . . . . . . . . . . . . . . 372

3.3.7 Random Rectangular Array . . . . . . . . . . . . . . 373 
3.3.8 Cantilever Arrays . . . . . . . . . . . . . . . . . . . . 373

3.3 .9 Verniers . . . . . . . . . . . . . . . . 374

3.3 .10 Fractals . . . . . . . . . . . . . . . 374

3.3.11 Grayscale Image To GDS . . . . . . . . . . . . . 374

Programming Reference 376

4.1 Programming Examples . . . . . . . . . . . . . . . 376

4.1.1 Template . . . . . . . . . . . . . . . 376

4.1.2 Script Method Access . . . . . . . . . . . . . . . 377

4.1.3 Boolean Operations and Affine Transformations . . . 379

4.1 .4 Labeled Arrays . . . . . . . . . . . . . . . . . . 381

$4.1 .5 \quad$ MEMS Perforated Flexures . . . . . . . . . . 383

4.1.6 Custom Class Methods . . . . . . . . . . . . . . . . . . . 385

4.1.7 GDS Area Objects - Layers and Data-types . . . . . . . . 387

4.1.8 Centering GDS Area Objects . . . . . . . . . . . . . . 388

4.1 .9 PostScript to GDS . . . . . . . . . . . . . . . . . . . 389

4.1.10 Curved Fluidic Channels . . . . . . . . . . . . . . . . . . . 391

4.2 Reference Methods . . . . . . . . . . . . . . . . . . . . . . . 394

4.2.1 Accessor and Mutator Methods . . . . . . . . . . . 394

4.2.2 Miscellaneous GDS Area and Struct Methods . . . . . . 395

4.2.3 Shape Methods . . . . . . . . . . . . . . . . . 396

4.2 .4 Array Methods . . . . . . . . . . . . . . . . . . . . 398

4.2.5 Text, Labels, PostScript and Logo Methods . . . . . . . . 401

4.2.6 Miscellaneous Object Methods . . . . . . . . . . . . . . . 404

4.2.7 Alignment and Reticle Element Methods . . . . . . . . . 414

4.2.8 Nanophotonics Library Methods . . . . . . . . . . . . . . 417

4.2.9 MEMS NEMS Library Methods . . . . . . . . . . . . . . . 453

$\begin{array}{ll}\text { Bibliography } & 479\end{array}$ 


\subsection{Overview}

latform independent CNST Nanolithography toolbox for scripted layout generation and complex processing was designed to address aggressively scaled nanoscale device architectures. Within the layout design phase, imprecise representation of curved objects [1-37] at nanoscale dimensions results in increased line-edge roughness. Scattering events from the consequent asperities along device peripheries leads to enhanced dissipative effects. To circumvent these dilemmas, along with standard commercial layout tools, we have developed a custom CAD scripting software package for directly streaming complex shapes to GDSII.

At CNST, we have strong research efforts in nanoscale optics and photonics [38-69]. Consequently, we have developed a library of nanophotonics components (the CNST nanophotonics library contains microrings, S-bends based on Bezier curves, photonic crystals, variety of tapers, grating couplers [7075] and other complex structures) with precise control of vertex location in the GDSIl file. Accurate shape representation is of particular importance to high-resolution lithography, for example, using the either of the two NanoFab $100 \mathrm{keV}$ tools with sub-nm grid snapping capability. The flexible scripting interface enables significant user customization to lay out structures outside of the standard available designs. Furthermore, the toolbox is platform independent and will run on any operating system (Linux, Windows and MacOS X) with Java standard edition (SE) 8. In addition to standard Java libraries, the toolbox utilizes the freely available JGDS library for encoding shapes to GDS objects [76].

The CNST nanolithography toolbox also contains primitives (ellipses, torus, rectangles, etc), pillar-hole arrays (rectangular, hexagonal), a variety of spirals, fractals, gratings, verniers, arbitrary function generator (shapes defined by a mathematical function), label makers, text to GDS, postScript to GDS, zone plates, grayscale objects [77-82], images to GDS, polar arrays (also, hexagonal and rectangular), and many more customized shapes. Additionally, we include a MEMS library of elements containing variety of actuators, flexures, clamped beams, and interacting array structures[83-103]. The package also contains CNST-developed reticle generators for various steppers (barcode, label, reticle marks), a CNST ebeam lithography write time estimator, and a ebeam lithography job and schedule file generator with a graphical display of pattern placement (Figure 1.1). 


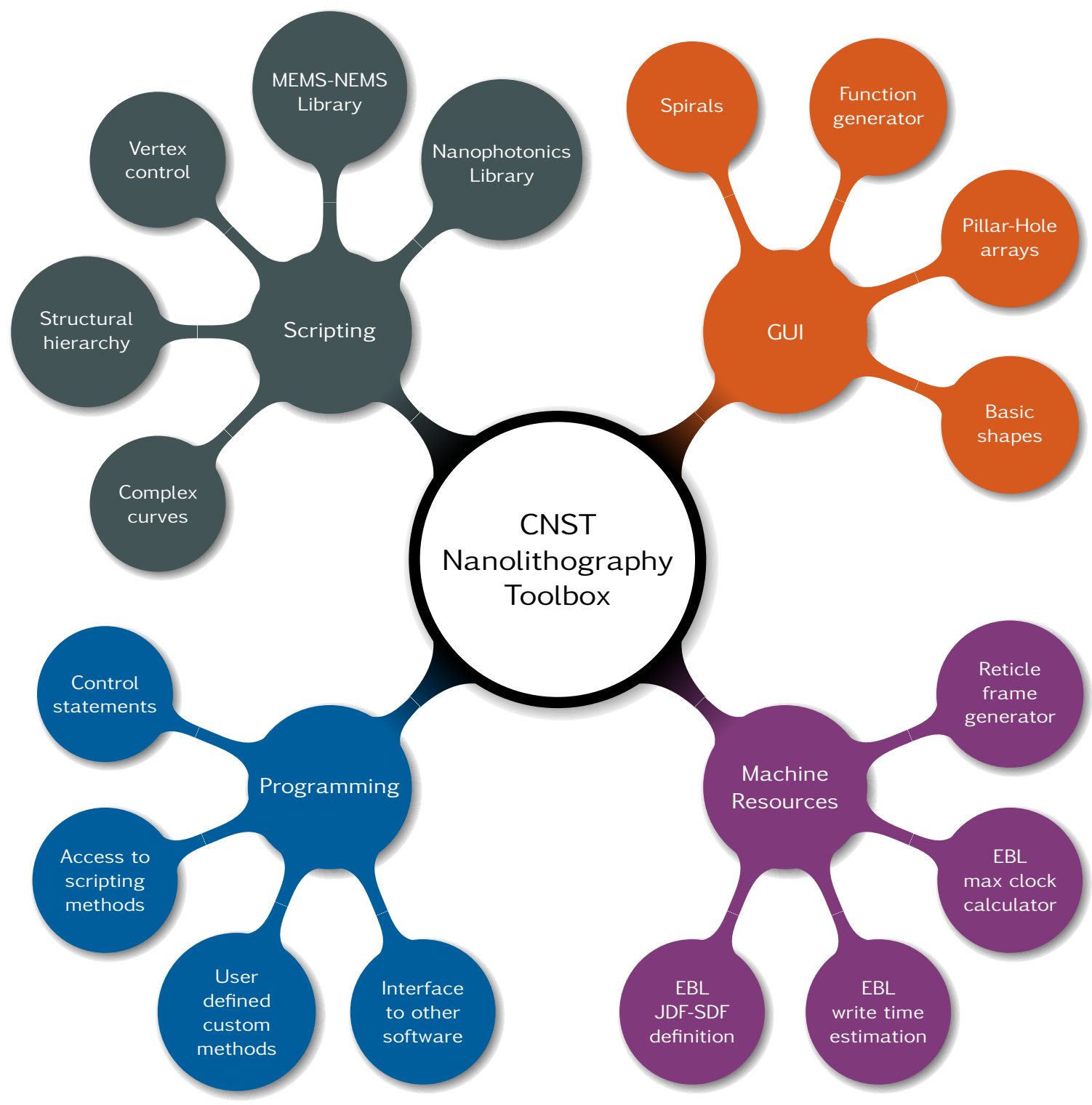

Figure 1.1: CNST Nanolithography Toolbox chart illustrating a myriad of available complex shapes, objects and functions. 


\subsubsection{Terms Of Use}

This software was developed at the National Institute of Standards and Technology (NIST) by employees of the Federal Government in the course of their official duties. Pursuant to title 17 Section 105 of the United States Code this software is not subject to copyright protection and is in the public domain. The NIST CNST nanolithography toolbox is an experimental system. NIST assumes no responsibility whatsoever for its use by other parties, and makes no guarantees, expressed or implied, about its quality, reliability, or any other characteristic. We would appreciate acknowledgment if the software is used. This software can be redistributed and/or modified freely provided that any derivative works bear some notice that they are derived from it, and any modified versions bear some notice that they have been modified.

\subsubsection{Disclaimer}

This manual identifies certain commercial equipment, instruments, and materials to specify the experimental procedure. Such identification does not imply recommendation or endorsement by the National Institute of Standards and Technology, nor does it imply that the equipment, instruments, and materials identified are necessarily the best available for the purpose.

\subsubsection{Acknowledgements}

If you developed layouts using the CNST nanolithography toolbox, please acknowledge its use by including the following reference:

The Nanolithography Toolbox, K. C. Balram, D. A. Westly, M. Davanco, K. Grutter, Q. Li, T. Michels, C. H. Ray, L. Yu, R. Kasica, C. B. Wallin, I. Gilbert, B. A. Bryce, G. Simelgor, J. Topolancik, N. Lobontiu , Y. Liu, P. Neuzil, V. Svatos, K. A. Dill, N. A. Bertrand, M. Metzler, G. Lopez, D. A. Czaplewski, L. Ocola, K. Srinivasan, S. Stavis, V. Aksyuk, J. A. Liddle, S. Krylov and B. R. Ilic, J. Res. Natl. Inst. Stand. 121, pp. 464-475 (2016). http://dx.doi.org/10.6028/jres.121.024

\subsubsection{Development Team}

Many researchers at NIST, CNST and external collaborators have made substantial contributions to the nanolithography toolbox. The development team most notably consists of Krishna C. Balram (University of Maryland), Daron A. Westly, Marcelo Davanco, Karen Grutter, Qing Li (University of Maryland), Thomas Michels, Christopher H. Ray, Liya Yu, Richard Kasica, Christopher B. Wallin (University of Maryland), lan Gilbert (University of Maryland), Brian A. Bryce (Harvey Mudd College), Gregory Simelgor (Edico Genome), Juraj Topolancik (Roche Sequencing Solutions), Nicolae Lobontiu (University of Alaska), Yuxiang Liu (Worcester Polytechnic Institute), Pavel Neuzil (Brno University of Technol-

NIST • CNST Nanolithography Toolbox v2016.09.01 • http://www.nist.gov/cnst/

page 10 of 488 
ogy, KIST-Europe), Vojta Svatos (Brno University of Technology),Kristen A. Dill, Neal A. Bertrand, Meredith Metzler (University of Pennsylvania), Gerald Lopez (University of Pennsylvania), David A. Czaplewski (Argonne National Laboratory), Leonidas Ocola (Argonne National Laboratory), Kartik Srinivasan, Samuel Stavis, Vladimir Aksyuk, J. Alexander Liddle, Slava Krylov (Tel-Aviv University) and B. Robert llic.

\subsubsection{Future Developments}

The CNST nanolithography toolbox is broad in scope and continuously growing within the CNST community. The toolbox is distributed with the hope that it will be useful, but without any warranty, without even an implied warranty for any particular purpose. All efforts have been made to ensure that the CNST nanolithography toolbox is supported on Linux, Windows and MacOS $X$. Please direct comments, suggestions, encountered bugs to Nanolithography.Toolbox@nist.gov. The software will evolve over time, implementing features as the CNST identifies and prioritizes new applications. 


\subsubsection{Java 8 Requirements}

The graphical user interface (GUI) was constructed using JavaFX components. The code employs a variety of elements contained in latest java standard edition (SE) 8, hence earlier versions will not work correctly. To determine the $\mathrm{SE}$ version, at the (windows command or linux/mac terminal) prompt type:

$$
\text { java -version }
$$

At the terminal prompt the following would appear:

java version "1.8.0_40"

Java(TM) SE Runtime Environment (build 1.8.0_40-b26)

Java HotSpot(TM) 64-Bit Server VM (build 25.40-b25, mixed mode)

1.8.X_XX indicates Java version 8 is available. To run the CNST NanoLithography Toolbox, either double click on the CNSTnanoToolboxVXXXX.jar file ( $X$ 's represent version numbers) or run from a command prompt in a following manner:

$$
\text { java -jar CNSTnanoToolboxVXXXX.jar }
$$

Upon execution of the java code, the CNST Nanolithography Toolbox will appear in a similar fashion as seen in figure 1.2. The menu appearance may vary slightly depending on the operating system the interface is running under. Figure 1.2 shows the toolbox as it appears under 64-bit Windows 7.

\subsubsection{Distribution Package}

The package contains the following files and directories:

CNSTnanoToolboxVXXXX.jar The CNST Nanolithography Toolbox executable Java JAR file. X's represent version numbers.

CNSTdefaultValues.xml Default parameter values for the CNST ebeam tool module, load and save file directories.

Examples

Directory containing syntax coloring XML language definition file, numerous scripting, GUI module and programming examples.

\subsubsection{Modes of Operation}

\subsubsection{CNST Scripting}

The most powerful feature of the CNST nanolithography toolbox is scripting. Virtually any shape can be constructed using the CNST Scripting features. The scripting feature is listed as the first option within the resource menu. The scripting module reads in an ASCII file with a .cnst extension and converts

NIST • CNST Nanolithography Toolbox v2016.09.01 • http://www.nist.gov/cnst/

page 12 of 488 


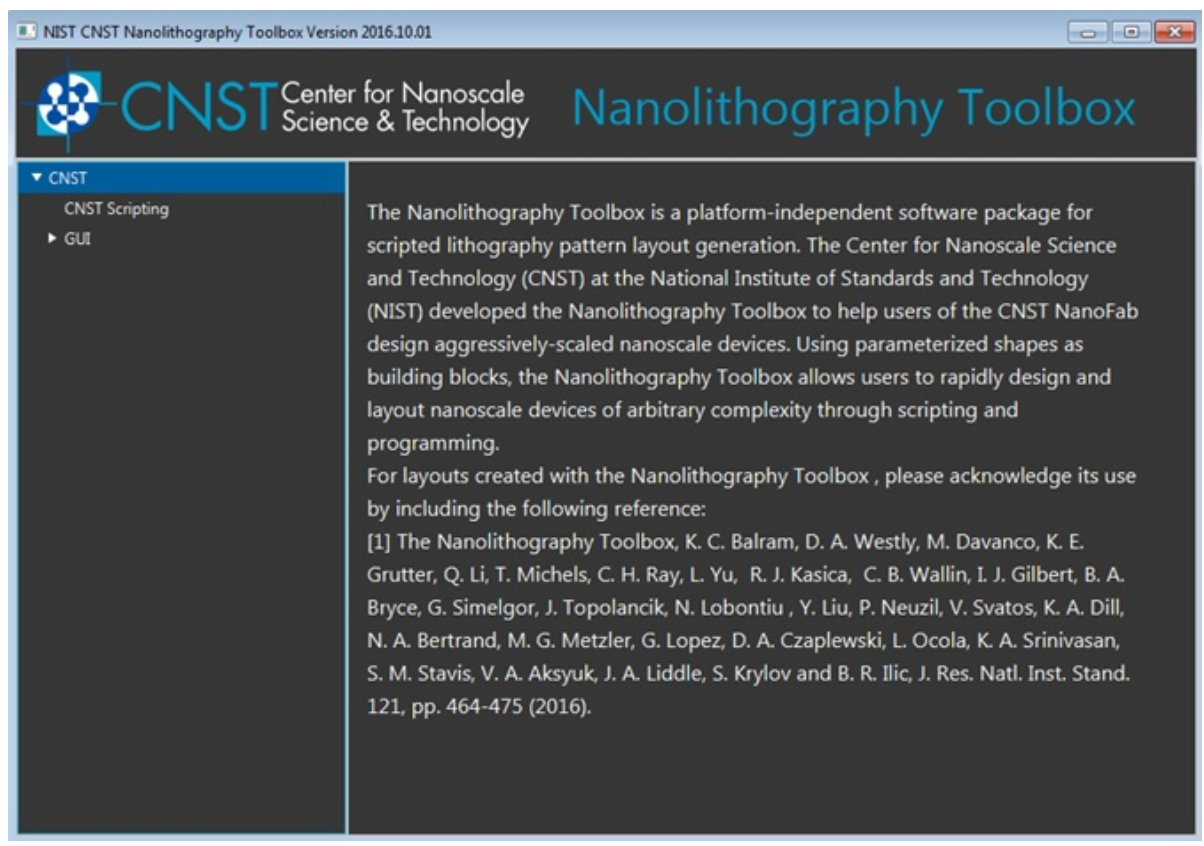

Figure 1.2: CNST nanolithography toolbox graphical user interface running under Windows 7. Various features are displayed within the left panel in a collapsible tree menu. Highlighting a node of a particular branch will display feature content in the main panel display.

complex objects directly to GDS. We also provide an extensive library of objects including numerous nanophotonic elements. During the pattern design phase, space is broken up into grid points between which the CNST scripting objects are rendered. Figure 1.3 shows various points connecting a variety of generated nanophotonic elements (exponential taper, photonic crystal waveguide, $y$-splitters, waveguides, s-bends and rings). Syntax coloring for .cnst files is available using NotePad++. This feature improves readability-structure and errors become visually distinct.

CNST scripting offers a myriad of options that are either not found or extremely difficult to implement within standard CAD packages that produce GDS files. The full command reference for CNST scripting is included in section 1.1.8.2. Figure 1.4 illustrates a variety of generated GDS shapes. CNST scripting is either carried out from the GUI or from the terminal command prompt (see section 2.1). Additionally, running batch execution of many .cnst files is described in section 2.1.5. 


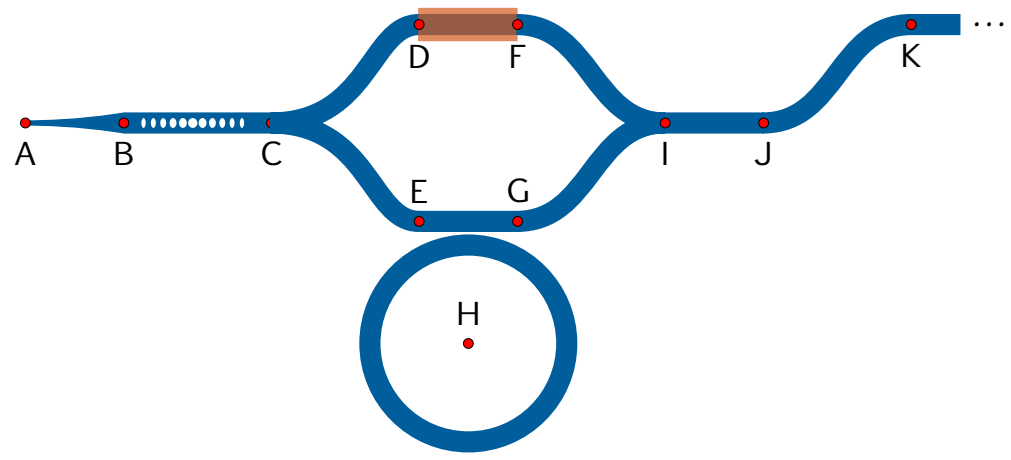

Figure 1.3: Pattern layout design schematic highlighting various available nanophotonic elements.

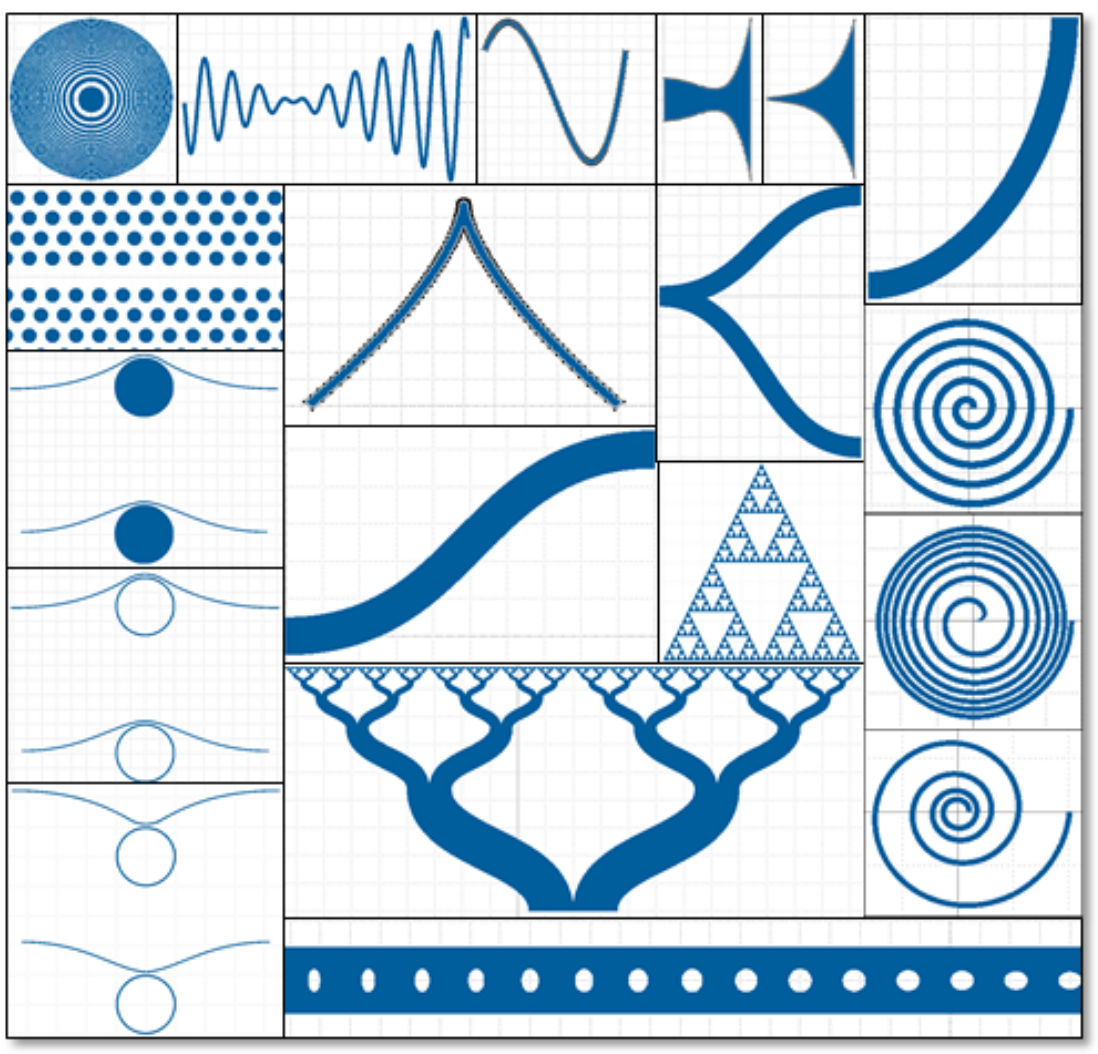

Figure 1.4: CNST Scripting example illustrating a variety of available shapes streamed directly to GDSII. 


\subsubsection{Graphical User Interface}

The main window of the graphical used interface is divided into three parts. The top title panel has the CNST logo and is static. The left panel contains a collapsible tree with options representing available toolbox features. The large right panel is the dynamic canvas displaying selected module options.

Double clicking on a module feature or single clicking on the triangle directly to the left that node expands the menu options. Consequently, all module elements within that branch node are displayed. For instance, the Basic Shapes branch has several objects including polygon-based pillar-hole arrays, arrays of tori, grating couplers, spirals and gratings. Highlighting any of the accessible feature nodes will display available options of the specified module. In case pillar-hole arrays, user defined parameters within a text field such as GDS file name, number of sides (shape vertices), GDS layer, rotation, radius, object array options (position, number of elements and pitch) along with buttons to generate a GDS file with the specified options, an about button, and an Exit button. Figure 1.5 shows a highlighted Pillar-Hole (Square/Hex) Array branch node within the left panel. The main panel shows various available module parameters within the toolbox GUl.

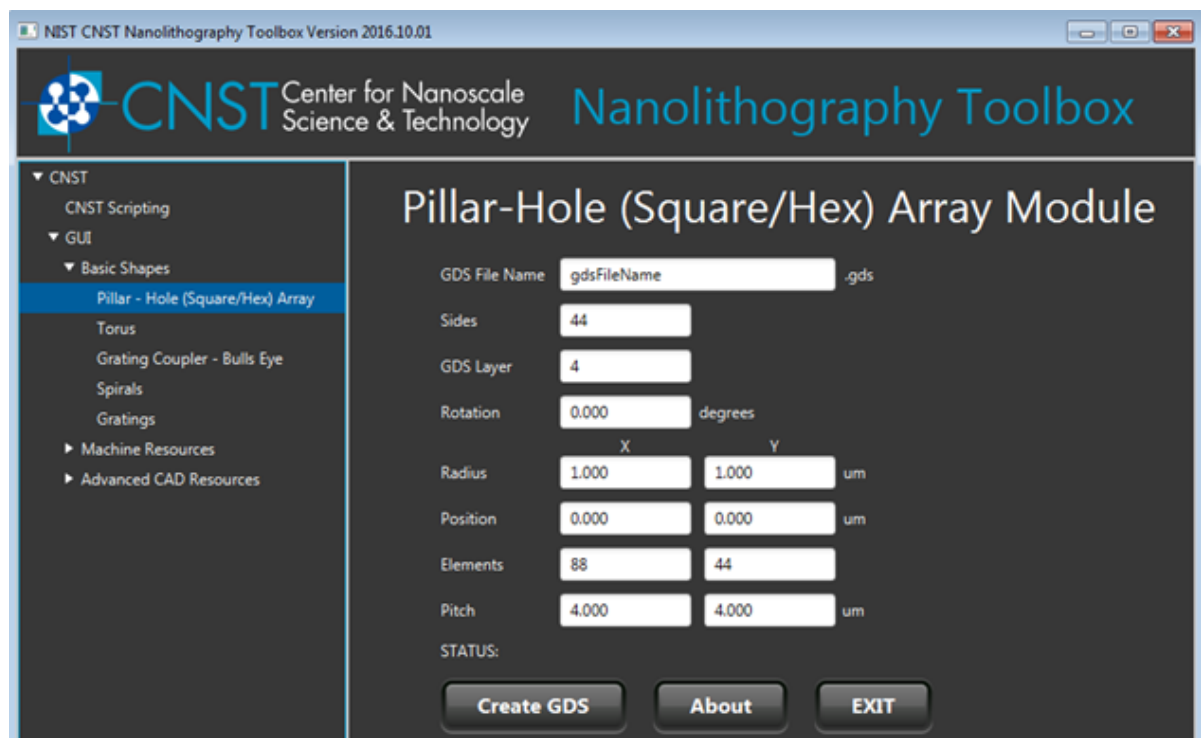

Figure 1.5: Pillar-hole array module example showing various available options within the main panel of the toolbox GUl. 


\subsection{Setup, Execution and Examples}

Scripting execution takes place either from the graphical user interface or the terminal command prompt.

\subsubsection{Quick Start Setup}

1. Change the $<$ OpenDirectory $>$ and $<$ SaveToDirectory $>$ parameters in the CNSTdefaultValues . $x m l$ setup file (see section 2.1.2).

2. Setup syntax coloring within NotePad++ (see section 2.1.8).

3. Create script files with extension .cnst. See scripting examples in section 2.1.9.6 and constructors in this chapter.

4. Run scripts either within the graphical user interface (section 2.1.3) or via command prompt (section 2.1.4). 


\subsubsection{Setup - CNSTdefaultValues.xml open and save direc- tories}

The CNST NanoLithography ToolBox requires that the CNSTdefaultValues . xml setup file resides in the same directory as the Java executable . jar file.

The first step in running and executing scripts requires the modification of the open and save directory variables within the CNSTdefaultValues. xml file. The toolbox will not function on some operating systems if these values are not properly changed.

Open the CNSTdefaultValues.xml file within a text editor and change the $<$ OpenDirectory $>$ and <SaveToDirectory $>$ parameters to a directory where . cnst script and GDS files are respectively read and saved. Figures 2.6 and 2.6 illustrate modifications for Windows and Mac/Unix/Linux platforms.

$<$ openDirectory>C: \Users \user \CNSTnanoToolBox \loadFiles $\backslash</$ OpenDirectory>

$<$ SaveToDirectory>C: \Users \user \CNSTnanoToolBox \saveFiles $</$ SaveToDirectory $>$

(a)

<OpenDirectory>/Users/user/CNSTnanoToolBox/loadFiles/</OpenDirectory>

<SaveToDirectory>/Users/user/CNSTnanoToolBox/saveFiles/</SaveToDirectory $>$

(b)

Figure 2.6: Modification to open and save directory variables within the CNSTdefaultValues. xml setup file for (a) Windows and (b) Mac/Unix/Linux platforms. 


\subsubsection{Graphical User Interface Execution}

Start the graphical user interface by either double clicking on the executable jar file or at the prompt type:

$$
\text { java -jar CNSTnanoToolboxVXXXX.jar }
$$

When the interface appears, in the left panel click CNST Scripting (step 1) and then click the Load Script button (step 2). Within the file manager locate the .cnst script file (step 3), click 0pen (step 4) and the full path of the script file will appear in the Script File: label. Type in the desired GDS file name and click the Create GDS button. Under the STATUS: label, the full path of the saved GDS file will appear.
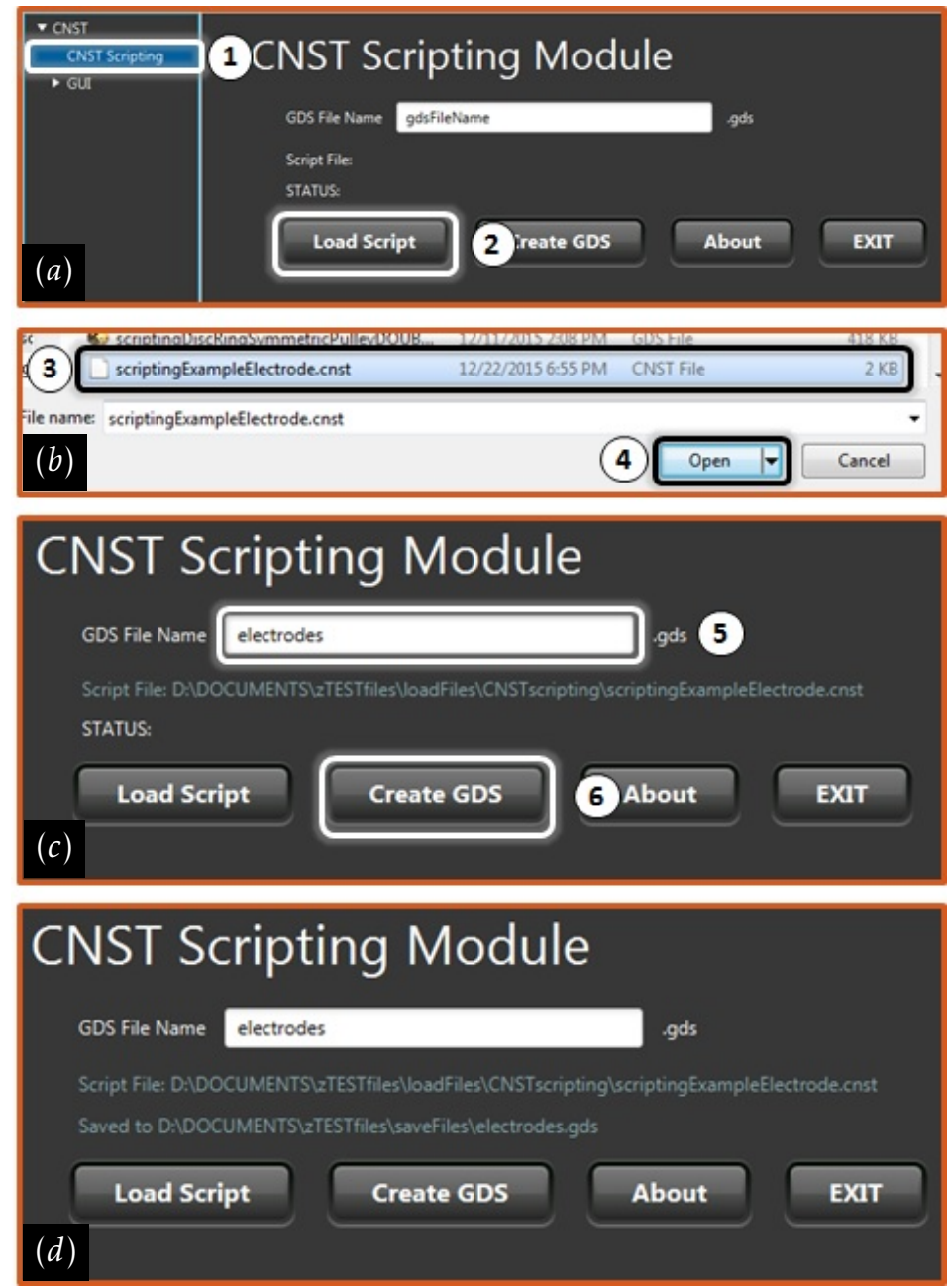

Figure 2.7: GDS generation using the graphical scripting interface. 


\subsubsection{Running CNST Scripts From a Terminal Command Prompt}

At the terminal command prompt, the command below allows script execution without the graphical user interface. Additionally, using the getfonts option allows the extraction of all available system font names to be stored into atime/date stamped ASCII file.

$$
\text { java -jar CNSTnanoToolboxVXXXX.jar [option] }
$$

[option]

cnstscripting inputFileName.cnst outputFileName.gds

Process a CNST scripting file inputFileName.cnst and cast the resulting structures into an outputFileName.gds file.

getfonts

Obtain system fonts and store them into a time/date stamped file. Useful for correctly spelling font names within various available text constructors.

\subsubsection{Running Multiple CNST Scripts - Batch File Mode}

Batch mode execution of many CNST script files is performed from a terminal command prompt. In Windows, to open the command prompt, click Start then type cmd within the 'search programs and files'. Within the Linux and Mac environments open a terminal command prompt. First create a text file where each line entry has the format defined within the previous section (2.1.4). In Windows, name the file with an extension BAT, i.e. CNSTbatchFile.BAT. For instance, the text file would have the following entries:

java -jar CNSTnanoToolboxVXXXX.jar cnstscripting scriptFile01.cnst output01.gds java -jar CNSTnanoToolboxVXXXX.jar cnstscripting scriptFile02.cnst output02.gds

java -jar CNSTnanoToolboxVXXXX.jar cnstscripting scriptFileN.cnst outputN.gds

To execute the ASCII batch file, at the windows DOS prompt type the file name:

$C: \backslash$ Users $\backslash$ robilic $\backslash$ CNSTscripting $>$ CNSTbatchFile.BAT

In Linux or Mac type dot then backslash followed by the filename:

robilic \CNSTscripting $\$>$. \CNSTbatchFile.BAT

NIST • CNST Nanolithography Toolbox v2016.09.01 • http://www.nist.gov/cnst/

page 19 of 488 


\subsubsection{Running CNST Scripts Within Matlab}

Matlab allows for execution of system commands and return outputs. Figure 2.1.6 shows how matlab generates a matlabScript.cnst file. Subsequently, using a system command, matlab runs java to create a GDS file. This was accomplished by using commands described in section 2.1.4. Lastly, another system command executes kLayout, an open source layout viewer and editor, in order to display the output GDS file. The generated file has two GDS structures. The first has a single ellipse, and the second has 100 ellipses, each cast into a different GDS layer, along a diagonal. The latter was generated using the for loop control statement.

The return status shows the command prompt output during code execution. In this case, the directory where the output GDS resides (see save directory information in section 2.1.2).

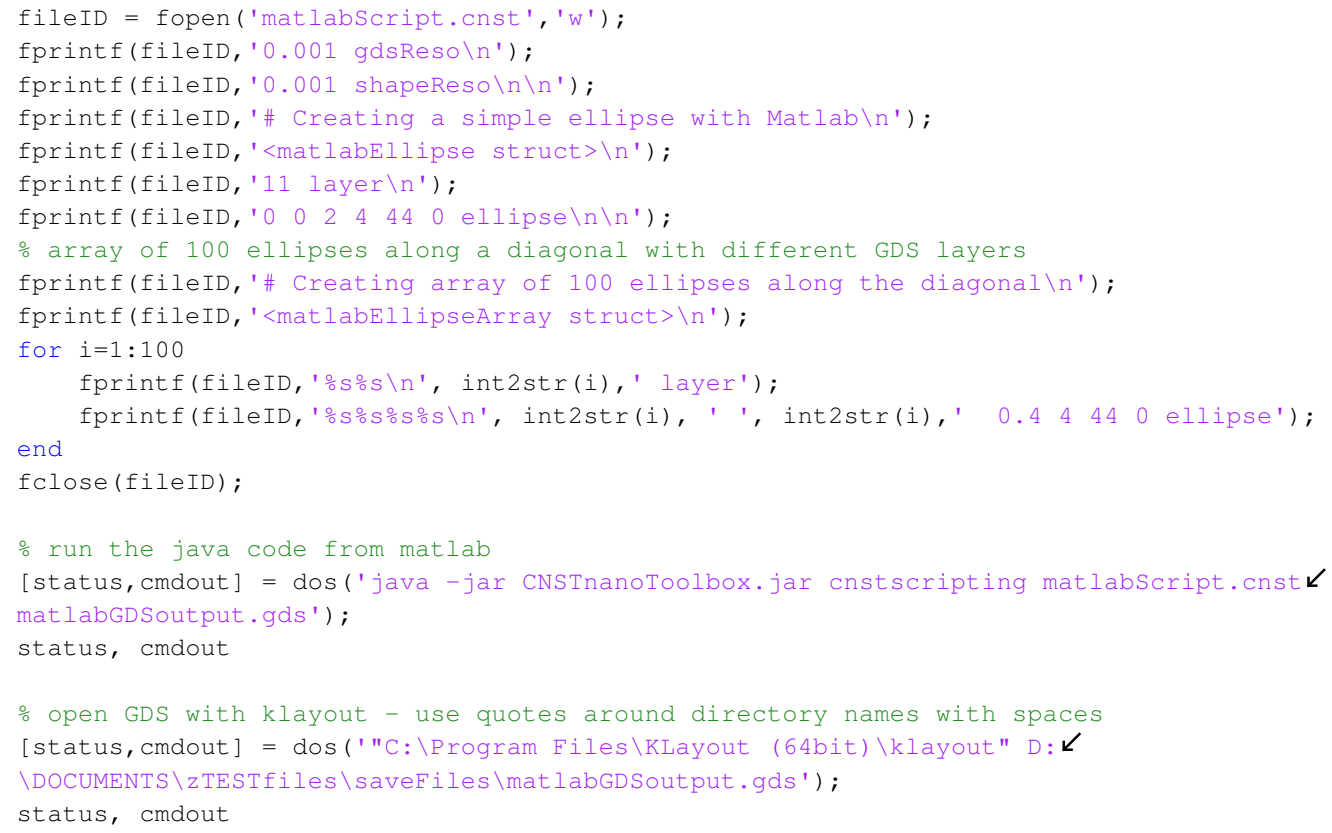

Figure 2.8: GDS generation and viewing using a Matlab script.

Example matlab files are located in \EXAMPLES \CNSTscripting \Matlab. 


\subsubsection{Python Script Generation}

Python programming can be used to create complex CNST scripting files. Programming in general allows for variable initialization, loops, Booleans, branching, control statements, conditionals and other methods to be used when algorithmically constructing script files. The program shown in figure $2.9 \mathrm{cre}-$ ates a pythonScript.cnst script file that contains two GDS structures, one with a single ellipse, and another with an array of circles along a circular path in another GDS structure. The latter is accomplished within a for loop repetition statement. Subsequently the nanolithography toolbox is used to create an output GDS file seen as an inset in figure 2.9. Example python files are located in $\backslash$ EXAMPLES $\backslash$ CNSTscripting $\backslash$ Python.

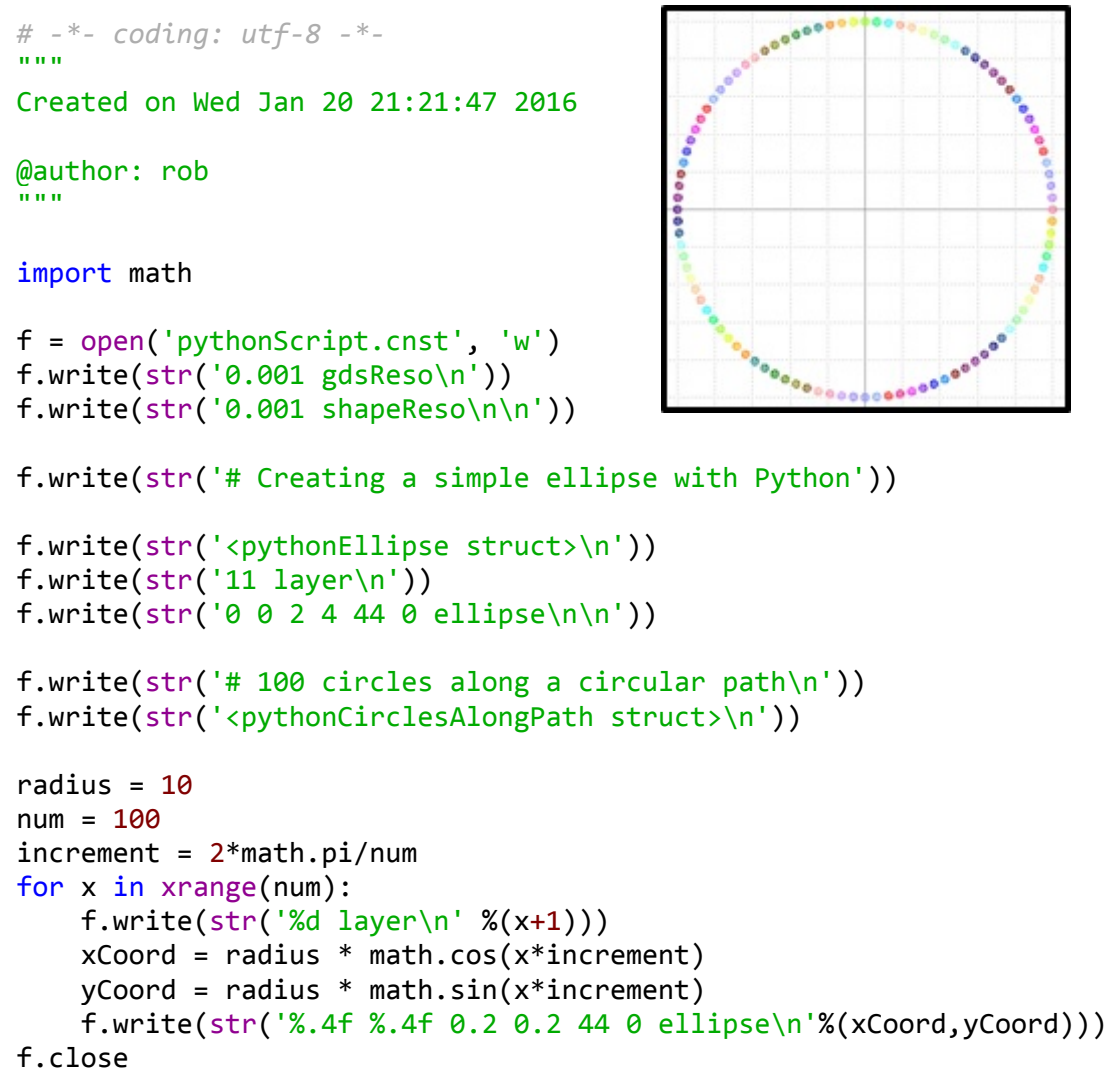

Figure 2.9: Python programming example used to generate an array of 100 circles, each within a distint GDS layer, along a circular path. Inset shows the rendered GDS output of circles along a circular path. 


\subsubsection{NotePad++ Syntax Coloring}

The package provides a language definition XML file for Notepad++. The constructor keywords listed within the XML file could be used to create a language definition file for any text editor. Syntax coloring within NotePad++ is accomplished by reading in the provided CNSTscripting.xml language definition file. Subsequently, all files with extension .cnst will have proper syntax coloring (Figure 2.10). This is useful when visualizing and debugging scripts. The following is a procedure for NotePad++ syntax coloring:

1. Language -> Define your language.... -> click Import....

2. choose $\backslash$ EXAMPLES $\backslash$ CNSTscripting $\backslash$ NotePad++XML $\backslash C N S T$ scripting.xml language definition file and click open

3. close NotePad++ and reopen a file with extension .cnst

4. Some versions may require users to repeat this procedure

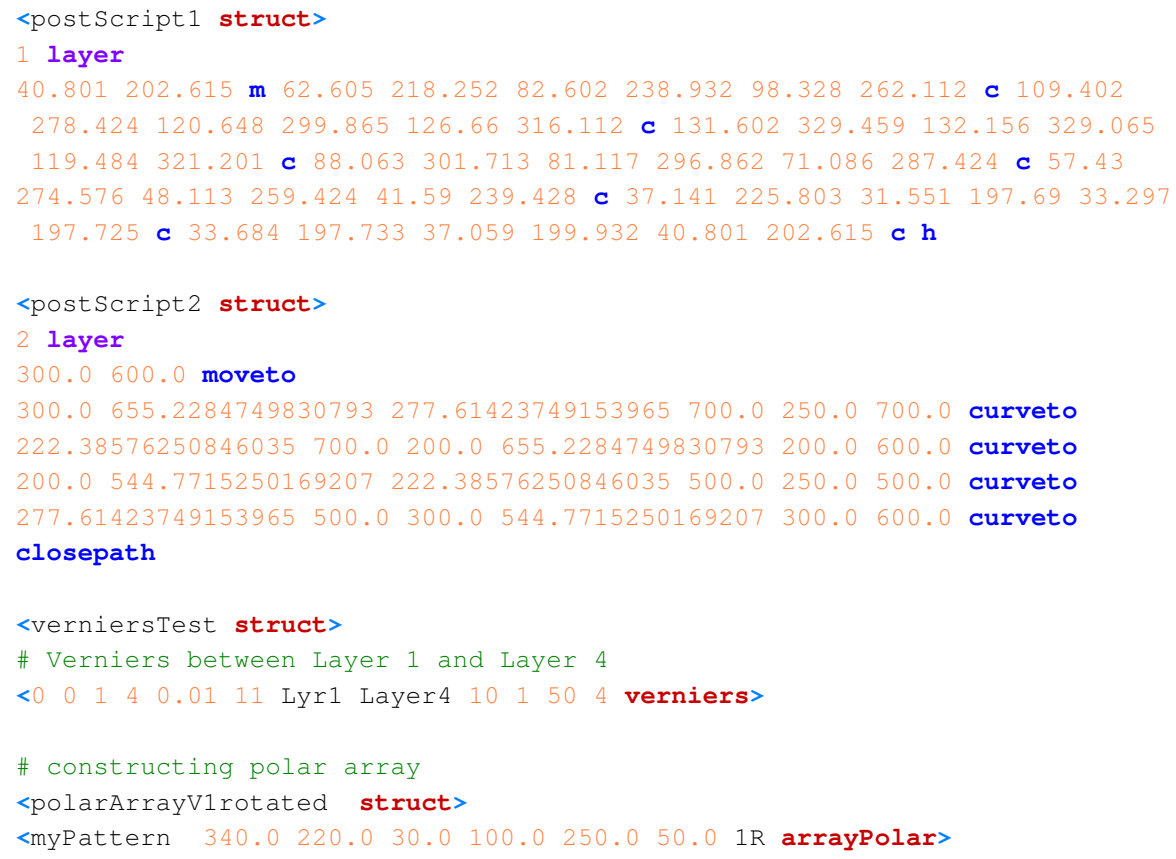

Figure 2.10: Notepad++ screenshot illustrating syntax coloring of .cnst script files defined by the CNSTscripting.xml language definition file. 


\subsubsection{Scripting Examples}

\subsubsection{Basic Scripting Example}

Figure 2.11 shows a simple script that creates two structures, one with a rectangle and the other with two ellipses. The procedure for creating script files is the following:

1. gdsReso defines the output rendering resolution (in $\mu \mathrm{m}$ ) of the GDS file. This parameter is included at the top of each file.

2. shapeReso defines the rendering resolution (in $\mu \mathrm{m}$ ) of vectorized shapes. This parameter can be placed anywhere in the file and can be changed for each vectorized shape.

\section{Create structures.}

4. Initialize layers and dataTypes, then place objects into structures. The following subsections introduce a variety of constructors for creating complex shapes.

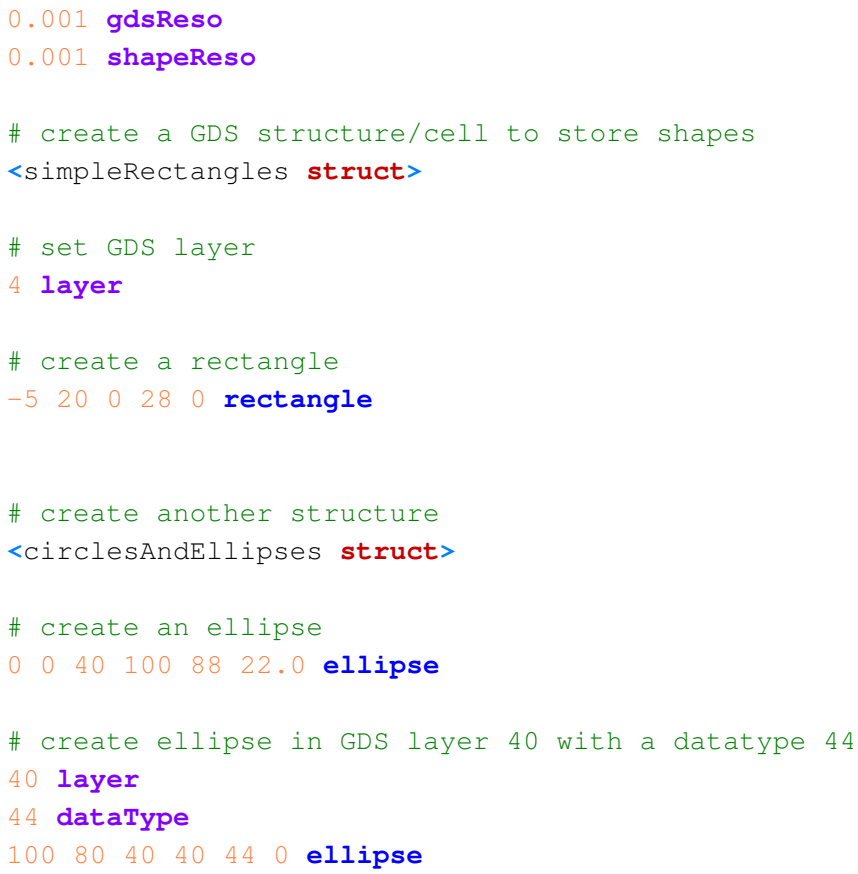

Figure 2.11: Basic scripting example (scriptingExampleBasicScriptingExample.cnst). 


\subsubsection{Scripting Example - Labeled Calibration Ruler}

The example script creates a labeled calibration ruler. Tick marks are $250 \mathrm{~nm}$ wide and $5 \mu \mathrm{m}$ long. Markers labeled 5 and 10 markers are $7.5 \mu \mathrm{m}$ and $10 \mu \mathrm{m}$ long. This type structure is useful for calibrating (optical and electron) microscopes.

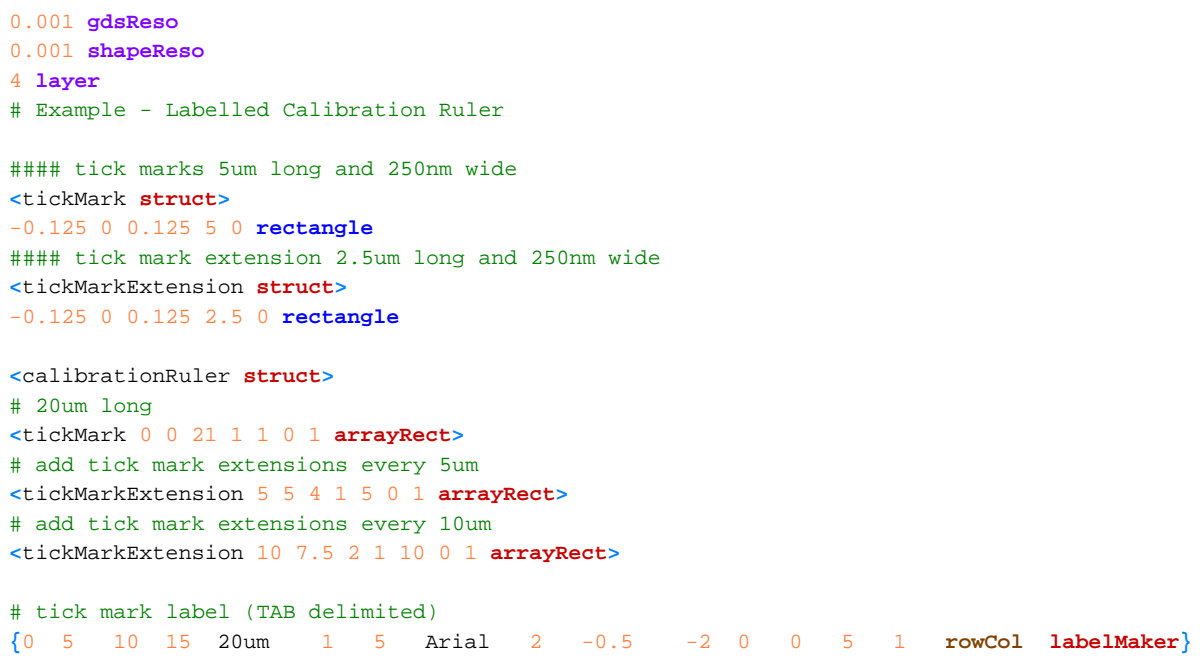

(a)

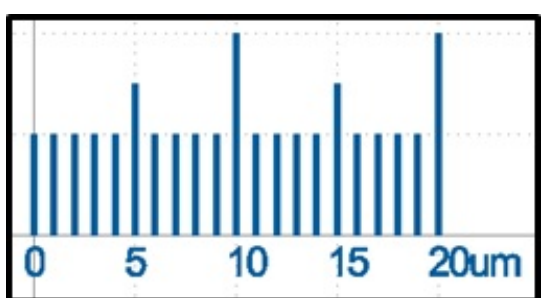

(b)

Figure 2.12: Labeled calibration ruler example. (a) Example script (scriptingExampleLabeledCalibrationRuler.cnst) illustrates the construction of (b) a labeled calibration ruler. 


\subsubsection{Labeled Electrodes}

The script initiates with a $250 \mu \mathrm{m}$ bond pad. The structure is then instantiated into a $5 \times 1$ rectangular array, Bezier curves and alignment crosses are then added. This structure (electrodeSegment) is then instantiated to construct the electrodeHalf, which is then used to form the electrodes structure. Bond pad labels are then added and electrodes structure is instantiated into top using the instanceSym constructor, ensuring symmetric extents around the origin.

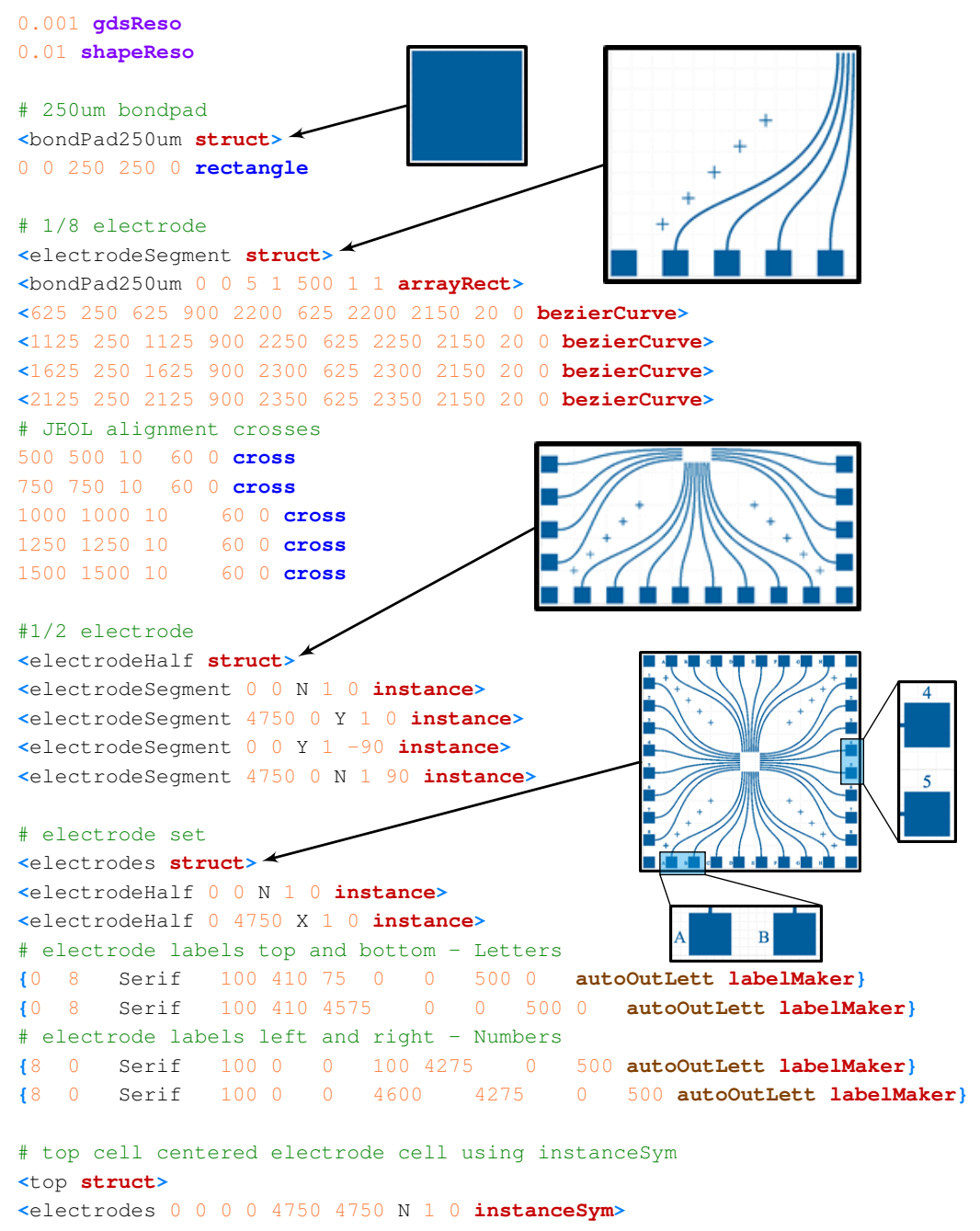

Figure 2.13: Scripting example (scriptingExampleElectrode.cnst) illustrates the construction of electrodes with labels and 5 levels of alignment crosses. 


\subsubsection{MEMS Comb Drive Flexures}

The example script below first creates a microelectromechanical systems (MEMS) flexure with two anchored pads using the flexure2E constructor. Subsequently, two linear comb drive structures are connected to the flexure element.

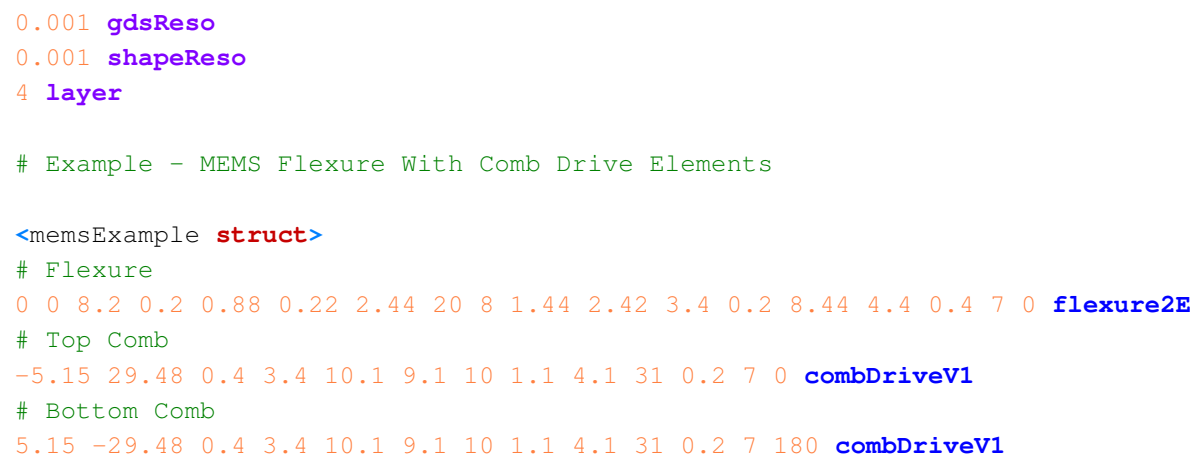

(a)

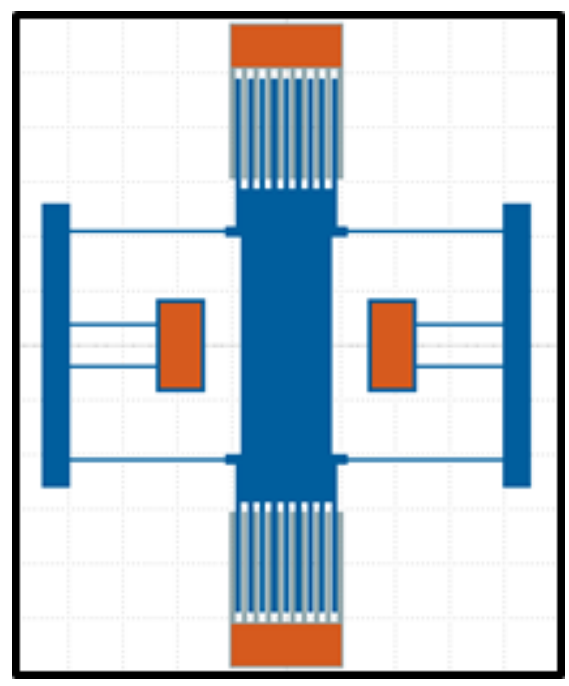

(b)

Figure 2.14: MEMS linear comb drive actuating flexure example. (a) Example script (scriptingExampleMEMSv1.cnst) illustrates the construction of (b) comb drive elements connected to a movable flexure. 


\subsubsection{MEMS Radial Comb Drive Circular Hub}

The example script below first creates four radial comb structures connected to a circular hub.

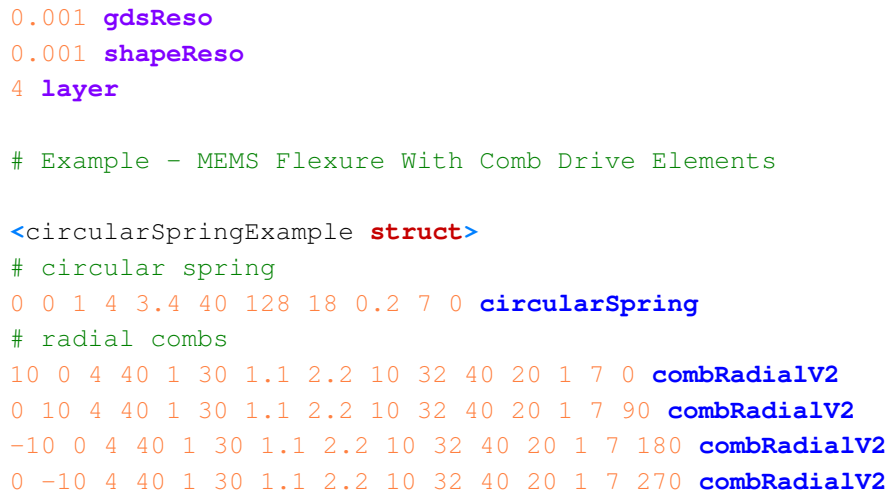

(a)

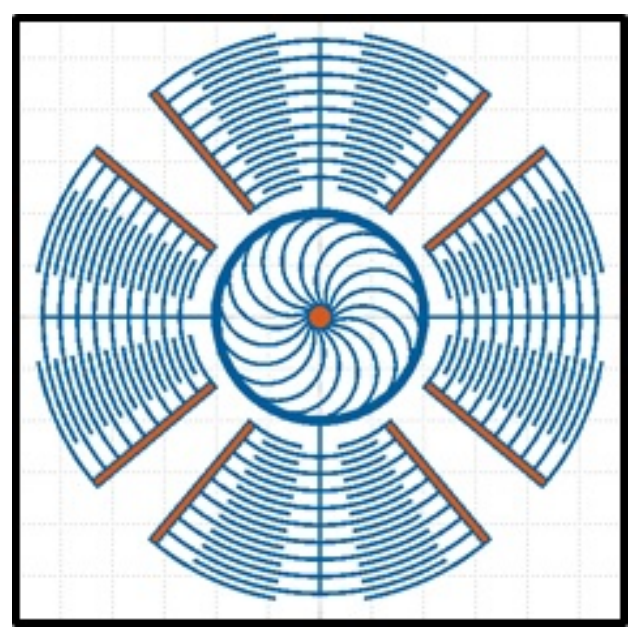

(b)

Figure 2.15: MEMS radial comb drive actuator example. (a) Example script (scriptingExampleMEMSv2.cnst) used to create (b) four radial comb drive elements connected to a circular hub. 


\subsubsection{Scripting Example Files Description}

CNST scripting offers a variety of constructor objects that allow for any conceivable shape to be rendered into a GDS output. This chapter includes a number of CNST scripting examples designed to elaborate on the functionality of these objects. Overall, the example collection covers each CNST scripting constructor. To improve the readability of the script, we included brief constructor documentation comments in each of the presented examples. The example scripts (.cnst files) and the corresponding GDS output files are contained in a folder $\backslash$ loadFiles $\backslash$ CNSTscripting. The folder also contains an Excel spreadsheet CNSTscripting. $x$ ls $x$ with data used in several presented examples. A brief description of each example script file is given below.

scriptingAlignmentFeatures.cnst contains examples of variety of multilevel preconfigured and custom user defined alignment mark strategies, verniers, and arrows. Details of these features are described in section 2.8 .

scriptingArraysAndInstancing. cnst creates a structure with the name myPattern constructed from various primitive elements, including text and spiral objects. The structure is then instantiated at a $10 \mathrm{X}$ reduced magnification. Furthermore, the structure is arranged into rectangular, polar and hexagonal arrays.

scriptingBasicShapes . cnst is a simple script demonstrating the use of primitive shapes. First, we introduce line comments. Then, resolution features of the GDS rendered output and vectorized shapes is explained. Next, we present constructors for creating GDS structures (cells) and layers. Directly following, ellipses, vectorized ellipses, tori (arcs), rectangles, rounded rectangles, polygons, star shapes, and crosses are constructed within initialized GDS structures and layers.

scriptingBezierCurve.cnst illustrates the use of the Bezier curve constructor.

scriptingBooleansGenAreasBiasTransformations . cnst is an example demonstrating extraction of layer shapes from a GDS structure and casting the resulting shapes into a generalized area object. Using these objects boolean operations, general area copies, shape biasing and affine transformation of the areas is demonstrated.

scriptingCNSTasmlContactLabelBarcodeGenerator. cnst demonstrates the use of various constructors used to generate contact lithography (label and logo) and CNST ASML PAS5500 i-line stepper reticles (reticle marks, label, barcode and logo).

scriptingCircleTorusWave . cnst example demonstrates circular (section 2.3.3) and torus (section 2.3.16) structures with sinusoidally varying boundaries.

NIST • CNST Nanolithography Toolbox v2016.09.01 • http://www.nist.gov/cnst/

page 28 of 488 
scriptingCustomTaper.cnst example employs data from the CNSTscripting. $\mathrm{xls} x$ file (CustomTaper sheet) to create a taper defined by the $(x, y)$ point pairs. Data was simply copied and pasted into the cnst script file, then terminated by $T_{x} \quad T_{y} \quad \theta_{\left(T_{x}, T_{y}\right)}$ customTaper.

scriptingDiscRingPulleysBezier . cnst(Bezier - angle defined coupling), scriptingDiscRingPulleysBezierLC.cnst (Bezier - coupling length defined) and scriptingDiscRingPulleysArc . cnst (Arcs - both angle and coupling length defined) show various examples of the disc pulley system described in sections 2.9.9.2 to 2.9.9.16.

scriptingDiscRingSymmetricPulleyDOUBLE1.cnst, scriptingDiscRingSymmetricPulleyDOUBLE2.cnst, scriptingDiscRingSymmetricPulleyDOUBLE3. cnst and scriptingDiscRingSymmetricPulleyDOUBLE4. cnst show various examples of the ring-disc systems with two coupling regions described in sections 2.9.9.2 to 2.9.9.16.

scriptingExampleBasicScriptingExample.cnst is an example of a basic script shown in section 2.1.9.1.

scriptingExampleElectrode.cnst is an example script of labeled electrodes with alignment crosses as shown in section 2.1.9.3.

scriptingExampleLabeledCalibrationRuler.cnst example script creates the labeled calibration ruler in section 2.1.9.2.

scriptingExampleMEMSv1. cnst script creates a MEMS flexure with two linear comb drive elements. Details of this scripts are included in section 2.1.9.4.

scriptingExampleMEMSv2. cnst script creates four MEMS radial comb drives connected to a circular hub as described in section 2.1.9.5.

scriptingFractals. cnst shows the use of various fractal constructors defined in section 2.7.3.

scriptingF unctionPlot. cnst shows several functional plotting examples using constructors defined in section 2.7.4.

scriptingGratingsAndGratingCoupler. cnst is an example using constructors from sections 2.9.7.1 and 2.9.7.3.

scriptingGrayscale.cnst is an example using a variety of grayscale constructors from section 2.7.5.

scriptingInstanceExamples. cnst are a collection of examples using the instance constructor section 2.4.4.

scriptingLabelMaker.cnst was used to create the GDS rendered output in Figure 2.45.

scriptingLabel0utline.cnst illustrates the use of constructors from section 2.6.4. Here, text outlines are used to generate chip labels.

NIST • CNST Nanolithography Toolbox v2016.09.01 • http://www.nist.gov/cnst/

page 29 of 488 
scriptingLogos. cnst example shows the use of the three logo constructors from section 2.6.6.

scriptingMeanderChannels.cnst illustrates various meandering channels with end reservoirs using constructors from section 2.7.8.

scriptingMEMSactuators . cnst shows various examples of MEMS actuators as described in section 2.10.1.

scriptingMEMSarraysFLAT . cnst and scriptingMEMSar raysHIERARCHY.cnst shows interacting MEMS arrays as described in section 2.10.7.

scriptingMEMScantilevers.cnst shows various examples of cantilevers of varying length extending from a base as described in section 2.10.5.

scriptingMEMSdoublyClampedBeams . cnst shows various examples of doubly clamped beams of varying length extending from a base as described in section 2.10.6.

scriptingMEMSflexures.cnst shows various MEMS accelerometer type anchored flexures with a proof mass as described in section 2.10.4.

scriptingMEMSstressStrainMeasurementStructures . cnst shows various MEMS stress-strain measurement devices including Guckel Rings (see section 2.10.8).

scriptingMISCob jects . cnst shows a variety of miscellaneous objects (interdigitated electrodes) found in section 2.7 .

scriptingMultiFile1.cnst, scriptingMultiFile2.cnst, scriptingMultiFile4 . cnst and scriptingMultiFile8. cnst are files used to generate scriptingMultiFile.gds. This is an example that calls multiple script files to generate a GDS output as seen in section 2.2.8.

scriptingPhotonicCrystalsHexArrays.cnst example demonstrates the use of photonic crystals defined in section 2.9.8.

scriptingPhotonicsCoupledWaveguideDiscTip.cnst example demonstrates the use of various coupled architectures of waveguides, discs and tips, as defined in section 2.9.15.

scriptingPhotonicsCouplersBendsMisc.cnst example demonstrates the use of various waveguides, slot waveguides and couplers defined in sections 2.9.1.1 to 2.9 .3 .15 .

scriptingPhotonicsGratingCouplersWi thWaveguides . cnst example highlights grating couplers with integrated waveguides as defined in section 2.9.7.4.

scriptingPillarHoleHexagonalSquareArrays.cnst example demonstrates the use of pillar-hole hexagonal and square arrays defined in section 2.4.6.

scriptingPoints2Instance. cnst is a script demonstrating structure instantiation at user defined $(x, y)$ point pairs. In this case a structure test is created and instantiated along a spiral path. Coordinates for the path were calcu-

NIST • CNST Nanolithography Toolbox v2016.09.01 • http://www.nist.gov/cnst/ page 30 of 488 
lated using Excel and presented in the supplementary CNSTscripting. $x$ ls $x$ file (SpiralArray sheet).

script ingPoints2 Instance. cnst script renders two user defined shapes. Both shapes are defined within the supplementary CNSTscripting. $x l s x$ file (CustomTaper and Gaussian sheets).

scriptingPolyPath.cnst script creates two distinct paths along user defined $(x, y)$ point pairs. Here, the two paths are defined within the supplementary CNSTscripting. xlsx file (CustomTaper and Gaussian sheets).

scriptingPostScript.cnst demonstrates postScript file conversion to a GDS rendered shape. Postscript statements were extracted from the supplementary file scriptingPostScript.eps using Figure 2.47 guidelines.

scriptingRaceTrack.cnst was used to create the GDS rendered output in Figure 2.131.

scriptingSpiralDelayLines.cnst describes the usage of spiral delay line constructors (section 2.9.5). The example illustrates the $S_{T}$ (number of skipped turns) parameter from the spiralDelayLineArchV2 constructor. In the example, $S_{T}$ varies from 0 to 8 while all other parameters remain constant.

scriptingSpiralDelayLinesInverse.cnst similar to the previous example, except the spirals form a slot waveguide of width $W$ defined by the exposure sleeve $W_{e}$. Example describes the inverse spiral delay line constructors (Archimedes and Fermat as defined in section 2.9.6) and illustrates the $S_{T}$ (number of skipped turns) parameter from the spiralDelayLineArchV2Inv constructor. In the example, $S_{T}$ varies from 0 to 4 while all other parameters remain constant.

scriptingSpirals.cnst demonstrates production of Archimedes, Fermat and Logarithmic spirals as defined in section 2.7.14.

scriptingText.cnst demonstrates the use of textgds constructor for creating text objects within GDS files.

scriptingText0utline.cnst example shows the use of the textOutline constructor. As shown in figure 2.44, the rendered fonts are defined by a shape outline of a user-specified width (fontOutline). This feature is useful when labeling devices defined by write-time-limited, electron beam lithography systems.

scriptingVerniers.cnst illustrates the use of verniers between two lithographic levels.

script ingWaveGui dePhCs . cnst example demonstrates the use of various photoniccrystal based waveguides defined in sections 2.9.10 to 2.9.14.

NIST • CNST Nanolithography Toolbox v2016.09.01 • http://www.nist.gov/cnst/ page 31 of 488 


\subsection{Interface Functions}

\subsubsection{Comments}

Line comments begins with \#. Comments begin with a new line. Comments cannot be appended within or at the end of a scripting command line.

\subsubsection{Creating Structures (Cells)}

The method creates a GDS structure (cell). Subsequent elements will be placed into the defined cell. If the cell already exists, then elements will be added to the existing structure. Structure names may be up to 32 characters long. Allowed structure character names are $A-Z, a-z, 0-9$, underscore (_), question mark (?) and dollar sign (\$).

$$
<\text { structureName struct }>
$$

\subsubsection{Layer}

The method defines a GDS layer for subsequent shapes. Allowed GDS layer numbers range from $0-255$. Any value outside this range will automatically be set to GDS layer 0.

$$
\text { layerNumber layer }
$$

\subsubsection{Data Type}

The method defines a GDS data-type for each layer. Allowed GDS data-type numbers range from $0-255$. Any value outside this range will automatically be set to GDS data-type 0 .

$$
\text { dataTypeNumber dataType }
$$

\subsubsection{GDS Rendering Resolution}

The method sets the snapping grid of the final GDS output. Default global value is $0.001 \mu \mathrm{m}$. Parameter $g d s R e s o l u t i o n$ is specified in units of micrometers.

$$
\text { gdsResolution gdsReso }
$$

0.001 gdsReso defines a $0.001 \mu \mathrm{m}$ rendering resolution. 


\subsubsection{Shape Resolution}

The method sets the rendering resolution resolution for subsequent shapes (primarily vector defined shapes, S-Bends, Y-Bends, postscript file conversions, Text elements, etc). Default global value is $0.001 \mu \mathrm{m}$. This value could be changed for each shape, providing flexibility on the number of points used to define a particular GDS shape. Parameter shapeResolution is specified in units of micrometers.

shapeResolution shapeReso

0.001 shapeReso defines a $0.001 \mu \mathrm{m}$ rendering resolution.

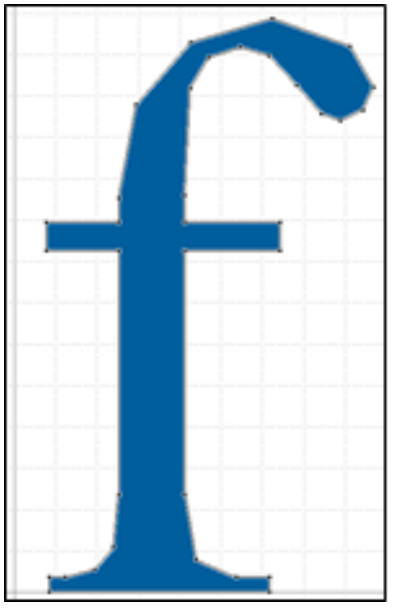

(a)

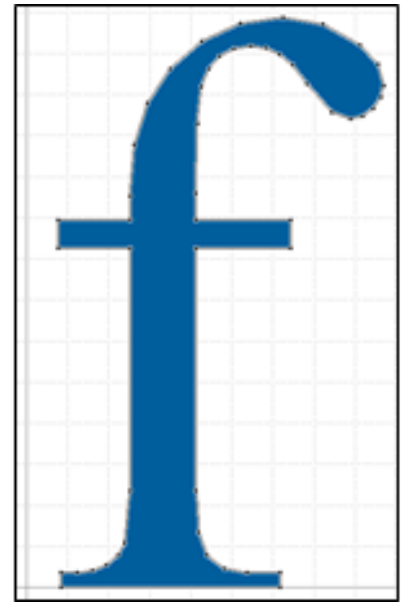

(b)

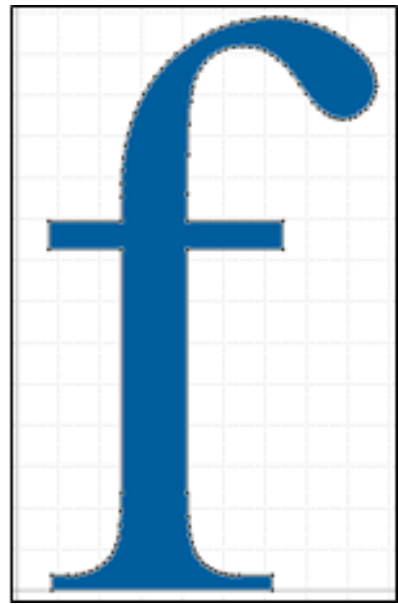

(c)

Figure 2.16: shapeReso example illustrating rendering a text object at (a) $1 \mu \mathrm{m}$ (b) $0.1 \mu \mathrm{m}$ and (c) $0.01 \mu \mathrm{m}$.

\subsubsection{Font Outline Width}

The method sets the font outline width (in micrometers). This is used for outline text and label objects (see sections 2.44 and 2.6.4). Default global value is $0.1 \mu \mathrm{m}$. This value could be changed for each outlined object

$$
\text { fontOutlineWidth fontOutline }
$$

0.400 fontOutline defines a $0.400 \mu \mathrm{m}$ font outline width. 


\subsubsection{Calls To Multiple CNST Script Files}

The fileName constructor allows script files to call other CNST script files. The called script contents (from file cnstScriptFileName) will be executed until the end of file is reached, then the execution process jumps back to the original script and continues to process shapes. Overall, this method allows users to break up the process flow over many script files.

$$
\text { <cnstScriptFileName fileName> }
$$

Script files residing in folders within the root directory can be called in the following manner:

Windows:

$$
\text { <dirName \cnstScriptFileName fileName> }
$$

Mac/Unix/Linux:

$$
<\text { dirName/cnstScriptFileName fileName> }
$$

NOTE: Directories and file names can not contain white characters such as spaces or tabs. This method assumes that cnstScriptFileName has the extension .cnst, i.e. cnstScriptFileName.cnst, hence . cnst must be omitted in the file label cnstScriptFileName. The root directory is defined by the $<$ OpenDirectory $>$ field within the CNSTdefaultValues . xml setup file. Also, backslash (Windows) and forward slash (MacOSX, Linux) characters are omitted prior to the first directory statement (dirName).

\subsubsection{Log File Time Date Stamp}

At the end of each GDS export a log file, containing a variety of information, including error messages, is created. The log filename assumes the name of the CNST script, with . log extension. During subsequent executions of a distinct script file, the log file will be overwritten. The constructor logFileTimeDate creates log files with filenames containing additional time and date information, hence each script execution will create a new log file.

NIST • CNST Nanolithography Toolbox v2016.09.01・http://www.nist.gov/cnst/

page 34 of 488 


\subsection{Shapes}

\subsubsection{Circles and Ellipses}

\subsubsection{Primitive Ellipse}

Elliptical shape is centered at $(x, y)$, defined by two radii $\left(r_{x}\right.$ and $\left.r_{y}\right)$, the number of sides $\left(N_{\text {sides }}\right)$ and rotated about the center at an angle $\theta_{(x, y)}$ expressed in degrees. In this scenario, the shape defining vertices are evenly distributed at angular increments of $2 \pi / N_{\text {sides }}$.

$$
\begin{array}{lllllll}
x & y & r_{x} & r_{y} & N_{\text {sides }} & \theta_{(x, y)} & \text { ellipse }
\end{array}
$$

\subsubsection{Vectorized Ellipse}

Elliptical shape is constructed from Bezier curves, centered at $(x, y)$, defined by two radii $\left(r_{x}\right.$ and $\left.r_{y}\right)$, and rotated about the center at an angle $\theta_{(x, y)}$ expressed in degrees. Rendering resolution of the vectorized shape (number of shape vertices) is controlled using the shapeReso parameter (see section 2.2.6). Unlike the primitive ellipse example where shape vertices are evenly distributed, since the vectorized form is constructed using Bezier curves, vertices are not regularly spaced. More vertices are allocated to regions of higher curvature.

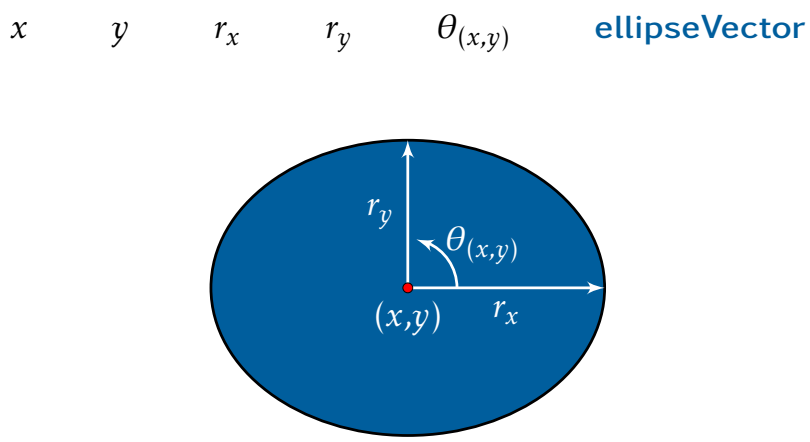

Figure 2.17: ellipse and ellipseVector constructor parameters. 


\subsubsection{Circle through three points}

The resulting shape is defined by the the three points and the number of sides $\left(N_{\text {sides }}\right)$.

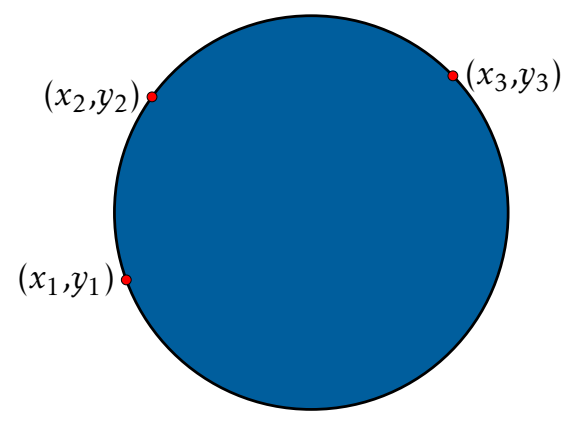

Figure 2.18: circlethree parameters.

$\begin{array}{llllllll}x_{1} & y_{1} & x_{2} & y_{2} & x_{3} & y_{3} & N_{\text {sides }} & \text { circlethree }\end{array}$




\subsubsection{Circle With a Wave Boundary}

Circular structure of radius $r$ with a sinusoidal boundary centered at $(x, y)$. A represents the sine wave amplitude, $n$ is an integer number of oscillations along the boundary extending from 0 to $2 \pi, N_{s}$ is the number of sides used to construct the boundary, and $\theta_{(x, y)}$ is the rotation about the center point.

$\begin{array}{llllllll}x & y & r & n & A & N_{s} & \theta_{(x, y)} & \text { circleWave }\end{array}$

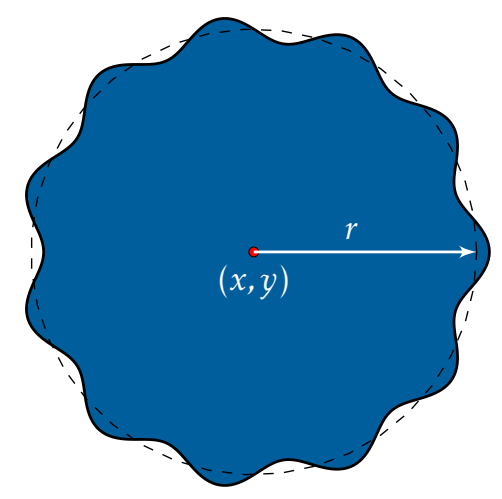

Figure 2.19: Circle with a sinusoidal boundary variation. 


\subsubsection{Cross}

Cross is defined by the center $(x, y)$, width $(W)$, length $(L)$ and rotation about the center at an angle $\theta_{(x, y)}$ expressed in degrees.

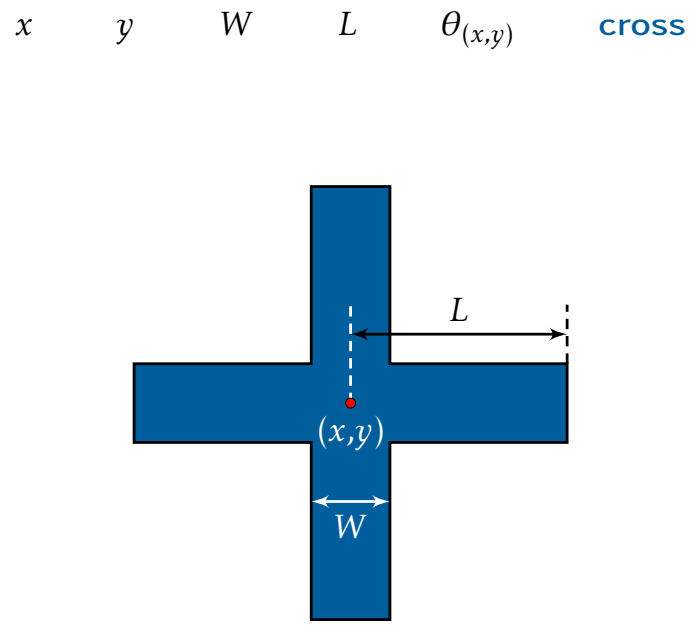

Figure 2.20: cross constructor parameters. 


\subsubsection{L-Shape}

L-shape is defined by the center $(x, y)$, widths $\left(W_{1}\right.$ and $\left.W_{2}\right)$, lengths $\left(L_{1}\right.$ and $\left.L_{2}\right)$ and rotation about the center at an angle $\theta_{(x, y)}$ expressed in degrees.

$\begin{array}{llllllll}x & y & W_{1} & L_{1} & W_{2} & L_{2} & \theta_{(x, y)} & \text { Lshape }\end{array}$

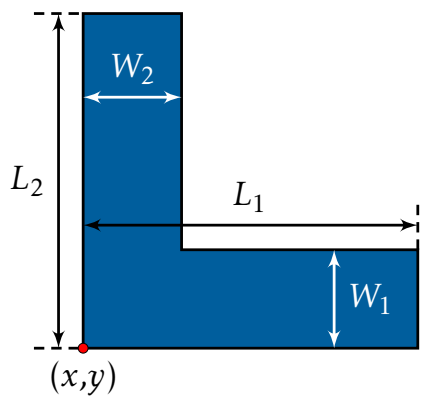

Figure 2.21: Lshape constructor parameters. 


\subsubsection{Pie Shaped Arc}

Arc shape is centered at $(x, y)$, defined by an two radii $\left(r_{x}\right.$ and $\left.r_{y}\right)$, sweep angles, the number of sides $\left(N_{\text {sides }}\right)$ and rotated about the center point by $\theta_{(x, y)}$. Sweep angles, start and end angles $\left(\theta_{s}\right.$ and $\left.\theta_{e}\right)$, and $\theta_{(x, y)}$ are expressed in degrees.

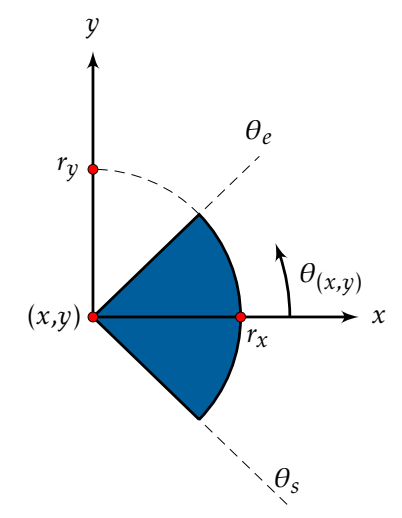

Figure 2.22: arc constructor used to create user defined pie-shaped arc sections.

$$
\begin{array}{lllllllll}
x & y & r_{x} & r_{y} & \theta_{s} & \theta_{e} & N_{\text {sides }} & \theta_{(x, y)} & \text { arc }
\end{array}
$$

\subsubsection{Pie Shaped Arc - Vector}

The vectorized pie shaped arc is constructed using Bezier curves. Rendering resolution of the defined shape is controlled using the shapeReso parameter defined in section 2.2.6.
$x$
$y \quad r_{x}$
$r_{y}$
$\theta_{s}$
$\theta_{e} \quad \theta_{(x, y)}$
arcVector

\section{Waveguide}

Figure 2.23: Illustration shows an arc section at one end of a waveguide. Rounding waveguide corners alleviates stress crowding at sharp corners. This is particularly useful when patterning thick stressed layers (resist, thin films, etc). 


\subsubsection{Polygon}

Polygon is defined by the center $(x, y)$, outer radius $(r)$, number of sides $\left(N_{\text {sides }}\right)$, and rotation about the center at an angle $\theta_{(x, y)}$ expressed in degrees. A shape is characterized by an arbitrary number of vertices to form a closed figure.

$\begin{array}{lllllll}x & y & r & N_{\text {sides }} & \theta_{(x, y)} & \text { polygon }\end{array}$

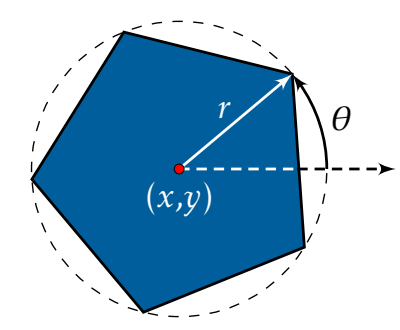

Figure 2.24: polygon constructor parameters. 


\subsubsection{Rectangle}

The shape consists of four $90^{\circ}$ corners. Three constructors are used to define the rectangular object.

$\begin{array}{cccccc}x_{L L} & y_{L L} & x_{U R} & y_{U R} & \theta_{\left(x_{L L}, y_{L L}\right)} & \text { rectangle } \\ x_{L L} & y_{L L} & L & H & \theta_{\left(x_{L L}, y_{L L}\right)} & \text { rectangleLH } \\ x_{C} & y_{C} & L & H & \theta_{\left(x_{C}, y_{C}\right)} & \text { rectangleC }\end{array}$
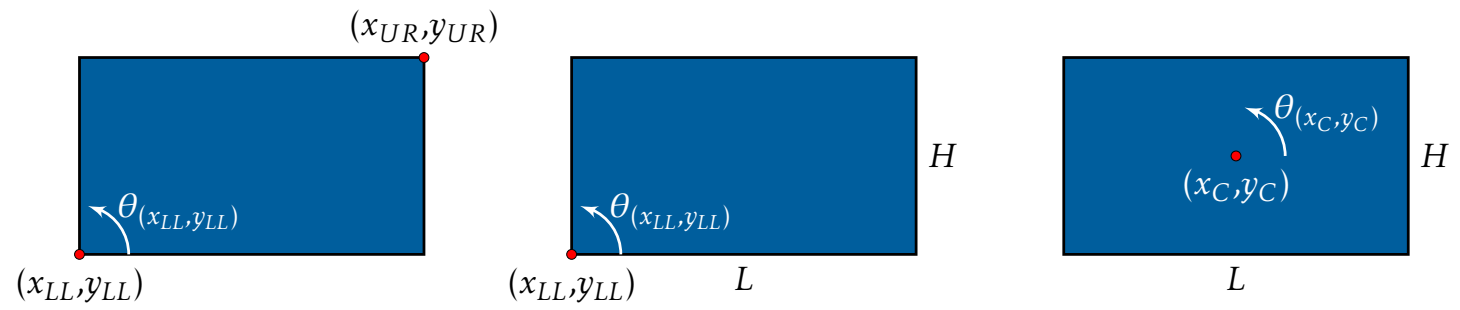

(a)

(b)

(c)

Figure 2.25: Three rectangle constructors. (a) rectangle, (b) rectangleLH, and (c) rectangleC 


\subsubsection{Rounded Rectangle}

Rounded rectangle constructor roundrect is defined using the lower left corner $\left(x_{L L}, y_{L L}\right)$, length $(L)$ and height values $(H)$. The curved sections of the rounded rectangle are defined by the two radii $\left(r_{x}\right.$ and $\left.r_{y}\right)$ and are rendered using the specified shapeReso resolution parameter (see section 2.2.6). Rotation of the rounded rectangle is about the lower left corner $\left(x_{L L}, y_{L L}\right)$. Rounded rectangles defined using roundrect $C$ are of length $L$, height $H$ and are centered at $\left(x_{C}, y_{C}\right)$.

$\begin{array}{llllllll}x_{L L} & y_{L L} & L & H & r_{x} & r_{y} & \theta_{\left(x_{L L}, y_{L L}\right)} & \text { roundrect } \\ x_{C} & y_{C} & L & H & r_{x} & r_{y} & \theta_{\left(x_{C}, y_{C}\right)} & \text { roundrectC }\end{array}$

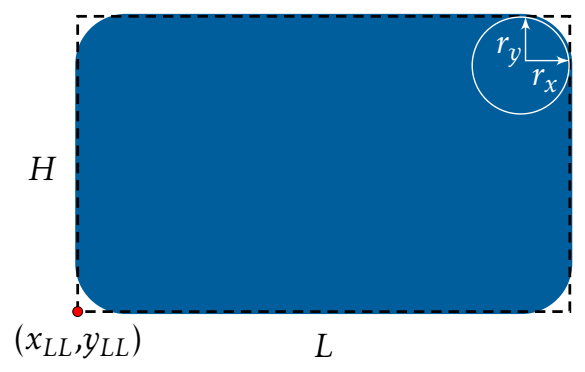

(a)

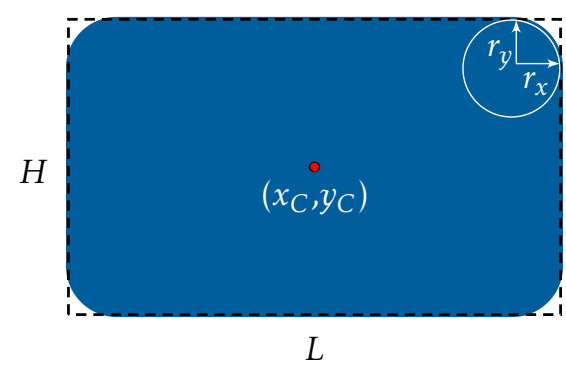

(b)

Figure 2.26: Rounded rectangle constructors defined by (a) lower left corner (roundrect) and (b) center coordinates (roundrectC). 


\subsubsection{Rectangular SU-Shape}

The below constructor creates a rectangular $\mathrm{S}$ - and $\mathrm{U}$-shape objects. Positive and negative values of the three lengths $\left(L_{i}\right)$ allow for a variety of S- and $\mathrm{U}$-shapes. These structures are useful for routing metal runners to bond pads.

$\begin{array}{llllllll}x & y & L_{1} & L_{2} & L_{3} & W & \theta_{(x, y)} & \text { rectSUshape }\end{array}$

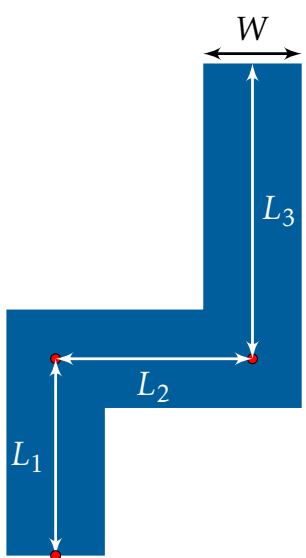

$(x, y)$

(a)

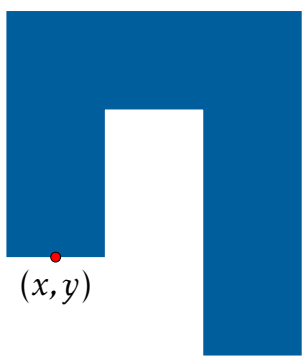

(c)

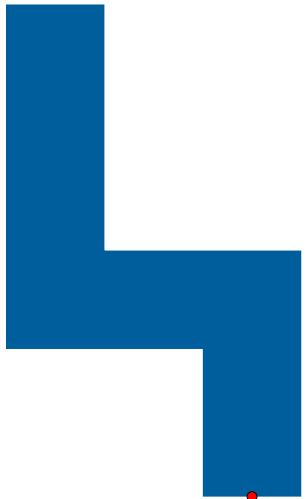

(b)

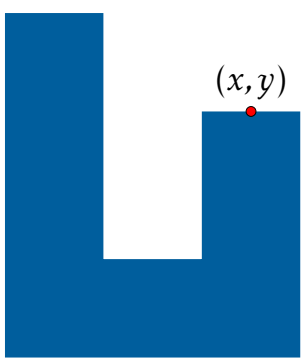

(d)

Figure 2.27: Rectangular SU-shapes constructed using various length values. (a) all lengths $L_{i}$ are positive,(b) $L_{1}, L_{3}$ positive, $L_{2}$ negative, (c) $L_{1}, L_{2}$ positive, $L_{3}$ negative and (d) $L_{1}, L_{2}$ negative, $L_{3}$ positive values. 


\subsubsection{Rectangle With a Linear Taper}

Below constructor creates a parametrized rectangle of width $w_{1}$ and length $L_{1}$ connected to a taper. Tapered region varies linearly over the taper length $L_{2}$ from a the rectangle width $w_{1}$ to the end taper width $w_{2}$.

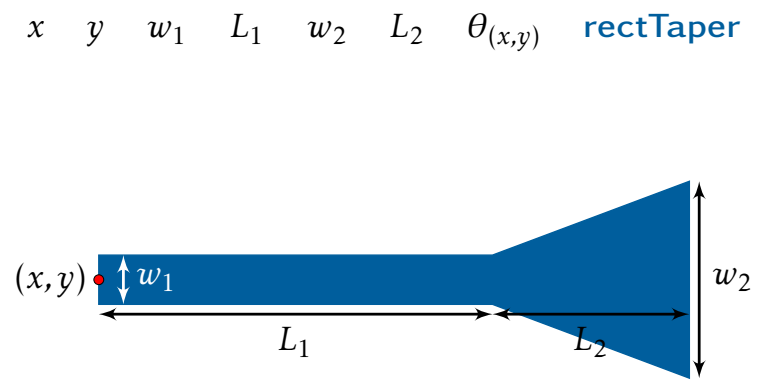

Figure 2.28: Rectangle with a taper at one end. 


\subsubsection{Star}

Star is defined by the center $(x, y)$, the two radii $\left(r_{i}\right.$ and $\left.r_{o}\right)$, number of points $\left(N_{\text {points }}\right)$, and rotation about the center at an angle $\theta_{(x, y)}$ expressed in degrees.

$\begin{array}{lllllll}x & y & r_{i} & r_{o} & N_{\text {points }} & \theta_{(x, y)} & \text { star }\end{array}$

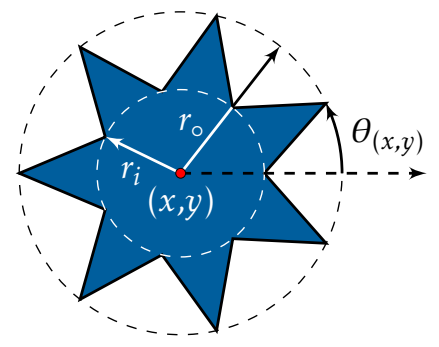

Figure 2.29: star constructor parameters. 


\subsubsection{Torus - Arc}

A shape defined by torus is centered at $(x, y)$, defined by an inner and outer radii $\left(r_{i}\right.$ and $\left.r_{o}\right)$, sweep angle, and the number of sides $\left(N_{\text {sides }}\right)$. Sweep angles, start and end angles $\left(\theta_{s}\right.$ and $\left.\theta_{e}\right)$ are expressed in degrees. The torusW constructor creates a similar shape defined by the midpoint radius $r$ and width $w$.

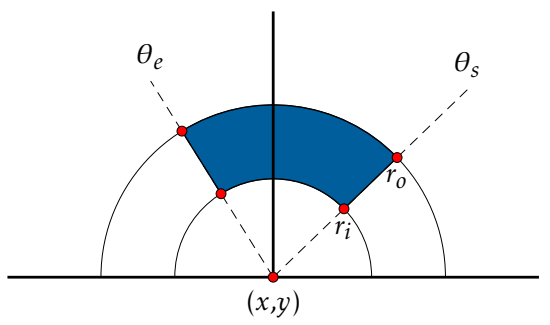

(a)

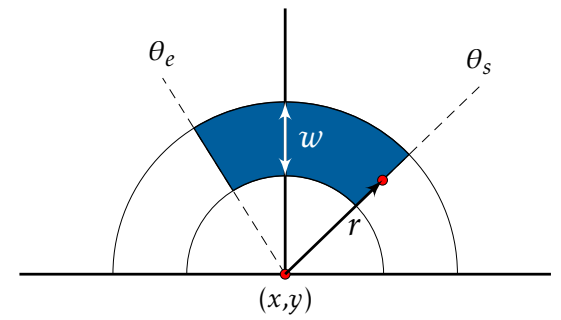

(b)

Figure 2.30: Example of arc sections defined by (a) torus and (b) torusW constructors.

$$
\begin{array}{llllllll}
x & y & r_{i} & r_{o} & \theta_{s} & \theta_{e} & N_{\text {sides }} & \text { torus } \\
x & y & r & w & \theta_{s} & \theta_{e} & N_{\text {sides }} & \text { torusW }
\end{array}
$$

\subsubsection{Torus - Vector}

The vectorized torus is centered at $(x, y)$ and defined by an inner and outer radii $\left(r_{i}\right.$ and $\left.r_{o}\right)$. The shape is constructed by subtracting two vectorized circles (vectorized ellipses with $r_{x}=r_{y}$, see section 2.3.1.2). Rendering resolution of the defined shape is controlled using the shapeReso parameter defined in section 2.2.6.

$$
\begin{array}{lllll}
x & y & r_{i} & r_{o} & \text { torusVector }
\end{array}
$$




\subsubsection{Torus With a Wave boundary}

Torus structure with a sinusoidal boundary of inner and outer radii $r_{i}$ and $r_{\circ}$. The structure is centered at $(x, y)$ with inner and outer sinusoidal boundaries in phase or $\frac{\pi}{2}$ out of phase. $A$ represents the sine wave amplitude, $n$ the number of oscillations along the inner and outer boundaries extending from 0 to $2 \pi$, $N_{s}$ is the number of sides used to construct each boundary, and $\theta_{(x, y)}$ is the rotation about the center point.

$$
\begin{array}{lllllllll}
x & y & r_{i} & r_{\circ} & n & A & N_{s} & \theta_{(x, y)} & \text { torusWaveln } \\
x & y & r_{i} & r_{\circ} & n & A & N_{s} & \theta_{(x, y)} & \text { torusWaveOut }
\end{array}
$$

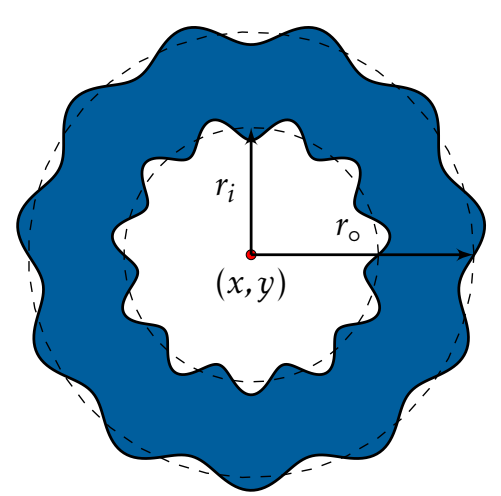

(a)

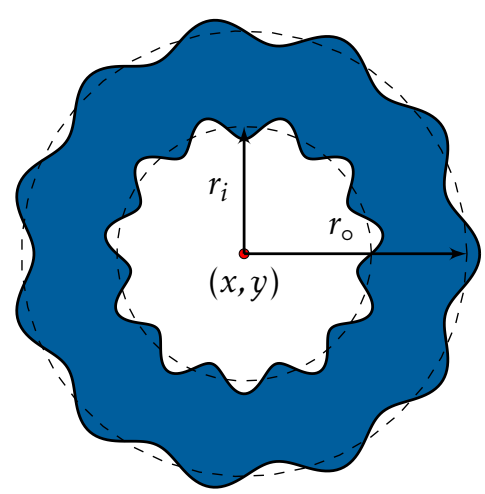

(b)

Figure 2.31: Torus wave with inner and outer radii (a) in phase (torus Waveln) and (b) $\frac{\pi}{2}$ out of phase (torusWaveOut). 


\subsection{Arrays and Instances}

\subsubsection{Rectangular Arrays}

The method instantiates and arrays a GDS structure on a periodic rectangular grid. 2 constructors are available. Both define the starting point of the array $(x, y)$ along with the number of columns $\left(N_{\text {columns }}\right)$ and rows $\left(N_{\text {rows }}\right)$. First (denoted as the 1 parameter prior arrayRect constructor) has a defined pitch of $\Delta x$ and $\Delta y$ in the $x$ and $y$ directions respectively. The second (denoted as the 2 parameter prior arrayRect constructor) is characterized by the number of elements distributed evenly between the start $(x, y)$ and end $\left(x_{e}, y_{e}\right)$ points of the array.

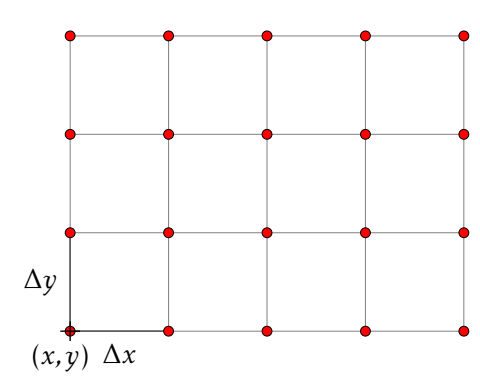

(a)

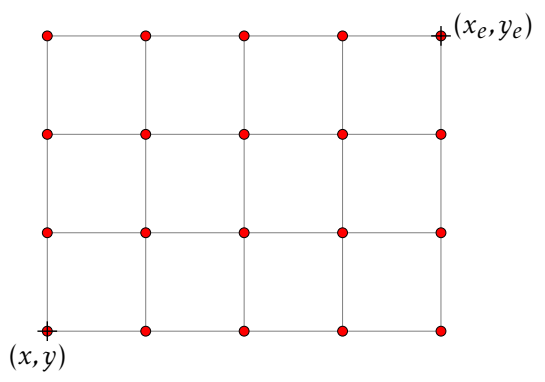

(b)

Figure 2.32: Two versions of the rectangular array constructor arrayRect (a) 1 (b) 2 .

$\begin{array}{llllllllll}\text { <structureToBeArrayed } & x & y & N_{\text {columns }} & N_{\text {rows }} & \Delta x & \Delta y & 1 & \text { arrayRect }> \\ \text { <structureToBeArrayed } & x & y & N_{\text {columns }} & N_{\text {rows }} & x_{e} & y_{e} & 2 & \text { arrayRect }>\end{array}$




\subsubsection{Hexagonal Arrays}

This method arrays GDS structures on a periodic hexagonal grid. The resulting arrayed structure consists of two rectangular arrays. Hexagonal arrays are characterized by the starting coordinate $(x, y)$, number of columns and rows ( $N_{\text {columns }}$ and $\left.N_{\text {rows }}\right)$ are specified, with previously defined structure instantiated on a hexagonal grid with spacing defined by $\Delta s$.

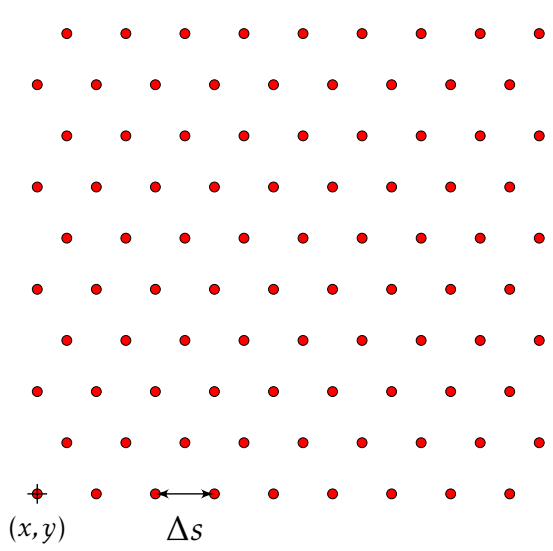

Figure 2.33: arrayHex constructor used to create hexagonal arrays.

$<$ structureToBeArrayed $x \quad y \quad N_{\text {columns }} \quad N_{\text {rows }} \quad \Delta s$ arrayHex> 


\subsubsection{Polar Arrays}

The array method instantiates GDS structures in polar coordinates. There are 2 main versions of the array polar constructor, each with two variants. Versions 1 and 1R: Start $\left(\theta_{s}\right)$ and end angles $\left(\theta_{e}\right)$, along with start $\left(r_{s}\right)$ and end $\left(r_{e}\right)$ radii, are specified, with a previously defined structure instantiated at angular and radial increments defined respectively by $\Delta \theta$ and $\Delta r$. In figure 2.34a, GDS structure named structureToBeArrayed is instantiated starting angularly at $\theta_{s}=60$ to $\theta_{e}=120$, in angular increments of 30 degrees, and radially starting at $r=2.0 \mu \mathrm{m}$ to $r=4.0 \mu \mathrm{m}$ with radial increments of $1.0 \mu \mathrm{m}$. Version $1 \mathrm{R}$ rotates each instance to point towards $r=0$.

Versions 2 and 2R: Start $\left(\theta_{s}\right)$ and end angles $\left(\theta_{e}\right)$, along with start $\left(r_{s}\right)$ and end $\left(r_{e}\right)$ radii, are specified, number of instances between the defined regions is defined by $\theta_{\#}$ and $r_{\#}$ in the angular and radial directions respectively. Region of space defined by the start and end parameters is uniformly subdivided. In figure $2.34 \mathrm{~b}$, structure structureToBeArrayed is instantiated $\theta_{\#}=5$ times starting angularly at $\theta_{s}=60$ to $\theta_{e}=120$, and radially $r_{\#}=5$ times starting at $r=2.0 \mu \mathrm{m}$ to $r=4.0 \mu \mathrm{m}$. Version 2R rotates each instance to point towards $r=0$.

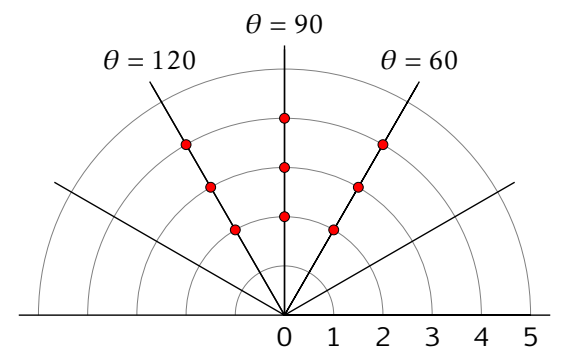

(a)

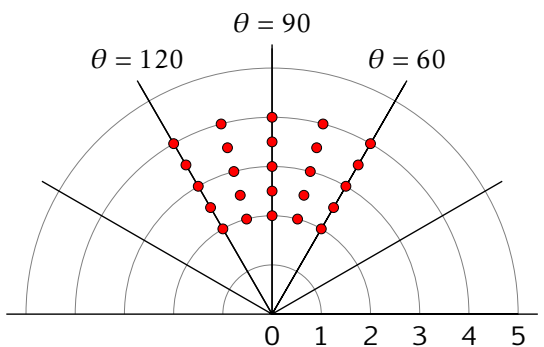

(b)

Figure 2.34: Four versions of the polar array constructor arrayPolar. (a) 1 and $1 R$, (b) 2 and $2 R$.

$\begin{array}{lccccccccc}\text { <structureToBeArrayed } & \theta_{s} & \theta_{e} & \Delta \theta & r_{s} & r_{e} & \Delta r & 1 & \text { arrayPolar> } \\ \text { <structureToBeArrayed } & \theta_{s} & \theta_{e} & \Delta \theta & r_{s} & r_{e} & \Delta r & 1 \mathrm{R} & \text { arrayPolar } \\ \text { <structureToBeArrayed } & \theta_{s} & \theta_{e} & \theta_{\#} & r_{s} & r_{e} & r_{\#} & 2 & \text { arrayPolar> } \\ \text { <structureToBeArrayed } & \theta_{s} & \theta_{e} & \theta_{\#} & r_{s} & r_{e} & r_{\#} & 2 \mathrm{R} & \text { arrayPolar }>\end{array}$




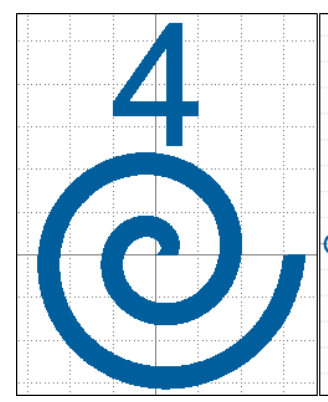

(a)

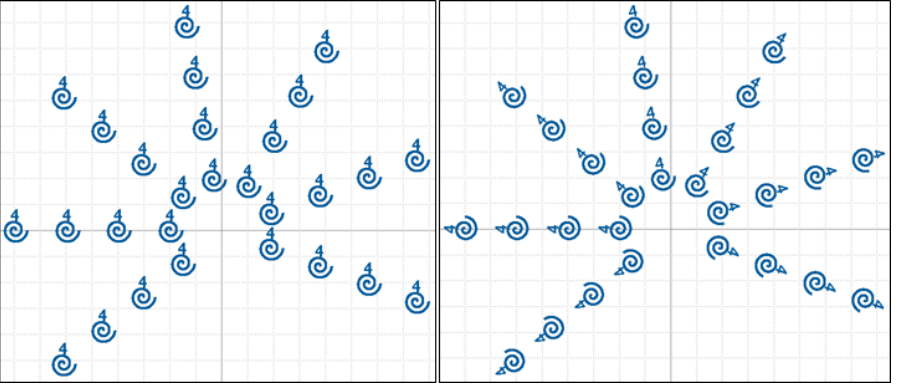

(b)

(c)

Figure 2.35: Polar Array example. (a) cell containing two objects, versions (b) 1 and (c) $1 R$ of the arrayPolar constructor.

\subsubsection{Instancing GDS Structures}

The method intantiates GDS structures at a point $(x, y)$ within other structures. The resulting instances are characterized by mirror, scaling and rotation parameters. Mirroring parameter MIR assumes values of $X$ and $Y$ for symmetric reflections around respective axes. Any other MIR values represent unmirrored structures (i.e. using a single character other than $X$ and $Y$, i.e. $N$ ). Magnification (MAG) and rotation $(\theta)$ are double values.

$$
\text { <structureToBelnstanced } x \text { y } \text { MIR MAG } \theta \text { instance> }
$$

Below constructor first symmetrically centers the structure around the origin by employing user-defined pattern boundary parameters then transforms the instantiated structure. The bounding box is defined by the lower left $\left(x_{L L}, y_{L L}\right)$ and upper right $\left(x_{U R}, y_{U R}\right)$ coordinates of the structural boundary. This method could be useful when instantiating various structures for a multi-image stepper reticle design. Transformation parameters, translation $(x, y)$, mirroring (MIR), magnification (MAG) and rotation $(\theta)$ are defined in an identical manner as for the instance constructor.

<structureToBelnstanced $x \quad y \quad x_{L L} \quad y_{L L} x_{U R} \quad y_{U R}$ MIR MAG $\theta$ instanceSym> 


\subsubsection{Points To Instance}

This method instantiates GDS structures along a list of user defined points. This methodology is accomplished within the following two step process: first, by defining the instanced structure in double brackets, then instantiating the structure at the specified coordinates, terminated by the constructor points 2 instance.

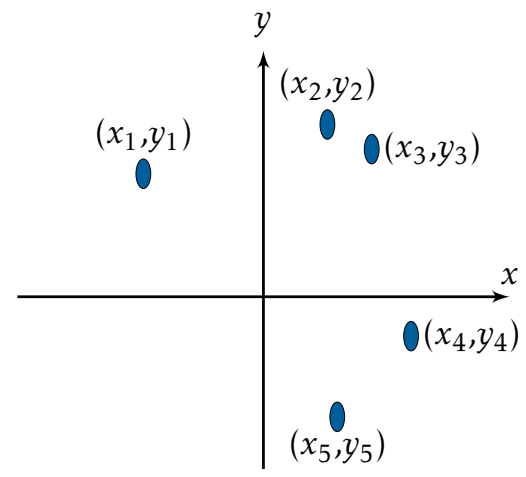

Figure 2.36: points2instance constructor used to instantiate structures (cells) at user defined $\left(x_{i}, y_{i}\right)$ positions.

$\ll$ structureToBelnstanced $\gg$

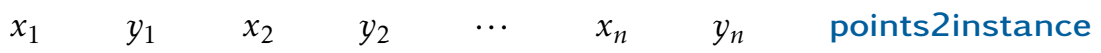

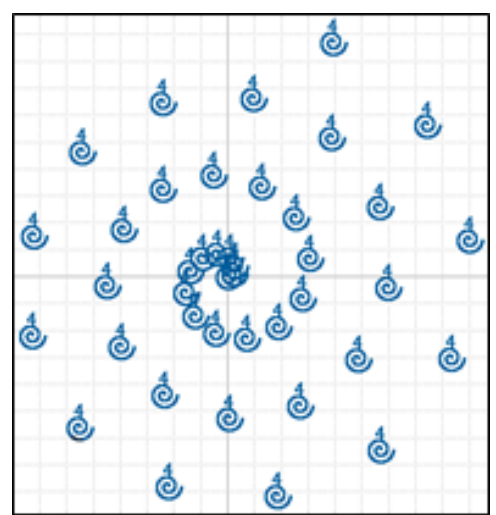

Figure 2.37: Points to instance example. Structure instanced along a path defined by a set of $(x, y)$ coordinate pairs. 


\subsubsection{Pillar-Hole Hexagonal and Square Arrays}

This method constructs hexagonal and square lattice $\left(N_{x} \times N_{y}\right)$ arrays of periodic structures. Number of vertex points $\left(N_{\text {sides }}\right)$ defines the polygon, i.e. $N_{\text {sides }}=8$ defines an octogon. Vertices of the vectorized shapes are defined by the shapeReso parameter. Vectorized array constructors are defined by the suffix V. Narrow elliptical shapes are best defined in vectorized forms. In this case, the curved section comprises a dense distribution of vertices. Each rendered shape has a rotational degree of freedom controlled by the $\theta$ parameter. Array positions are defined either by the center of the lower-left shape or by the array centroid (defined by the constructor suffix $\mathrm{C}$ ).

Hexagonal and square pillar-hole arrays:

$$
\begin{array}{lllllllllllll}
\text { <uniqueStructName } & x & y & r_{x} & r_{y} & N_{\text {sides }} & p_{x} & p_{y} & N_{x} & N_{y} & \theta & \text { sqrPillar> } \\
\text { <uniqueStructName } & x & y & r_{x} & r_{y} & N_{\text {sides }} & p_{x} & & N_{x} & N_{y} & \theta & \text { hexPillar> } \\
\text { <uniqueStructName } & x & y & r_{x} & r_{y} & N_{\text {sides }} & p_{x} & p_{y} & N_{x} & N_{y} & \theta & \text { sqrHole> } \\
\text { <uniqueStructName } & x & y & r_{x} & r_{y} & N_{\text {sides }} & p_{x} & & N_{x} & N_{y} & \theta & \text { hexHole> } \\
\text { <uniqueStructName } & x & y & r_{x} & r_{y} & N_{\text {sides }} & p_{x} & p_{y} & N_{x} & N_{y} & \theta & \text { sqrPillarC> } \\
\text { <uniqueStructName } & x & y & r_{x} & r_{y} & N_{\text {sides }} & p_{x} & N_{x} & N_{y} & \theta & \text { hexPillarC> } \\
\text { <uniqueStructName } & x & y & r_{x} & r_{y} & N_{\text {sides }} & p_{x} & p_{y} & N_{x} & N_{y} & \theta & \text { sqrHoleC> } \\
\text { <uniqueStructName } & x & y & r_{x} & r_{y} & N_{\text {sides }} & p_{x} & & N_{x} & N_{y} & \theta & \text { hexHoleC> }
\end{array}
$$

Vectorized hexagonal and square pillar-hole arrays:

$$
\begin{aligned}
& \begin{array}{llllllllllllll}
<\text { uniqueStructName } & x & y & r_{x} & r_{y} & p_{x} & p_{y} & N_{x} & N_{y} & \theta & \text { sqrPillarV }>
\end{array}
\end{aligned}
$$

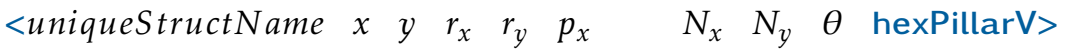

$$
\begin{aligned}
& \begin{array}{llllllllllllll}
<\text { uniqueStructName } & x & y & r_{x} & r_{y} & p_{x} & p_{y} & N_{x} & N_{y} & \theta & \text { sqrHoleV }>
\end{array}
\end{aligned}
$$

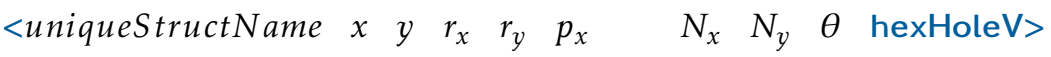

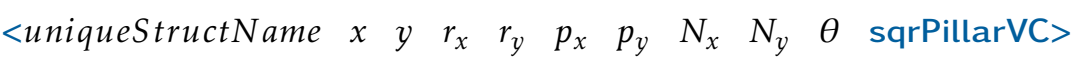

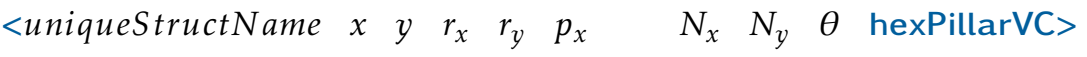

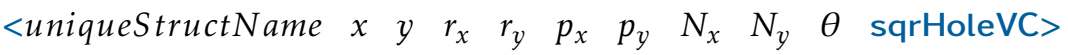

$$
\begin{aligned}
& \text { <uniqueStructName } \quad x \quad y \quad r \begin{array}{llllllll}
r_{x} & r_{y} & p_{x} & N_{x} & N_{y} & \theta & \text { hexHoleVC }
\end{array}
\end{aligned}
$$




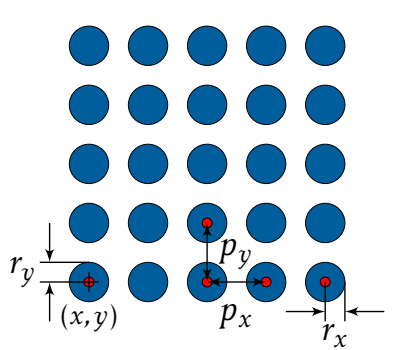

(a)

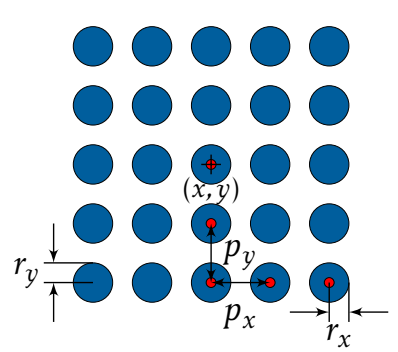

(c)

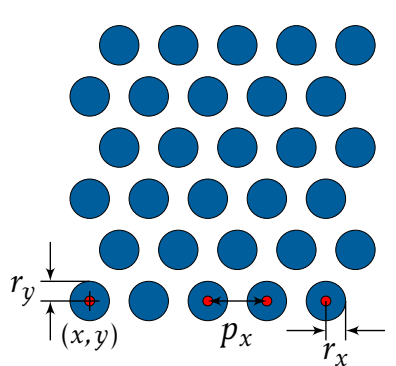

(b)

Figure 2.38: Square and hexagonal arrays of holes defined by the lower-left corner $((a)$ and $(b))$ and by the centroid $((c)$ and $(d))$.

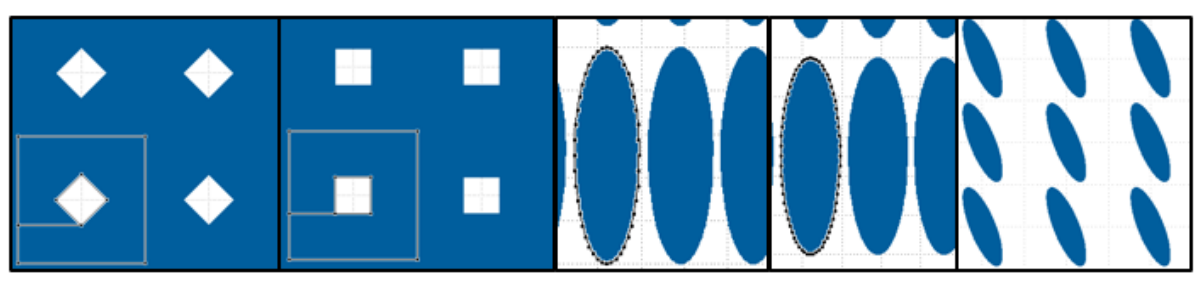

(a)

(b)

(c)

(d)

$(e)$

Figure 2.39: Various pillar-hole examples. (a) $N_{\text {sides }}=4$, (b) $N_{\text {sides }}=4$ and $\theta=45$ degrees, (c) ellipses with $N_{\text {sides }}$ defined, (d) vectorized ellipses with denser number of vertices at curved sections where shapeReso defines the rendered resolution, (e) ellipse array with $\theta=22$ degrees. 


\subsection{General Area Operations}

\subsubsection{Boolean Operations}

Constructive area geometry allows for the creation of complex shapes by means of Boolean operators between generalized areas. The processing strategy is to create objects within a variety of GDS structures. A collection of objects residing in a particular GDS Layer (extractedGDSLayer) are then extracted from an existing structure. Using the genArea constructor, the objects are then stored into a generalized area defined by a distinct string (genAreaName). This operation is repeated until two or more distinct generalized areas are created. Boolean operation between two generalized areas is then carried out in order to create desired compound geometries. The results of the Boolean operation are cast into a user defined GDS layer within an initialized GDS structure. Figure 2.40 illustrates the AND, OR, SUBTRACT and XOR operations between two general areas (A and B). Additionally, as shown in the following section (2.5.2)affine transformation could be implemented with genArea objects.

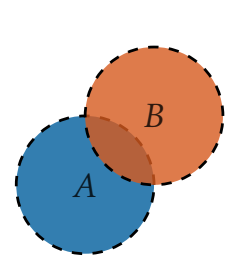

(a)

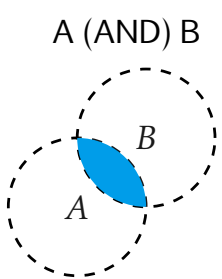

(b)

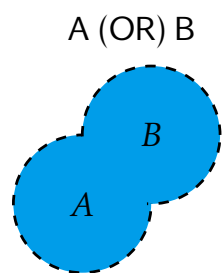

(c)

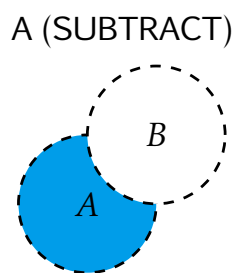

(d)

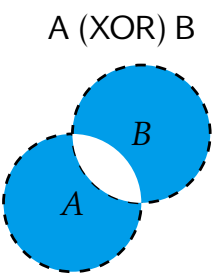

(e)

Figure 2.40: Boolean operations between two generalized area objects. (a) two general area objects $A$ and $B$, (b) $A$ (AND) B, (c) A (OR) B, (d) A (SUBTRACT) B, (e) $A(X O R) B$.

The following constructor extracts shapes residing in a GDS layer, from an existing GDS structure, and then stores the contents into a general area object. genAreaName is a string constructed form a continuous set of characters without spaces or tabs.

$$
\text { <genAreaName structName extractedGDSLayer genArea> }
$$

Boolean operations between two general area objects:

<genAreaName1 genAreaName2 resultGDSLayer OPERATION>

OPERATION constructor takes on the following values: AND, OR, SUBTRACT and XOR. Results from the operations are stored into a currently initialized GDS structure.

NIST • CNST Nanolithography Toolbox v2016.09.01 • http://www.nist.gov/cnst/

page 56 of 488 
IMPORTANT NOTES: 1) genAreaName string identifier can not contain spaces or tabs. 2) When extracting large instantiated GDS arrays, the genArea constructor flattens the data (i.e. the GDS structure hierarchy is not preserved). Similar to Boolean operations, the procedure is computationally intensive when large instantiated arrays are used. Consequently, these operations could take a considerable amount of time. For large periodic arrays, built using the instance constructor, the above process is considerably faster when operations are performed on a smaller instantiated GDS structure. This process circumvents a host of dillemas and preserves the hierarchy of the arrayed GDS structure. 3) Processing large, clear-field arrayed patterns could lead, under certain operations, to shapes with a large number of vertices. Some commercial packages have an upper vertex limit per GDS shape.

\section{Boolean SUBTRACT example:}

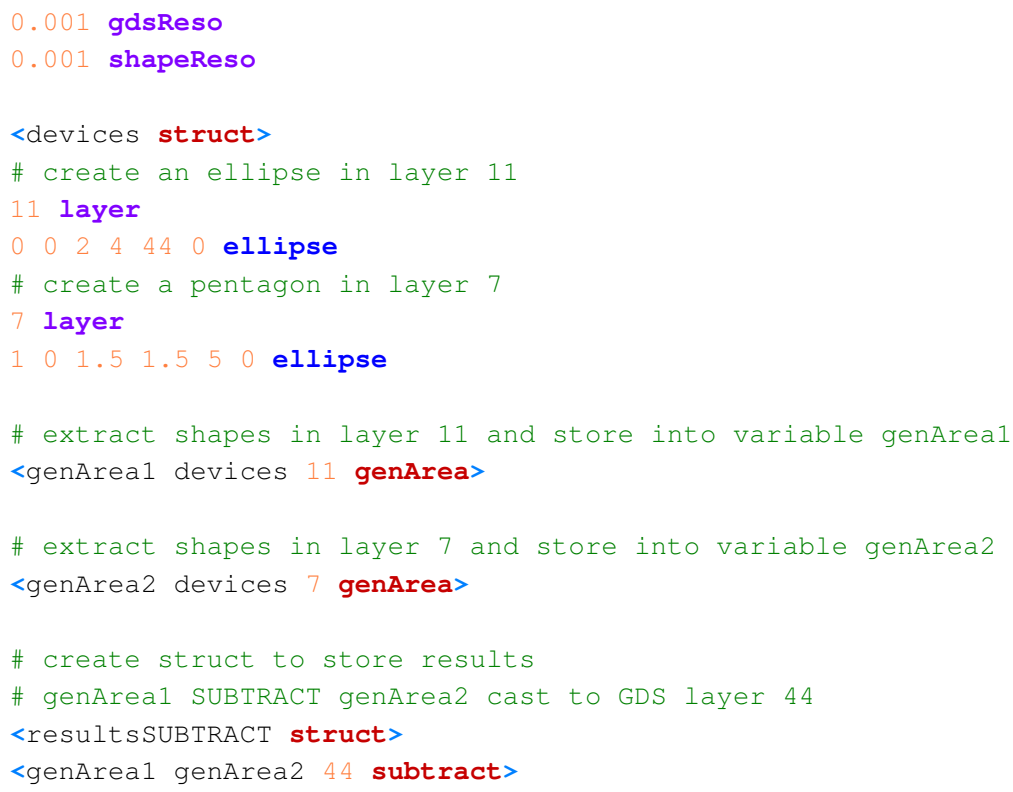

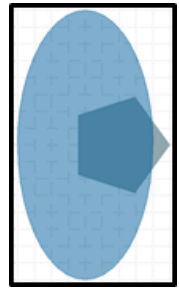

(b)

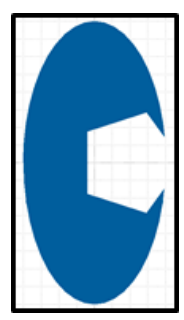

(c)

Figure 2.41: Boolean SUBTRACT operation between an ellipse and a pentagon. (a) CNST script, (b) ellipse and a pentagon GDS shapes, (c) resulting GDS shape following a Boolean subtraction between an ellipse and a pentagon. 


\subsubsection{General Area Copies, Shape Bias and Affine Transfor- mations}

This module copies general areas into a currently initialized GDS structure. The general areas are extracted using the genArea constructor. The objects then undergo affine transformations (translation, mirroring, scaling, rotation and shape bias). Resulting areas are then cast into a GDS layer and copied into a currently initialized GDS structure. The utility of this option is manifested in myriad forms, especially when casting multiple stepper images onto a single reticle from a multi-layer GDS structure design. Also, unlike instantiation, where layer properties are inherited from the parent structure, this module allows for users to define GDS layer numbers for each general area copy.

Variable genAreaName defines the areas extracted via genArea, $x$ and $y$ are translation directions in micrometers, MIRROR parameter is used to horizontally (around $y$-axis) or vertically (around x-axis) flip (mirror) the areas, MAG defines the pattern scaling, pattern rotation is defined by $\theta$, and resultGDSLayer defines the GDS layer of the resulting general area shapes. MIRROR parameter assumes values of $X$ and $Y$ for symmetric reflections around respective axes. Any other MIRROR values represent unmirrored structures (i.e. using a single character other than $X$ and $Y$, i.e. N). MAG is a positive double value indicating area magnification. MAG $=1$ omits area scaling. Rotation defined by the parameter $\theta$ is expressed in degrees

BIAS parameter defines the amount of shape perimeter expansion (positive bias) or contraction (negative bias) in micrometers (see Figure 2.42).

The module supplies the following two constructors:

genAreaCopy - translates objects relative to the structural coordinates of the general area objects.

genAreaCopyC - first centers the object around the origin, then performs affine transformations, i.e. translation is with respect to to $(0,0)$.

<genAreaName $x$ y MIRROR MAG $\theta$ BIAS resultGDSLayer genAreaCopy $>$
<genAreaName x y MIRROR MAG $\theta$ BIAS resultGDSLayer genAreaCopyC $>$

These constructors copy a defined general area (flattened area) into an initialized GDS structure. Unlike struct instantiation, the process of copying areas does not retain hierarchy. Consequently the process results in larger files (see Important Notes comment in previous section 2.5.1).

NIST • CNST Nanolithography Toolbox v2016.09.01 • http://www.nist.gov/cnst/

page 58 of 488 


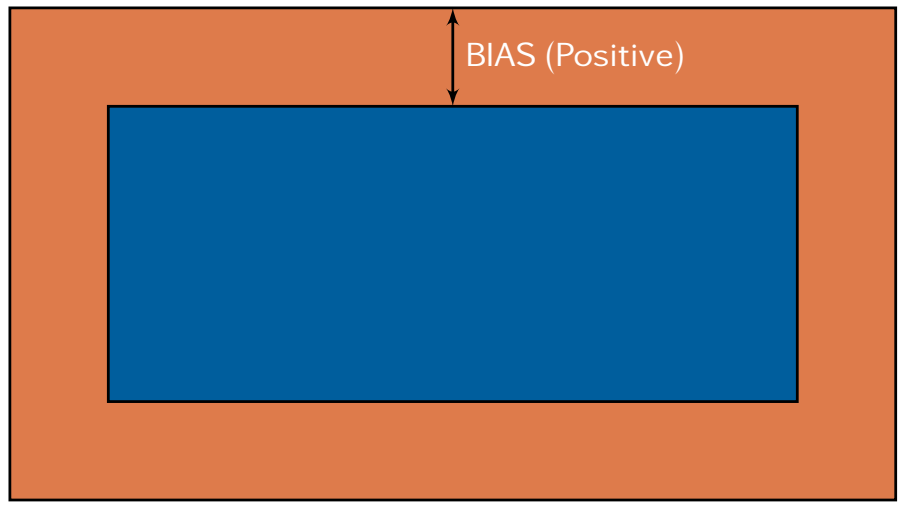

(a)

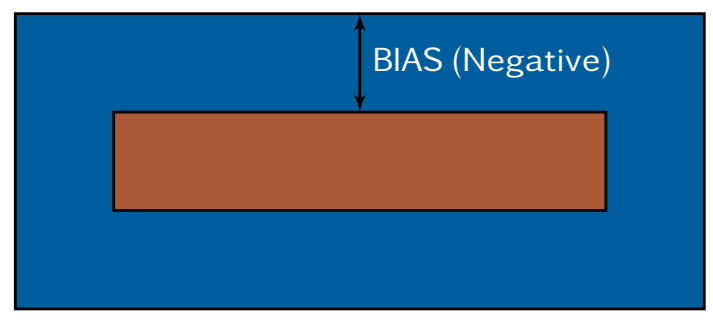

(b)

Figure 2.42: Shape biasing (a) expanding and (b) contracting an area using a positive and negative bias. 


\subsection{Text Labels, PostScript and Logos}

\subsubsection{Text}

Text shape is characterized by a string of characters using vector based system fonts. Text shapes are directly placed within the active GDS structure. There are two constructors for text shapes, where placement is either at the lower left corner (textgds) or at center (textgdsC) of the text string. Interface function parameter shapeReso defines the rendering resolution of the text. Parameter some T ext represents all printable characters. If the fontName does not exist, "Serif" will be used and a comment will be placed within the error log file. If "Serif" does not exist, then first encountered font within the system font list will be used. $(x, y)$ values are in micrometers. Font size is specified by conventional fontmetrics definitions, measured from the descender to the ascender line in micrometers.

$$
\begin{aligned}
& <\{\{\text { someText }\}\} \quad\{\{\text { fontName }\}\} \text { fontSize } x y \text { textgds }> \\
& <\{\{\text { someText }\}\} \quad\{\{\text { fontName }\}\} \text { fontSize } x y \text { textgdsC }>
\end{aligned}
$$

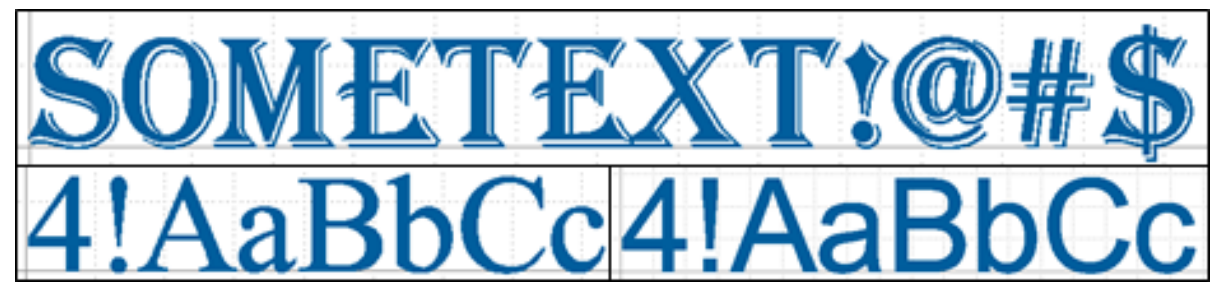

Figure 2.43: GDS rendered textgds example.

The following are scripts used to generate the text in Figure 2.43:

$$
\begin{array}{llllll}
<\{\{\text { SomeText!@\#\$\}\} } & \{\{\text { Algerian }\}\} & 20 & 0 & 0 & \text { textgds }> \\
<\{\{4 ! A a B b C c\}\} & \{\{\text { Serif }\}\} & 20 & 0 & 0 & \text { textgds }> \\
<\{\{4 ! A a B b C c\}\} & \{\{\text { Arial }\}\} & 20 & 0 & 0 & \text { textgds }>
\end{array}
$$

NIST • CNST Nanolithography Toolbox v2016.09.01・http://www.nist.gov/cnst/

page 60 of 488 


\subsubsection{Text Outline}

Text outline shapes are useful when lithographic write times (e.g. electron beam lithography) are of consideration. In a similar manner to Text shape in the previous section, the outline shape is characterized a string of text, font name and size, width of the outline and position $(x, y)$. There are two constructors for text shapes, where placement is either at the lower left corner (textOutline) or at center (textOutlineC) of the text string. Interface function parameter shapeReso defines the rendering resolution of the text. If the fontName does not exist, "Serif" will be used and a comment will be placed within the error log file. If "Serif" does not exist, then first encountered font within the system font list will be used. $(x, y)$ values are in micrometers. Font size is specified by conventional fontmetrics definitions, measured from the descender to the ascender line in micrometers. Outline width is specified using the fontOutline parameter (see section 2.2.7).

$$
\begin{aligned}
& <\{\{\text { someText }\}\} \quad\{\{\text { fontName }\}\} \text { fontSize } x \text { y textOutline }> \\
& <\{\{\text { someText }\}\} \quad\{\{\text { fontName }\}\} \text { fontSize } x \quad y \text { textOutlineC }>
\end{aligned}
$$

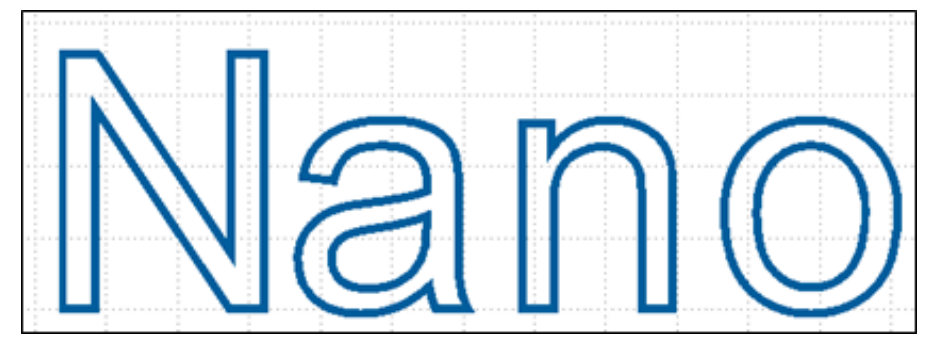

Figure 2.44: Text Outline GDS example.

IMPORTANT NOTE: Within this object, errors dealing with exceeding maximum number of GDS vertices could be encountered. To circumvent this dilemma, when using larger letters, increase the shapeReso parameter. 


\subsubsection{Label Maker}

Label maker has 6 available constructors. 4 constructors automatically generate labels based on the number of rows and columns. Chip labels are either numbers (autoOuter and autoRowColumn)or a combination of numbers and letters (autoOuterLetters and autoRowColumnLetters). 2 remaining constructors generate custom, user-defined, labels (outer and rowColumn). Each constructor pair has an outer and row-column option. Label placement for the outer option is along the top and left side of the chip array. Top side placement starts at $(x, y)$ and the left hand side placement initiates at $\left(x_{r}, y_{r}\right)$. Label placement for the row-column option initiates with the top-left label at a position $(x, y)$. In both cases number of rows $\left(N_{\text {row }}\right)$ and columns $\left(N_{\text {col }}\right)$, font name (fontName), font size (fontSize in $\mu \mathrm{m})$, and the pitch along the two directions $\left(\Delta_{x}\right.$ and $\Delta_{y}$ ) are specified. Similarly, custom labels are defined by an additional set of label parameters $\left(L_{1}, L_{2} \ldots L_{n}\right)$. In all cases, if the font name is not contained within the system font list, label maker will default to a Serif font.

4 auto labels:

$\left\{N_{\text {row }} N_{\text {col }}\right.$ fontName fontSize $x \quad y \quad x_{r} y_{r} \Delta_{x} \Delta_{y}$ autoOut labelMaker $\}$

$\left\{N_{\text {row }} N_{\text {col }}\right.$ fontName fontSize $x$ y $x_{r} y_{r} \Delta_{x} \Delta_{y}$ autoRowCol labelMaker $\}$

$\left\{N_{\text {row }} N_{\text {col }}\right.$ fontName fontSize $x \quad y \quad x_{r} y_{r} \Delta_{x} \Delta_{y}$ autoOutLett labelMaker $\}$

$\left\{N_{\text {row }} N_{\text {col }}\right.$ fontName fontSize $x \quad y \quad x_{r} y_{r} \Delta_{x} \Delta_{y}$ autoRowColLett labelMaker $\}$

2 custom labels where parameters are TAB SEPARATED:

$\left\{\begin{array}{llllllllllllll}L_{1} & L_{2} & \ldots & L_{n} & N_{\text {row }} & N_{\text {col }} \text { fontName fontSize } & x & y & x_{r} & y_{r} & \Delta_{x} & \Delta_{y} & \text { out } & \text { labelMaker }\}\end{array}\right.$

$\left\{\begin{array}{llllllllllll}L_{1} & L_{2} & \ldots & L_{n} & N_{\text {row }} & N_{\text {col }} \text { fontName fontSize } x & y & x_{r} & y_{r} & \Delta_{x} & \Delta_{y} & \text { rowCol labelMaker }\end{array}\right.$

IMPORTANT NOTES: 1) Labels $L_{1} \ldots L_{n}$ are constructed from any printable ASCll character, including the space character. Therefore, space separation between constructor parameters is NOT allowed. All specified parameters within above constructors must be TAB separated. 2) Carriage returns cannot be used within the script line constructor.

NIST • CNST Nanolithography Toolbox v2016.09.01 • http://www.nist.gov/cnst/

page 62 of 488 


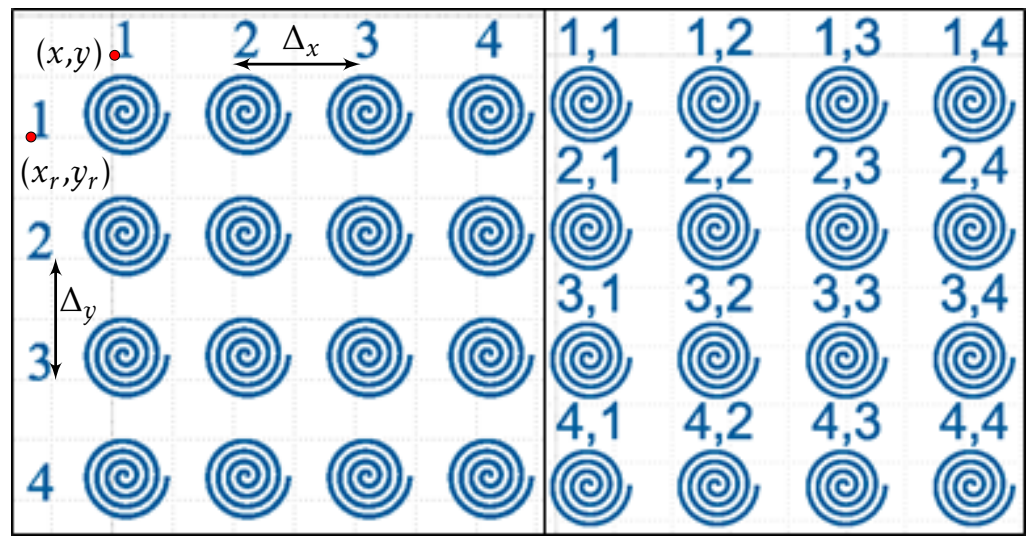

(a) (b)

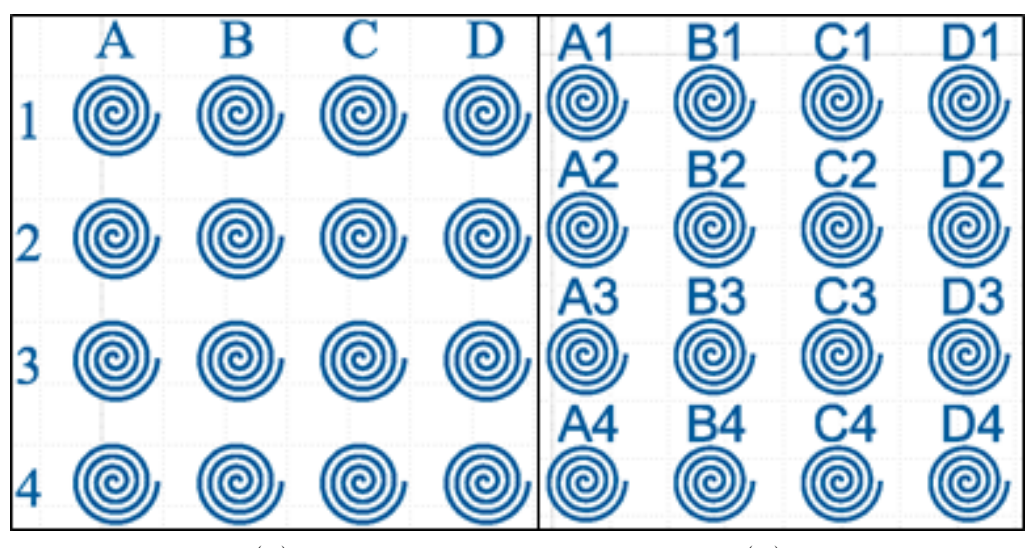

(c)

(d)

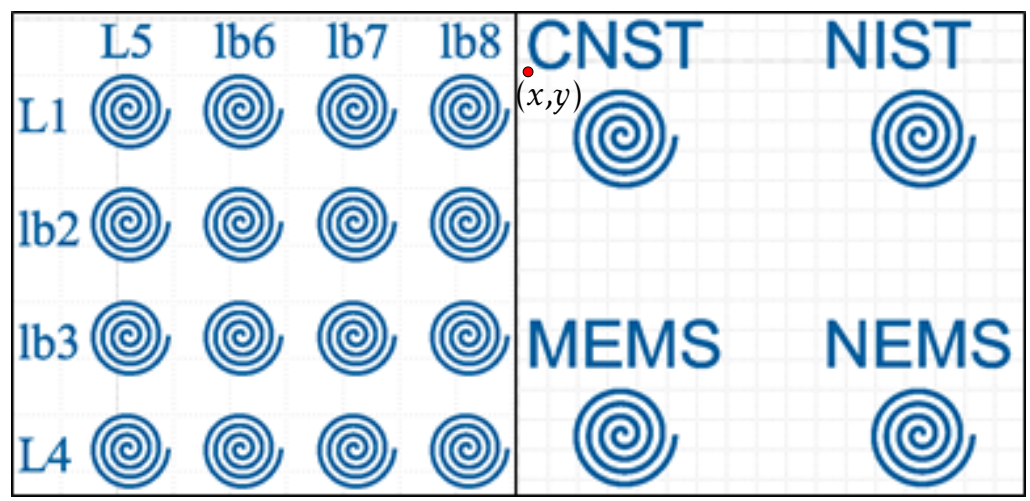

(e)

(f)

Figure 2.45: Label Maker Constructor Examples: (a) autoOut, (b) autoRowCol, (c) autoOutLett, (d) autoRowColLett, (e) out, and (f) rowCol. 


\subsubsection{Label Maker - Outline Text}

This method is identical to the previously defined label maker section (2.6.3) with the exception that rendered shapes are outlined text objects. Outline width is specified using the fontOutline parameter (see section 2.2.7).

4 auto labels:

$\left\{N_{\text {row }} N_{\text {col }}\right.$ fontName fontSize $x$ y $x_{r} y_{r} \Delta_{x} \Delta_{y}$ autoOut labelOutline $\}$

$\left\{N_{\text {row }} N_{\text {col }}\right.$ fontName fontSize $x$ y $x_{r} y_{r} \Delta_{x} \Delta_{y}$ autoRowCol labelOutline $\}$

$\left\{N_{\text {row }} N_{\text {col }}\right.$ fontName fontSize $x$ y $x_{r} y_{r} \Delta_{x} \Delta_{y}$ autoOutLett labelOutline $\}$

$\left\{N_{\text {row }} N_{\text {col }}\right.$ fontName fontSize $x$ y $x_{r} y_{r} \Delta_{x} \Delta_{y}$ autoRowColLett labelOutline $\}$

2 custom labels where parameters are TAB SEPARATED:

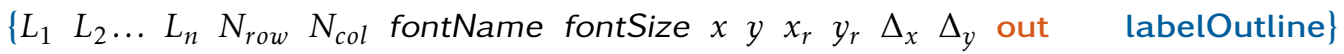

$\left\{\begin{array}{llllllllllllll}L_{1} & L_{2} & \ldots & L_{n} & N_{\text {row }} & N_{\text {col }} & \text { fontName fontSize } & x & y & x_{r} & y_{r} & \Delta_{x} & \Delta_{y} & \text { rowCol labelOutline }\end{array}\right.$

IMPORTANT NOTES: 1 ) Labels $L_{1} \ldots L_{n}$ are constructed from any printable ASCll character, including the space character. Therefore, space separation between constructor parameters is NOT allowed. All specified parameters within above constructors must be TAB separated. 2) Carriage returns cannot be used within the script line constructor. 3 ) Within this object, errors dealing with exceeding maximum number of GDS vertices could be encountered. To circumvent this dilemma, when using larger letters, increase the shapeReso parameter. 


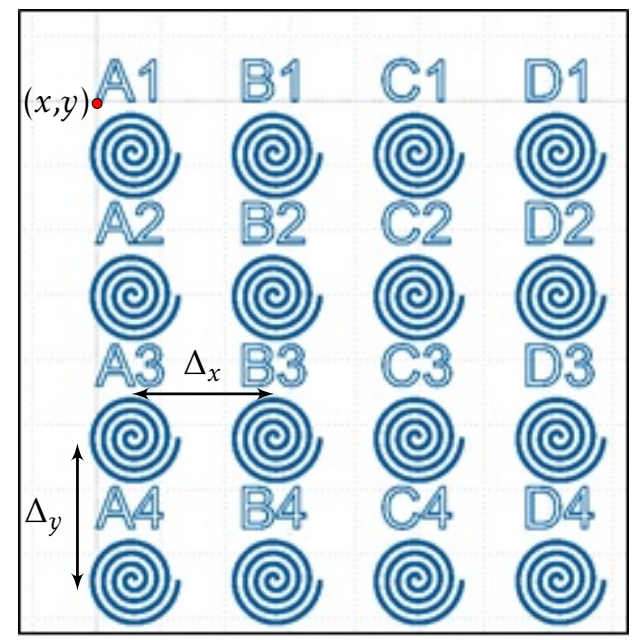

(a)

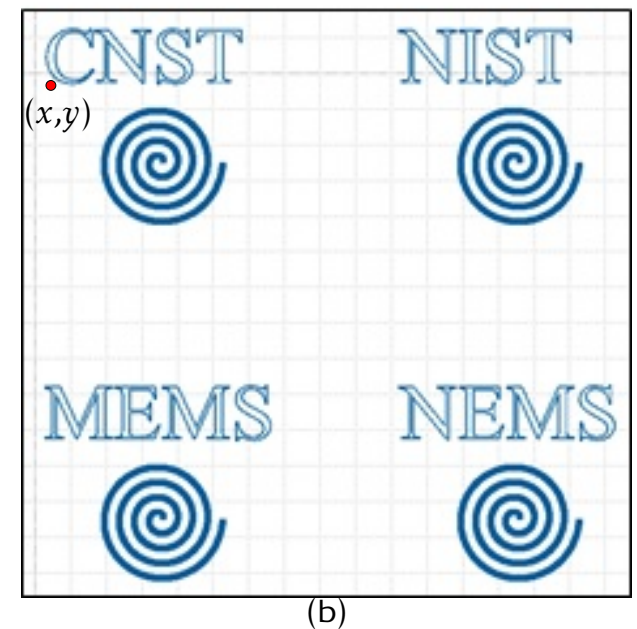

Figure 2.46: Label Maker Outlined Text Constructor Examples: (a) autoRowColLett and (b) rowCol. 


\subsubsection{PostScript to GDS}

\subsubsection{PostScript Pixel Value}

PostScript shapes are defined by double values in conjunction with constructors (moveTo $(\mathrm{m})$, lineTo $(\mathrm{l})$, curveTo $(\mathrm{c})$, etc). The pixel value defines scaling of these doubles. Default global value is 1.000 . This implies that the postscript coordinate values are unscaled and in units of micrometers. Parameter pixelScaling represents the scaling of the postscript coordinate values. For instance,

\subsection{0 psPixelValue}

scales each postscript value by 10 times. Alternatively,

$$
0.100 \text { psPixelValue }
$$

reduces the postscript image by 10 . Once the value is set, subsequent postscript shapes are rendered to this value. This value could be changed for each postscript shape.

$$
\text { pixelScaling psPixelValue }
$$

\subsubsection{PostScript Fracturing}

The following parameter defines the number of fractured postscript shapes. Default global value is 1 , implying that the shape is unfractured. The psFracElements parameter allows direct control of number of vertices per GDS shape. This is important since aggressively scaled, large, continuous postscript shapes could exceed the maximum number of vertices per GDS shape.

\section{0 psFracElements}

implies that the overall postscript shape area will be subdivided into 20 segments along the $y$-direction. This value could be changed for each postscript shape.

numberOfFracturedElements psFracElements

\subsubsection{Defining Postscript Shapes}

Bitmap images could be vectorized using either Illustrator, Inkscape (open source package) or some other package capable of exporting EPS files. Figure 2.47 illustrates the relevant portion of the EPS file. This information is copied and pasted within the CNST script file. 


\section{PostScript Example - PSshapeRaven.eps}

$\% \%$ EndProlog

$\% \%$ Page: 11

$\% \%$ BeginPageSetup

\%\% PageBoundingBox: 0 -1 15187

$\% \%$ EndPageSetup

q 0 -1 15188 rectclip q

0 $86.913151-87$ re W n

$0 \mathrm{~g}$

$143.8950 .167 \mathrm{~m} 142.4570 .456$ 140.961 0.925140 .039 1.378

138.0942 .331135 .9613 .824134 .7775 .058 с 134.1765 .687134 .035

5.913134 .0356 .249 с 134.0356 .593134 .096 .675134 .3636 .738 c

135.1056 .913136 .2976 .339137 .8135 .074 c 138.774 .277139 .641

3.843140 .8523 .562 с 142.5313 .175

4.046142 .6953 .468142 .6953 .335 с 142.6953 .066142 .2342 .917

141.6913 .015 с 139.9143 .327138 .773 .808137 .6884 .706 с 135.844

6.23134 .4226 .87134 .4226 .179 с 134.4265 .476138 .0512 .675

140.3361 .617 с 142.4690 .624146 .1090 .07147 .9220 .46 c h

Q Q

showpage

$\% \%$ Trailer

Figure 2.47: PostScript scriptingPostScript.eps example. The arrow denotes the relevant text to be copied and pasted into the CNST script file.

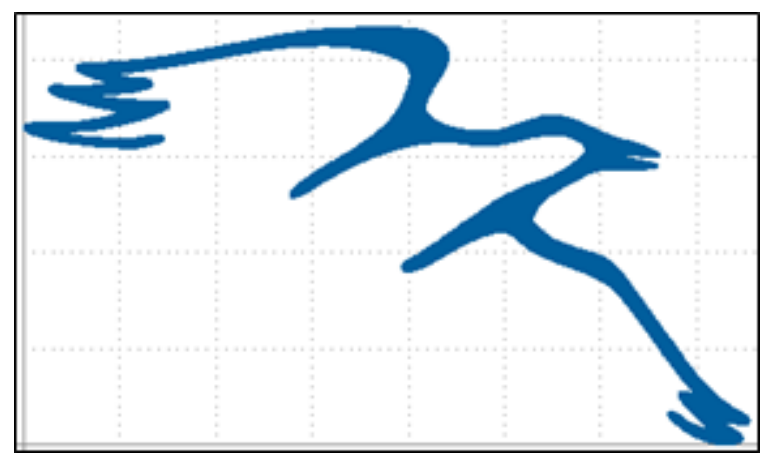

Figure 2.48: GDS shape of the rendered postscript shape. 


\subsubsection{CNST and NIST logos}

Three constructors are available for placement of CNST and NIST logos. Individual (cnstEmblem, cnstLogo, nistLogo ) or combined (nistCnstLogo) are placed at coordinates that define the centroid of the GDS logo shape. Since logos are cast using postscript values, the shapeReso parameter defines the rendering resolution of the logo shapes. Scaling of the resulting logo is set using the scale parameter.

$\begin{array}{llll}x & y & \text { scale } & \text { cnstEmblem } \\ x & y & \text { scale } & \text { cnstLogo } \\ x & y & \text { scale } & \text { nistLogo } \\ x & y & \text { scale } & \text { nistCnstLogo }\end{array}$

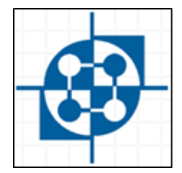

(a)

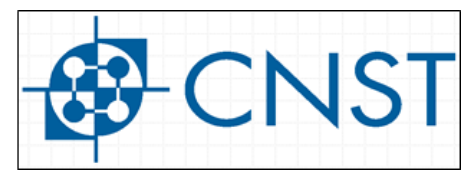

(b)

Figure 2.49: CNST logos created using the (a) cnstLogoEmblem and (b) cnstLogo constructors.

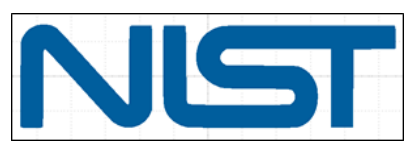

Figure 2.50: NIST logo created using the nistLogo constructor.

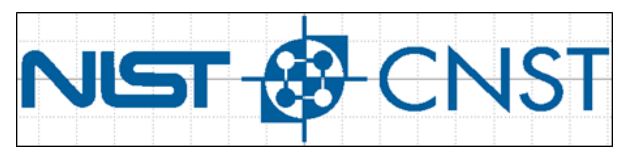

Figure 2.51: Combined NIST and CNST logos created using the nistCnstLogo constructor. 


\subsection{Objects}

\subsubsection{Arc (Torus-Circle) bounded square-hex arrays}

The following constructors create a square or hexagonal array of dots inside of an arc boundary defined by an inner and outer radii $\left(r_{i}\right.$ and $\left.r_{o}\right)$, start and end angles $\left(\theta_{s}\right.$ and $\left.\theta_{e}\right)$ and the number of vertices $(N)$. For a torus defined by $r_{i}$ and $r_{o}, \theta_{s}=0$ and $\theta_{e}=360$. A circular boundary is represented by a radius $r_{o}$ with $r_{i}=0, \theta_{s}=0$ and $\theta_{e}=360$. The circular dots of radius $r_{s}$ are either defined by a number of vertices $n_{s}$ (arcSquareFill and arcHexFill) or by the shapeReso parameter for vectorized shapes (arcSquareFillV and arcHexFillV). Parameter $\theta_{(x, y)}$ defines the shape rotation about the arc origin $(x, y)$.

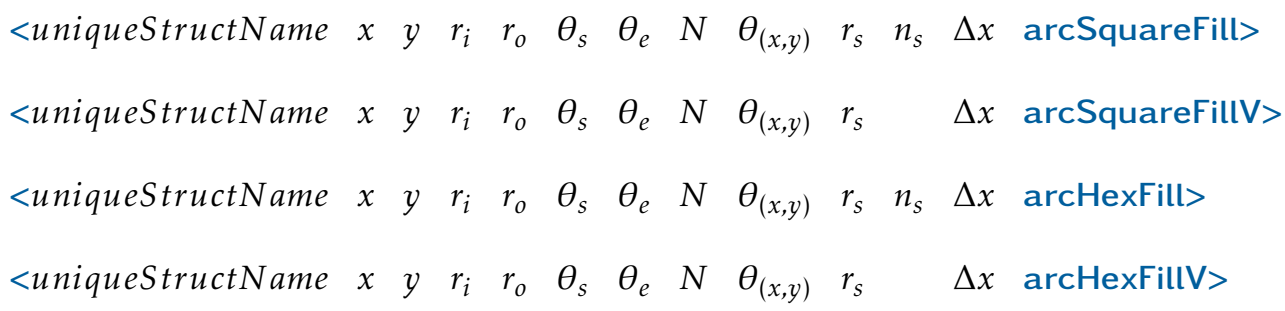

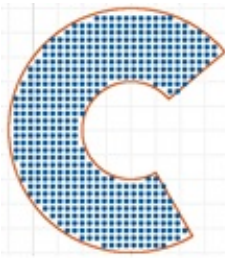

(a)

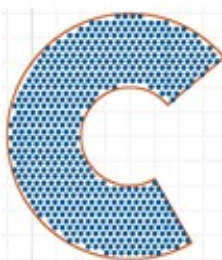

(d)

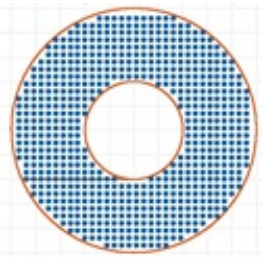

(b)

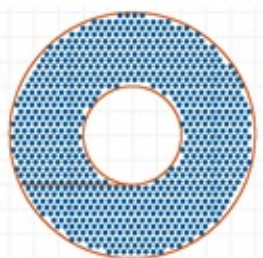

(e)

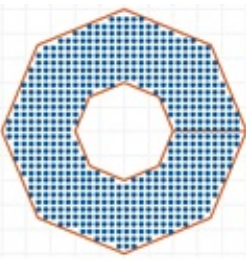

(c)

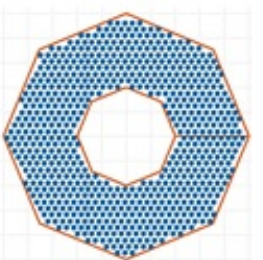

(f)

Figure 2.52: Square array inside (a) an arc, (b) circular and (c) hexagonal $(N=8)$ boundary. Hexagonal array of dots inside (d) an arc, (e) circular and (f) hexagonal $(N=8)$ boundary. 


\subsubsection{Bezier Curve}

The toolbox employs Bezier curves to represent complex curved objects. The shapes are rendered to a resolution defined by the shapeReso parameter. In general, Bezier curve is a parametrized curve used as a path in computer vector graphics. Concatenation of multiple Bezier paths can be used to model smooth curves of arbitrary complexity. A Bezier curve is mathematically expressed as

$$
B(t)=\sum_{k=0}^{n}{ }^{n} C_{k}(1-t)^{n-1} t^{k} P_{k}
$$

where $n$ is the polynomial degree, $k$ is the index, $t$ is a variable ranging $0 \leq t \leq 1$, and ${ }^{n} C_{k}$ is the binomial coefficient given by

$$
{ }^{n} C_{k}=\left(\begin{array}{l}
n \\
k
\end{array}\right)=\frac{n !}{k !(n-k) !}
$$

The nanolithography toolbox employs cubic Bezier curves for creating complex curved shapes. With with $n=3$ equation 2.1 becomes

$$
B(t)=(1-t)^{3} P_{0}+3(1-t)^{2} t P_{1}+3(1-t) t^{2} P_{2}+t^{3} P_{3}
$$

where the corresponding start and end points are $P_{0}$ and $P_{3}$, with respective control points $P_{1}$ and $P_{2}$. Equation 2.3 for the two coordinates is written as

$$
\begin{aligned}
& B(t)_{x}=(1-t)^{3} P_{0 x}+3(1-t)^{2} t P_{1 x}+3(1-t) t^{2} P_{2 x}+t^{3} P_{3 x} \\
& B(t)_{y}=(1-t)^{3} P_{0 y}+3(1-t)^{2} t P_{1 y}+3(1-t) t^{2} P_{2 y}+t^{3} P_{3 y}
\end{aligned}
$$

Figure 2.53 shows rendered curves at varying resolution. Rendered curves exhibit an increased vertex density at higher curvatures automatically. The points are uniformly spaced in $t$, but become closer together in $(x, y)$ space as curvature increases.

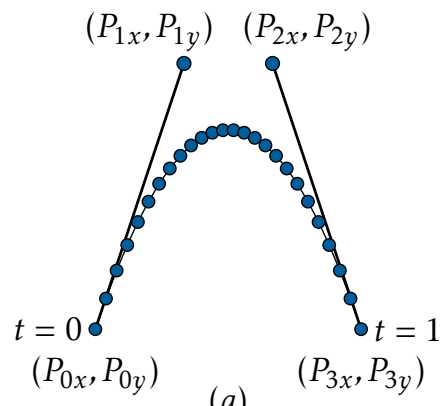

(a)

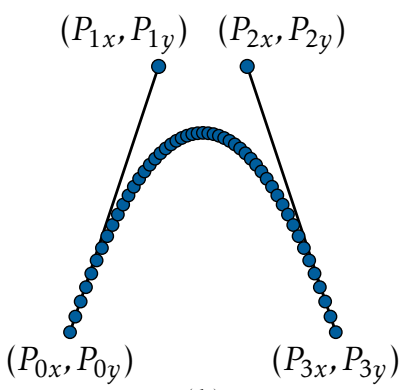

(b)

Figure 2.53: Cubic Bezier curves rendered at a (a) lower and (b) higher resolution. Dots represent rendered curve vertices.

NIST • CNST Nanolithography Toolbox v2016.09.01 • http://www.nist.gov/cnst/

page 70 of 488 
Bezier Curve is defined using a start $\left(x_{1}, y_{1}\right)$ and end $\left(x_{2}, y_{2}\right)$ points, two control points $\left(c x_{1}, c y_{1}\right)$ and $\left(c x_{2}, c y_{2}\right)$, curve width $(W)$ and $\theta_{\left(x_{1}, y_{1}\right)}$ rotation about $\left(x_{1}, y_{1}\right)$. shapeReso parameter defines the rendering resolution of the Bezier curve.

$\begin{array}{lllllllllll}<x_{1} & y_{1} & c x_{1} & c y_{1} & c x_{2} & c y_{2} & x_{2} & y_{2} & W & \theta_{\left(x_{1}, y_{1}\right)} & \text { bezierCurve }>\end{array}$

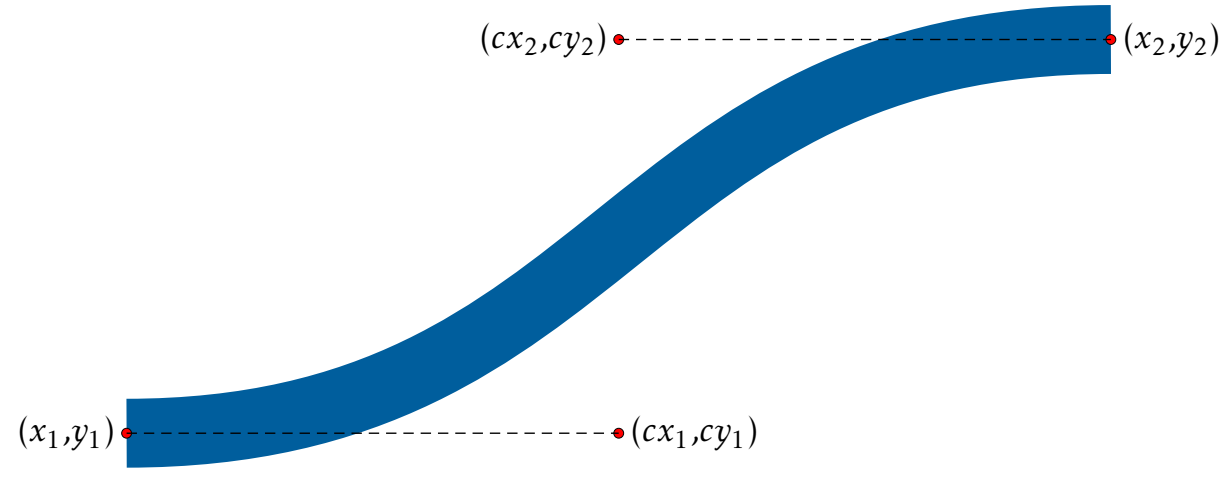

Figure 2.54: Bezier Curve example showing the start and end points along with the corresponding control points.

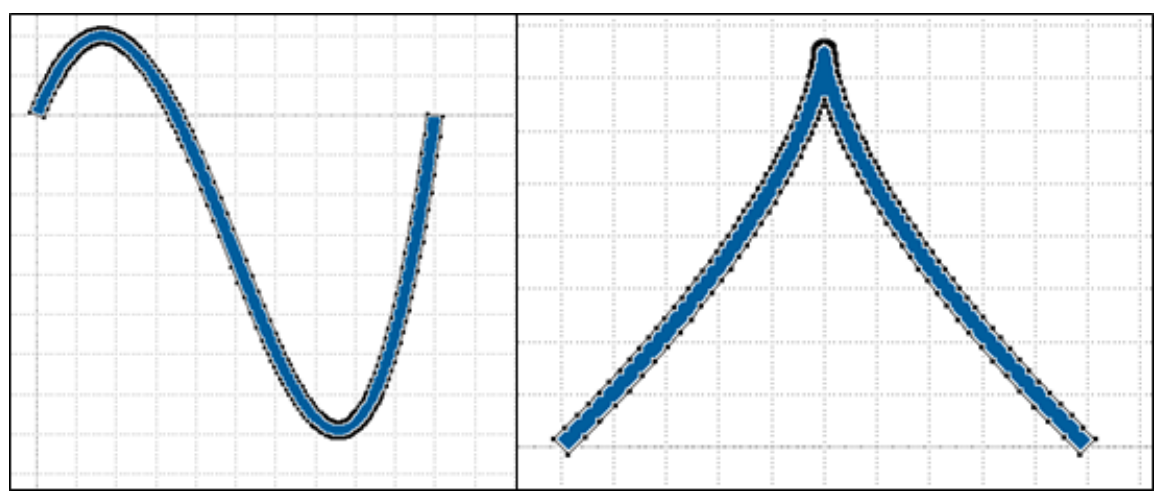

Figure 2.55: Two example GDS shapes of the bezierCurve constructor. Highlighted periphery shows curved sections with densely packed vertices. 
$\begin{array}{llllllllllll}<x_{1} & y_{1} & c x_{1} & c y_{1} & c x_{2} & c y_{2} & x_{2} & y_{2} & W_{e} & W_{s} & \theta_{\left(x_{1}, y_{1}\right)} & \text { bezierCurvelnv }>\end{array}$

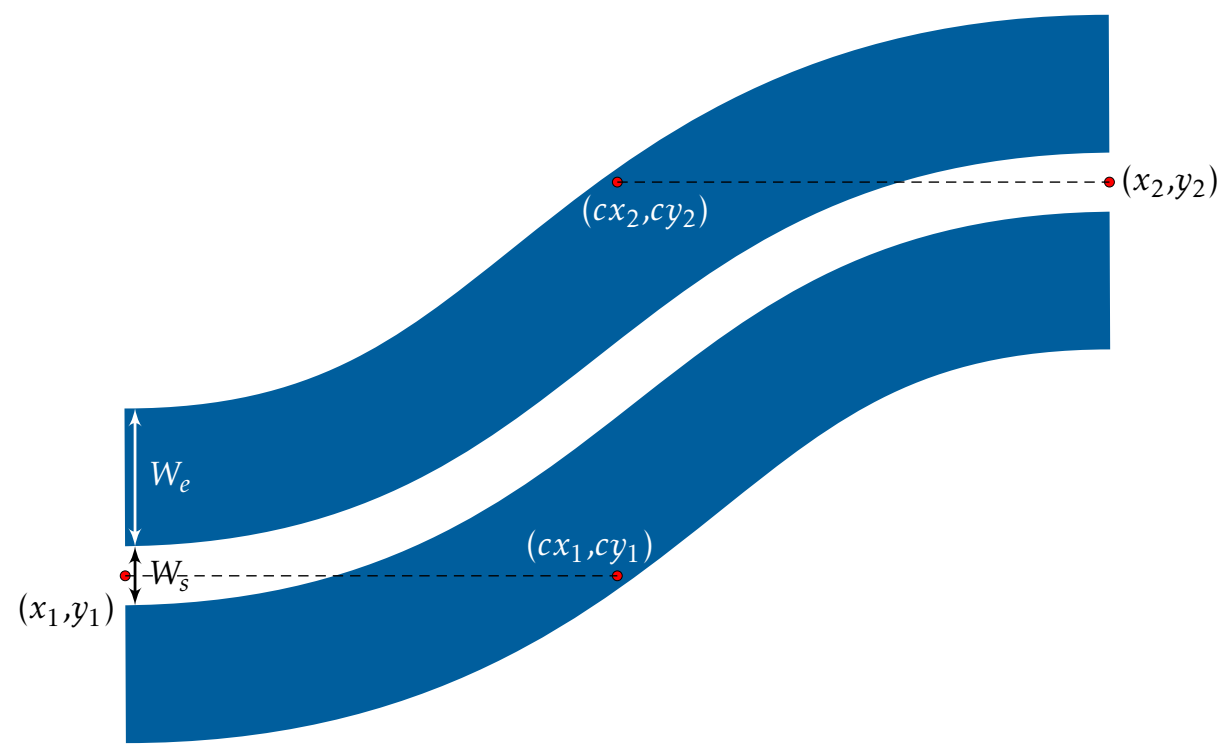

Figure 2.56: Schematic illustration showing various parameters from the bezierCurvelnv constructor. 
$\begin{array}{lllllllllllll}<x_{1} & y_{1} & c x_{1} & c y_{1} & c x_{2} & c y_{2} & x_{2} & y_{2} & W_{e} & W_{s} & g & \theta_{\left(x_{1}, y_{1}\right)} & \text { bezierCurvelnvSlot }>\end{array}$

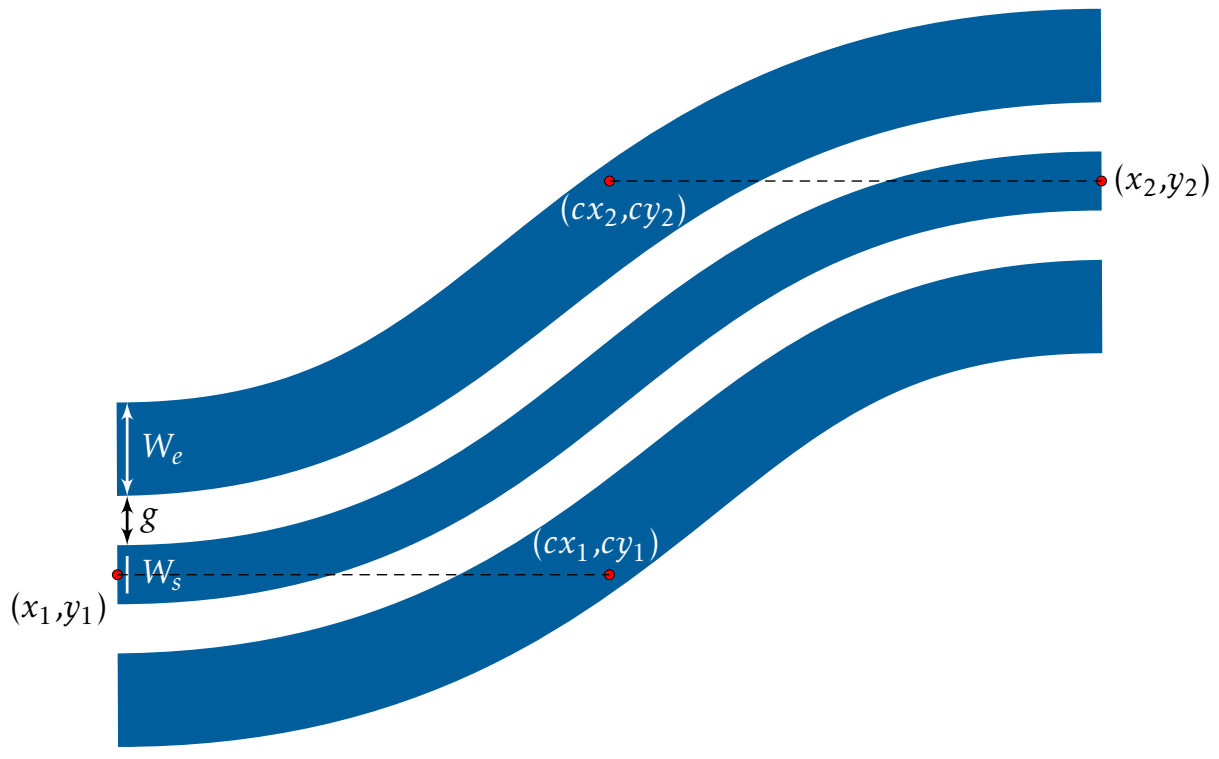

Figure 2.57: Schematic illustration of the bezierCurvelnvSlot constructor. 


\subsubsection{Fractals}

Several constructor methods are available for creating self-similar structures, including Sierpinski triangle and carpet, Vicsek saltire and cross, curved trees and various other tree-like structures. The last GDS structure iteration constructed by the recursive fractal generator is instantiated at $(x, y)$. Iteration numbers are postfix appended to the shortStructName parameter.

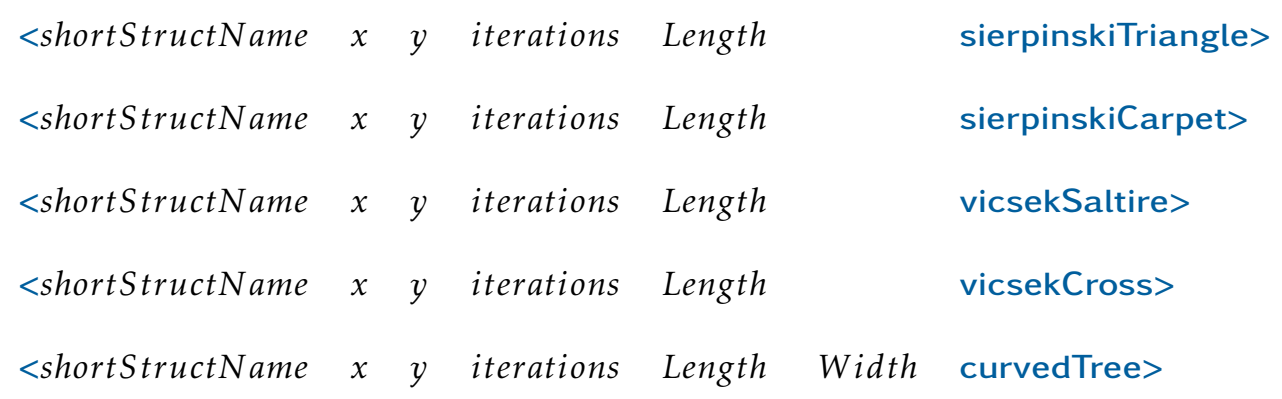

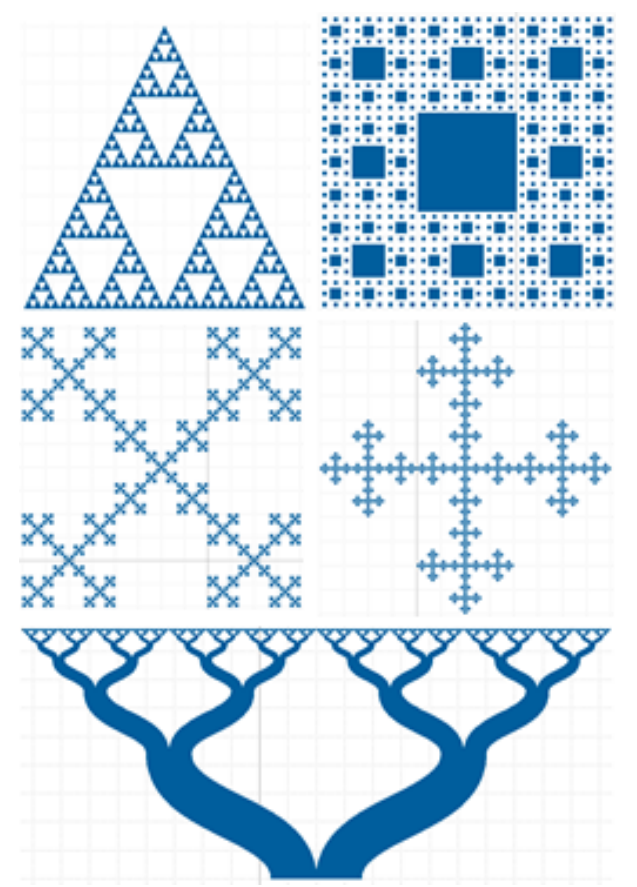

Figure 2.58: Generated GDS fractal examples shapes. 


\subsubsection{Function Plot}

Functional plot method allows functional representation in $(x, y)$ and $(r, \theta)$ coordinate systems. Generated curves are defined by a function, translation $(x, y)$, lower $\left(x_{L}\right.$ and $\left.\theta_{L}\right)$ and upper limits $\left(x_{U}\right.$ and $\left.\theta_{U}\right)$, number of segments $(N)$, width $(W)$, cap and join parameters. Cap is represented by the following integer values $B U T T=0, R O U N D=1$, or $S Q U A R E=2$. Join is represented by the following integer values $M I T E R=0, R O U N D=1, B E V E L=2$. In order to avoid exceeding GDS shape point limits, each function is fractured horizontally and vertically about the centroid.

$$
\begin{array}{llllllllllll}
<\{\{y(x)\}\} & x & y & x_{L} & x_{U} & N & W & C A P & J O I N & \theta_{(x, y)} & \text { functionXY }> \\
<\{\{r(t)\}\} & x & y & \theta_{L} & \theta_{U} & N & W & C A P & J O I N & \theta_{(x, y)} & \text { functionRT }>
\end{array}
$$

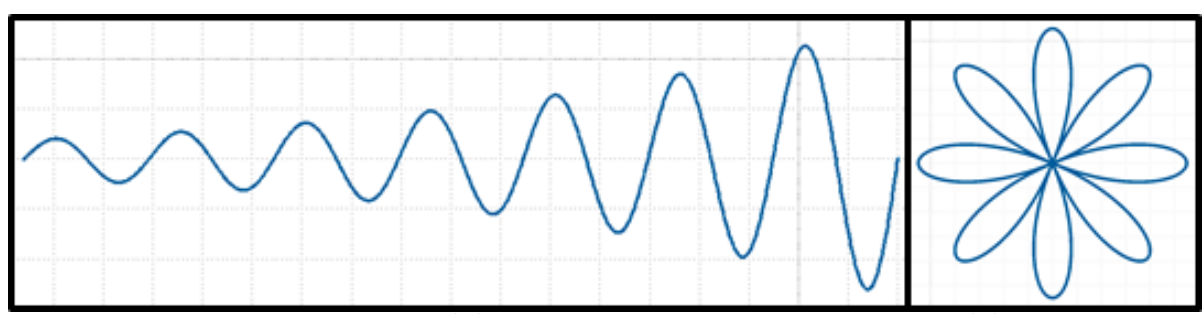

(a)

(b)

Figure 2.59: Function plots (a) $y(x)$ and (b) $r(\theta)$.

Functional plots in figures 2.59 (a) and $2.59 \mathrm{~b}$ are respectively rotated by $180^{\circ}$ and $22.5^{\circ}$ about $(10,-10)$. Both curves were constructed using $N=1000$, a width of $0.22 \mu \mathrm{m}, C A P=R O U N D$ and JOIN $=$ MITER. Functional representation for the two curves is correspondingly given by

$$
\begin{gathered}
y(x)=14 \sin \left(\frac{x}{2}\right) e^{-\frac{x}{44}} \\
r(\theta)=11 \sin (4 \theta)
\end{gathered}
$$

The following scripts (see example file scriptingF unctionPlot.cnst) to generate functional plots in Figure 2.59:

$<\{\{14 * M a t h \cdot \sin (x / 2) * M a t h \cdot \exp (-x / 44)\}\} \quad 10-1008810000.2210180$ functionXY>

$<\{\{11 *$ Math.sin $(4 * t)\}\} \quad 10-1006.2810000 .221022 .5$ functionRT>

As seen above, in polar coordinates $(r, \theta), \theta$ parameter is represented as a variable $t$. Mathematical functions are represented in terms of the Java 
Math class methods. For instance $\sin (x)$ is represented as Math.sin $(x)$. Several methods from the Java Math class are shown below. The entire Java Math class method summary documentation, including argument limits, is available online.

$\begin{array}{llll}\text { Math.sin}(\mathrm{x}) & \sin (x) & \text { Math.sinh }(\mathrm{x}) & \sinh (x) \\ \text { Math.cos }(\mathrm{x}) & \cos (x) & \text { Math.cosh }(\mathrm{x}) & \cosh (x) \\ \text { Math.tan }(\mathrm{x}) & \tan (x) & \text { Math.tanh }(\mathrm{x}) & \tanh (x) \\ \text { Math.exp }(\mathrm{x}) & e^{x} & \text { Math.expm }(\mathrm{x}) & e^{x}-1 \\ \text { Math.log}(\mathrm{x}) & \ln (x) & \text { Math.log10(x) } & \log _{10}(x) \\ \text { Math.sqrt }(\mathrm{x}) & \sqrt{x} & \text { Math.pow }(\mathrm{x}, \mathrm{n}) & x^{n} \\ \text { Math.PI } & \pi & \text { Math.random( }) \quad \text { random number in [0,1) }\end{array}$

NIST • CNST Nanolithography Toolbox v2016.09.01 • http://www.nist.gov/cnst/

page 76 of 488 


\subsubsection{Grayscale}

\subsubsection{Polygons}

This section offers a variety of means for constructing grayscale objects from segmented polygons. GDS layers are assigned to each of the sections with layer numbers ranging from $n-1$ (outer) to 0 (inner). Figures 2.60a, b and c show evenly distributed grayscale structure are centered at $(x, y)$, with $n$ number of segmentations, the outermost dimensions defined by $d_{x}, d_{y}, r_{x}$ and $r_{y}$, and $\theta_{(x, y)}$ rotation about the origin. Rectangular structures (figure $2.60 \mathrm{a}$ ) are equivalent to 45 degree rotated n-gon structures where the number of sides $N_{s}=4$ (figure 2.60c).

$$
\begin{array}{lllllllll}
x & y & d_{x} & d_{y} & n & & \theta_{(x, y)} & \text { grayER } \\
x & y & r_{x} & r_{y} & n & N_{s} & \theta_{(x, y)} & \text { grayENgon }
\end{array}
$$

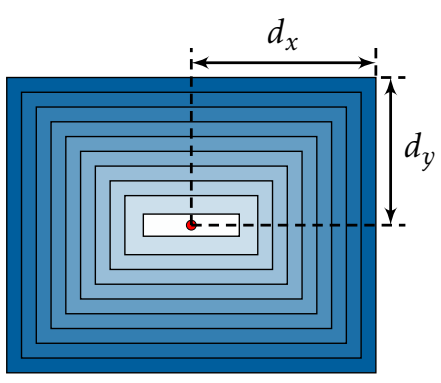

(a)

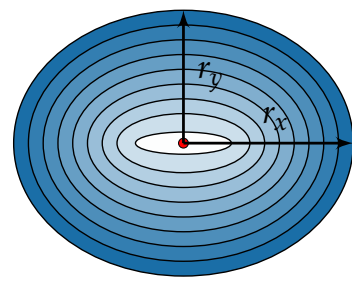

(b)

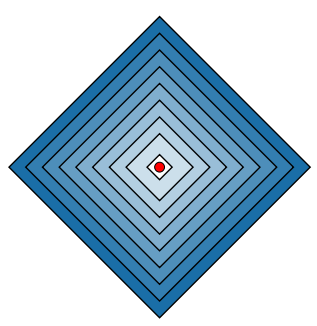

(c)

Figure 2.60: Evenly distributed grayscale segments for (a) rectangles and n-gons with (b) large number of vertices $\left(N_{s}\right)$ and (c) $N_{s}=4, r_{x}=r_{y}$. 
User defined grayscale segments are shown in figures $2.61 \mathrm{a}, \mathrm{b}$ and $\mathrm{c}$. The respective constructors are defined by $\left(d_{x i}, d_{y i}\right)$ (or $\left.\left(r_{x_{i}}, r_{y_{i}}\right)\right)$ coordinate pairs. The outer most segment is defined by $\left(d_{x_{1}}, d_{y_{1}}\right)\left(\operatorname{or}\left(r_{x_{1}}, r_{y_{1}}\right)\right)$. The constructors further assume that $d_{x_{1}}>d_{x_{2}}>d_{x_{3}} \cdots>d_{x_{n}}$ and $d_{y_{1}}>d_{y_{2}}>d_{y_{3}} \cdots>d_{y_{n}}$. Similarly, for $\mathrm{n}$-gon segments $r_{x_{1}}>r_{x_{2}}>r_{x_{3}} \cdots>r_{x_{n}}$ and $r_{y_{1}}>r_{y_{2}}>r_{y_{3}} \cdots>r_{y_{n}}$.

$\begin{array}{llllllllllll}x & y & d_{x_{1}} & d_{y_{1}} & d_{x_{2}} & d_{y_{2}} & \cdots & d_{x_{n}} & d_{y_{n}} & \theta_{(x, y)} & \text { grayUDR }\end{array}$

$\begin{array}{llllllllllll}x & y & r_{x_{1}} & r_{y_{1}} & r_{x_{2}} & r_{y_{2}} & \cdots & r_{x_{n}} & r_{y_{n}} & N_{s} & \theta_{(x, y)} & \text { grayUDNgon }\end{array}$

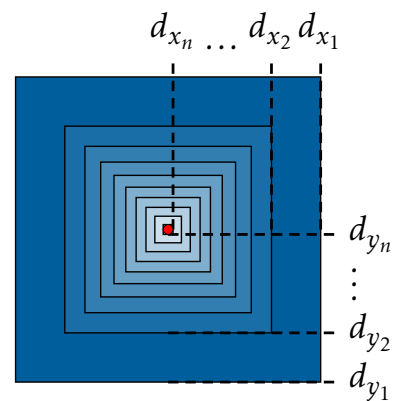

(a)

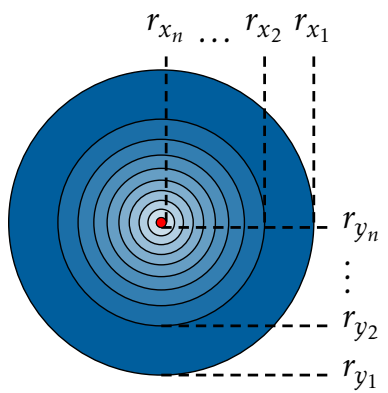

(b)

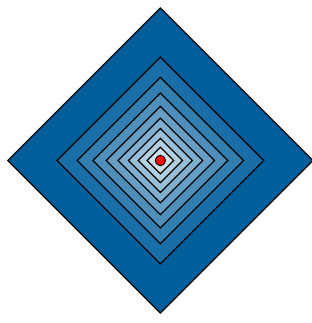

(c)

Figure 2.61: User defined distribution of grayscale segments for (a) rectangles and $n$-gons with (b) large number of vertices $\left(N_{s}\right)$ and (c) $N_{s}=4, r_{x_{i}}=r_{y_{i}}$. 


\subsubsection{Ramp}

grayERamp constructor allows ramping with either UP $=1$, where the $d_{x}$ is subdivided into $n$ segments with GDS layer numbers range from 0 to $n-1$. Alternatively, with UP $=0$, the GDS layer numbers range from $n-1$ to 0 . gray ERamp2 constructor forms a double ramp (up then down) with evenly divided segments where GDS layers range from

$$
L= \begin{cases}L_{\frac{n}{2}} \ldots L_{0} \ldots L_{\frac{n}{2}-1} & n \text { is even } \\ L_{\frac{n-1}{2}} \ldots L_{0} \ldots L_{\frac{n-1}{2}} & n \text { is odd }\end{cases}
$$

$$
\begin{array}{lllllllll}
x & y & d_{x} & d_{y} & n & \text { UP } & \theta_{(x, y)} & \text { grayERamp } \\
x & y & d_{x} & d_{y} & n & & \theta_{(x, y)} & \text { grayERamp2 }
\end{array}
$$

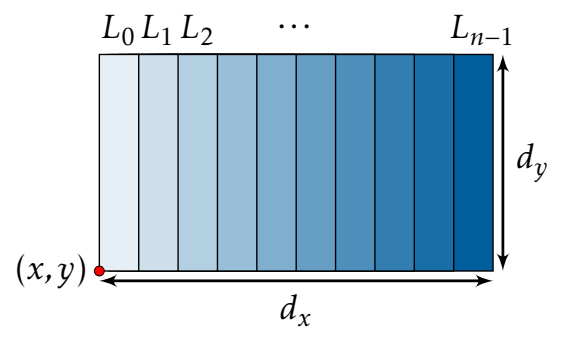

(a)

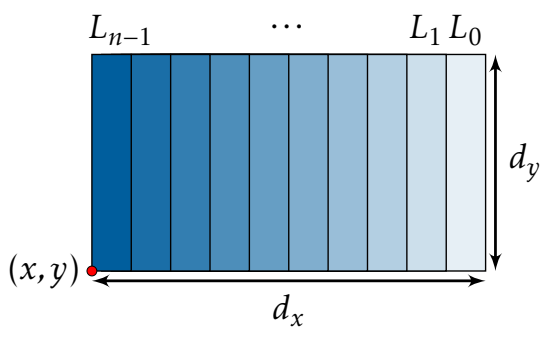

(b)

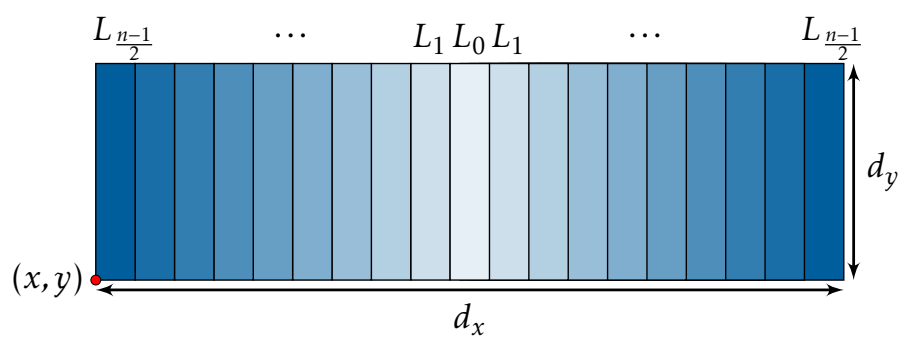

(c)

Figure 2.62: Evenly distributed grayscale ramp segments using grayERamp with UP values of (a) 1 and (b) 0 . (c) Grayscale ramp up/down segments using the grayERamp2 constructor. 
Similarly, user defined ramp segments are defined using the below constructors. Number of constructed shapes is equal to $n-1$, where $n$ is the number of defined segment positions. Grayscale segments undergo a translation by $\left(x_{t}, y_{t}\right)$ and rotation $\theta_{\left(x_{t}, y_{t}\right)}$. Constructors assume $x_{1}<x_{2}<x_{3} \cdots<d_{x_{n}}, n>1$ with GDS layers for grayUDRamp2 defined:

$$
L= \begin{cases}L_{\frac{n}{2}-1} \ldots L_{0} \ldots L_{\frac{n}{2}-1} & n \text { is even } \\ L_{\frac{n-3}{2}} \ldots L_{0} \ldots L_{\frac{n-1}{2}} & n \text { is odd and } n>3 \\ L_{0}, L_{1} & n=3 \\ L_{0} & n=2\end{cases}
$$

$$
\begin{array}{llllllllll}
x_{t} & y_{t} & x_{1} & x_{2} & \ldots & x_{n} & d_{y} & \text { UP } & \theta_{\left(x_{t}, y_{t}\right)} & \text { grayUDRamp } \\
x_{t} & y_{t} & x_{1} & x_{2} & \ldots & x_{n} & d_{y} & & \theta_{\left(x_{t}, y_{t}\right)} & \text { grayUDRamp2 }
\end{array}
$$

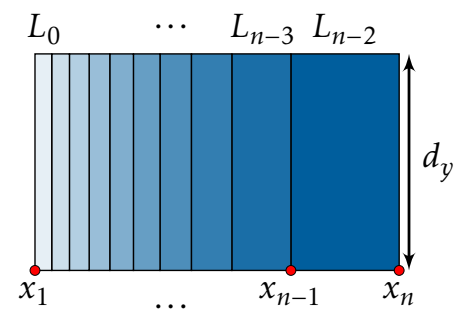

(a)

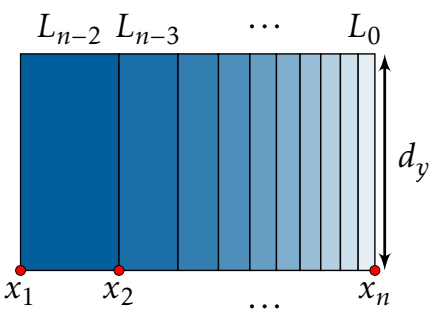

(b)

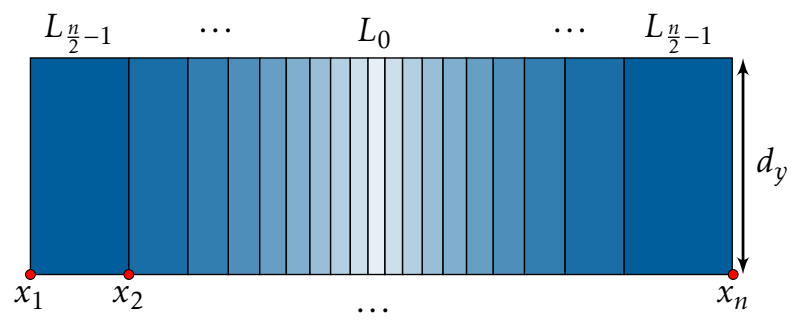

(c)

Figure 2.63: User defined grayscale ramp segments using grayURRamp with UP values of (a) 1 and (b) 0 . (c) Grayscale ramp up/down segments using the grayURRamp 2 constructor with an even number of points $n$. 


\subsubsection{Spiral Staircase}

The spiral structure is constructed using overlapping arc segments cast to different GDS layers $\left(L_{i}\right)$. The resulting structure is defined by the center position $(x, y)$, inner and outer radii $\left(r_{i}\right.$ and $\left.r_{\circ}\right)$, number of arc segments $(n)$, number of sides of each segment $\left(N_{\text {sides }}\right)$ and the rotation angle $\left(\theta_{(x, y)}\right)$.

$\begin{array}{lllllllll}x & y & r_{i} & r_{\circ} & n & N_{\text {sides }} & \theta_{(x, y)} & \text { graySpiralStairOverlap }\end{array}$

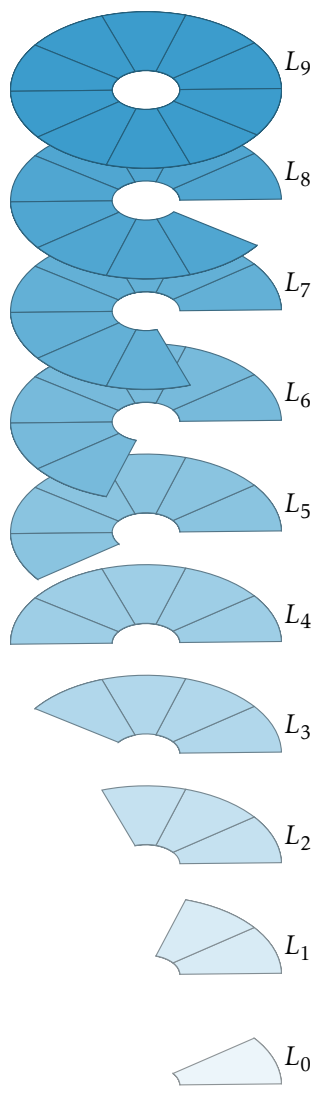

(a)

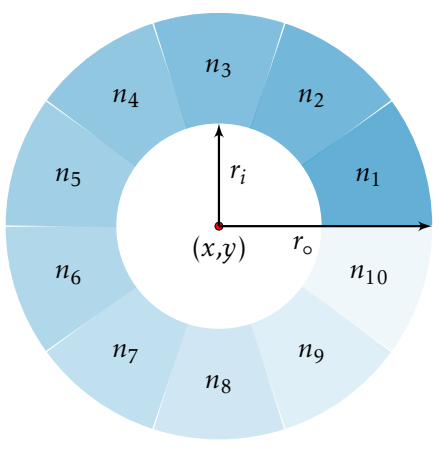

(b)

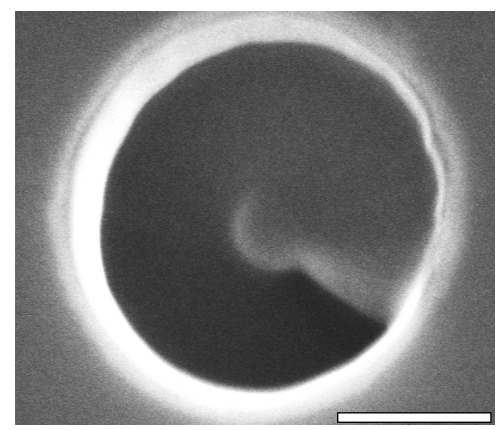

(c)

Figure 2.64: Grayscale spiral staircase with overlapping segments. (a) 3D projection and (b) 2D illustration showing various constructor parameters. (c) Scanning electron micrograph of a focused ion beam fabricated structure. Scale bar corresponds to $200 \mathrm{~nm}$. (Courtesy of L. Ocola [82]) 
The following constructor creates a grayscale spiral staircase structure without overlapping arc segments.

$$
\begin{array}{llllllllll}
x & y & r_{i} & r_{\circ} & n & N_{\text {sides }} & \theta_{(x, y)} & \text { graySpiralStair }
\end{array}
$$

Here, each segment $\left(n_{i}\right)$ is represented by a distinct GDS layer.

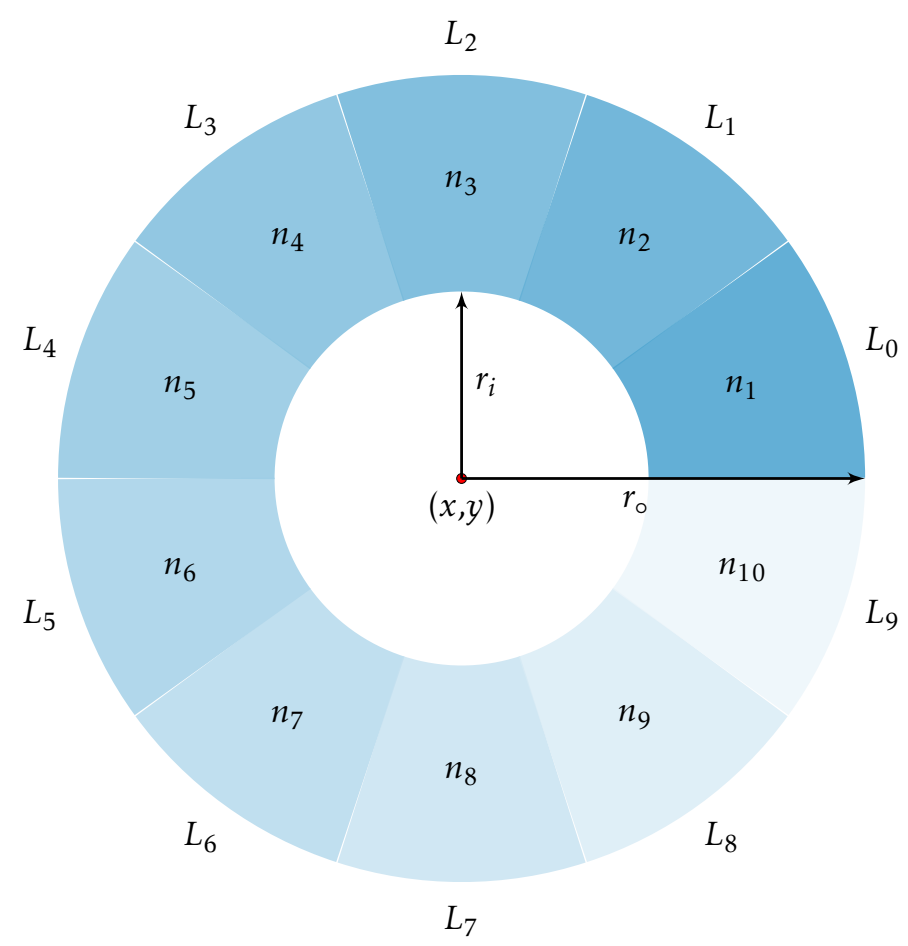

(b)

Figure 2.65: Grayscale spiral staircase. 


\subsubsection{Interdigitated Electrodes}

$$
\begin{array}{lllllllllllll}
x & y & w_{1} & w_{2} & l_{1} & l_{2} & l_{3} & N & p & b_{H} & b_{W} & \theta_{(x, y)} & \text { intElec1 } \\
x & y & w_{1} & w_{2} & l_{1} & l_{2} & l_{3} & N & p & b_{H} & b_{W} & \theta_{(x, y)} & \text { intElec2 }
\end{array}
$$
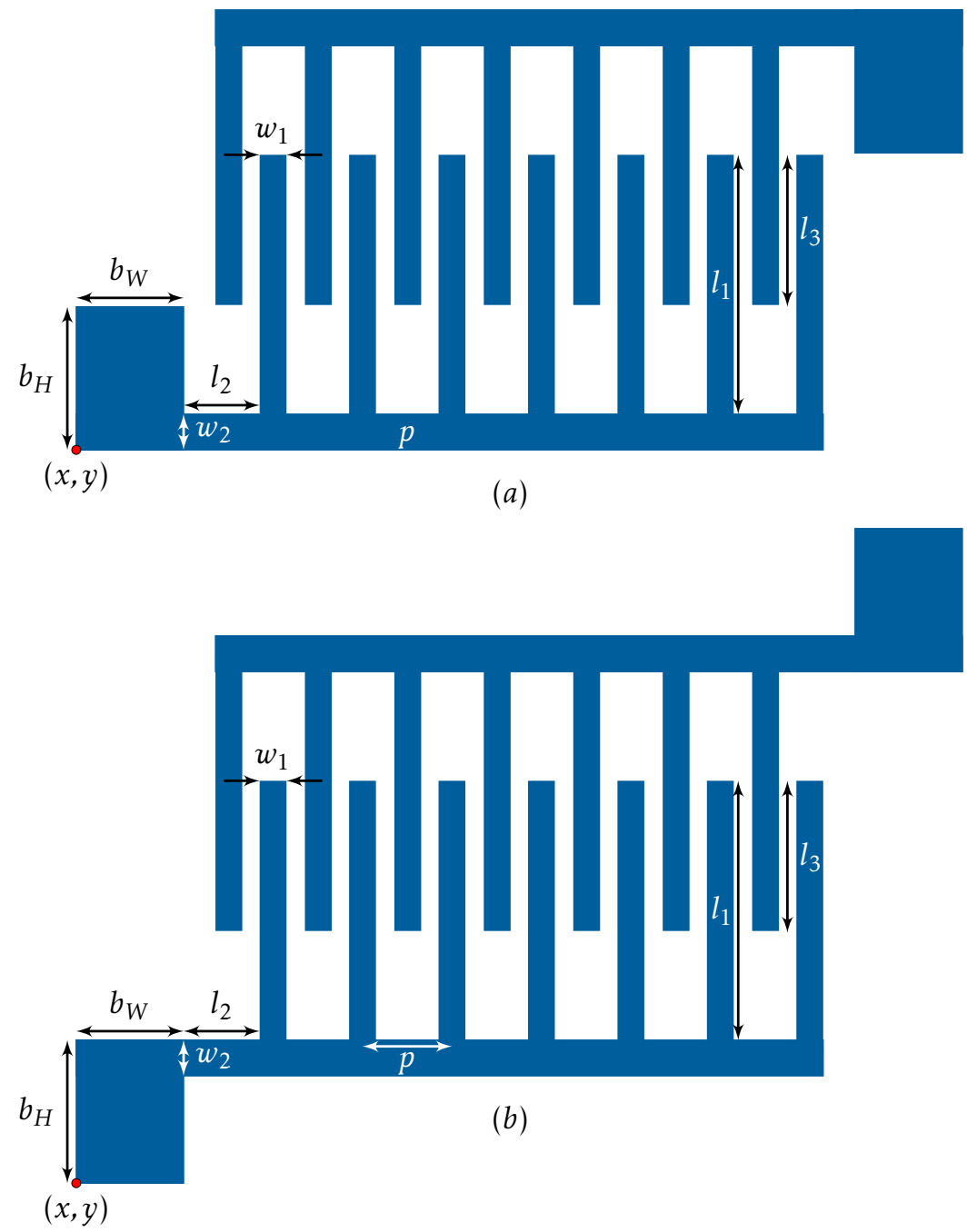

Figure 2.66: Interdigitated electrodes (a) intElec1 and (b) intElec2. 
$\begin{array}{llllllllllllll}x & y & w_{1} & w_{2} & l_{1} & l_{2} & l_{3} & N & p & b_{H} & b_{W} & \theta_{(x, y)} & \text { intElec3 }\end{array}$

$\begin{array}{llllllllllllll}x & y & w_{1} & w_{2} & l_{1} & l_{2} & l_{3} & N & p & b_{H} & b_{W} & \theta_{(x, y)} & \text { intElec4 }\end{array}$

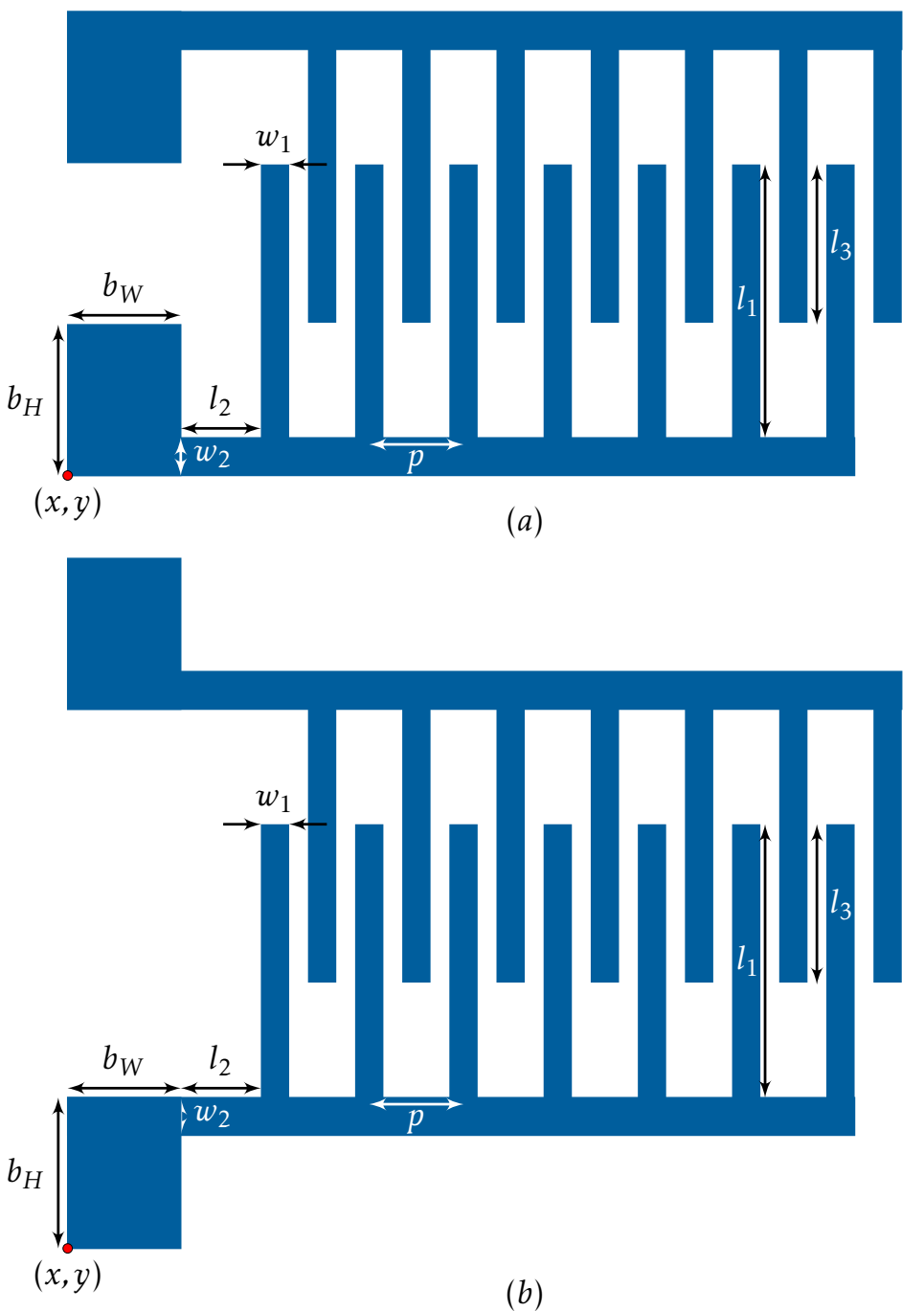

Figure 2.67: Interdigitated electrodes (a) intElec3 and (b) intElec4. 
$\begin{array}{lllllllllllll}x & y & w_{1} & w_{2} & l_{1} & l_{2} & l_{3} & N & p & b_{H} & b_{W} & \theta_{(x, y)} & \text { intElec5 }\end{array}$

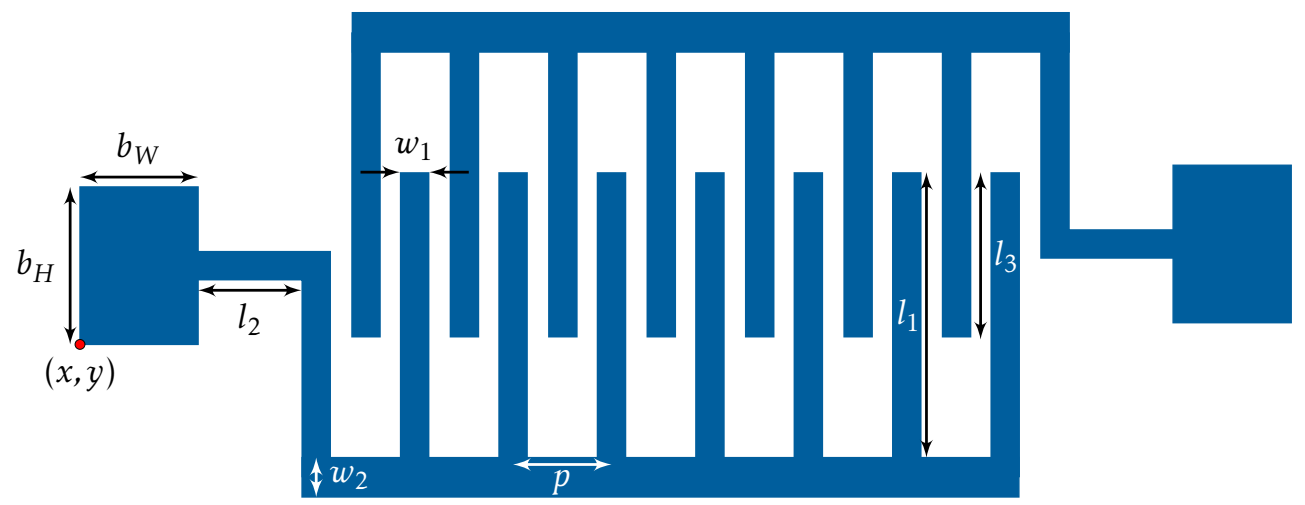

Figure 2.68: Interdigitated electrodes intElec5. 


\subsubsection{Junctions}

\subsubsection{T Junction}

$\begin{array}{llllllllll}x & y & w_{1} & w_{2} & L_{1} & L_{2} & r & N_{\text {sides }} & \theta_{(x, y)} & \text { tJunction }\end{array}$

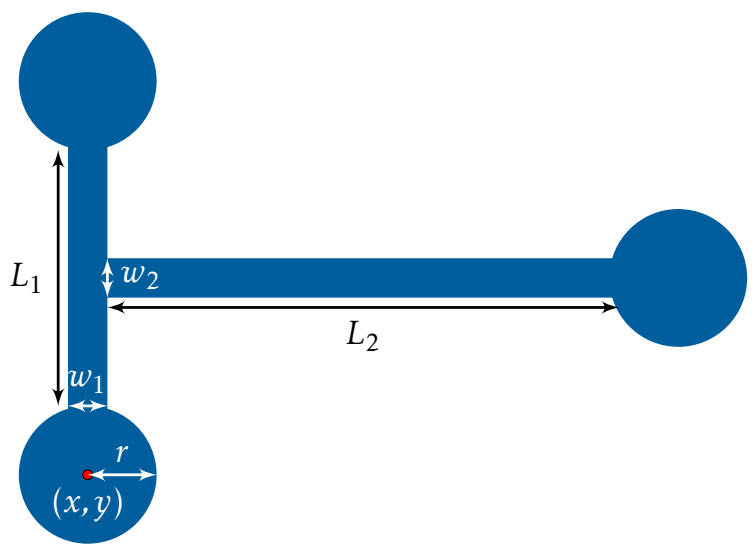

(a)

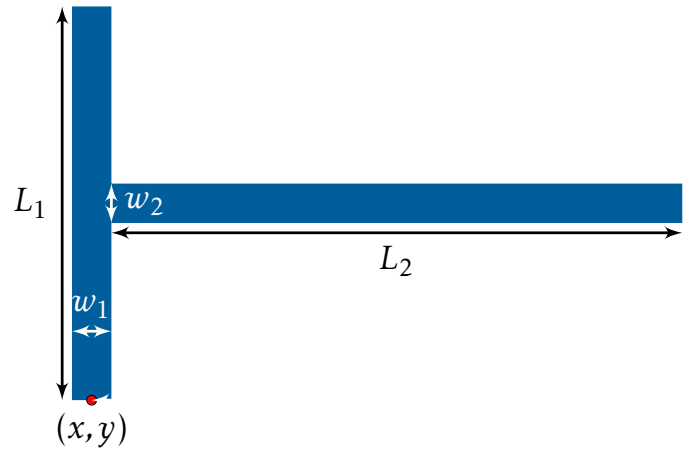

(a)

Figure 2.69: $T$ junction (a) with and (b) without $(r=0)$ circular ports. 


\subsubsection{H Junction}

$\begin{array}{llllllllll}x & y & w_{1} & w_{2} & L_{1} & L_{2} & r & N_{\text {sides }} & \theta_{(x, y)} & \text { hJunction }\end{array}$

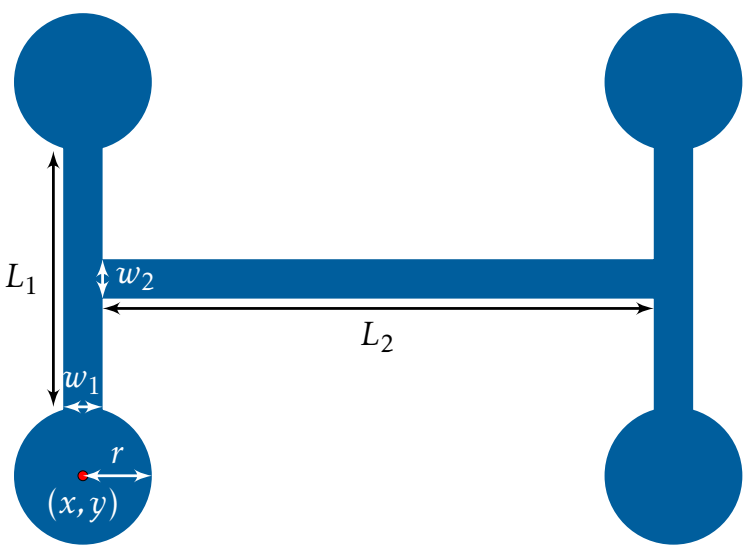

(a)

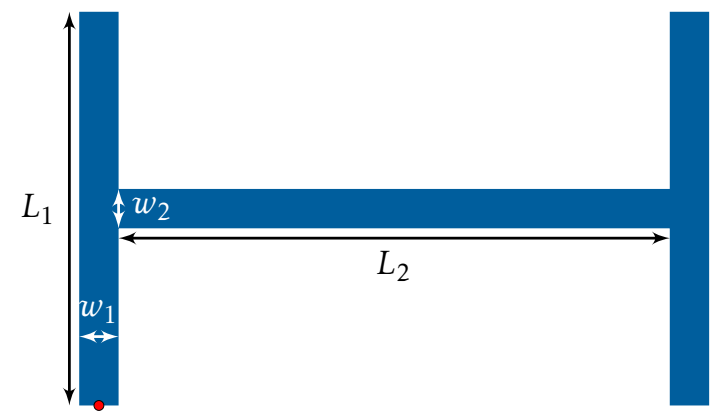

(b)

Figure 2.70: H junction (a) with and (b) without $(r=0)$ circular ports. 


\subsubsection{Arrow Junction}

$\begin{array}{lllllllllll}x & y & w_{1} & w_{2} & L_{1} & L_{2} & r & N_{\text {sides }} & \theta & \theta_{(x, y)} & \text { arrowJunction }\end{array}$

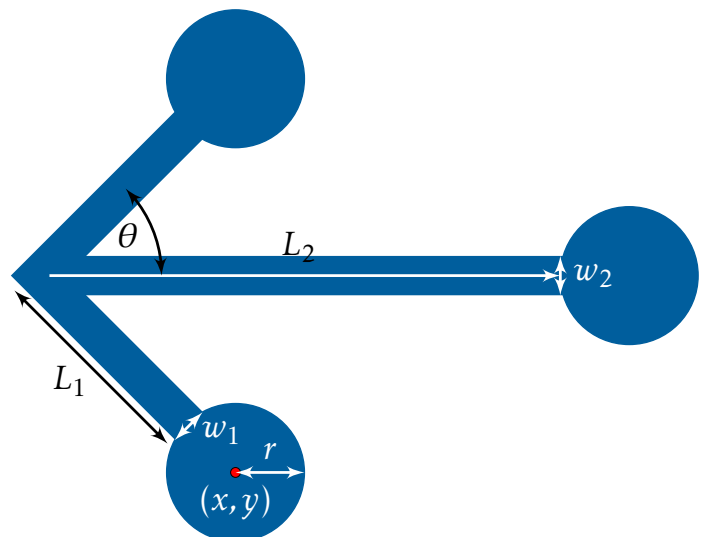

(a)

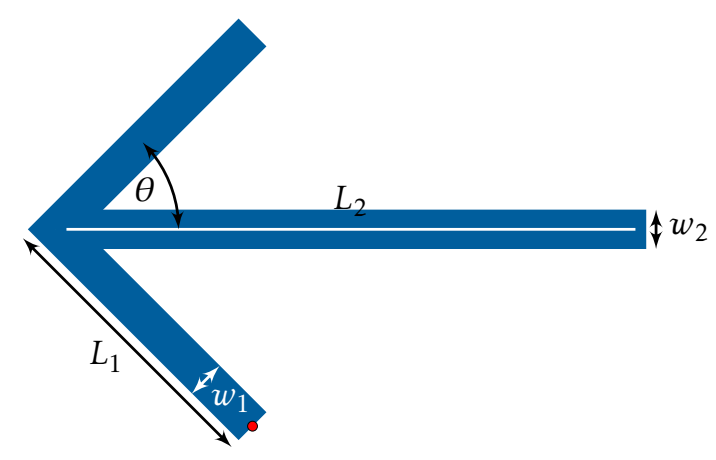

(b)

Figure 2.71: Arrow junction (a) with and (b) without $(r=0)$ circular ports. 


\subsubsection{Meander Channel}

The method constructs a meandering channel with rectangular ports. These structures find potential use in fluidic and gas delivery system applications. Four available constructors represent various wavelike structures including sine, square, ramp and triangle. Rectangular ports, residing at each channel end, are defined by the length $\left(L_{1}\right.$ and $\left.L_{2}\right)$ and height $\left(H_{1}\right.$ and $\left.H_{2}\right)$ parameters. Connections to the reservoirs are made through segments $a$ and $b$ of width $W$. Wave structures initiate at $x=a$ and are of length $b$. Number of wave periods is defined by $N$. The sine wave structure is constructed from a number of segments defined by the parameter $N_{\text {segments }}$.

$\begin{array}{lllllllllllllll}<x & y & L_{1} & H_{1} & L_{2} & H_{2} & W & A & N & N_{\text {segments }} & a & b & c & \theta_{(x, y)} & \text { meanderSin> } \\ <x & y & L_{1} & H_{1} & L_{2} & H_{2} & W & A & N & & a & b & c & \theta_{(x, y)} & \text { meanderSqr> } \\ <x & y & L_{1} & H_{1} & L_{2} & H_{2} & W & A & N & & a & b & c & \theta_{(x, y)} & \text { meanderRamp }> \\ <x & y & L_{1} & H_{1} & L_{2} & H_{2} & W & A & N & & a & b & c & \theta_{(x, y)} & \text { meanderTri }\end{array}$

Figure 2.72 illustrates the four meander channel configurations with respective parameters. As shown in FigurerefmeanderPortsExample, rectangular reservoirs at each channel end can be eliminated by setting respective length and height parameters equal to 0 , i.e. $L_{1}=H_{1}=0$ and/or $L_{2}=H_{2}=0$. 


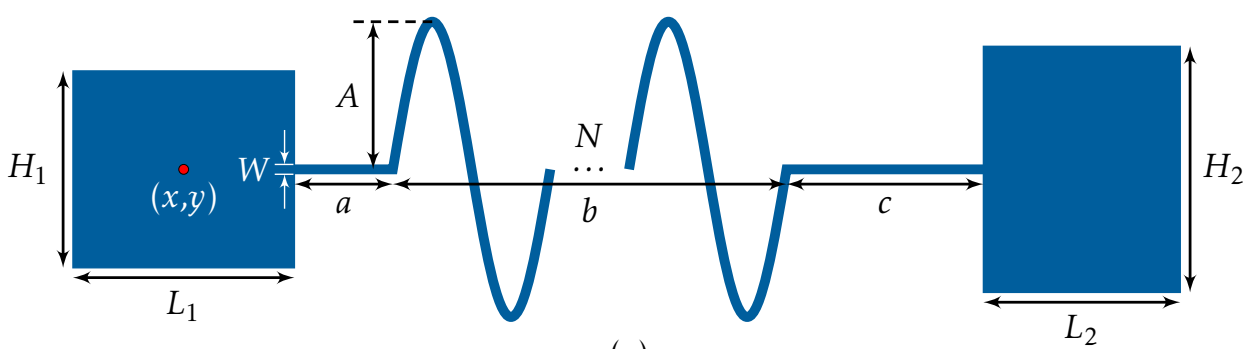

(a)

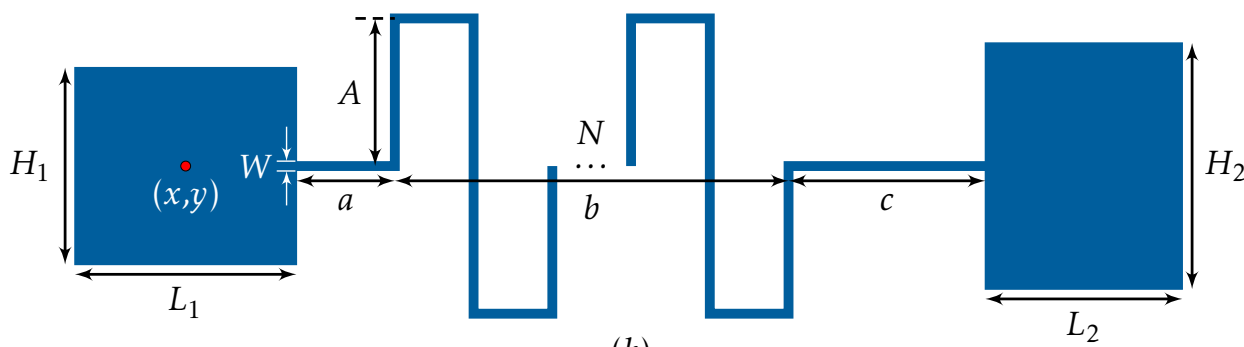

(b)

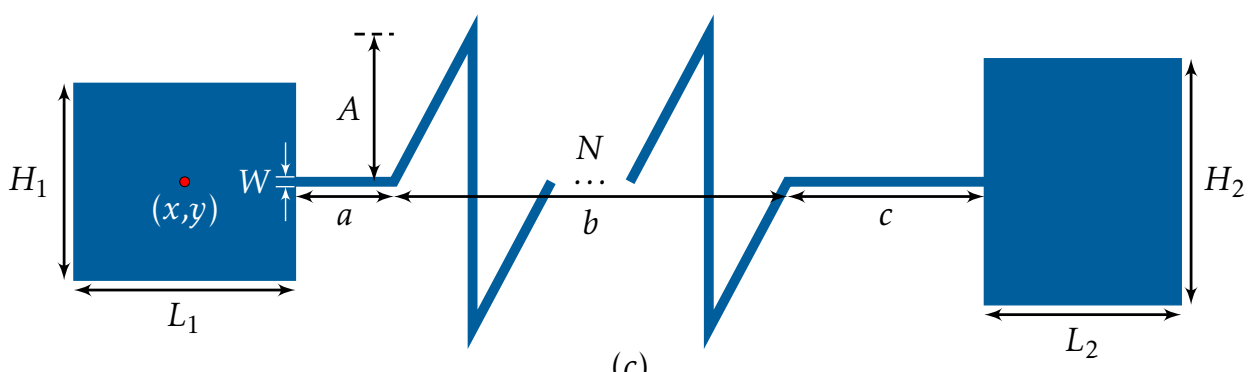

(c)

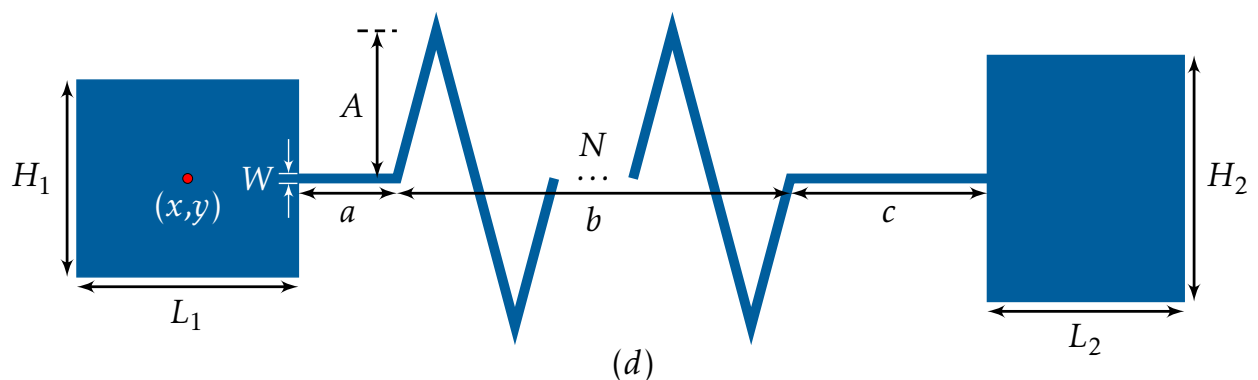

Figure 2.72: Example shapes illustrating various parameters from the (a) meanderSin, (b) meanderSqr, (c) meanderRamp and (d) meanderTri constructors. 


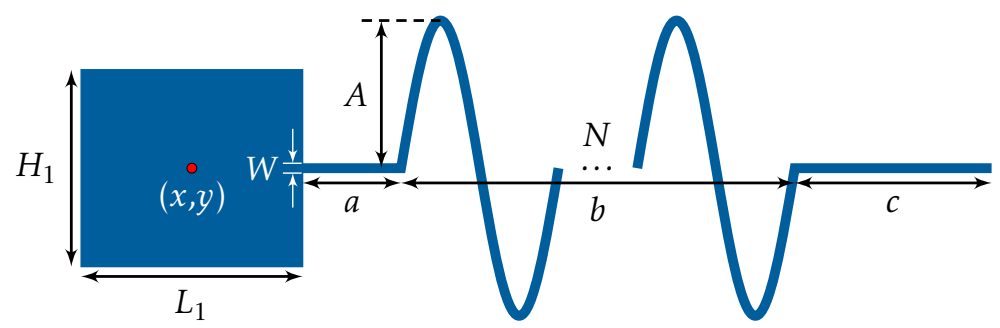

(a)

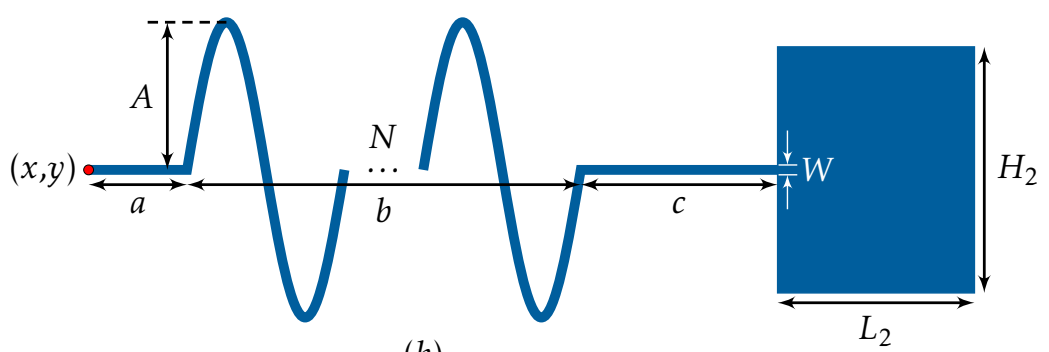

(b)

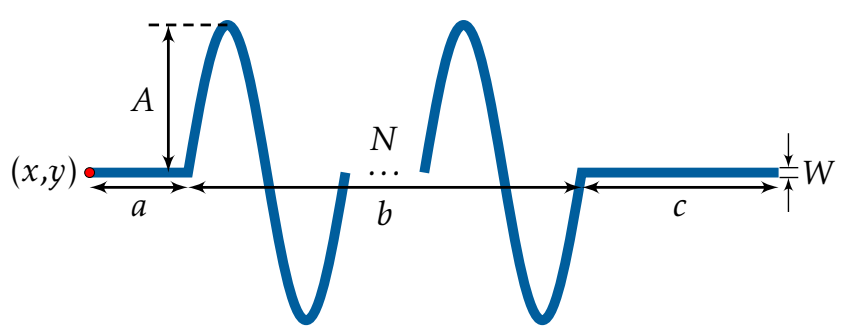

(c)

Figure 2.73: Meander channel example illustrating various rectangular reservoir configurations. Structures without (a) right reservoir using $\mathrm{L}_{2}=\mathrm{H}_{2}=0$, (b) left reservoir using $L_{1}=H_{1}=0$ and (c) reservoirs using $L_{1}=H_{1}=L_{2}=H_{2}=0$. 


\subsubsection{Points To Shape}

The method creates a polygon from user defined $(x, y)$ point pairs. Last point $\left(x_{n}, y_{n}\right)$ connects the first $\left(x_{1}, y_{1}\right)$ to close the shape.

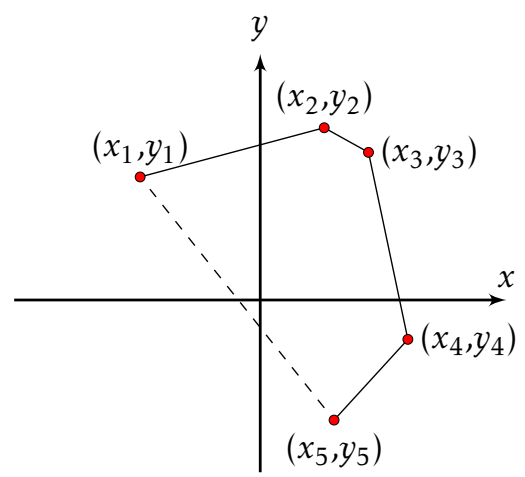

Figure 2.74: Example of the points2shape constructor illustrating a closed shape constructed from points 5 points, $\left(x_{1}, y_{1}\right) \ldots\left(x_{5}, y_{5}\right)$.

$\begin{array}{llllllll}x_{1} & y_{1} & x_{2} & y_{2} & \cdots & x_{n} & y_{n} & \text { points2shape }\end{array}$

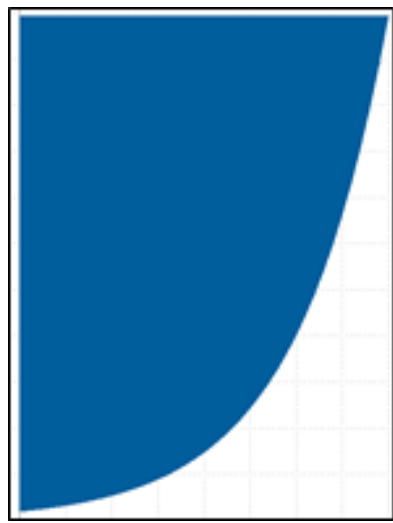

Figure 2.75: points2shape example illustrating a GDS rendered polygon. 


\subsubsection{Polygon Along a Path}

The method creates a polygon of a specified width $(W)$ along a path defined by $(x, y)$ point pairs. Cap is represented by the following integer values $B U T T=0$, $R O U N D=1$, or $S Q U A R E=2$. Join is represented by the following integer values MITER $=0, R O U N D=1, B E V E L=2$.

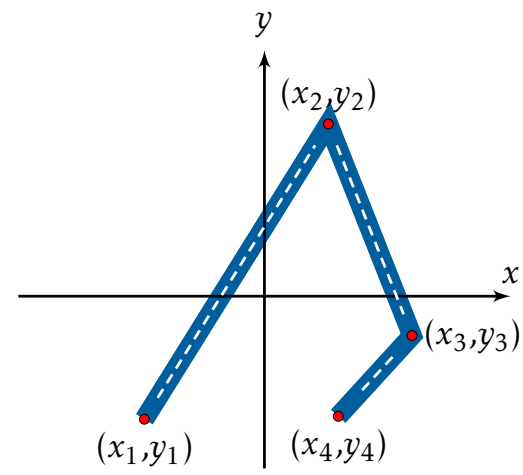

Figure 2.76: Example of the polypath constructor illustrating a rendered shape constructed from points 5 points, $\left(x_{1}, y_{1}\right) \ldots\left(x_{5}, y_{5}\right)$.

$\begin{array}{llllllllll}x_{1} & y_{1} & x_{2} & y_{2} & \cdots & x_{n} & y_{n} & W & \text { Cap Join polypath }\end{array}$

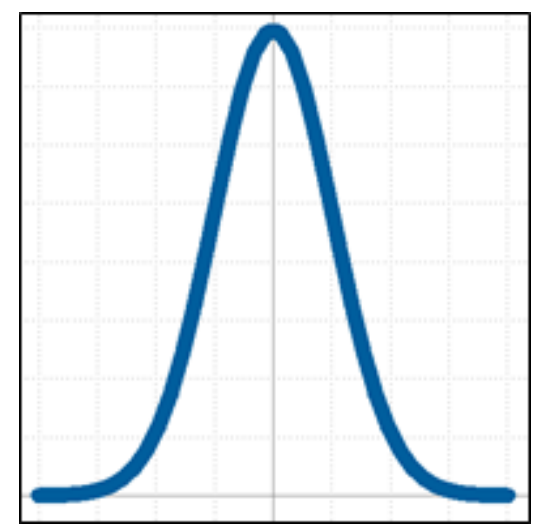

Figure 2.77: polypath example illustrating a GDS polygon along a Gaussian path. 


\subsubsection{Random Polygons}

The below constructor creates randomly placed polygons into a user defined area. Single polygon of radius $r$ with $N_{s}$ sides is created and cast into a GDS struct with the name uniqueStructName. The $N_{e}$ number of shapes are then instantiated into an area of width $W$ and height $H$. Separation parameter $S$ defines the minimum separation between the outer radial perimeter of the objects. If the randomly generated coordinate violates the minimum separation distance, the module will keep generating random coordinates until the maximum number of failures (defined by the parameter iteration parameters $I$ ) is reached. The lower left corner of the random array is positioned at $(x, y)$.

Random rotation is enabled if $R_{R}$ is set to 1 , each shape will be randomly rotated within the current GDS structure. Any other numeric value or $R_{R}$ will leave the shape element unchanged. Enabling the $R_{R}$ option for for features with many sides is not particularly useful, however, with $S=3$ and $R_{R}=1$ produces randomly oriented triangles (see Figure 2.78).

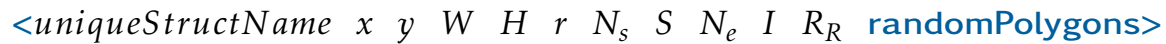

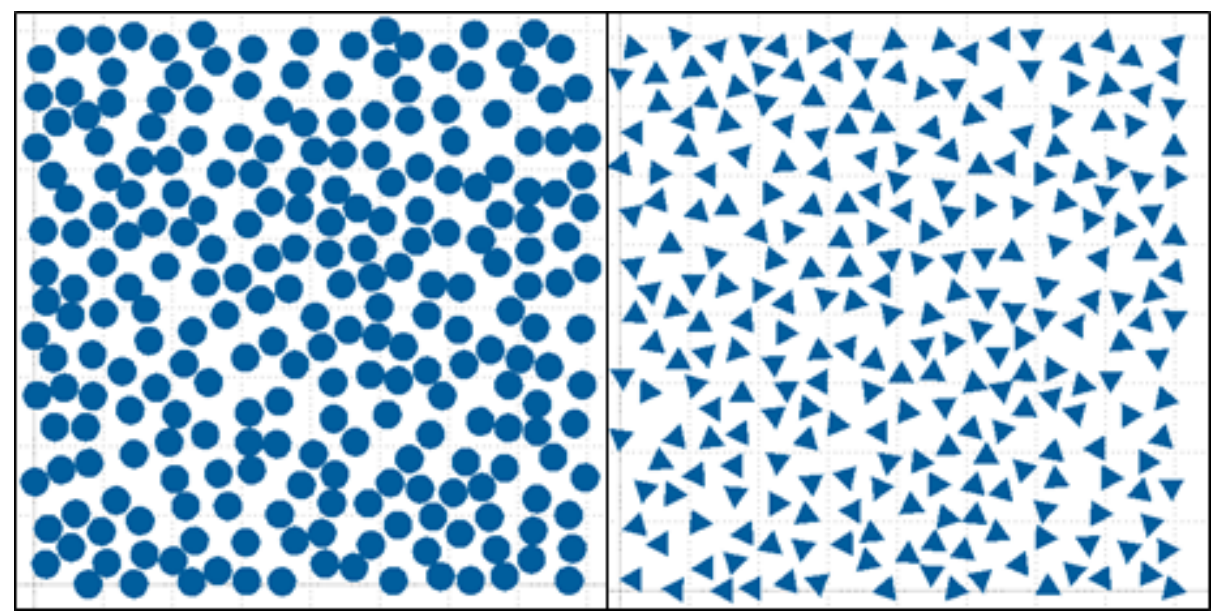

Figure 2.78: View of a generated GDS file using the Random Polygons module. The area was $40 \mu \mathrm{m} \times 40 \mu \mathrm{m}$, number of elements and iterations were set to 400 , with an element radius and separation of $1 \mu \mathrm{m}$ and $2 \mu \mathrm{m}$ respectively. Generated shapes had (a) 44 sides and (b) 3 sides with random rotation enabled. 


\subsubsection{Random Ellipses and Vectorized Ellipses}

Similar to the random polygon example, the following constructors create random ellipses (randomEllipses) and vectorized ellipses (randomEllipsesV). shapeReso defines the rendering resolution of randomEllipsesV.

$$
\begin{array}{lllllllllllllll}
\text { <uniqueStructName } & x & y & W & H & r_{x} & r_{y} & N_{s} & S & N_{e} & I & R_{R} & \text { randomEllipses }> \\
\text { <uniqueStructName } & x & y & W & H & r_{x} & r_{y} & & S & N_{e} & I & R_{R} & \text { randomEllipsesV> }
\end{array}
$$

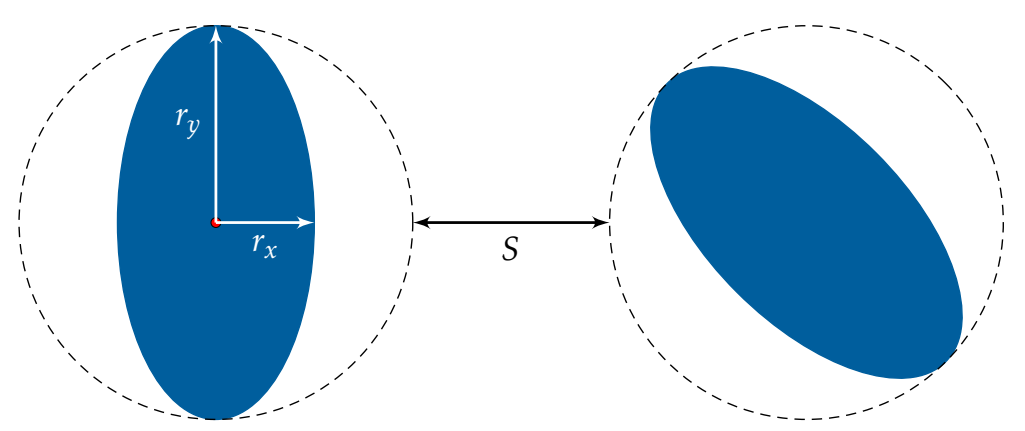

Figure 2.79: Random ellipse example showing separation (S) between adjacent circular boundaries defined by the radius $r_{y}$.

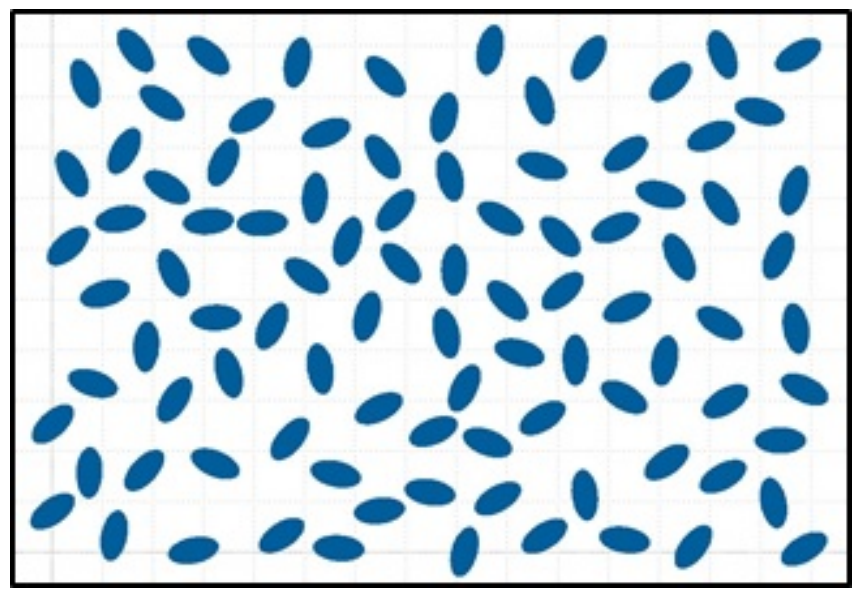

Figure 2.80: GDS rendered random ellipses with random rotation enabled $\left(R_{R}=1\right)$. 


\subsubsection{Resolution Test Pattern}

Radial pattern below is useful for characterizing the stigmation of a lithography tool. Using the resoPattern constructor, the structure is defined by the center origin $(x, y)$, radius $(r)$ and width $(w)$. In this scenario, to ensure pattern symmetry, $360 / w=i$, where $i=2,4, \ldots, n$ is an even positive integer. For even distribution of segments across the $2 \pi r$ circumference, $i$ can be any positive integer greater than zero. The second constructor (resoPatternPi) creates a similar figure where the end-segment is an integer of $\pi$ width.

$\begin{array}{llllll}x & y & r & w & \theta_{(x, y)} & \text { resoPattern } \\ x & y & r & n & \theta_{(x, y)} & \text { resoPatternPi }\end{array}$

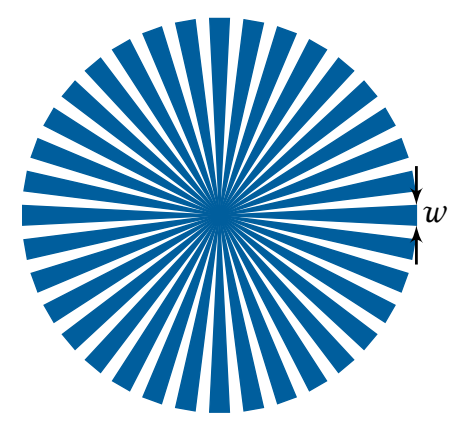

(a)

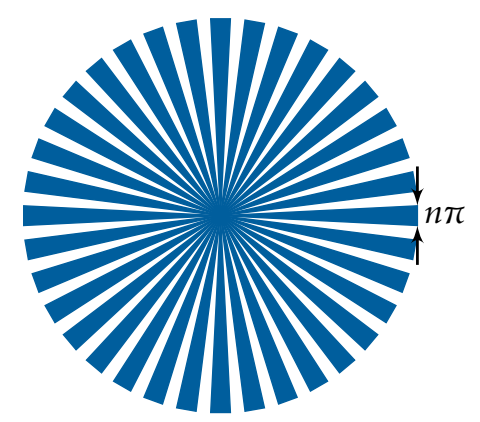

(b)

Figure 2.81: Resolution patterns using (a) resoPattern and (b) resoPatternPi constructors. 
Resolution pattern formed using two rows of rectangles of width $W$, height $H$, pitch $2 W$ and $N$ elements in each row. A text label is placed directly above the array of lines indicating the value of $W$. The font resolution is defined using the global shapeReso parameter. The font height is equal to the value of $H$ (text label in Figure 2.82 is not shown to scale).

$$
\begin{array}{lllllll}
x & y & W & H & N & \theta_{(x, y)} & \text { resoPatternRS }
\end{array}
$$

The following constructor creates arrays of rectangular shaped resolution patterns using a start and end width values $\left(W_{s}\right.$ and $\left.W_{e}\right)$, with a $\delta$ incremental variation. The space between the arrays is defined by the parameter $S$ in micrometer units.
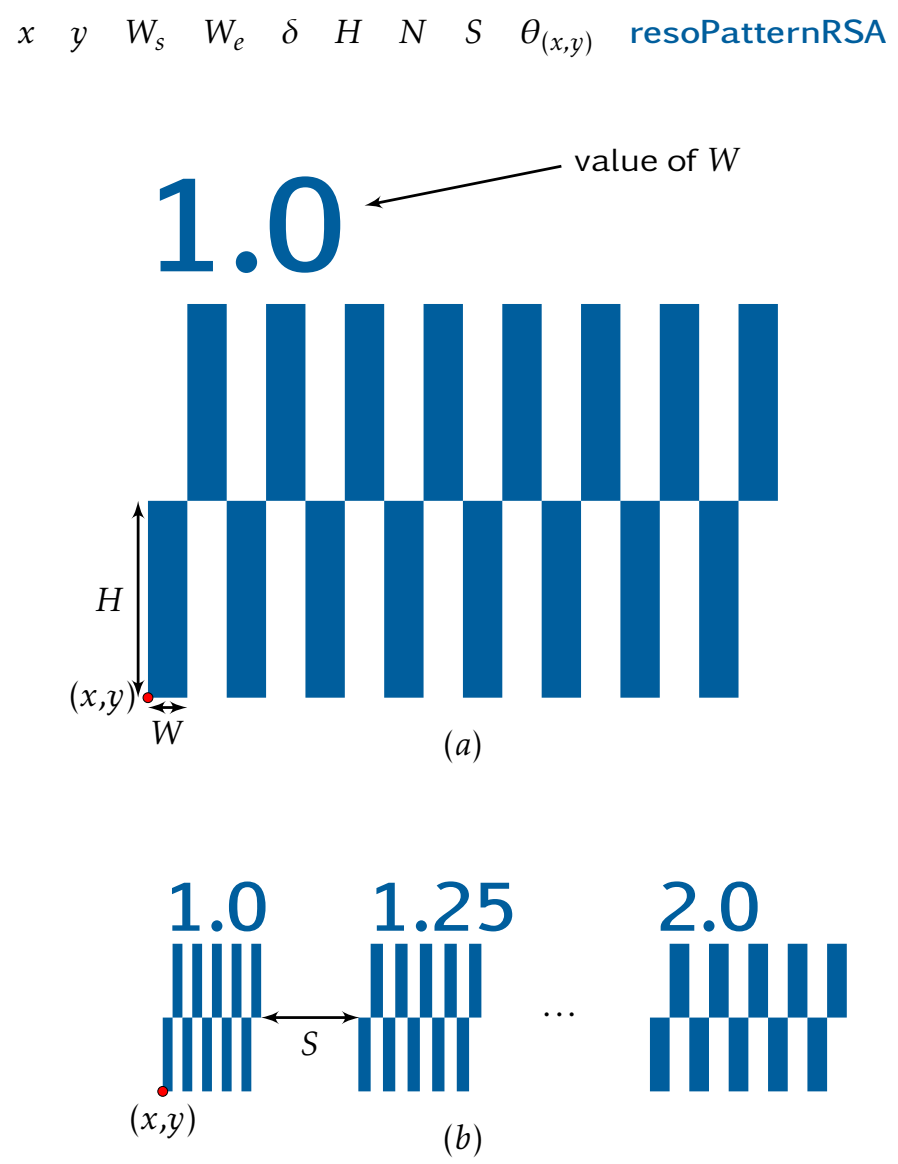

Figure 2.82: Resolution pattern of rectangular lines using (a) resoPatternRS and (b) resoPatternRSA constructors. 
The following resolution patter is formed using L-shapes of width $W$, height $H$, pitch $2 W$ and $N$ number of shapes. The maximum number of generated shapes is defined when $W=H$. Text label definition is identical to one described for the resoPatternRS constructor (previous example).

$$
\begin{array}{lllllll}
x & y & W & H & N & \theta_{(x, y)} & \text { resoPatternLS }
\end{array}
$$

An arrayed version of the above constructor is defined by a start and end width values $\left(W_{s}\right.$ and $\left.W_{e}\right)$, with a $\delta$ incremental variation. The space between the arrays is defined by the parameter $S$ in micrometer units.

$$
\begin{array}{llllllllll}
x & y & W_{s} & W_{e} & \delta & H & N & S & \theta_{(x, y)} & \text { resoPatternLSA }
\end{array}
$$

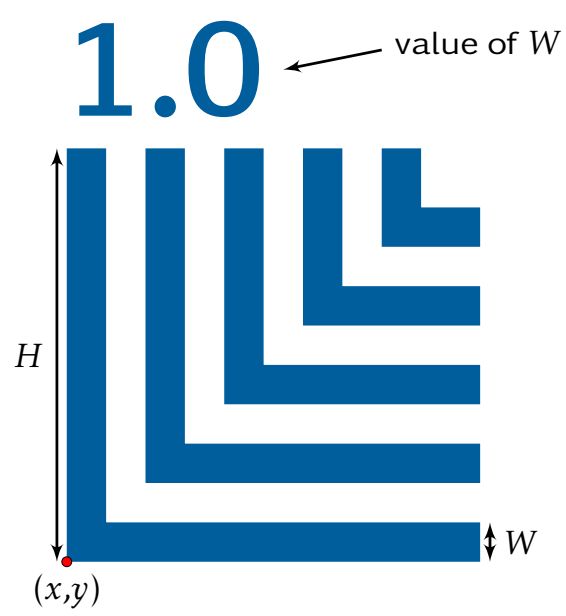

(a)

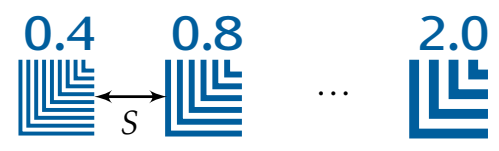

(b)

Figure 2.83: Resolution pattern of rectangular lines using (a) resoPatternLS and (b) resoPatternLSA constructors. 


\subsubsection{Spirals}

Spiral objects are characterized by uniform, converging and diverging spacing between subsequent turns. Constant spacing represents an Archimedes spiral where $r=m \theta$, where $m=($ separation + width $) /(2 \pi)$ (Figure 2.84a). Separation parameter defines the pitch between subsequent spiral turns. Fermats spiral, defined as $r=\sqrt{a^{2} \theta}$, is shown in Figure 2.84b. The logarithmic spiral, defined as $r=a e^{b \theta}$, is shown in Figure 2.84c. Center of each spiral is defined by the point $(x, y)$.

\begin{tabular}{|c|c|c|c|c|c|c|}
\hline$x$ & $y$ & Width & $N_{\text {turns }}$ & Separation & increment & spiralArch \\
\hline$x$ & $y$ & Width & $N_{\text {turns }}$ & $a$ & increment & spiralFermat \\
\hline & $y$ & Width & $N_{\text {turns }}$ & $b$ & increment & spiralLog \\
\hline
\end{tabular}

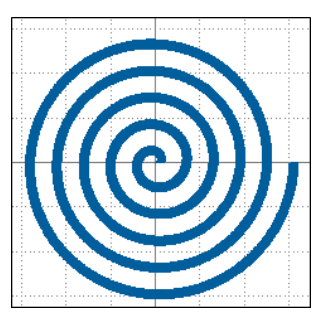

(a)

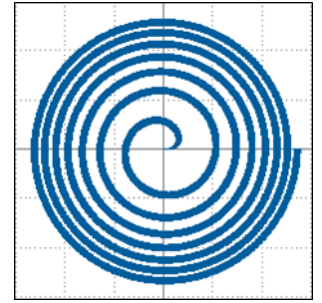

(b)

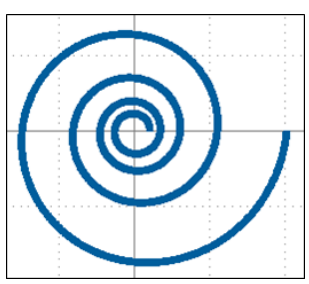

(c)

Figure 2.84: GDS rendered spiral examples. (a) Archimedes (b) Fermat and (c) Logarithmic spiral.

The following scripts were used to generate the spirals centered around the origin $(x=0, y=0)$ in Figure 2.84:
$\begin{array}{lllll}0 & 0 & 1.0 & 5 & 2.0\end{array}$
0.010 spiralArch
$\begin{array}{lllll}0 & 0 & 2.5 & 7 & 8\end{array}$
0.010 spiralFermat
$\begin{array}{lllll}0 & 0 & 4.4 & 4 & 8.0\end{array}$
$0.1 \quad 0.010 \quad$ spiralLog 


\subsubsection{Spiral - Rectangular}

Below constructor creates a rectangular spiral of $N_{t} u r n s$, of width $w$, start length $L_{s}$ and pitch $p$ between turns.

$\begin{array}{llllllllll}x & y & w & L_{s} & p & N_{t u r n s} & \theta_{(x, y)} & \text { spiralRect }\end{array}$

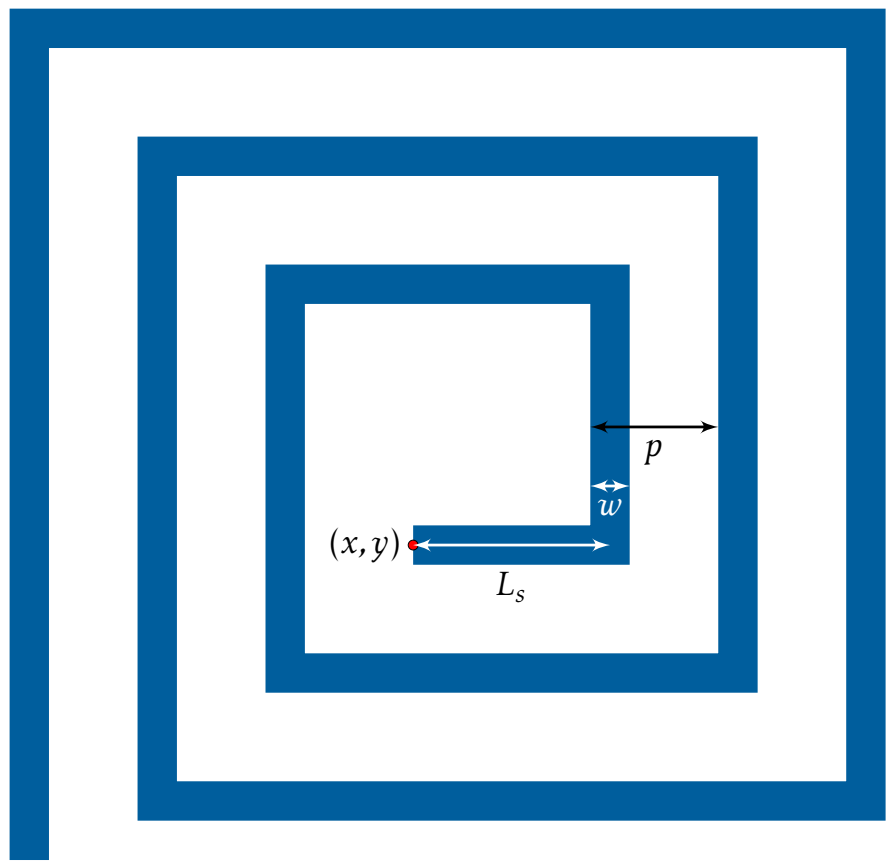

Figure 2.85: Rectangular spiral. 


\subsection{Alignment and Reticle Elements}

\subsubsection{Alignment Marks - Predefined}

The following section includes a variety of predefined, multilevel alignment mark strategies for lithographic applications. Within these methods, alignment mark size and shape values are predefined. The central dark circular aperture within each of the structures defines the $(x, y)$ position. The $\theta_{(x, y)}$ defines the rotation (in degrees) about $(x, y)$.

The following are constructors for two- and three-level alignment marks shown in figures 2.86 and 2.87. $L_{a}, L_{b}$ and $L_{c}$ are integer values $(0-255)$ representing GDS layer numbers. Pattern extents for alignFFFB1 and alignFFFB2 alignment patterns in figure 2.86$)$ range from $(-80 \mu \mathrm{m},-80 \mu \mathrm{m})$ to $(80 \mu \mathrm{m}, 80 \mu \mathrm{m})$. For the three-level alignment mark system (align3Level), pattern extents range from $(-1000 \mu \mathrm{m},-1000 \mu \mathrm{m})$ to $(1000 \mu \mathrm{m}, 1000 \mu \mathrm{m})$.

$$
\begin{array}{ccccccc}
<x & y & L_{a} & L_{b} & & \theta_{(x, y)} & \text { alignFFFB1> } \\
<x & y & L_{a} & L_{b} & & \theta_{(x, y)} & \text { alignFFFB2> } \\
<x & y & L_{a} & L_{b} & L_{c} & \theta_{(x, y)} & \text { align3Level }>
\end{array}
$$

The following defines a constructor for a two level alignment system with verniers and options for tone reversal of layers within a specified region (figure 2.88). As defined above, $L_{a}$ and $L_{b}$ are integer values defining the two rendered GDS layer numbers. Parameter vernReso defines the resolution between the two verniers in units of $\mu \mathrm{m}$. Layers $L_{a}$ and/or $L_{b}$ are tone-reversed within an area defined by the inversion length $\left(I_{L}\right)$ and width $\left(_{W}\right)$ if their respective boolean flags $I N V_{a}$ and $I N V_{b}$ are activated. The boolean inversion parameters are activated using any integer value other than 0. Pattern extents range from $(-900 \mu \mathrm{m},-900 \mu \mathrm{m})$ to $(900 \mu \mathrm{m}, 900 \mu \mathrm{m})$.

$$
<x \quad y \quad L_{a} \quad L_{b} \text { vernReso } I_{N} V_{a} \quad I N V_{b} \quad I_{L} \quad I_{W} \quad \theta_{(x, y)} \text { alignVern> }
$$

Two-level alignment strategies defined in figure 2.89 contains verniers and GDS layer number labels. As defined directly above, $L_{a}$ and $L_{b}$ are GDS layer numbers, and vernReso parameter defines the resolution between the two verniers. alignVernLb1 pattern extents in range from $(-1000 \mu \mathrm{m},-1000 \mu \mathrm{m})$ to $(1000 \mu \mathrm{m}, 1000 \mu \mathrm{m})$, whereas alignVernLb2 pattern extents in range from $(-230 \mu \mathrm{m},-230 \mu \mathrm{m})$ to $(117 \mu \mathrm{m}, 155 \mu \mathrm{m})$.

$$
\begin{array}{lllllll}
<x & y & L_{a} & L_{b} & \text { vernReso } & \theta_{(x, y)} & \text { alignVernLb1> } \\
<x & y & L_{a} & L_{b} & \text { vernReso } & \theta_{(x, y)} & \text { alignVernLb2> }
\end{array}
$$

NIST • CNST Nanolithography Toolbox v2016.09.01 • http://www.nist.gov/cnst/ page 101 of 488 

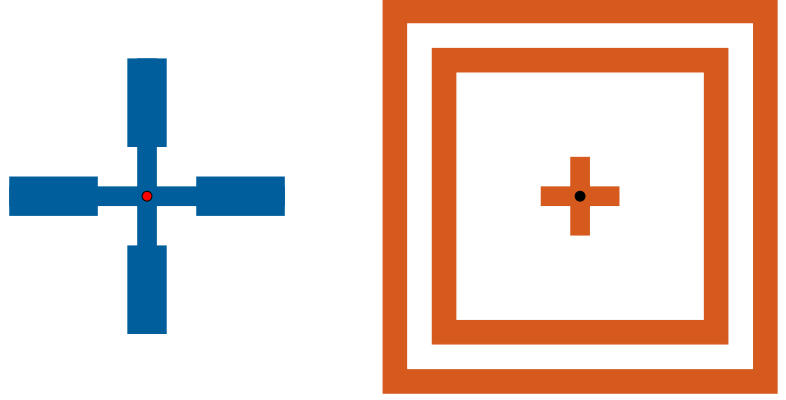

(a)
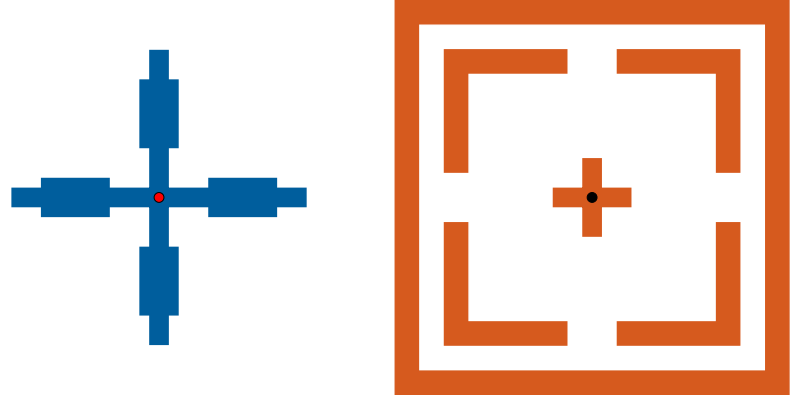

(b)

Figure 2.86: Two level lithography alignment mark strategies constructed using (a) alignFFFB1 and (b) alignFFFB2. Two layers $L_{a}$, and $L_{b}$ are shown separately as blue (left) and orange (right). These methods could be used for front-to-front or front-to-back alignment. 


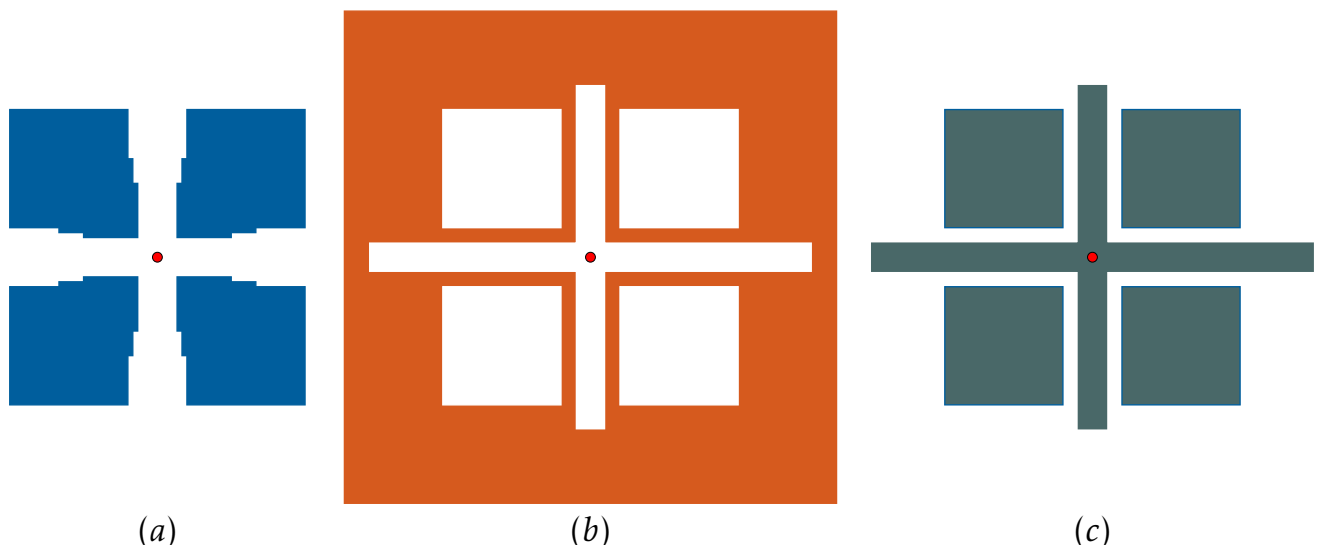

Figure 2.87: Three-level, front-side, lithography alignment mark strategy constructed using align 3 Level with layers (a) $L_{a}$, (b) $L_{b}$ and (c) $L_{c}$.

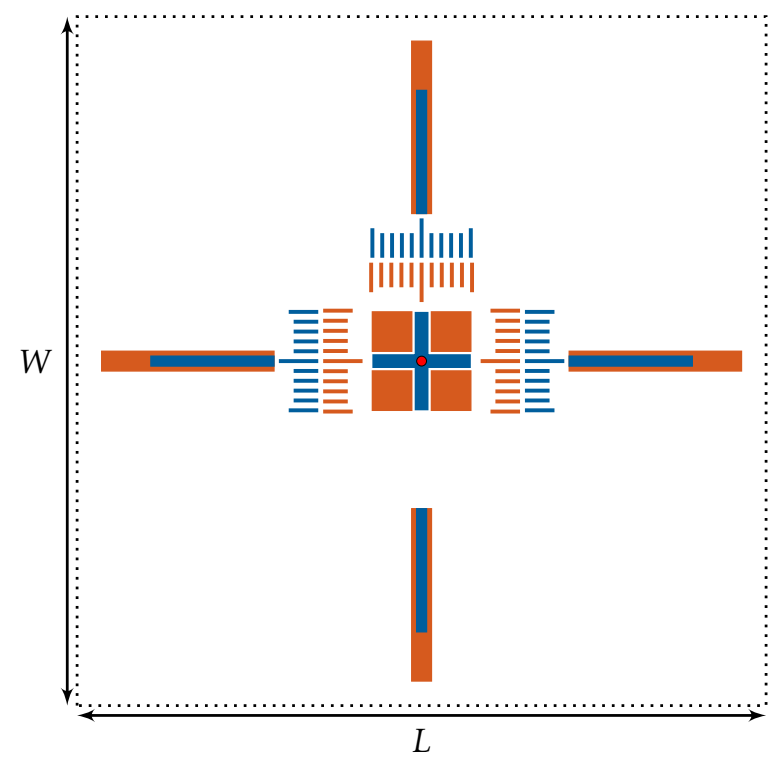

Figure 2.88: Two-level alignment mark strategy with $x$ and $y$ verniers constructed using alignVern. Blue and orange layers are $L_{a}$ and $L_{b}$ respectively. Length $\left(I_{L}\right)$ and width $\left(I_{W}\right)$ parameters define the region extent used for tone reversal of the specified alignment layers. This method is suitable for conventional overlay lithography applications. 


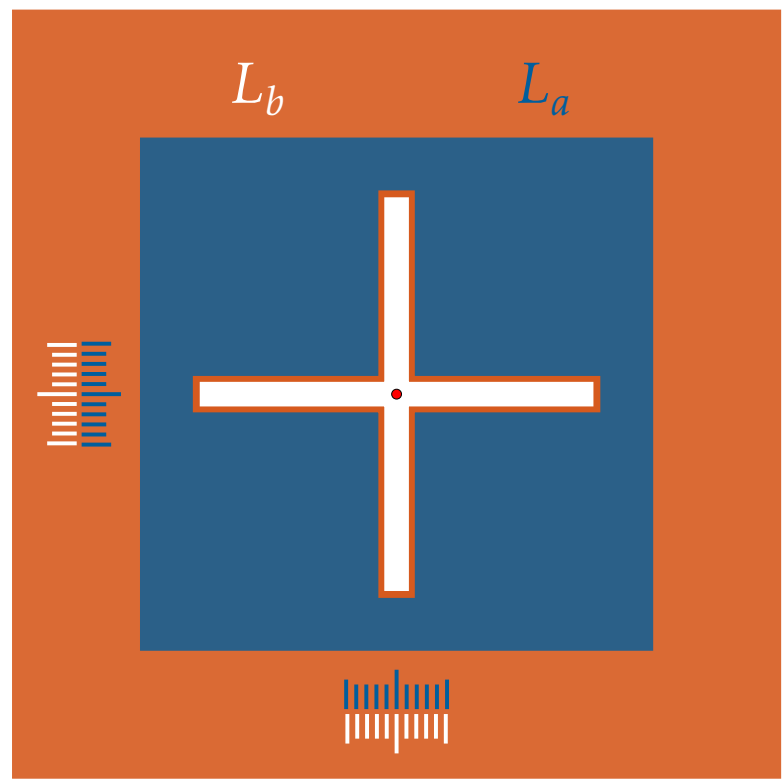

(a)

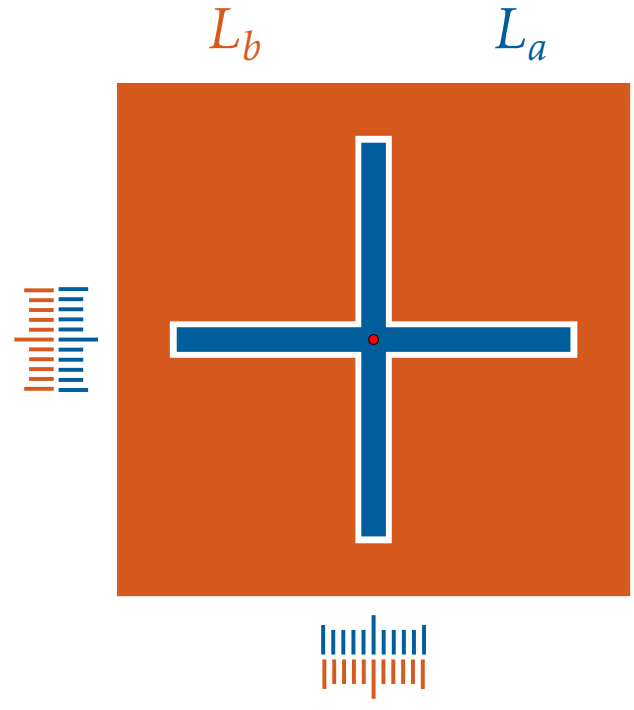

(b)

Figure 2.89: Two-level alignment mark strategies with $x$ and $y$ verniers, and layer labels constructed using (a) alignVernLb1 and (b) alignVernLb2. Blue and orange layers are $L_{a}$ and $L_{b}$ respectively. These method is suitable for conventional overlay lithography applications. 


\subsubsection{Alignment Marks - Custom}

The following section includes 4 types of cross-based marks for multilevel lithographic alignment. Unlike section 2.8.1 with predefined marks, the following mark definitions are fully customizable by user defined parameters. Figure 2.90 shows a small circle at the center of each shape denoting the translation point $(x, y)$. Each shape undergoes a rotation by $\theta_{(x, y)}$ about the center point $(x, y)$. The dotted box of length $I_{L}$ and width $I_{W}$ denotes the boundary within which layer inversion takes place. Boolean tone reversal (inversion) is activated by the parameter $I N V$. A value $I N V=0$ signifies that tone reversal within the specified boundary will not take place. Any other integer value for INV will invert the tone of the mark. Layers are set by the usual layer command (see section 2.2.4).

Figure 2.90a shows a simple cross defined by a width $(W)$, a horizontal and vertical length segments $\left(L_{1}\right.$ and $\left.L_{2}\right)$.

$$
\begin{array}{lllllllllll}
<x & y & L_{1} & W & L_{2} & I N V & I_{L} & I_{W} & \theta_{(x, y)} & \text { alignCustC1 }
\end{array}
$$

Figure 2.90b shows a cross of width $(W)$, length $(L)$, paddle length $\left(P_{L}\right)$ and width $\left(P_{W}\right)$.

$$
\begin{array}{lllllllllllll}
<x & y & L & W & P_{L} & P_{W} & I N V & I_{L} & I_{W} & \theta_{(x, y)} & \text { alignCustC2 }
\end{array}
$$

Figure 2.90c shows a cross of width $(W)$, length $(L)$, paddle length $\left(P_{L}\right)$ and width $\left(P_{W}\right)$ offset by a length $\left(L_{X}\right)$ from the edge.

$$
\begin{array}{llllllllllllll}
< & y & L_{1} & W & P_{L} & P_{W} & L_{X} & I N V & I_{L} & I_{W} & \theta_{(x, y)} & \text { alignCustC3 }
\end{array}
$$

Figure $2.90 \mathrm{~d}$ shows a cross defined by a width $(W)$, a horizontal and vertical length segments $\left(L_{1}\right.$ and $\left.L_{2}\right)$, and a rectangle of length $\left(B_{L}\right)$ and width $\left(B_{W}\right)$ at a distance $(d)$ from the cross edge.

$$
\begin{array}{llllllllllllll}
<x & y & L_{1} & W & L_{2} & d & B_{L} & B_{W} & I N V & I_{L} & I_{W} & \theta_{(x, y)} & \text { alignCustC4 }
\end{array}
$$




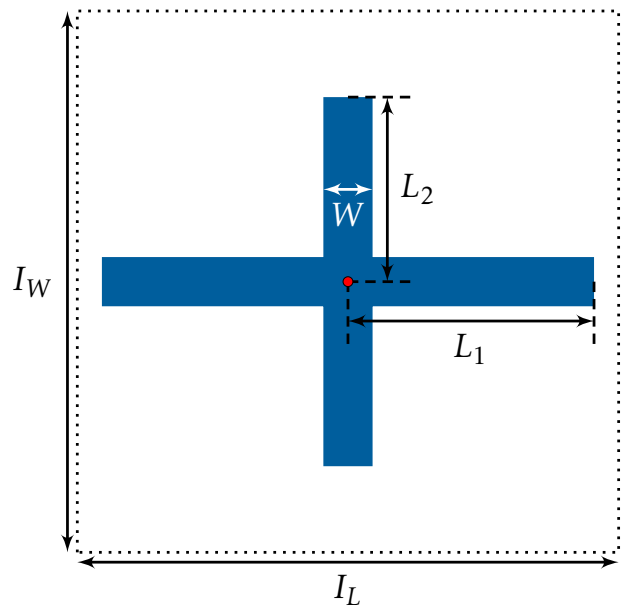

(a)

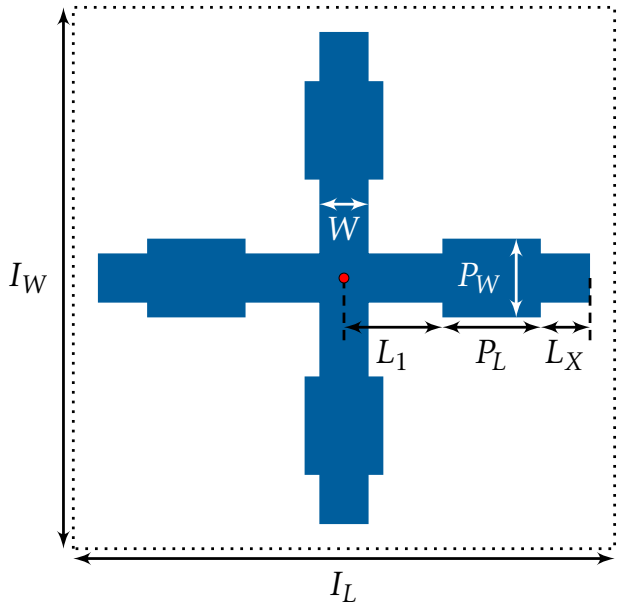

(c)

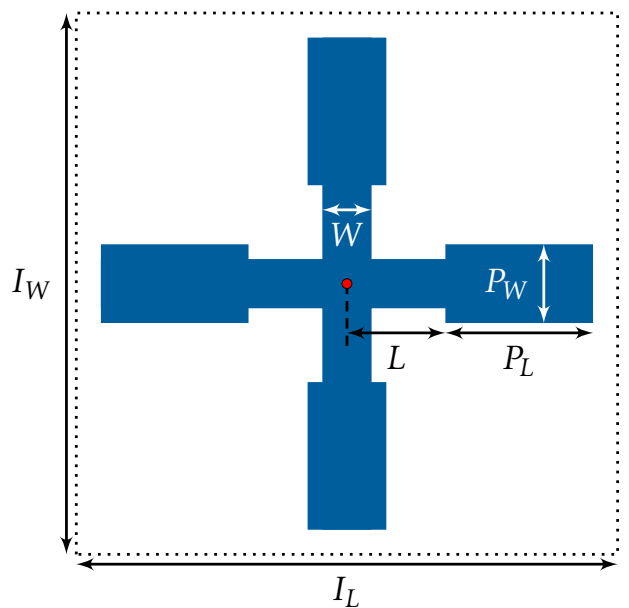

(b)

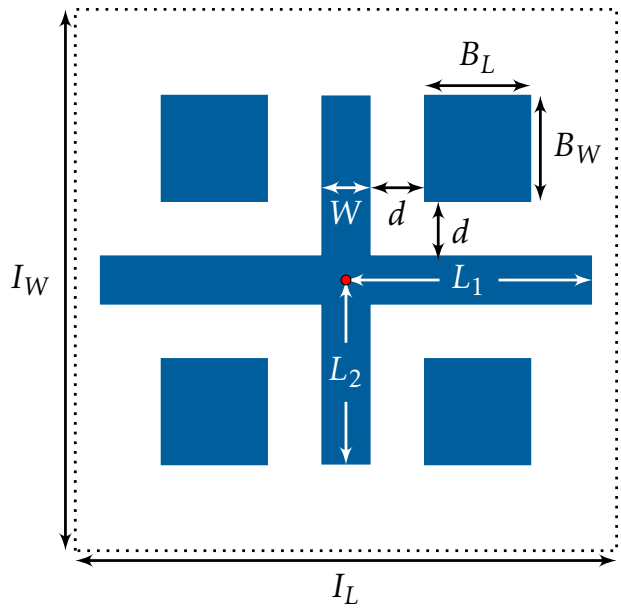

(d)

Figure 2.90: Custom alignment crosses using (a) alignCustC1, (b) alignCustC2, (c) alignCustC3, (d) alignCustC 4 constructors. 


\subsubsection{Reticle Barcode and Label Frames}

The following methods create reticle marks, barcodes and labels for the CNST ASMLPAS5500 i-line stepper and reticle labels for contact lithography tools (5 inch and 7 inch). Contact reticle frames contain a label (up to 22 characters) along with a NIST and CNST logos. In all cases MIRROR is parameter that defines if the final structure will be mirrored. Any integer other than 0 will mirror the resulting reticle frame.

CNST ASML PAS5500 stepper frame with reticle marks, barcode, label and logo:

$$
<\{\{\text { labelText }\}\} \quad\{\{\text { barCode }\}\} \text { MIRROR cnstASML }>
$$

Contact Frames:

$$
\begin{aligned}
& <\{\{\text { labelText }\}\} \text { MIRROR cnstContact5 }> \\
& <\{\{\text { labelText }\}\} \text { MIRROR cnstContact7 }>
\end{aligned}
$$

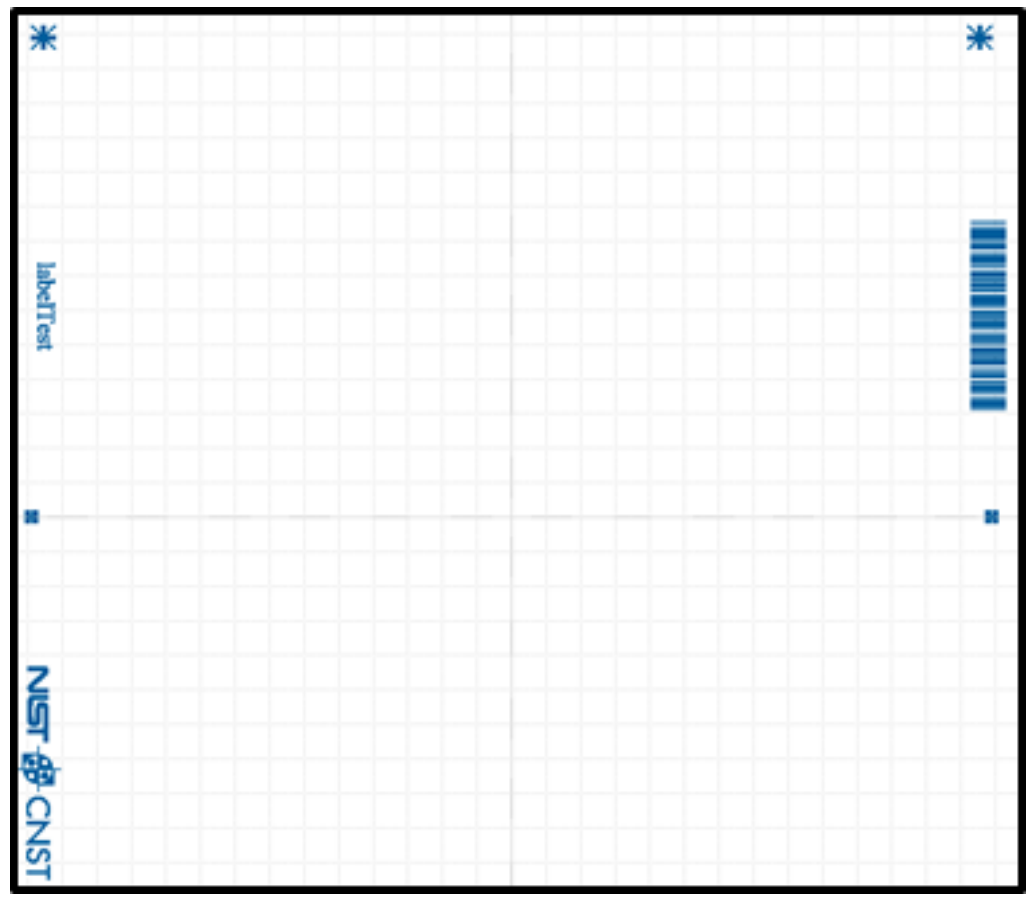

Figure 2.91: CNST ASML PAS5500 reticle frame with MIRROR $=0$. 


\subsubsection{Vernier}

Figure 2.92 shows a vernier structure with a central element of length $(L)$, width $(W)$, and pitch $(p)$. Dotted rectangle of length $\left(I_{L}\right)$ and width $\left(I_{W}\right)$ represent a boundary within which layer tone reversal takes place. With inversion parameters set to 0 , i.e. $I_{a}=0$ and/or $I_{b}=0$, boolean inversion of layers $L_{a}$ and/or $L_{b}$ will not take place. Any other integer value will invert the pattern within the bounding box. Parameter vernReso defines the resolution of the verniers.

$\begin{array}{lllllllllllllllll}< & y & L_{a} & L_{b} & L & W & p & \text { vernReso } & s & I_{a} & I_{b} & I_{L} & I_{W} & \theta_{(x, y)} & \text { alignCustVern }>\end{array}$

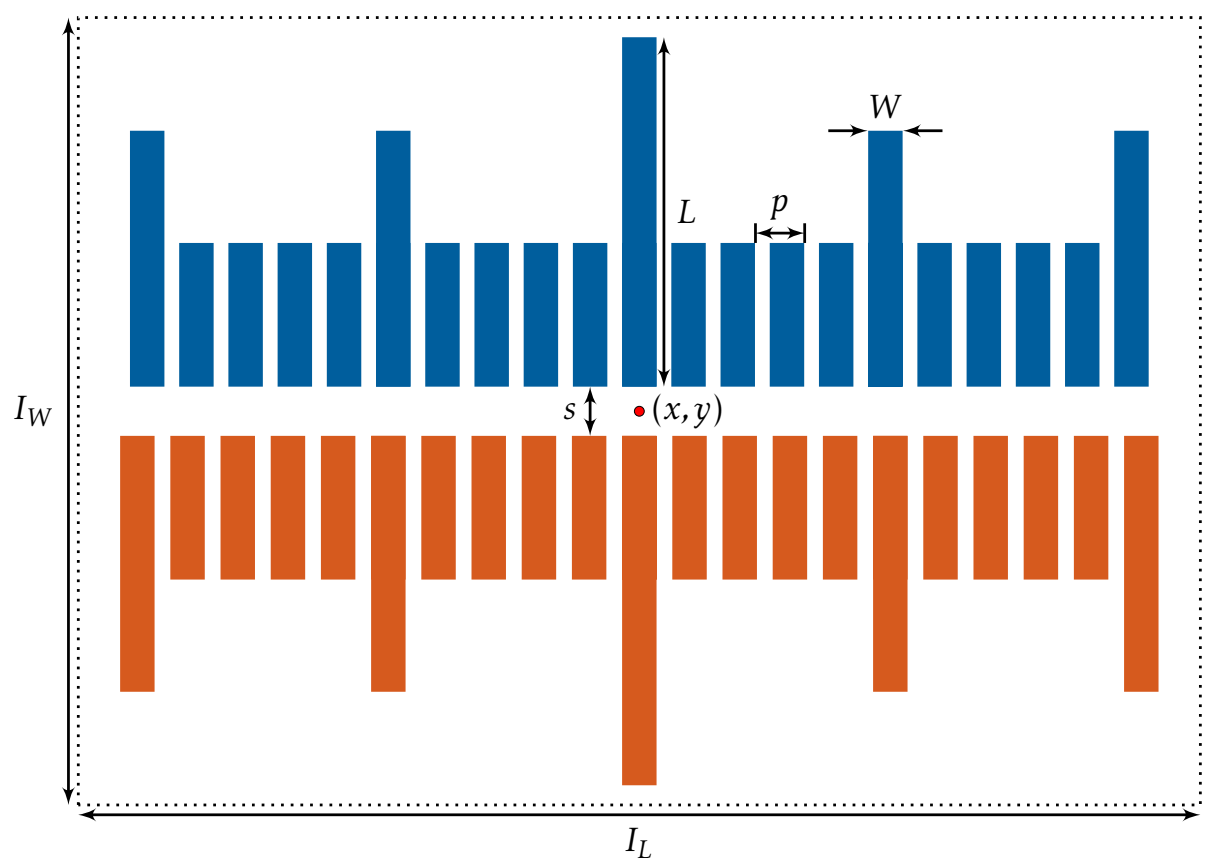

Figure 2.92: Verniers between layers $L_{a}$ (blue) and $L_{b}$ (orange) created using the alignCustVern constructor. 


\subsubsection{Vernier With Labels}

The method constructs verniers between two lithographic levels. Text resolution is set by shapeReso. Text font used is Serif. The verniers are centered at position $(x, y)$. Vernier resolution is in micrometers. The two layer parameters, LyrA and LyrB are GDS layer numbers (integers from 0 to 255), Parameter $N_{\text {ticks }}$ represents the total number of vernier bars. Text labels are defined by the fontSize and string parameters $L b l A$ and $L b l B$. These strings cannot have white characters (spaces/tabs) and are generally short layer descriptions. The width, length and pitch parameters define the vernier bars.

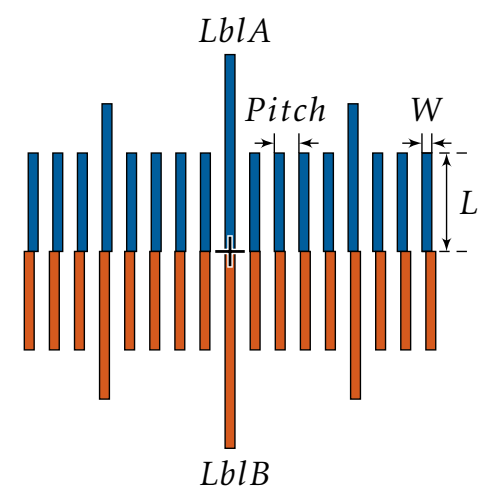

Figure 2.93: Example of the veniers constructor illustrating parameters between two layers.

$<x$ y LyrA LyrB vernierReso $N_{t i c k s}$ LblA LblB fontSize $W$ L Pitch verniers>

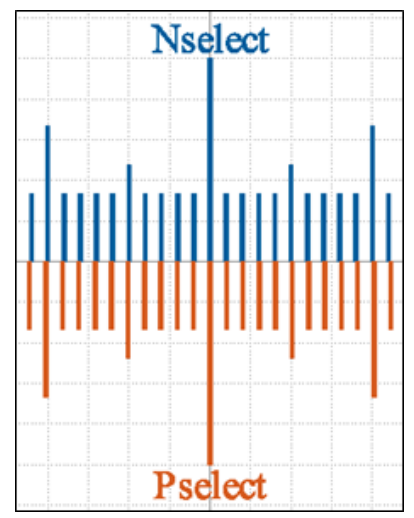

Figure 2.94: GDS rendered output of the verniers example between two lithographic levels. 


\subsubsection{Arrows}

This method constructs arrow structures that are useful in a variety of lithographic applications including labeling features and as an aid in finding features of interest, for instance, arrows pointing towards alignment marks or small, isolated devices. Each constructor is defined by parameters in the below table. An isolated arrow head is used to construct the arrow element. Constructor arrowArray defines a linear array of $N$ arrow features.

$$
\begin{array}{lllllllll}
<x & y & W_{a} & L_{a} & W & & & \theta_{(x, y)} & \text { arrowHead }> \\
<x & y & W_{a} & L_{a} & W & L & & \theta_{(x, y)} & \text { arrow }> \\
<x & y & W_{a} & L_{a} & W & L & N & \theta_{(x, y)} & \text { arrowArray }>
\end{array}
$$

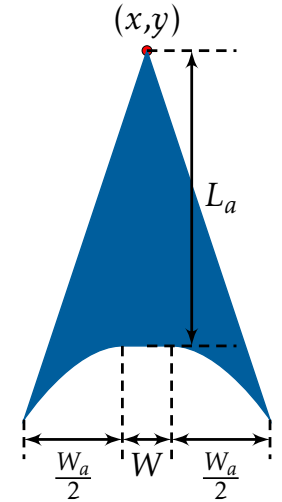

(a)

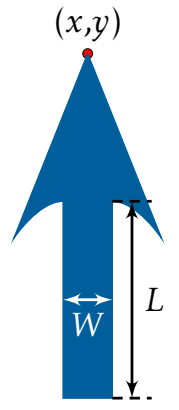

(b)

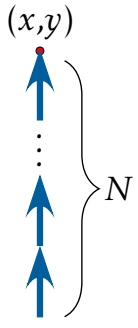

(c)

Figure 2.95: Example shapes illustrating various parameters from the (a) arrowHead, (b) arrow and (c) arrowArray constructors.

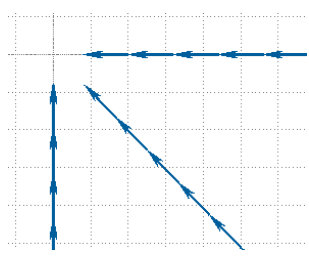

Figure 2.96: Rendered GDS file example with arrowArray structures. 


\subsection{CNST Nanophotonics Library}

This section contains a variety of shapes commonly encountered nanophotonic applications. Many of these shapes (tapers, S-bends, Y-bends, etc) could be used for micro- and nano-fluidics, MEMS/NEMS and other fields of applied sciences. Each element has several constructor definitions, allowing the user to create structures using either positive or negative tone resists. Additionally, constructors allow for addition of endcaps at the structural termination points. This in turn alleviates stresses at sharp field crowding corners, thereby preventing undesired formation of stress cracks. Figure 2.97 shows an example of $600 \mathrm{~nm}$ patterned ZEP layer on top of $700 \mathrm{~nm}$ stoichiometric silicon nitride deposited using low pressure chemical vapor deposition. In this case, cracks initiated at the sharp edges within the nitride layer during the reactive ion etch step (figure $2.97 \mathrm{a}, \mathrm{b}$ ). The dilemma was circumvented by corner rounding (figure 2.97c, d).
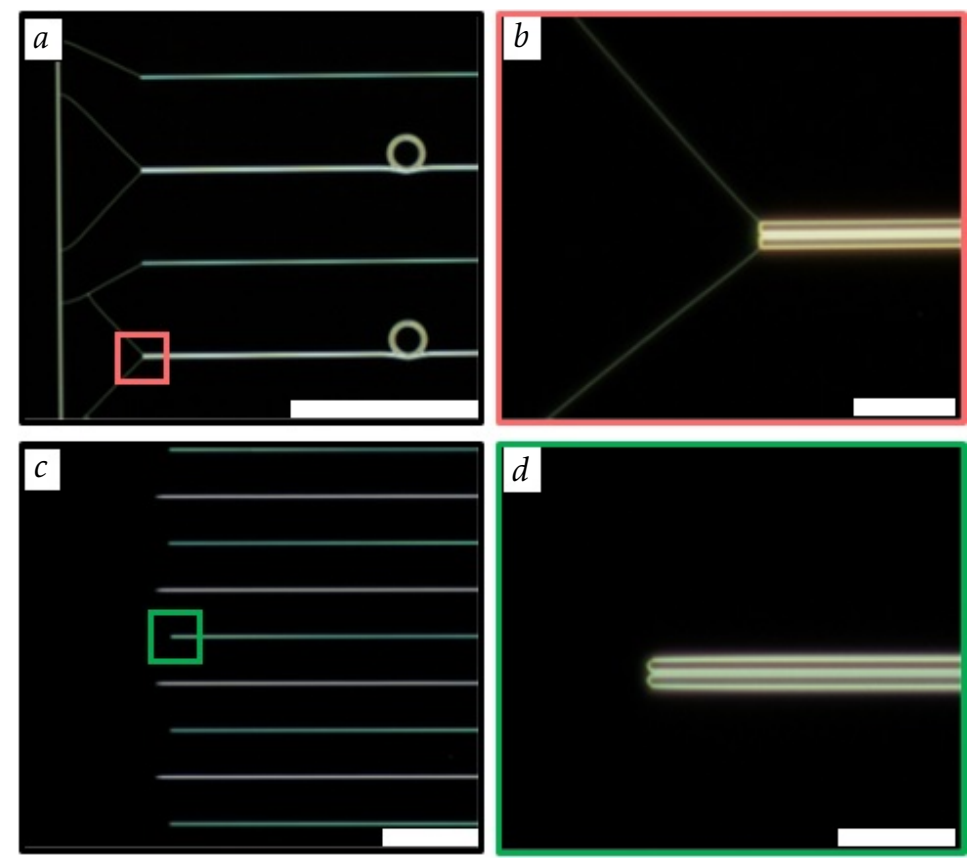

Figure 2.97: Silicon nitride waveguides with square ((a) and (b)) and rounded corners ((c) and (d)). Waveguides with square corners show cracks initiating at the waveguide edges. Waveguides with rounded corners alleviate stresses at sharp corners, thereby preventing cracks formation. Scale bar in (a) and (c) represents $250 \mu \mathrm{m}$ and in (b) and (d) represents $20 \mu \mathrm{m}$. 


\subsubsection{Waveguides}

\subsubsection{Waveguide}

This method is useful when creating waveguides with a negative tone resist. Rectangular shape characterized by start point $\left(x_{1}, y_{1}\right)$, end point $\left(x_{2}, y_{2}\right)$, width $(W)$ and rotation about point $\left(x_{1}, y_{1}\right)$. The waveguide is defined in the horizontal direction, condition i.e. $x_{1}=x_{2}$ must be satisfied. For vertical waveguides, choose $\theta_{\left(x_{1}, y_{1}\right)}=90$. Waveguide endcaps are defined by parameters $E C_{l e f t}$ and $E C_{\text {right }}$. Endcap values of 0 and 1 correspond to slot waveguides without and with endcaps.

$$
<x_{1} \quad y_{1} \quad x_{2} \quad y_{2} \quad W \quad \theta_{\left(x_{1}, y_{1}\right)} \quad E C_{\text {left }} \quad E C_{\text {right }} \text { waveguide }>
$$

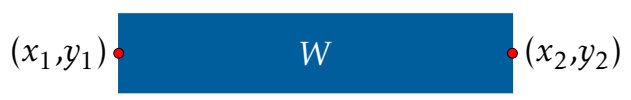

(a)

\section{W}

(b)

\section{W}

(c)

Figure 2.98: Waveguide example using the waveguide constructor. The method is beneficial when creating waveguides from a negative tone resist. Waveguides examples (a) without endcaps $\left(E_{\text {left }}=E_{\text {right }}=0\right)$, (b) with left end cap $\left(E_{\text {left }}=1, E_{\text {right }}=0\right)$, (c) with right endcap $\left(E_{\text {left }}=0, E_{\text {right }}=1\right)$. 


\subsubsection{Waveguide Slot}

This method is useful when creating slot waveguides with a negative tone resist. Positive-tone resist exposure creates a waveguide of width $W_{\text {slot }}$. The shape characterized by start point $\left(x_{1}, y_{1}\right)$, end point $\left(x_{2}, y_{2}\right)$, waveguide width $(W)$, slot width $\left(W_{\text {slot }}\right)$ and rotation about point $\left(x_{1}, y_{1}\right)$. The waveguide is defined in the horizontal direction, condition i.e. $x_{1}=x_{2}$ must be satisfied. For vertical waveguides, choose $\theta_{\left(x_{1}, y_{1}\right)}=90$. Waveguide endcaps are defined by parameters $E C_{\text {left }}$ and $E C_{\text {right }}$. Endcap values of 0 and 1 correspond to slot waveguides without and with endcaps.

$$
<x_{1} \quad y_{1} \quad x_{2} \quad y_{2} \quad W \quad W_{\text {slot }} \quad \theta_{\left(x_{1}, y_{1}\right)} \quad E C_{\text {left }} \quad E C_{\text {right }} \text { waveguideSlot }>
$$

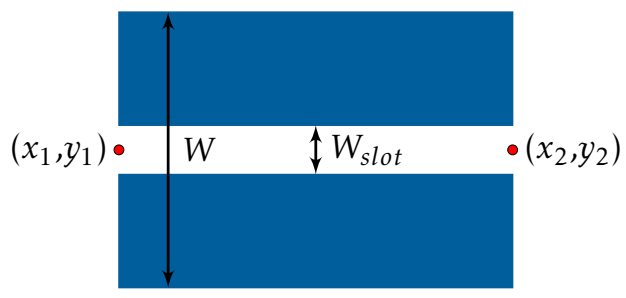

(a)

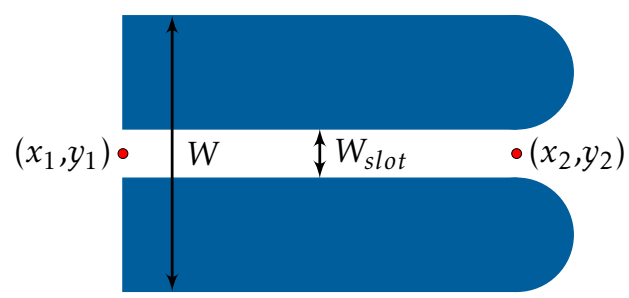

(b)

Figure 2.99: Slot waveguide example using the waveguideSlot constructor. The method is beneficial when creating waveguides from a negative tone resist. Positive-tone resist exposure creates a waveguide of width $W_{\text {slot }}$. Slot waveguide examples (a) without endcaps $\left(E_{\text {left }}=E_{\text {right }}=0\right)$, (b) with right end cap $\left(E_{\text {left }}=0, E_{\text {right }}=1\right)$. 


\subsubsection{Waveguide Inverse}

This method is useful when creating waveguides with a positive tone resist. The shape characterized by start point $\left(x_{1}, y_{1}\right)$, end point $\left(x_{2}, y_{2}\right)$, waveguide width $(W)$, exposed sleeve width $\left(W_{e}\right)$ and rotation about point $\left(x_{1}, y_{1}\right)$. The waveguide is defined in the horizontal direction, condition i.e. $y_{1}=y_{2}$ must be satisfied. For vertical waveguides, choose $\theta_{\left(x_{1}, y_{1}\right)}=90$. Waveguide endcaps are defined by parameters $E C_{\text {left }}$ and $E C_{\text {right }}$. Endcap values of 0 and 1 correspond to slot waveguides without and with endcaps.

$$
<x_{1} \quad y_{1} \quad x_{2} \quad y_{2} \quad W \quad W_{e} \quad \theta_{\left(x_{1}, y_{1}\right)} \quad E C_{\text {left }} \quad E C_{\text {right }} \text { waveguidelnv }>
$$

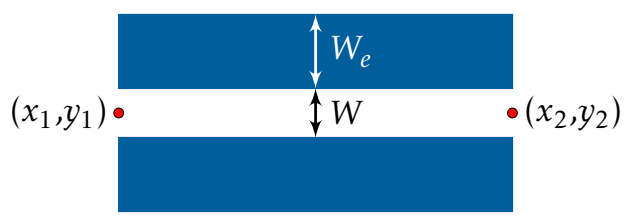

(a)

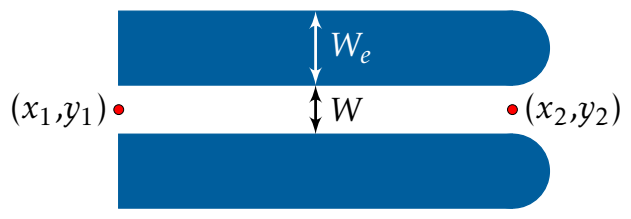

(b)

Figure 2.100: Waveguide example using the waveguidelnv constructor. The method is beneficial when creating waveguides from a positive tone resist. Inverse waveguide examples (a) without endcaps $\left(E_{\text {left }}=E_{\text {right }}=0\right)$, (b) with right end cap $\left(E_{\text {left }}=0, E_{\text {right }}=1\right)$. 


\subsubsection{Waveguide Inverse Slot}

This method is useful when creating slot waveguides with a positive tone resist. The shape characterized by start point $\left(x_{1}, y_{1}\right)$, end point $\left(x_{2}, y_{2}\right)$, waveguide width $(W)$, slot width $\left(W_{s}\right)$, exposed sleeve width $\left(W_{e}\right)$ and rotation about point $\left(x_{1}, y_{1}\right)$. The waveguide is defined either in the horizontal or vertical direction, condition i.e. $x_{1}=x_{2}$ must be satisfied.

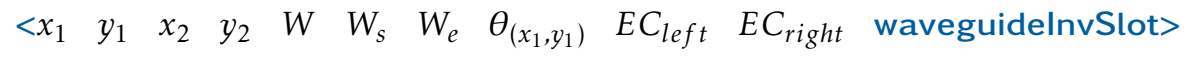

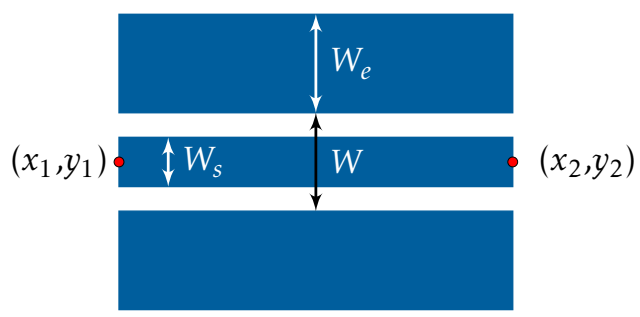

(a)

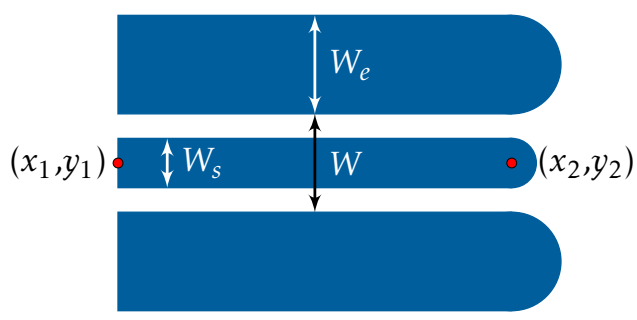

(b)

Figure 2.101: Inverse slot waveguide example using the waveguidelnvSlot constructor. The method is beneficial when creating waveguides from a positive tone resist. Inverse slot waveguide examples (a) without endcaps $\left(E_{\text {left }}=\right.$ $\left.E_{\text {right }}=0\right)$, (b) with right end cap $\left(E_{\text {left }}=0, E_{\text {right }}=1\right)$. 


\subsubsection{Waveguide Expander}

The waveguide expander is defined by width $w$, length $L$, length division $\Delta L$, TE mode angle $\theta$, beam waist $w_{0}$, wavelength $\lambda$, base height $b_{H}$ and two dimensionless parameters $a$ and $b$. Wavelength $\lambda$ is represented in nanometers. All other parameters $\left(L, \Delta L, w, w_{0}, b_{H}\right)$ are in micrometers. The variable gap is defined by

$$
g(x)=\frac{1}{b} \ln \left(\frac{2 \lambda \pi^{1.5}}{a w} \exp \left(\frac{\frac{-x^{2}}{w^{2}}}{1-\operatorname{erf}\left(\frac{x}{w}\right)}\right)\right)
$$

where

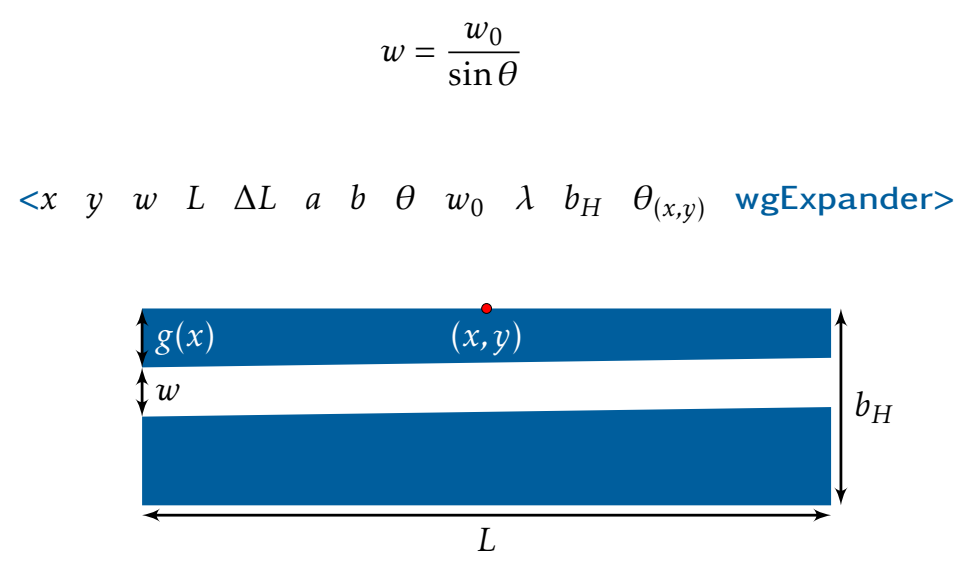

Figure 2.102: Waveguide expander 


\subsubsection{Tapers}

\subsubsection{Linear Taper}

The linear waveguide taper method is beneficial with negative tone resists. Trapezoidal shape characterized by start point $\left(x_{1}, y_{1}\right)$, end point $\left(x_{2}, y_{2}\right)$, width at start $\left(W_{1}\right)$ and end $\left(W_{2}\right)$ points, and rotation about point $\left(x_{1}, y_{1}\right)$. The linear taper shape is defined either in the horizontal or vertical direction, condition i.e. $x_{1}=x_{2}$ or $y_{1}=y_{2}$ must be satisfied.

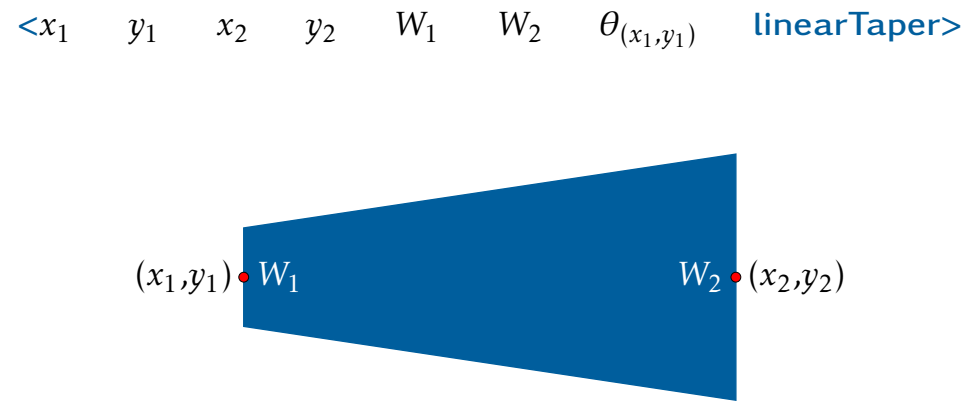

Figure 2.103: Linear waveguide taper generated using the linearTaper constructor. Structure is useful with negative tone resists. 


\subsubsection{Linear Taper Slot}

The slot linear waveguide taper is beneficial with negative tone resists. With positive tone resists, this structure would result in a tapered waveguide structure. Trapezoidal shape characterized by start point $\left(x_{1}, y_{1}\right)$, end point $\left(x_{2}, y_{2}\right)$, width at start $\left(W_{1}\right)$ and end $\left(W_{2}\right)$ points, slot width at start $\left(W_{s 1}\right)$ and end $\left(W_{s 2}\right)$ points, and rotation about point $\left(x_{1}, y_{1}\right)$. The linear taper shape is defined either in the horizontal or vertical direction, condition i.e. $x_{1}=x_{2}$ or $y_{1}=y_{2}$ must be satisfied.

$$
\begin{array}{llllllllll}
x_{1} & y_{1} & x_{2} & y_{2} & W_{1} & W_{2} & W_{s 1} & W_{s 2} & \theta_{\left(x_{1}, y_{1}\right)} & \text { linearTaperSlot }>
\end{array}
$$

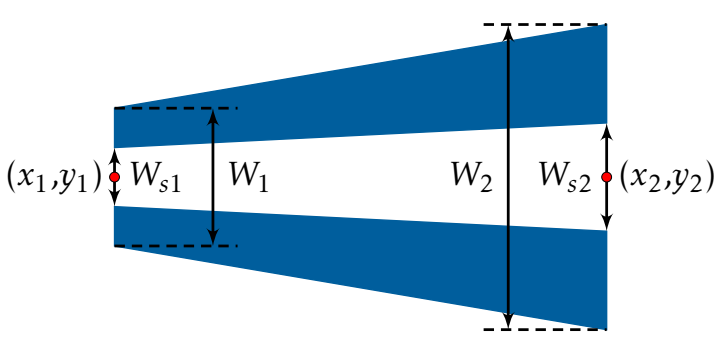

Figure 2.104: Linear taper slot waveguide structure created using the linearTaperSlot constructor. Structure is useful with negative tone resists. Positive tone resists generate a tapered waveguide structure of start and end with of $W_{s 1}$ and $W_{s 3}$ respectively. 


\subsubsection{Linear Taper Inverse Slot}

The inverse slot linear waveguide taper is beneficial with negative tone resists. With positive tone resists, this structure would result in a tapered slot waveguide structure. Trapezoidal shape characterized by start point $\left(x_{1}, y_{1}\right)$, end point $\left(x_{2}, y_{2}\right)$, width at start $\left(W_{1}\right)$ and end $\left(W_{2}\right)$ points, slot width at start $\left(W_{s 1}\right)$ and end $\left(W_{s 2}\right)$ points, gap $(g)$, and rotation about point $\left(x_{1}, y_{1}\right)$. The linear taper shape is defined either in the horizontal or vertical direction, condition i.e. $x_{1}=x_{2}$ or $y_{1}=y_{2}$ must be satisfied.

$\begin{array}{lllllllllll}x_{1} & y_{1} & x_{2} & y_{2} & W_{1} & W_{2} & g & W_{s 1} & W_{s 2} & \theta_{\left(x_{1}, y_{1}\right)} & \text { linearTaperlnvSlot }>\end{array}$

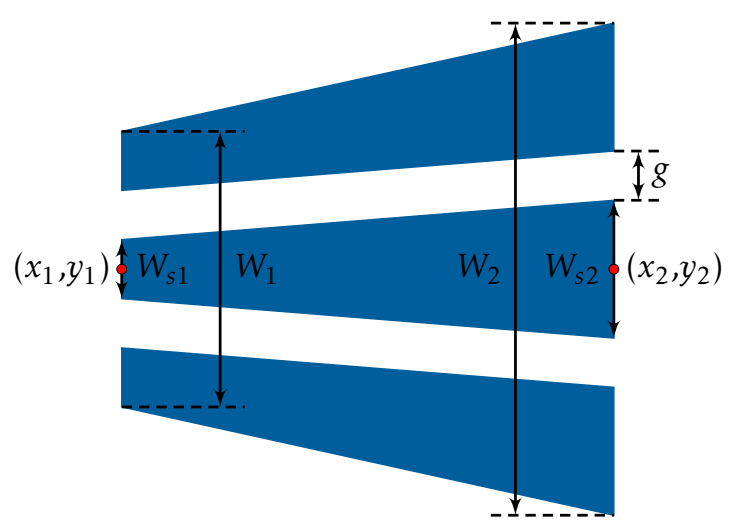

Figure 2.105: Example of the linearTaper constructor. 


\subsubsection{Exponential Taper}

Tapered shape characterized by a width $\left(W_{1}\right)$ at start point $\left(x_{1}, y_{1}\right)$ exponentially increasing to $\left(W_{2}\right)$ at a length $L$ from $\left(x_{1}, y_{1}\right)$, and rotation about point $\left(x_{1}, y_{1}\right)$. The exponential curve is constructed from $N$ segments.

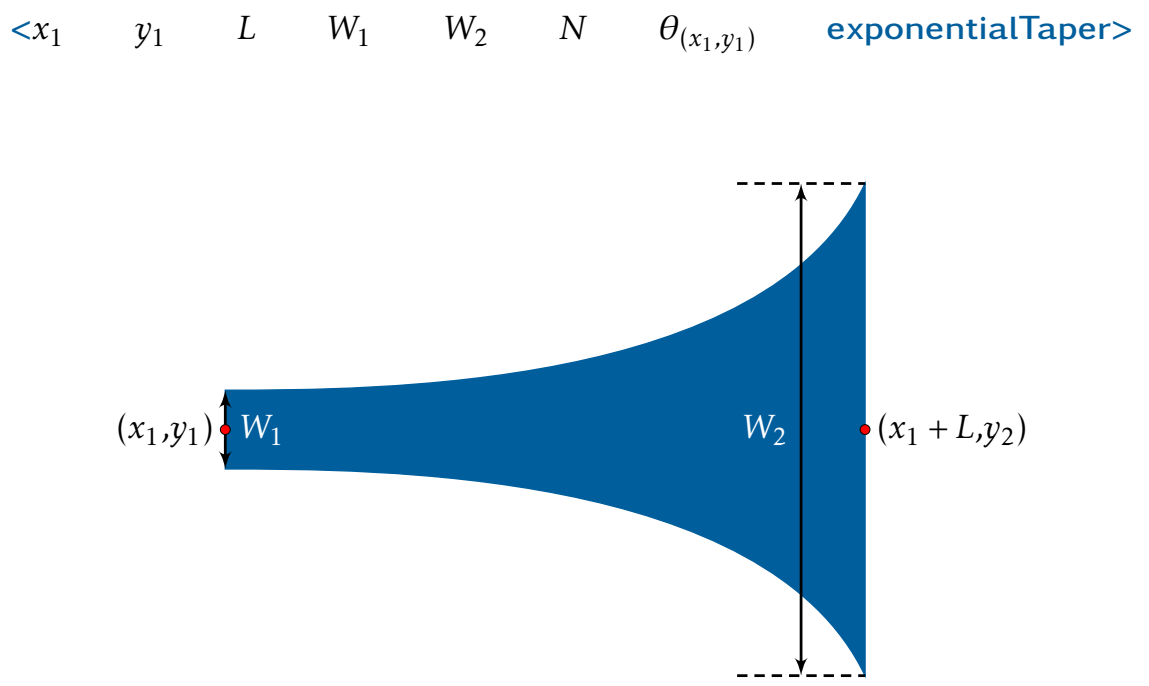

Figure 2.106: Example of the exponentialTaper constructor. 


\subsubsection{Exponential Taper Inverse}

Tapered shape characterized by a width $\left(W_{1}\right)$ at start point $\left(x_{1}, y_{1}\right)$ exponentially increasing to $\left(W_{2}\right)$ at a length $L$ from $\left(x_{1}, y_{1}\right)$, exposed sleeve width $\left(W_{e}\right)$ and rotation about point $\left(x_{1}, y_{1}\right)$. The exponential curve is constructed from $N$ segments.

$$
<x_{1} \quad y_{1} \quad L \quad W_{1} \quad W_{2} \quad N \quad W_{e} \quad \theta_{\left(x_{1}, y_{1}\right)} \text { exponentialTaperlnv> }
$$

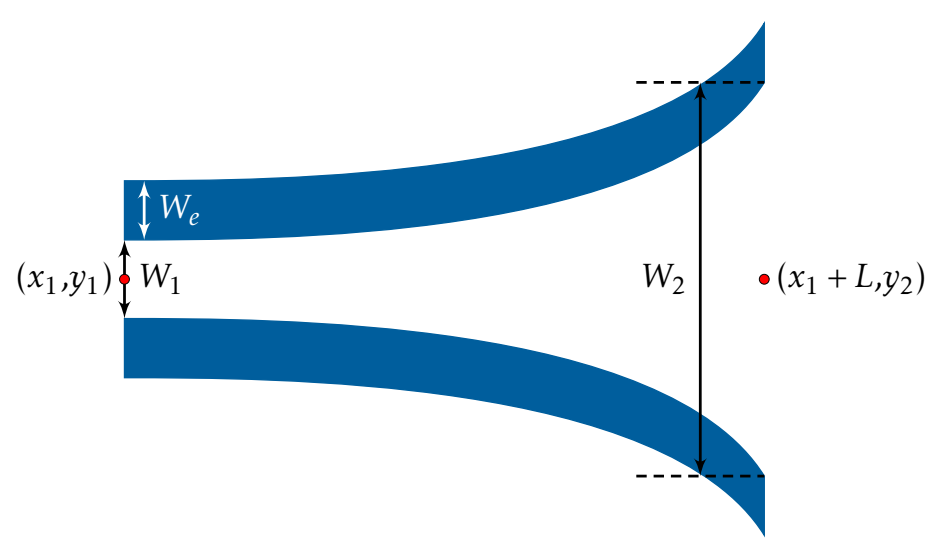

Figure 2.107: Tapered exponential shape created using the exponentialTaperInv constructor. 


\subsubsection{Exponential Taper Inverse Slot}

Tapered shape characterized by a width $\left(W_{1}\right)$ at start point $\left(x_{1}, y_{1}\right)$ exponentially increasing to $\left(W_{2}\right)$ at a length $L$ from $\left(x_{1}, y_{1}\right)$, start and end slot widths $\left(W_{s 1}\right.$ and $\left.W_{s 2}\right)$, exposed sleeve width $\left(W_{e}\right)$ and rotation about point $\left(x_{1}, y_{1}\right)$. The exponential curve is constructed from $N$ segments.

$\begin{array}{lllllllllll}<x_{1} & y_{1} & L & W_{1} & W_{2} & N & W_{e} & W_{s 1} & W_{s 2} & \theta_{\left(x_{1}, y_{1}\right)} & \text { exponentialTaperInvSlot }>\end{array}$

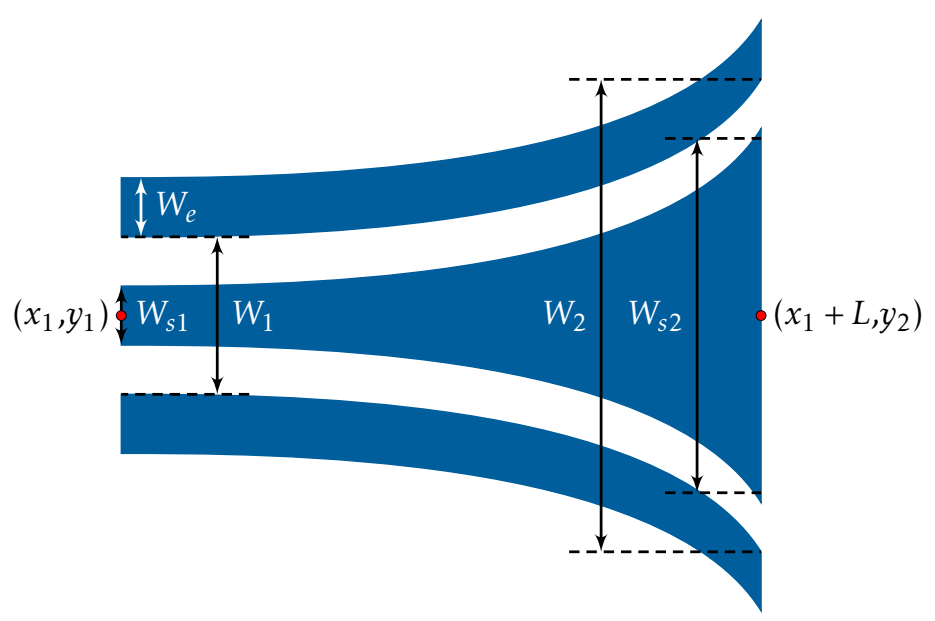

Figure 2.108: Tapered exponential slot waveguide shape created using the exponentialTaperlnvSlot constructor. 


\subsubsection{Custom Taper}

Custom tapered shape characterized by user-defined $(x, y)$ coordinates. Points $(\mathrm{x} 1, \mathrm{y} 1)$ to $(\mathrm{xn}, \mathrm{yn})$ are generated (top portion of the curve) and mirrored around $y=0$, closing the tapered shape that's translated to $\left(T_{x}, T_{y}\right)$ and rotated about the translation point $\theta_{\left(T_{x}, T_{y}\right)}$.

$$
\begin{array}{lllllllllll}
x_{1} & y_{1} & x_{2} & y_{2} & \cdots & x_{n} & y_{n} & T_{x} & T_{y} & \theta_{\left(T_{x}, T_{y}\right)} & \text { customTaper }
\end{array}
$$

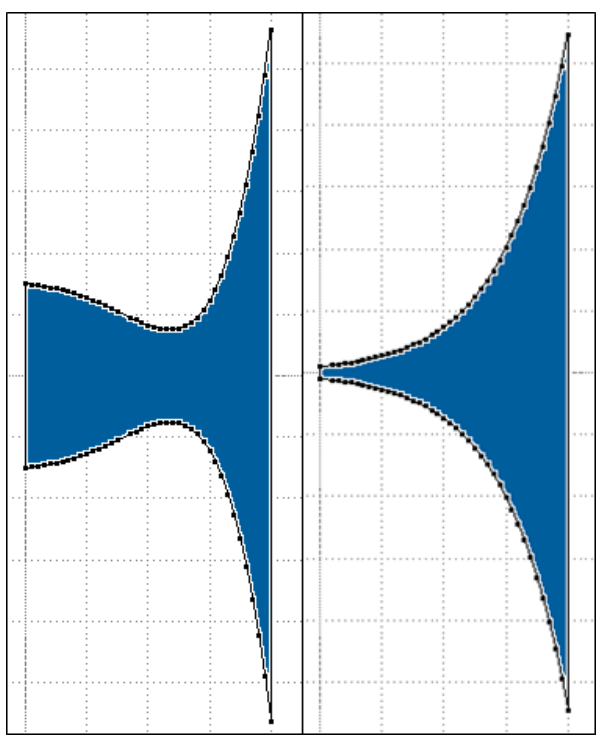

Figure 2.109: Two example GDS shapes of the customTaperExamples constructor with highlighted user defined vertices. 


\subsubsection{Couplers}

\subsubsection{Directional Couplers}

$\begin{array}{llllllllllllll}x & y & L_{1} & w_{1} & w_{e 1} & L_{2} & L_{3} & w_{2} & w_{e 2} & g & r & N_{s i d e s} & \theta_{(x, y)} & \text { directionalCoupler } 1>\end{array}$

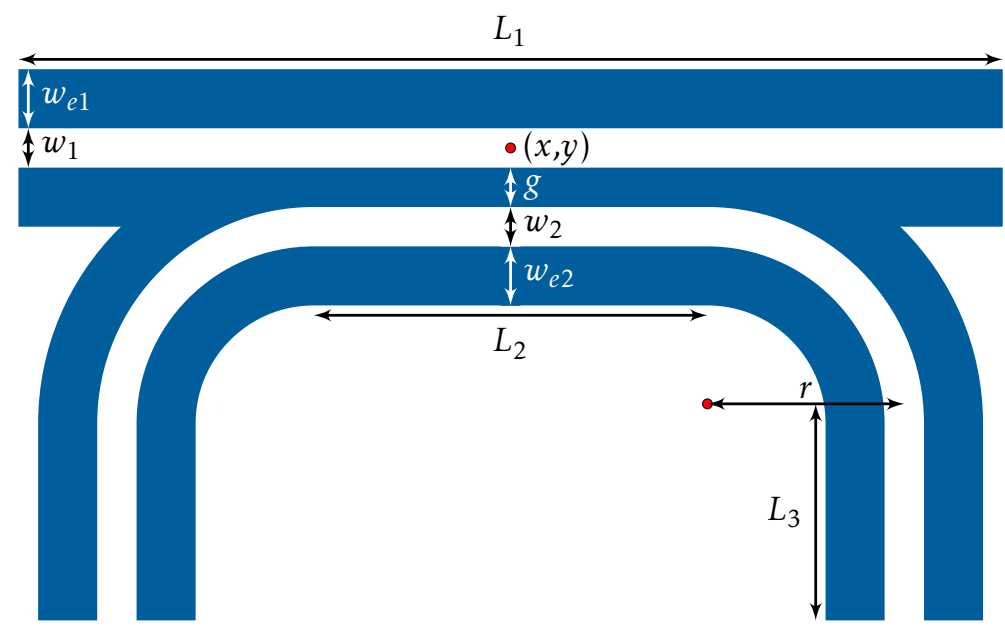

Figure 2.110: Directional coupler example using the directionalCoupler1 constructor. 
$<x \quad y \quad L_{1} \quad w_{1} \quad w_{e 1} \quad L_{2} \quad L_{3} \quad w_{2} \quad w_{e 2} \quad g \quad r \quad N_{\text {sides }} \theta_{(x, y)}$ directionalCoupler2>

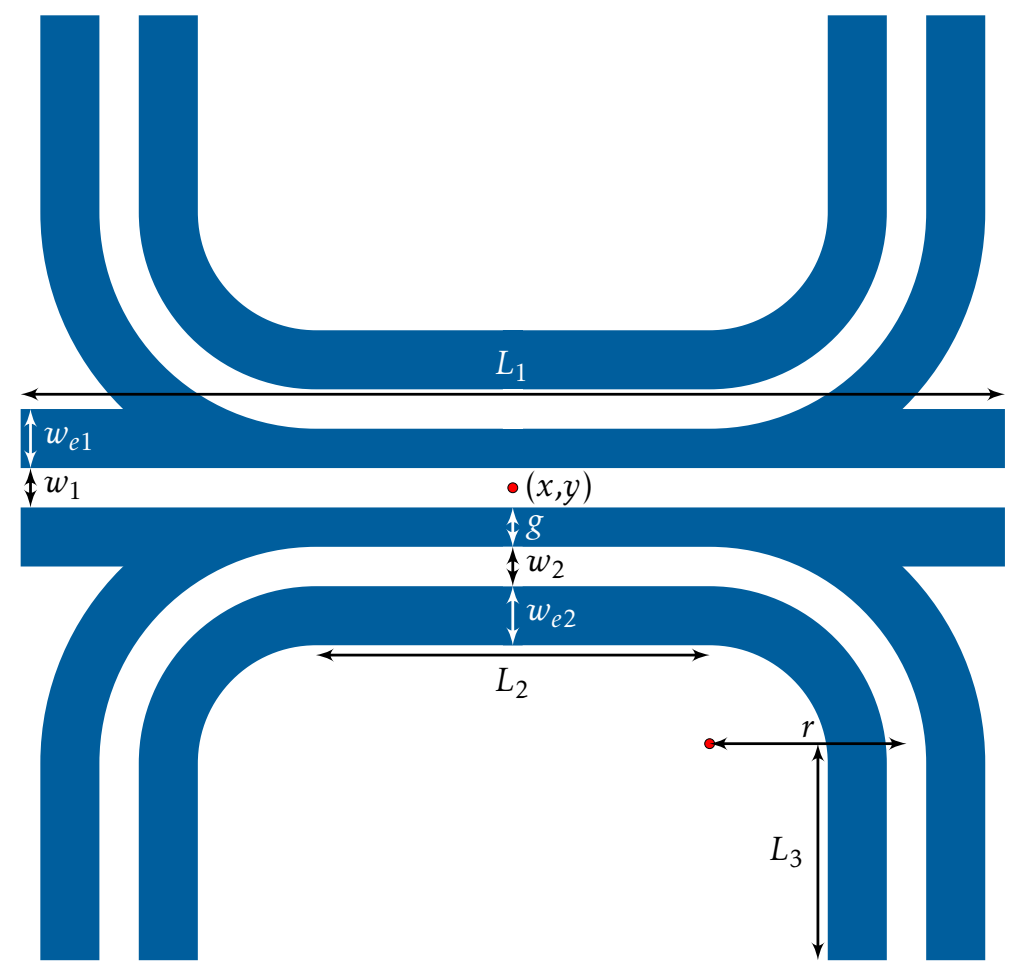

Figure 2.111: Directional coupler example using the directionalCoupler2 constructor. 
$<x \quad y \quad w \quad w_{e} \quad g \quad L_{1} \quad L_{2} \quad r \quad N_{\text {sides }} \theta_{(x, y)}$ directionalCoupler3 $>$

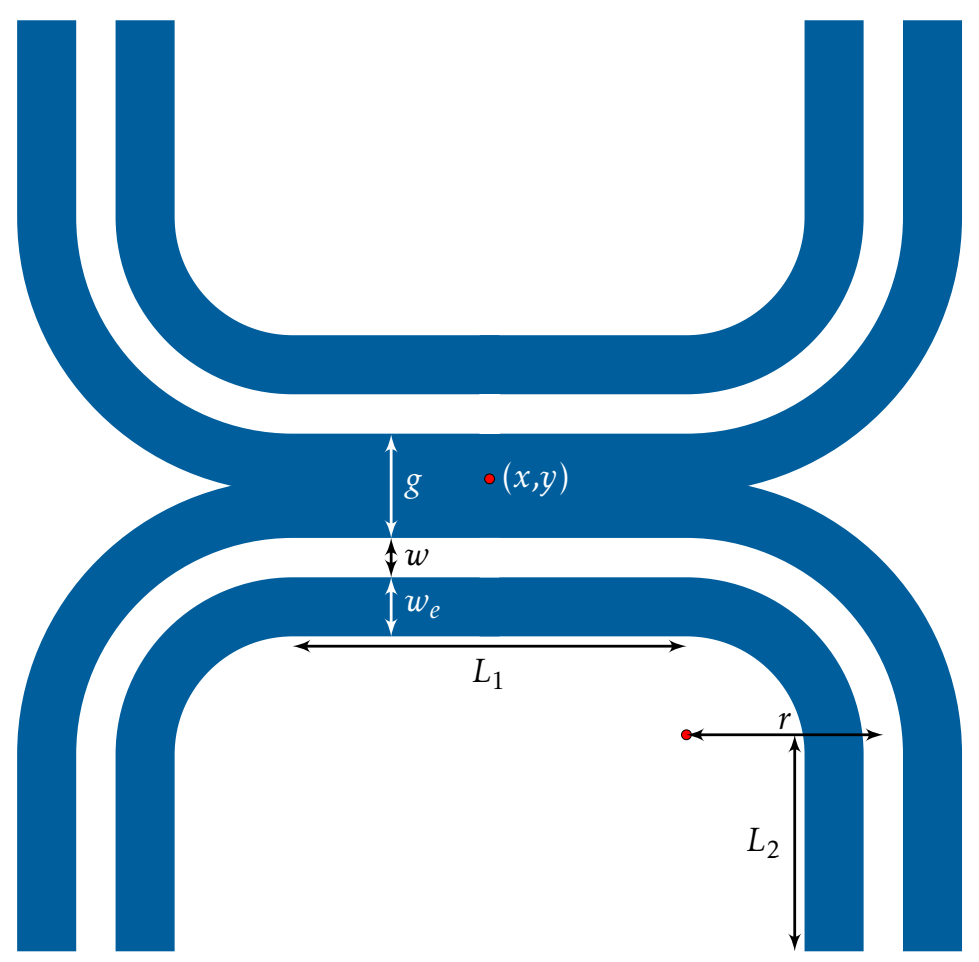

Figure 2.112: Directional coupler example using the directionalCoupler3 constructor. 
$<x \quad y \quad w \quad w_{e} \quad g \quad L_{1} \quad L_{B} \quad H_{B} \quad \theta_{(x, y)}$ directionalCoupler4>

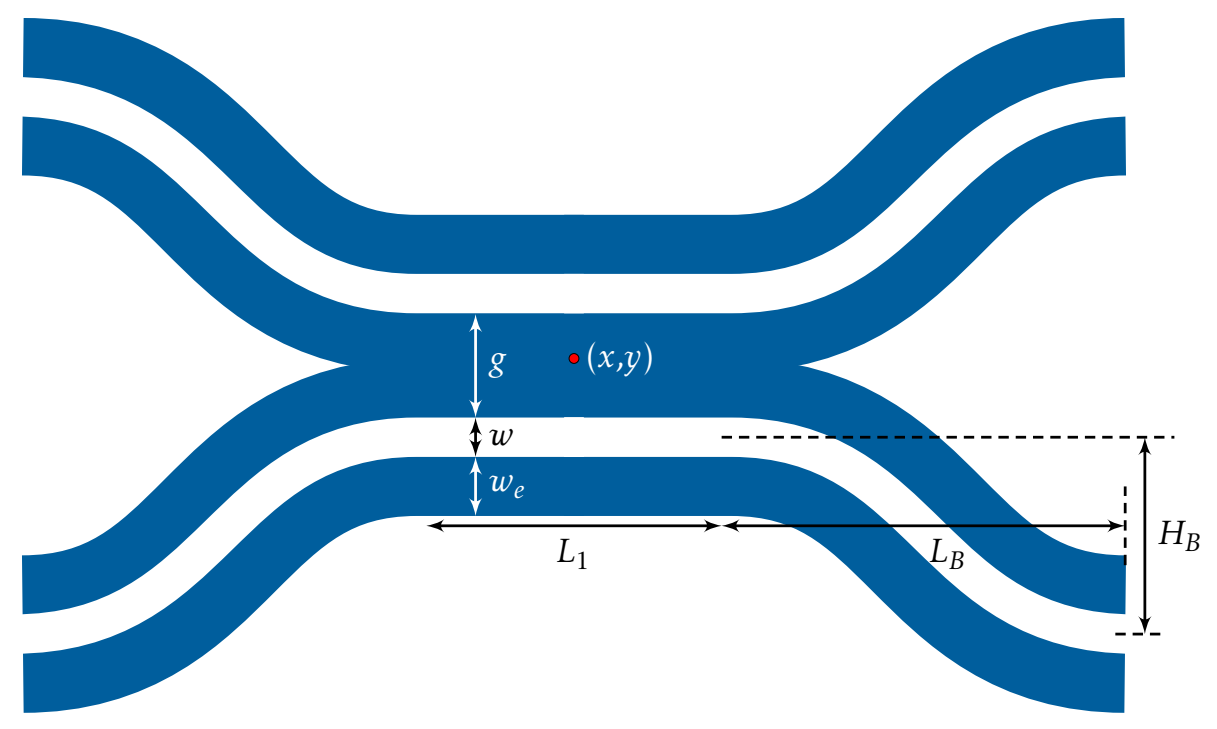

Figure 2.113: Directional coupler example using the directionalCoupler4 constructor. 


\subsubsection{S-Bend Taper}

S-Bend taper is characterized by the start coordinate $(x, y)$, length $(L)$, height $(H)$, start $\left(W_{\text {start }}\right)$ and end $\left(W_{\text {end }}\right)$ waveguide widths, and rotation about point $(x, y)$. shapeReso parameter defines the rendering resolution of the S-bend (Bezier based) curve.

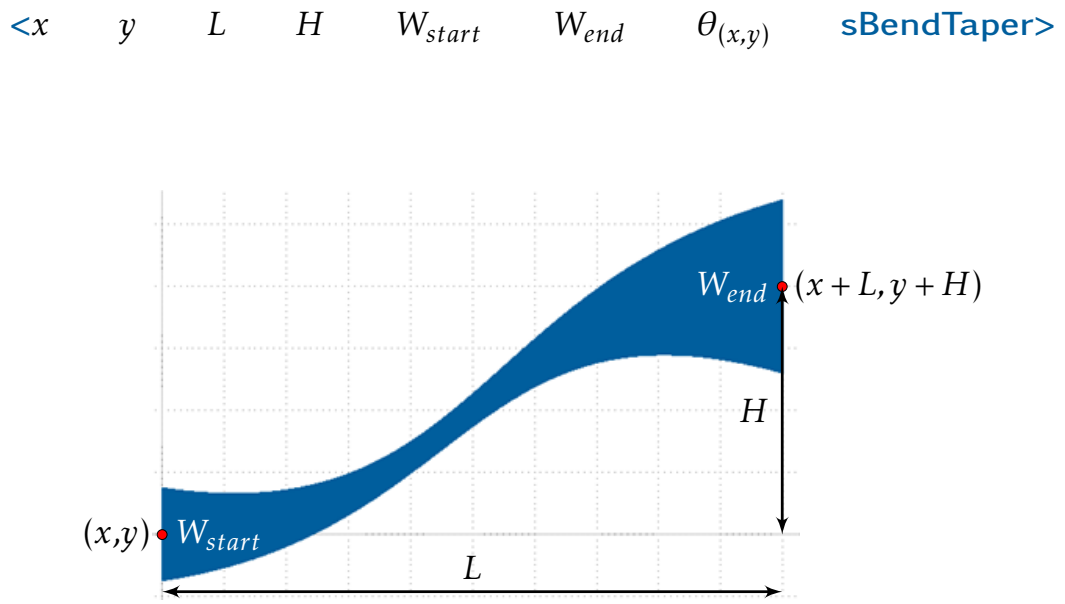

Figure 2.114: An example GDS shape of the sBendTaper constructor. 


\subsubsection{S-Bend Funnel}

S-Bend funnel is characterized by the start coordinate $(x, y)$, length $(L)$, height $(H)$, start $(W)$ and rotation about point $(x, y)$. shape Reso parameter defines the rendering resolution of the S-bend (Bezier based) curve.

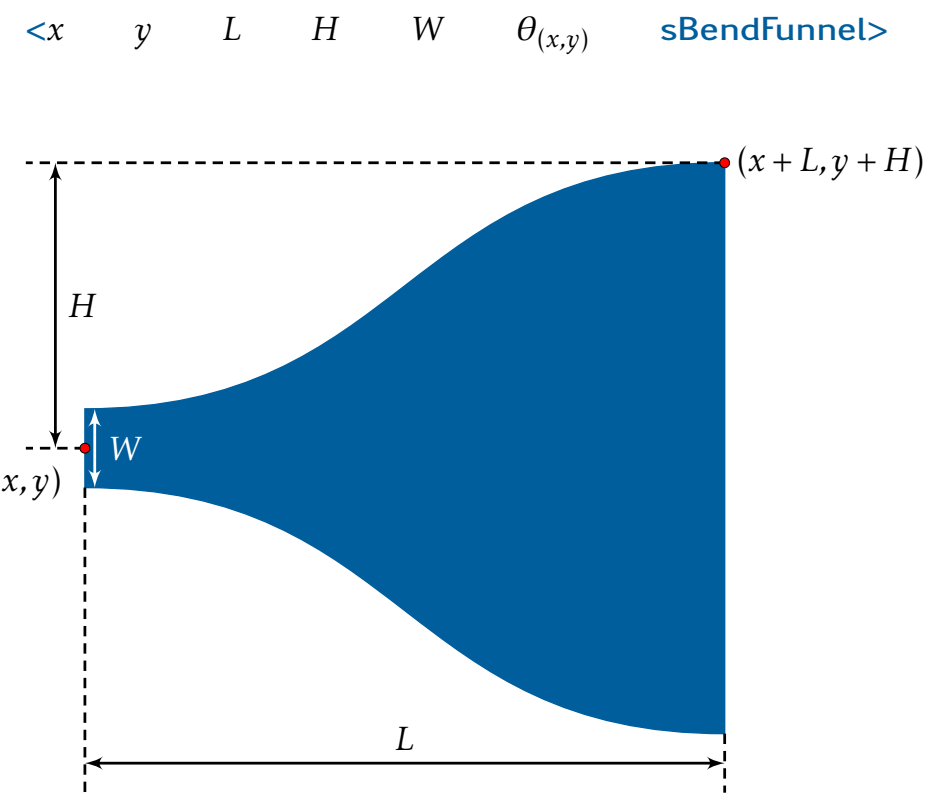

Figure 2.115: Schematic illustration showing various parameters from the sBendFunnel constructor. 


\subsubsection{S-Bend}

S-Bends are Bezier curves characterized by two constructors. First consists of the start $\left(x_{1}, y_{1}\right)$ and end $\left(x_{2}, y_{2}\right)$ points. The second is characterized by the start point $\left(x_{1}, y_{1}\right)$, length $(L)$ and height $(H)$ parameters. Both constructors are characterized by a waveguide width $(W)$ and the rotation about the start point $\left(x_{1}, y_{1}\right)$. shape Reso parameter defines the rendering resolution of the S-bend (Bezier based) curve. Both $L$ and $H$ parameters are defined by positive and negative double precision values relative to the start coordinate point $\left(x_{1}, y_{1}\right)$. For instance, a negative value of $H$ would place the end point at $y_{1}-H$.

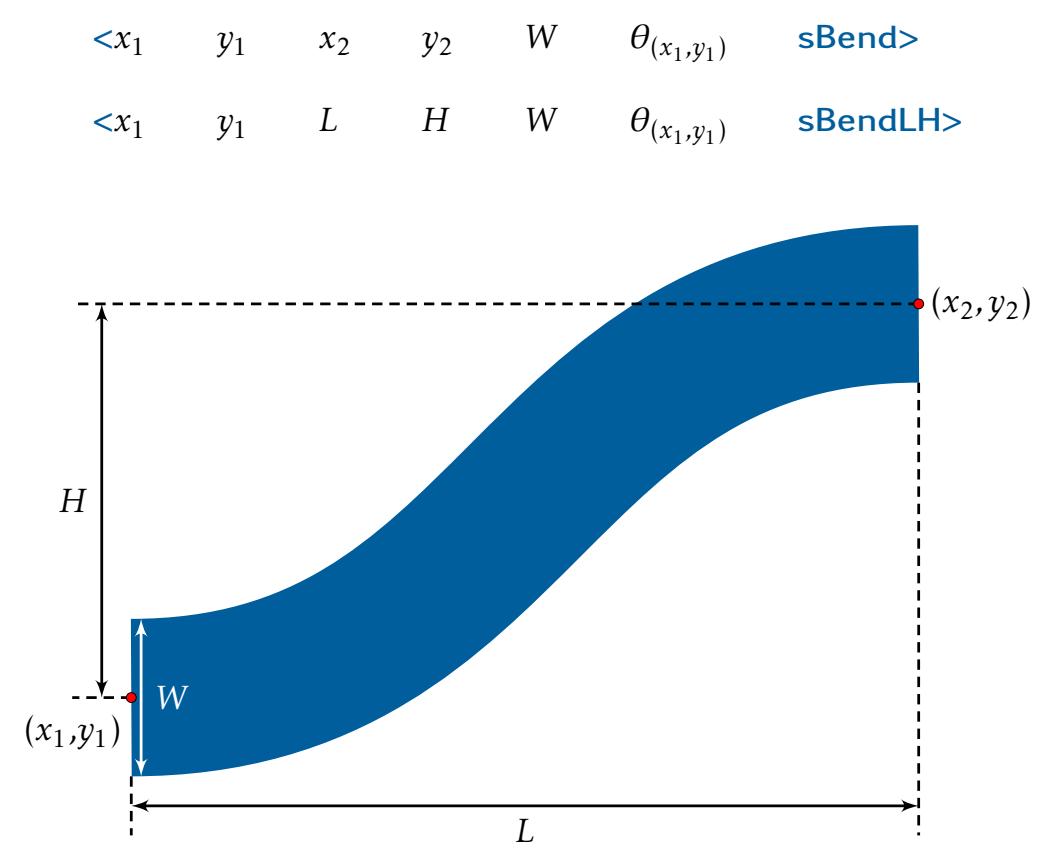

Figure 2.116: An example S-Bend GDS shape illustrating various parameters from the two sBend constructors. 


\subsubsection{S-Bend Inverse}

Similar to S-Bends (section 2.9.3.4), sBendlnv constructor is used to create s-bend waveguides using a positive tone resist. Alternatively, using a negative tone resist, a slot-waveguide of width $W+W_{e}$ and a slot of width $W$ is constructed. The shape is characterized by the start point $\left(x_{1}, y_{1}\right)$, length $(L)$, height $(H)$, waveguide width $(W)$, exposure sleeve width $\left(W_{e}\right)$ and the rotation about the start point $\left(x_{1}, y_{1}\right)$. shapeReso parameter defines the rendering resolution of the S-bend (Bezier based) curve. Both $L$ and $H$ parameters are defined by positive and negative double precision values relative to the start coordinate point $\left(x_{1}, y_{1}\right)$. For instance, a negative value of $H$ would place the end point $y$ value at $y_{1}-H$.

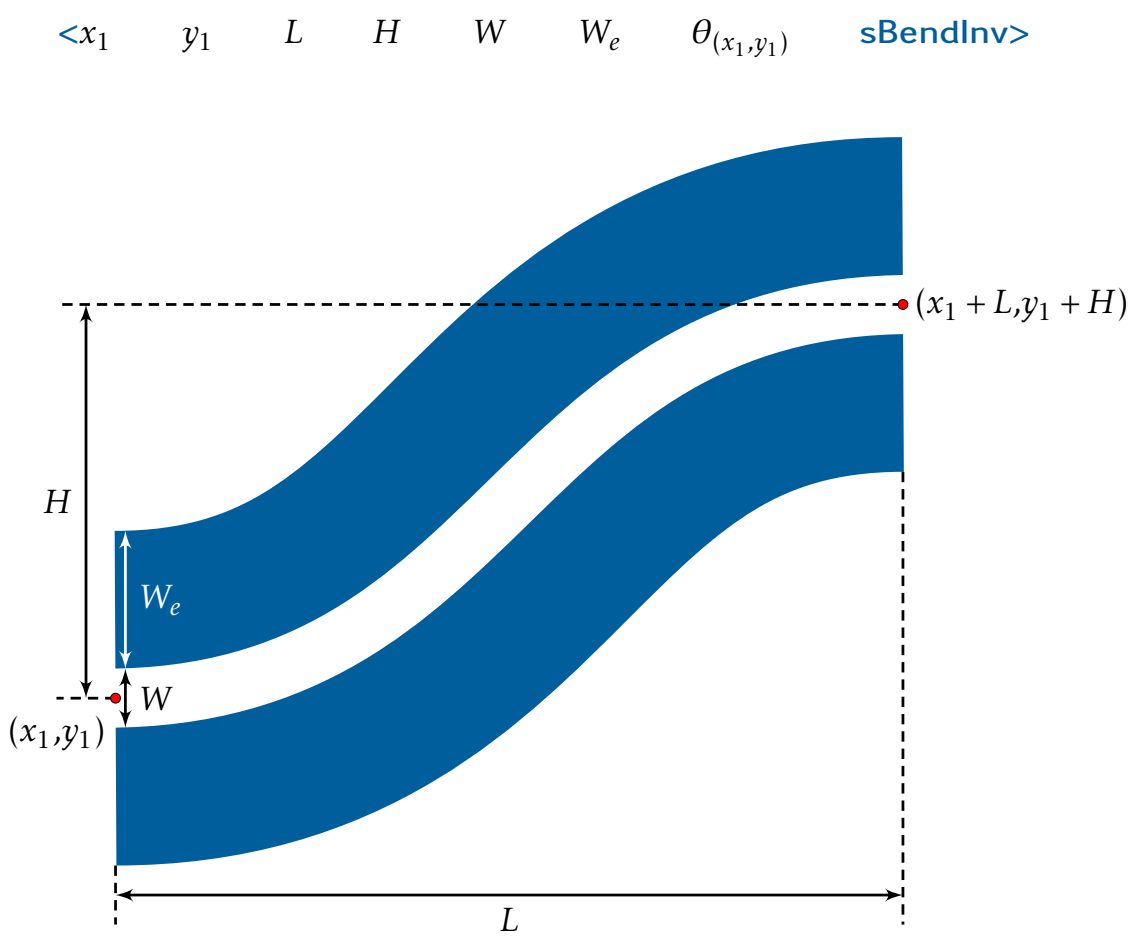

Figure 2.117: Schematic illustration showing various parameters from the sBendlnv constructor. 


\subsubsection{S-Bend Inverse Slot}

Similar to S-Bends (section 2.9.3.4), sBendlnvSlot constructor is used to create $\mathrm{s}$-bend slot waveguides using a positive tone resist. The shape is characterized by the start point $\left(x_{1}, y_{1}\right)$, length $(L)$, height $(H)$, slot width $\left(W_{s}\right)$, gap $(g)$, exposure sleeve width $\left(W_{e}\right)$ and the rotation about the start point $\left(x_{1}, y_{1}\right)$ parameters. shapeReso parameter defines the rendering resolution of the S-bend (Bezier based) curve. Both $L$ and $H$ parameters are defined by positive and negative double precision values relative to the start coordinate point $\left(x_{1}, y_{1}\right)$. For instance, a negative value of $H$ would place the end point $y$ value at $y_{1}-H$.

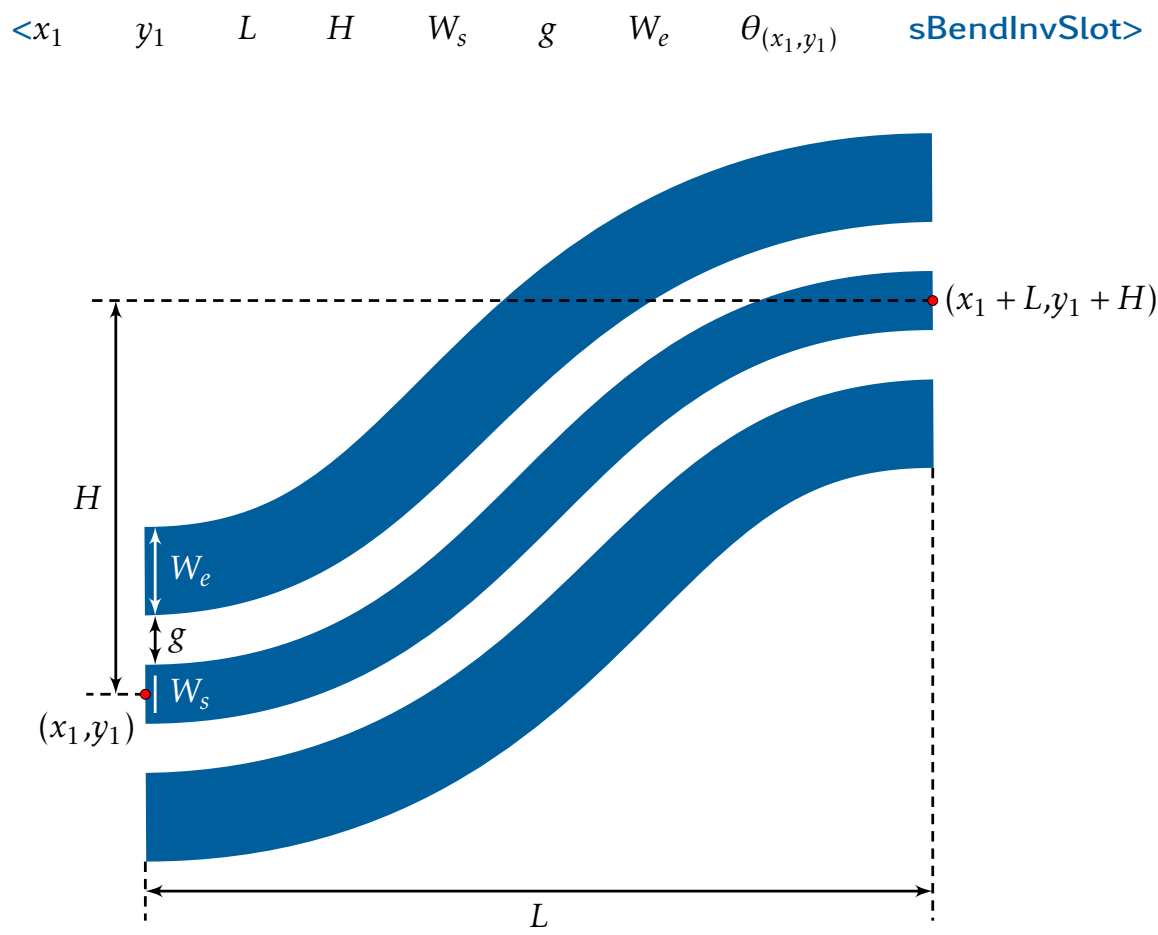

Figure 2.118: Schematic illustration showing various parameters from the sBendlnvSlot constructor. 


\subsubsection{Y-Bend}

Y-Bends are constructed by merging two S-Bend curves. Similarly to the SBend curve defined in the section 2.9.3.4, Y-Bends are characterized by two constructors. First consists of the start $\left(x_{1}, y_{1}\right)$ and two end $\left(x_{2}, y_{2}\right)$ points $\left(\left(x_{2}, y_{2}\right)\right.$ and $\left.\left(x_{3}, y_{3}\right)\right)$. The second is characterized by the start point $\left(x_{1}, y_{1}\right)$, pair of length $\left(L_{2}\right.$ and $\left.L_{3}\right)$ and pair of height $\left(H_{2}\right.$ and $\left.H_{3}\right)$ parameters. Both constructors are characterized by a waveguide width $(W)$ and the rotation about the start point $\left(x_{1}, y_{1}\right)$. shapeReso parameter defines the rendering resolution of the Y-bend (Bezier based) curve. The length and height parameters are defined by positive and negative double precision values relative to the start coordinate point $\left(x_{1}, y_{1}\right)$. For instance, a negative value of $H_{3}$ would place the end point $y$ value at $y_{1}-H_{3}$. Figure 2.119 shows a negative $H_{3}$ value.

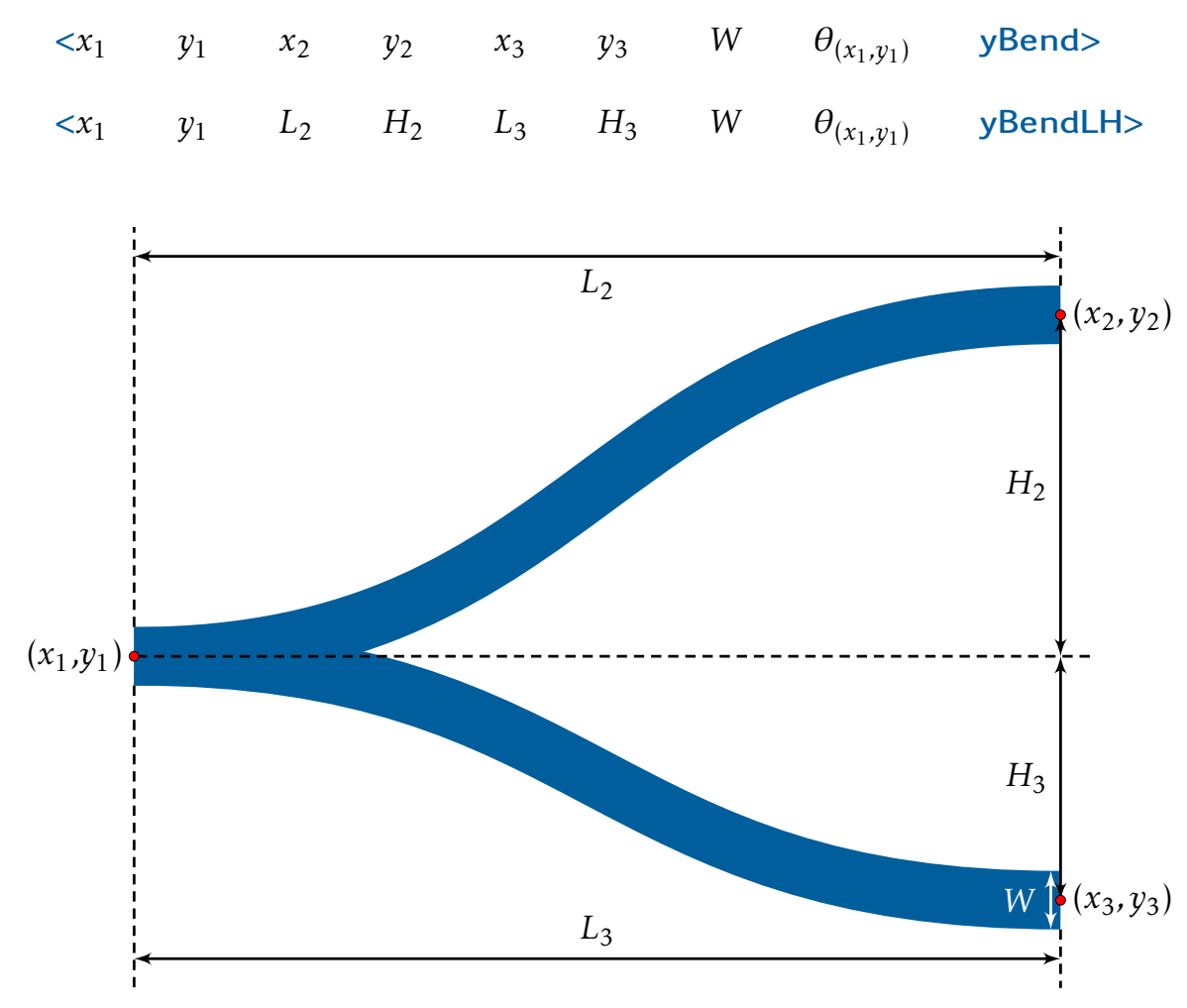

Figure 2.119: An example Y-Bend GDS shape illustrating various parameters from the two yBend constructors. 


\subsubsection{Y-Bend Inverse}

Similar to Y-Bends (section 2.9.3.7), yBendlnv constructor is used to create a $y$-bend waveguide bifurcation using a positive tone resist. Alternatively, using a negative tone resist, a slot-waveguide of width $W+W_{e}$ and a slot of width $W$ is constructed. The shape characterized is characterized by the start coordinate point $\left(x_{1}, y_{1}\right)$, a pair of lengths $\left(L_{2}\right.$ and $\left.L_{3}\right)$, a pair of heights $\left(H_{2}\right.$ and $\left.H_{3}\right)$, waveguide width $(W)$, exposure sleeve $\left(W_{e}\right)$ and the rotation about the start point $\left(x_{1}, y_{1}\right)$ parameters. shapeReso parameter defines the rendering resolution of the Y-bend (Bezier based) curve. Both length and height parameters are defined by positive and negative double precision values relative to the start coordinate point $\left(x_{1}, y_{1}\right)$. For instance, a negative value of $\mathrm{H}_{3}$ would place the end point $y$ value at $y_{1}-H_{3}$, hence value of $H_{3}$ value in Figure 2.120 is negative.

$$
\begin{array}{clllllllll}
<x_{1} & y_{1} & L_{2} & H_{2} & L_{3} & H_{3} & W & W_{e} & \theta_{\left(x_{1}, y_{1}\right)} & \text { yBendlnv }>
\end{array}
$$

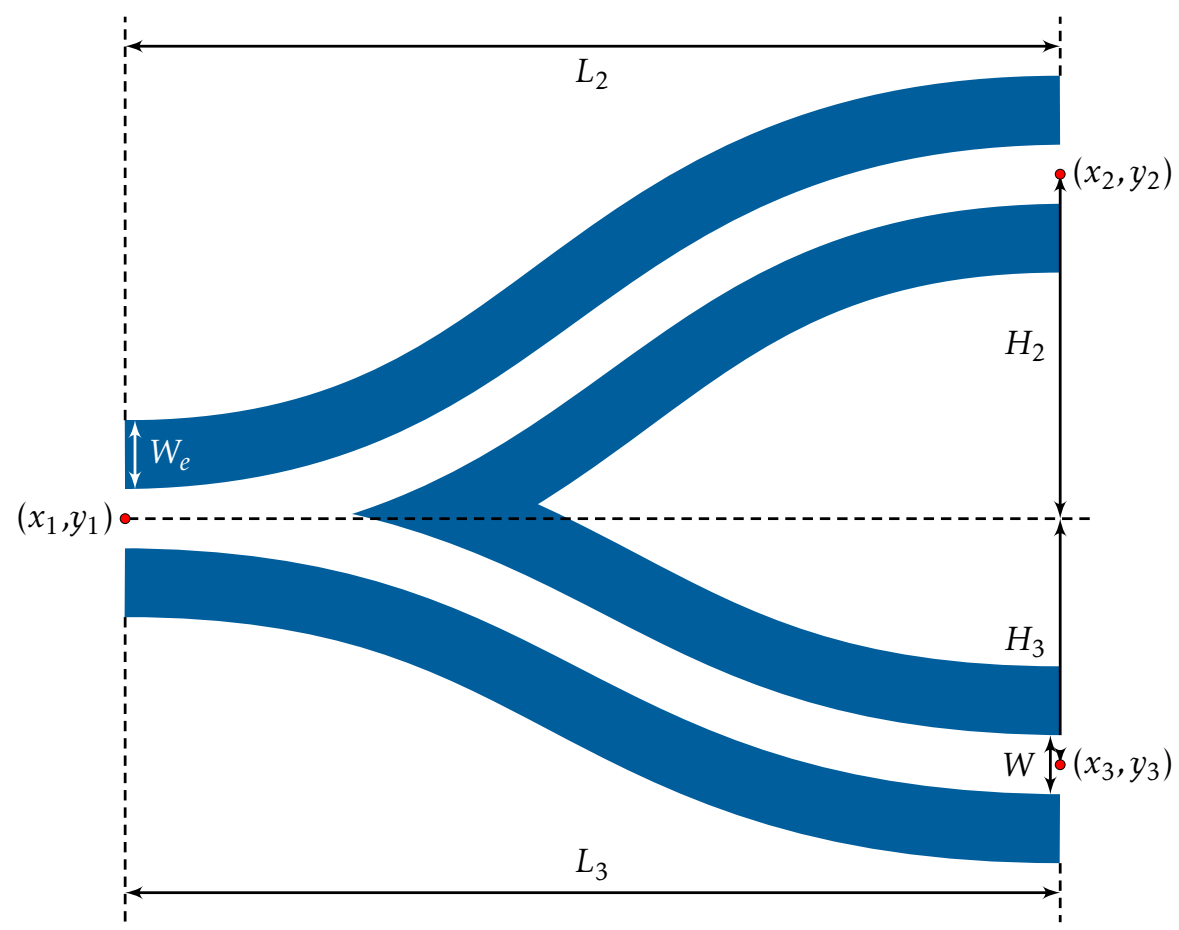

Figure 2.120: Schematic illustration showing various parameters from the yBendlnv constructor. 


\subsubsection{Y-Bend Inverse Slot}

Similar to Y-Bends (section 2.9.3.7), yBendlnv constructor is used to create a $y$-bend slot waveguide bifurcation using a positive tone resist. The shape characterized is characterized by the start coordinate point $\left(x_{1}, y_{1}\right)$, a pair of lengths $\left(L_{2}\right.$ and $\left.L_{3}\right)$, a pair of heights $\left(H_{2}\right.$ and $\left.H_{3}\right)$, slot width $\left(W_{s}\right)$, gap $(g)$, exposure sleeve width $\left(W_{e}\right)$ and the rotation about the start point $\left(x_{1}, y_{1}\right)$ parameters. shapeReso parameter defines the rendering resolution of the Y-bend (Bezier based) curve. Both length and height parameters are defined by positive and negative double precision values relative to the start coordinate point $\left(x_{1}, y_{1}\right)$. For instance, a negative value of $\mathrm{H}_{3}$ would place the end point $y$ value at $y_{1}-H_{3}$, hence value of $H_{3}$ value in Figure 2.121 is negative .

$$
<x_{1} \quad y_{1} \quad L_{2} \quad H_{2} \quad L_{3} \quad H_{3} \quad W_{s} \quad g \quad W_{e} \quad \theta_{\left(x_{1}, y_{1}\right)} \quad \text { yBendlnvSlot }>
$$

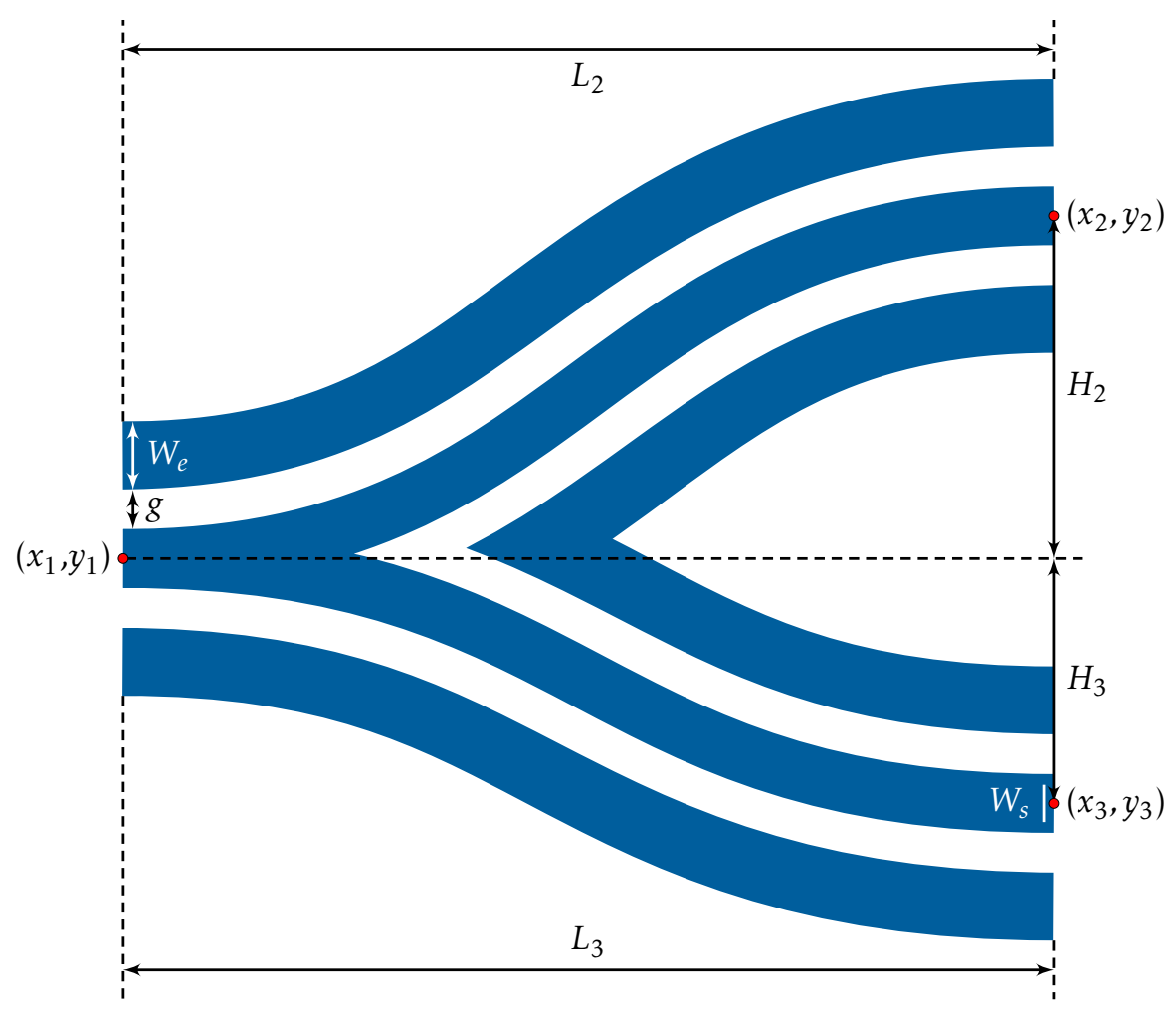

Figure 2.121: Schematic illustration showing various parameters from the yBendlnvSlot constructor. 


\subsubsection{Y-Bend - 90 degree}

$$
\begin{array}{llllllll}
< & y & r_{1} & r_{2} & w & N_{\text {sides }} & \theta_{(x, y)} & \text { yBend90 }
\end{array}
$$

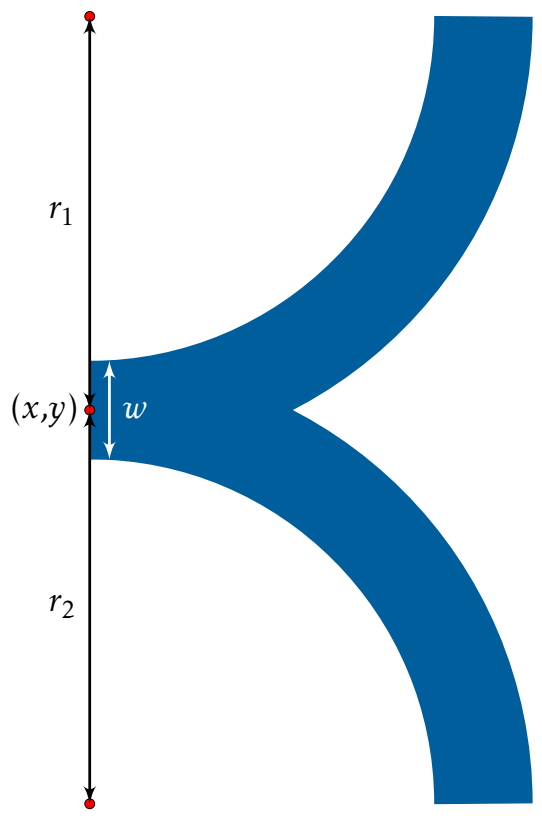

Figure 2.122: An example of a 90 degree Y-Bend. 


\subsubsection{Y-Bend Inverse - 90 degree}

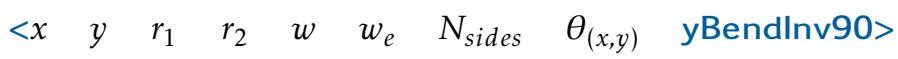

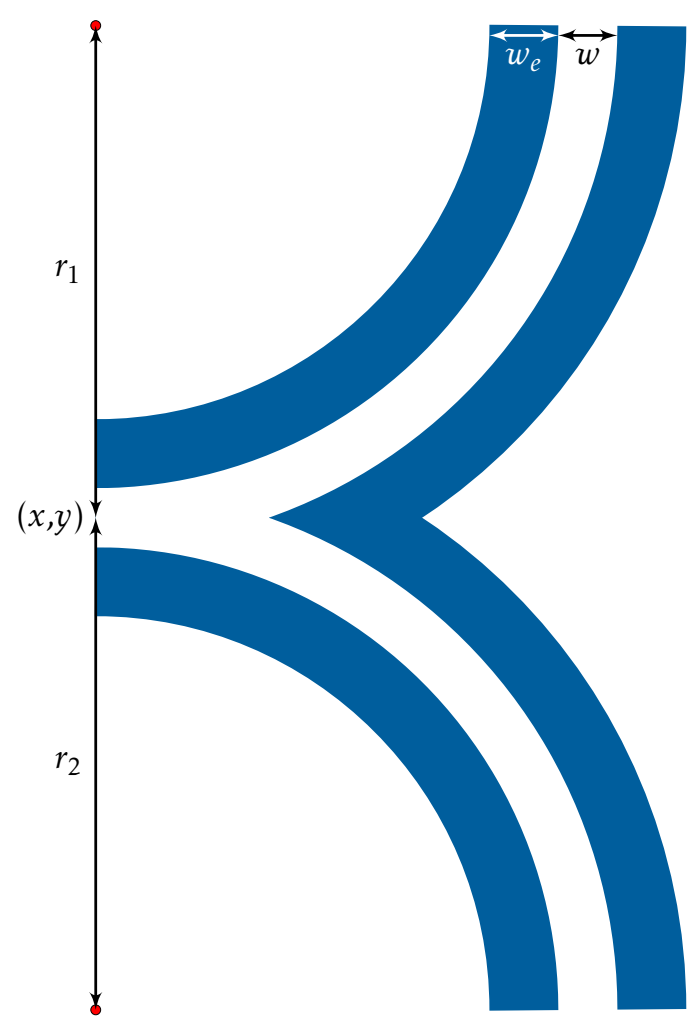

Figure 2.123: An example of a 90 degree Y-Bend inverse shape. 


\subsubsection{Y-Bend Inverse Slot - 90 degree}

$\begin{array}{llllllllll}< & y & r_{1} & r_{2} & w_{s} & g & w_{e} & N_{\text {sides }} & \theta_{(x, y)} & \text { yBendlnvSlot90 }>\end{array}$

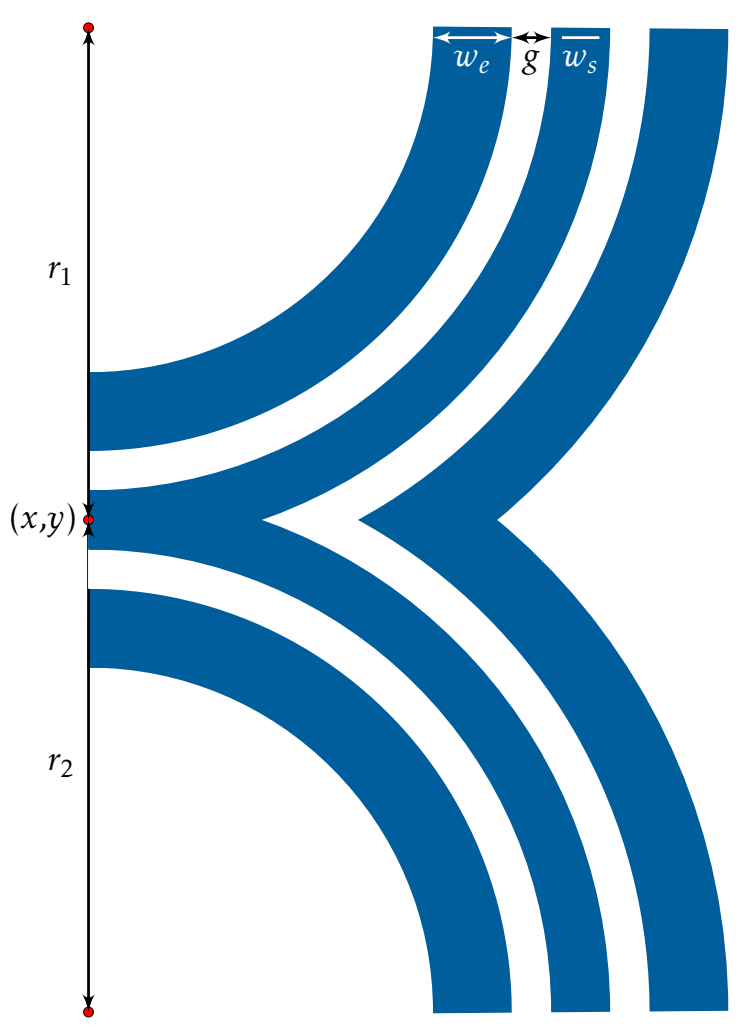

Figure 2.124: An example of a 90 degree Y-Bend inverse slot shape. 


\subsubsection{90 Degree Bend}

90 Degree Bends are Bezier curves characterized by two constructors. First consists of the start $\left(x_{1}, y_{1}\right)$ and end $\left(x_{2}, y_{2}\right)$ points. The second is characterized by the start point $\left(x_{1}, y_{1}\right)$, length $(L)$ and height $(H)$ parameters. Both constructors are characterized by a waveguide width $(W)$ and the rotation about the start point $\left(x_{1}, y_{1}\right)$. shapeReso parameter defines the rendering resolution of the 90 Degree Bend (Bezier-based) curve.

$\begin{array}{lllllll}<x_{1} & y_{1} & x_{2} & y_{2} & W & \theta_{\left(x_{1}, y_{1}\right)} & \text { 90degreeBend }> \\ <x_{1} & y_{1} & L & H & W & \theta_{\left(x_{1}, y_{1}\right)} & 90 \text { degreeBendLH }>\end{array}$

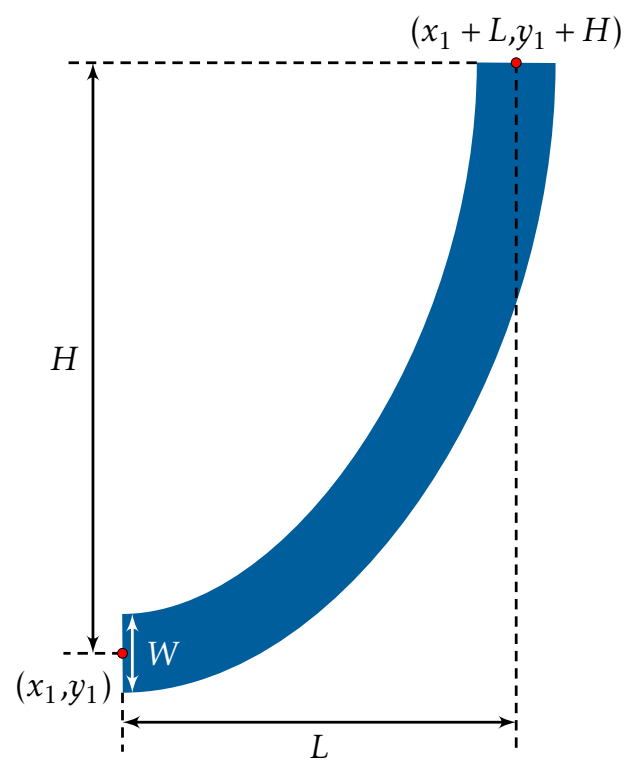

Figure 2.125: An example 90 degree bend GDS shape illustrating various parameters from the two constructors. 


\subsubsection{90 Degree Bend Inverse}

Similar to 90 Degree Bends (section 2.9.3.13), 90degreeBendlnv constructor is used to create a 90 degree bend waveguide structure using a positive tone resist. Alternatively, using a negative tone resist, a slot-waveguide of width $W+W_{e}$ and a slot of width $W$ is constructed. The shape is characterized by the start coordinate point $\left(x_{1}, y_{1}\right)$, length $(L)$, height $(H)$, waveguide width $(W)$, exposure sleeve $\left(W_{e}\right)$ and the rotation about the start point $\left(x_{1}, y_{1}\right)$ parameters. shapeReso parameter defines the rendering resolution of the 90 degree (Bezier based) curve. Both length and height parameters are defined by positive and negative double precision values relative to the start coordinate point $\left(x_{1}, y_{1}\right)$. For instance, a negative value of $H$ would place the end point $y$ value at $y_{1}-H$, hence value of $H$ in Figure 2.126 is positive .

$\begin{array}{llllllll}<x_{1} & y_{1} & L & H & W & W_{e} & \theta_{\left(x_{1}, y_{1}\right)} & \text { 90degreeBendlnv }>\end{array}$

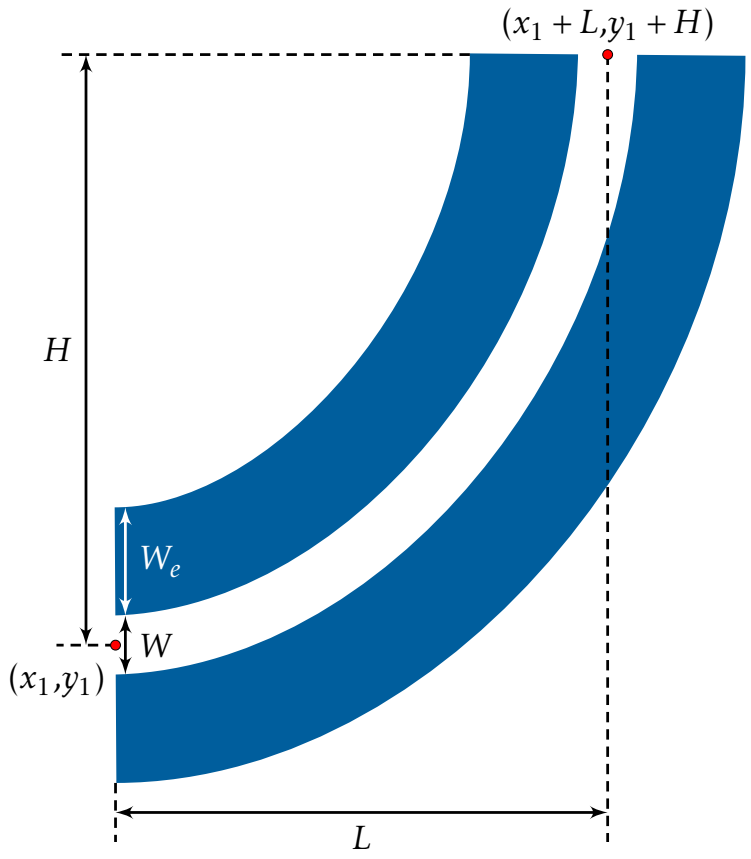

Figure 2.126: Schematic illustration showing various parameters from the 90degreeBendlnv constructor. 


\subsubsection{90 Degree Bend Inverse Slot}

Similar to 90 Degree Bends (section 2.9.3.13), 90degreeBendlnv constructor is used to create a 90 degree bend slot waveguide structure using a positive tone resist. The shape characterized is characterized by the start coordinate point $\left(x_{1}, y_{1}\right)$, length $(L)$, height $(H)$, slot width $\left(W_{s}\right)$, gap $(g)$, exposure sleeve width $\left(W_{e}\right)$ and the rotation about the start point $\left(x_{1}, y_{1}\right)$ parameters. shapeReso parameter defines the rendering resolution of the 90 degree (Bezier-based) curve. Both length and height parameters are defined by positive and negative double precision values relative to the start coordinate point $\left(x_{1}, y_{1}\right)$. For instance, a negative value of $H$ would place the end point $y$ value at $y_{1}-H$, hence value of $H$ in Figure 2.127 is positive.

$\begin{array}{lllllllll}<x_{1} & y_{1} & L & H & W & g & W_{e} & \theta_{\left(x_{1}, y_{1}\right)} & \text { 90degreeBendlnvSlot }>\end{array}$

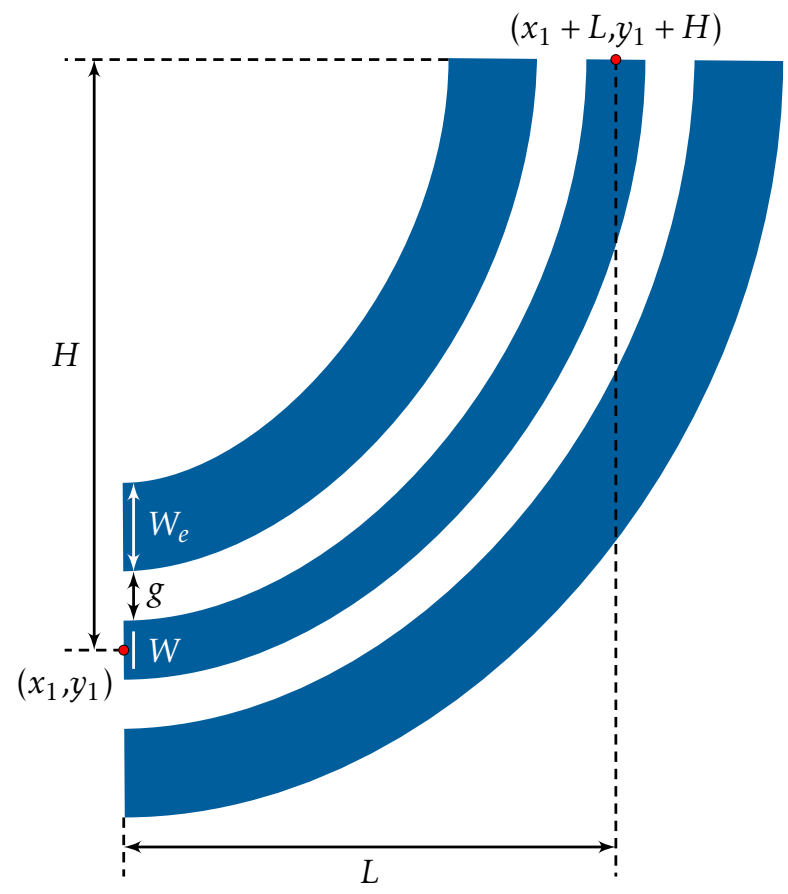

Figure 2.127: Schematic illustration showing various parameters from the 90degreeBendlnvSlot constructor.

NIST • CNST Nanolithography Toolbox v2016.09.01 • http://www.nist.gov/cnst/

page 141 of 488 


\subsubsection{180 Degree Bend}

The 180 degree, $\mathrm{u}$-shaped, bend is characterized by a lengths $L_{1}$ and $L_{2}$ with a coupled half torus of diameter $D$. The number of vertices constructing the torus is represented by the $N$ parameter. $D$ is measured from the midpoints of the two length segments.

$\begin{array}{lllllllll} & y & L_{1} & L_{2} & D & W & N & \theta_{(x, y)} & \text { 180degreeBend }>\end{array}$

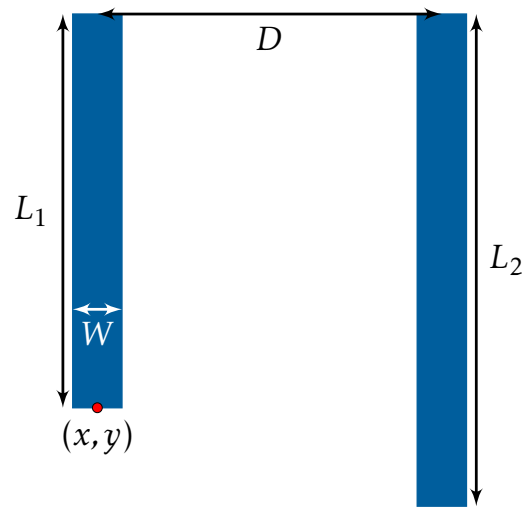

Figure 2.128: 180 degree bend example. 


\subsubsection{180 Degree Bend Inverse}

Similar to the 180 degree bend in the previous section (2.9.3.16), the inverse device has an additional exposure sleeve parameter $W_{e}$.

$\begin{array}{lllllllllll} & y & y & L_{1} & L_{2} & D & W & W_{e} & N & \theta_{(x, y)} & 180 \text { degreeBendlnv }>\end{array}$

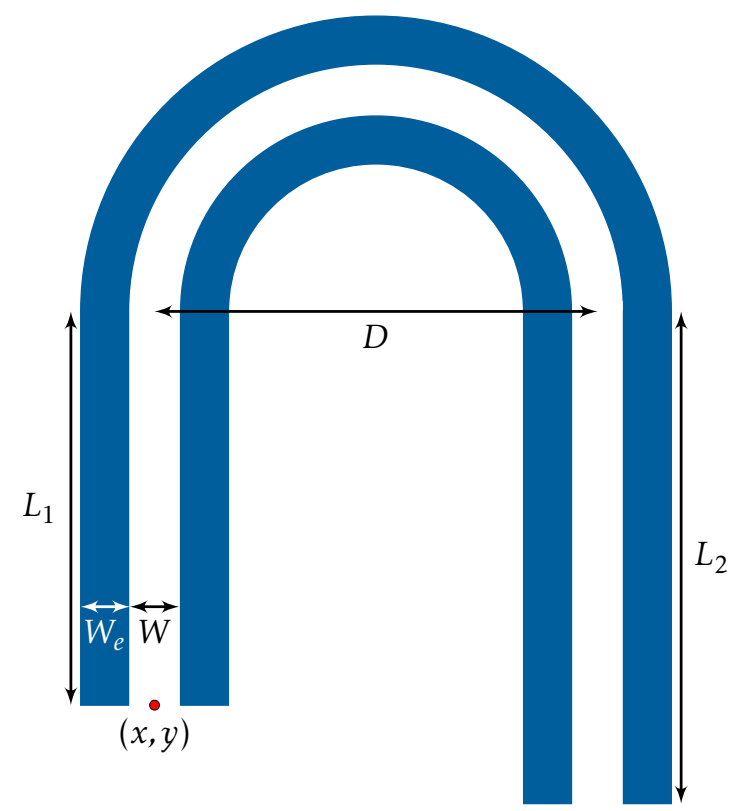

Figure 2.129: 180 degree bend inverse example. 


\subsubsection{180 Degree Bend Inverse Slot}

Similar to the 180 degree inverse bend in the previous section (2.9.3.17), the inverse slot device has an additional gap parameter $g$.

$$
\begin{array}{ccccccccccc}
<x & y & L_{1} & L_{2} & D & W & g & W_{e} & N & \theta_{(x, y)} & 180 \text { degreeBendlnvSlot }>
\end{array}
$$

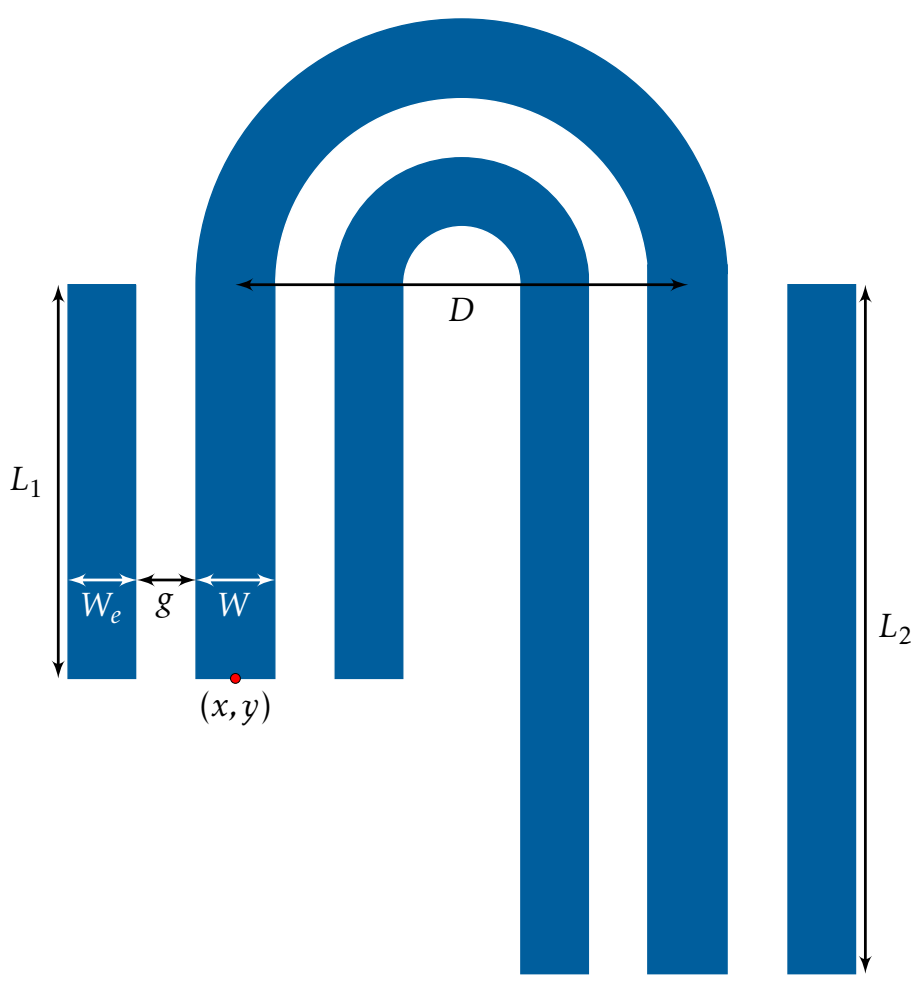

Figure 2.130: 180 degree bend inverse slot example. 


\subsubsection{Racetrack}

Racetrack object is characterized by the center coordinate $(x, y)$, length of the straight section $(L)$, track width $(W)$, inner radius $\left(r_{i n}\right)$, rotation about the center point $\left(\theta_{(x, y)}\right)$, and the number of segments creating each of the curved sections $(N)$.

$$
\begin{array}{llllllll}
<x & y & L & W & r_{i n} & \theta_{(x, y)} & N & \text { raceTrack }>
\end{array}
$$

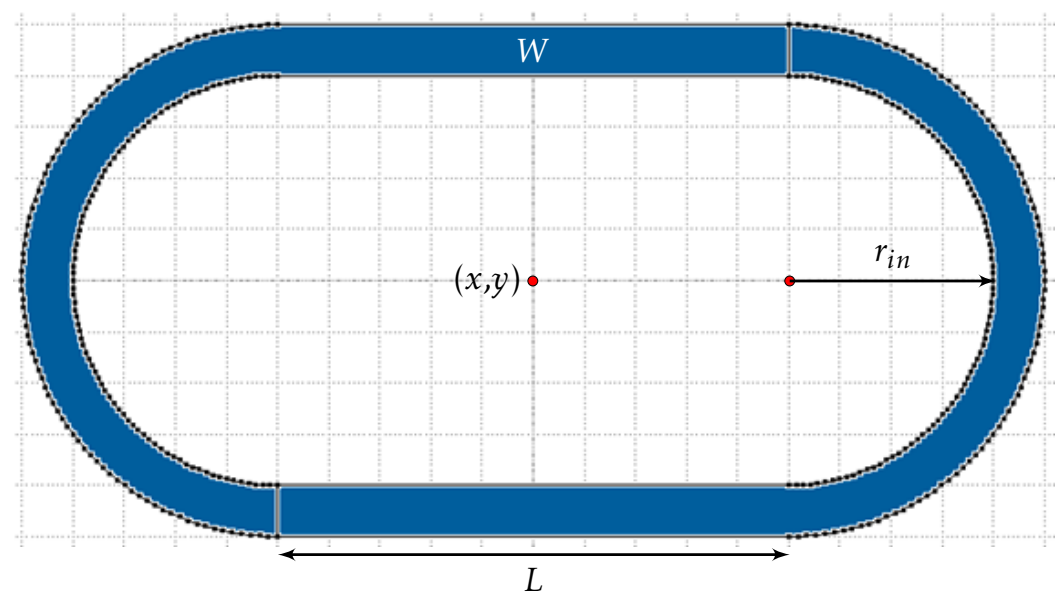

Figure 2.131: An example GDS shape illustrating various parameters from the raceTrack constructors. Dots along the structural periphery represent the number of points $(N)$ used to construct the curved racetrack sections. 


\subsubsection{Spiral Delay Line}

Interdigitated spiral delay line objects are characterized by either uniform (Archimedes) and converging (Fermat's) spacing between subsequent turns. Constant spacing Archimedes spiral delay line illustrated in Figure 2.9.5a is represented by

$$
r(\theta)=\left\{\begin{array}{c}
m \theta \\
-m \theta
\end{array}\right.
$$

where $m=(S+W) /(2 \pi)$, and the separation parameter $S$ defines the pitch between subsequent spiral turns. Spiral is further defined by $\Delta_{R}$ defining the resolution of the spiral, the length of the coupling waveguide $(L)$, the rotation about center $\left(\theta_{(x, y)}\right)$, and $E C=1$ or 0 corresponding to the waveguide coupling segment without and with semi-circular endcaps. Fermat's spiral spiral delay line illustrated in Figure 2.9.5b is represented by

$$
r(\theta)=\left\{\begin{array}{c}
\sqrt{a^{2} \theta} \\
-\sqrt{a^{2} \theta}
\end{array}\right.
$$

$$
\begin{array}{llllllllll}
x & y & W & N_{\text {turns }} & S & \Delta_{R} & L & \theta_{(x, y)} & E C & \text { spiralDelayLineArch } \\
x & y & W & N_{\text {turns }} & a & \Delta_{R} & L & \theta_{(x, y)} & E C & \text { spiralDelayLineFermat }
\end{array}
$$

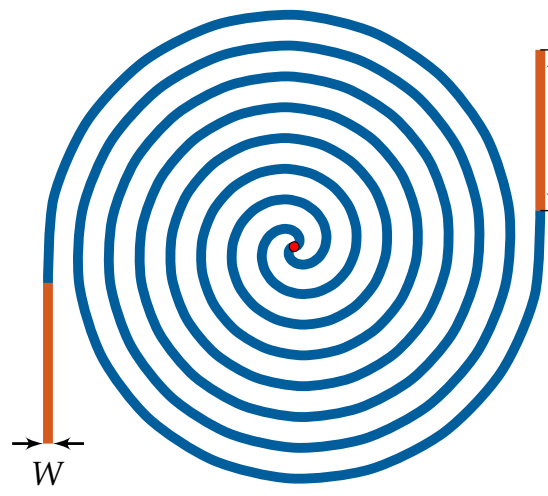

(a)

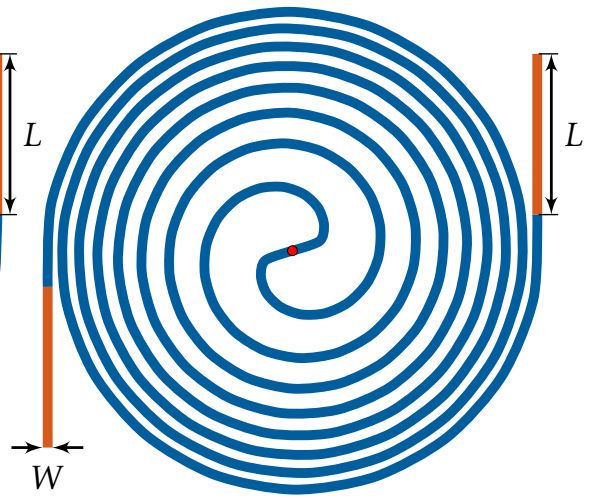

(b)

Figure 2.132: Spiral delay lines for the (a) Archimedes spiral constructor spiralDelayLineArch and (b) Fermat spiral constructor spiralDelayLineFermat. Central dot represents the coordinate defined by $(x, y)$.

IMPORTANT NOTE: The above interdigitated spirals are created and fractured along the $y$-axis. On the one hand, this action eliminates any possibility of exceeding maximum number of allowed points per GDS shape. On the opposite 
end of the spectrum, this action is computation intensive, consuming large amounts of RAM and takes a considerable amount of time for spirals of many turns defined by a large collection points.

The following is an interdigitated Archimedes spiral delay line that alleviates some of the computational strains of the previous version. The delay line illustrated in Figure 2.133 is characterized by a center point $(x, y)$, spiral width, the number of turns $\left(N_{\text {turns }}\right)$, separation $(S)$ between subsequent turns, points per turn $\left(P_{T}\right)$, and the number of skipped turns before spiral rendering initiates $\left(S_{T}\right)$. In this case, points per spiral turn are controlled, hence for spirals containing many turns, the resolution of the outermost ring will be coarser than the internal rings. This spiral is generated much faster than ones shown in Figure 2.132.

\section{$\begin{array}{llllllllllll}x & y & W & N_{\text {turns }} & S & P_{T} & S_{T} & L & \theta_{(x, y)} & E C & \text { spiralDelayLineArchV2 }\end{array}$}

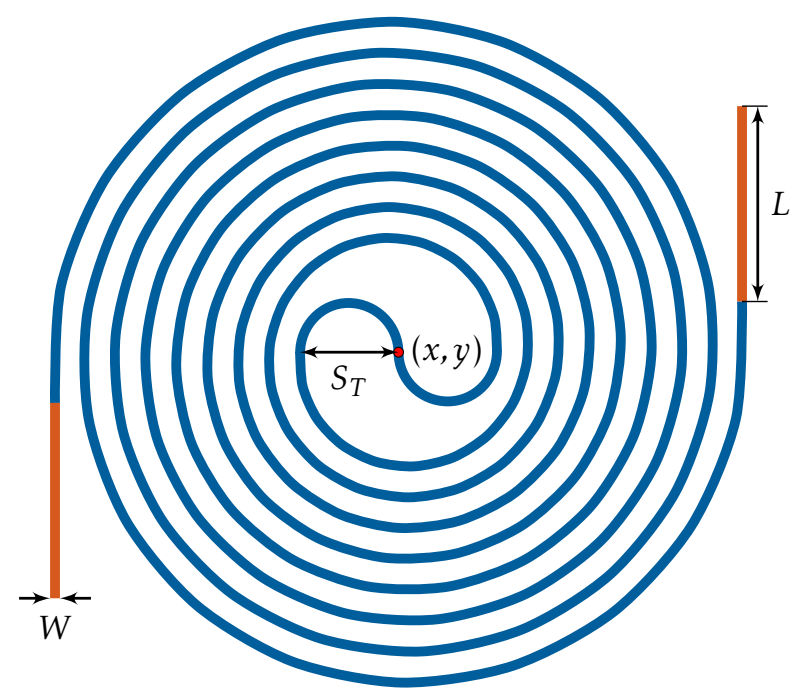

Figure 2.133: Archimedes interdigitated spiral delay line constructed by spiralDelayLineArchV2 constructor. Parameter $S_{T}$ is an integer $\left(S_{T} \geq 0\right)$ defining the number of skipped turns before rendering of the spiral initiates. 


\subsubsection{Inverse Spiral Delay Line}

Inverse spiral delay line objects are characterized by either uniform (Archimedes) and converging (Fermat's) spacing between subsequent turns. Section 2.9.5 shows details for the construction of the two spirals. These spirals are defined by the center point $(x, y)$, slot width $W$, exposure sleeve width $W_{e}$, number of turns $\left(N_{\text {turns }}\right)$, separation $(S)$ between turns, and the $\Delta_{R}$ value defining resolution rendering of the spiral.
$\begin{array}{llllllllllll}x & y & W & W_{e} & N_{t u r n s} & S & \Delta_{R} & L & \theta_{(x, y)} & E C & \text { spiralDelayLineArchlnv }\end{array}$
$\begin{array}{lllllllllll}x & y & W & W_{e} & N_{\text {turns }} & a & \Delta_{R} & L & \theta_{(x, y)} & E C & \text { spiralDelayLineFermatlnv }\end{array}$

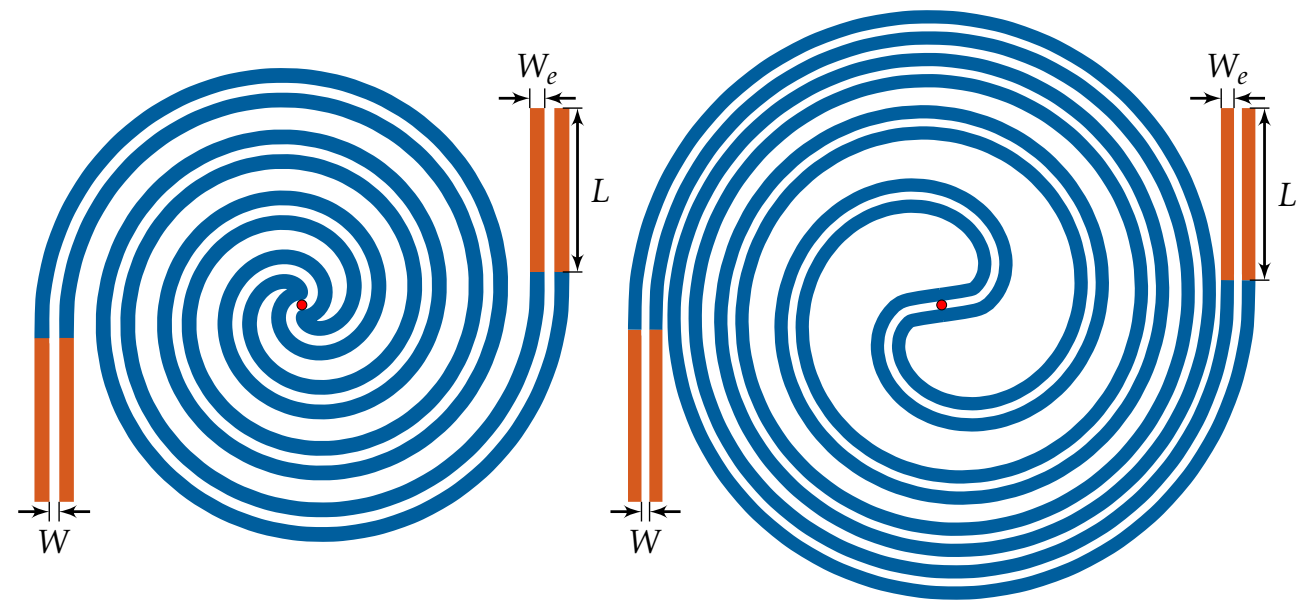

(a)

(b)

Figure 2.134: Inverse spiral delay lines for the (a) Archimedes spiral constructor spiralDelayLineArchlnv and (b) Fermat spiral constructor spiralDelayLineFermatlnv. Central dot represents the coordinate defined by $(x, y), W$ and $W_{e}$ are the corresponding slot and exposure sleeve widths. 
The following is another version of the inverted Archimedes spiral delay line. The delay line illustrated in Figure 2.135 is characterized by a center point $(x, y)$, spiral width, exposure sleeve width, the number of turns $\left(N_{\text {turns }}\right)$, separation $(S)$ between subsequent turns, points per turn $\left(P_{T}\right)$, and the number of skipped turns before spiral rendering initiates $\left(S_{T}\right)$. In this case, points per spiral turn are controlled, hence for spirals containing many turns, the resolution of the outermost ring will be coarser than the internal rings.

$\begin{array}{lllllllllllll}x & y & W & W_{e} & N_{t u r n s} & S & P_{T} & S_{T} & L & \theta_{(x, y)} & E C & \text { spiralDelayLineArchV2Inv }\end{array}$

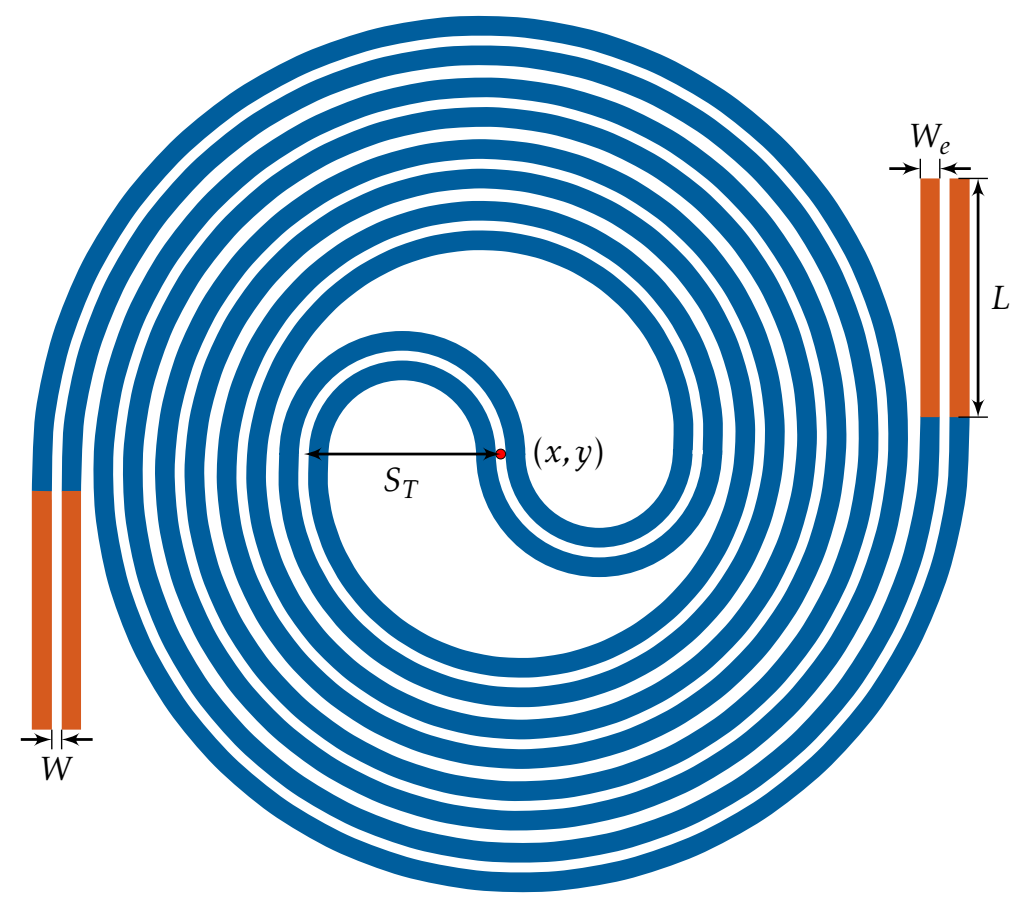

Figure 2.135: Archimedes interdigitated spiral delay line constructed by spiralDelayLineArchV2Inv constructor. Parameter $S_{T}$ is an integer $\left(S_{T} \geq 0\right)$ defining the number of skipped turns before rendering of the spiral initiates.

IMPORTANT NOTE: The above interdigitated spirals (Figures 2.134- 2.135) are created and fractured along the $y$-axis. As mentioned in the previous section, fracturing eliminates any possibility of exceeding maximum number of allowed points per GDS shape. Unfortunately, the fracturing is computationally intensive, consuming large amounts of RAM for spirals of many turns defined by a large collection points. 


\subsubsection{Gratings}

\subsubsection{Grating}

Grating structure is defined by the line width, length and pitch. A single line is cast into a GDS structure singleRectangleStructName and arrayed (instantiated) at the specified pitch within the existing GDS structure. $N_{\text {lines }}$ parameter represents the number of instantiated lines.

$$
\text { <singleRectangleStructName } \quad x \quad y \quad W \quad L \quad \text { Pitch } N_{\text {lines }} \text { grating> }
$$

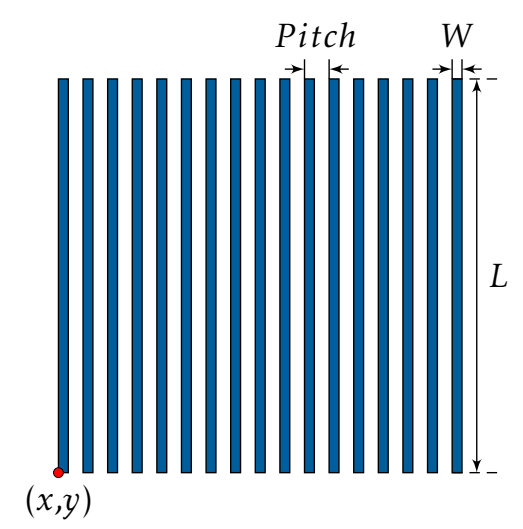

Figure 2.136: Example of the grating constructor. 


\subsubsection{Apodized Grating}

The apodized grating structure is defined by the grating length $\left(G_{L}\right)$, the increment $\left(\Delta G_{L}\right)$, grating line height $(H)$, duty cycle cutoff $\left(d_{C C}\right)$, and grating parameters $p_{i}$ and $d_{i}$. The grating extends from $\left(-\frac{G_{L}}{2}\right)$ to $\left(+\frac{G_{L}}{2}\right)$ with the lower edge centered around $(x, y)$.

$\begin{array}{llllllllllllllllll}x & y & G_{L} & \Delta G_{L} & H & d_{C C} & p_{1} & p_{2} & p_{3} & p_{4} & d_{1} & d_{2} & d_{3} & d_{4} & d_{5} & \theta_{(x, y)} & \text { apodizedGrating }\end{array}$

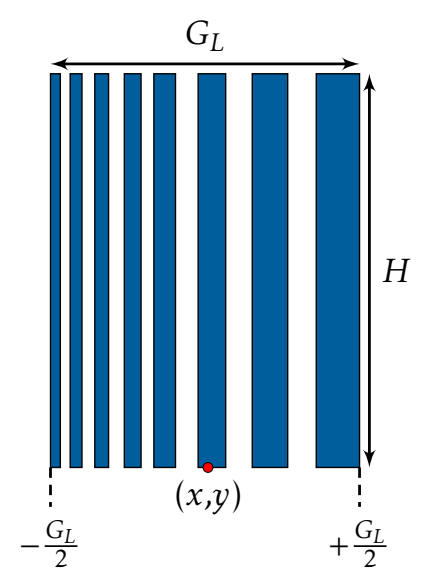

Figure 2.137: Example of the apodizedGrating constructor. 


\subsubsection{Grating Coupler}

Structure characterized by a center $(x, y)$, distance $(r)$ from center to the midpoint of the first arc section, width $(W)$ and pitch $(p)$ of the arc section, start $\left(\theta_{s}\right)$ and end angles $\left(\theta_{e}\right)$, number of sides $\left(N_{\text {sides }}\right)$ for each of the arc sections and number of elements $\left(N_{\text {elements }}\right)$.

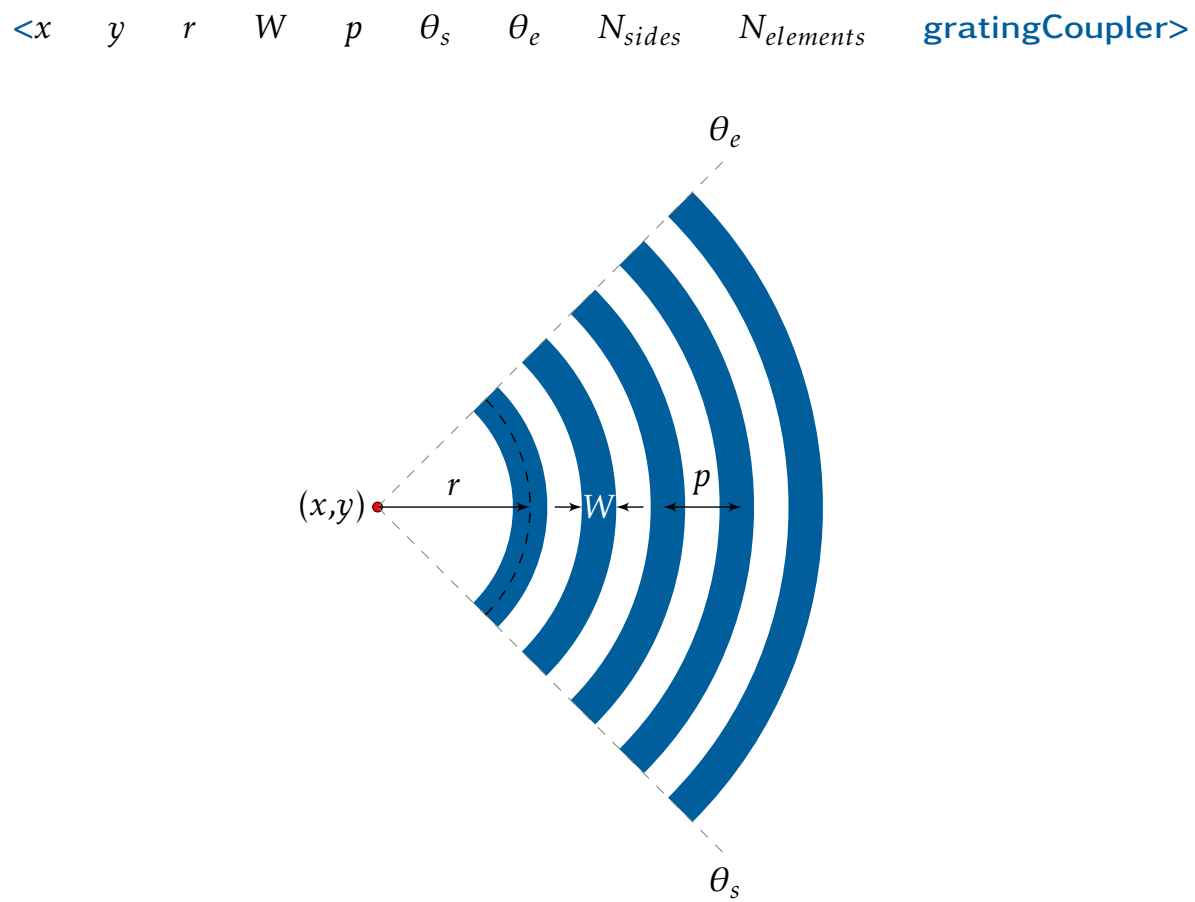

Figure 2.138: Example illustrating various parameters of the gratingCoupler constructor. 


\subsubsection{Grating Couplers With Waveguides}

The following constructors generate grating couplers with integrated waveguides within the $x-y$ plane using

$$
q \lambda_{\circ}=n_{e f f} \sqrt{x^{2}+y^{2}}-x n_{c} \cos \theta_{c}
$$

where the focal point is located at the origin $(x, y)$ with $x$ along the waveguide axis, $q$ is an integer for each grating line, $\theta_{c}$ is the grating angle span, $n_{c}$ and $n_{\text {eff }}$ are the respective cladding and effective refractive index values, and $\lambda_{\circ}$ is the wavelength. The following are the constructors for the waveguide (GDS Layer $L_{w g}$ ) with an integrated grating coupler (GDS Layer $L_{g}$ )

$<x y w L H s \lambda_{\circ} n_{e f f} n_{c} \theta_{c} R g_{p}$ ratio $N_{g} N_{s} L_{w g} L_{g} E C \theta_{(x, y)}$ gratingCWGinv $>$

$<x y w L H \quad \lambda_{\circ} n_{e f f} n_{c} \theta_{c} R g_{p}$ ratio $N_{g} N_{s} L_{w g} L_{g} E C \theta_{(x, y)}$ gratingCWG $>$

As labeled in Figures 2.139 and 2.140, the structure is placed and rotated $\left(\theta_{(x, y)}\right)$ relative to the origin $(x, y)$. Waveguides are constructed using a bezier s-bend curve and defined by a width $(w)$, length $(L)$ and height $(H)$. A negative value of $H$ will extend the waveguide below the $y$-axis. Sleeve width for inverse waveguides (Figures 2.139a and 2.140a) is defined by $s, R$ is the distance to the outermost grating, $g_{p}$ is the grating pitch and the duty cycle is defined by the ratio value. Consequently the grating line width is given by

$$
g_{w}=g_{p} * \text { ratio }
$$

$N_{g}$ defines the number of generated grating lines with the maximum number grating lines within $R$ given by

$$
N_{\max }=\text { ceil } \frac{n_{e f f} R}{\lambda_{\circ}}
$$

The outer and inner radius of each ring is defined by

$$
\begin{gathered}
r_{q(\text { out })}=\frac{q \lambda_{\circ}}{n_{e f f}-n_{c} \cos \alpha_{i} \cos \theta} \\
r_{q(\text { in })}=r_{q(\text { out })}-\left(g_{p} * \text { ratio }\right)=r_{q(\text { out })}-g_{w}
\end{gathered}
$$

where

$$
\theta=\arccos n_{e f f}-\frac{\lambda_{\circ}}{g_{p}}
$$

and $\alpha_{i}$ is the angular range from $-\theta_{x}$ to $\theta_{c}$. Parameter $\left(x, y, w, L, H, s, \lambda_{\circ}, R, g_{p}\right)$ dimensions are expressed in micrometers.

NIST • CNST Nanolithography Toolbox v2016.09.01 • http://www.nist.gov/cnst/

page 153 of 488 

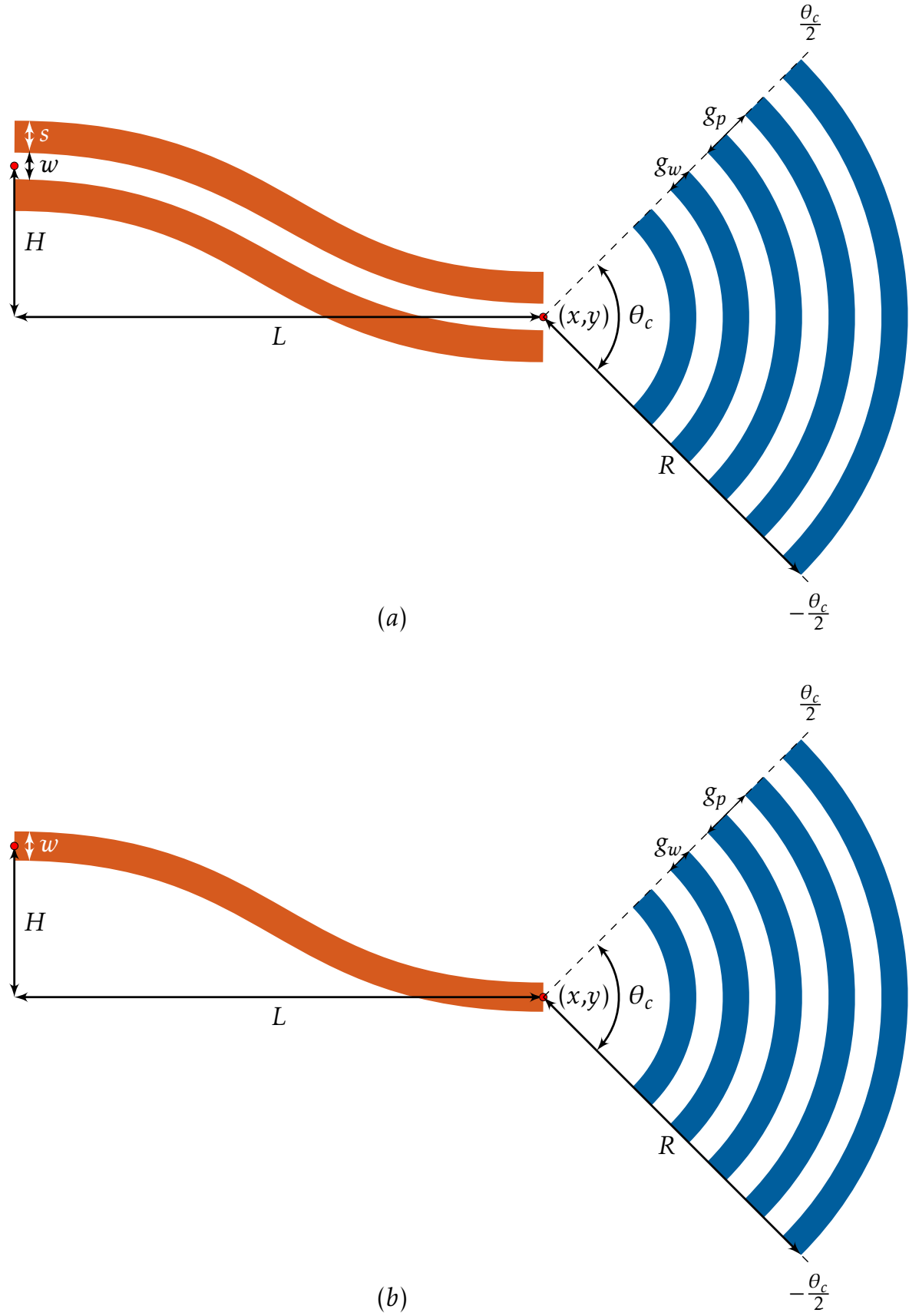

Figure 2.139: Grating couplers (a) waveguide with sleeve and (b) waveguide with $E C=0$. 

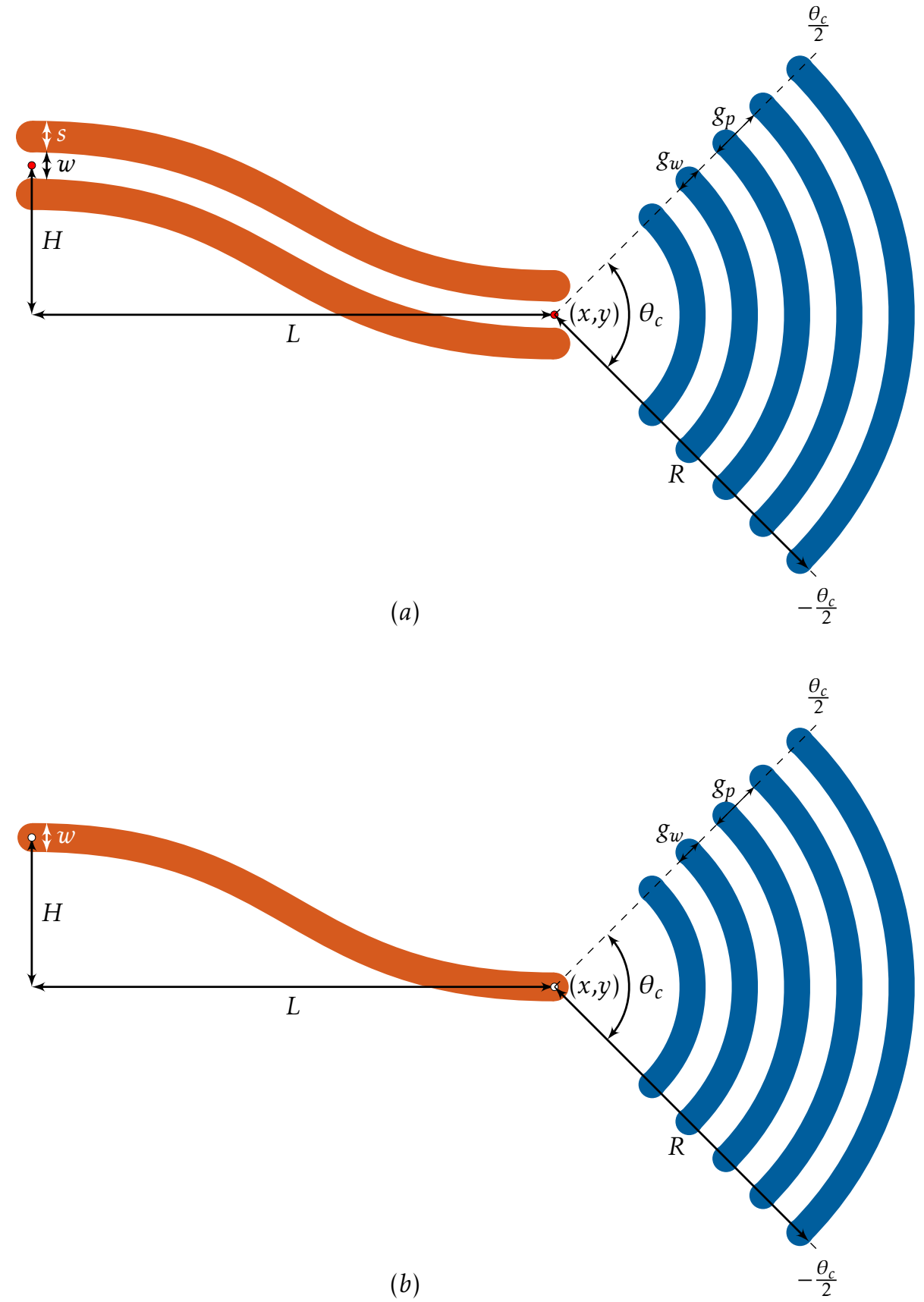

Figure 2.140: Grating couplers with endcaps. (a) waveguide with sleeve and (b) waveguide with $E C=1$. 


\subsubsection{Photonic Crystals and Hexagonal Arrays}

Photonic crystal and hexagonal array structures characterized by a center $(x, y)$, circle radius $(r)$, pitch $\left(x_{p}\right)$, number of elements $N_{x}$ and $N_{y}$ in the corresponding $x$ and $y$ directions, and spacing $(\delta)$ between the two arrays. Parameter uniqueStructName represents the name for the structure (cell) for the circular element. The circular element is then arrayed to construct the photonic crystal structure.

Within constructors phC and hex, parameter $N_{\text {sides }}$ defines the number of sides of the individual polygon/circular apertures. When using vectorized constructors ( $\mathrm{phCV}$ and hexV), resulting structures are circles with number of sides determined by the shape rendering resolution parameter shapeReso.

$\begin{array}{lllllllllll}\text { <uniqueStructName } & x & y & r & x_{p} & N_{x} & N_{y} & N_{\text {sides }} & \delta & \text { phC> } \\ \text { <uniqueStructName } & x & y & r & x_{p} & N_{x} & N_{y} & & \delta & \text { phCV > } \\ \text { <uniqueStructName } & x & y & r & x_{p} & N_{x} & N_{y} & N_{\text {sides }} & & \text { hex> } \\ \text { <uniqueStructName } & x & y & r & x_{p} & N_{x} & N_{y} & & & \text { hexV> }\end{array}$

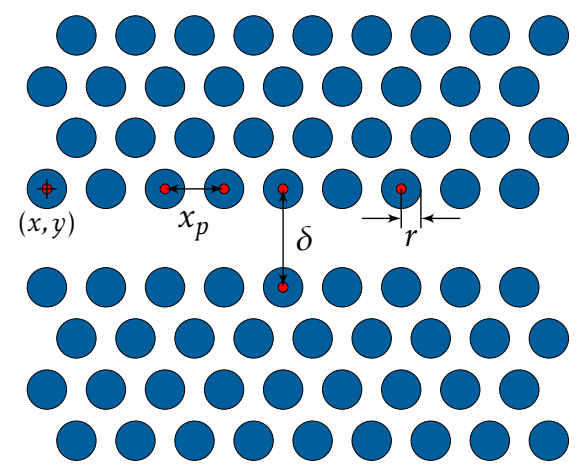

(a)

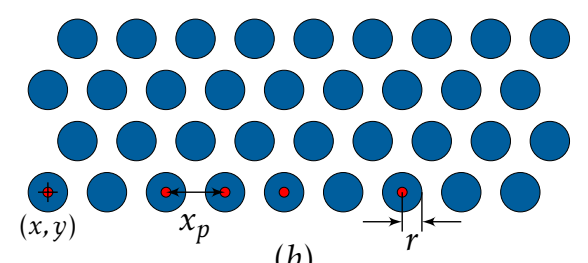

(b)

Figure 2.141: (a) Photonic crystal and (b) hexagonal array examples illustrating various constructor parameters.

NIST • CNST Nanolithography Toolbox v2016.09.01 • http://www.nist.gov/cnst/

page 156 of 488 


\subsubsection{Disc-Ring Architectures}

\subsubsection{Disc-Ring - Bezier Curves, Arcs and Endcaps}

The following sections illustrate coupled waveguides to disc and ring structures. The coupling region is defined by an arc section that symmetrically interacts with the disc/ring structure. The waveguide region leading away from the coupling region is defined by either a Bezier or an arc segment.

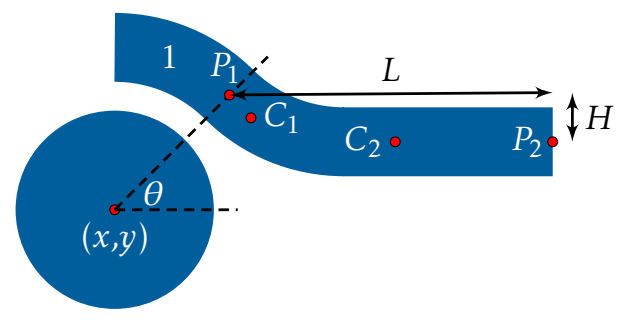

(a)

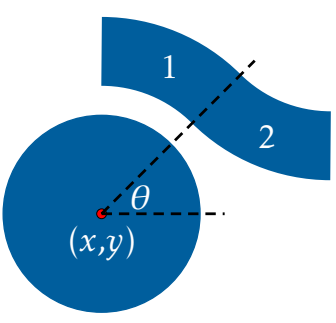

(b)

Figure 2.142: Schematic illustration highlighting the waveguide coupling region constructed using (a) Bezier and (b) arc segments.

Figure 2.142a illustration has coupling region (labeled as 1 ) defined by an arc. The continuing segment ending at a distance $L$ is defined by a Bezier curve consisting of start and end points $\left(P_{1}\right.$ and $\left.P_{2}\right)$ and their corresponding control points $C_{1}$ and $C_{2}$. Control points are defined as,

$$
\begin{aligned}
& C_{1 x}=P_{1 x}+\frac{R}{4} * \cos (\phi) \\
& C_{1 y}=P_{1 x}-\frac{R}{4} * \sin (\phi) \\
& C_{2 x}=P_{2 x}-\frac{L}{2} \\
& C_{2 y}=P_{2 y}
\end{aligned}
$$

where $R=\sqrt{H^{2}+L^{2}}$, and $\phi=90-\theta$.

Figure $2.142 \mathrm{~b}$ shows a coupling region defined by two arc segments. For this segment, the straight waveguide portion of length $L$ is omitted. In both cases, the drawn structures are mirrored around the $y$-axis.

NIST • CNST Nanolithography Toolbox v2016.09.01 • http://www.nist.gov/cnst/

page 157 of 488 


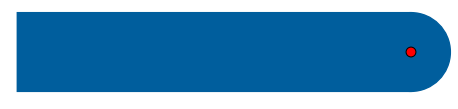

(a)

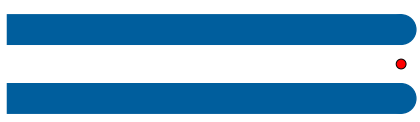

(b)

Figure 2.143: Illustrations showing semicircular encaps on the right hand side of (a) a waveguide and (b) an inverse and inverse positive waveguide. Endcaps are included on both left and right sides of the waveguide when parameter $E C=1$. The red circle denotes the end point of the waveguide structure. Semicircular endcap radius of the equals half of the waveguide width.

Figure 2.143 shows shows waveguide structures with semicircular endcaps. The structures are encountered in subsequent sections and are useful when stressed thin film underlayers are patterned using thick resists. The corner rounding at the waveguide termination end alleviates the stress field, thereby mitigating resist and thin film cracking during subsequent reactive ion etch processing steps. 


\subsubsection{Disc-Ring Infinite}

Shapes characterized by a straight waveguide $\left(R_{w g}=\infty\right)$ above a disc and ring objects. Both objects are described by the center coordinate $(x, y)$, number of segments $(N)$, gap between the object and shape $(g)$, waveguide length $(L)$ and width $(W)$. Disc object is defined by a radius $\left(r_{d}\right)$ and the ring is characterized by an inner radius $r_{i}$ and ring width $\left(W_{r}\right)$. Boolean parameter $E C$ controls if semicircular endcaps are incorporated in the waveguide structure. $E C$ is an integer value with zero representing false, hence waveguides without endcaps, i.e. $E C=0$ and $E C=1$ for waveguides without and with endcaps, respectively.

$$
\begin{array}{llllllllll}
<x & y & r_{d} & & N & g & L & W & E C & \text { disclnfinite }> \\
<x & y & r_{i} & W_{r} & N & g & L & W & E C & \text { ringInfinite }>
\end{array}
$$

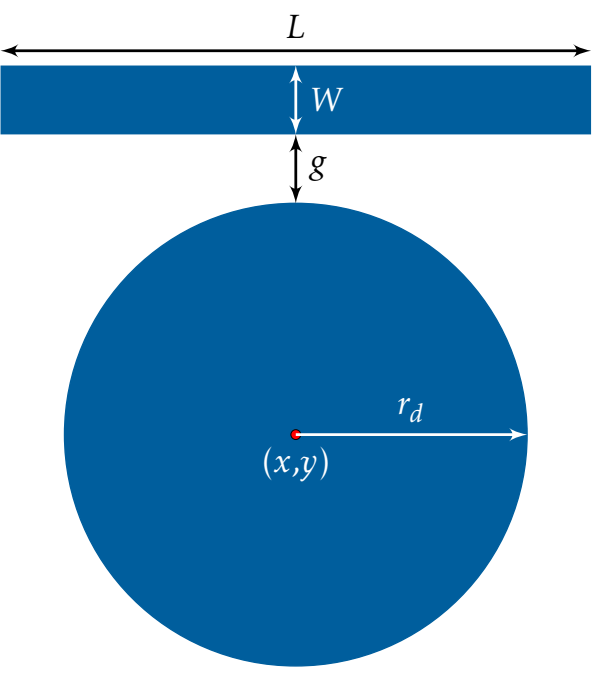

(a)

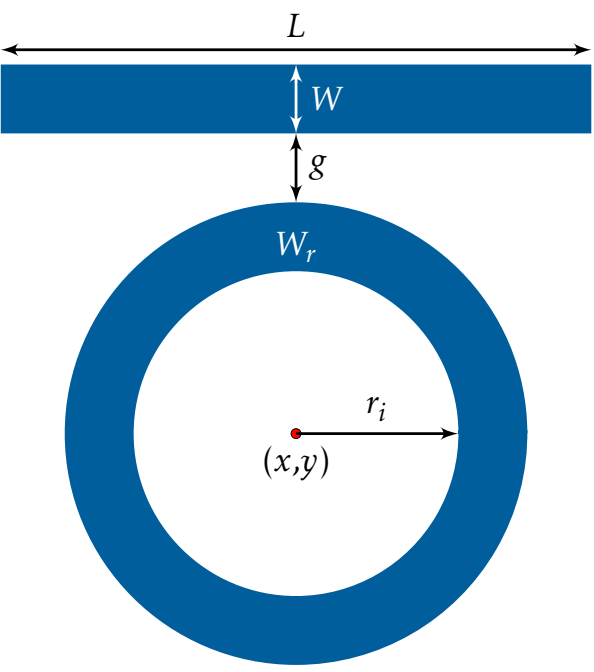

(b)

Figure 2.144: Examples illustrating various parameters of the (a) disclnfinite and (b) ringInfinite constructors. 
Infinite disc and ring structures with an additional coupling waveguide.

$$
\begin{array}{lllllllllllll}
<x & y & r_{d} & & N & g_{1} & L_{1} & W_{1} & g_{2} & L_{2} & W_{2} & E C & \text { disclnfDS }> \\
<x & y & r_{i} & W_{r} & N & g_{1} & L_{1} & W_{1} & g_{2} & L_{2} & W_{2} & E C & \text { ringlnfDS }>
\end{array}
$$

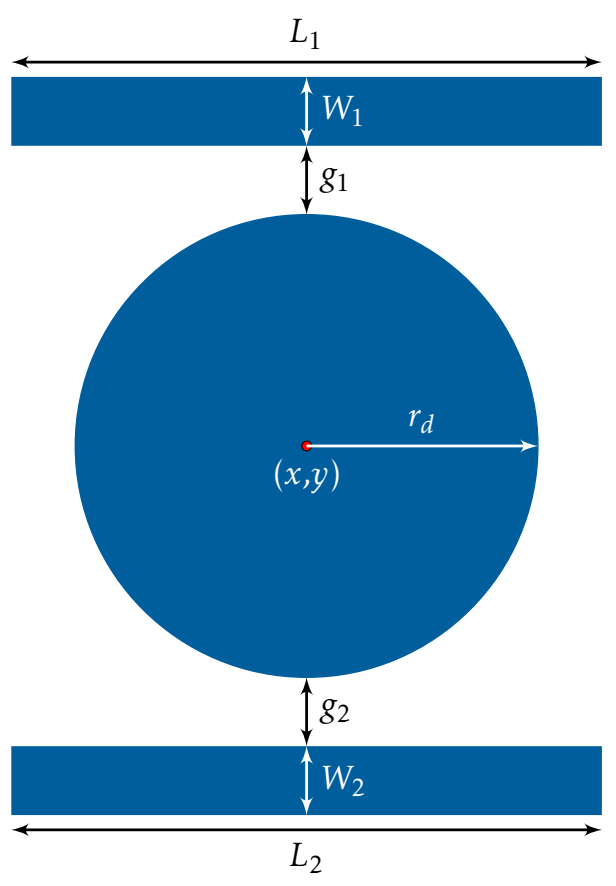

(a)

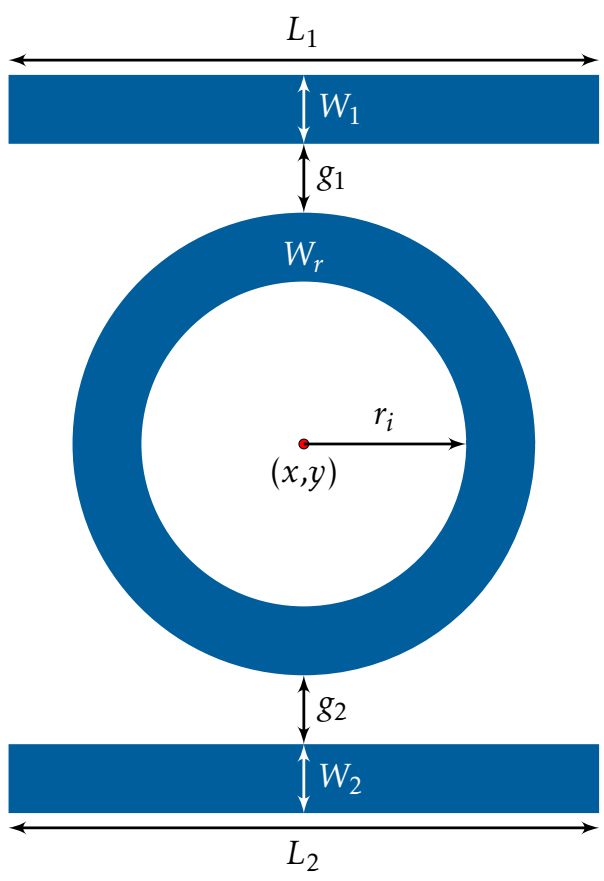

(b)

Figure 2.145: Examples illustrating various parameters of the (a) disclnfDS and (b) ringInfDS constructors. 


\subsubsection{Disc-Ring Infinite Inverse}

The structure is similar to the disc-ring waveguide infinite structure defined in the previous section 2.9.9.2. Here, a slot waveguide of width $W$ is formed by exposing a surrounding rectangular region of width $W_{e}$. The overall shapes are characterized by a straight waveguide $\left(R_{w g}=\infty\right)$ above a disc and ring objects. Both objects are described by the center coordinate $(x, y)$, number of segments $(N)$, gap between the object and shape $(g)$, waveguide length $(L)$, waveguide width $(W)$ and exposure sleeve width $\left(W_{e}\right)$. Disc object is defined by a radius $\left(r_{d}\right)$ and the ring is characterized by an inner radius $\left(r_{i}\right)$ and ring width $\left(W_{r}\right)$. Boolean parameter $E C$ controls if semicircular endcaps are incorporated in the waveguide structure $(E C=0$ and $E C=1$ for waveguides without and with endcaps, respectively).

$$
\begin{array}{lllllllllll}
<x & y & r_{d} & & N & g & L & W & W_{e} & E C & \text { disclnfinitelnv }> \\
<x & y & r_{i} & W_{r} & N & g & L & W & W_{e} & E C & \text { ringlnfinitelnv }>
\end{array}
$$

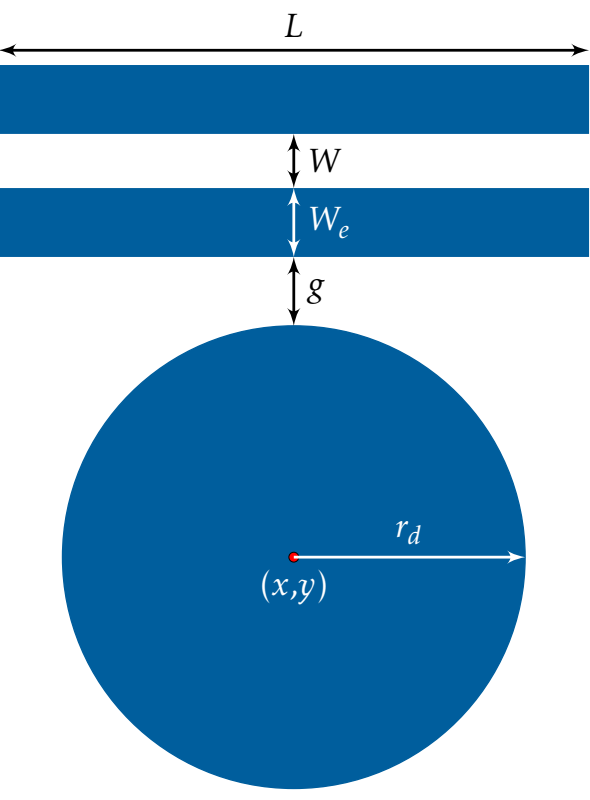

(a)

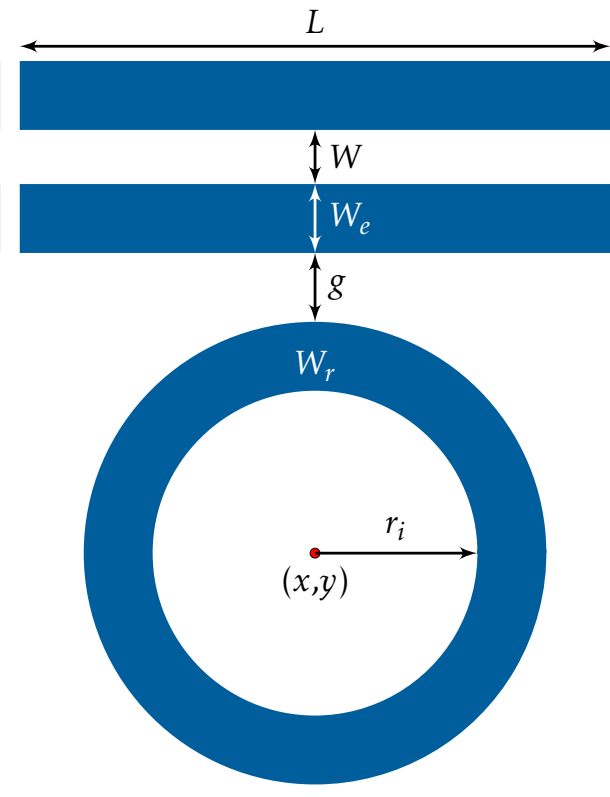

(b)

Figure 2.146: Examples illustrating various parameters of the (a) disclnfinitelnv and (b) ringInfinitelnv constructors. 
Infinite inverse disc and ring structures with an additional waveguide.

$$
\begin{array}{lllllllllllllll}
<x & y & r_{d} & & N & g_{1} & L_{1} & W_{1} & W_{e 1} & g_{2} & L_{2} & W_{2} & W_{e 2} & E C & \text { disclnflnvDS }> \\
<x & y & r_{i} & W_{r} & N & g_{1} & L_{1} & W_{1} & W_{e 1} & g_{2} & L_{2} & W_{2} & W_{e 2} & E C & \text { ringlnflnvDS }>
\end{array}
$$

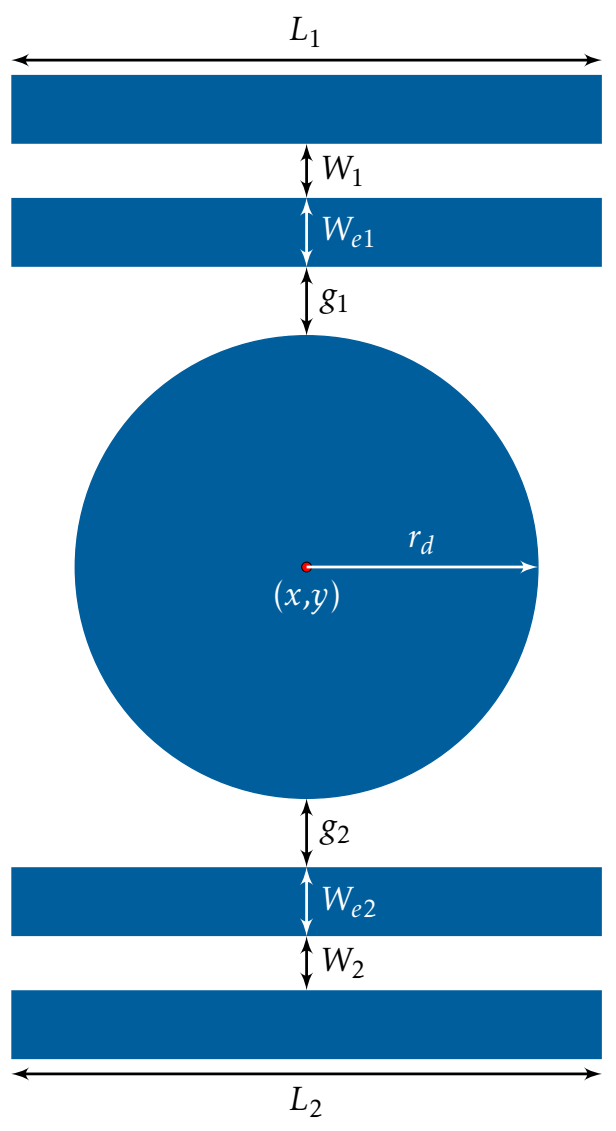

(a)

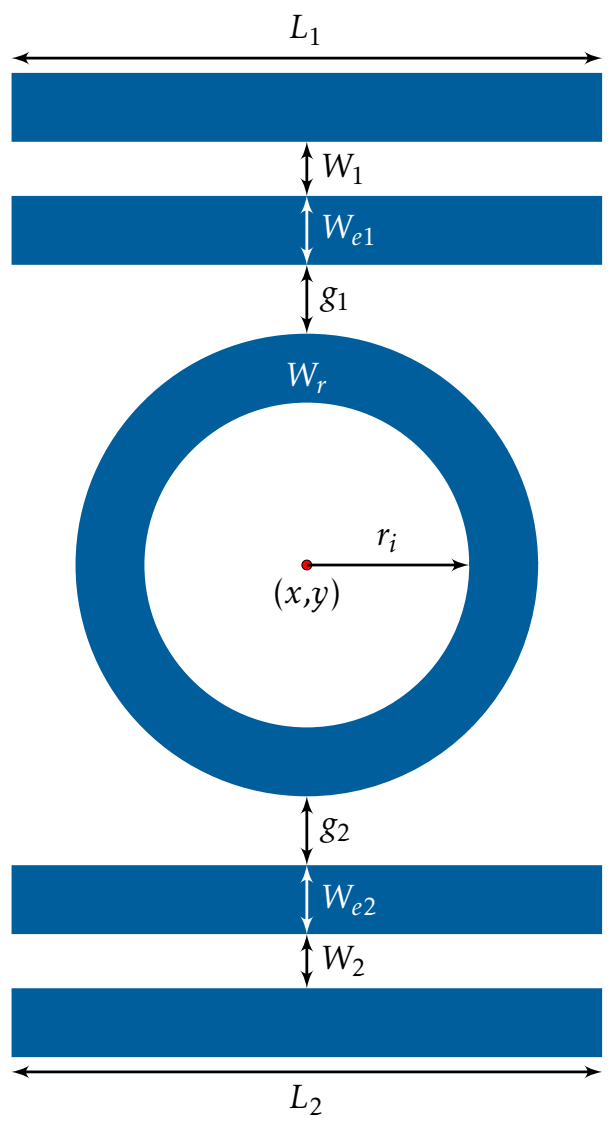

(b)

Figure 2.147: Examples illustrating various parameters of the (a) disclnflnvDS and (b) ringInfInvDS constructors. 


\subsubsection{Disc-Ring Infinite Inverse Positive Tone}

The structure is similar to the disc-ring waveguide structure defined in section 2.9.9.2. Here, a waveguide of width $W$ and either a ring of width $W_{r}$ or disc of radius $r_{d}-r_{e}$ are formed by positive tone resist exposure of the respective exposure sleeve regions $W_{e}$ and $r_{e}$. The overall shapes are characterized by a straight waveguide $\left(R_{w g}=\infty\right)$ above a disc and ring objects. Both objects are described by the center coordinate $(x, y)$, number of segments $(N)$, gap between the object and shape $(g)$, waveguide length $(L)$, waveguide width $(W)$ and exposure sleeve width $\left(W_{e}\right)$. Disc object is defined by a radius $\left(r_{d}\right)$ and the ring is characterized by a radius $\left(r_{i}\right)$ and ring width $\left(W_{r}\right)$.

$$
\begin{aligned}
& \begin{array}{lllllllllllll}
<x & y & r_{d} & & r_{e} & N & g & L & W & W_{e} & E C & \text { disclnfinitelnvPos }>
\end{array} \\
& \begin{array}{llllllllllll}
< & y & r_{i} & W_{r} & r_{e} & N & g & L & W & W_{e} & E C & \text { ringlnfinitelnvPos }>
\end{array}
\end{aligned}
$$

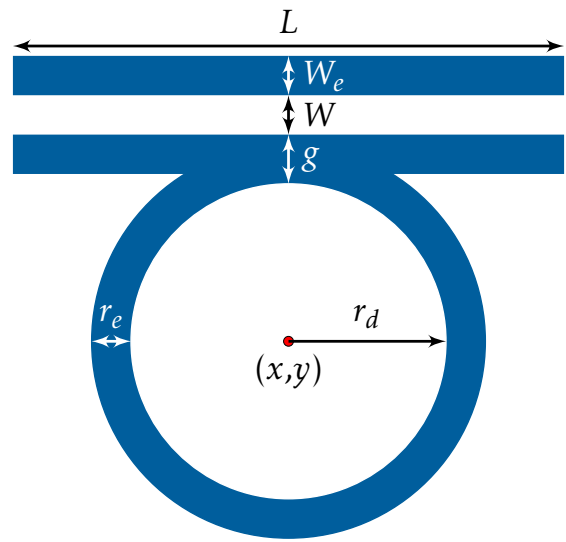

(a)

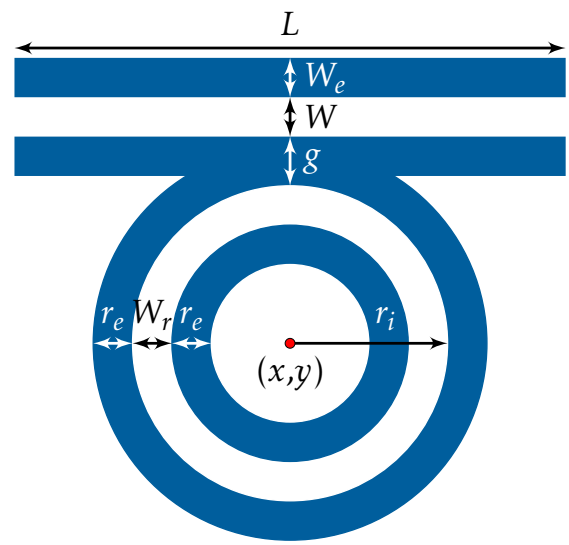

(b)

Figure 2.148: Examples illustrating various parameters of the (a) disclnfinitelnvPos and (b) ringInfinitelnvPos constructors. 
Infinite inverse positive disc and ring structures with an additional coupling waveguide.

$\begin{array}{llllllllllllllllllllllllllll}x & y & r_{d} & & r_{e} & N & g_{1} & L_{1} & W_{1} & W_{e 1} & g_{2} & L_{2} & W_{2} & W_{e 2} & E C & \text { disclnflnvPosDS }\end{array}$

$\begin{array}{lllllllllllllllllllll}x & y & r_{i} & W_{r} & r_{e} & N & g_{1} & L_{1} & W_{1} & W_{e 1} & g_{2} & L_{2} & W_{2} & W_{e 2} & E C & \text { ringlnflnvPosDS }>\end{array}$

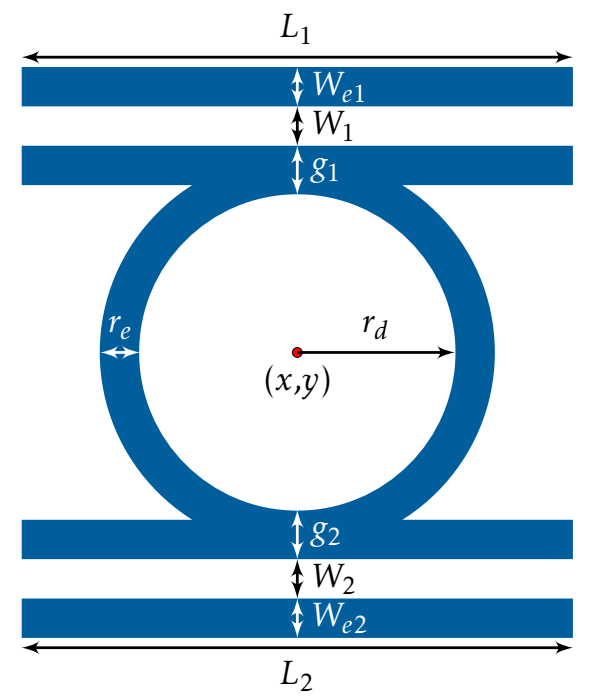

(a)

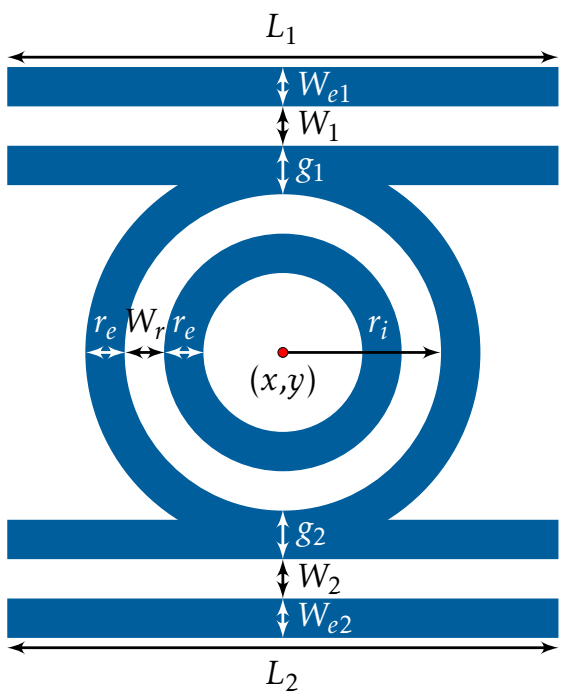

(b)

Figure 2.149: Examples illustrating various parameters of the (a) disclnflnvPosDS and (b) ringInfInvPosDS constructors. 


\subsubsection{Disc-Ring Symmetric Bezier}

Shapes characterized by a curved waveguide above a disc $\left(R_{\text {waveGuide }}=-R_{\text {Disc }}\right)$ and ring $\left(R_{\text {waveGuide }}=-R_{\text {Ring }}\right)$ objects. Both objects (disc/ring) are described by the center coordinate $(x, y)$, number of segments $(N)$, gap between the object and waveguide $(g)$, waveguide opening angle $(\theta)$, number of sides $\left(N_{w g}\right)$, width $(W)$, length $(L)$, height $(H)$, disc radius, $\left(r_{d}\right)$, ring radius $\left(r_{i}\right)$ and width $\left(W_{r}\right)$. $E C=0$ and $E C=1$ for waveguides without and with endcaps, respectively.

$$
\begin{array}{lllllllllllll}
<x & y & r_{d} & & N & g & \theta & N_{w g} & W & L & H & E C & \text { discSymmetric }> \\
<x & y & r_{i} & W_{r} & N & g & \theta & N_{w g} & W & L & H & E C & \text { ringSymmetric }>
\end{array}
$$

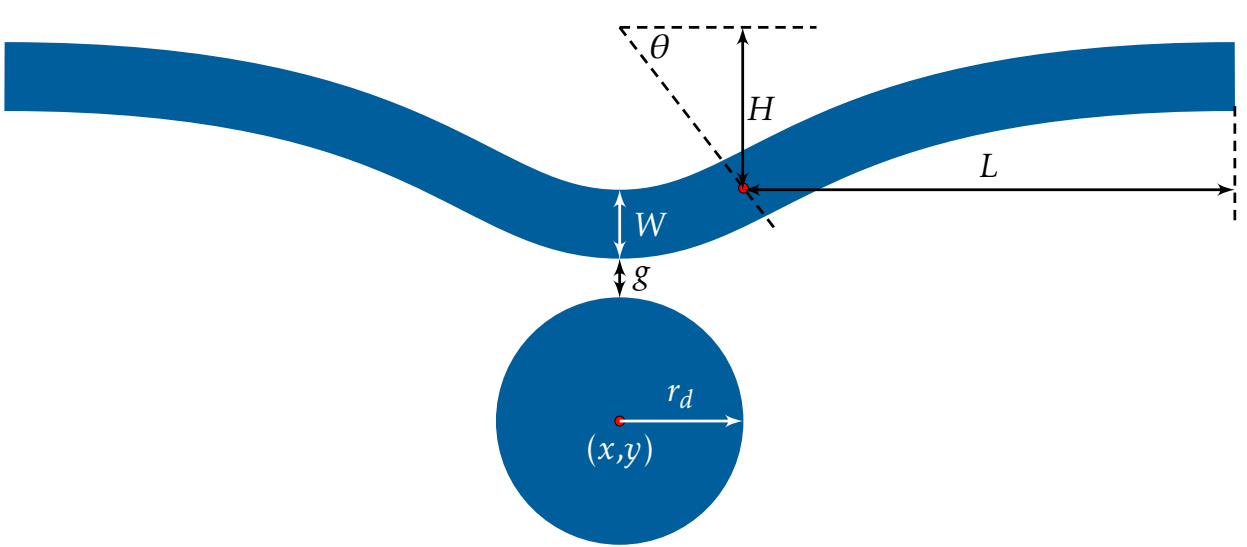

(a)

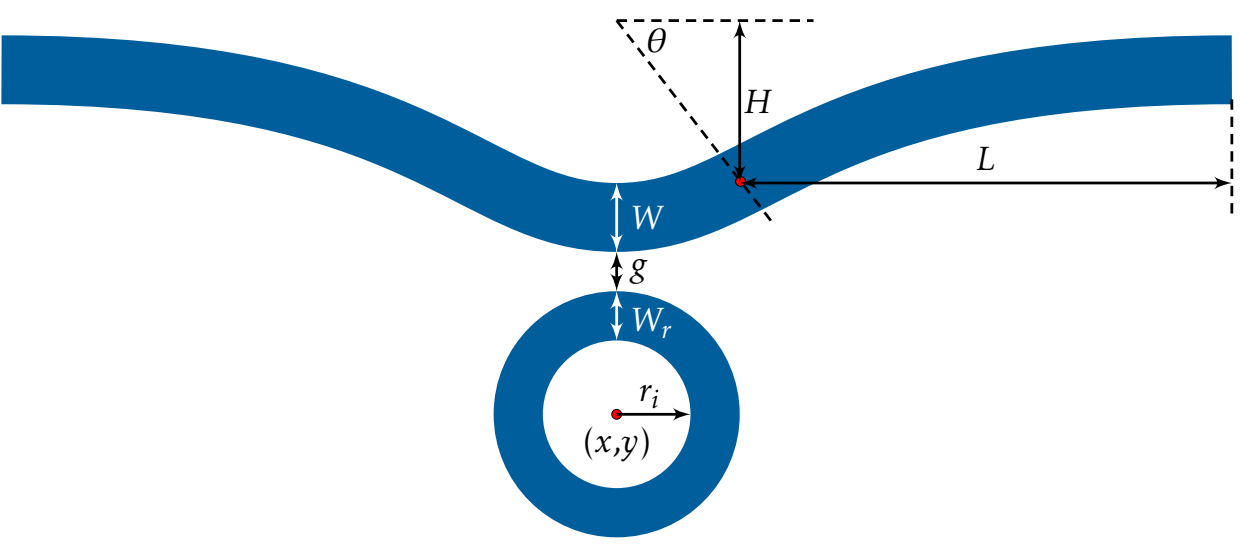

(b)

Figure 2.150: Example shapes illustrating various parameters from the (a) discSymmetric and (b) ringSymmetric constructors. 
Disc symmetric structure with an additional coupling waveguide.

$$
\begin{array}{lllllllllllllll}
x & y & r_{d} & N & g_{1} & \theta & N_{w g} & W_{1} & L_{1} & H & g_{2} & W_{2} & L_{2} & E C & \text { discSymDS }>
\end{array}
$$

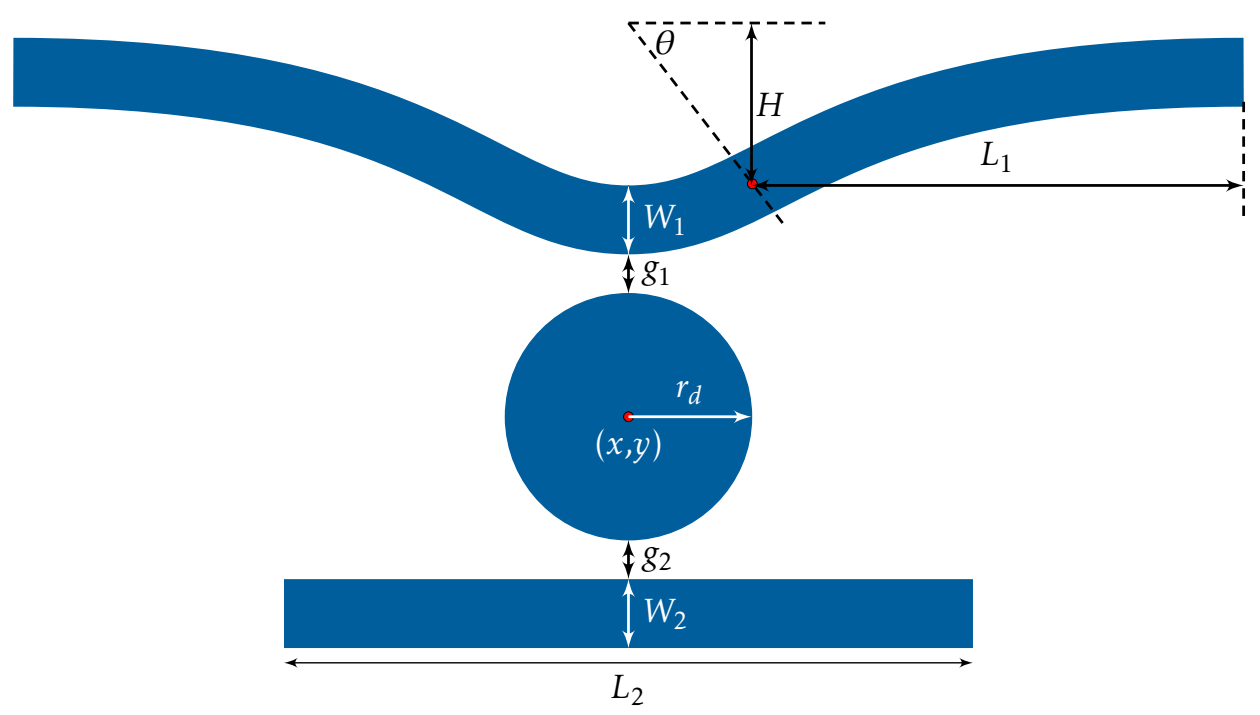

Figure 2.151: Example shape illustrating various parameters from the discSymDS constructor. 
Disc symmetric structure with an additional coupling pulley.

$\begin{array}{llllllllllllllllllllll}x & y & r_{d} & N & g_{1} & \theta_{1} & N_{w g} & W_{1} & L_{1} & H_{1} & g_{2} & \theta_{2} & W_{2} & L_{2} & H_{2} & E C & \text { discSymPul }\end{array}$

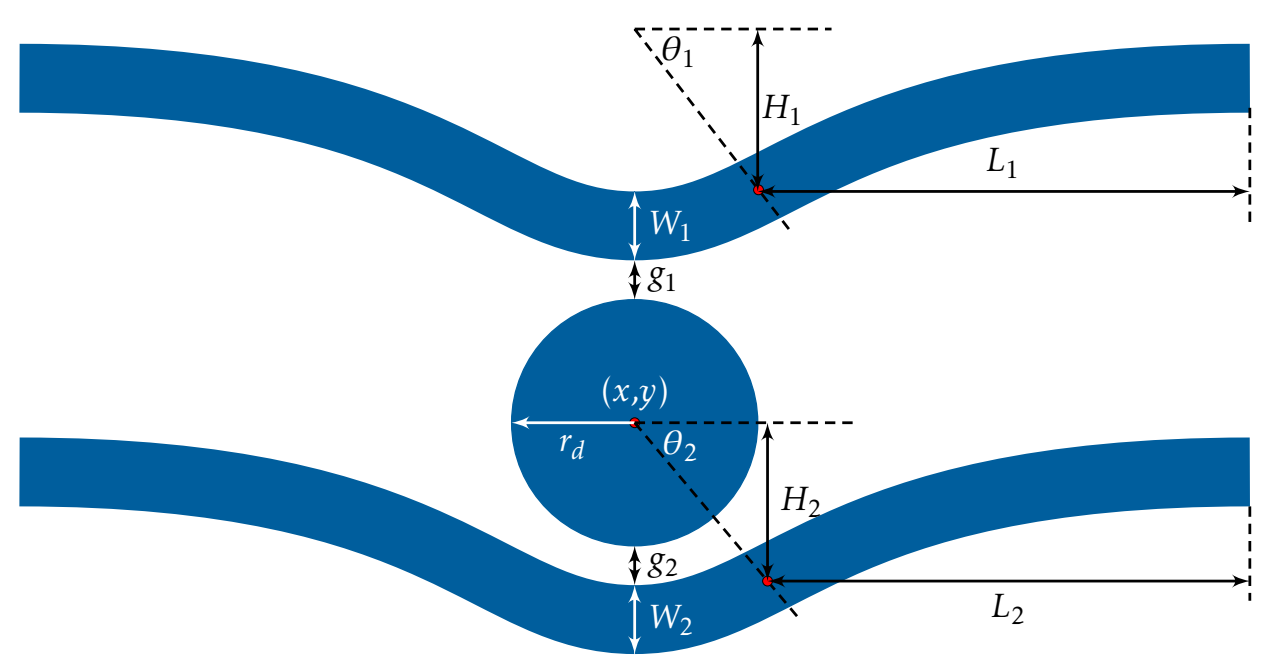

Figure 2.152: Example shape illustrating various parameters from the discSymPul constructors. 
Ring symmetric structure with an additional coupling waveguide.

$\begin{array}{llllllllllllllll}<x & y & r_{i} & W_{r} & N & g_{1} & \theta & N_{w g} & W_{1} & L_{1} & H & g_{2} & W_{2} & L_{2} & E C & \text { ringSymDS }>\end{array}$

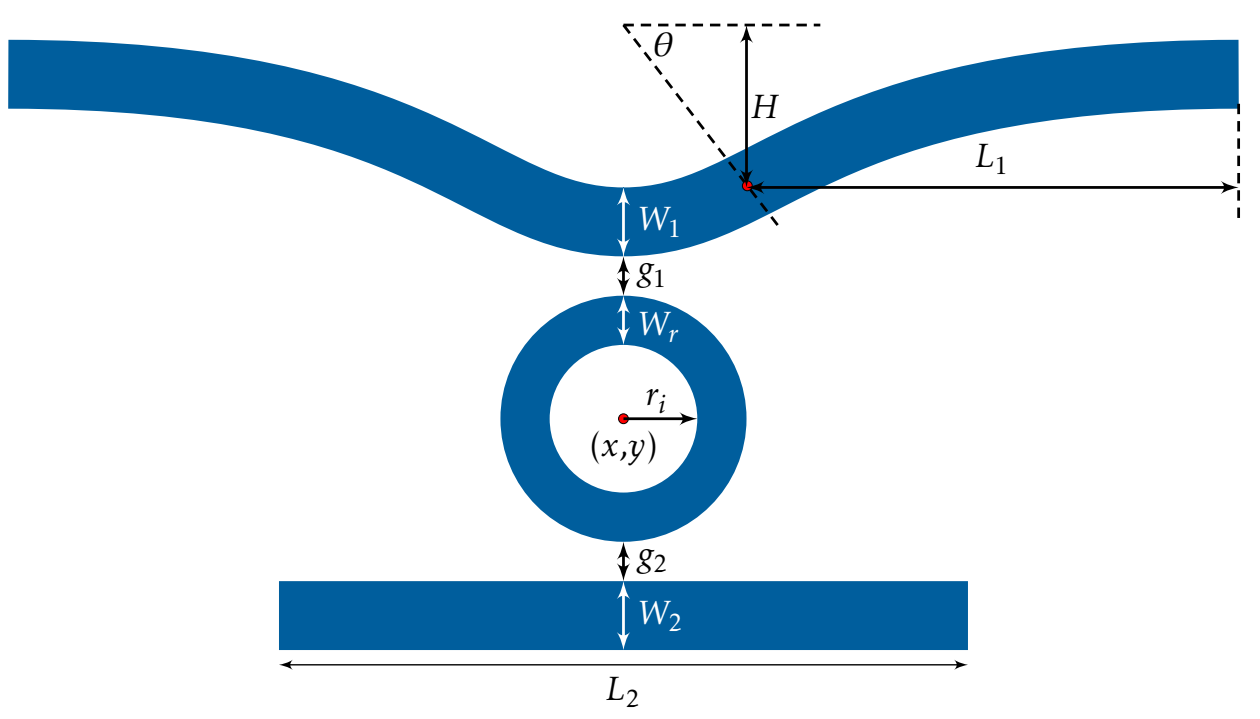

Figure 2.153: Example shape illustrating various parameters from the ringSymDS constructor. 
Ring symmetric structure with an additional coupling pulley.

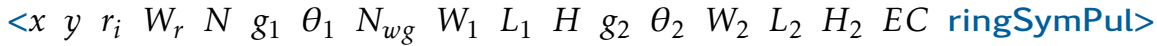

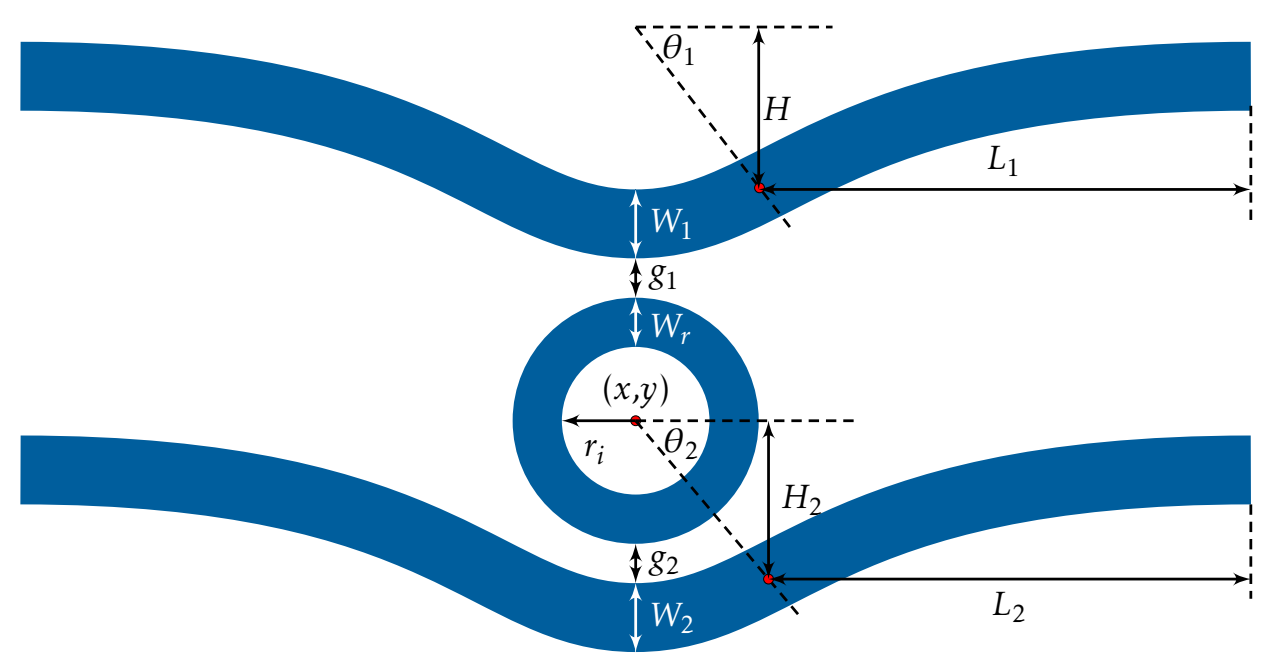

Figure 2.154: Example shape illustrating various parameters from the ringSymPul constructor. 
The following disc-ring waveguide symmetric structures are defined by the coupling length parameter $L_{c}$.

$$
\begin{array}{lllllllllllll}
<x & y & r_{d} & & N & g & L_{c} & N_{w g} & W & L & H & E C & \text { discSymmetricLC> } \\
<x & y & r_{i} & W_{r} & N & g & L_{c} & N_{w g} & W & L & H & E C & \text { ringSymmetricLC> }
\end{array}
$$

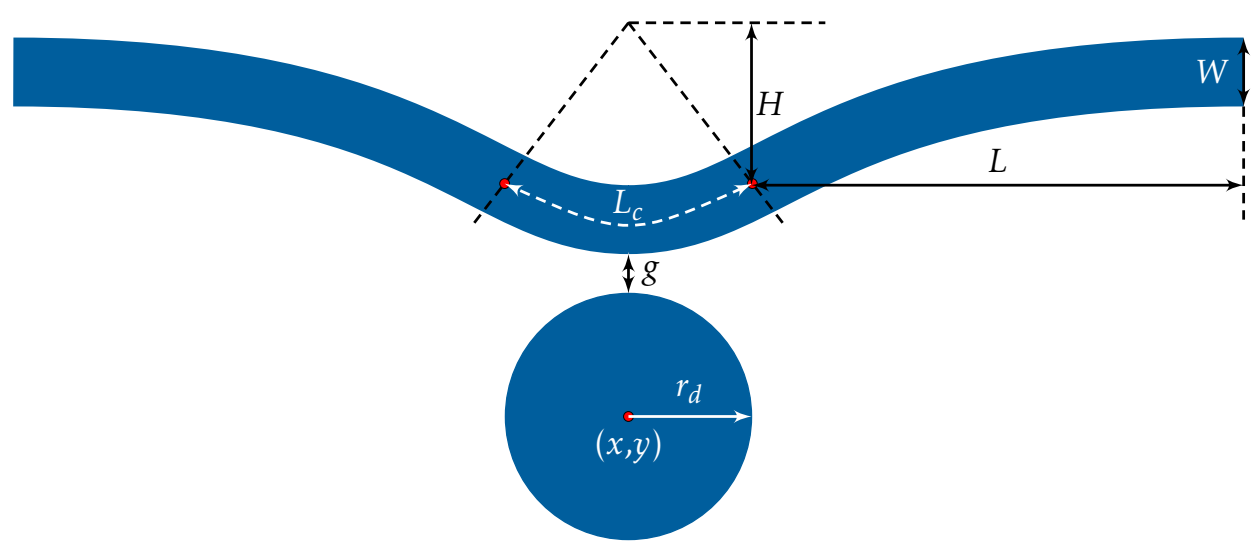

(a)

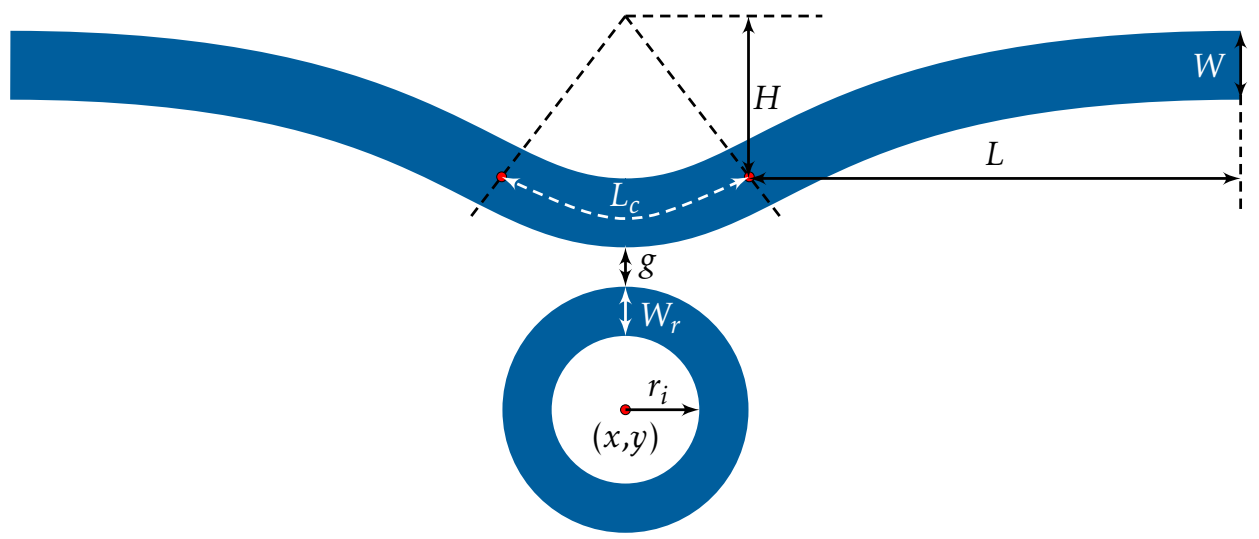

(b)

Figure 2.155: Example shapes illustrating various parameters from the (a) discSymmetricLC and (b) ringSymmetricLC constructors. 
Disc symmetric structure with an additional coupling waveguide.

$\begin{array}{llllllllllllllllll}< & y & r_{d} & N & g_{1} & L_{c} & N_{w g} & W_{1} & L_{1} & H & g_{2} & W_{2} & L_{2} & E C & \text { discSymLCDS }>\end{array}$

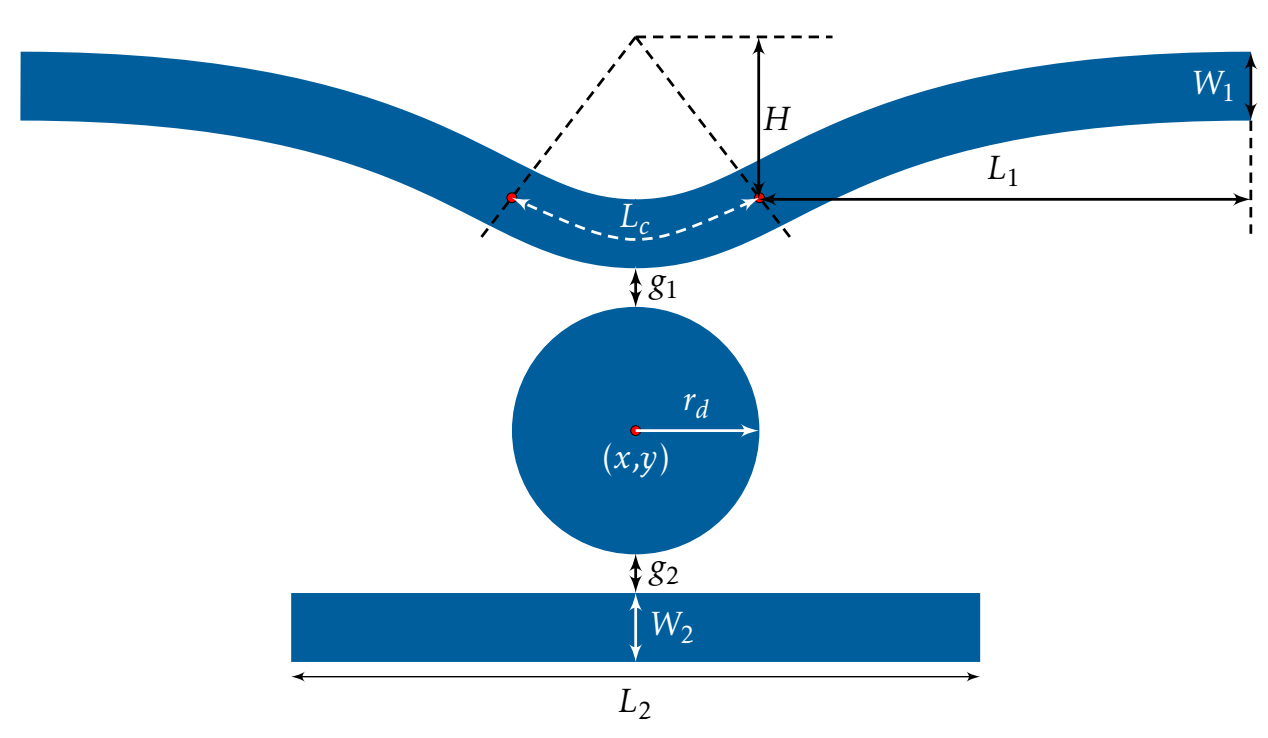

Figure 2.156: Example shape illustrating various parameters from the discSymLCDS constructor. 
Disc symmetric structure with an additional coupling pulley.

$\begin{array}{lllllllllllllllllllll} & y & r_{d} & N & g_{1} & L_{c 1} & N_{w g} & W_{1} & L_{1} & H_{1} & g_{2} & L_{c 2} & W_{2} & L_{2} & H_{2} & E C & \text { discSymLCPul }\end{array}$

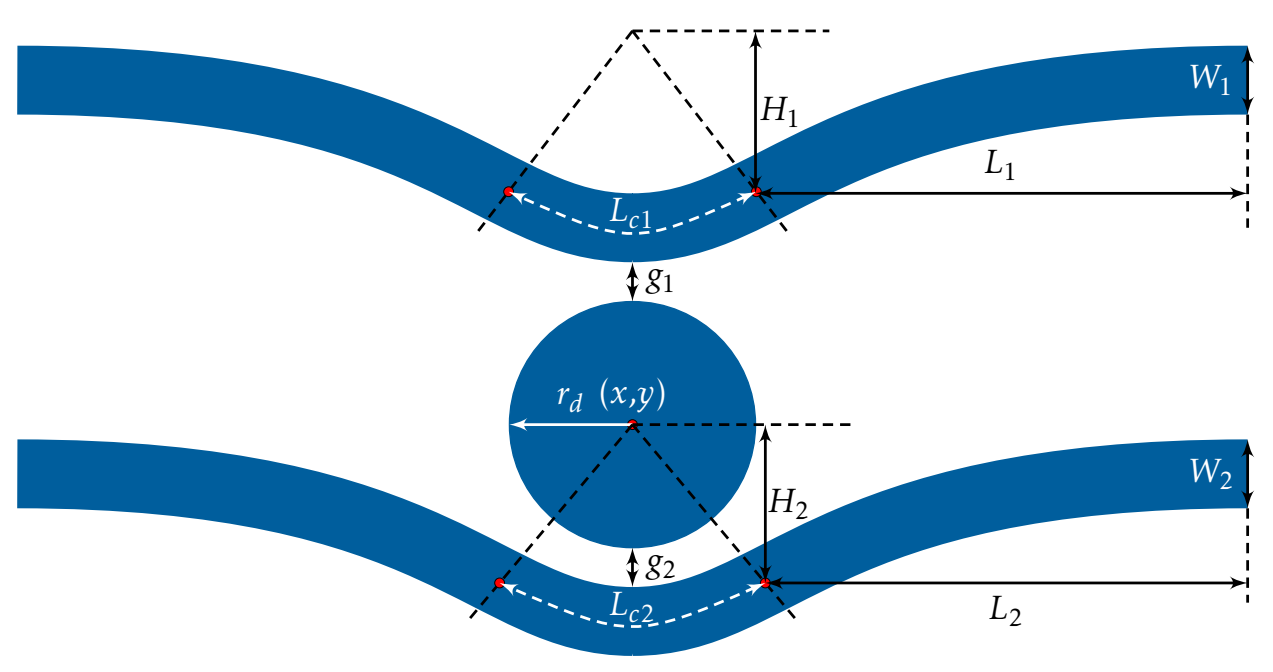

Figure 2.157: Example shape illustrating various parameters from the discSymLCPul constructor. 
Ring symmetric structure with an additional coupling waveguide.

$$
\begin{array}{llllllllllllllll}
<x & y & r_{i} & W_{r} & N & g_{1} & L_{c} & N_{w g} & W_{1} & L_{1} & H & g_{2} & W_{2} & L_{2} & E C & \text { ringSymLCDS }>
\end{array}
$$

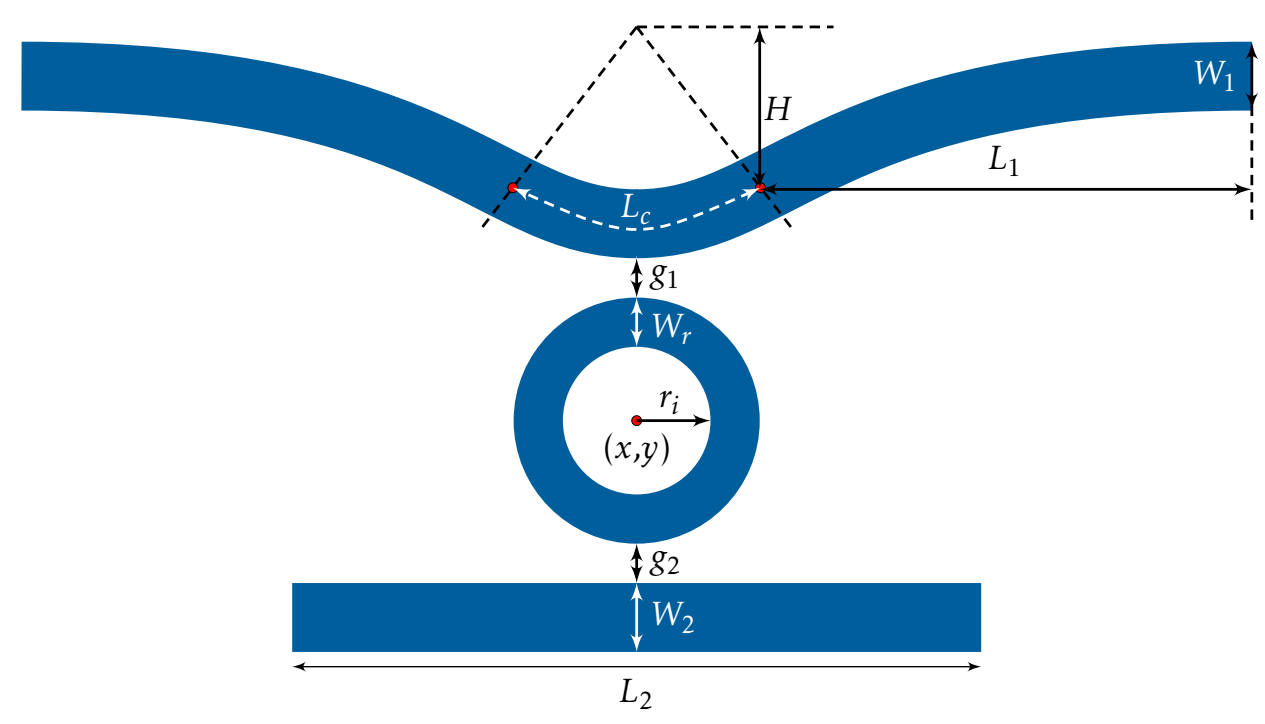

Figure 2.158: Example shape illustrating various parameters from the ringSymLCDS constructor. 
Ring symmetric structure with an additional coupling pulley.

$\begin{array}{lllllllllllllllllllllllll}<x & y & r_{i} & W_{r} & N & g_{1} & L_{c 1} & N_{w g} & W_{1} & L_{1} & H_{1} & g_{2} & L_{c 2} & W_{2} & L_{2} & H_{2} & E C & \text { ringSymLCPul }\end{array}$

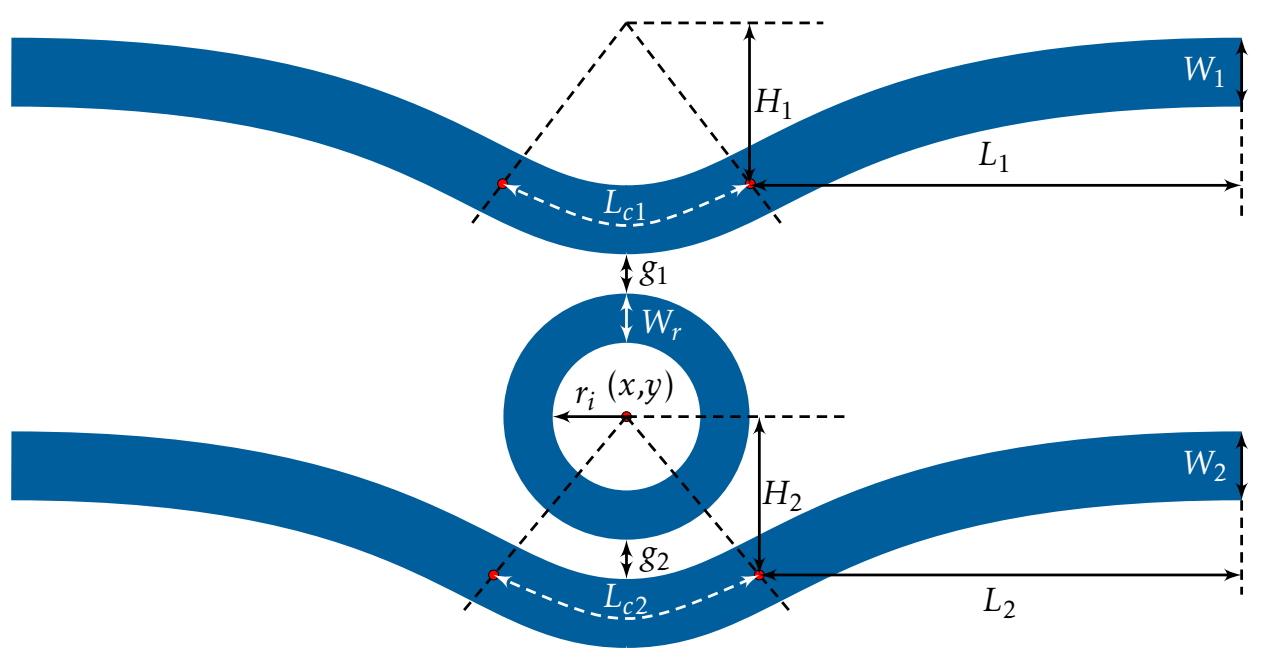

Figure 2.159: Example shape illustrating various parameters from the ringSymLCPul constructor. 


\subsubsection{Disc-Ring Symmetric Arc}

Symmetric structures similar to ones described in section 2.9.9.5 with coupling regions constructed using arcs. Both objects (disc/ring) are described by the center coordinate $(x, y)$, number of segments $(N)$, gap between the object and waveguide $(g)$, waveguide opening angle $(\theta)$, number of sides $\left(N_{w g}\right)$, width $(W)$, and length of the connecting straight waveguide section $\left(L_{S}\right)$, disc radius, $\left(r_{d}\right)$, ring radius $\left(r_{i}\right)$ and width $\left(W_{r}\right)$. EC $=0$ and $E C=1$ for waveguides without and with endcaps, respectively.

$$
\begin{array}{llllllllllll}
<x & y & r_{d} & & N & g & \theta & N_{w g} & W & L_{S} & E C & \operatorname{disc} S y m m e t r i c A> \\
<x & y & r_{i} & W_{r} & N & g & \theta & N_{w g} & W & L_{S} & E C & \text { ringSymmetricA }>
\end{array}
$$

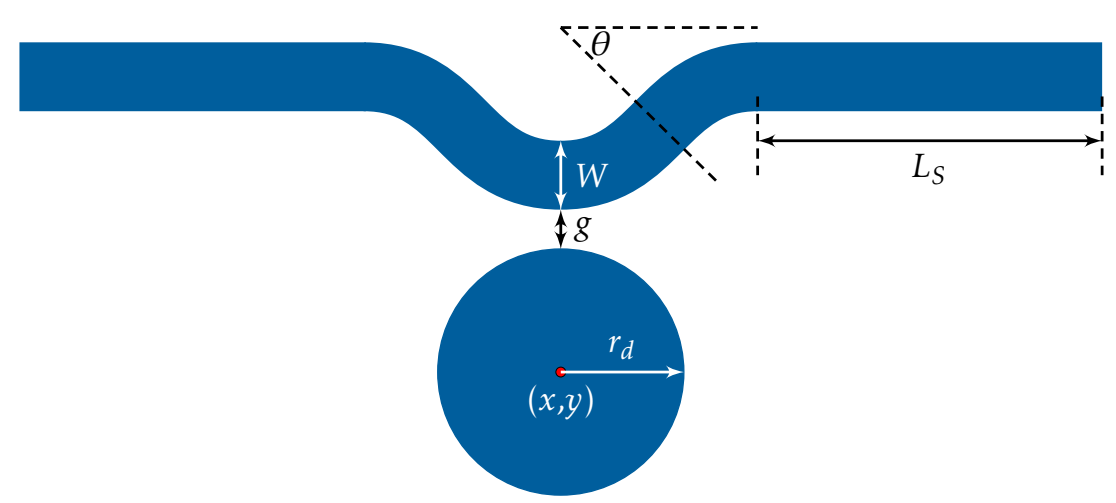

(a)

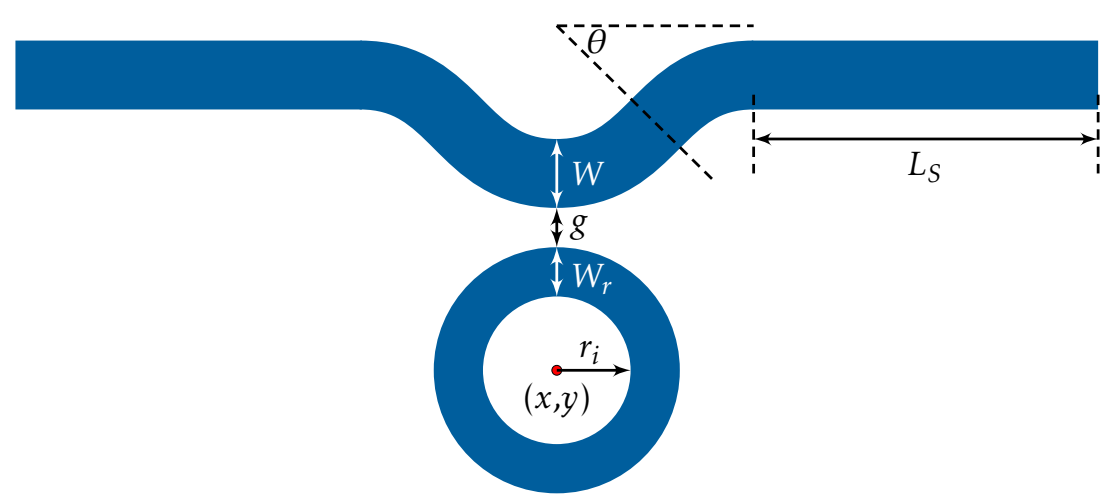

(b)

Figure 2.160: Example shapes illustrating various parameters from the (a) discSymmetricA and (b) ringSymmetricA constructors. 
Disc symmetric arc structure with an additional coupling waveguide.

$\begin{array}{lllllllllllllllll}x & y & r_{d} & N & g_{1} & \theta & N_{w g} & W_{1} & L_{S} & g_{2} & W_{2} & L_{2} & E C & \text { discSymADS } & \end{array}$

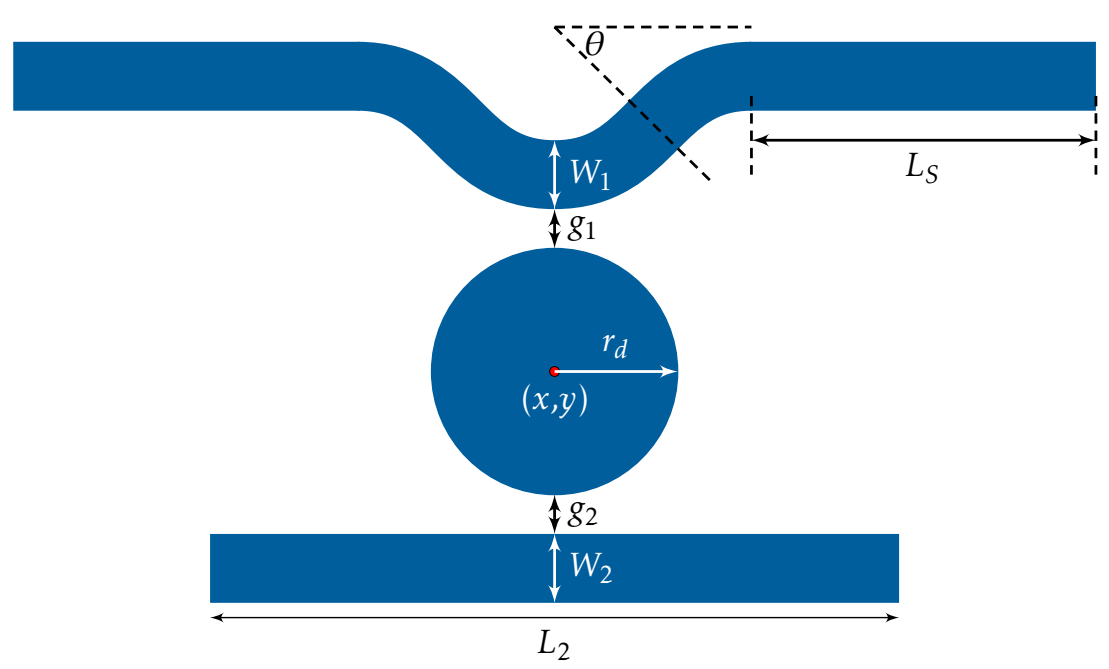

Figure 2.161: Example shape illustrating various parameters from the discSymADS constructor. 
Disc symmetric arc structure with an additional coupling pulley.

$<x \quad y \quad r_{d} N \begin{array}{lllllllllllll} & g_{1} & \theta_{1} & N_{w g} & W_{1} & L_{S 1} & g_{2} & \theta_{2} & W_{2} & L_{S 2} & E C & \operatorname{disc} S y m A P u l\end{array}$

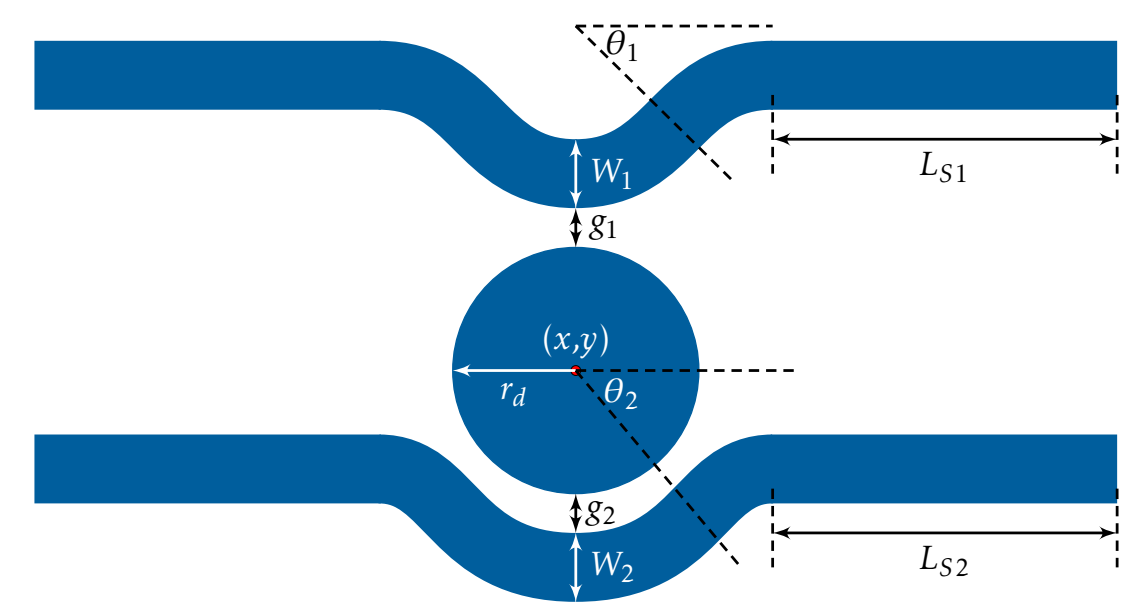

Figure 2.162: Example shape illustrating various parameters from the discSymAPul constructor. 
Ring symmetric arc structure with an additional coupling waveguide.

$\begin{array}{llllllllllllllllll}< & y & r_{i} & W_{r} & N & g_{1} & \theta & N_{w g} & W_{1} & L_{S} & g_{2} & W_{2} & L_{2} & E C & \text { ringSymADS }\end{array}$

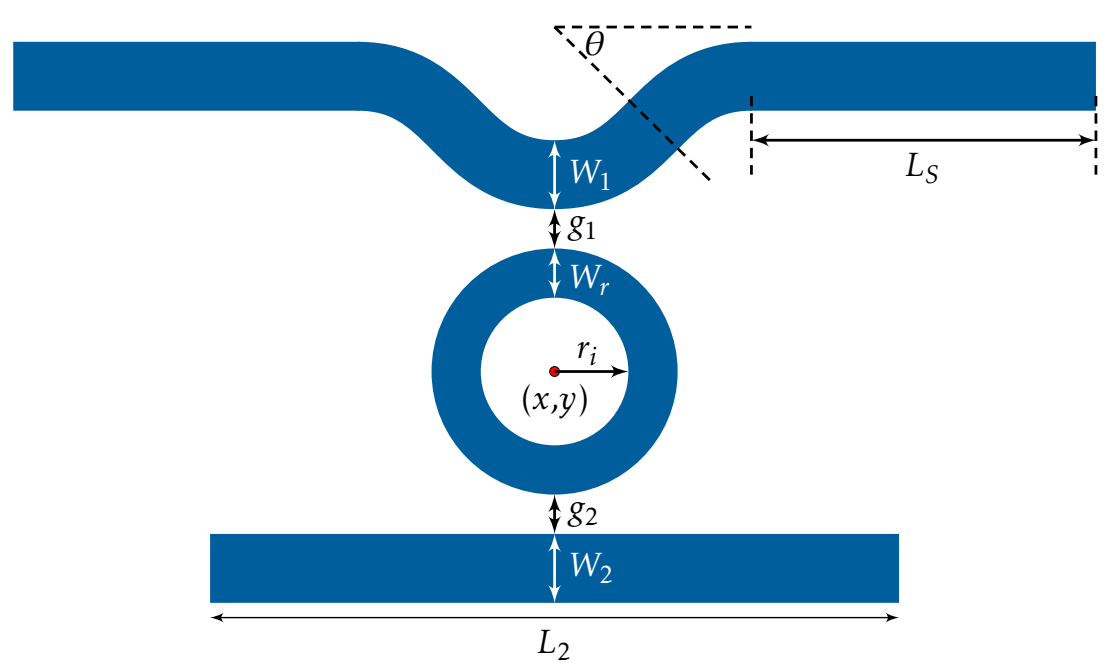

Figure 2.163: Example shape illustrating various parameters from the ringSymADS constructor. 
Ring symmetric arc structure with an additional coupling pulley.

$\begin{array}{lllllllllllllllllllll}< & y & r_{i} & W_{r} & N & g & \theta_{1} & N_{w g} & W & L_{S} & g_{2} & \theta_{2} & W_{2} & L_{S 2} & E C & \text { ringSymAPul }\end{array}$

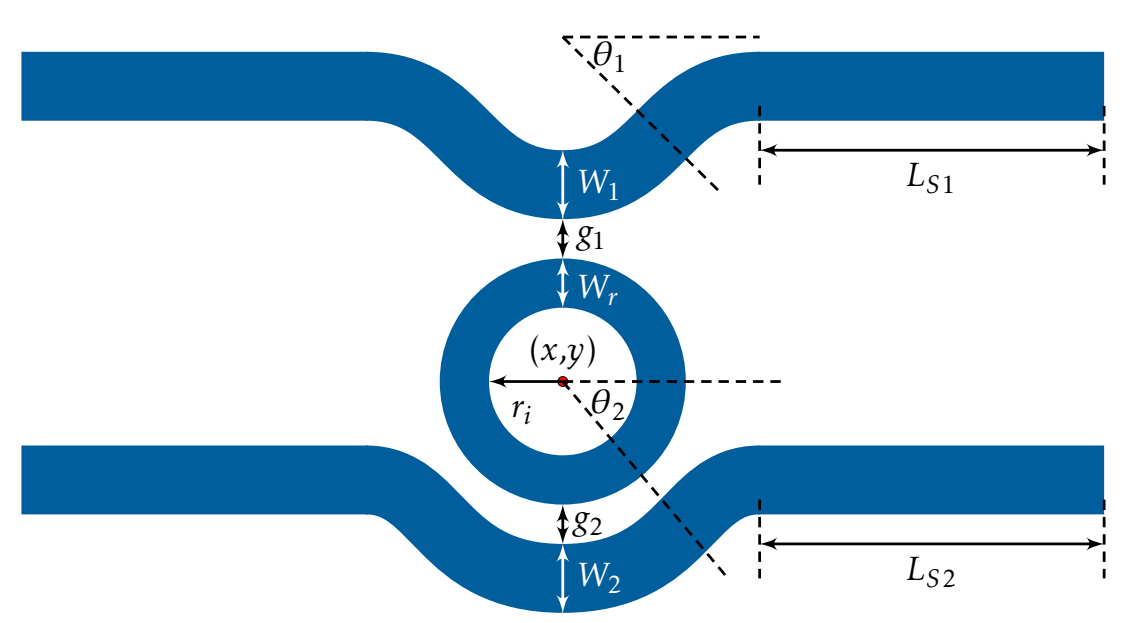

Figure 2.164: Example shape illustrating various parameters from the ringSymAPul constructor. 
The following arc based, disc-ring waveguide symmetric structures are defined by the coupling length parameter $L_{c}$.

$$
\begin{array}{llllllllllll}
<x & y & r_{d} & & N & g & L_{c} & N_{w g} & W & L_{S} & E C & \operatorname{discSymmetricLCA}> \\
<x & y & r_{i} & W_{r} & N & g & L_{c} & N_{w g} & W & L_{S} & E C & \text { ringSymmetricLCA }>
\end{array}
$$

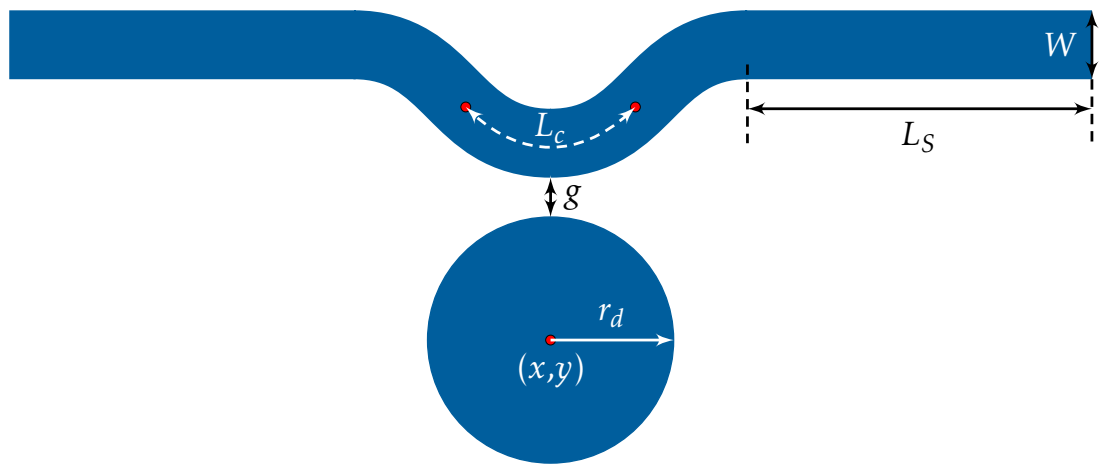

(a)

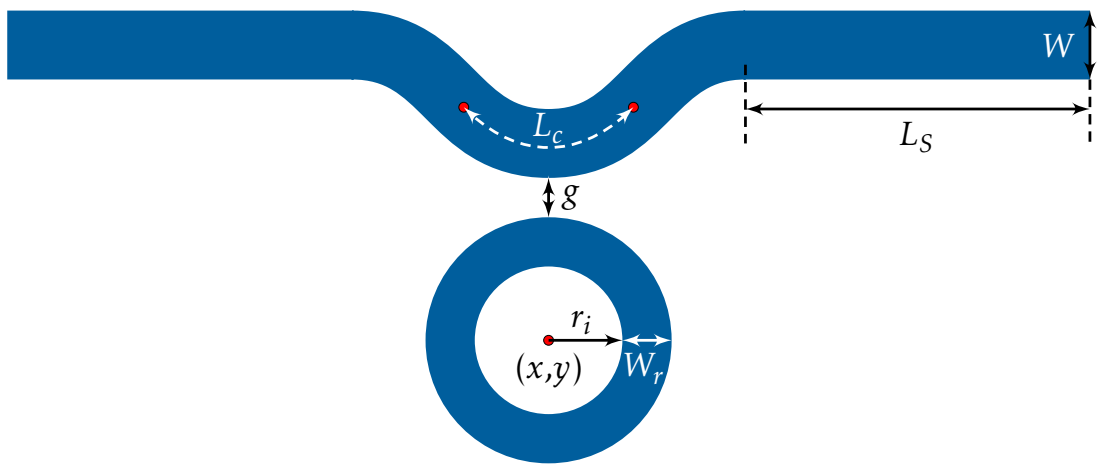

(b)

Figure 2.165: Example shapes illustrating various parameters from the (a) discSymmetricLCA and (b) ringSymmetricLCA constructors. 
Disc symmetric arc structure with an additional coupling waveguide.

$\begin{array}{lllllllllllllll}x & y & r_{d} & N & g_{1} & L_{c} & N_{w g} & W_{1} & L_{S} & g_{2} & W_{2} & L_{2} & E C & \text { discSymLCADS }>\end{array}$

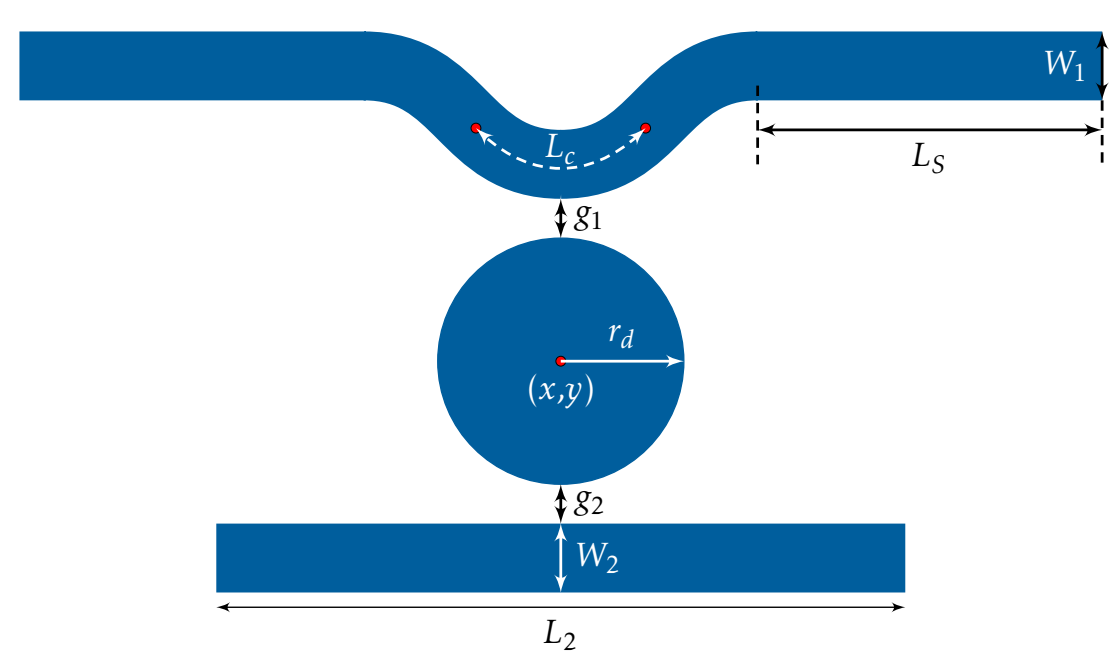

Figure 2.166: Example shape illustrating various parameters from the (a) discSymLCADS constructor. 
Disc symmetric arc structure with an additional coupling pulley.

$\begin{array}{llllllllllllllllll}x & y & r_{d} & N & g_{1} & L_{c 1} & N_{w g} & W_{1} & L_{S 1} & g_{2} & L_{c 2} & W_{2} & L_{S 2} & E C & \text { discSymLCAPul }\end{array}$

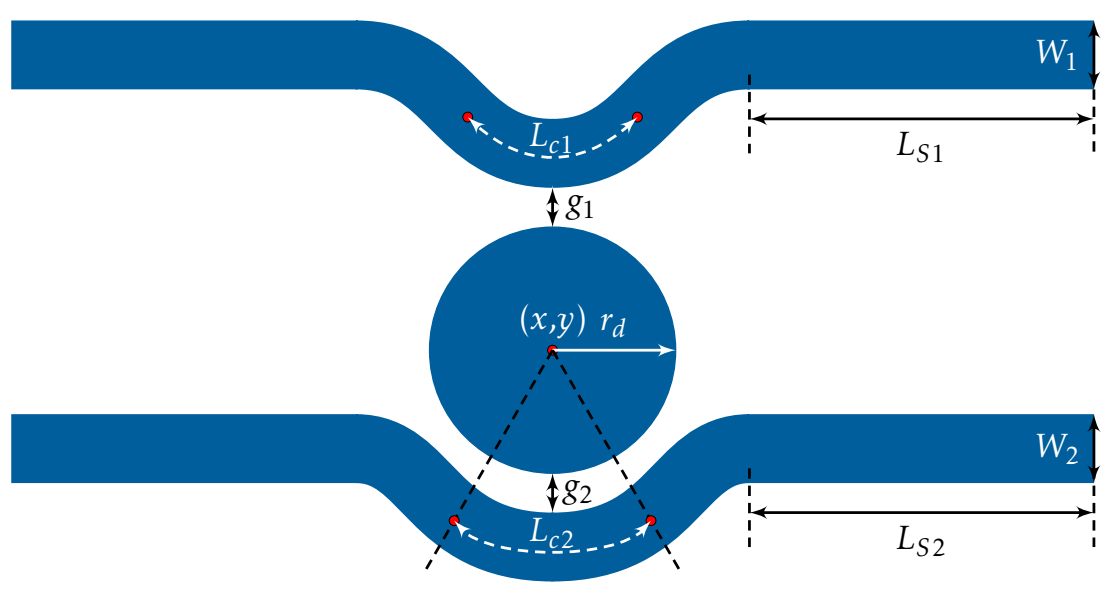

Figure 2.167: Example shape illustrating various parameters from the (a) discSymLCAPul constructor. 
Ring symmetric arc structure with an additional coupling waveguide.

$\begin{array}{lllllllllllllllll}<x & y & r_{i} & W_{r} & N & g_{1} & L_{c} & N_{w g} & W_{1} & L_{S} & g_{2} & W_{2} & L_{2} & E C & \text { ringSymLCADS }>\end{array}$

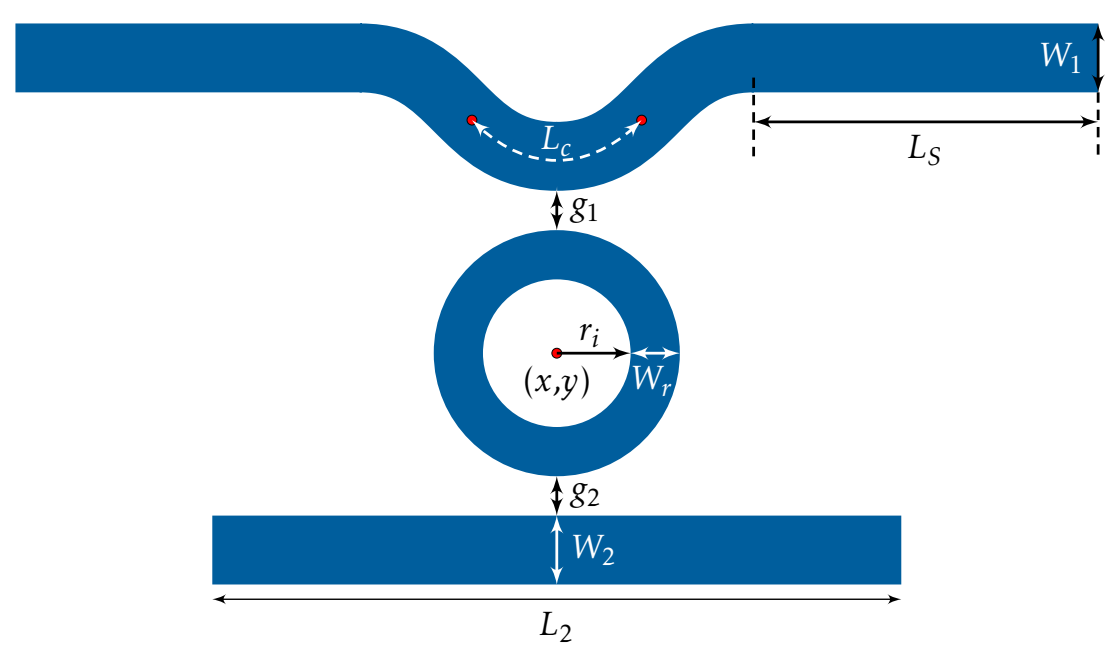

Figure 2.168: Example shape illustrating various parameters from the ringSymLCADS constructor. 
Ring symmetric arc structure with an additional coupling pulley.

$\begin{array}{lllllllllllllllllllllll}< & y & r_{i} & W_{r} & N & g_{1} & L_{c 1} & N_{w g} & W_{1} & L_{S 1} & g_{2} & L_{c 2} & W_{2} & L_{S 2} & E C & \text { ringSymLCAPul }\end{array}$

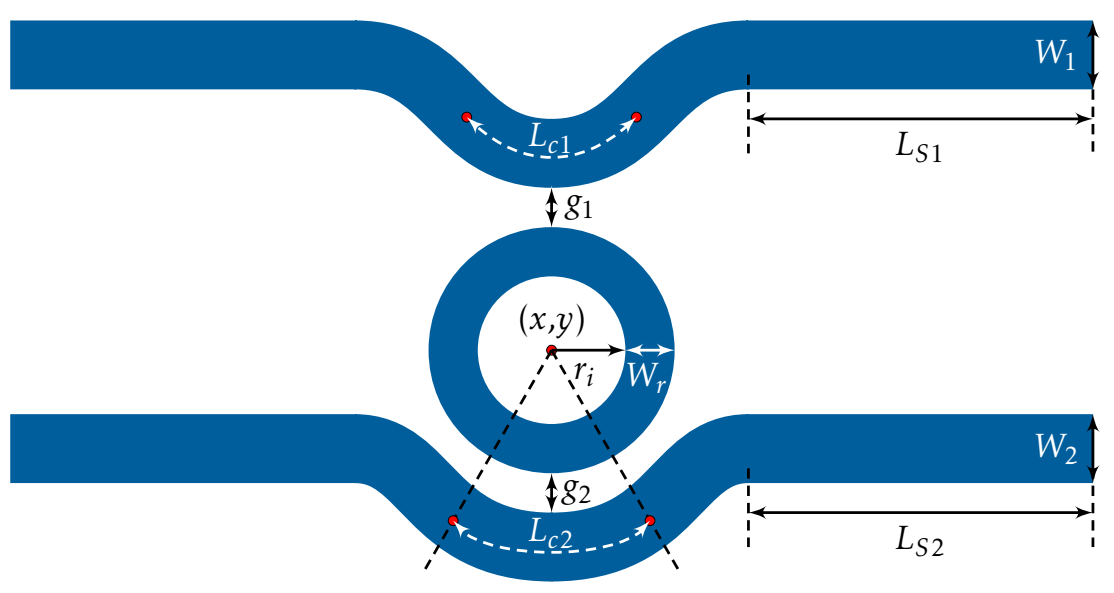

Figure 2.169: Example shape illustrating various parameters from the ringSymLCAPul constructor. 


\subsubsection{Disc-Ring Symmetric Inverse Bezier}

The structure is similar to the disc-ring waveguide symmetric structure defined in the previous section (2.9.9.5). Here, a slot waveguide of width $W$ is formed by exposing a surrounding rectangular region of width $W_{e}$. Using a negative tone resist, the resulting structure would produce a disc or ring shape at a distance $g$ to a slotted waveguide.

$$
\begin{array}{llllllllllllllll}
<x & y & r_{d} & & N & g & \theta & N_{w g} & W & W_{e} & L & H & E C & \text { discSymmetriclnv }> \\
<x & y & r_{i} & W_{r} & N & g & \theta & N_{w g} & W & W_{e} & L & H & E C & \text { ringSymmetriclnv }>
\end{array}
$$

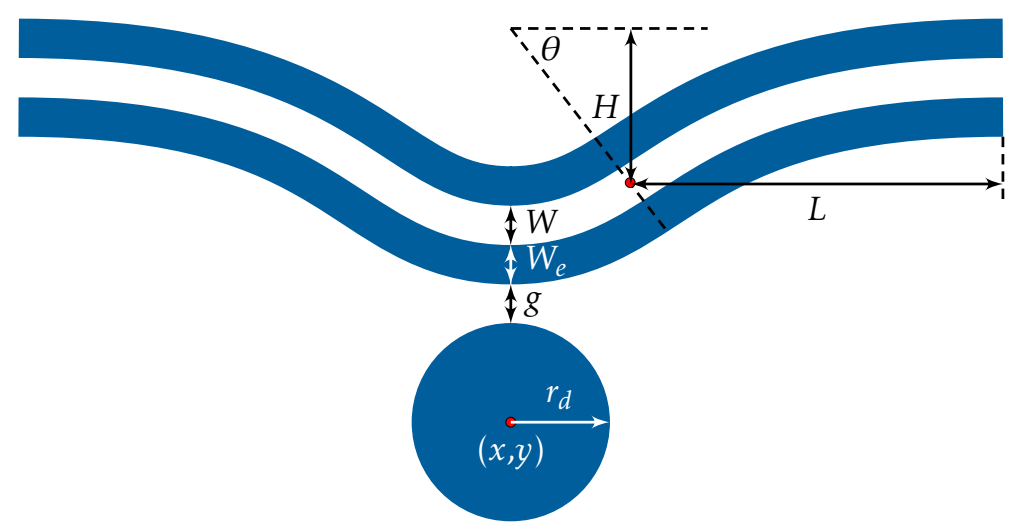

(a)

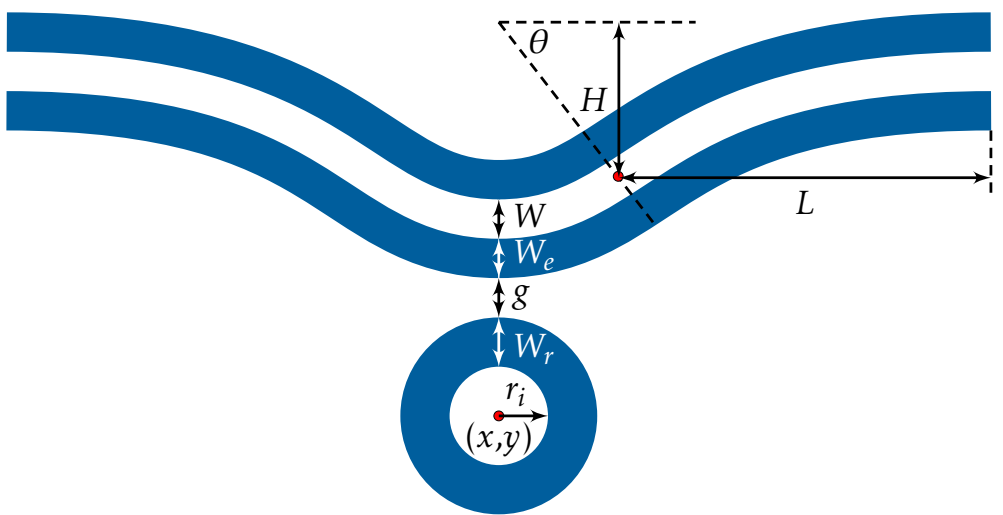

(b)

Figure 2.170: Example shapes illustrating various parameters from the (a) discSymmetriclnv and (b) ringSymmetriclnv constructors. 
Disc symmetric inverse structure with an additional coupling waveguide.

$\begin{array}{llllllllllllllllllllll} & y & r_{d} & N & g_{1} & \theta & N_{w g} & W_{1} & W_{e 1} & L_{1} & H & g_{2} & W_{2} & W_{e 2} & L_{2} & E C & \text { discSymlnvDS }\end{array}$

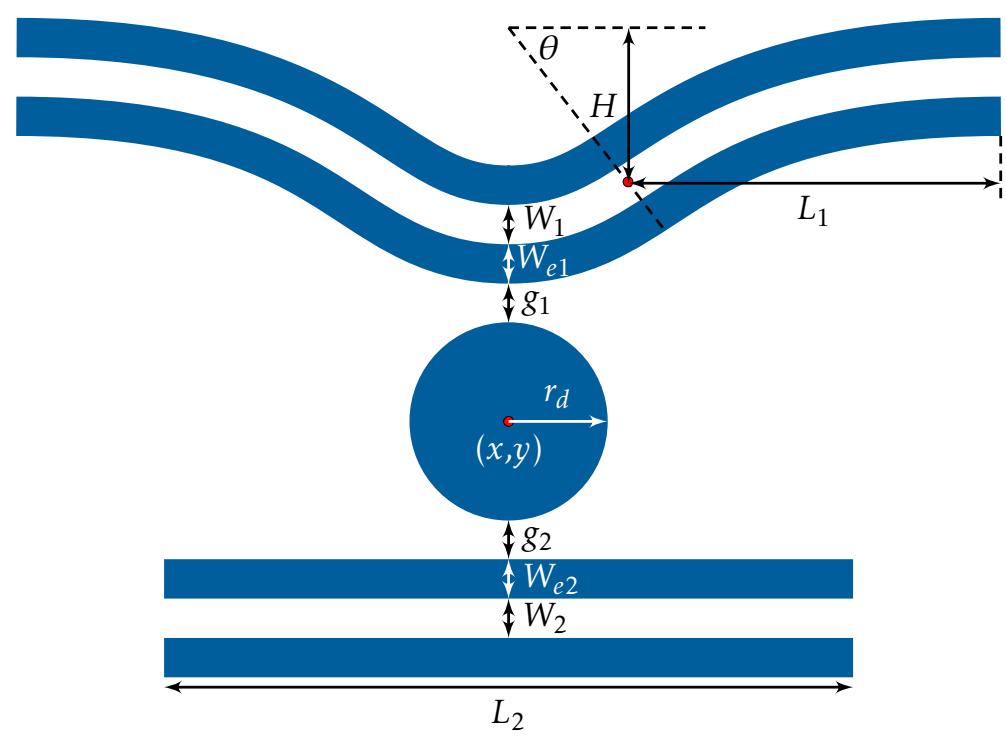

Figure 2.171: Example shape illustrating various parameters from the (a) discSymlnvDS constructor. 
Disc symmetric inverse structure with an additional coupling pulley.

$\begin{array}{lllllllllllllllllll}x & y & r_{d} & N & g_{1} & \theta_{1} & N_{w g} & W_{1} & W_{e 1} & L_{1} & H_{1} & g_{2} & \theta_{2} & W_{2} & W_{e 2} & L_{2} & H_{2} & E C & \text { discSymlnvPul }\end{array}$

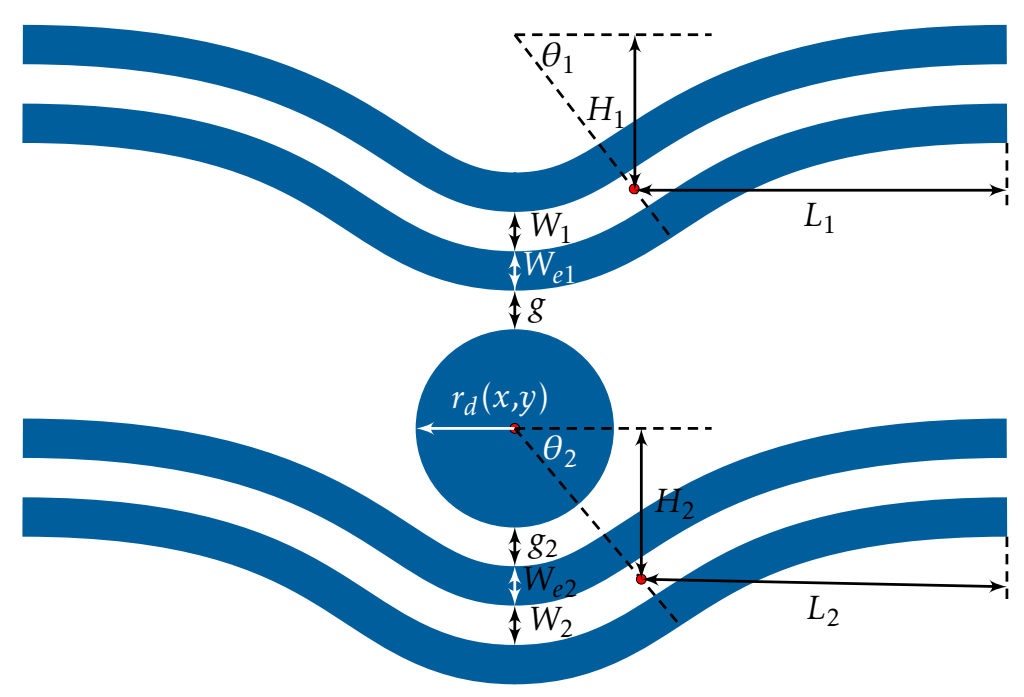

Figure 2.172: Example shape illustrating various parameters from the (a) discSymlnvPul constructor. 
Ring symmetric inverse structure with an additional coupling waveguide.

$\begin{array}{lllllllllllllllllllllllll}x & y & r_{i} & W_{r} & N & g_{1} & \theta & N_{w g} & W_{1} & W_{e 1} & L_{1} & H & g_{2} & W_{2} & W_{e 2} & L_{2} & E C & \text { ringSymlnvDS } & \end{array}$

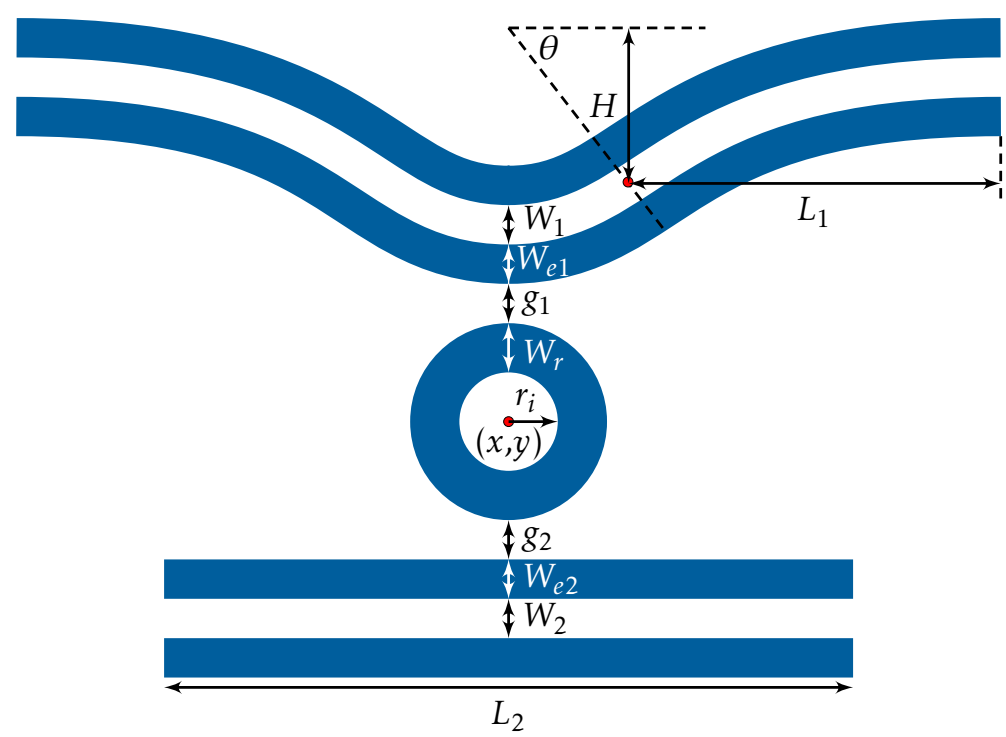

Figure 2.173: Example shape illustrating various parameters from the ringSymlnvDS constructor. 
Ring symmetric inverse structure with an additional coupling pulley.

$\begin{array}{lllllllllllllllllllllllllllll} & y & r_{i} & W_{r} & N & g_{1} & \theta_{1} & N_{w g} & W_{1} & W_{e 1} & L_{1} & H_{1} & g_{2} & \theta_{2} & W_{2} & W_{e 2} & L_{2} & H_{2} & E C \text { ringSymlnvPul }\end{array}$

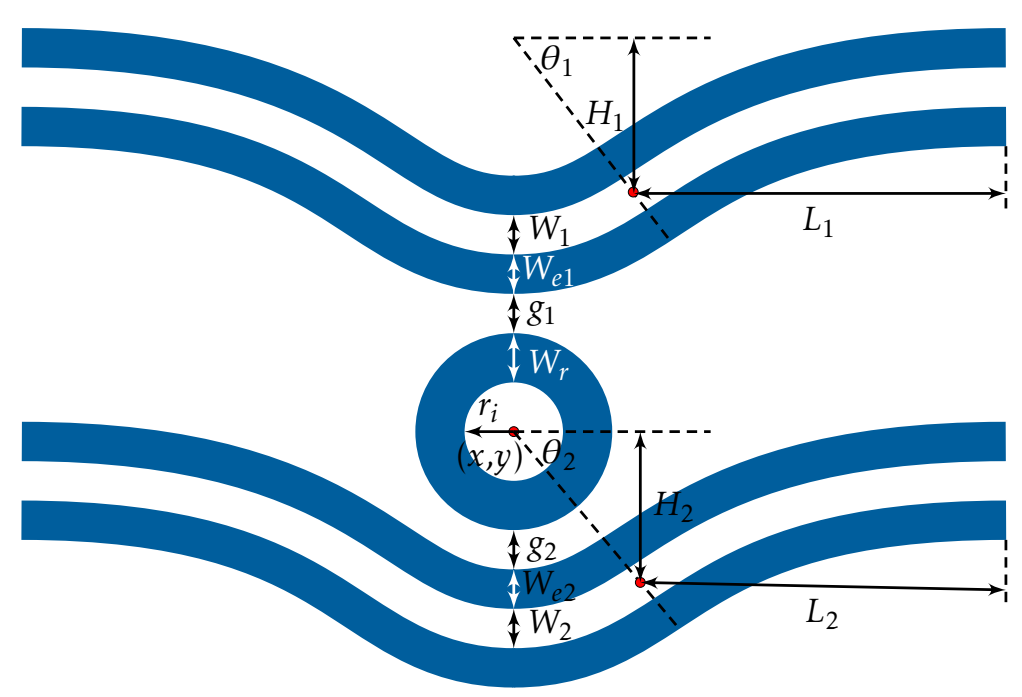

Figure 2.174: Example shape illustrating various parameters from the ringSymlnvPul constructor. 
The following disc-ring waveguide symmetric inverse structures are defined by the coupling length parameter $L_{c}$.

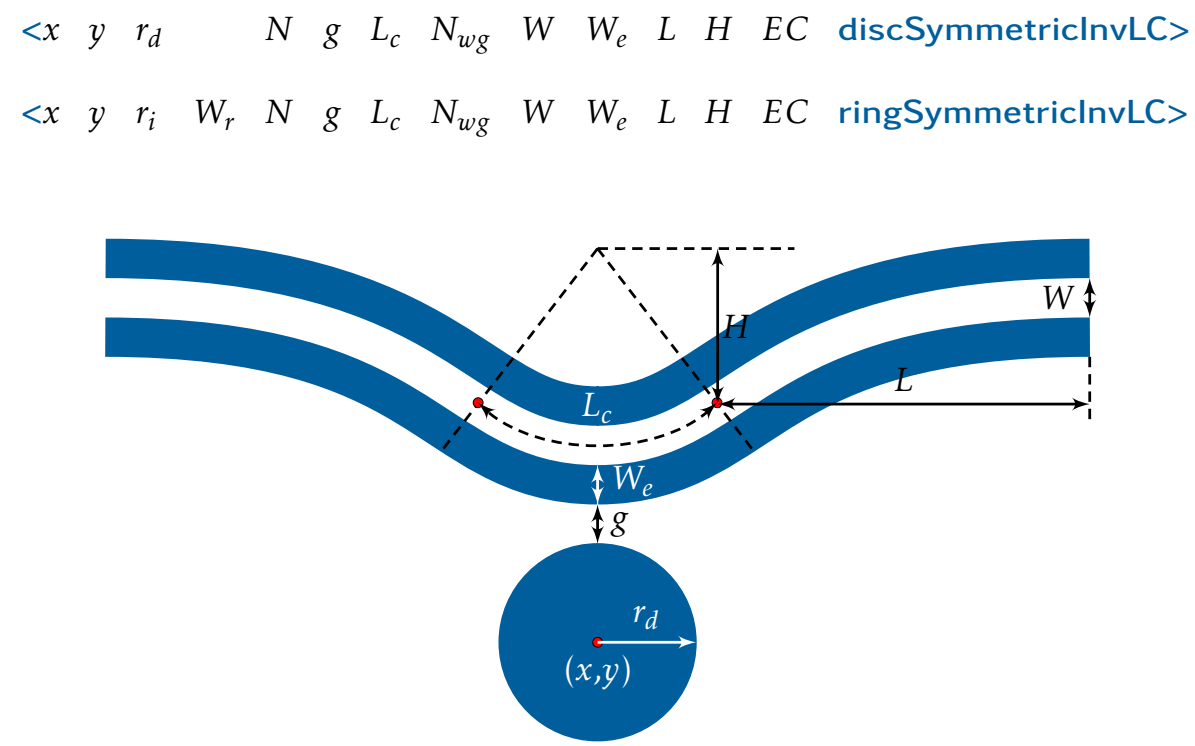

(a)

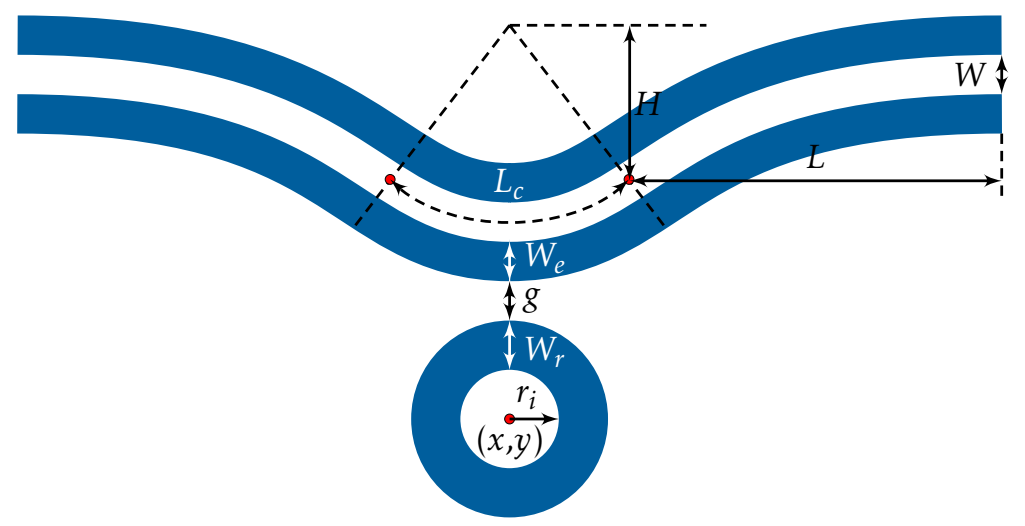

(b)

Figure 2.175: Example shapes illustrating various parameters from the (a) discSymmetriclnvLC and (b) ringSymmetriclnvLC constructors. 
Disc symmetric inverse structure with an additional coupling waveguide.

$\begin{array}{lllllllllllllllllllll}x & y & r_{d} & N & g_{1} & L_{c} & N_{w g} & W_{1} & W_{e 1} & L_{1} & H & g_{2} & W_{2} & W_{e 2} & L_{2} & E C & \text { discSymlnvLCDS }>\end{array}$

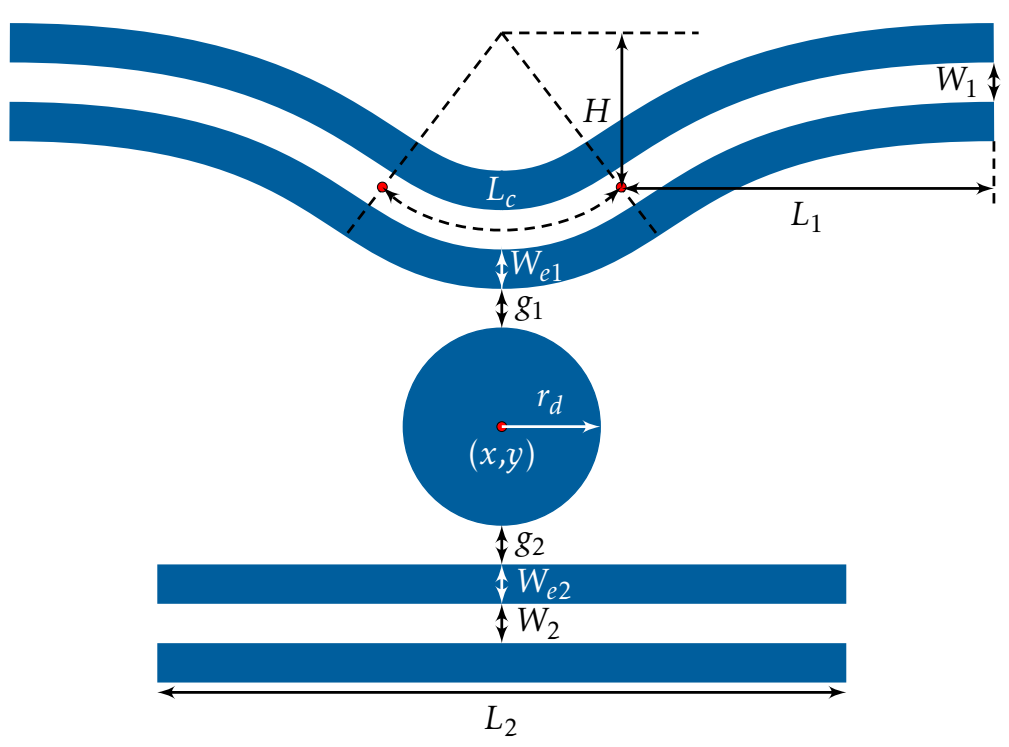

Figure 2.176: Example shape illustrating various parameters from the (a) discSymlnvLCDS constructor. 
Disc symmetric inverse structure with an additional coupling pulley.

$\begin{array}{lllllllllllllllllllll} & y & r_{d} & N & g_{1} & L_{c 1} & N_{w g} & W_{1} & W_{e 1} & L_{1} & H_{1} & g_{2} & L_{c 2} & W_{2} & W_{e 2} & L_{2} & H_{2} & E C & \text { discSymlnvLCPul }\end{array}$

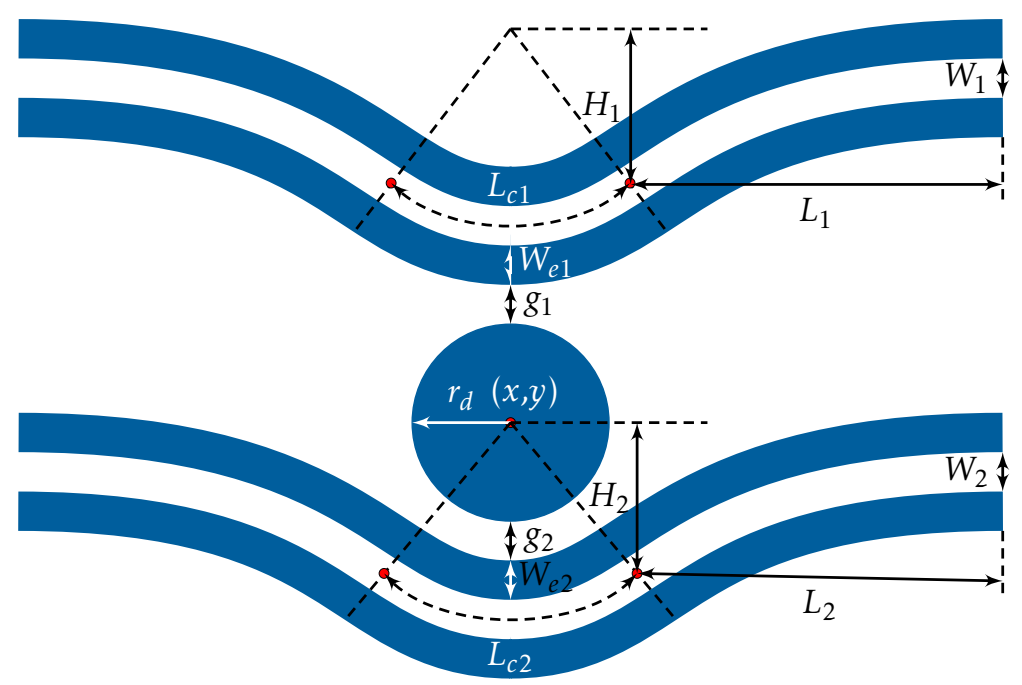

Figure 2.177: Example shape illustrating various parameters from the (a) discSymlnvLCPul constructor. 
Ring symmetric inverse structure with an additional coupling waveguide.

$\begin{array}{llllllllllllllllllllll}< & y & r_{i} & W_{r} & N & g_{1} & L_{c} & N_{w g} & W_{1} & W_{e 1} & L_{1} & H & g_{2} & W_{2} & W_{e 2} & L_{2} & E C & \text { ringSymlnvLCDS }>\end{array}$

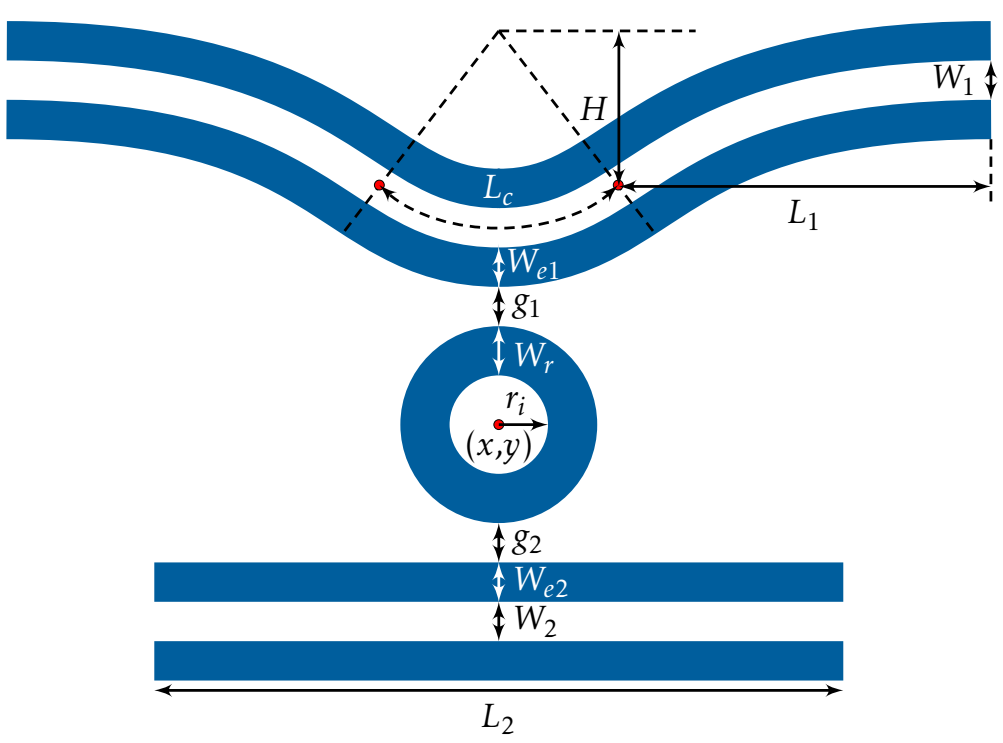

Figure 2.178: Example shape illustrating various parameters from the ringSymlnvLCDS constructor. 
Ring symmetric inverse structure with an additional coupling pulley.

$\begin{array}{llllllllllllllllllllllllllllllllll} & y & r_{i} & W_{r} & N & g_{1} & L_{c 1} & N_{w g} & W_{1} & W_{e 1} & L_{1} & H_{1} & g_{2} & L_{c 2} & W_{2} & W_{e 2} & L_{2} & H_{2} & E C & \text { ringSymlnvLCPul }\end{array}$

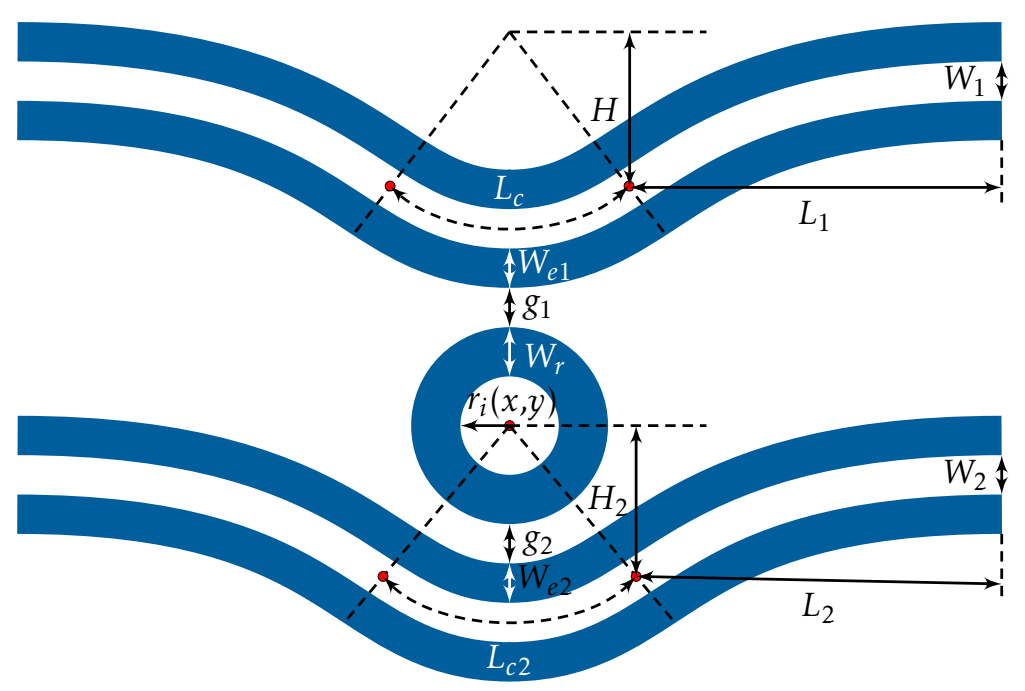

Figure 2.179: Example shape illustrating various parameters from the ringSymlnvLCPul constructor. 


\subsubsection{Disc-Ring Symmetric Inverse Arc}

Symmetric inverse structures constructed using arcs are similar to ones described in section 2.9.9.6. Here, a slot waveguide of width $W$ is formed by exposing a surrounding rectangular region of width $W_{e}$. Using a negative tone resist, the resulting structure would produce a disc or ring shape at a distance $g$ to a slotted waveguide.

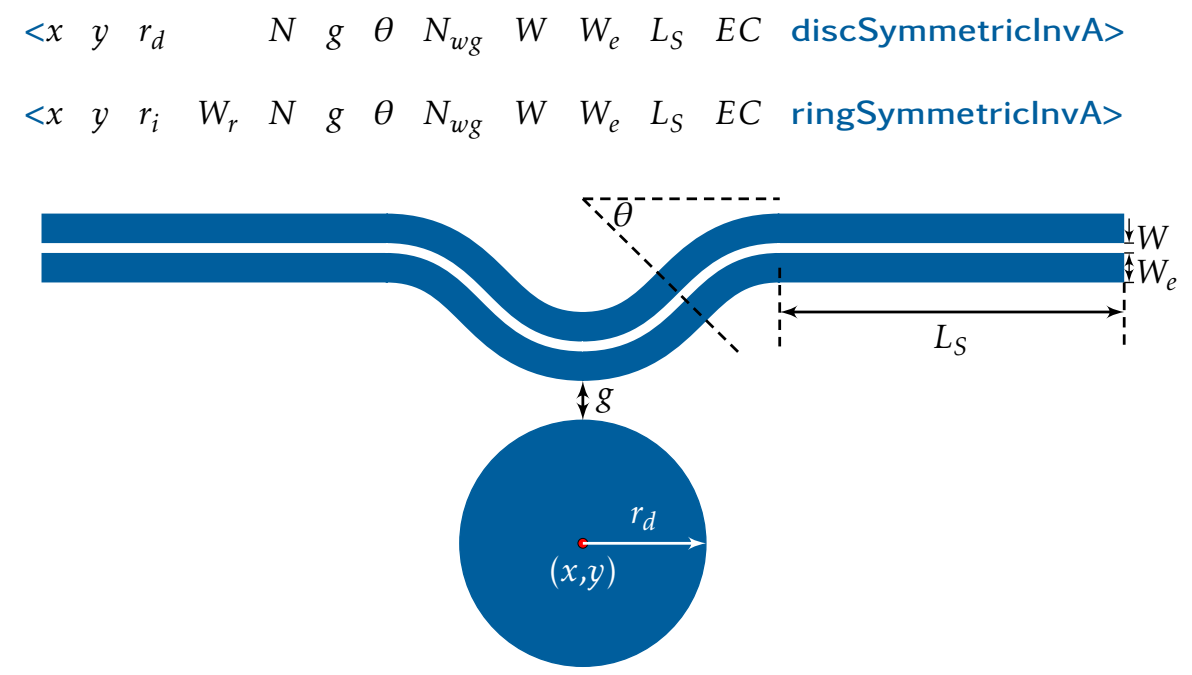

(a)

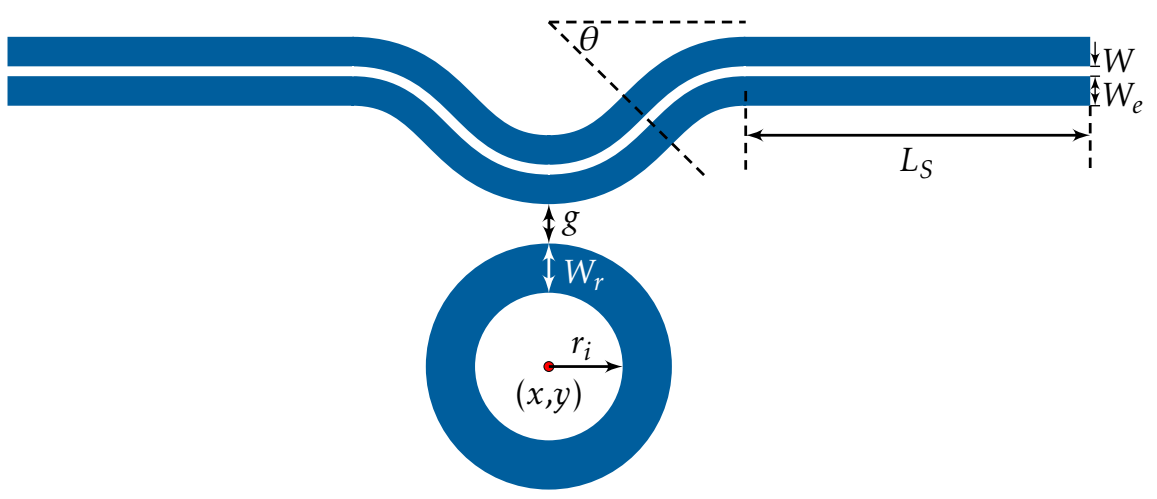

(b)

Figure 2.180: Example shapes illustrating various parameters from the (a) discSymmetriclnvA and (b) ringSymmetriclnvA constructors. 
Disc symmetric inverse arc structure with an additional coupling waveguide.

$\begin{array}{llllllllllllllllllllll}x & y & r_{d} & N & g_{1} & \theta & N_{w g} & W_{1} & W_{e 1} & L_{S} & g_{2} & W_{2} & W_{e 2} & L_{2} & E C & \text { discSymlnvADS } & \end{array}$

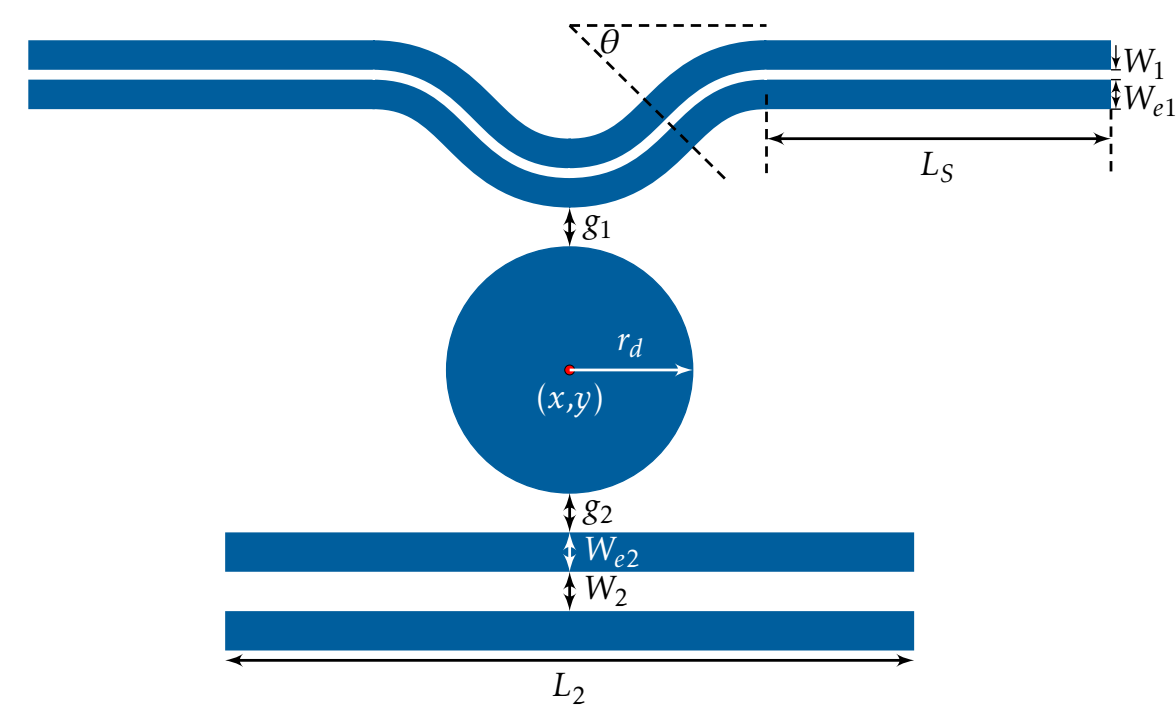

Figure 2.181: Example shape illustrating various parameters from the discSymlnvADS constructor. 
Disc symmetric inverse arc structure with an additional coupling pulley.

$\begin{array}{lllllllllllllllllll} & y & r_{d} & N & g_{1} & \theta_{1} & N_{w g} & W_{1} & W_{e 1} & L_{S 1} & g_{2} & \theta_{2} & W_{2} & W_{e 2} & L_{S 2} & E C & \text { discSymlnvaPul }\end{array}$

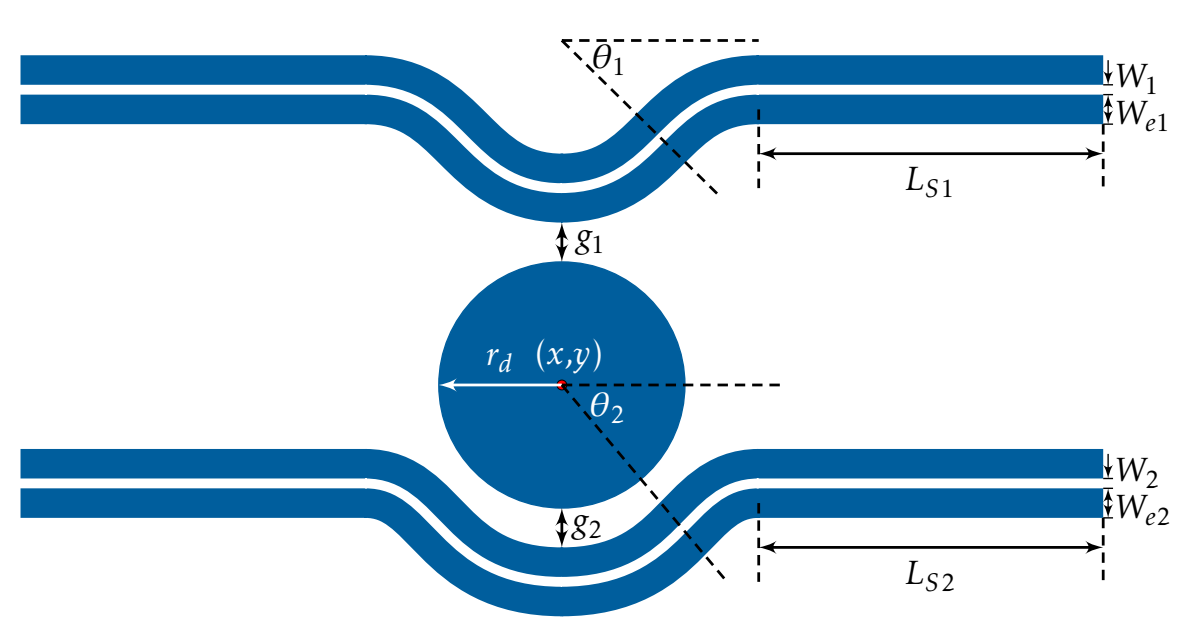

Figure 2.182: Example shape illustrating various parameters from the discSymInvAPul constructor. 
Ring symmetric inverse arc structure with an additional coupling waveguide.

$\begin{array}{llllllllllllllllllllll}x & y & r_{i} & W_{r} & N & g_{1} & \theta & N_{w g} & W_{1} & W_{e} & L_{S} & g_{2} & W_{2} & W_{e 2} & L_{2} & E C & \text { ringSymlnvADS }>\end{array}$

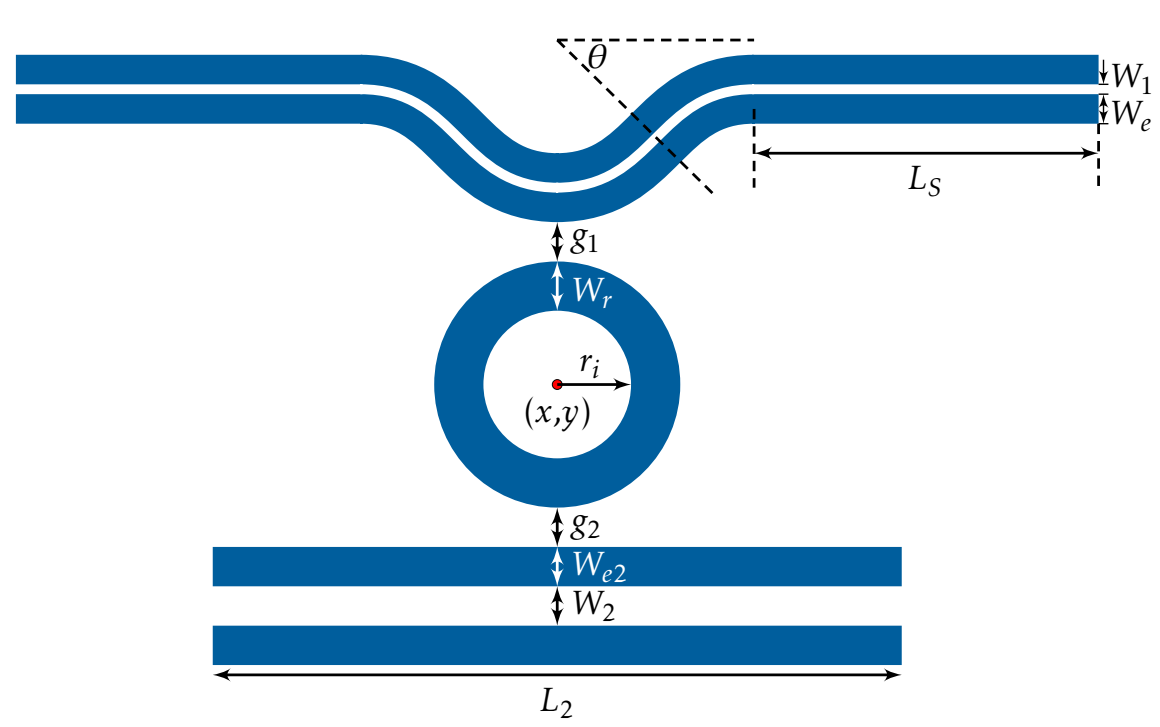

Figure 2.183: Example shapes illustrating various parameters from the ringSymlnvADS constructor. 
Ring symmetric inverse arc structure with an additional coupling pulley.

$\begin{array}{llllllllllllllllllll} & y & r_{i} & W_{r} & N & g_{1} & \theta_{1} & N_{w g} & W_{1} & W_{e 1} & L_{S 1} & g_{2} & \theta_{2} & W_{2} & W_{e 2} & L_{S 2} & E C & \text { ringSymlnvAPul }\end{array}$

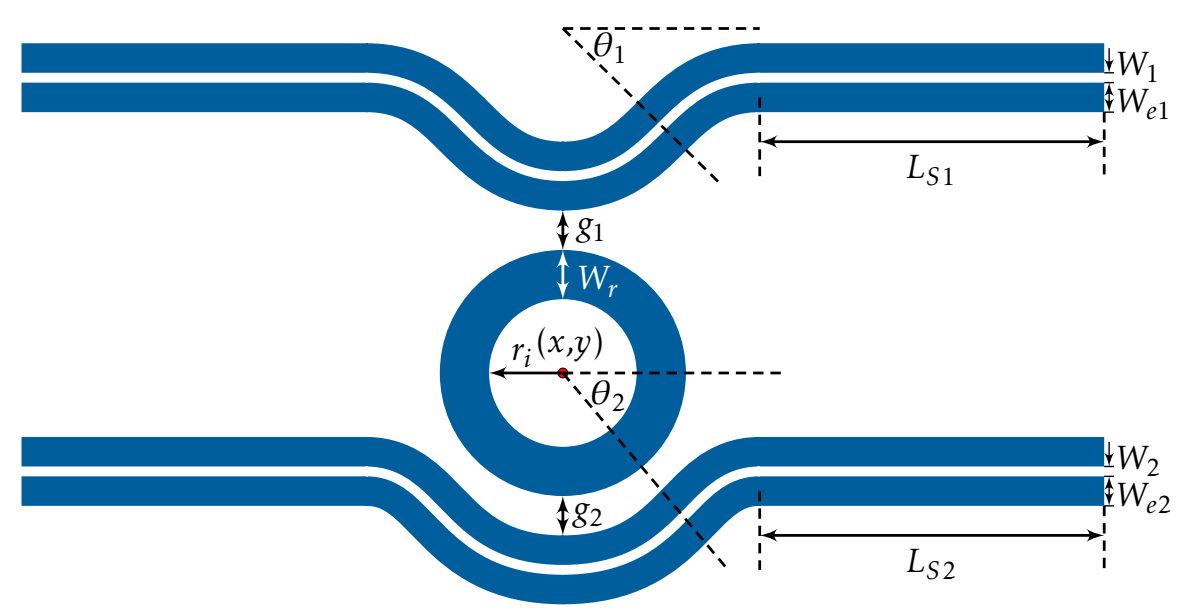

Figure 2.184: Example shapes illustrating various parameters from the ringSymlnvAPul constructor. 
The following arc based, disc-ring waveguide symmetric inverse structures are defined by the coupling length parameter $L_{c}$.

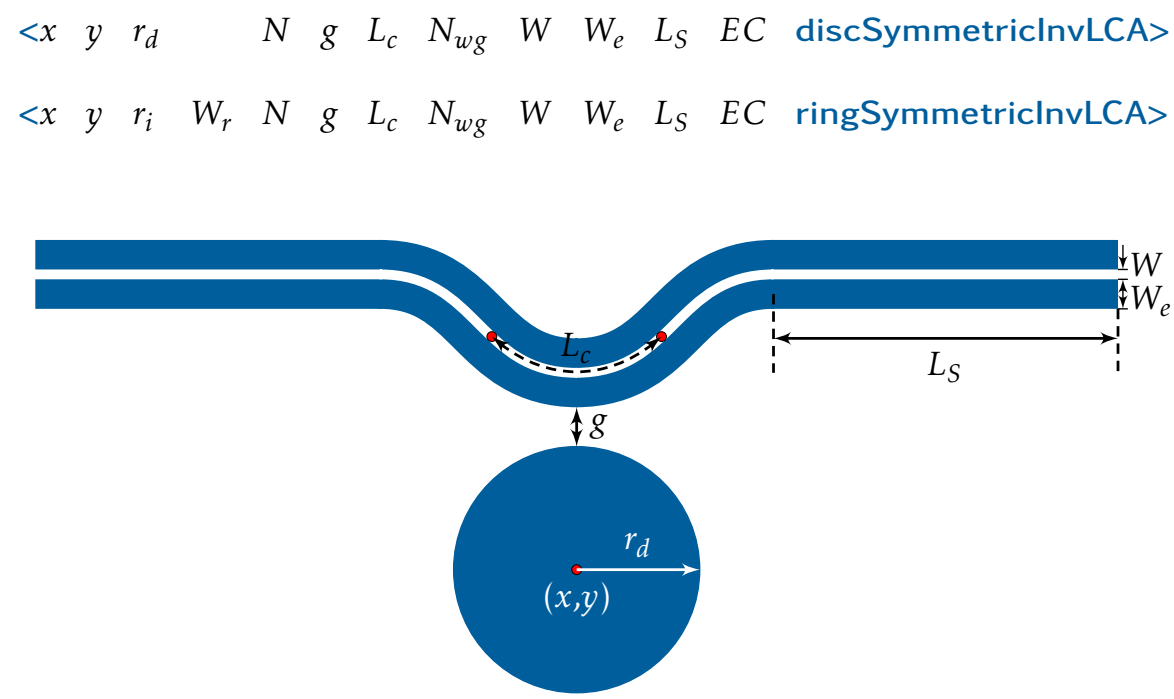

(a)

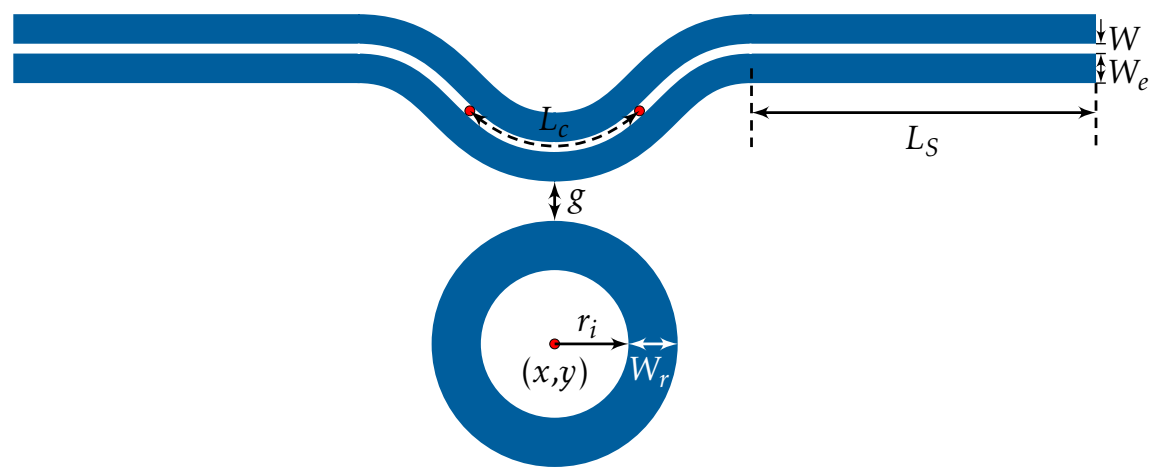

(b)

Figure 2.185: Example shapes illustrating various parameters from the (a) discSymmetriclnvLCA and (b) ringSymmetriclnvLCA constructors. 
Disc symmetric inverse arc structure with an additional coupling waveguide.

$\begin{array}{lllllllllllllllllllll}x & y & r_{d} & N & g_{1} & L_{c} & N_{w g} & W_{1} & W_{e 1} & L_{S} & g_{2} & W_{2} & W_{e 2} & L_{2} & E C & \text { discSymlnvLCADS }>\end{array}$

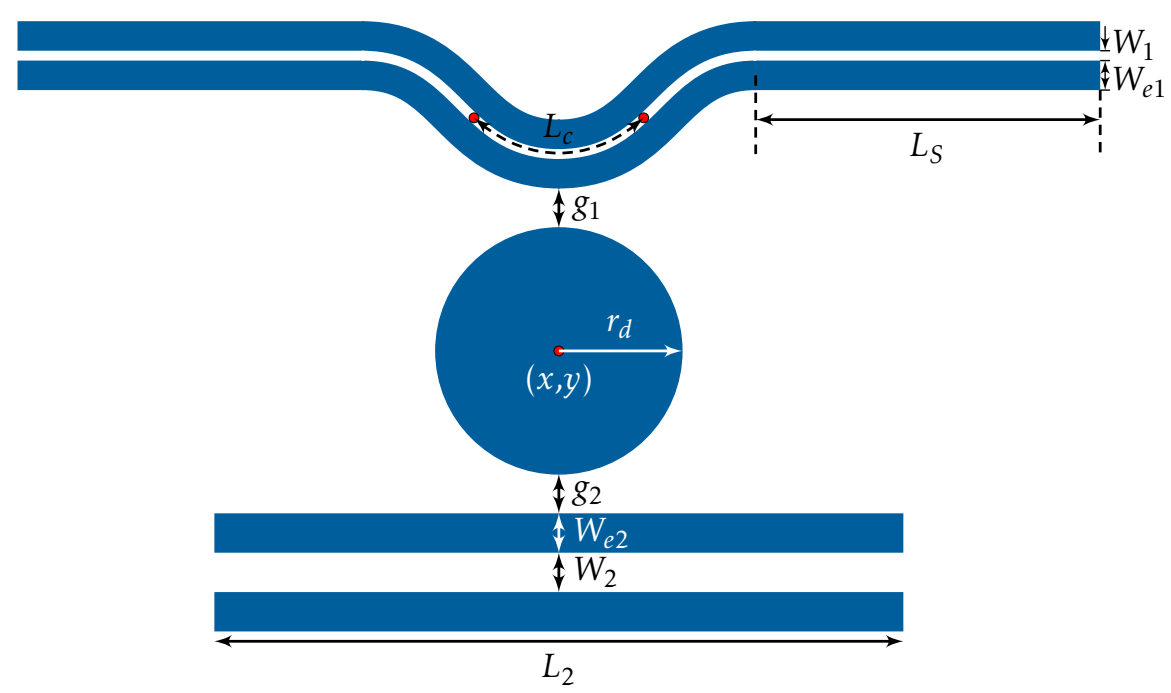

Figure 2.186: Example shape illustrating various parameters from the discSymlnvLCADS constructor. 
Disc symmetric inverse arc structure with an additional coupling pulley.

$\begin{array}{lllllllllllllllllllll} & y & r_{d} & N & g_{1} & L_{c} & N_{w g} & W_{1} & W_{e 1} & L_{S 1} & g_{2} & L_{c 2} & W_{2} & W_{e 2} & L_{S 2} & E C & \text { discSymlnvLCAPul }\end{array}$

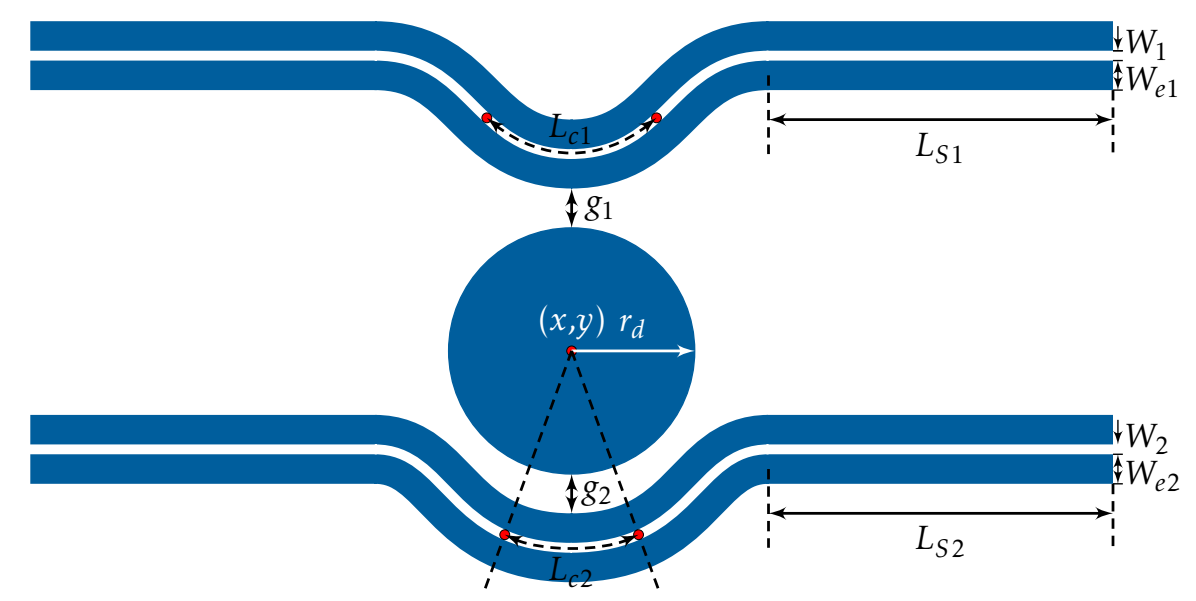

Figure 2.187: Example shape illustrating various parameters from the discSymlnvLCAPul constructor. 
Ring symmetric inverse arc structure with an additional coupling waveguide.

$\begin{array}{llllllllllllllllllllll} & y & r_{i} & W_{r} & N & g_{1} & L_{c} & N_{w g} & W_{1} & W_{e 1} & L_{S} & g_{2} & W_{2} & W_{e 2} & L_{2} & E C & \text { ringSymlnvLCADS }>\end{array}$

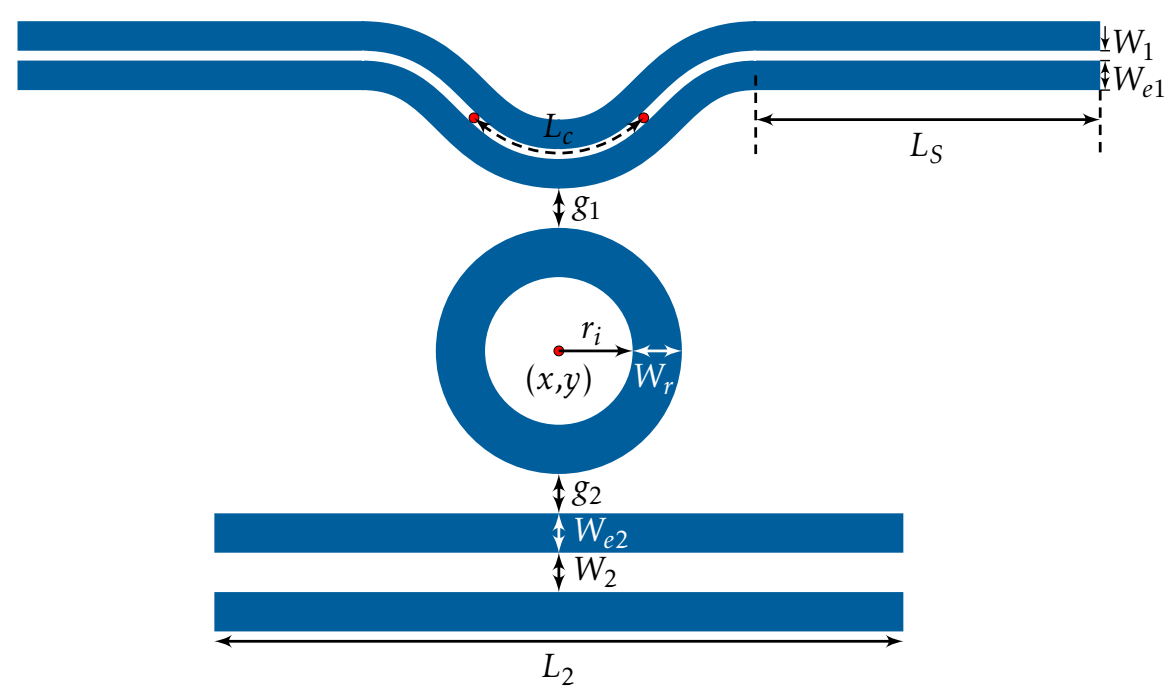

Figure 2.188: Example shape illustrating various parameters from the ringSymlnvLCADS constructor. 
Ring symmetric inverse arc structure with an additional coupling pulley.

$\begin{array}{llllllllllllllllllllll}x & y & r_{i} & W_{r} & N & g_{1} & L_{c 1} & N_{w g} & W_{1} & W_{e 1} & L_{S 1} & g_{2} & L_{c 2} & W_{2} & W_{e 2} & L_{S 2} & E C & \text { ringSymlnvLCAPul }\end{array}$

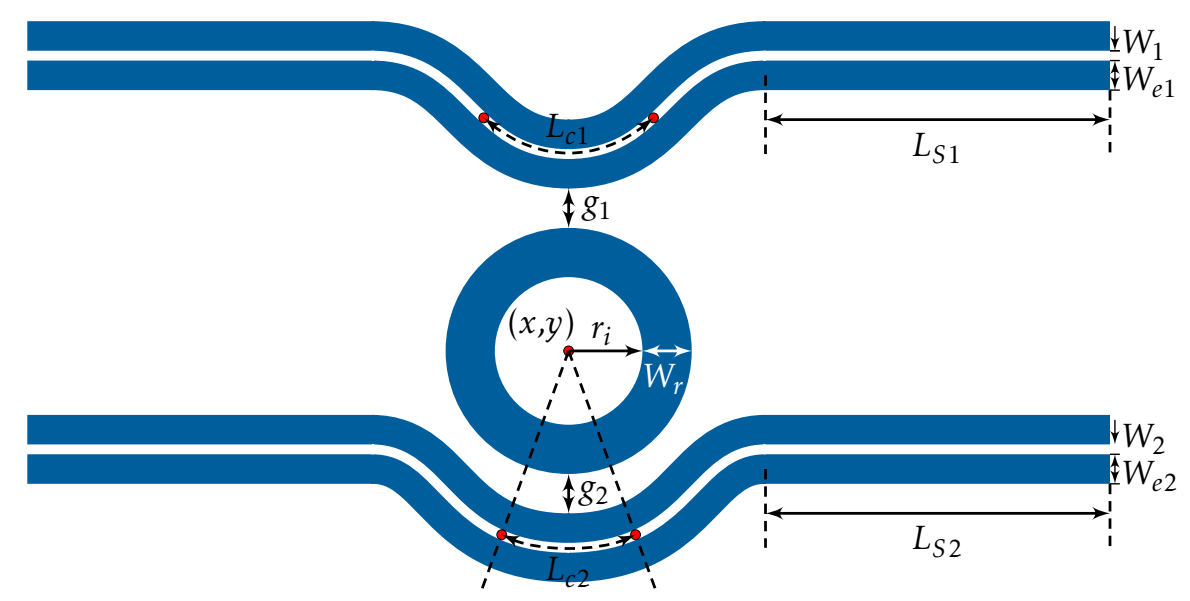

Figure 2.189: Example shape illustrating various parameters from the ringSymlnvLCAPul constructor. 


\subsubsection{Disc-Ring Symmetric Inverse Positive Tone Bezier}

The structure is similar to the disc-ring waveguide symmetric structure defined in section 2.9.9.5. Here, a slot waveguide is formed at a distance $g$ away from a disc or a ring structure using a positive resist exposure of the exposure sleeve elements $W_{e}$ and $r_{e}$.

$$
\begin{array}{llllllllllllllll}
<x & y & r_{d} & & r_{e} & N & g & \theta & N_{w g} & W & W_{e} & L & H & E C & \text { discSymmetriclnvPos }> \\
<x & y & r_{i} & W_{r} & r_{e} & N & g & \theta & N_{w g} & W & W_{e} & L & H & E C & \text { ringSymmetriclnvPos }>
\end{array}
$$

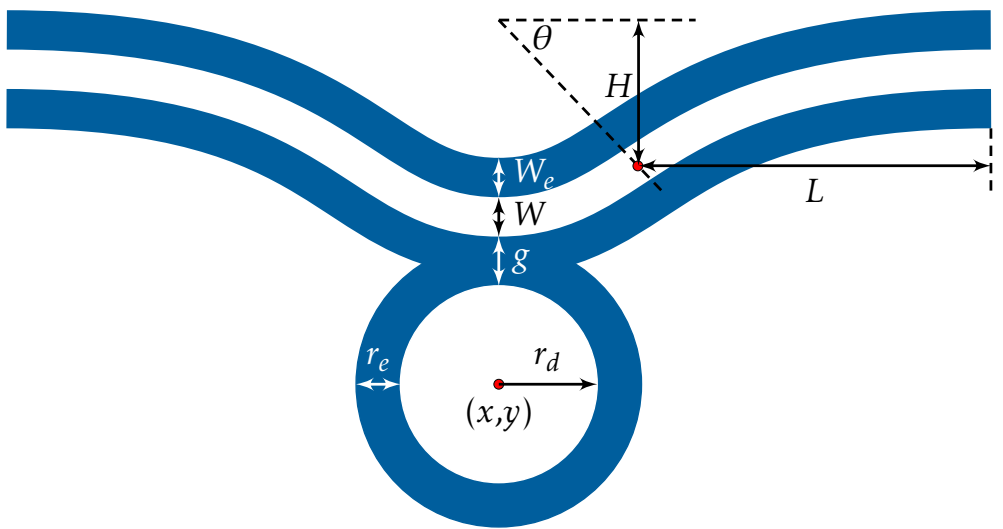

(a)

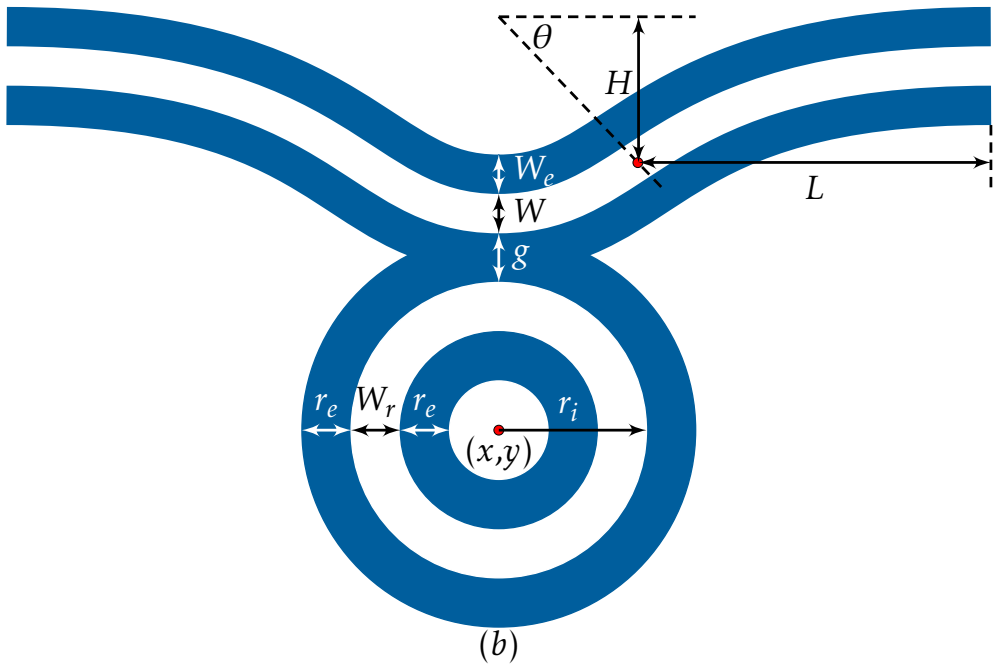

Figure 2.190: Example shapes illustrating various parameters from the (a) discSymmetriclnvPos and (b) ringSymmetriclnvPos constructors. 
Disc symmetric inverse positive structure with an additional coupling waveguide.

$\begin{array}{llllllllllllllllllllllll}< & y & r_{d} & r_{e} & N & g_{1} & \theta & N_{w g} & W_{1} & W_{e 1} & L_{1} & H & g_{2} & W_{2} & W_{e 2} & L_{2} & E C & \text { discSymlnvPosDS } & \end{array}$

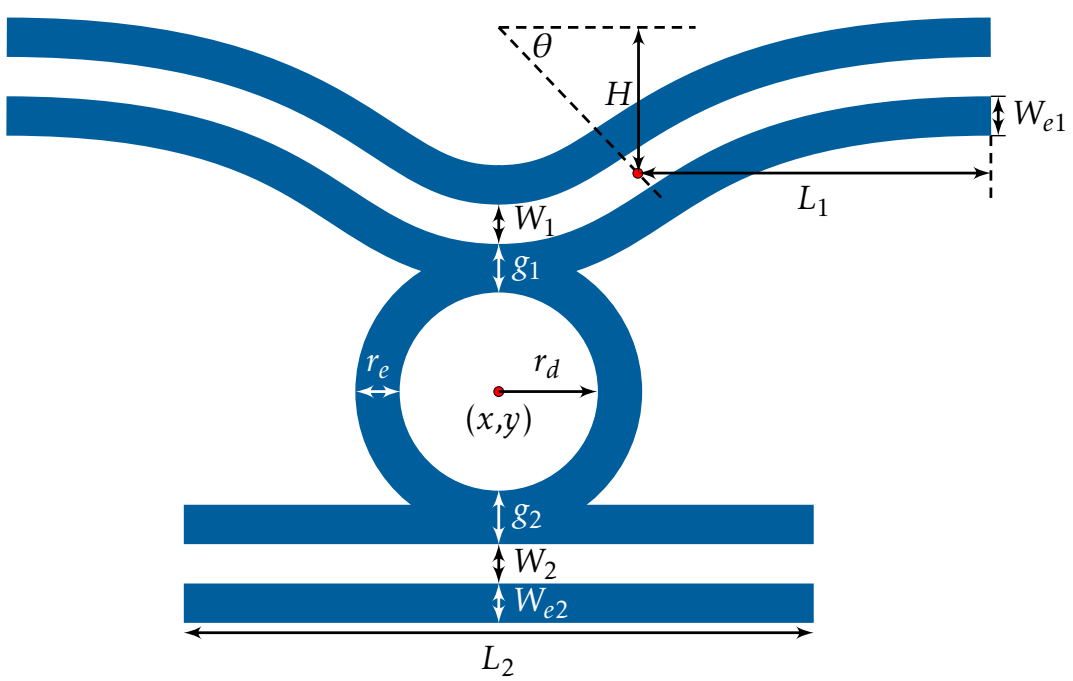

Figure 2.191: Example shape illustrating various parameters from the discSymlnvPosDS constructor. 
Disc symmetric inverse positive structure with an additional coupling pulley.

$<x y r_{d} r_{e} N g_{1} \theta_{1} N_{w g} W_{1} W_{e 1} L_{1} H_{1} g_{2} \theta_{2} W_{2} W_{e 2} L_{2} H_{2} E C$ discSymlnvPosPul $>$

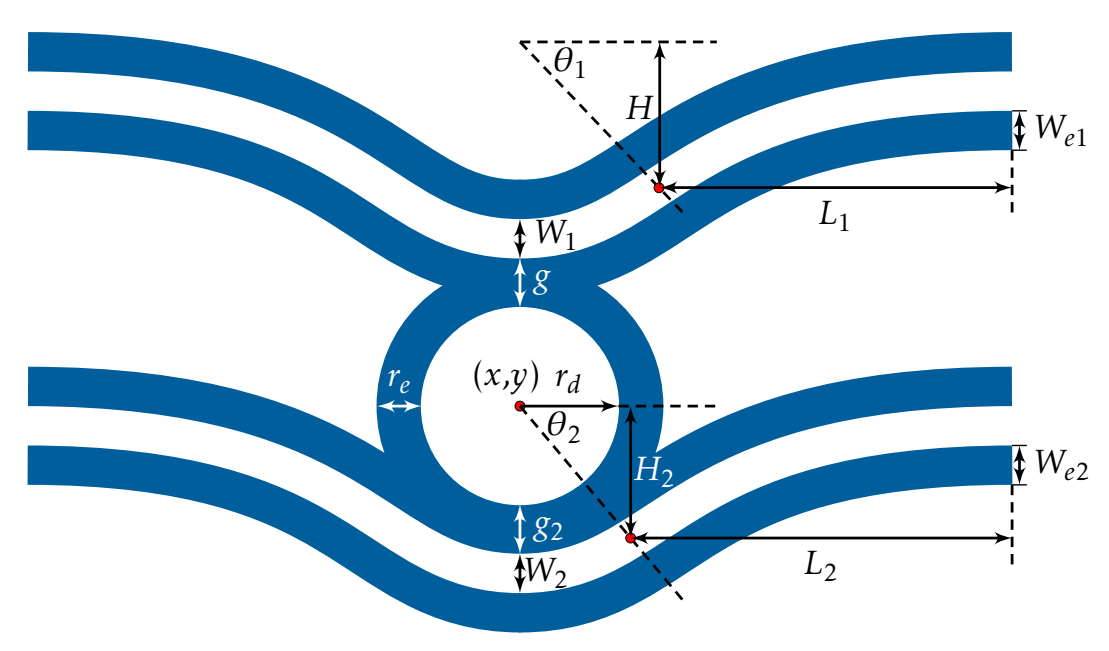

Figure 2.192: Example shape illustrating various parameters from the discSymlnvPosPul constructor. 
Ring symmetric inverse positive structure with an additional coupling waveguide.

$\begin{array}{llllllllllllllllllllllllllll}< & y & r_{i} & W_{r} & r_{e} & N & g_{1} & \theta & N_{w g} & W_{1} & W_{e 1} & L_{1} & H & g_{2} & W_{2} & W_{e 2} & L_{2} & E C & \text { ringSymlnvPosDS } & \end{array}$

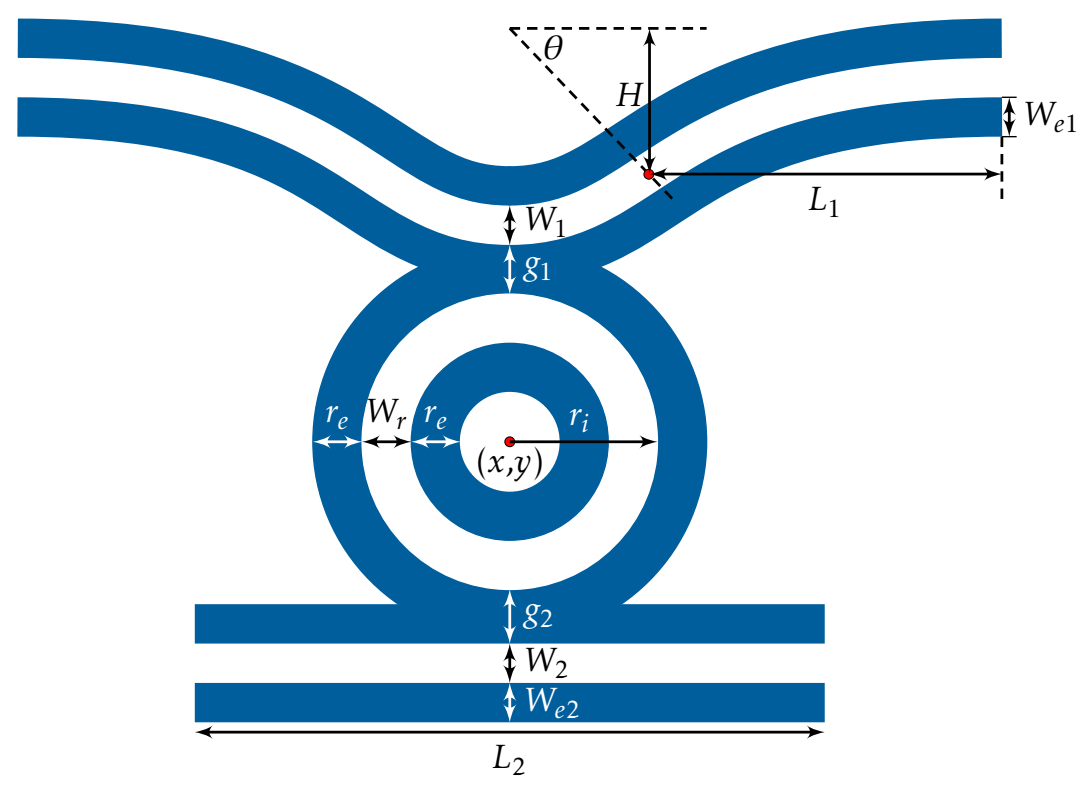

Figure 2.193: Example shape illustrating various parameters from the ringSymlnvPosDS constructor. 
Ring symmetric inverse positive structure with an additional coupling pulley.

$<x y r_{i} W_{r} r_{e} N g_{1} \theta_{1} N_{w g} W_{1} W_{e 1} L_{1} H_{1} g_{2} \theta_{2} W_{2} W_{e 2} L_{2} H_{2} E C$ ringSymlnvPosPul $>$

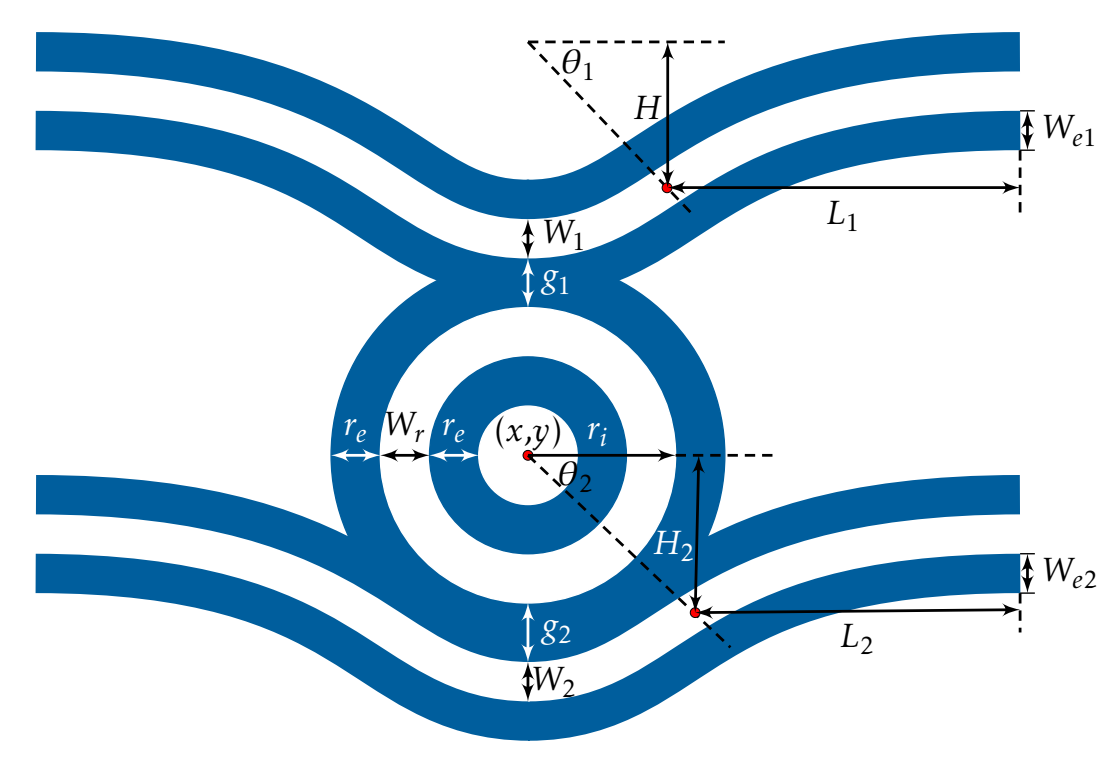

Figure 2.194: Example shape illustrating various parameters from the ringSymlnvPosPul constructor. 
The following disc-ring waveguide symmetric inverse positive tone structures are defined by the coupling length parameter $L_{c}$.

$\begin{array}{llllllllllllllllll}x & y & r_{d} & & r_{e} & N & g & L_{c} & N_{w g} & W & W_{e} & L & H & E C & \text { discSymmetriclnvPosLC }\end{array}$ $\begin{array}{lllllllllllllllllll}< & y & r_{i} & W_{r} & r_{e} & N & g & L_{c} & N_{w g} & W & W_{e} & L & H & E C & \text { ringSymmetriclnvPosLC }\end{array}$

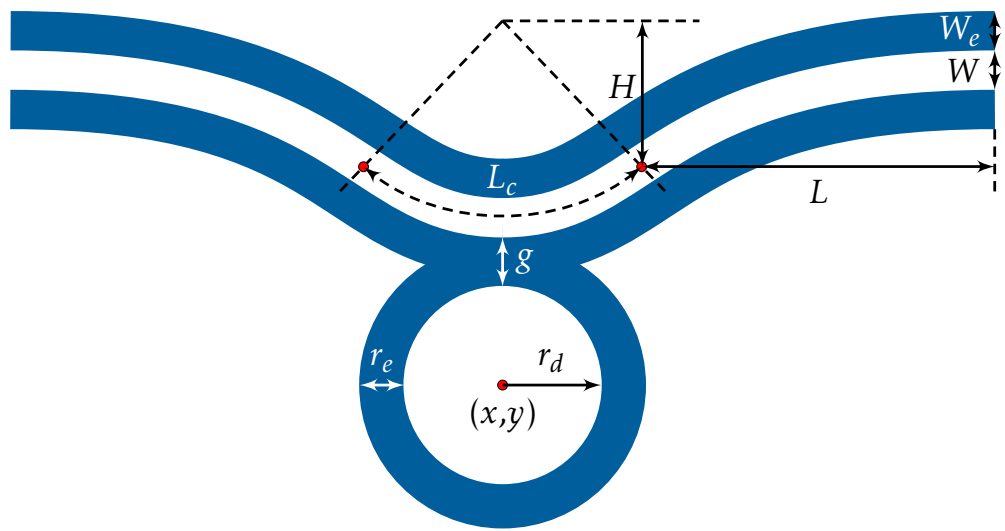

(a)

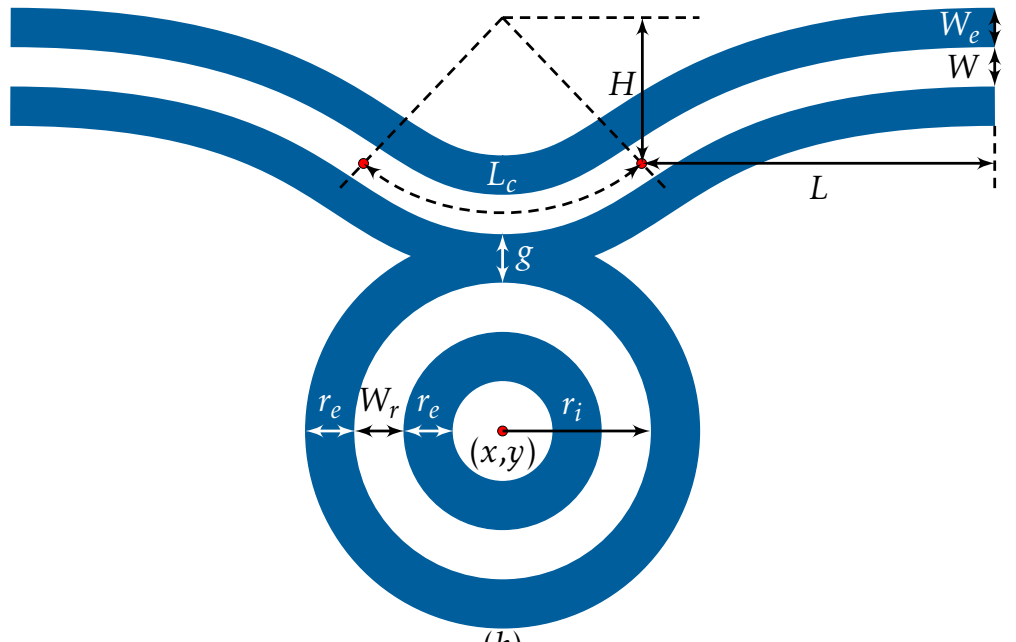

(b)

Figure 2.195: Example shapes illustrating various parameters from the (a) discSymmetriclnvPosLC and (b) ringSymmetriclnvPos $L C$ constructors. 
Disc symmetric inverse positive structure with an additional coupling waveguide.

$\begin{array}{llllllllllllllllllll} & y & r_{d} & r_{e} & N & g_{1} & L_{c} & N_{w g} & W_{1} & W_{e 1} & L_{1} & H & g_{2} & W_{2} & W_{e 2} & L_{2} & E C & \text { discSymlnvPosLCDS }\end{array}$

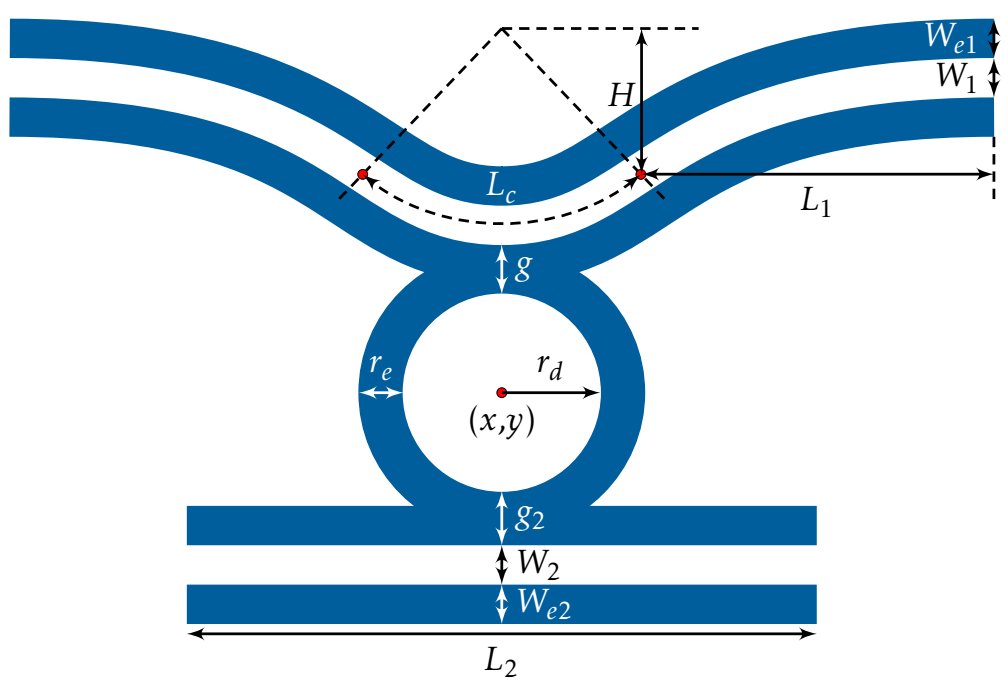

Figure 2.196: Example shape illustrating various parameters from the discSymln$\checkmark P$ osLCDS constructor. 
Disc symmetric inverse positive structure with an additional coupling pulley.

$<x y r_{d} r_{e} N g_{1} L_{c 1} N_{w g} W_{1} W_{e 1} L_{1} H_{1} g_{2} L_{c 2} W_{2} W_{e 2} L_{2} H_{2} E C$ discSymlnvPosLCPul $>$

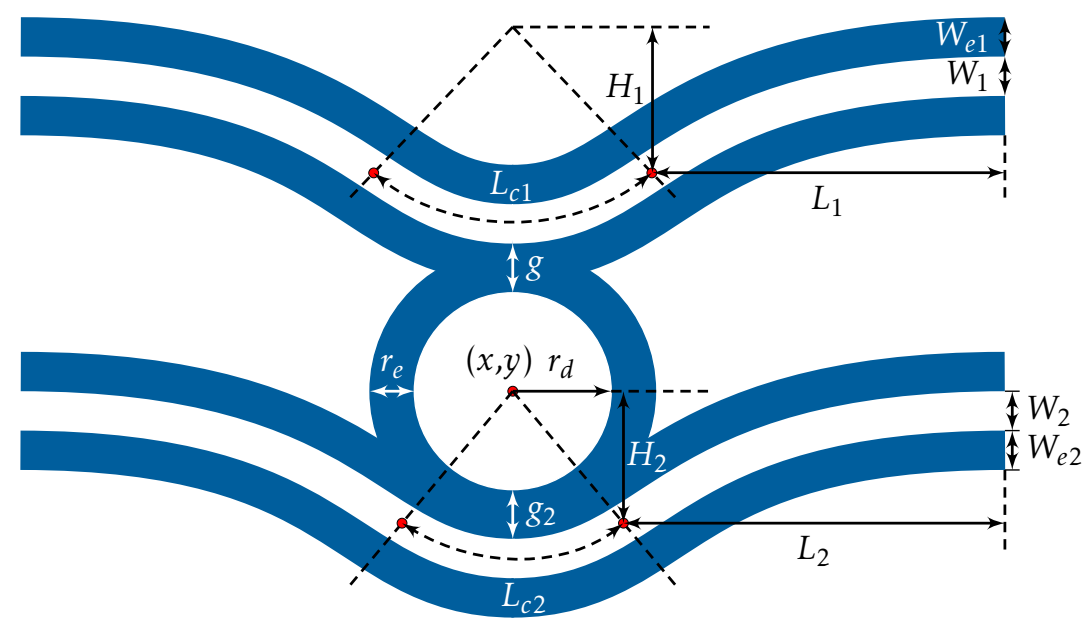

Figure 2.197: Example shape illustrating various parameters from the discSymlnvPosLCPul constructor. 
Ring symmetric inverse positive structure with an additional coupling waveguide.

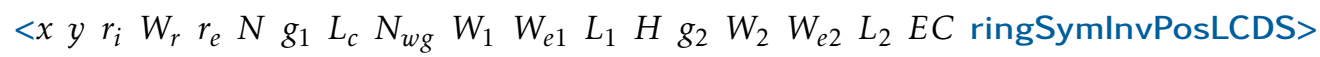

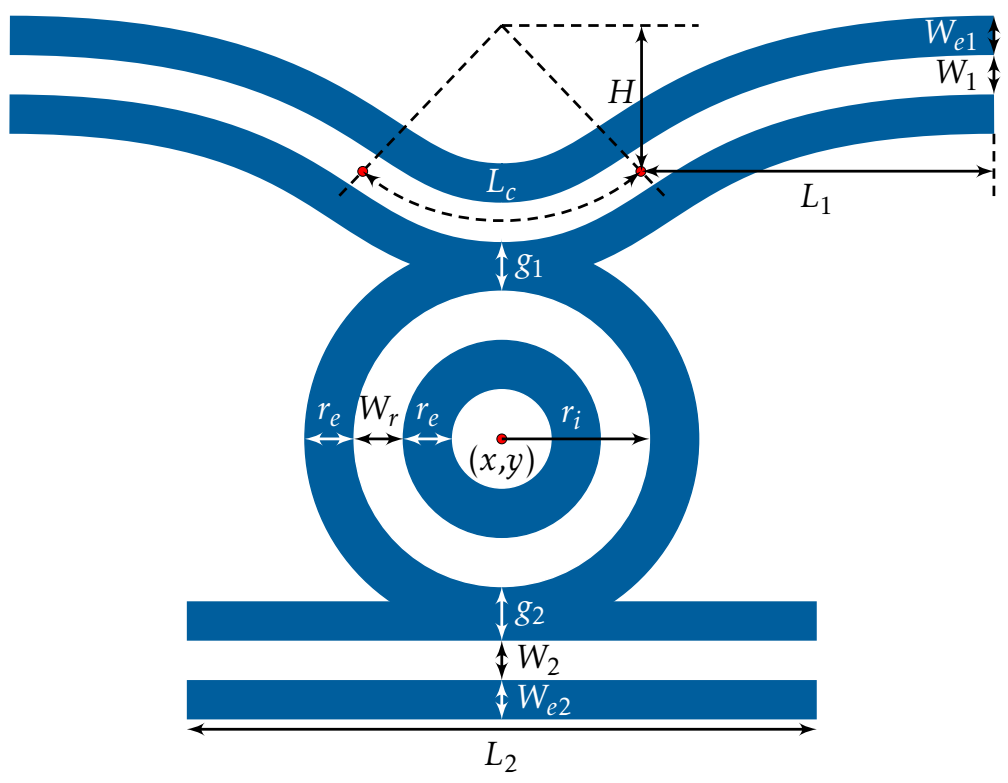

Figure 2.198: Example shape illustrating various parameters from the ringSymln$\checkmark P o s L C D S$ constructor. 
Ring symmetric inverse positive structure with an additional coupling pulley.

$<x y r_{i} W_{r} r_{e} N g_{1} L_{c 1} N_{w g} W_{1} W_{e 1} L_{1} H_{1} g_{2} L_{c 2} W_{2} W_{e 2} L_{2} H_{2} E C$ ringSymlnvPosLCPul $>$

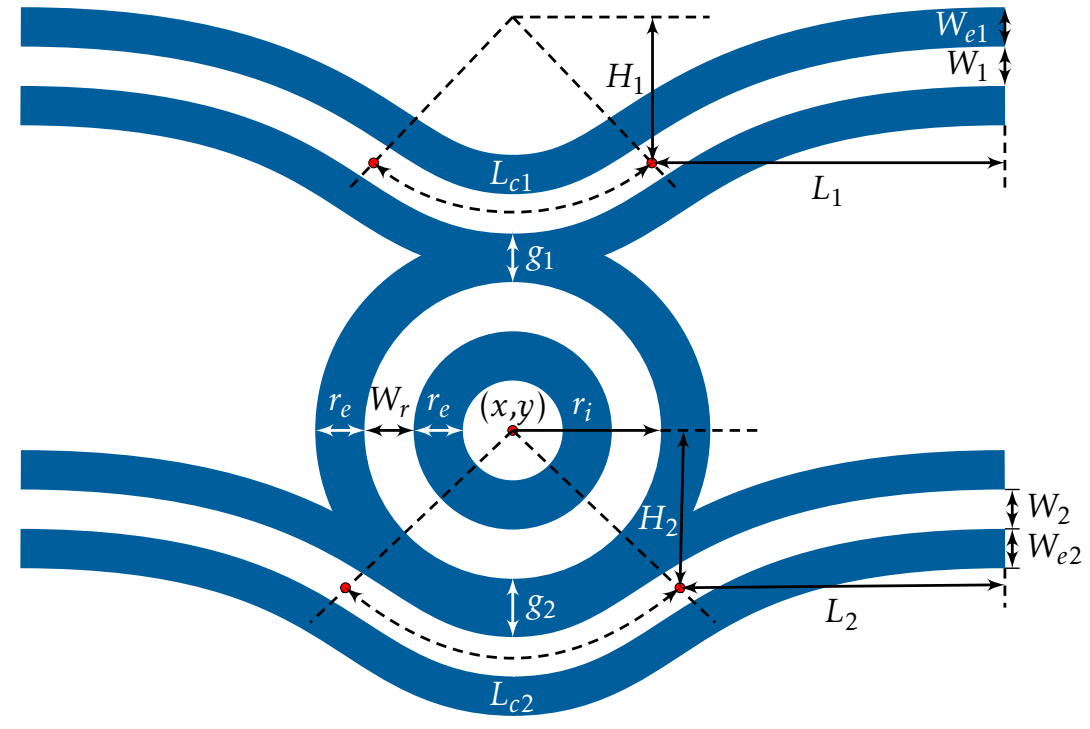

Figure 2.199: Example shape illustrating various parameters from the ringSymlnvPosLCPul constructor. 


\subsubsection{Disc-Ring Symmetric Inverse Positive Tone Arc}

Symmetric inverse positive tone structures constructed using arcs are similar to ones described in section 2.9.9.6. Here, a slot waveguide is formed at a distance $g$ away from a disc or a ring structure using a positive resist exposure of the exposure sleeve elements $W_{e}$ and $r_{e}$.

$$
\begin{array}{llllllllllllllll}
<x & y & r_{d} & & & r_{e} & N & g & \theta & N_{w g} & W & W_{e} & L_{S} & E C & \operatorname{discS} y m m e t r i c l n v P o s & > \\
<x & y & r_{i} & W_{r} & r_{e} & N & g & \theta & N_{w g} & W & W_{e} & L_{S} & E C & \text { ringSymmetriclnvPosA }>
\end{array}
$$

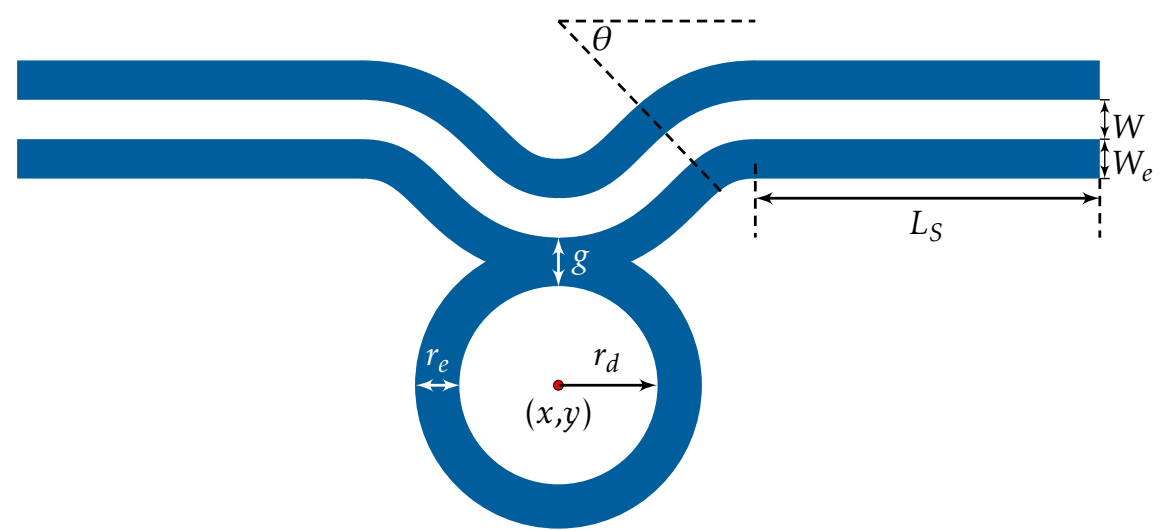

(a)

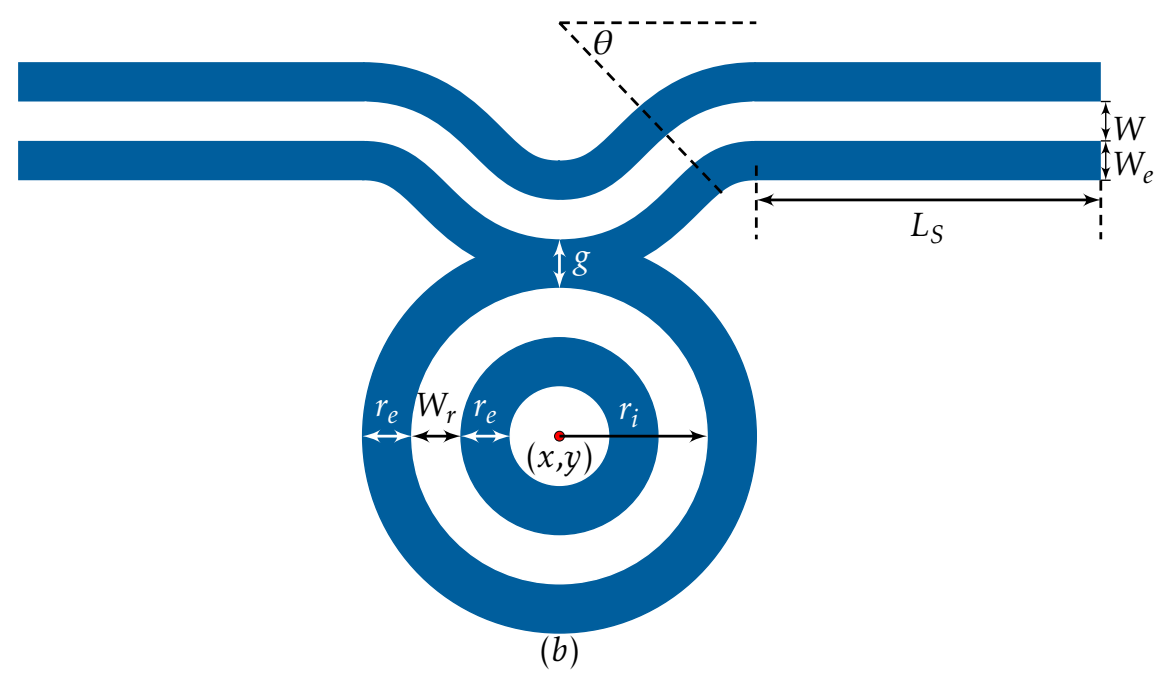

Figure 2.200: Example shapes illustrating various parameters from the (a) discSymmetriclnvPosA and (b) ringSymmetriclnvPosA constructors. 
Disc symmetric inverse positive arc structure with an additional coupling waveguide.

$\begin{array}{lllllllllllllllllllllll}< & y & r_{d} & r_{e} & N & g_{1} & \theta & N_{w g} & W_{1} & W_{e 1} & L_{S} & g_{2} & W_{2} & W_{e 2} & L_{2} & E C & \text { discSymlnvPosADS }\end{array}$

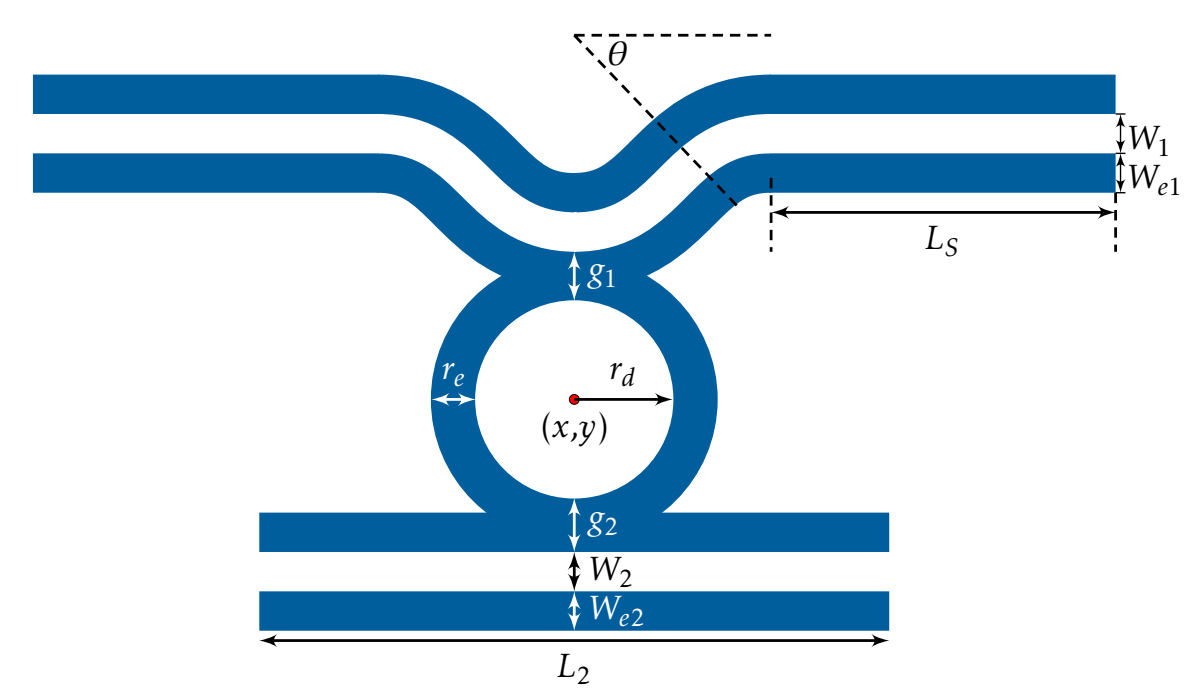

Figure 2.201: Example shapes illustrating various parameters from the (a) discSymln$v$ PosADS and (b) ringSymlnvPosADS constructors. 
Disc symmetric inverse positive arc structure with an additional coupling pulley.

$<x y r_{d} r_{e} N g_{1} \theta_{1} N_{w g} W_{1} W_{e 1} L_{S 1} g_{2} \theta_{2} W_{2} W_{e 2} L_{S 2} E C$ discSymlnvPosAPul $>$

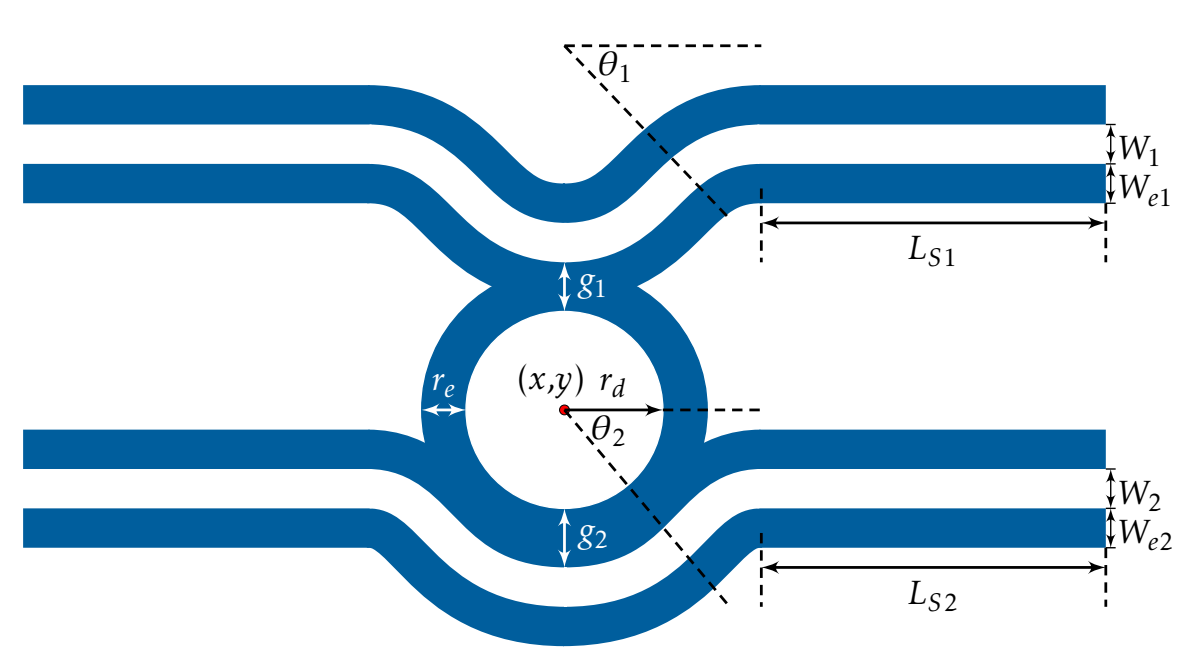

Figure 2.202: Example shape illustrating various parameters from the discSymInvPosAPul constructor. 
Ring symmetric inverse positive arc structure with an additional coupling waveguide.

$\begin{array}{llllllllllllllllll} & y & r_{i} & W_{r} & r_{e} & N & g & \theta & N_{w g} & W & W_{e} & L_{S} & g_{2} & W_{2} & W_{e 2} & L_{2} & E C & \text { ringSymlnvPosADS }>\end{array}$

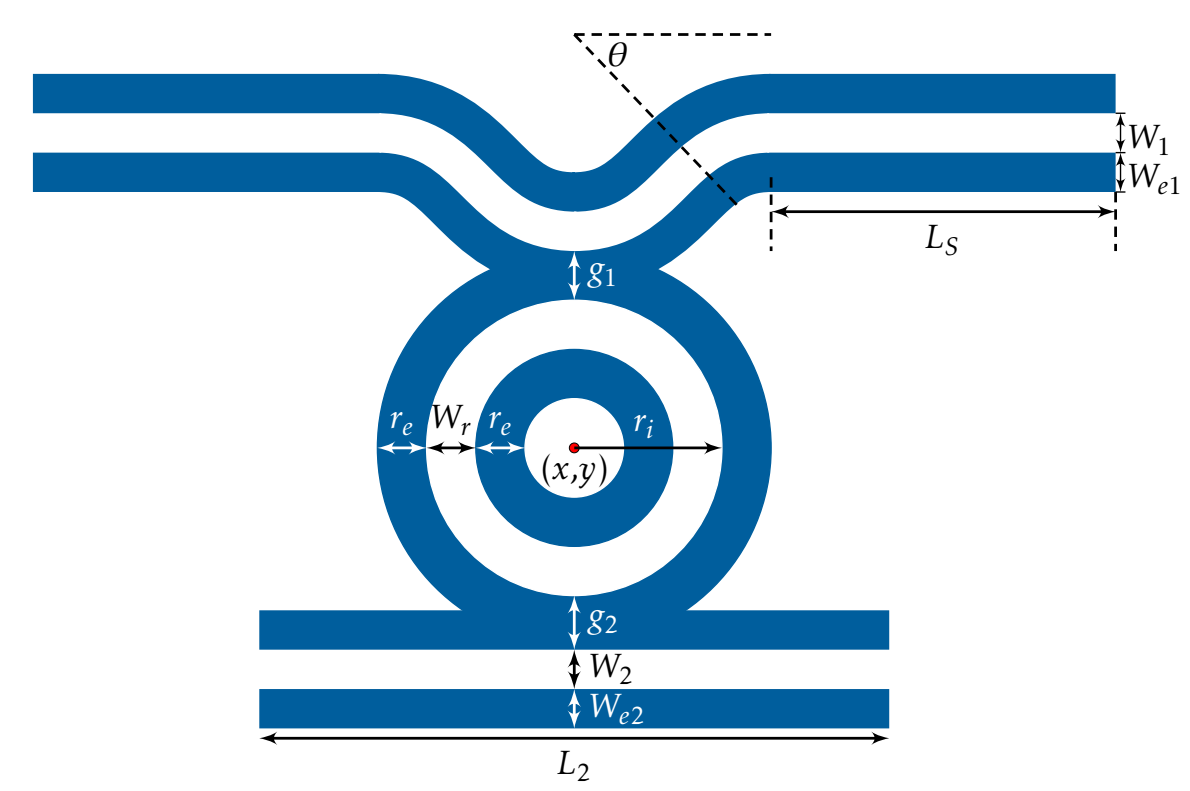

Figure 2.203: Example shape illustrating various parameters from the ringSymlnvPosADS constructor. 
Ring symmetric inverse positive arc structure with an additional coupling pulley.

$<x y r_{i} W_{r} r_{e} N g_{1} \theta_{1} N_{w g} W_{1} W_{e 1} L_{S 1} g_{2} \theta_{2} W_{2} W_{e 2} L_{S 2} E C$ ringSymlnvPosAPul $>$

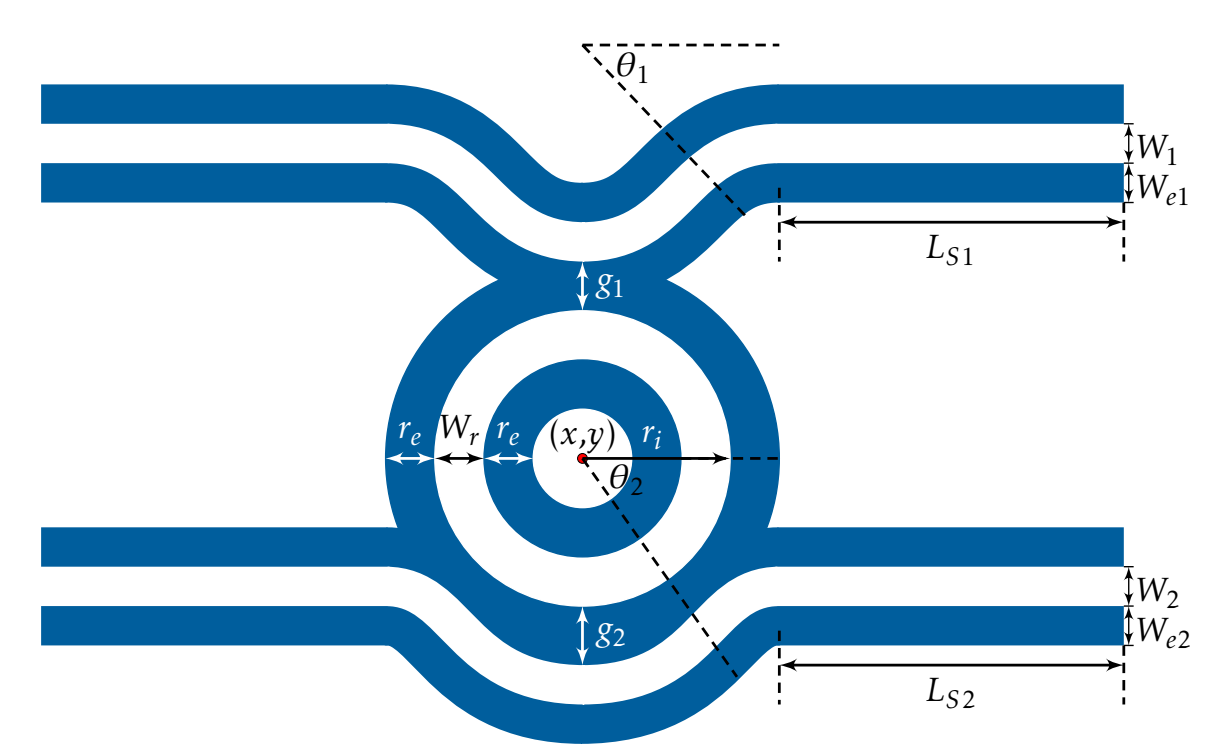

Figure 2.204: Example shape illustrating various parameters from the ringSymlnvPosAPul constructor. 
The following arc based, disc-ring waveguide symmetric inverse positive tone structures are defined by the coupling length parameter $L_{c}$.

$\begin{array}{llllllllllllllll}< & y & r_{d} & r_{e} & N & g & L_{c} & N_{w g} & W & W_{e} & L_{S} & E C & \text { discSymmetriclnvPosLCA }\end{array}$

$\begin{array}{lllllllllllllllll}x & y & r_{i} & W_{r} & r_{e} & N & g & L_{c} & N_{w g} & W & W_{e} & L_{S} & E C & \text { ringSymmetriclnvPosLCA }\end{array}$

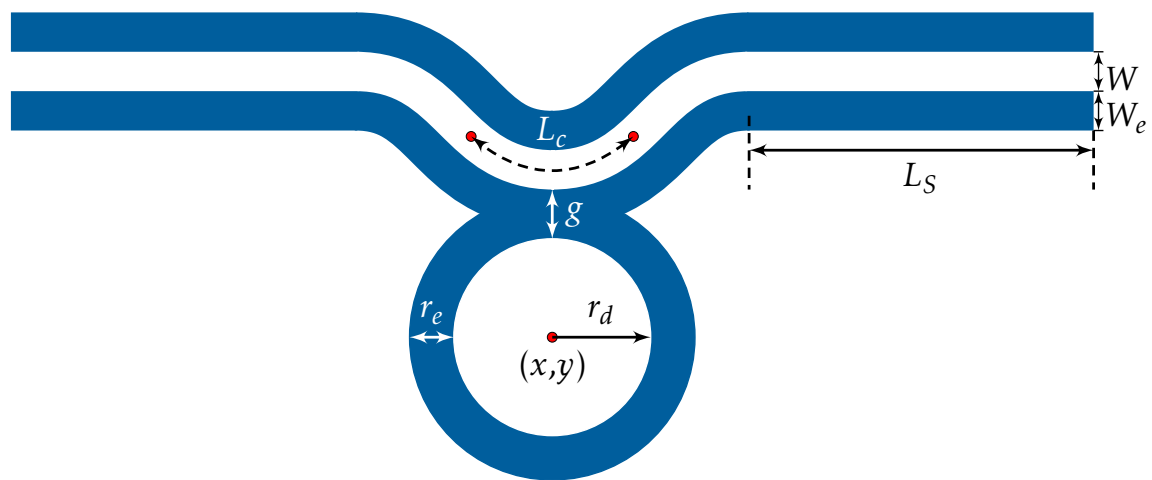

(a)

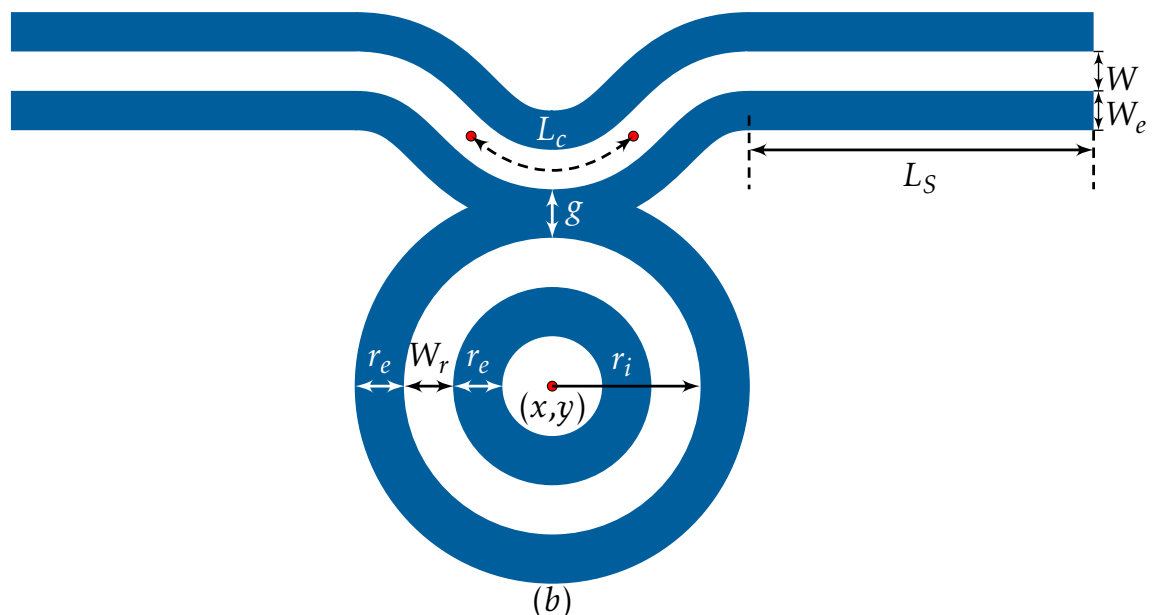

Figure 2.205: Example shapes illustrating various parameters from the (a) discSymmetriclnvPosLCA and (b) ringSymmetricInvPosLCA constructors. 
Disc symmetric inverse positive arc structure with an additional coupling waveguide.

$\begin{array}{llllllllllllllllllllll}x & y & r_{d} & r_{e} & N & g_{1} & L_{c} & N_{w g} & W_{1} & W_{e 1} & L_{S} & g_{2} & W_{2} & W_{e 2} & L_{2} & E C & \text { discSymlnvPosLCADS }\end{array}$

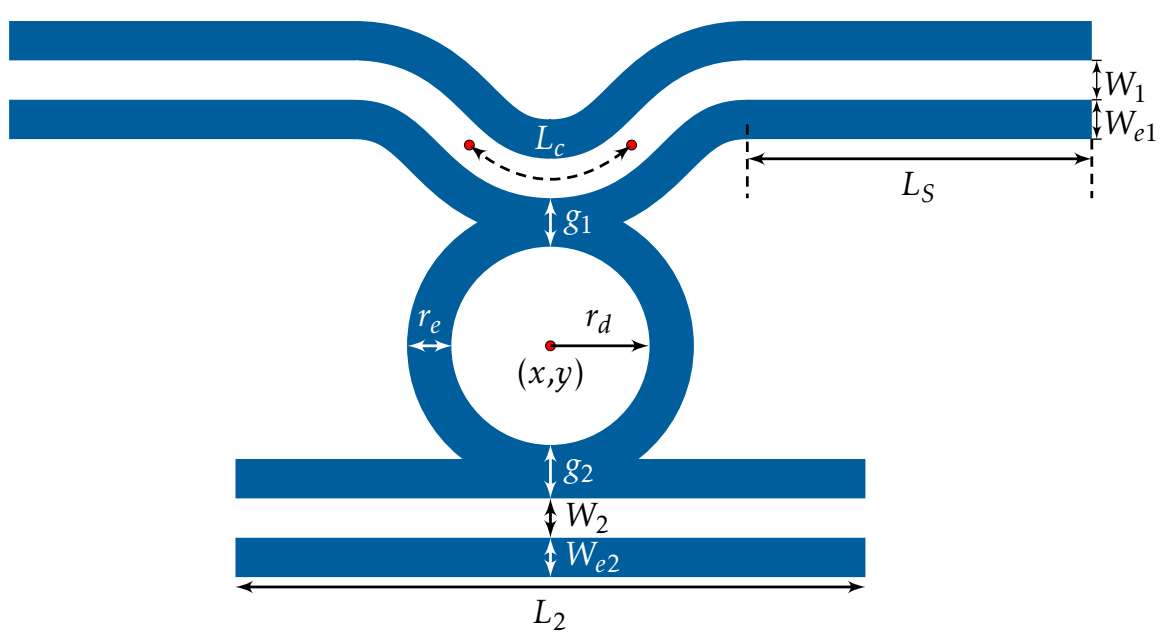

Figure 2.206: Example shape illustrating various parameters from the discSymlnvPosLCADS constructor. 
Disc symmetric inverse positive arc structure with an additional coupling pulley.

$<x y r_{d} r_{e} N g_{1} L_{c 1} N_{w g} W_{1} W_{e 1} L_{S 1} g_{2} L_{c 2} W_{2} W_{e 2} L_{S 2} E C$ discSymlnvPosLCAPul $>$

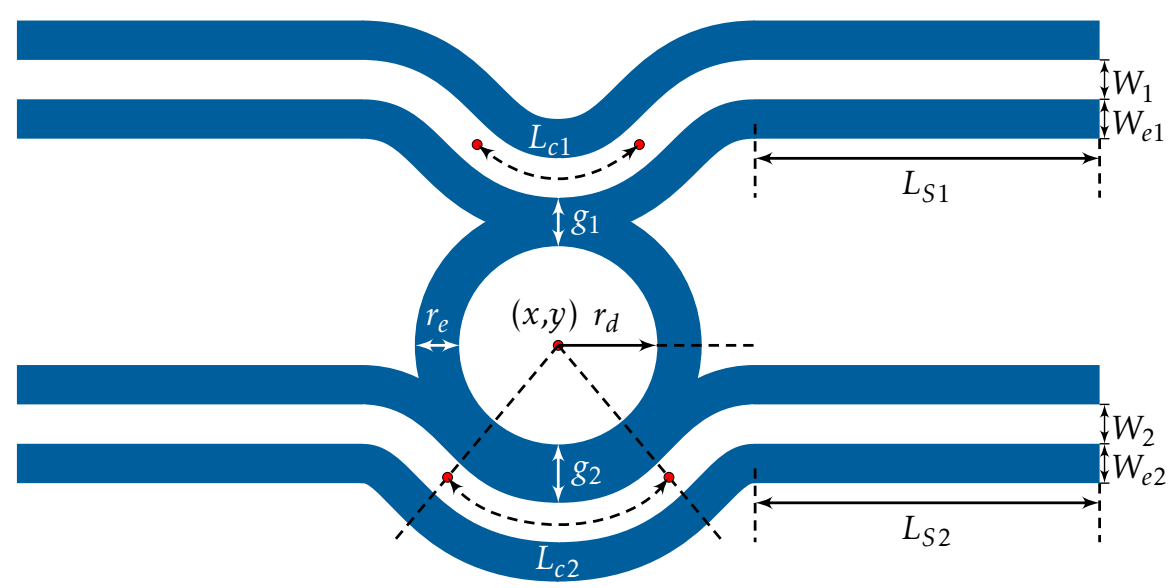

Figure 2.207: Example shape illustrating various parameters from the discSymlnvPosLCAPul constructor. 
Disc symmetric inverse positive arc structure with an additional coupling waveguide.

$\begin{array}{llllllllllllllllllllllllll}x & y & r_{i} & W_{r} & r_{e} & N & g_{1} & L_{c} & N_{w g} & W_{1} & W_{e 1} & L_{S} & g_{2} & W_{2} & W_{e 2} & L_{2} & E C & \text { ringSymlnvPosLCADS }\end{array}$

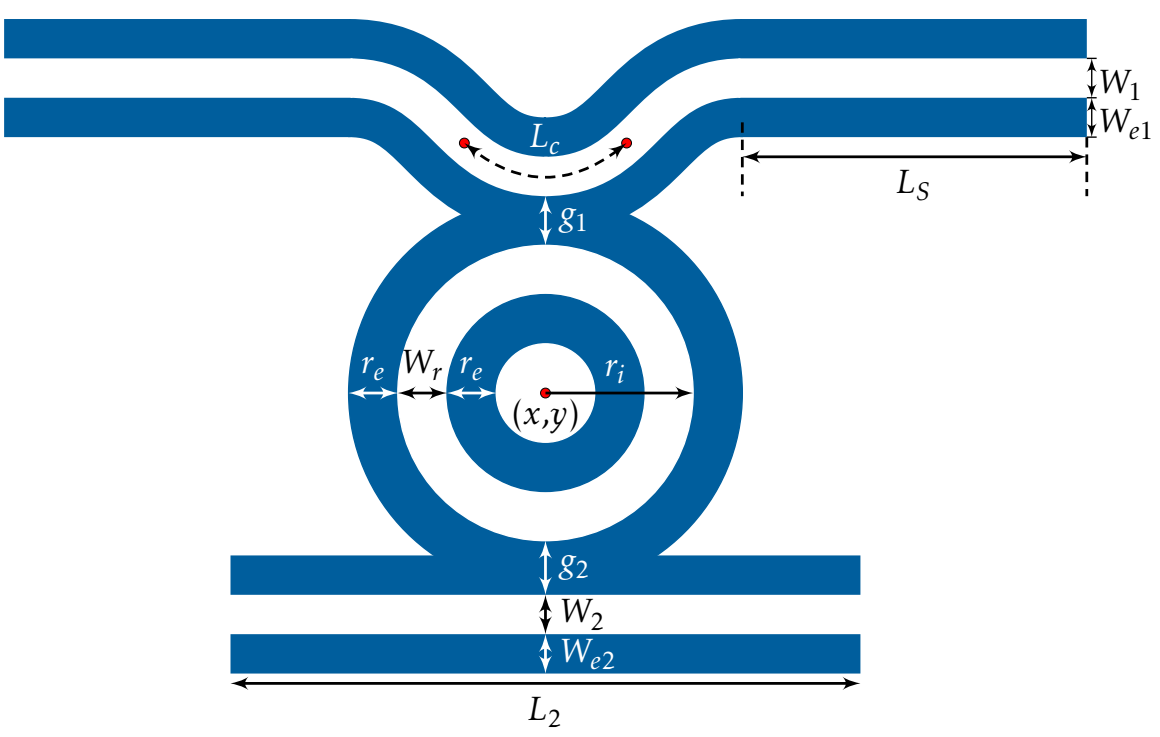

Figure 2.208: Example shape illustrating various parameters from the ringSymlnvPosLCADS constructor. 
Disc symmetric inverse positive arc structure with an additional coupling waveguide.

$<x y r_{i} W_{r} r_{e} N g_{1} L_{c 1} N_{w g} W_{1} W_{e 1} L_{S 1} g_{2} L_{c 2} W_{2} W_{e 2} L_{S 2} E C$ ringSymlnvPosLCAPul $>$

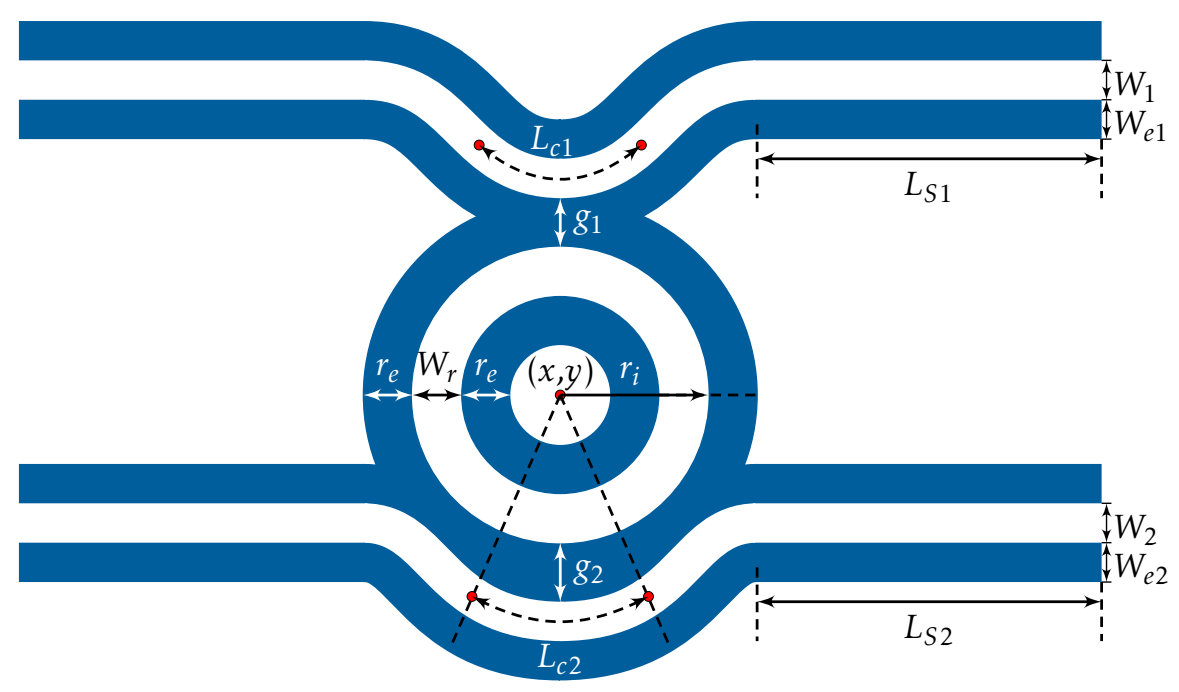

Figure 2.209: Example shape illustrating various parameters from the ringSymlnvPosLCAPul constructor. 


\subsubsection{Disc-Ring Pulley Bezier}

Shapes characterized by a curved waveguide around a disc $\left(R_{\text {waveGuide }}>\right.$ $\left.-R_{\text {Disc }}\right)$ and ring $\left(R_{\text {waveGuide }}>-R_{\text {Ring }}\right)$ objects. Both objects (disc/ring) are described by the center coordinate $(x, y)$, number of segments $(N)$, gap between the object and waveguide $(g)$, waveguide opening angle $(\theta)$, number of sides $\left(N_{w g}\right)$, width $(W)$, length $(L)$ and height $(H)$. Disc object is defined by a radius $\left(r_{d}\right)$ and the ring is characterized by an inner radius $\left(r_{i}\right)$ and ring width $\left(W_{r}\right)$. $E C=0$ and $E C=1$ for waveguides without and with endcaps, respectively.

$$
\begin{array}{lllllllllllll}
<x & y & r_{d} & & N & g & \theta & N_{w g} & W & L & H & E C & \text { discPulley }> \\
<x & y & r_{i} & W_{r} & N & g & \theta & N_{w g} & W & L & H & E C & \text { ringPulley }>
\end{array}
$$

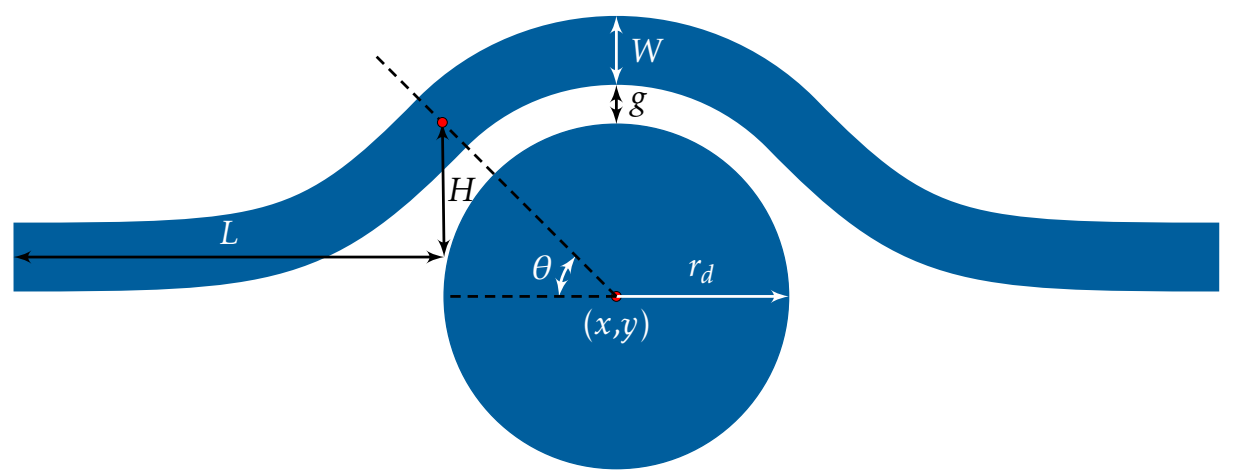

(a)

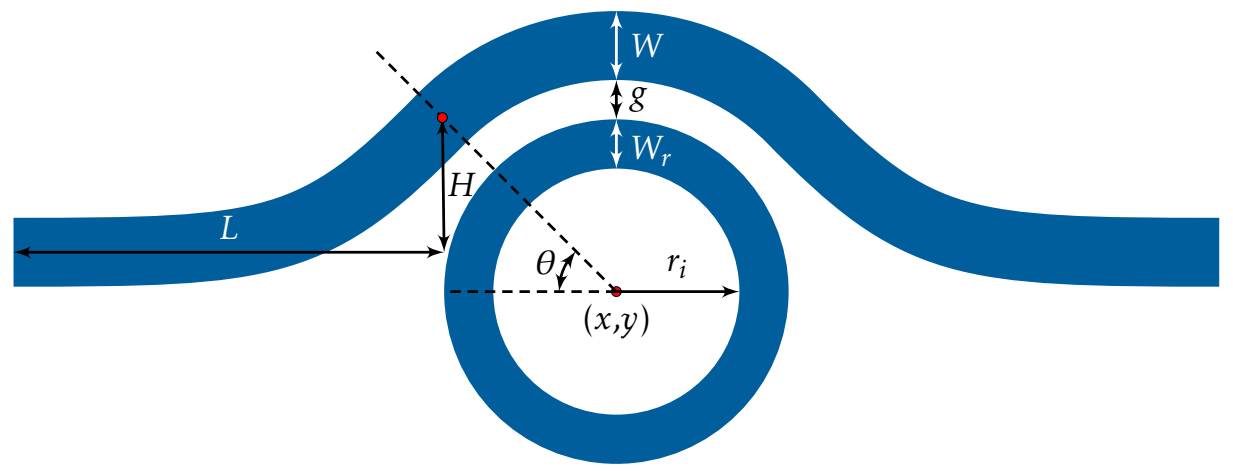

(b)

Figure 2.210: Example shapes illustrating various parameters of the (a) discPulley and (b) ringPulley constructors. 
Disc pulley structure with an additional coupling waveguide.

$\begin{array}{lllllllllllllll}x & y & r_{d} & N & g_{1} & \theta & N_{w g} & W_{1} & L_{1} & H & g_{2} & W_{2} & L_{2} & E C & \text { discPulDS }>\end{array}$

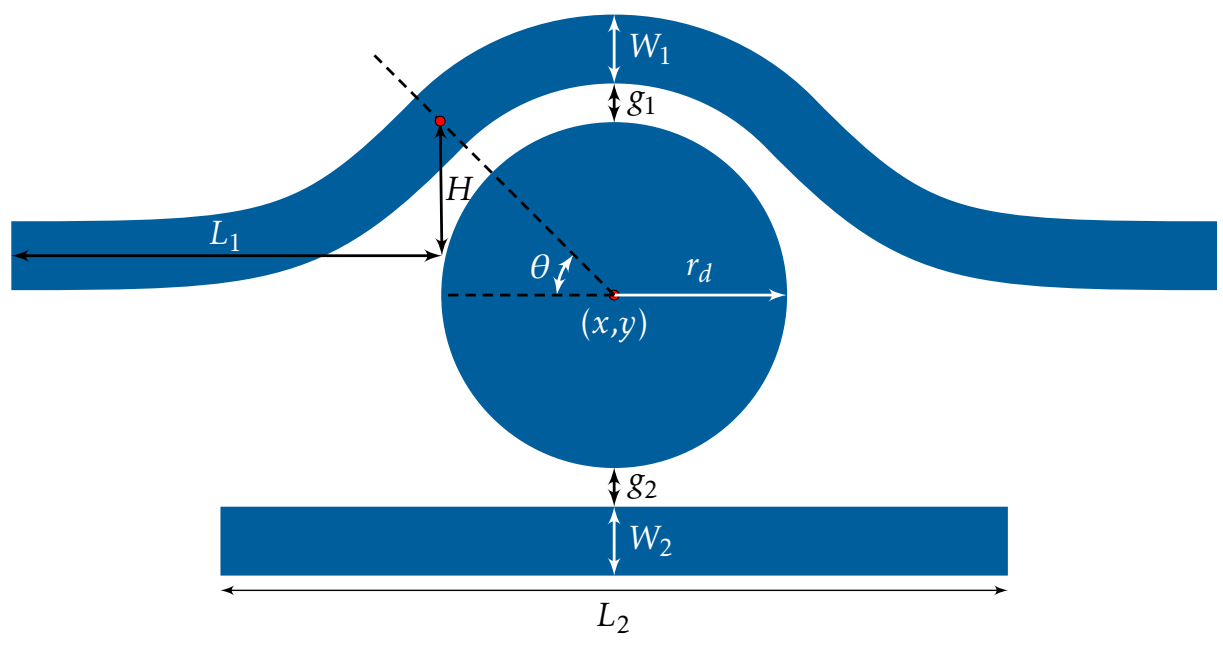

Figure 2.211: Example shape illustrating various parameters of the discPulDS constructor. 
Disc pulley structure with an additional coupling pulley.

$\begin{array}{llllllllllllllllllll}x & y & r_{d} & N & g_{1} & \theta_{1} & N_{w g} & W_{1} & L_{1} & H_{1} & g_{2} & \theta_{2} & W_{2} & L_{2} & H_{2} & E C & \text { discPulPul }\end{array}$

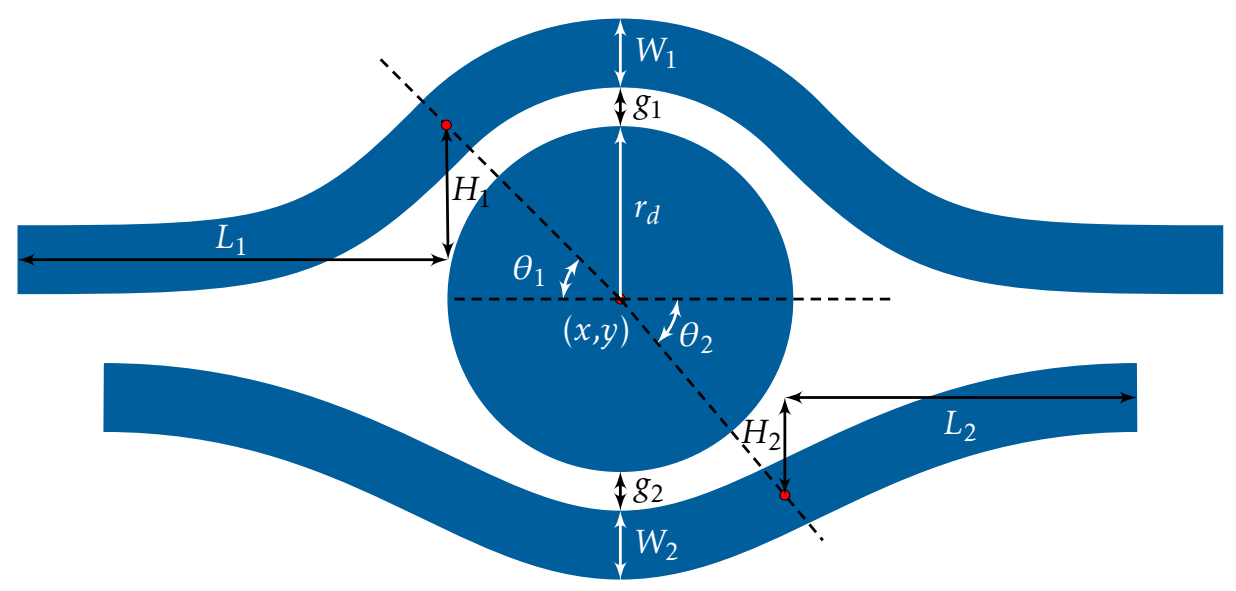

Figure 2.212: Example shape illustrating various parameters of the discPulPul constructor. 
Ring pulley structure with an additional coupling waveguide.

$\begin{array}{llllllllllllllll}x & y & r_{i} & W_{r} & N & g_{1} & \theta & N_{w g} & W_{1} & L_{1} & H & g_{2} & W_{2} & L_{2} & E C & \text { ringPulDS }>\end{array}$

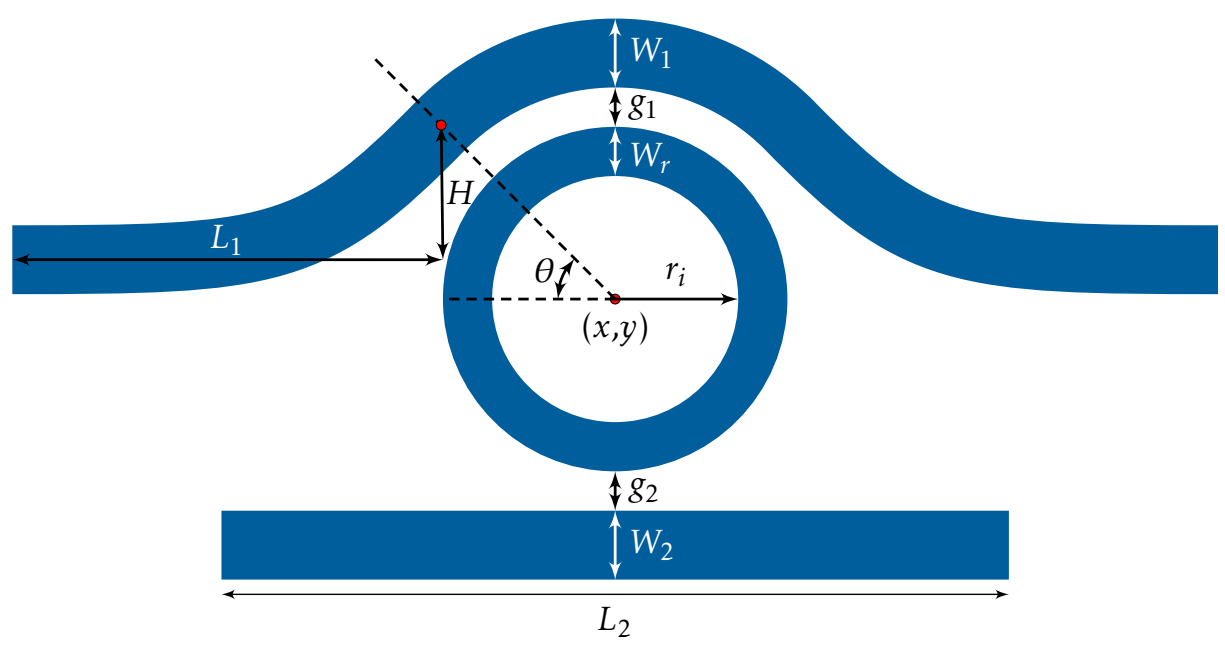

Figure 2.213: Example shape illustrating various parameters of the ringPulDS constructor. 
Ring pulley structure with an additional coupling pulley.

$\begin{array}{lllllllllllllllllll}<x & y & r_{i} & W_{r} & N & g_{1} & \theta_{1} & N_{w g} & W_{1} & L_{1} & H_{1} & g_{2} & \theta_{2} & W_{2} & L_{2} & H_{2} & E C & \text { ringPulPul }>\end{array}$

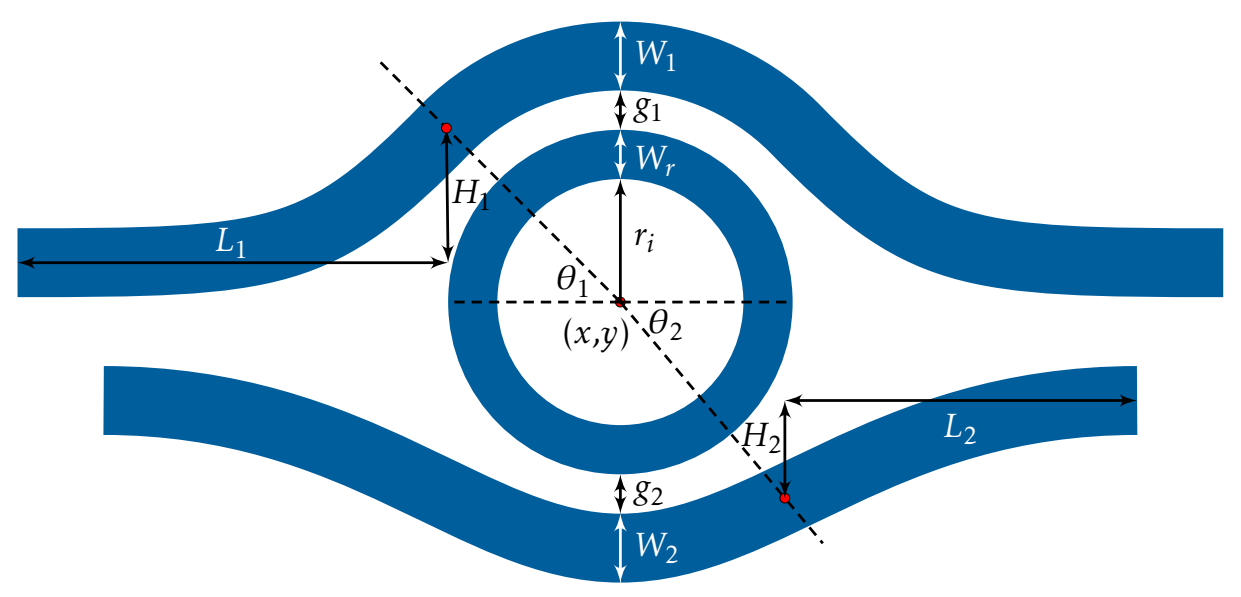

Figure 2.214: Example shape illustrating various parameters of the ringPulPul constructor. 
The following disc-ring waveguide pulley structures are defined by the coupling length parameter $L_{c}$.

$\begin{array}{lllllllllllll}<x & y & r_{d} & & N & g & L_{c} & N_{w g} & W & L & H & E C & \operatorname{discPulleyLC}> \\ <x & y & r_{i} & W_{r} & N & g & L_{c} & N_{w g} & W & L & H & E C & \text { ringPulleyLC }>\end{array}$

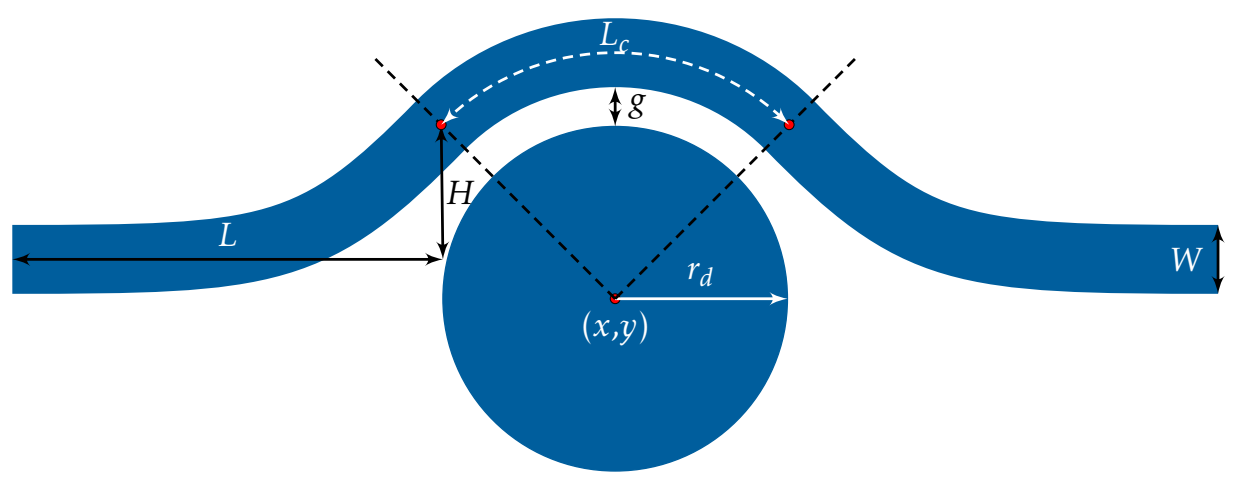

(a)

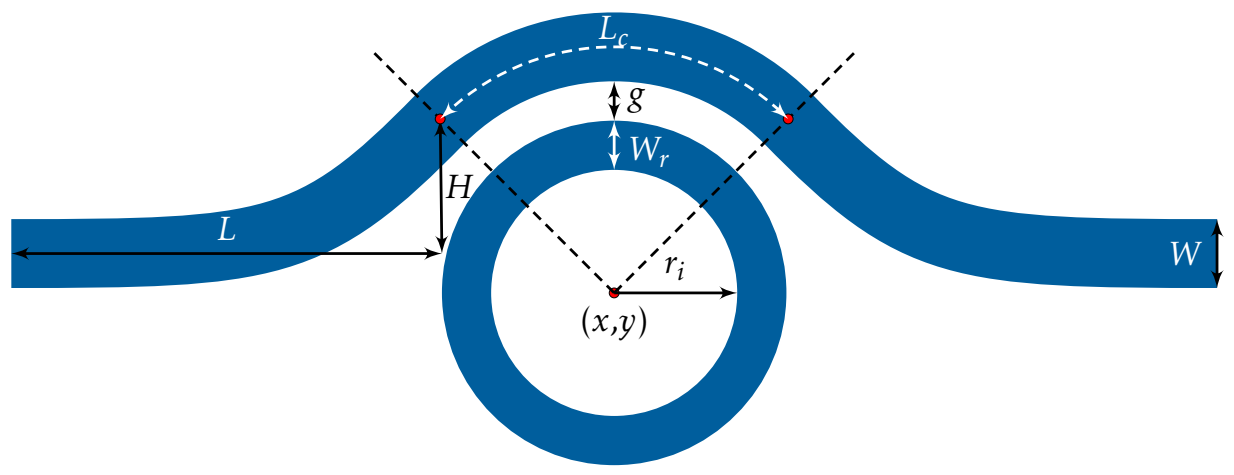

(b)

Figure 2.215: Example shapes illustrating various parameters of the (a) discPulleyLC and (b) ringPulleyLC constructors. 
Disc pulley structure with an additional coupling waveguide.

$\begin{array}{lllllllllllllll}x & y & r_{d} & N & g_{1} & L_{c} & N_{w g} & W_{1} & L_{1} & H & g_{2} & W_{2} & L_{2} & E C & \text { discPulLCDS }>\end{array}$

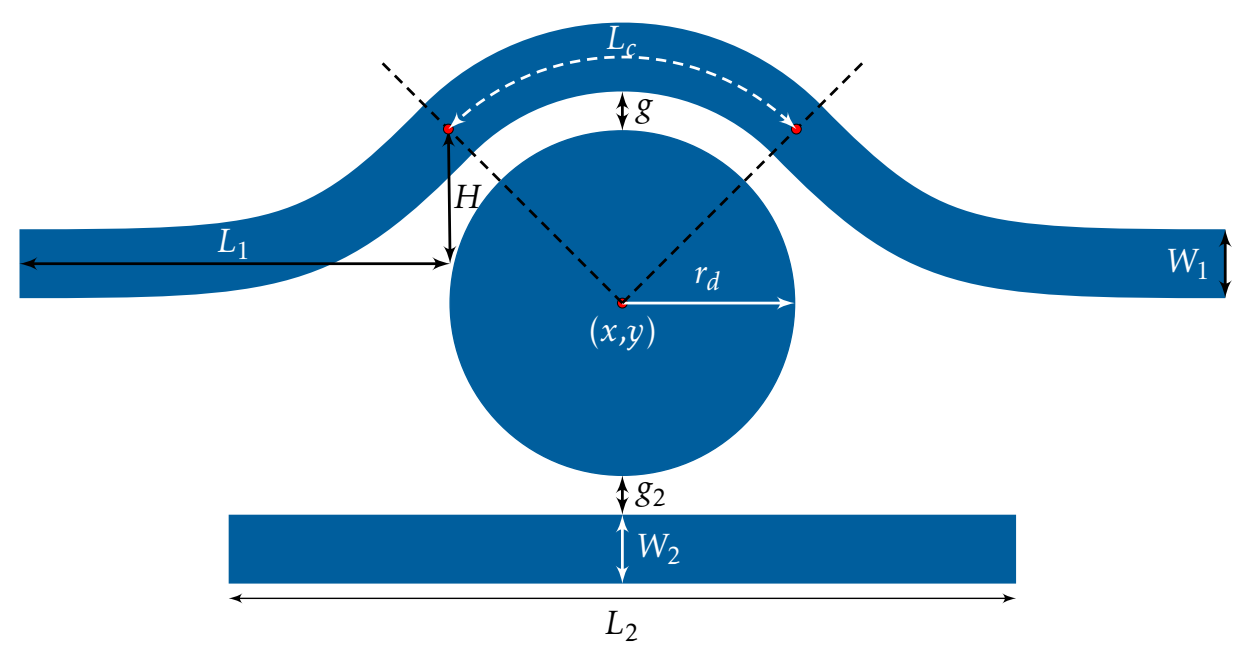

Figure 2.216: Example shape illustrating various parameters of the discPulLCDS constructor. 
Disc pulley structure with an additional coupling pulley.

$\begin{array}{lllllllllllllllllllllll}< & y & r_{d} & N & g_{1} & L_{c 1} & N_{w g} & W_{1} & L_{1} & H_{1} & g_{2} & L_{c 2} & W_{2} & L_{2} & H_{2} & E C & \text { discPullCPul }\end{array}$

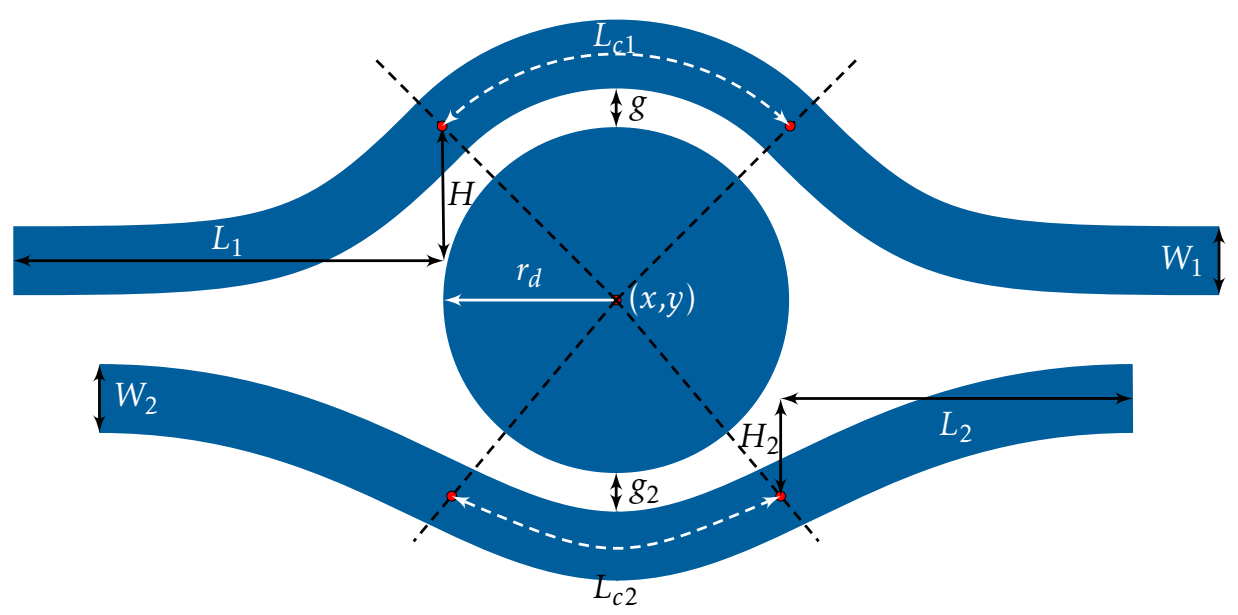

Figure 2.217: Example shape illustrating various parameters of the discPulLCPul constructor. 
Ring pulley structure with an additional coupling waveguide.

$\begin{array}{llllllllllllllll}<x & y & r_{i} & W_{r} & N & g_{1} & L_{c} & N_{w g} & W_{1} & L_{1} & H & g_{2} & W_{2} & L_{2} & E C & \text { ringPulLCDS }>\end{array}$

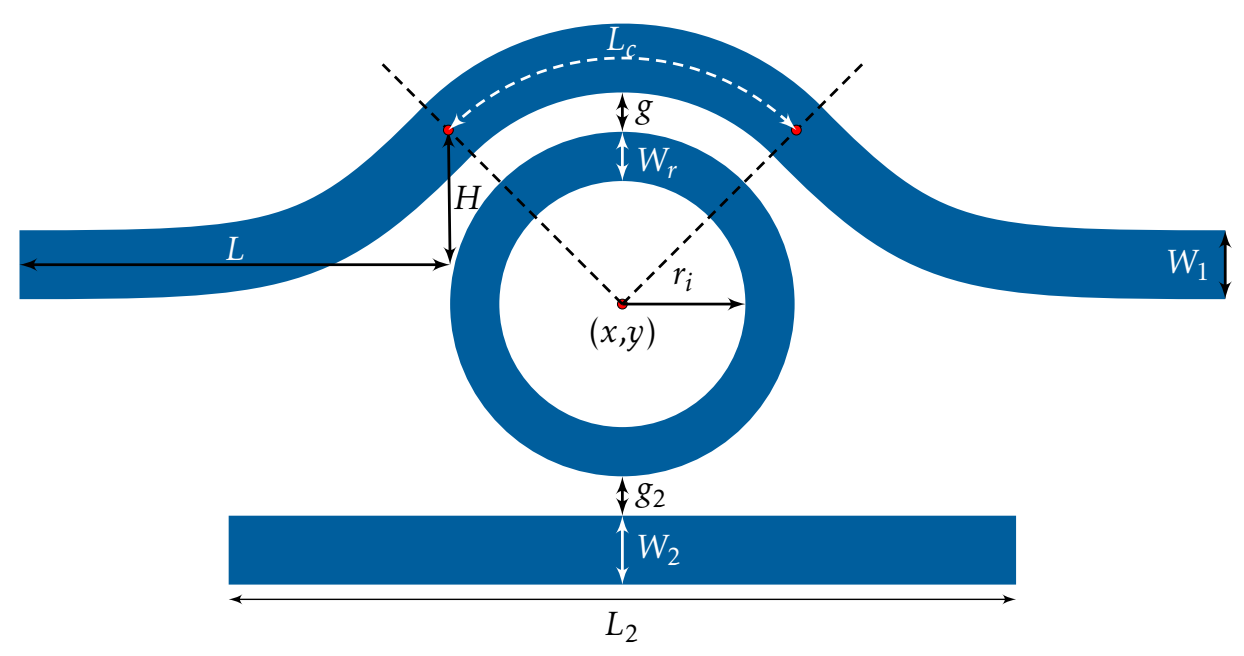

Figure 2.218: Example shape illustrating various parameters of the ringPulLCDS constructor. 
Ring pulley structure with an additional coupling pulley.

$\begin{array}{lllllllllllllllllllllllll}<x & y & r_{i} & W_{r} & N & g_{1} & L_{c 1} & N_{w g} & W_{1} & L_{1} & H & g_{2} & L_{c 2} & W_{2} & L_{2} & H_{2} & E C & \text { ringPulLCPul }\end{array}$

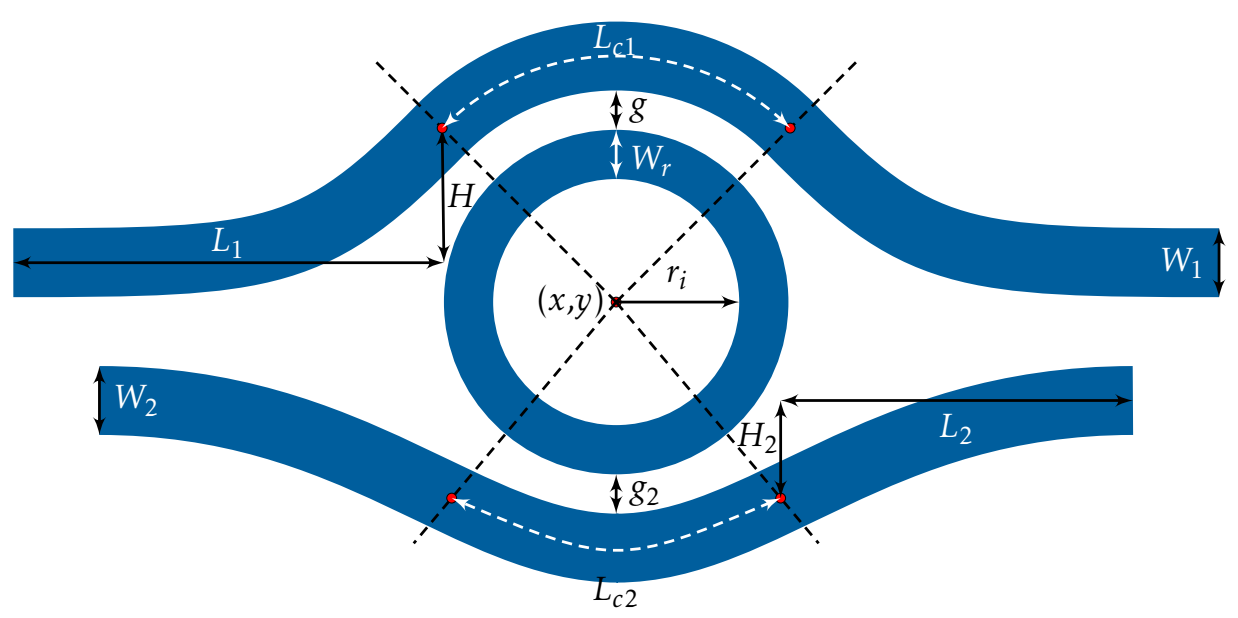

Figure 2.219: Example shape illustrating various parameters of the ringPulLCPul constructor. 


\subsubsection{Disc-Ring Pulley Arc}

Symmetric structures constructed using arcs are similar to ones described in section 2.9.9.11. Both objects (disc/ring) are described by the center coordinate $(x, y)$, number of segments $(N)$, gap between the object and waveguide $(g)$, waveguide opening angle $(\theta)$, number of sides $\left(N_{w g}\right)$, width $(W)$, and length of the connecting straight waveguide section $\left(L_{S}\right)$. Disc object is defined by a radius $\left(r_{d}\right)$ and the ring is characterized by an inner radius $\left(r_{i}\right)$ and ring width $\left(W_{r}\right) . E C=0$ and $E C=1$ for waveguides without and with endcaps, respectively.

$$
\begin{array}{llllllllllll}
<x & y & r_{d} & & N & g & \theta & N_{w g} & W & L_{S} & E C & \operatorname{discP} P u l l e y A> \\
<x & y & r_{i} & W_{r} & N & g & \theta & N_{w g} & W & L_{S} & E C & \text { ringPulleyA }>
\end{array}
$$

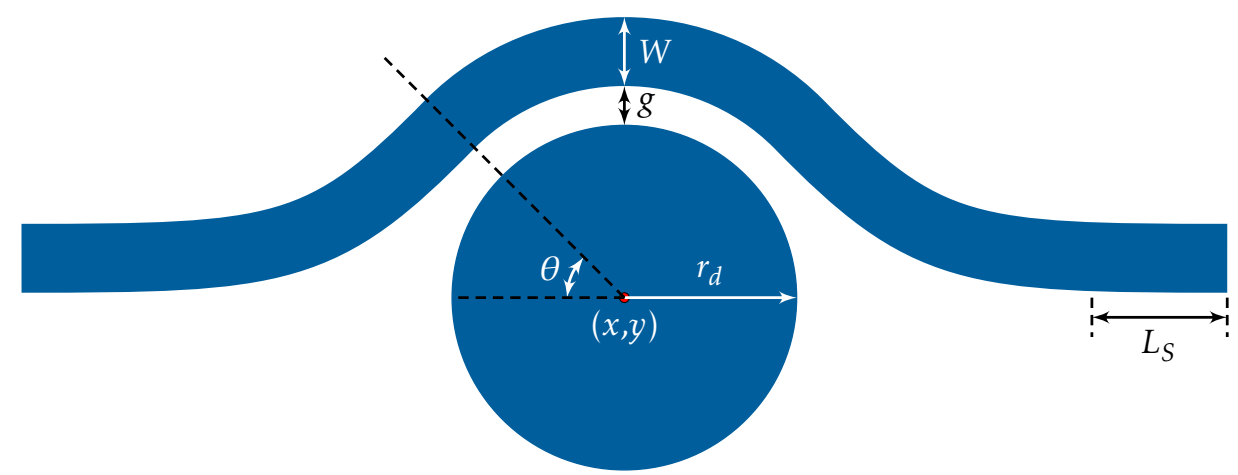

(a)

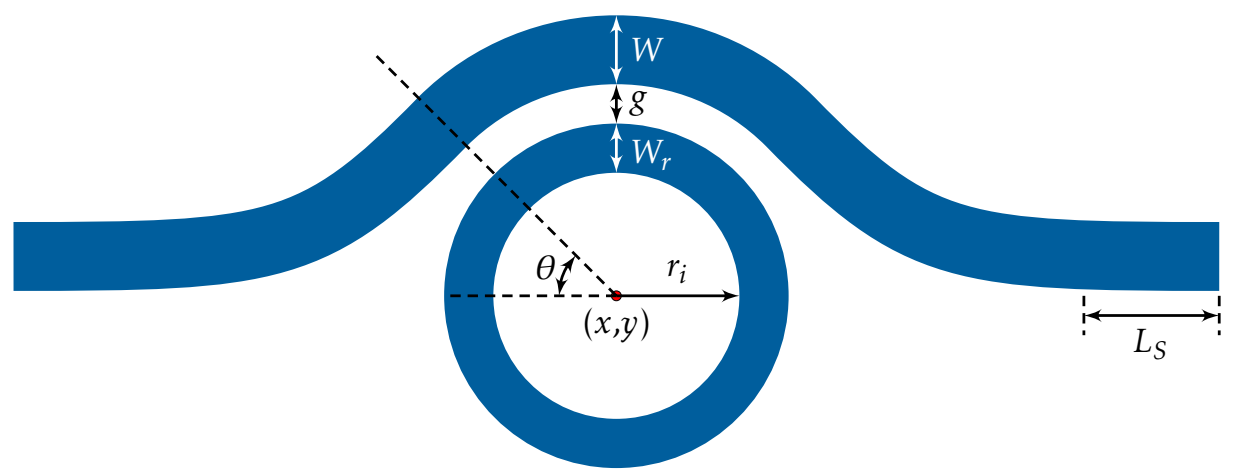

(b)

Figure 2.220: Example shapes illustrating various parameters of the (a) discPulleyA and (b) ringPulleyA constructors. 
Disc pulley arc structure with an additional coupling waveguide.

$\begin{array}{llllllllllllll}< & y & r_{d} & N & g_{1} & \theta & N_{w g} & W_{1} & L_{S} & g_{2} & W_{2} & L_{2} & E C & \text { discPulADS }>\end{array}$

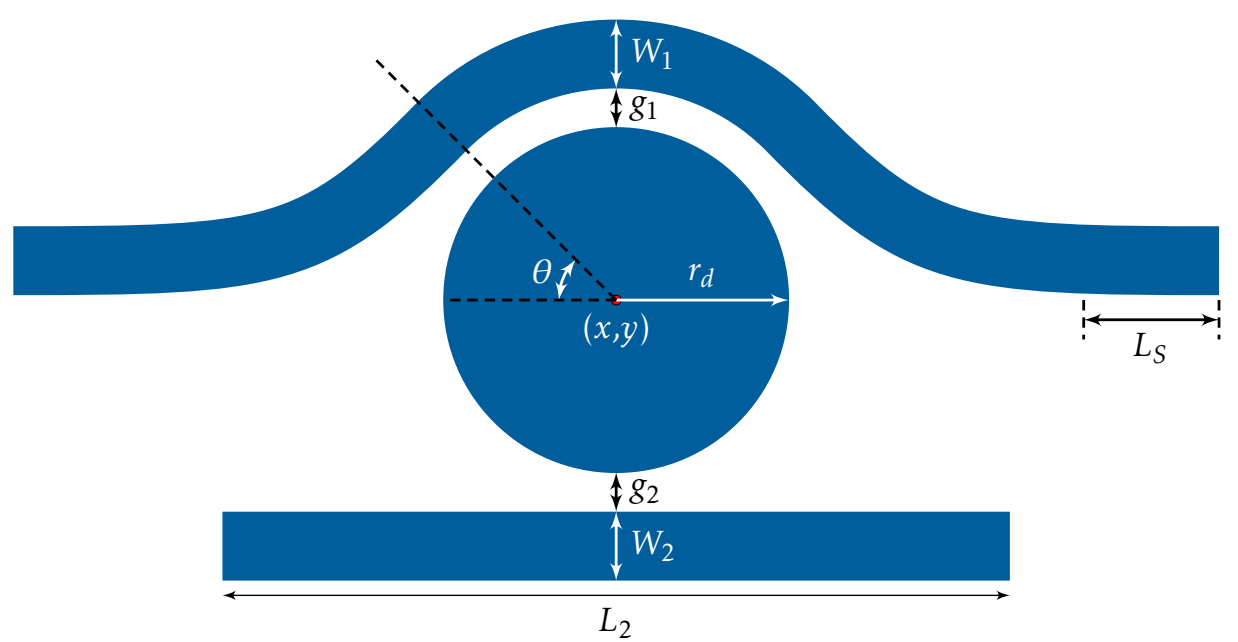

Figure 2.221: Example shape illustrating various parameters of the discPulADS constructor. 
Disc pulley arc structure with an additional coupling pulley.

$\begin{array}{llllllllllllllll}x & y & r_{d} & N & g_{1} & \theta_{1} & N_{w g} & W_{1} & L_{S 1} & g_{2} & \theta_{2} & W_{2} & L_{S 2} & E C & \text { discPulAPul }\end{array}$

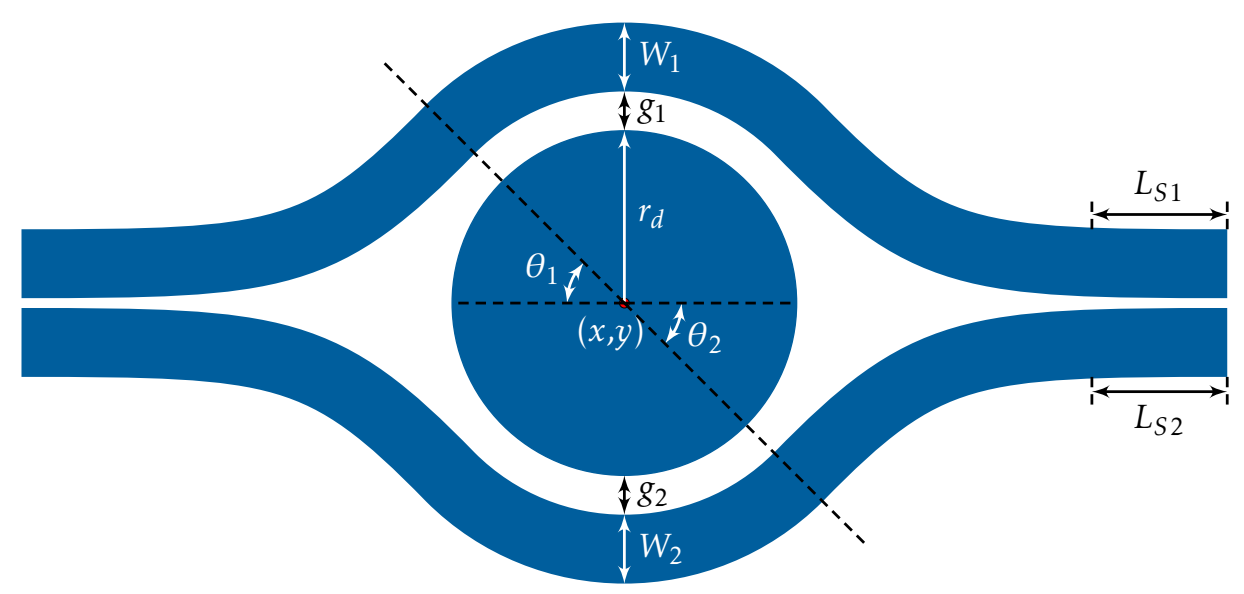

Figure 2.222: Example shape illustrating various parameters of the discPulAPul constructor. 
Ring pulley arc structure with an additional coupling waveguide.

$\begin{array}{lllllllllllllll}<x & y & r_{i} & W_{r} & N & g_{1} & \theta & N_{w g} & W_{1} & L_{S} & g_{2} & W_{2} & L_{2} & E C & \text { ringPulADS }>\end{array}$

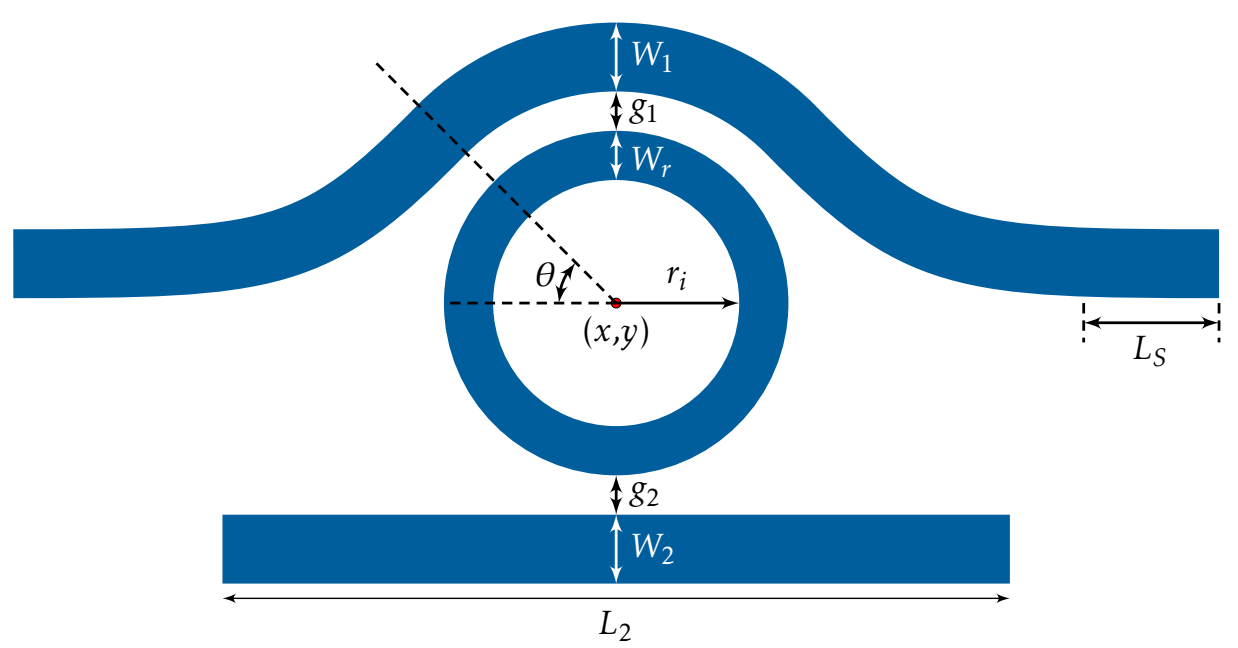

Figure 2.223: Example shape illustrating various parameters of the ringPulADS constructor. 
Ring pulley arc structure with an additional coupling pulley.

$\begin{array}{lllllllllllllllllll}<x & y & r_{i} & W_{r} & N & g_{1} & \theta_{1} & N_{w g} & W_{1} & L_{S 1} & g_{2} & \theta_{2} & W_{2} & L_{S 2} & E C & \text { ringPulAPul }\end{array}$

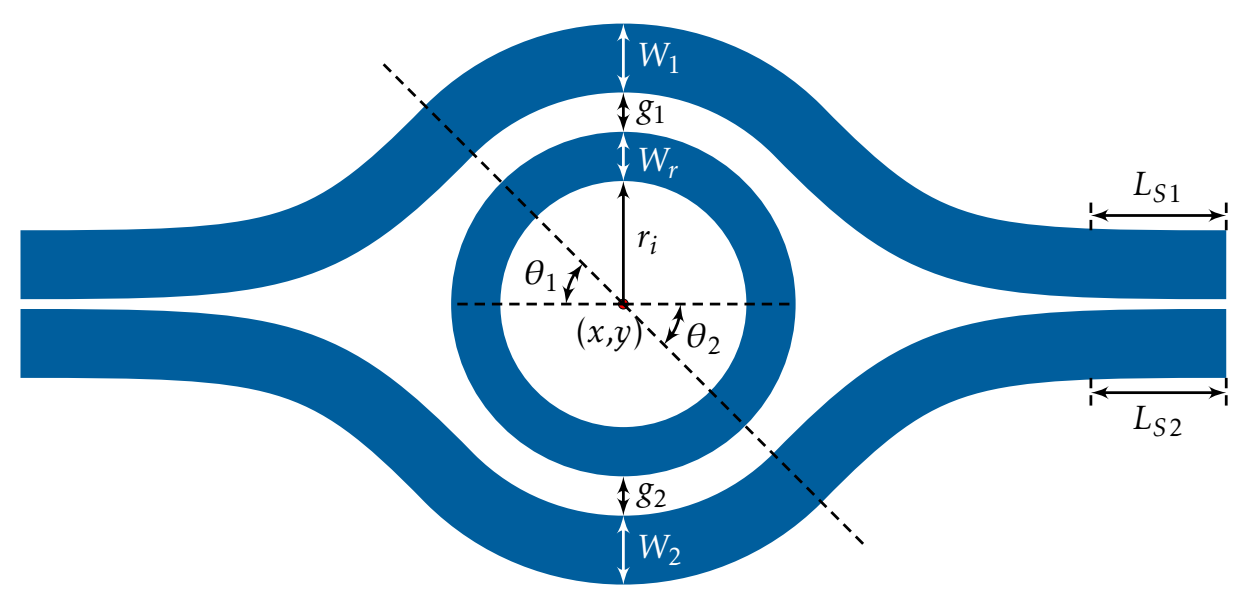

Figure 2.224: Example shape illustrating various parameters of the ringPulAPul constructor. 
The following arc based, disc-ring waveguide pulley structures are defined by the coupling length parameter $L_{c}$.

$$
\begin{array}{llllllllllll}
<x & y & r_{d} & & N & g & L_{c} & N_{w g} & W & L_{S} & E C & \operatorname{discPulleyLCA}> \\
<x & y & r_{i} & W_{r} & N & g & L_{c} & N_{w g} & W & L_{S} & E C & \text { ringPulleyLCA }>
\end{array}
$$

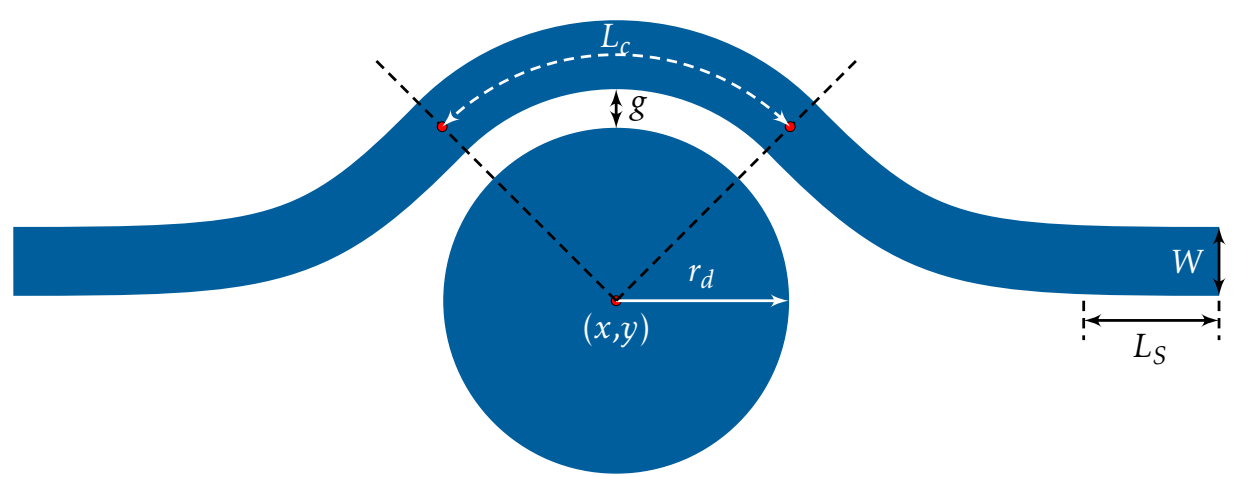

(a)

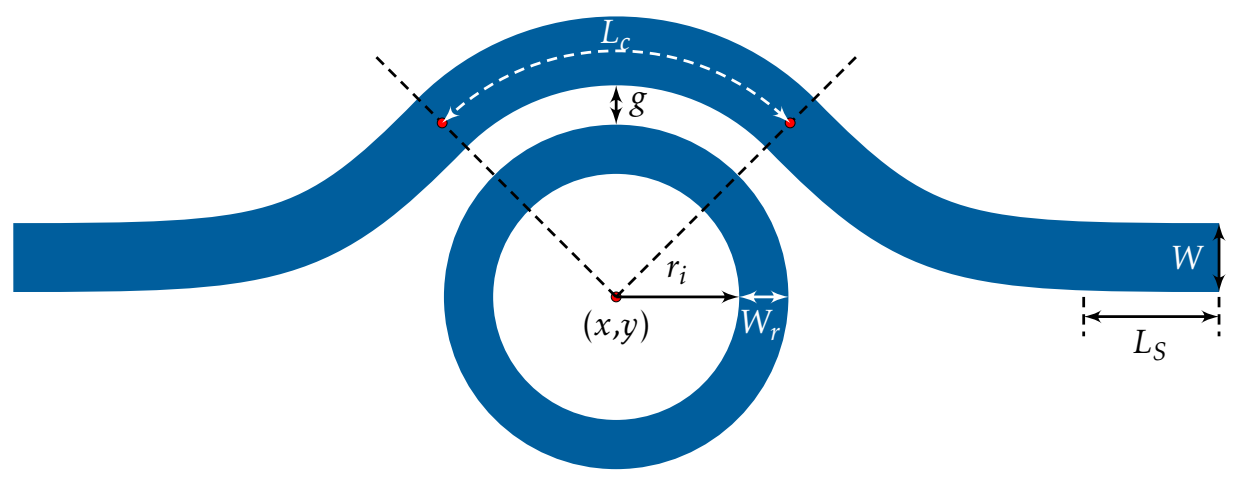

(b)

Figure 2.225: Example shapes illustrating various parameters of the (a) discPulleyLCA and (b) ringPulleyLCA constructors. 
Disc pulley arc structure with an additional coupling waveguide.

$\begin{array}{llllllllllllll}x & y & r_{d} & N & g_{1} & L_{c} & N_{w g} & W_{1} & L_{S} & g_{2} & W_{2} & L_{2} & E C & \text { discPulLCADS }>\end{array}$

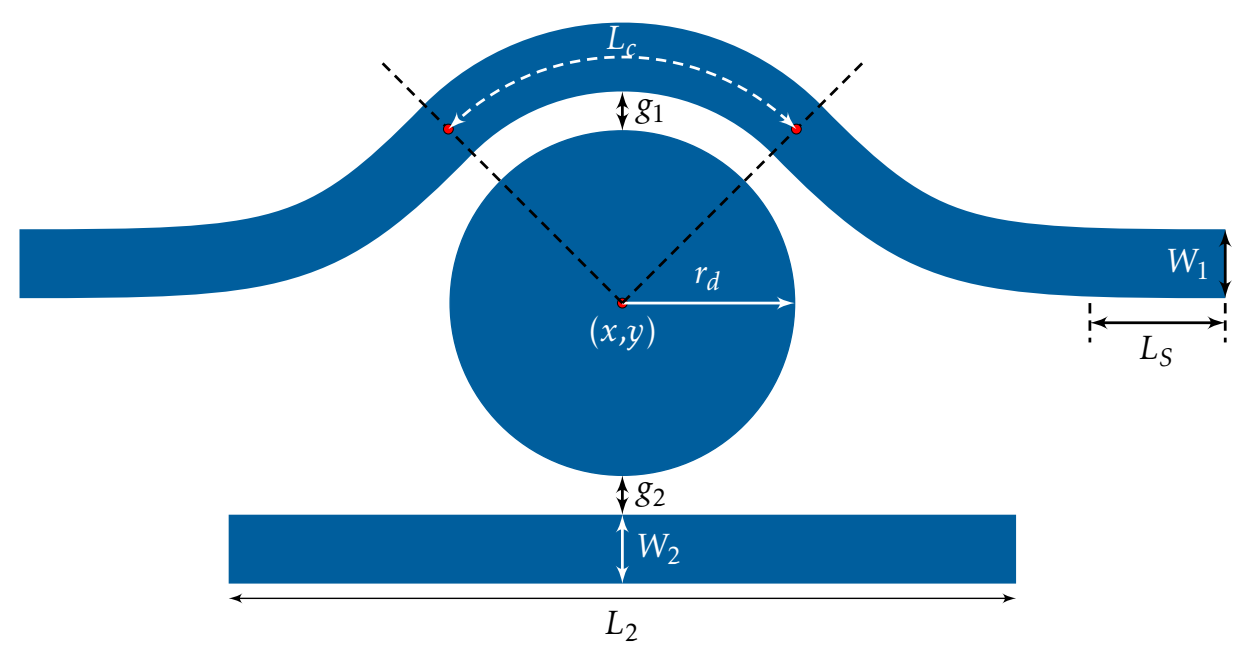

Figure 2.226: Example shape illustrating various parameters of the (a) discPulLCADS constructor. 
Disc pulley arc structure with an additional coupling pulley.

$\begin{array}{lllllllllllllll}<x & y & r_{d} & N & g_{1} & L_{c 1} & N_{w g} & W_{1} & L_{S 1} & g_{2} & L_{c 1} & W_{2} & L_{S 2} & E C & \text { discPullCAPul }\end{array}$

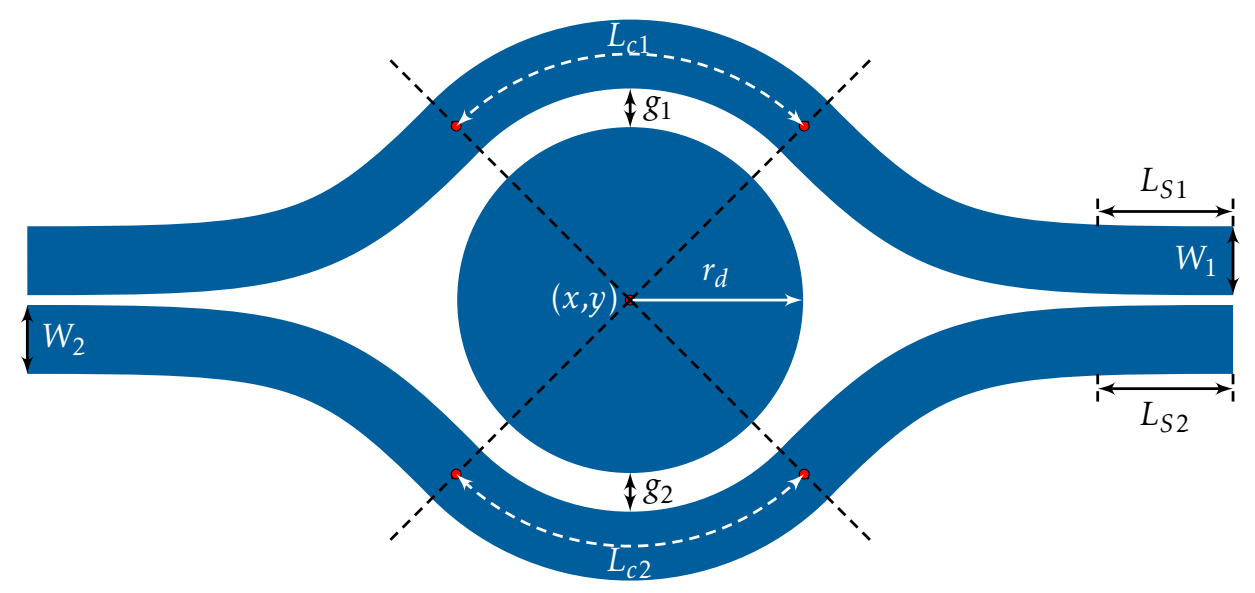

Figure 2.227: Example shape illustrating various parameters of the (a) discPulLCAPul constructor. 
Ring pulley arc structure with an additional coupling waveguide.

$\begin{array}{lllllllllllllll}<x & y & r_{i} & W_{r} & N & g_{1} & L_{c} & N_{w g} & W_{1} & L_{S} & g_{2} & W_{2} & L_{2} & E C & \text { ringPulLCADS }>\end{array}$

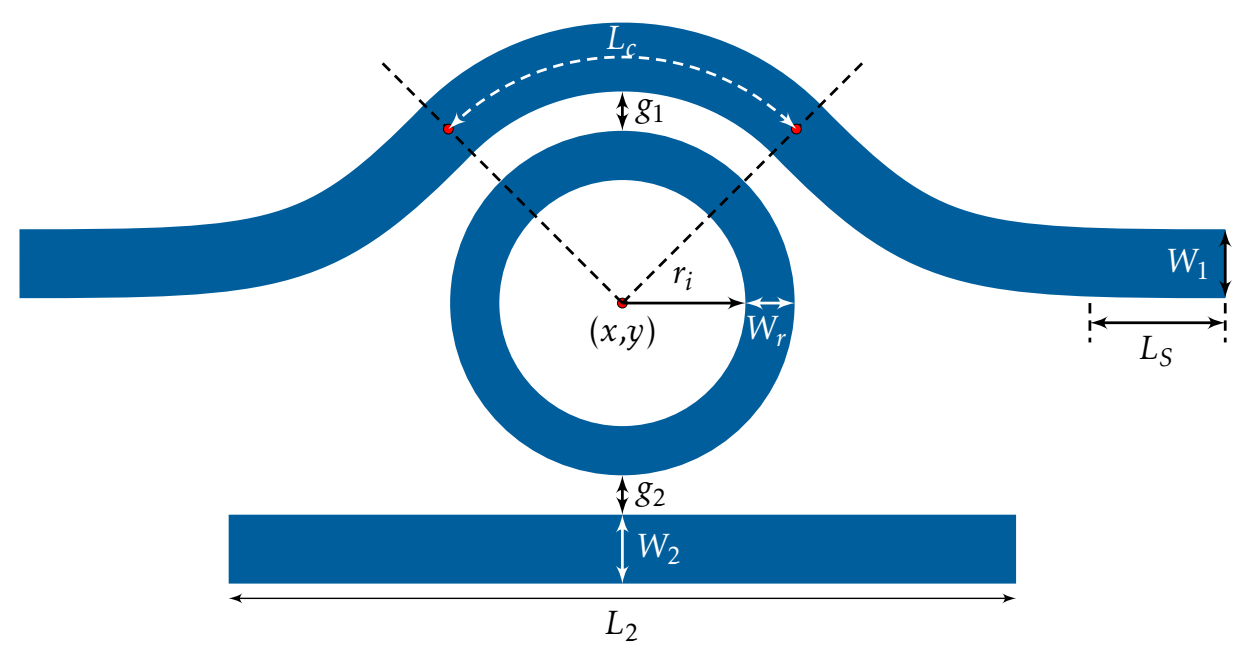

Figure 2.228: Example shape illustrating various parameters of the ringPulLCADS constructor. 
Ring pulley arc structure with an additional coupling pulley.

$\begin{array}{lllllllllllllllllll}< & y & r_{i} & W_{r} & N & g_{1} & L_{c 1} & N_{w g} & W_{1} & L_{S 1} & g_{2} & L_{c 1} & W_{2} & L_{S 2} & E C & \text { ringPulLCAPul }\end{array}$

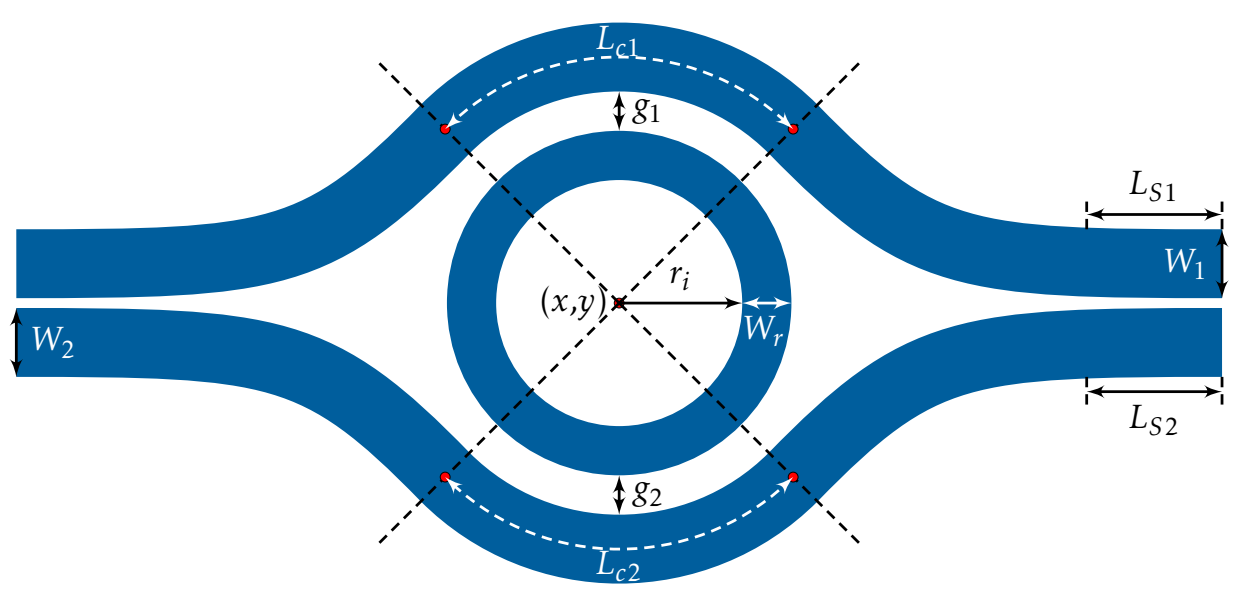

Figure 2.229: Example shape illustrating various parameters of the ringPulLCAPul constructor. 


\subsubsection{Disc-Ring Pulley Inverse Bezier}

The structure is similar to the disc-ring waveguide symmetric structure defined in the previous section (2.9.9.11). Here, a slot waveguide of width $W$ is formed by exposing a surrounding rectangular region of width $W_{e}$. Using a negative tone resist, the resulting structure would produce a disc or ring shape at a distance $g$ to a slotted waveguide.

$$
\begin{array}{lllllllllllllll}
<x & y & r_{d} & & N & g & \theta & N_{w g} & W & W_{e} & L & H & E C & \text { discPulleylnv }> \\
<x & y & r_{i} & W_{r} & N & g & \theta & N_{w g} & W & W_{e} & L & H & E C & \text { ringPulleylnv }>
\end{array}
$$

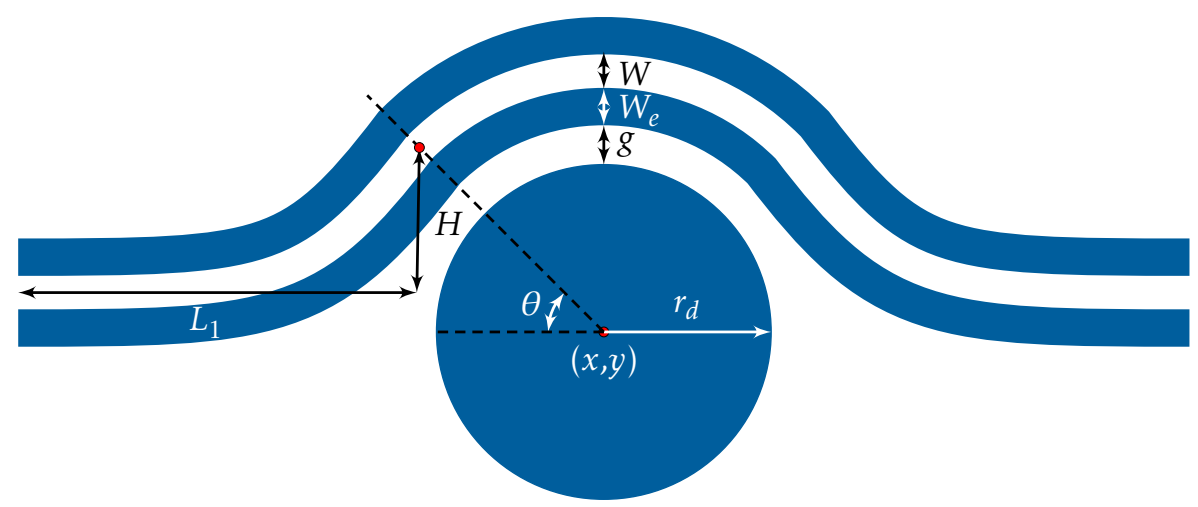

(a)

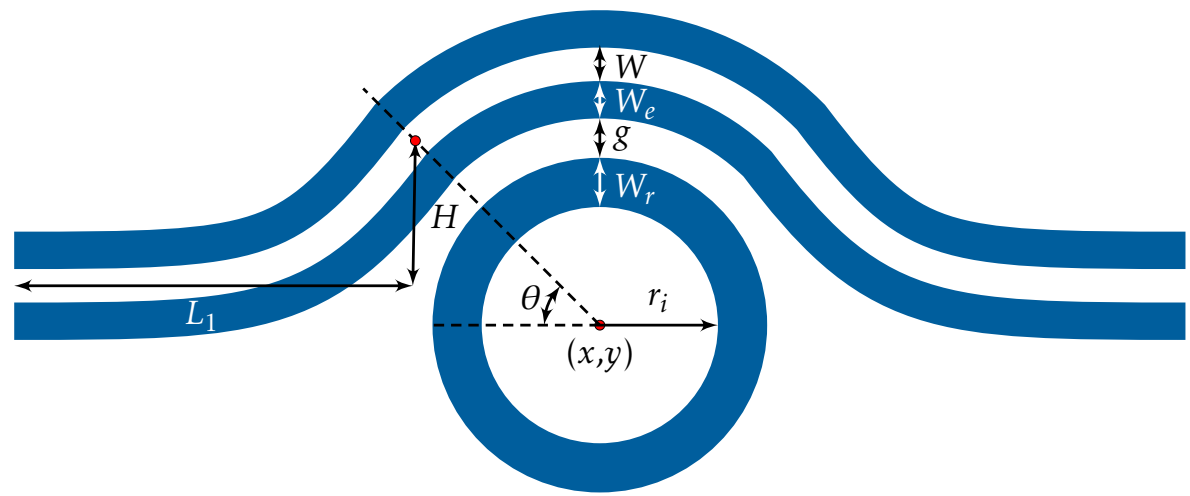

(b)

Figure 2.230: Example shapes illustrating various parameters of the (a) discPulleylnv and (b) ringPulleylnv constructors. 
Disc pulley inverse structure with an additional coupling waveguide.

$\begin{array}{llllllllllllllllllllll}x & y & r_{d} & N & g_{2} & \theta & N_{w g} & W_{2} & W_{e 2} & L_{2} & H & g_{2} & W_{2} & W_{e 2} & L_{2} & E C & \text { discPullnvDS }\end{array}$

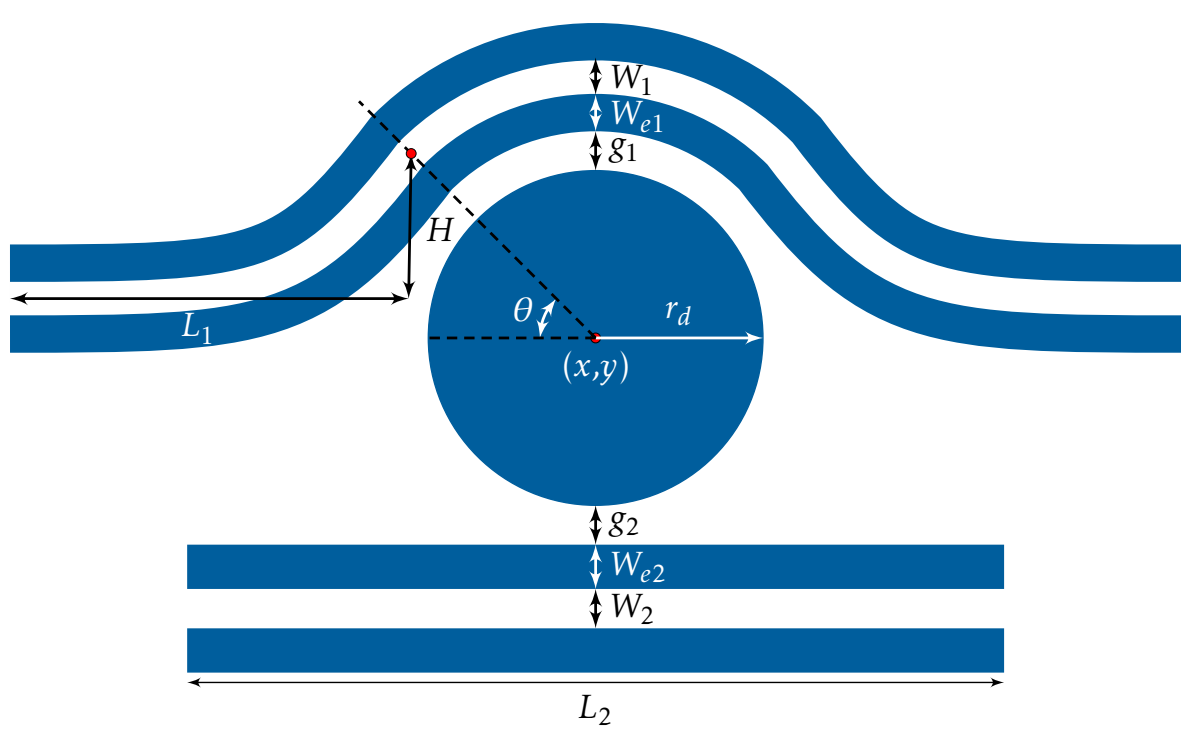

Figure 2.231: Example shape illustrating various parameters of the discPullnvDS constructor. 
Disc pulley inverse structure with an additional coupling pulley.

$\begin{array}{lllllllllllllllllllllllllllllllll}x & y & r_{d} & N & g_{2} & \theta_{1} & N_{w g} & W_{2} & W_{e 2} & L_{2} & H_{2} & g_{2} & \theta_{2} & W_{2} & W_{e 2} & L_{2} & H_{2} & E C & \text { discPullnvPul }\end{array}$

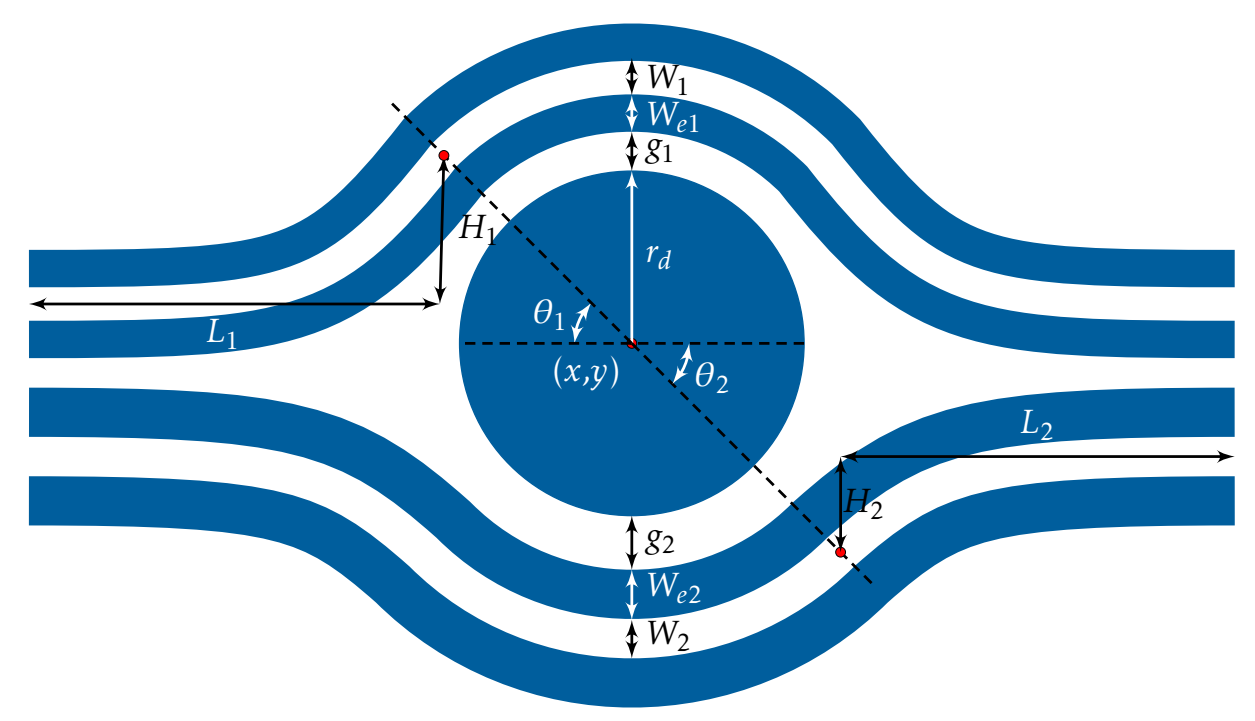

Figure 2.232: Example shape illustrating various parameters of the discPullnvPul constructor. 
Ring pulley inverse structure with an additional coupling waveguide.

$\begin{array}{llllllllllllllllllll}x & y & r_{i} & W_{r} & N & g_{1} & \theta & N_{w g} & W_{1} & W_{e 1} & L_{1} & H & g_{2} & W_{2} & W_{e 2} & L_{2} & E C & \text { ringPullnvDS }\end{array}$

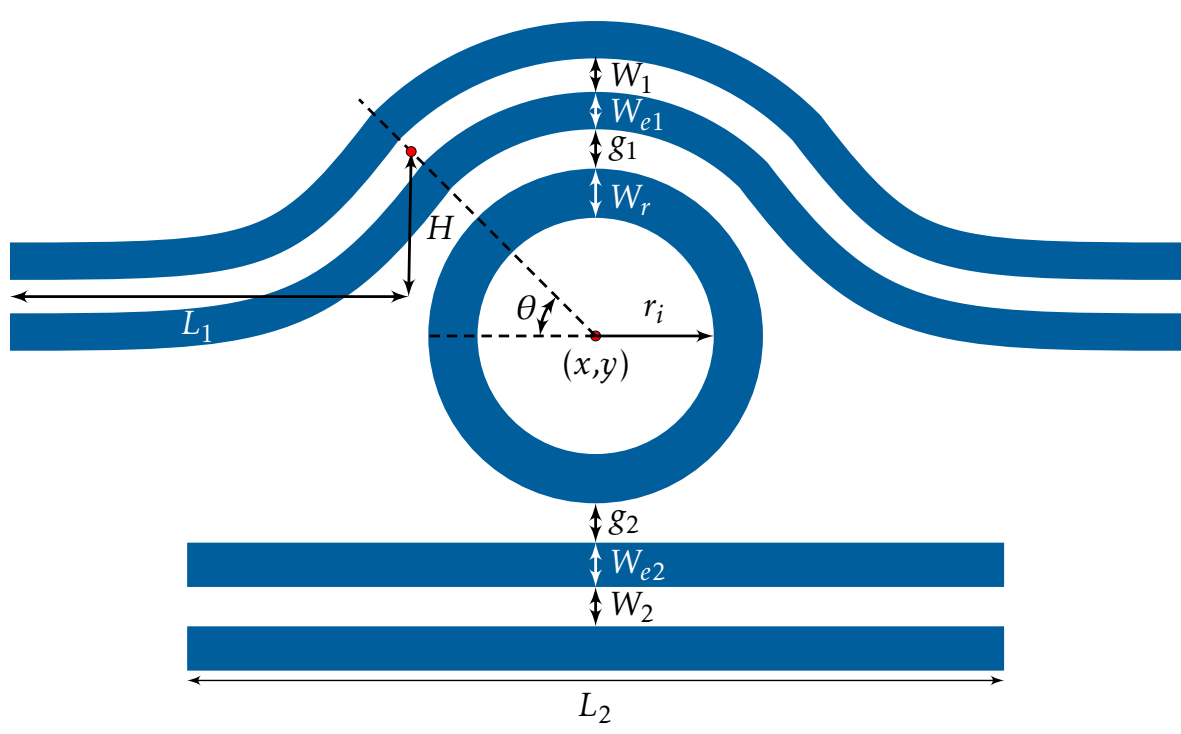

Figure 2.233: Example shape illustrating various parameters of the ringPullnvDS constructor. 
Ring pulley inverse structure with an additional coupling pulley.

$\begin{array}{llllllllllllllllllllllllllllllll}< & y & r_{i} & W_{r} & N & g_{1} & \theta_{1} & N_{w g} & W_{1} & W_{e 1} & L_{1} & H & g_{2} & \theta_{2} & W_{2} & W_{e 2} & L_{2} & H_{2} & E C & \text { ringPullnvPul }\end{array}$

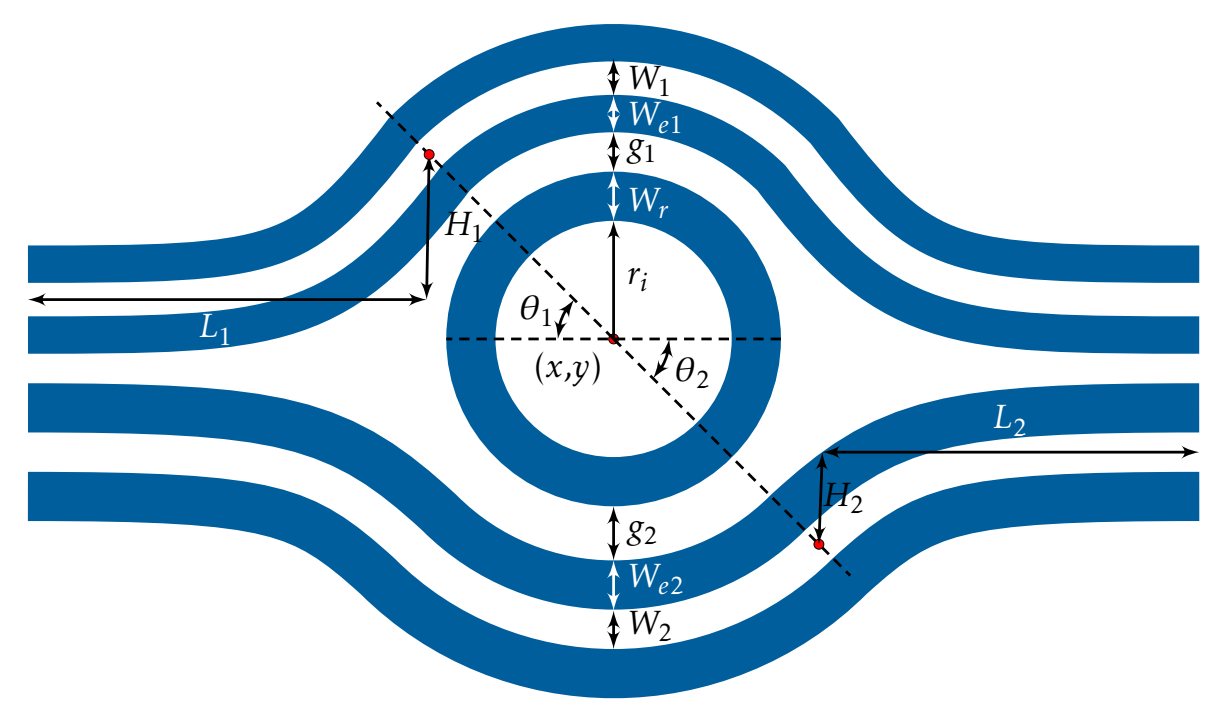

Figure 2.234: Example shape illustrating various parameters of the ringPullnvPul constructor. 
The following disc-ring waveguide pulley inverse structures are defined by the coupling length parameter $L_{c}$.

$$
\begin{array}{llllllllllllllll}
<x & y & r_{d} & & N & g & L_{c} & N_{w g} & W & W_{e} & L & H & E C & \text { discPulleylnvLC }> \\
<x & y & r_{i} & W_{r} & N & g & L_{c} & N_{w g} & W & W_{e} & L & H & E C & \text { ringPulleylnvLC }>
\end{array}
$$

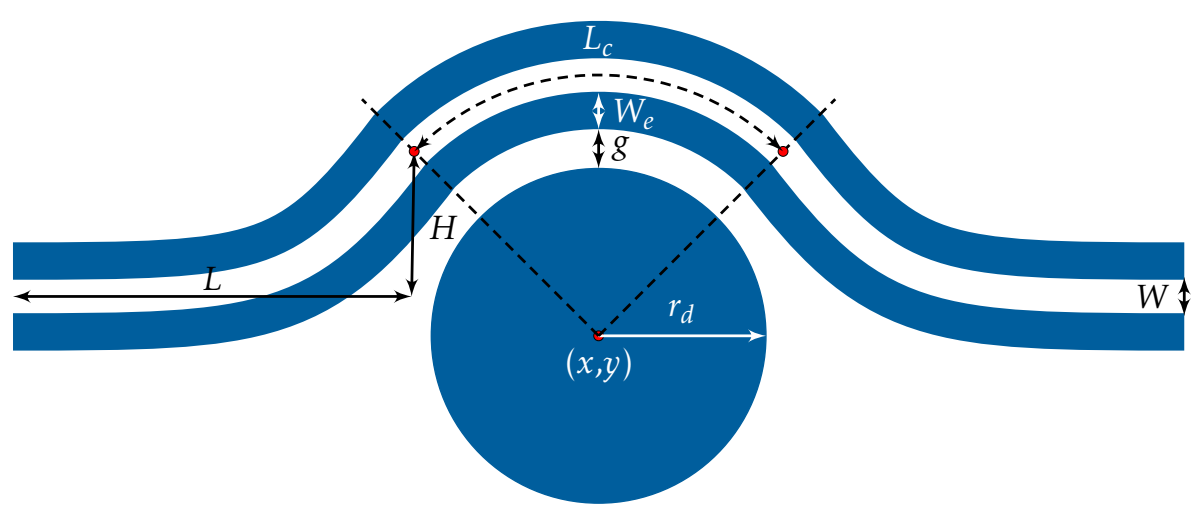

(a)

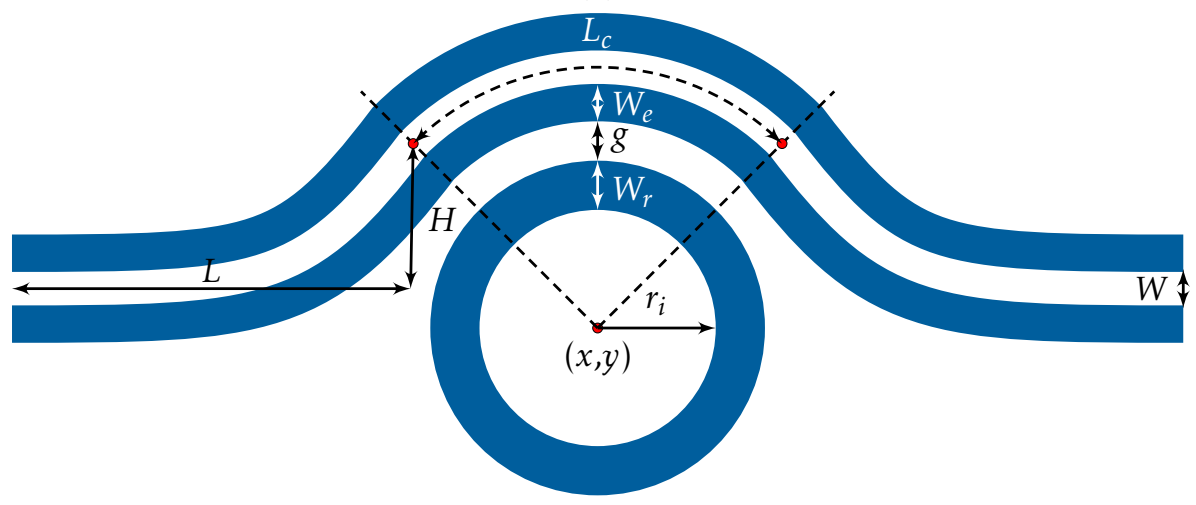

(b)

Figure 2.235: Example shapes illustrating various parameters of the (a) discPulleyln$v L C$ and (b) ringPulleylnvLC constructors. 
Disc pulley inverse structure with an additional coupling waveguide.

$\begin{array}{lllllllllllllllllllllll}x & y & r_{d} & N & g_{1} & L_{c} & N_{w g} & W_{1} & W_{e 1} & L_{1} & H & g_{2} & W_{2} & W_{e 2} & L_{2} & E C & \text { discPullnvLCDS }>\end{array}$

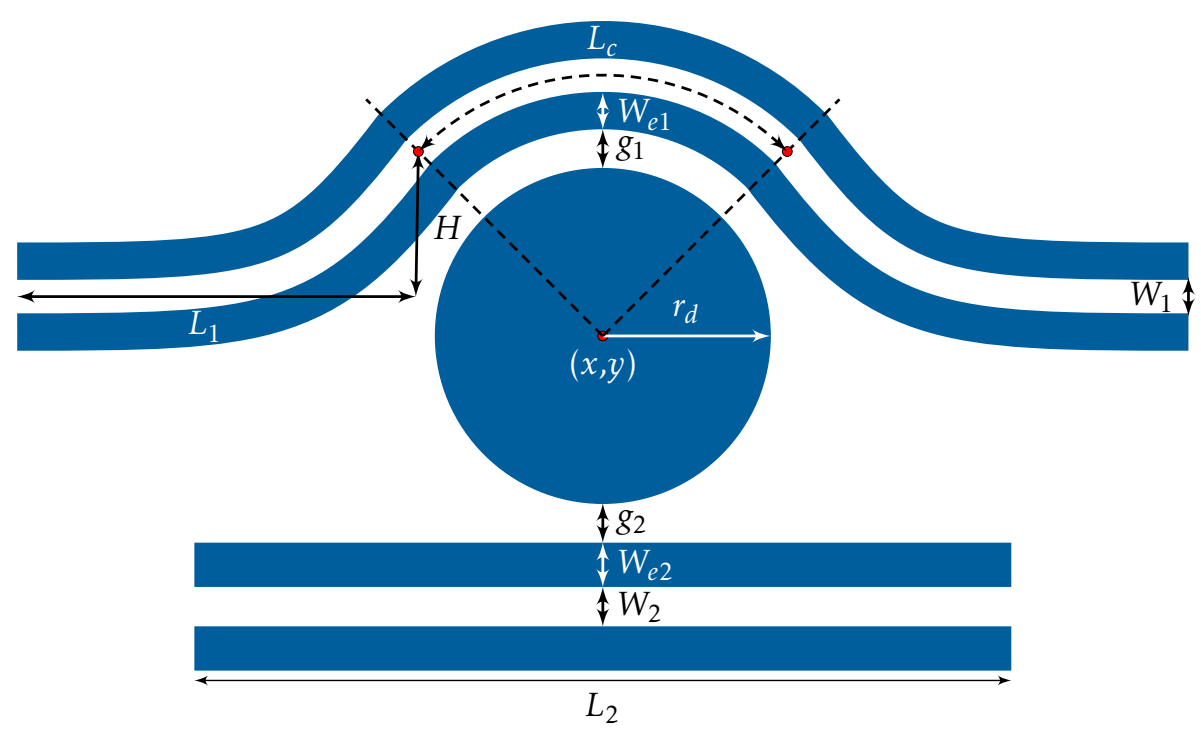

Figure 2.236: Example shape illustrating various parameters of the discPullnvLCDS constructor. 
Disc pulley inverse structure with an additional coupling pulley.

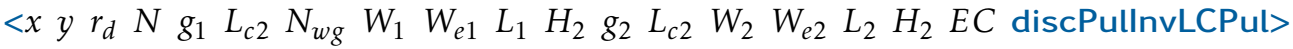

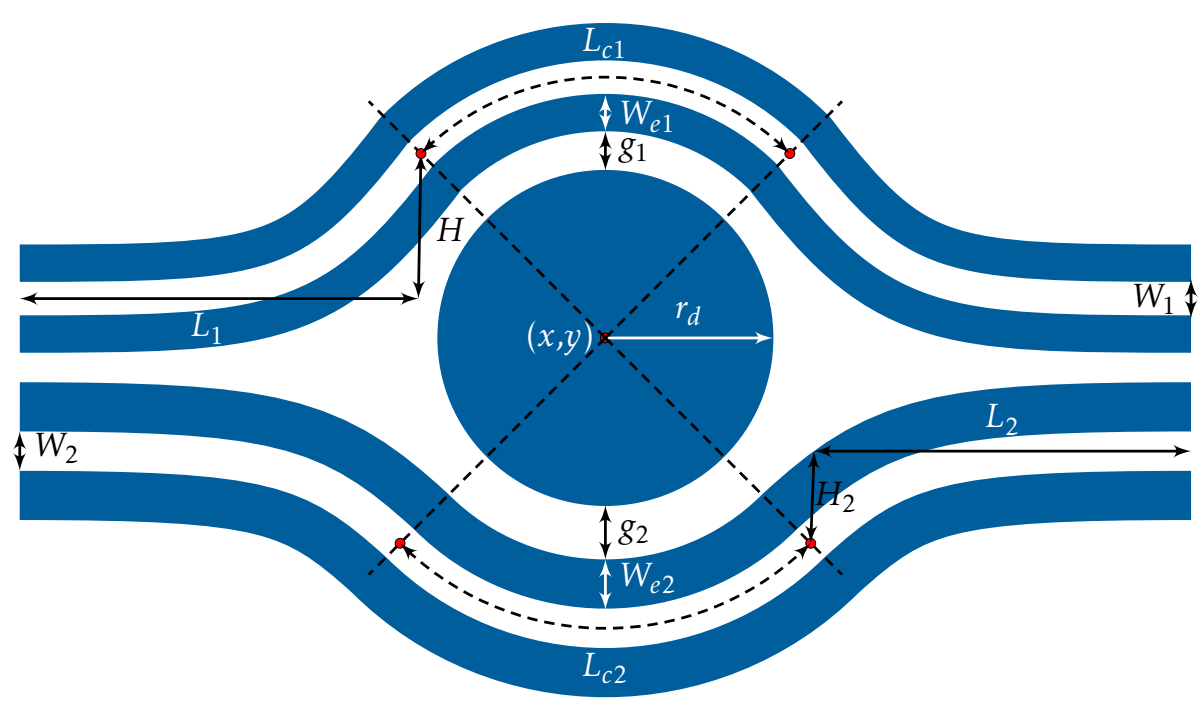

Figure 2.237: Example shape illustrating various parameters of the discPullnvLCPul constructor. 
Ring pulley inverse structure with an additional coupling waveguide.

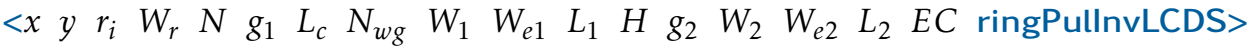

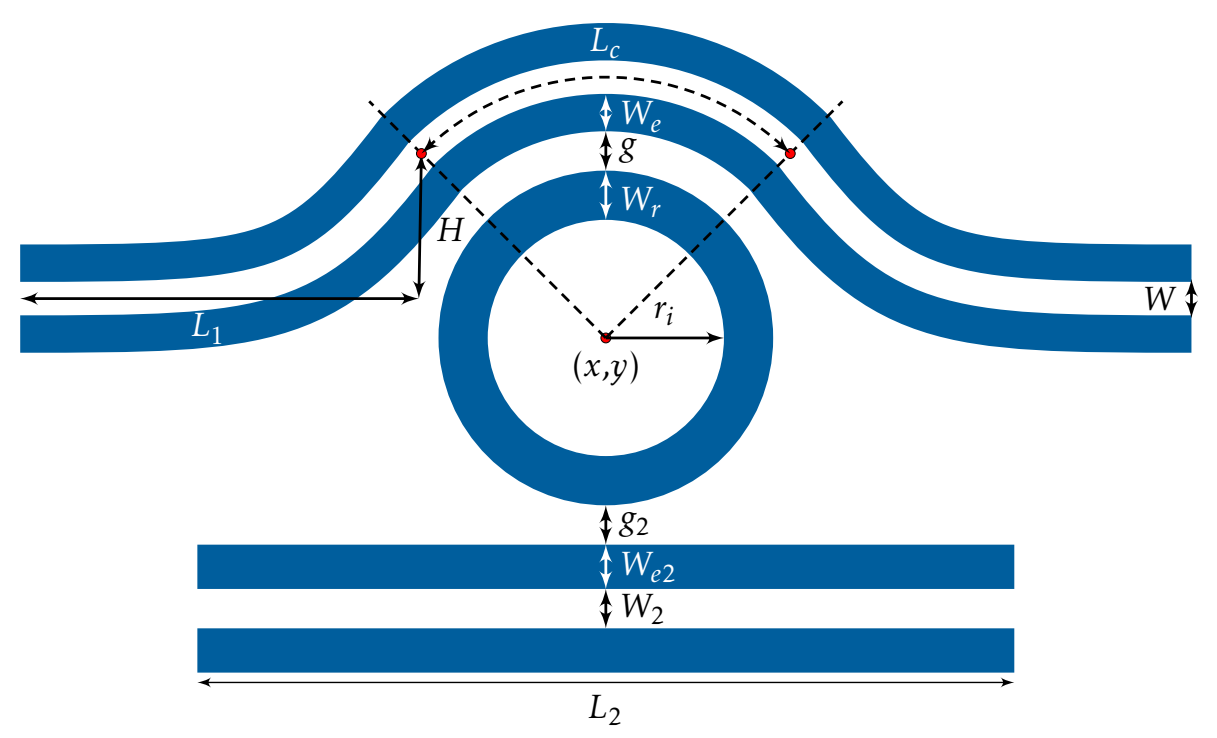

Figure 2.238: Example shape illustrating various parameters of the ringPullnvLCDS constructor. 
Ring pulley inverse structure with an additional coupling pulley.

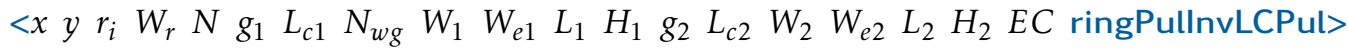

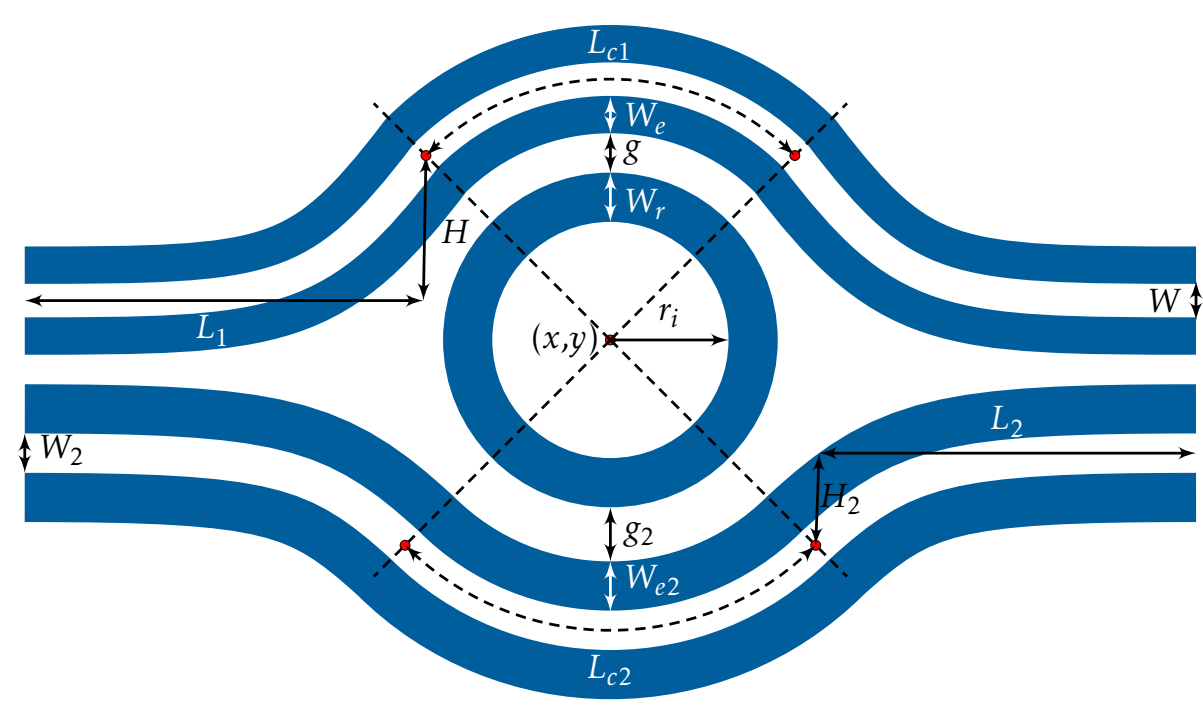

Figure 2.239: Example shape illustrating various parameters of the ringPullnvLCPul constructor. 


\subsubsection{Disc-Ring Pulley Inverse Arc}

Pulley inverse structures constructed using arcs are similar to ones described in section 2.9.9.12. Here, a slot waveguide of width $W$ is formed by exposing a surrounding rectangular region of width $W_{e}$. Using a negative tone resist, the resulting structure would produce a disc or ring shape at a distance $g$ to a slotted waveguide.

$$
\begin{array}{llllllllllllll}
<x & y & r_{d} & & N & g & \theta & N_{w g} & W & W_{e} & L_{S} & E C & \operatorname{discPulleylnv} A> \\
<x & y & r_{i} & W_{r} & N & g & \theta & N_{w g} & W & W_{e} & L_{S} & E C & \text { ringPulleylnvA }>
\end{array}
$$

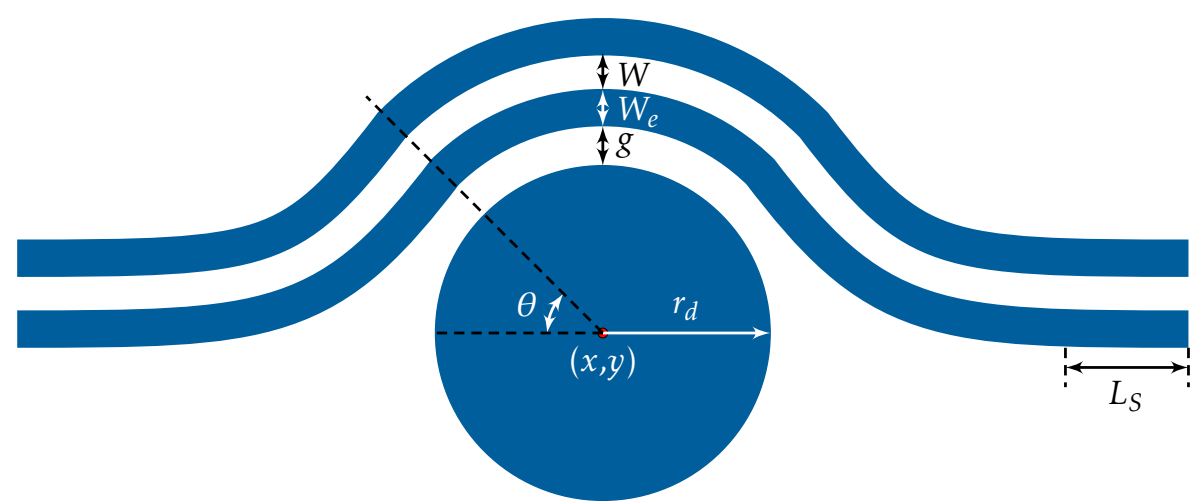

(a)

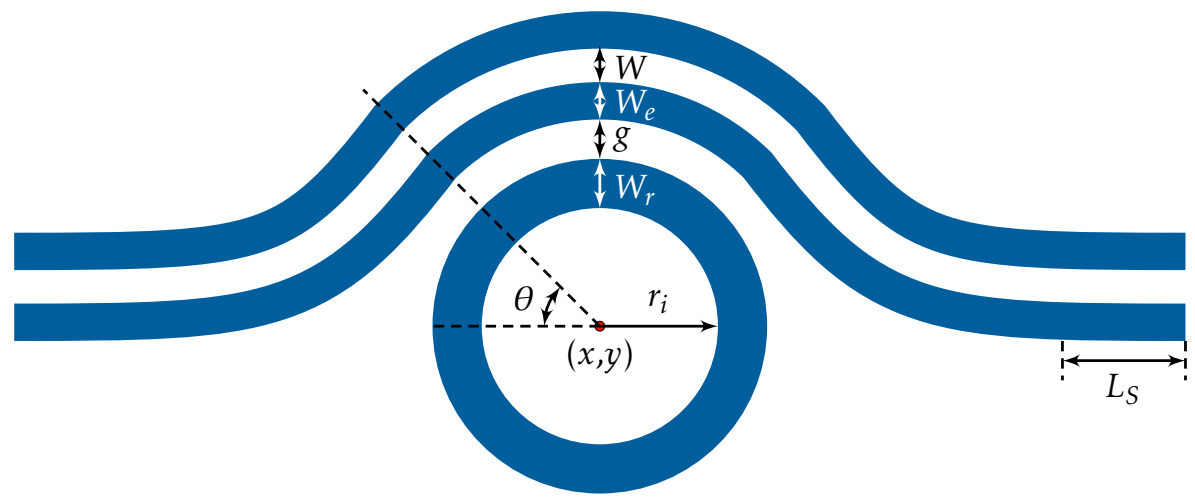

(b)

Figure 2.240: Example shapes illustrating various parameters of the (a) discPulleylnvA and (b) ringPulleylnvA constructors. 
Disc pulley inverse arc structure with an additional coupling waveguide.

$\begin{array}{llllllllllllllllll}x & y & r_{d} & N & g_{1} & \theta & N_{w g} & W_{1} & W_{e 1} & L_{S} & g_{2} & W_{2} & W_{e 2} & L_{2} & E C & \text { discPullnvADS }>\end{array}$

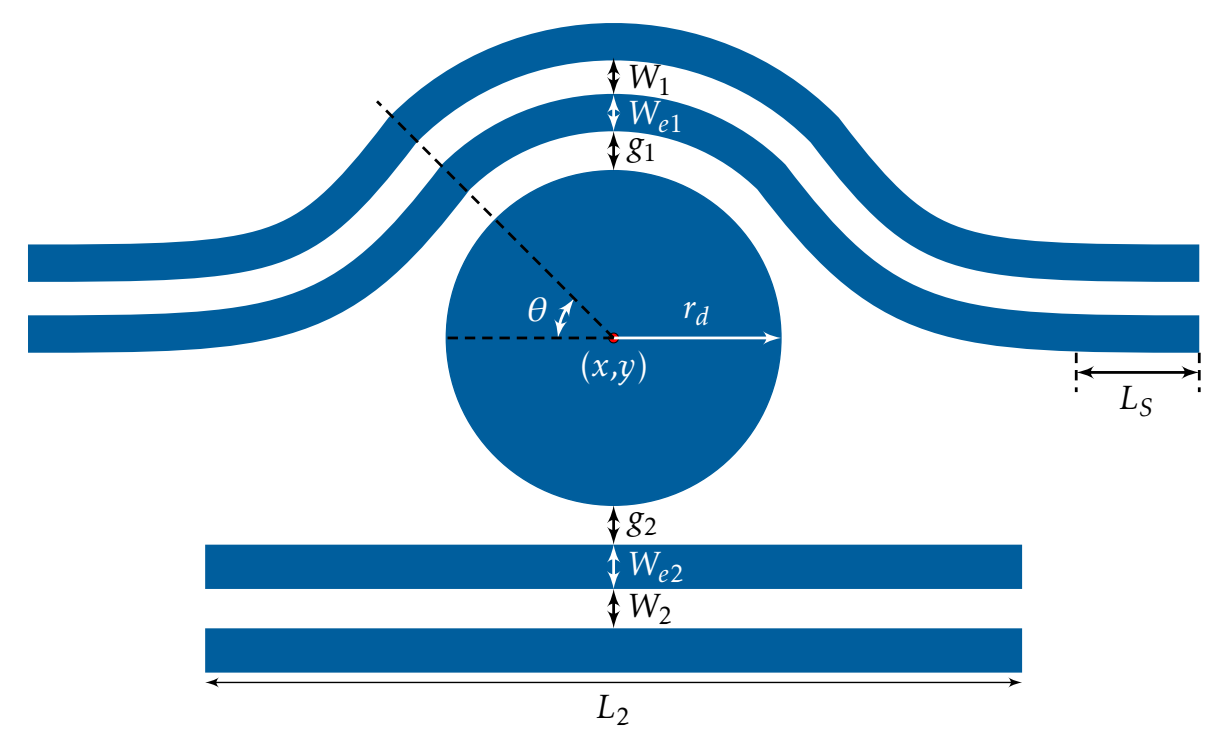

Figure 2.241: Example shape illustrating various parameters of the discPullnvADS constructor. 
Disc pulley inverse arc structure with an additional coupling pulley.

$\begin{array}{llllllllllllllllllll}<x & y & r_{d} & N & g_{1} & \theta_{1} & N_{w g} & W_{1} & W_{e 1} & L_{S 1} & g_{2} & \theta_{2} & W_{2} & W_{e 2} & L_{S 2} & E C & \text { discPullnvAPul }\end{array}$

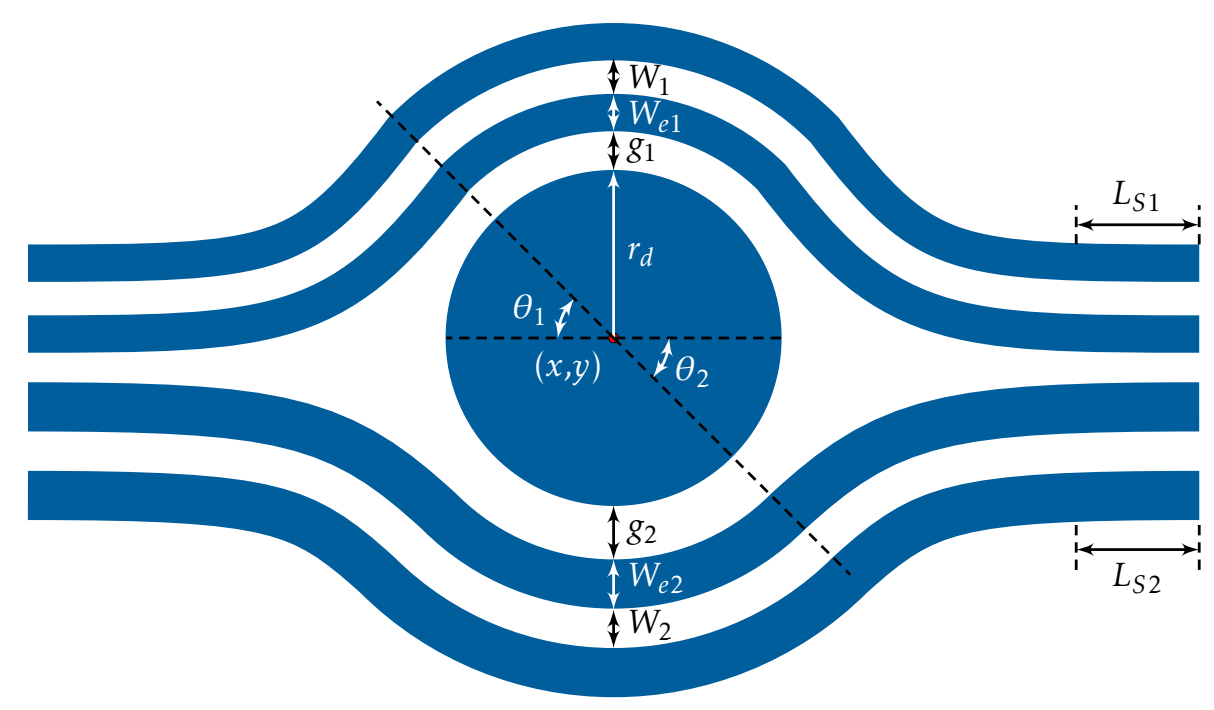

Figure 2.242: Example shape illustrating various parameters of the discPullnvAPul constructor. 
Ring pulley inverse arc structure with an additional coupling waveguide.

$\begin{array}{llllllllllllllllllllll}<x & y & r_{i} & W_{r} & N & g_{1} & \theta & N_{w g} & W_{1} & W_{e 1} & L_{S} & g_{2} & W_{2} & W_{e 2} & L_{2} & E C & \text { ringPullnvADS }>\end{array}$

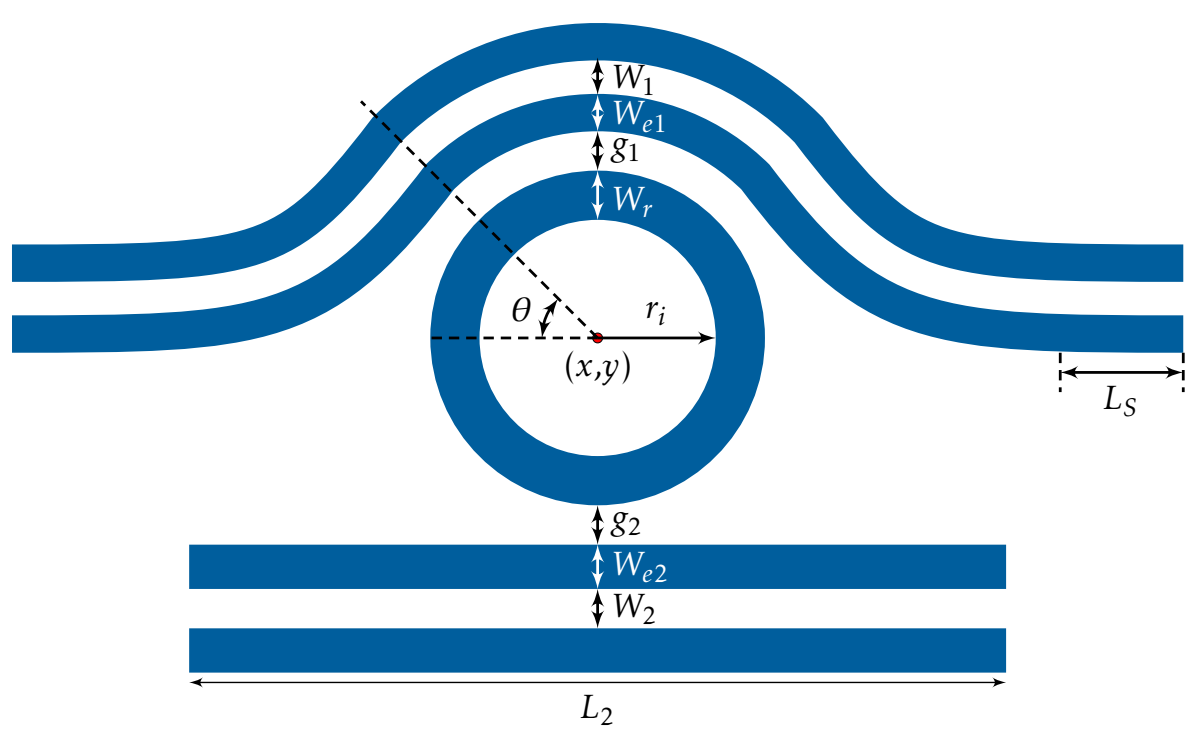

Figure 2.243: Example shape illustrating various parameters of the ringPullnvADS constructor. 
Ring pulley inverse arc structure with an additional coupling pulley.

$\begin{array}{llllllllllllllllllllll}x & y & r_{i} & W_{r} & N & g_{1} & \theta_{1} & N_{w g} & W_{1} & W_{e 1} & L_{S} & g_{2} & \theta_{2} & W_{2} & W_{e 2} & L_{2} & E C & \text { ringPullnvAPul }\end{array}$

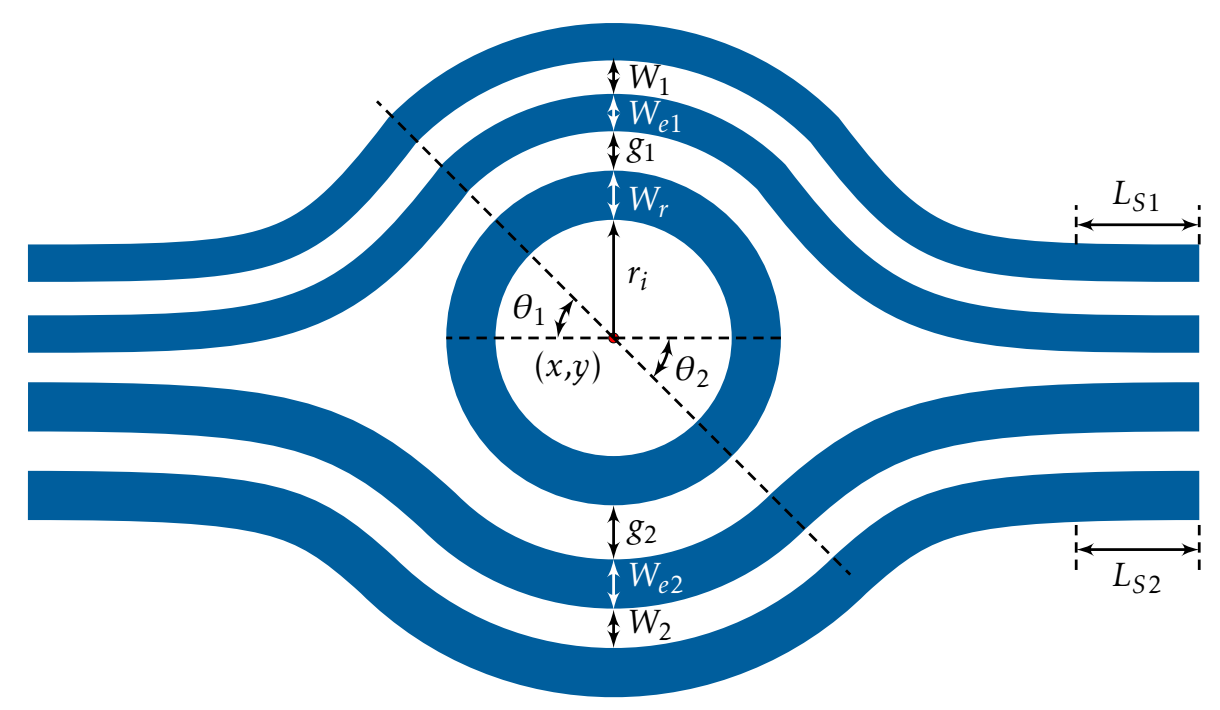

Figure 2.244: Example shape illustrating various parameters of the ringPullnvAPul constructor. 
The following arc based, disc-ring waveguide pulley inverse structures are defined by the coupling length parameter $L_{c}$.

$$
\begin{array}{llllllllllllll}
<x & y & r_{d} & & N & g & L_{c} & N_{w g} & W & W_{e} & L_{S} & E C & \text { discPulleylnvLCA }> \\
<x & y & r_{i} & W_{r} & N & g & L_{c} & N_{w g} & W & W_{e} & L_{S} & E C & \text { ringPulleylnvLCA }>
\end{array}
$$

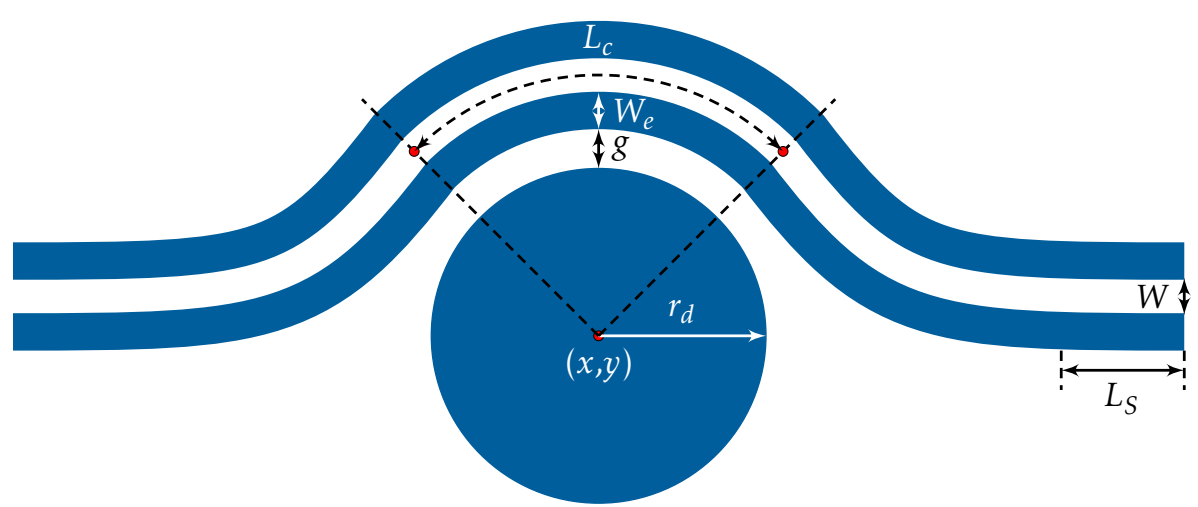

(a)

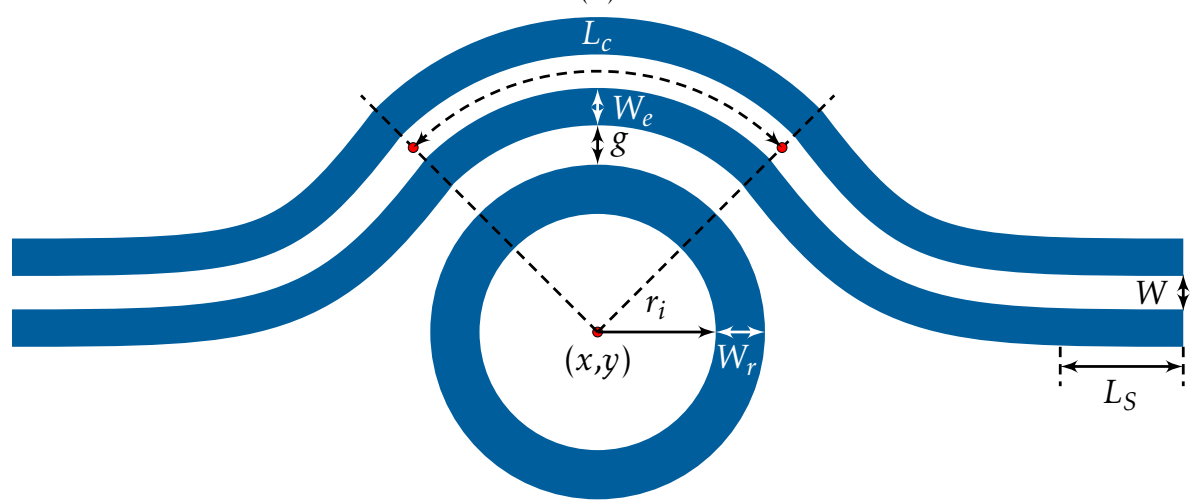

(b)

Figure 2.245: Example shapes illustrating various parameters of the (a) discPulleyln$v L C A$ and $(b)$ ringPulleylnvLCA constructors. 
Disc pulley inverse arc structure with an additional coupling waveguide.

$\begin{array}{llllllllllllllllllll}< & y & r_{d} & N & g_{1} & L_{c} & N_{w g} & W_{1} & W_{e 1} & L_{S} & g_{2} & W_{2} & W_{e 2} & L_{2} & E C & \text { discPullnvLCADS }>\end{array}$

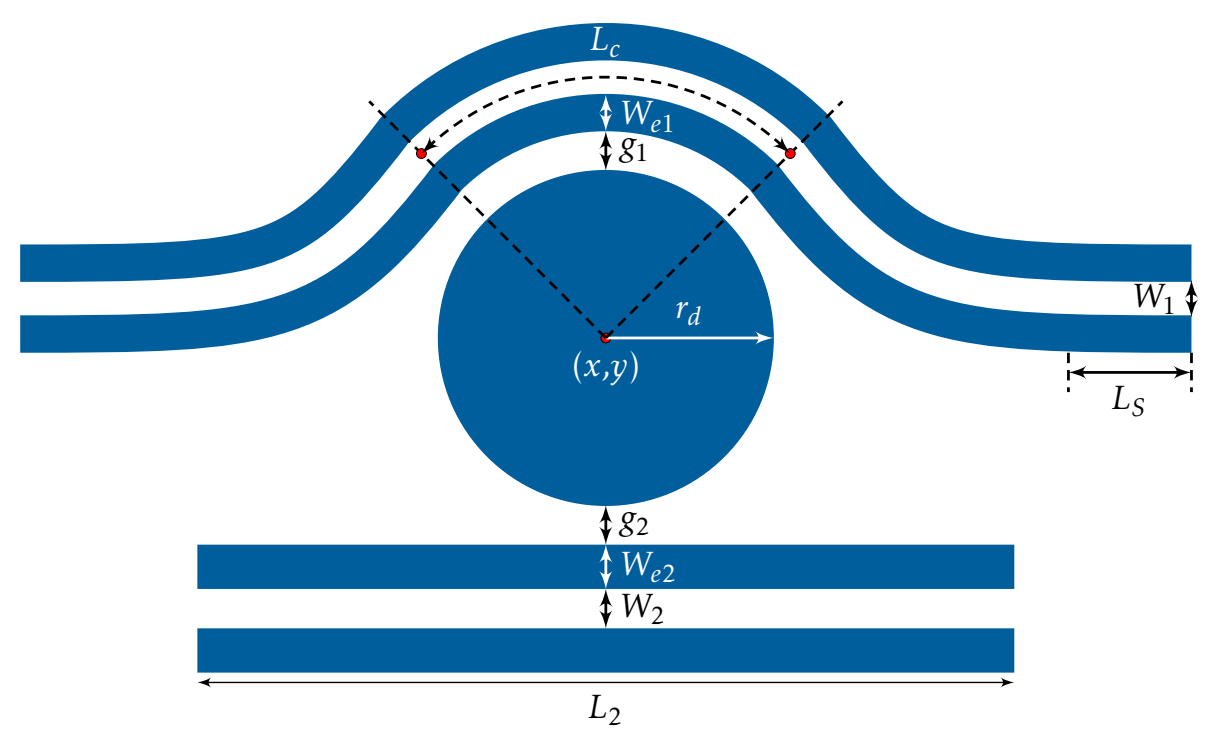

Figure 2.246: Example shape illustrating various parameters of the discPullnvLCADS constructor. 
Disc pulley inverse arc structure with an additional coupling pulley.

$\begin{array}{llllllllllllllllllllll}x & y & r_{d} & N & g_{1} & L_{c 1} & N_{w g} & W_{1} & W_{e 1} & L_{S 1} & g_{2} & L_{c 2} & W_{2} & W_{e 2} & L_{S 2} & E C & \text { discPullnvLCAPul }\end{array}$

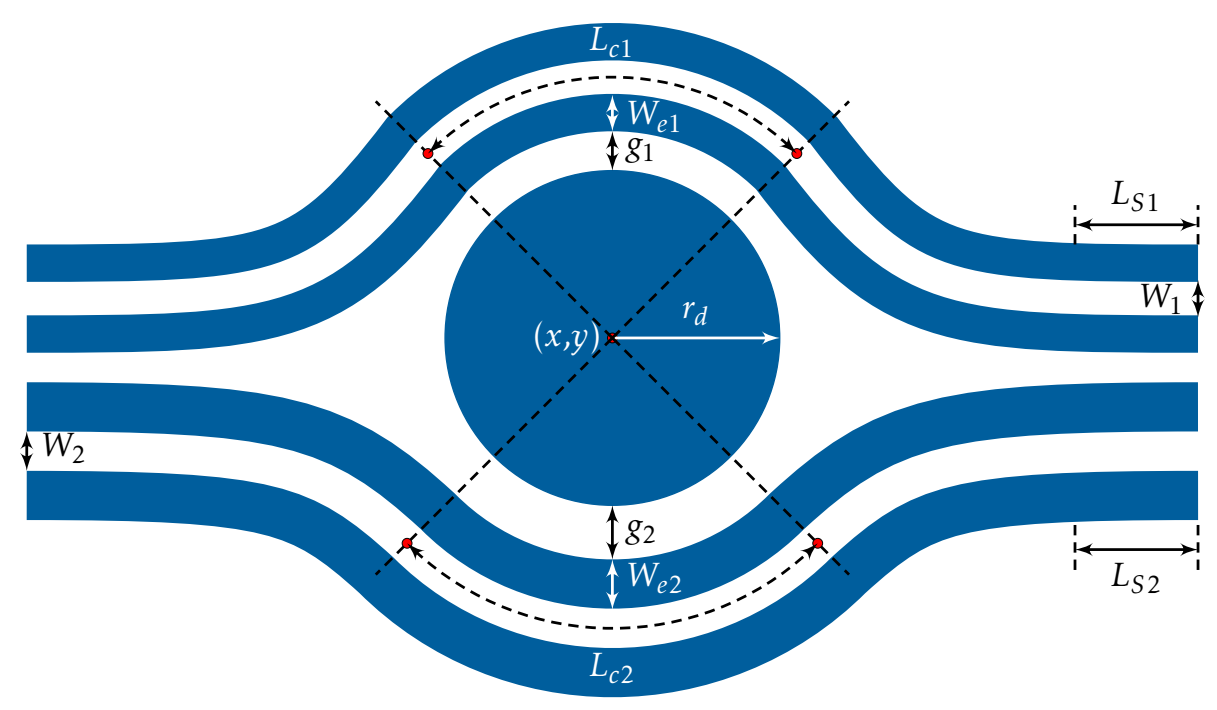

Figure 2.247: Example shape illustrating various parameters of the discPullnvLCAPul constructor. 
Ring pulley inverse arc structure with an additional coupling waveguide.

$\begin{array}{lllllllllllllllllll}x & y & r_{i} & W_{r} & N & g_{1} & L_{c} & N_{w g} & W_{1} & W_{e 1} & L_{S} & g_{2} & W_{2} & W_{e 2} & L_{2} & E C & \text { ringPullnvLCADS }>\end{array}$

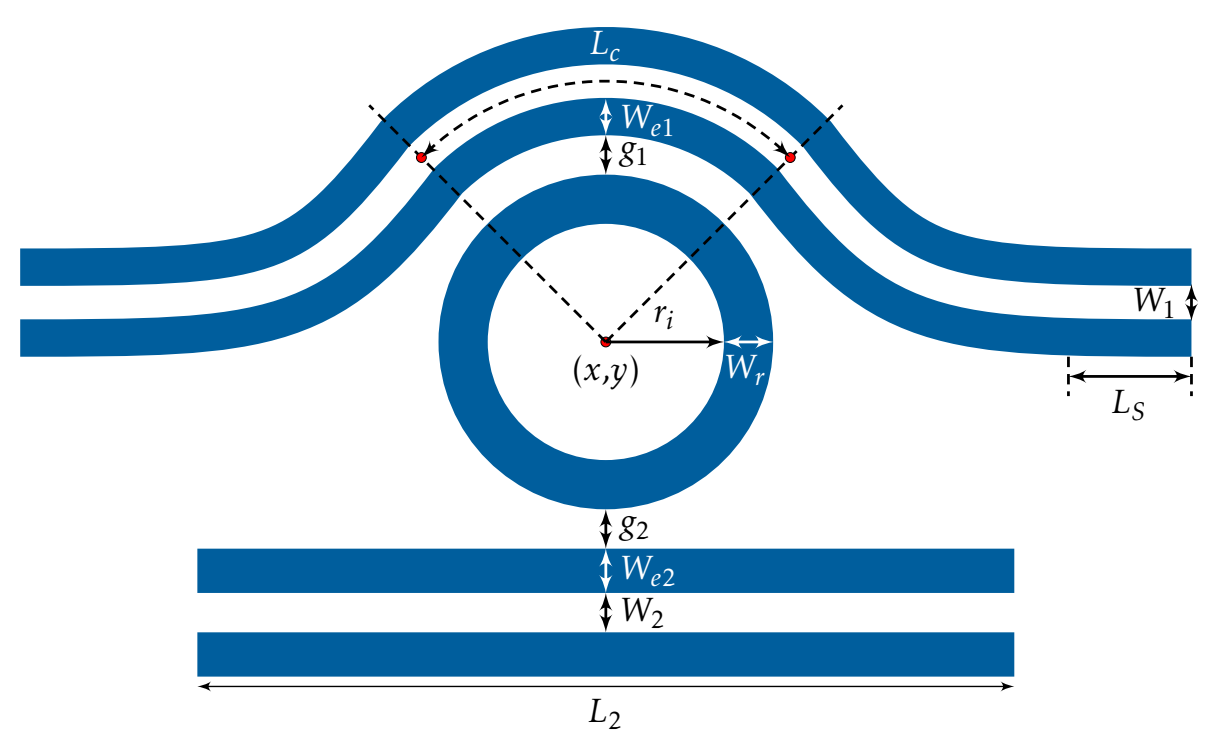

Figure 2.248: Example shape illustrating various parameters of the ringPullnvLCADS constructor. 
Ring pulley inverse arc structure with an additional coupling pulley.

$\begin{array}{llllllllllllllllllllllll} & y & r_{i} & W_{r} & N & g_{1} & L_{c 1} & N_{w g} & W_{1} & W_{e 1} & L_{S 1} & g_{2} & L_{c 2} & W_{2} & W_{e 2} & L_{S 2} & E C \text { ringPullnvLCADPul }\end{array}$

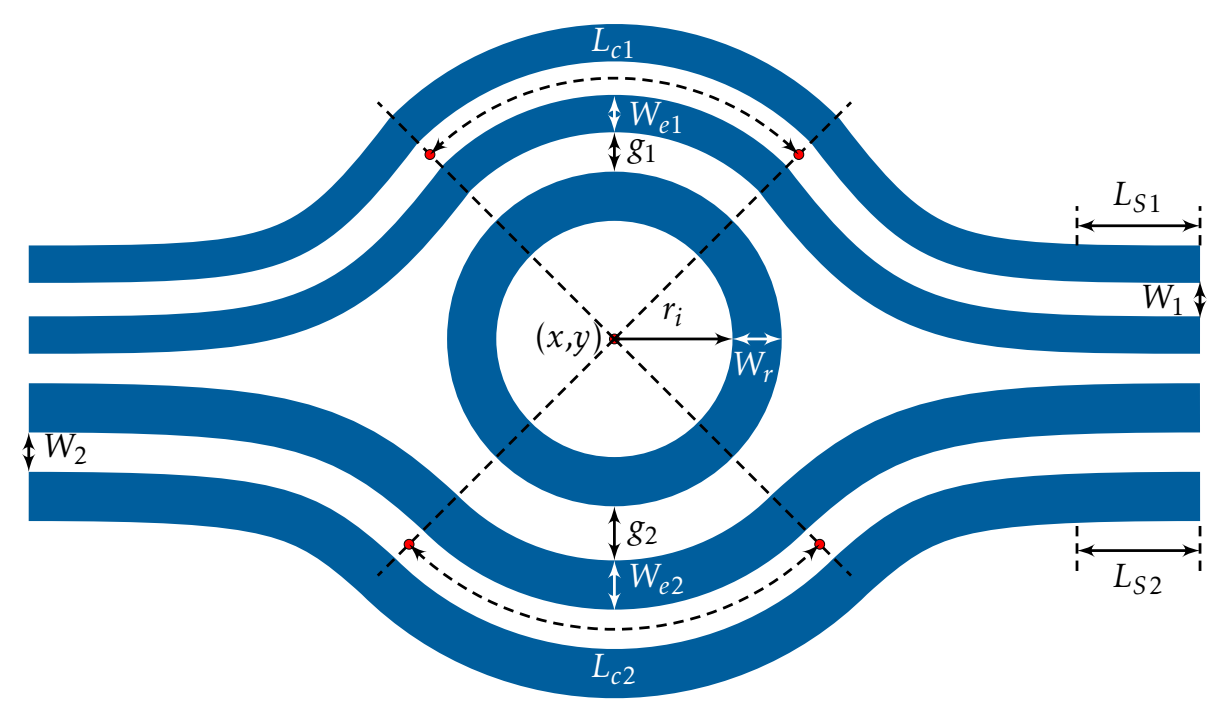

Figure 2.249: Example shape illustrating various parameters of the ringPullnvLCAPul constructor. 


\subsubsection{Disc-Ring Pulley Inverse Positive Tone Bezier}

The structure is similar to the disc-ring waveguide symmetric structure defined in section 2.9.9.11. Here, a slot waveguide is formed at a distance $g$ away from a disc or a ring structure using a positive resist exposure of the exposure sleeve elements $W_{e}$ and $r_{e}$.

$$
\begin{array}{llllllllllllllll}
<x & y & r_{d} & & & r_{e} & N & g_{1} & \theta & N_{w g} & W_{1} & W_{e 1} & L_{1} & H & E C & \text { discPulleylnvPos }> \\
<x & y & r_{i} & W_{r} & r_{e} & N & g_{1} & \theta & N_{w g} & W_{1} & W_{e 1} & L_{1} & H & E C & \text { ringPulleylnvPos }>
\end{array}
$$

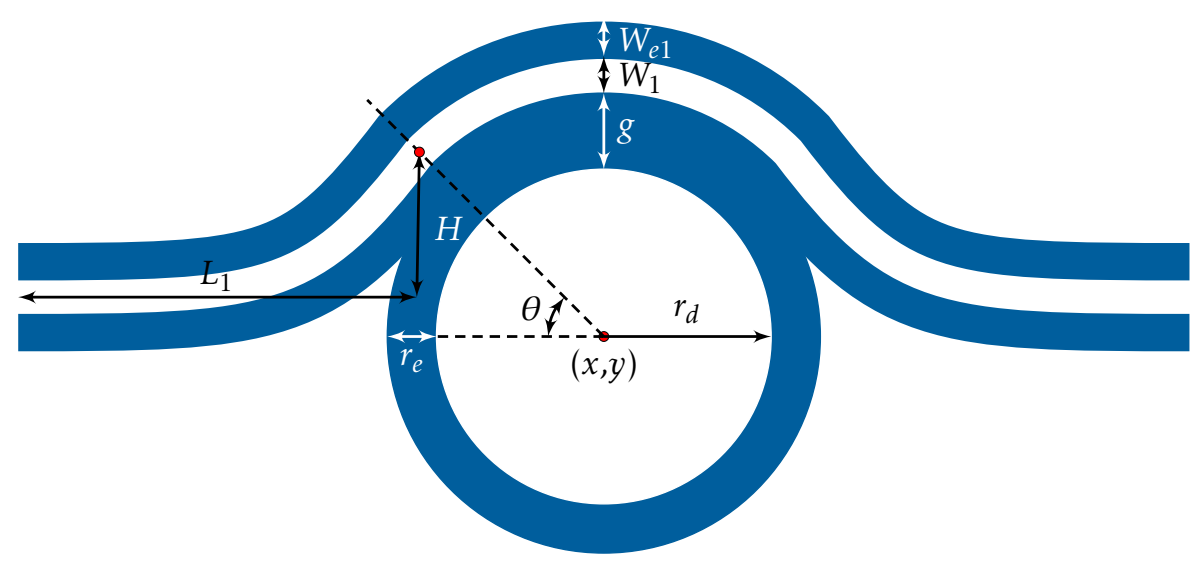

(a)

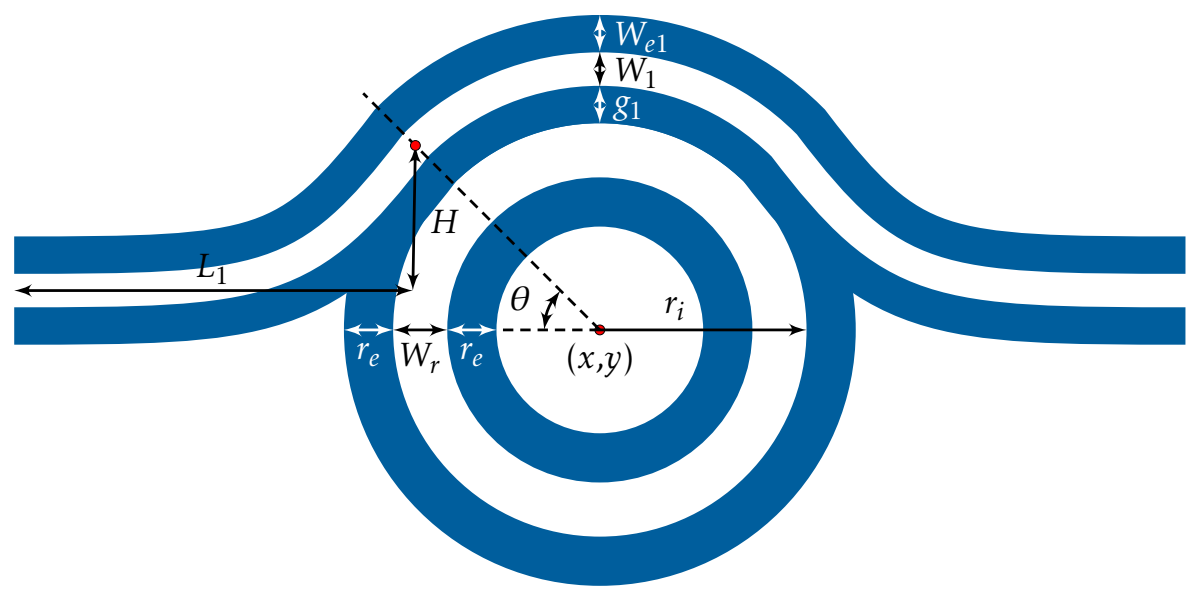

(b)

Figure 2.250: Example shapes illustrating various parameters of the (a) discPulleylnvPos and (b) ringPulleylnvPos constructors. 
Disc pulley inverse positive structure with an additional coupling waveguide.

$\begin{array}{lllllllllllllllllllllllll}x & y & r_{d} & r_{e} & N & g_{1} & \theta & N_{w g} & W_{1} & W_{e 1} & L_{1} & H & g_{2} & W_{2} & W_{e 2} & L_{2} & E C & \text { discPullnvPDS }\end{array}$

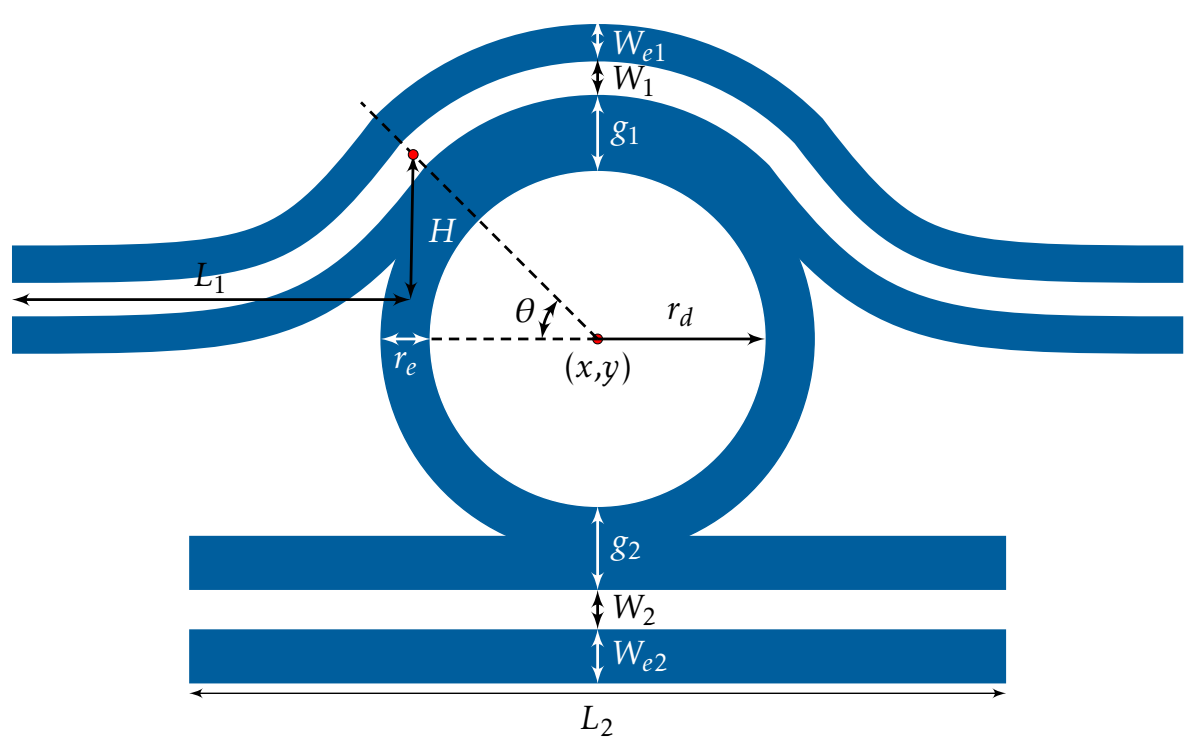

Figure 2.251: Example shape illustrating various parameters of the discPullnvPDS constructor. 
Disc pulley inverse positive structure with an additional coupling pulley.

$<x y r_{d} r_{e} N g_{1} \theta_{1} N_{w g} W_{1} W_{e 1} L_{1} H g_{2} \theta_{2} W_{2} W_{e 2} L_{2} H_{2} E C$ discPullnvPPul $>$

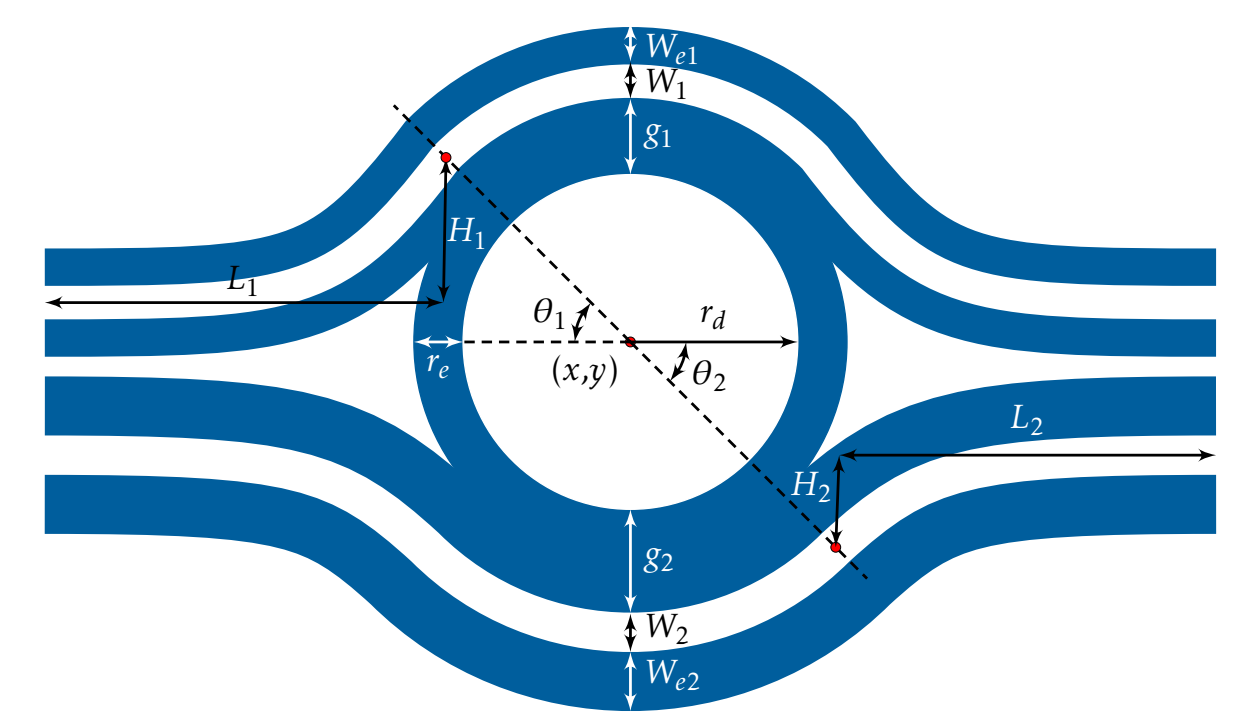

Figure 2.252: Example shape illustrating various parameters of the discPullnvPPul constructor. 
Ring pulley inverse positive structure with an additional coupling waveguide.

$\begin{array}{lllllllllllllllllllllllllll}< & y & r_{i} & W_{r} & r_{e} & N & g_{1} & \theta & N_{w g} & W_{1} & W_{e 1} & L_{1} & H & g_{2} & W_{2} & W_{e 2} & L_{2} & E C & \text { ringPullnvPDS }\end{array}$

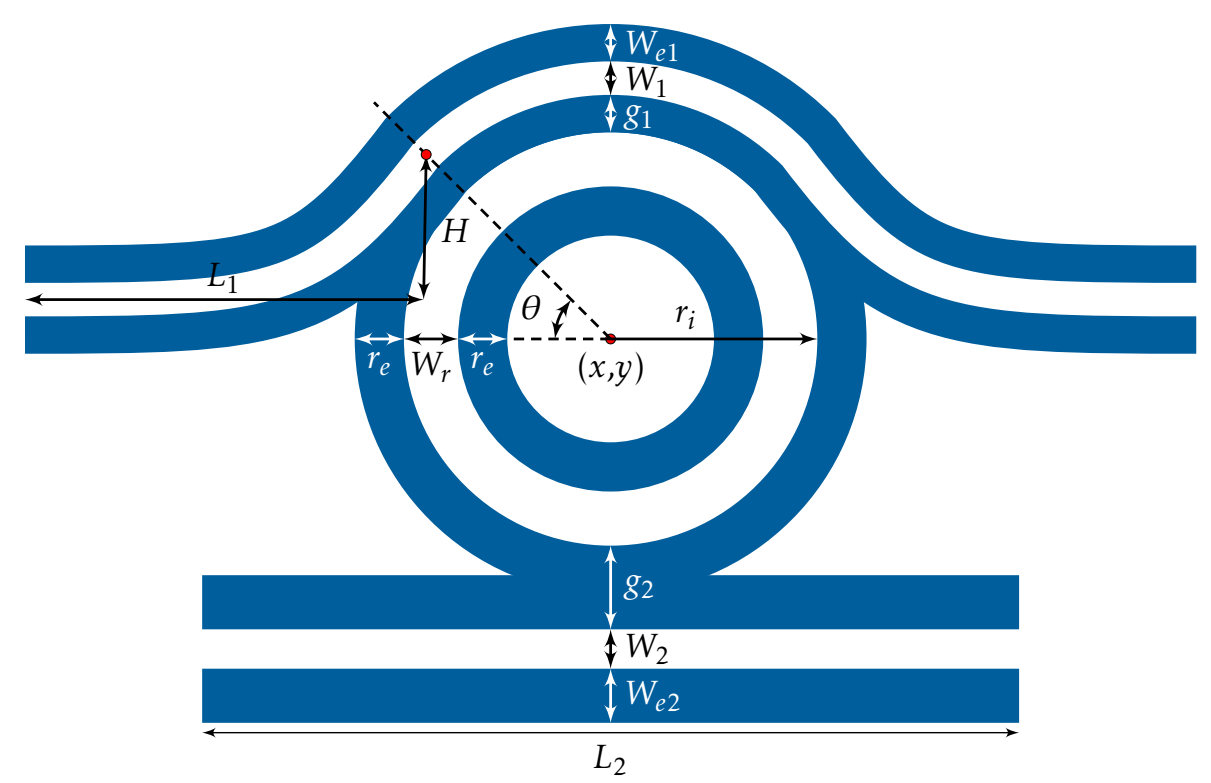

Figure 2.253: Example shape illustrating various parameters of the ringPullnvPDS constructor. 
Ring pulley inverse positive structure with an additional coupling pulley.

$<x y r_{i} W_{r} r_{e} N g_{1} \theta_{1} N_{w g} W_{1} W_{e 1} L_{1} H_{1} g_{2} \theta_{2} W_{2} W_{e 2} L_{2} H_{2} E C$ ringPullnvPPul $>$

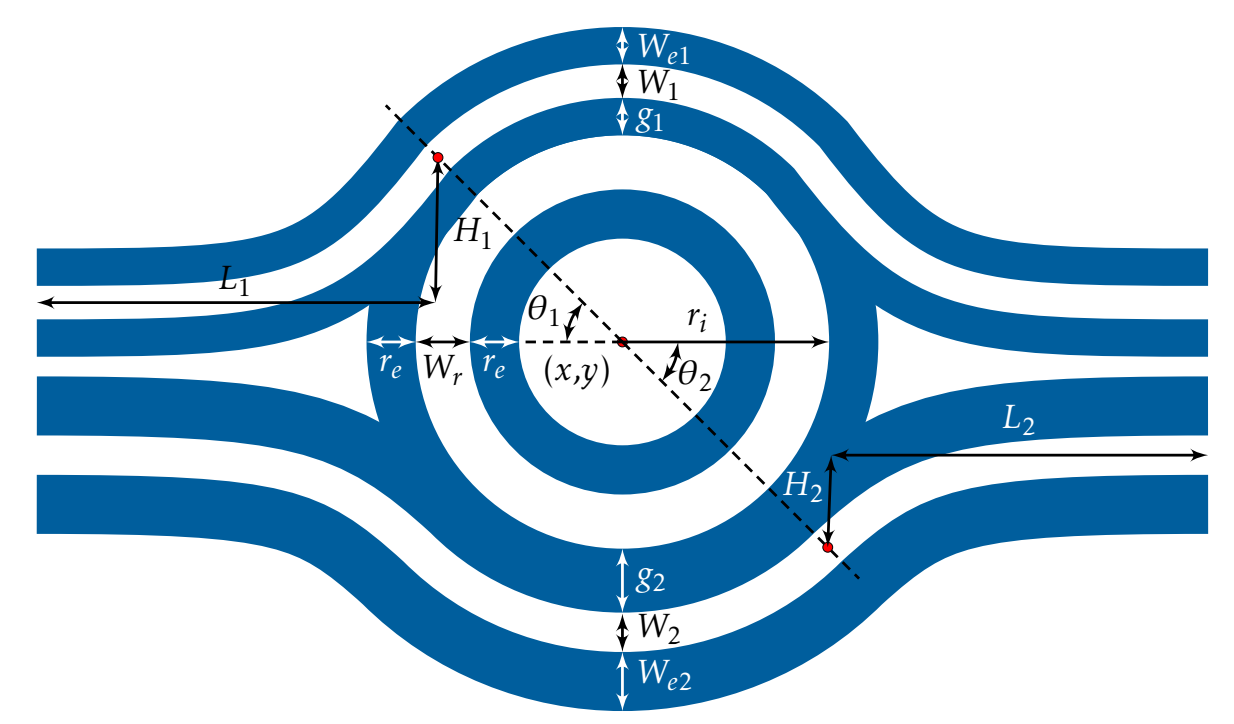

Figure 2.254: Example shape illustrating various parameters of the ringPullnvPPul constructor. 
The following disc-ring waveguide pulley inverse positive tone structures are defined by the coupling length parameter $L_{c}$.

$$
\begin{array}{llllllllllllllll}
<x & y & r_{d} & & & r_{e} & N & g & L_{c} & N_{w g} & W & W_{e} & L & H & E C & \text { discPulleylnvPosLC } \\
<x & y & r_{i} & W_{r} & r_{e} & N & g & L_{c} & N_{w g} & W & W_{e} & L & H & E C & \text { ringPulleylnvPosLC> }
\end{array}
$$

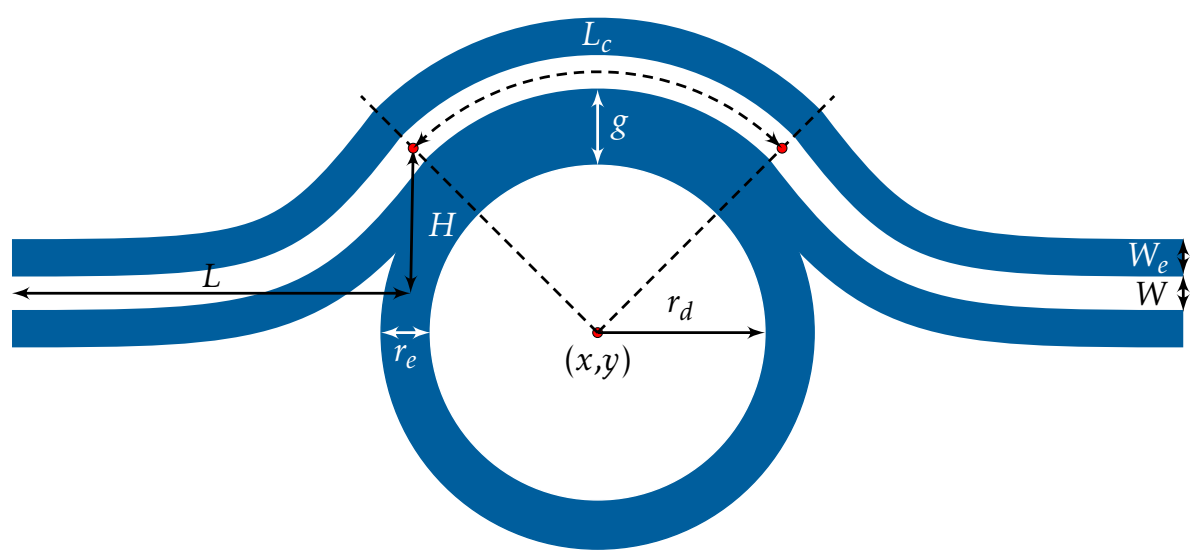

(a)

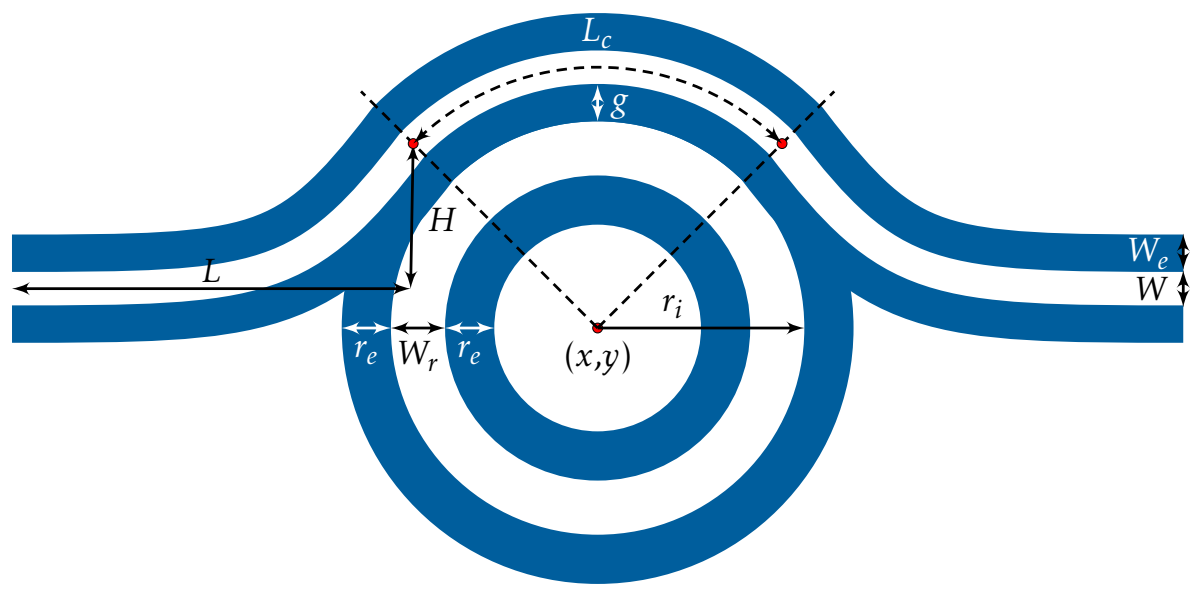

(b)

Figure 2.255: Example shapes illustrating various parameters of the (a) discPulleyln$v$ PosLC and (b) ringPulleylnvPosLC constructors. 
Disc pulley inverse positive structure with an additional coupling waveguide.

$\begin{array}{llllllllllllllllllllllll}x & y & r_{d} & r_{e} & N & g_{1} & L_{c} & N_{w g} & W_{1} & W_{e 1} & L_{1} & H & g_{2} & W_{2} & W_{e 2} & L_{2} & E C & \text { discPullnvPLCDS }\end{array}$

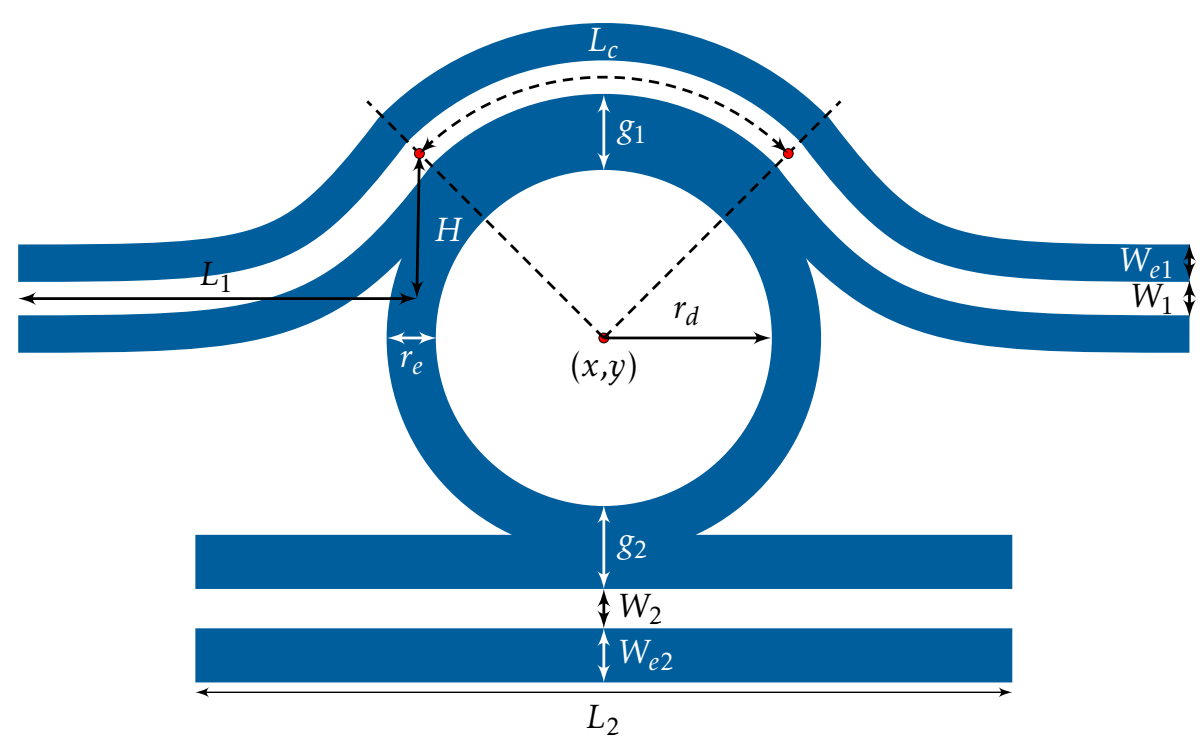

Figure 2.256: Example shape illustrating various parameters of the discPullnvPLCDS constructor. 
Disc pulley inverse positive structure with an additional coupling pulley.

$<x y r_{d} r_{e} N g_{1} L_{c} N_{w g} W_{1} W_{e 1} L_{1} H_{1} g_{2} L_{c 2} W_{2} W_{e 2} L_{2} H_{2} E C$ discPullnvPLCPul $>$

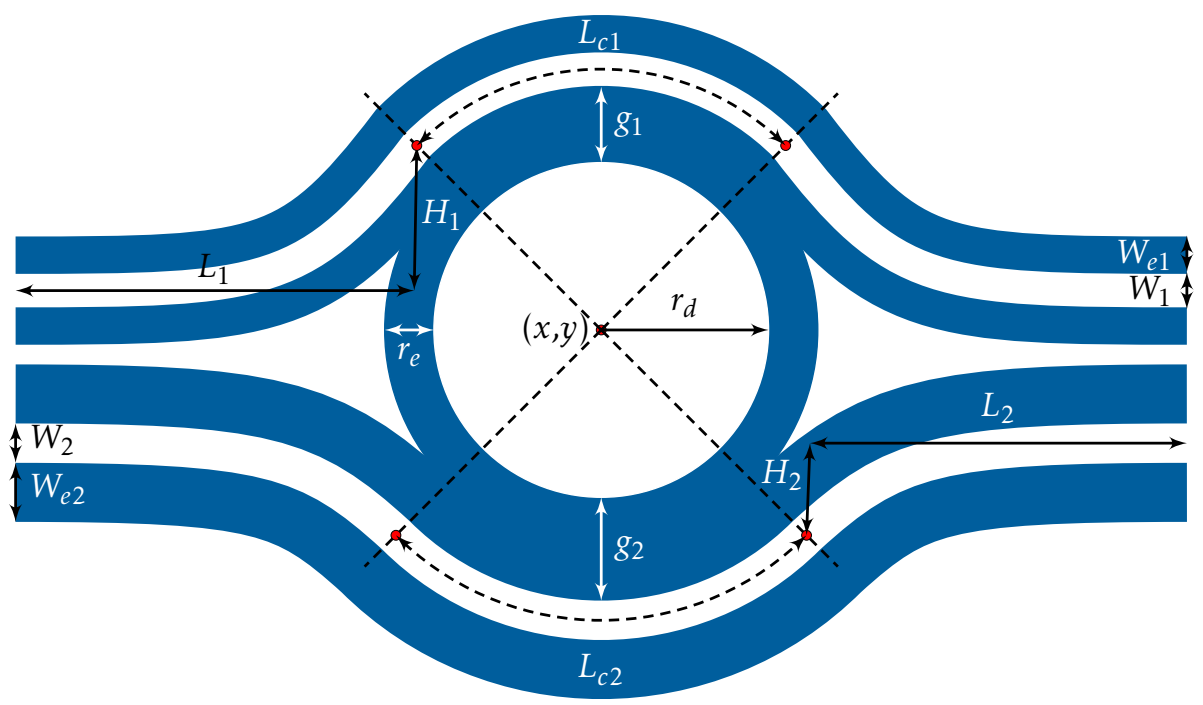

Figure 2.257: Example shape illustrating various parameters of the discPullnvPLCPul constructor. 
Disc pulley inverse positive structure with an additional coupling waveguide.

$\begin{array}{llllllllllllllllllllllllll}x & y & r_{i} & W_{r} & r_{e} & N & g_{1} & L_{c} & N_{w g} & W_{1} & W_{e 1} & L_{1} & H & g_{2} & W_{2} & W_{e 2} & L_{2} & E C & \text { ringPullnvPLCDS }\end{array}$

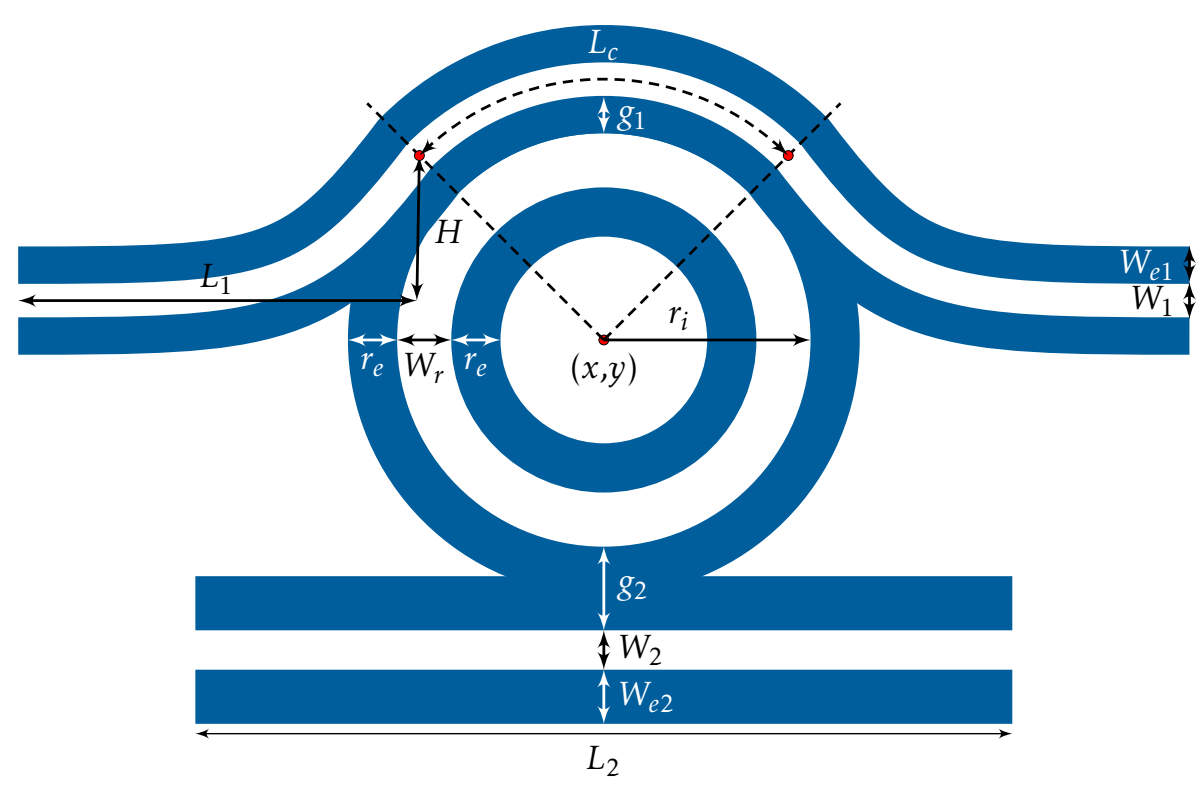

Figure 2.258: Example shape illustrating various parameters of the ringPullnvPLCDS constructor. 
Disc pulley inverse positive structure with an additional coupling pulley.

$<x y r_{i} W_{r} r_{e} N g_{1} L_{c 1} N_{w g} W_{1} W_{e 1} L_{1} H_{1} g_{2} L_{c 2} W_{2} W_{e 2} L_{2} H_{2} E C$ ringPullnvPLCPul $>$

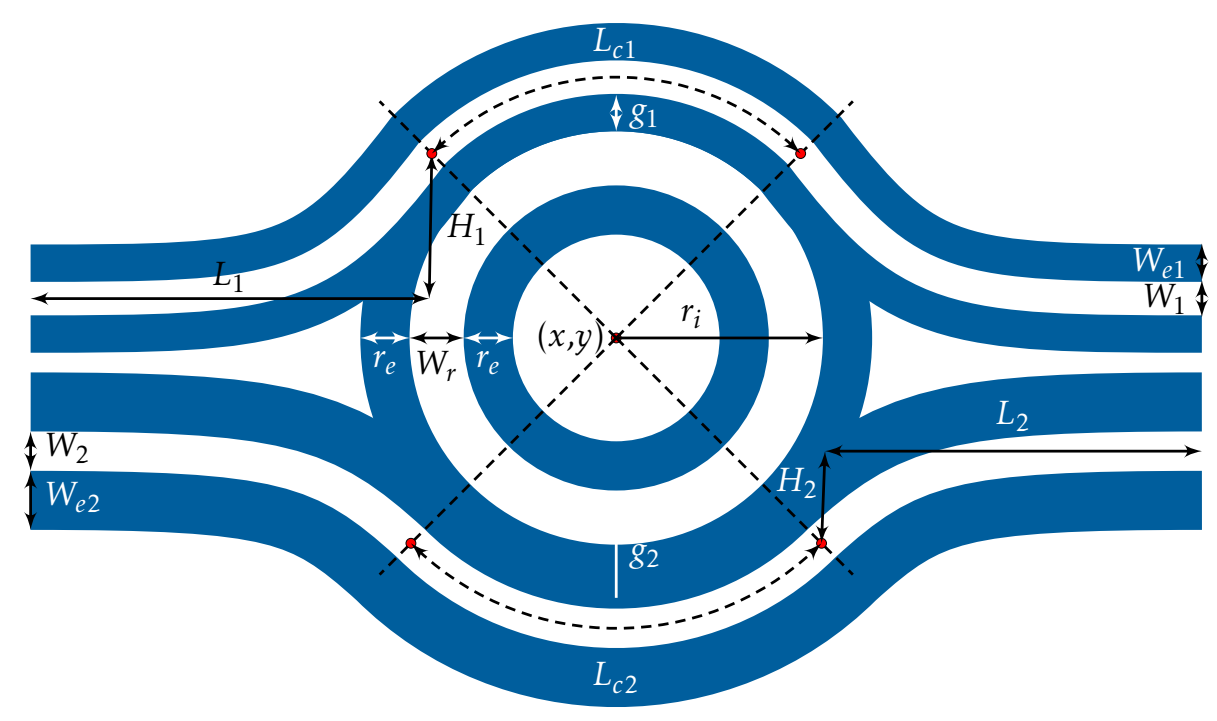

Figure 2.259: Example shape illustrating various parameters of the ringPullnvPLCPul constructor. 


\subsubsection{Disc-Ring Pulley Inverse Positive Tone Arc}

Pulley inverse positive tone structures constructed using arcs are similar to ones described in section 2.9.9.12. Here, a slot waveguide is formed at a distance $g$ away from a disc or a ring structure using a positive resist exposure of the exposure sleeve elements $W_{e}$ and $r_{e}$.

$$
\begin{array}{lllllllllllllll}
<x & y & r_{d} & & r_{e} & N & g & \theta & N_{w g} & W & W_{e} & L_{S} & E C & \operatorname{discPulleylnvPos} \mathrm{A}> \\
<x & y & r_{i} & W_{r} & r_{e} & N & g & \theta & N_{w g} & W & W_{e} & L_{S} & E C & \operatorname{ringPulleylnvPos} \mathrm{A}>
\end{array}
$$

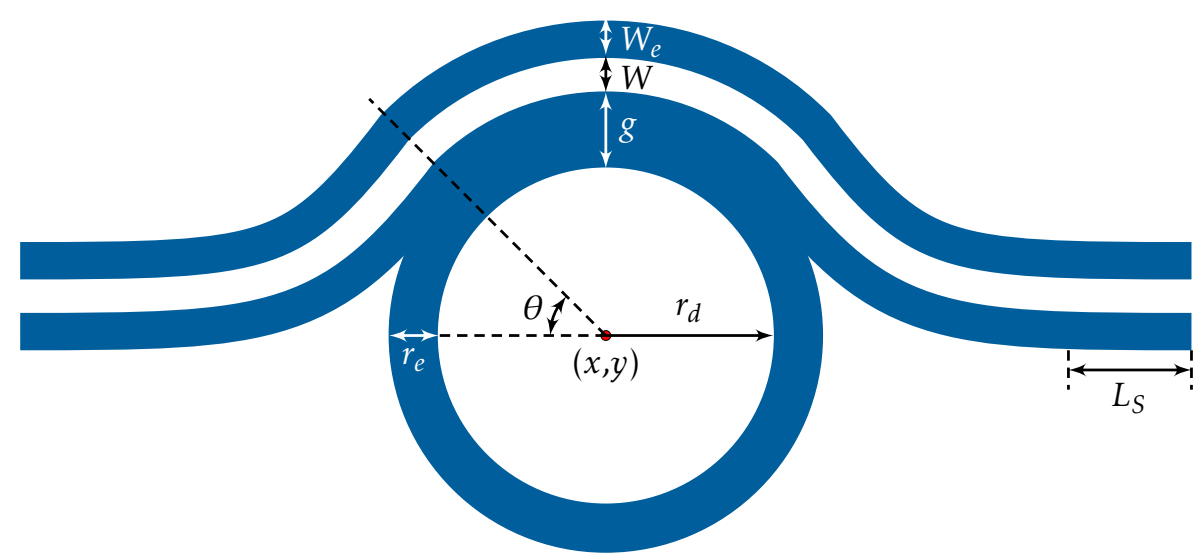

(a)

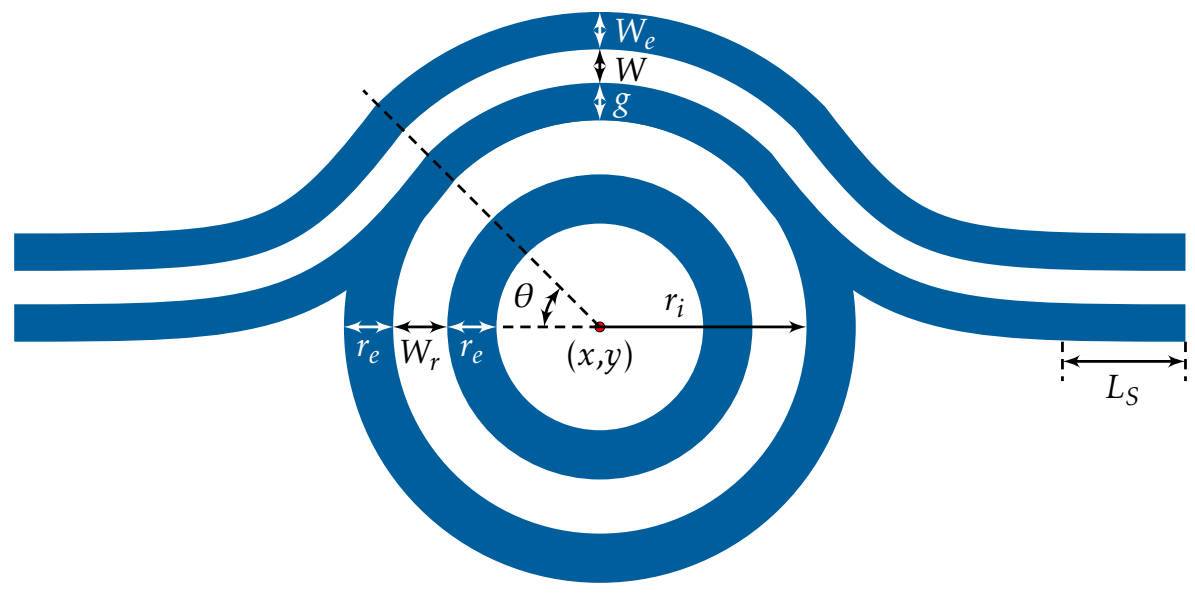

(b)

Figure 2.260: Example shapes illustrating various parameters of the (a) discPulleyln$v$ PosA and (b) ringPulleylnvPosA constructors. 
Disc pulley inverse positive arc structure with an additional coupling waveguide.

$\begin{array}{llllllllllllllllllllll}x & y & r_{d} & r_{e} & N & g_{1} & \theta & N_{w g} & W_{1} & W_{e 1} & L_{S} & g_{2} & W_{2} & W_{e 2} & L_{2} & E C & \text { discPullnvPADS }>\end{array}$

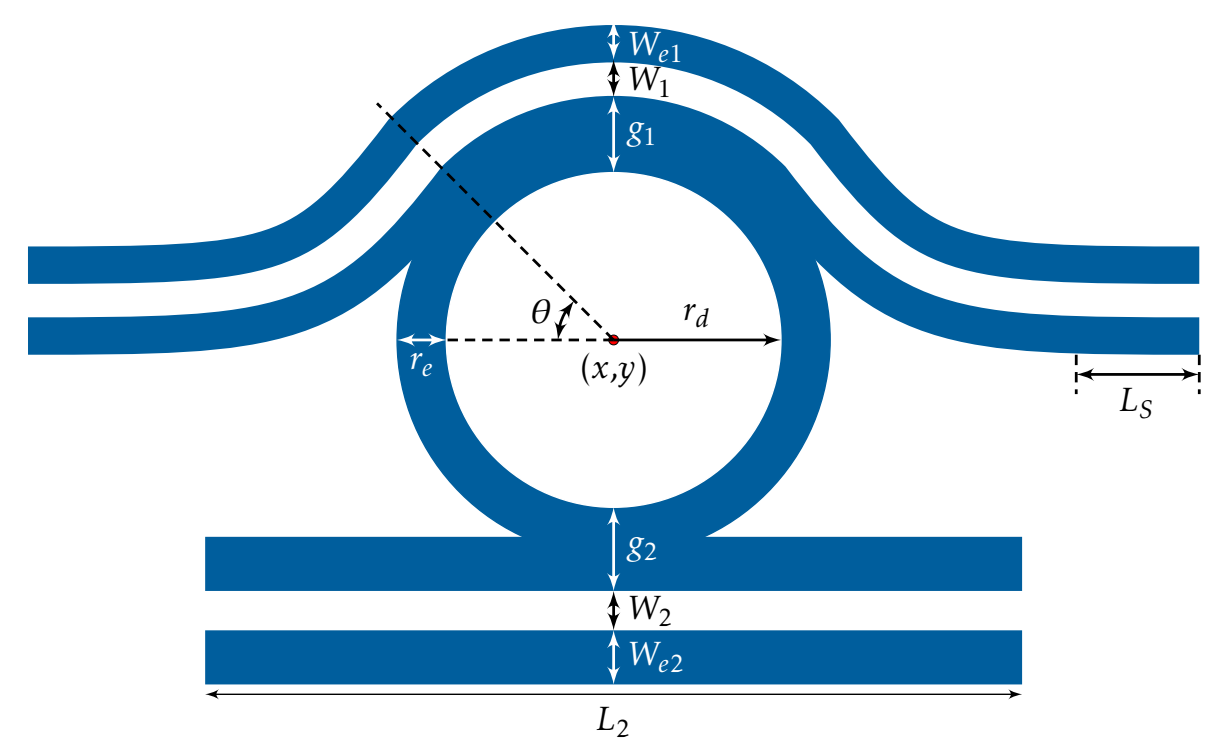

Figure 2.261: Example shape illustrating various parameters of the discPullnvPADS constructor. 
Disc pulley inverse positive arc structure with an additional coupling pulley.

$\begin{array}{lllllllllllllllllllllll}x & y & r_{d} & r_{e} & N & g_{1} & \theta_{1} & N_{w g} & W_{1} & W_{e 1} & L_{S 1} & g_{2} & \theta_{2} & W_{2} & W_{e 2} & L_{S 2} & E C & \text { discPullnvPAPul }\end{array}$

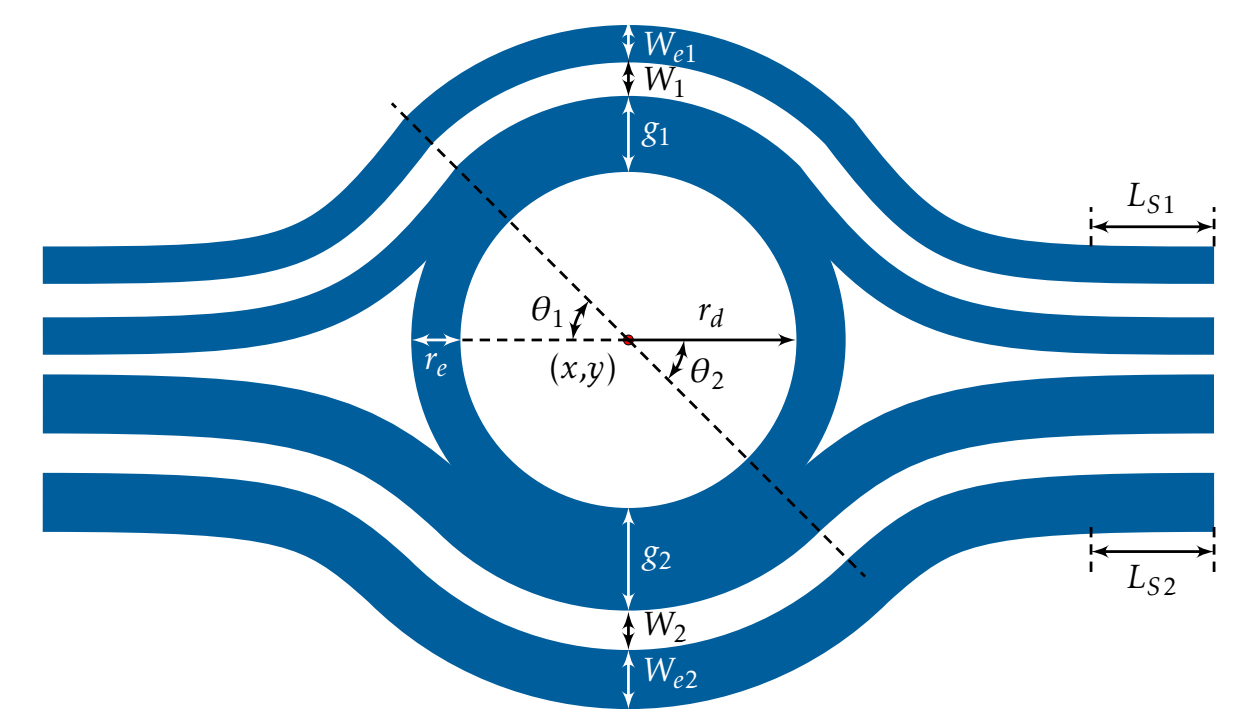

Figure 2.262: Example shape illustrating various parameters of the discPullnvPAPul constructor. 
Ring pulley inverse positive arc structure with an additional coupling waveguide.

$\begin{array}{llllllllllllllllllllllll}<x & y & r_{i} & W_{r} & r_{e} & N & g_{1} & \theta & N_{w g} & W_{1} & W_{e 1} & L_{S} & g_{2} & W_{2} & W_{e 2} & L_{2} & E C & \text { ringPullnvPADS }\end{array}$

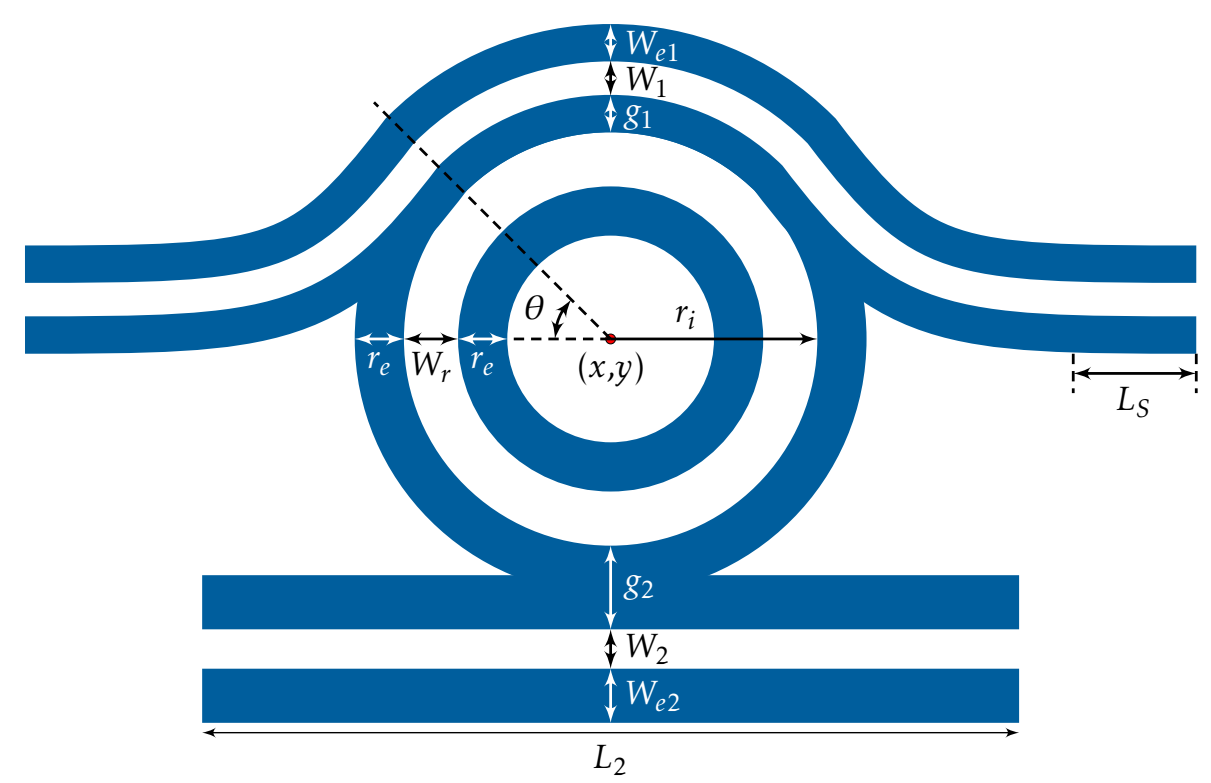

Figure 2.263: Example shape illustrating various parameters of the ringPullnvPADS constructor. 
Ring pulley inverse positive arc structure with an additional coupling pulley.

$\begin{array}{llllllllllllllllllllllll}< & y & r_{i} & W_{r} & r_{e} & N & g_{1} & \theta_{1} & N_{w g} & W_{1} & W_{e 1} & L_{S 1} & g_{2} & \theta_{2} & W_{2} & W_{e 2} & L_{2} & E C & \text { ringPullnvPAPul }\end{array}$

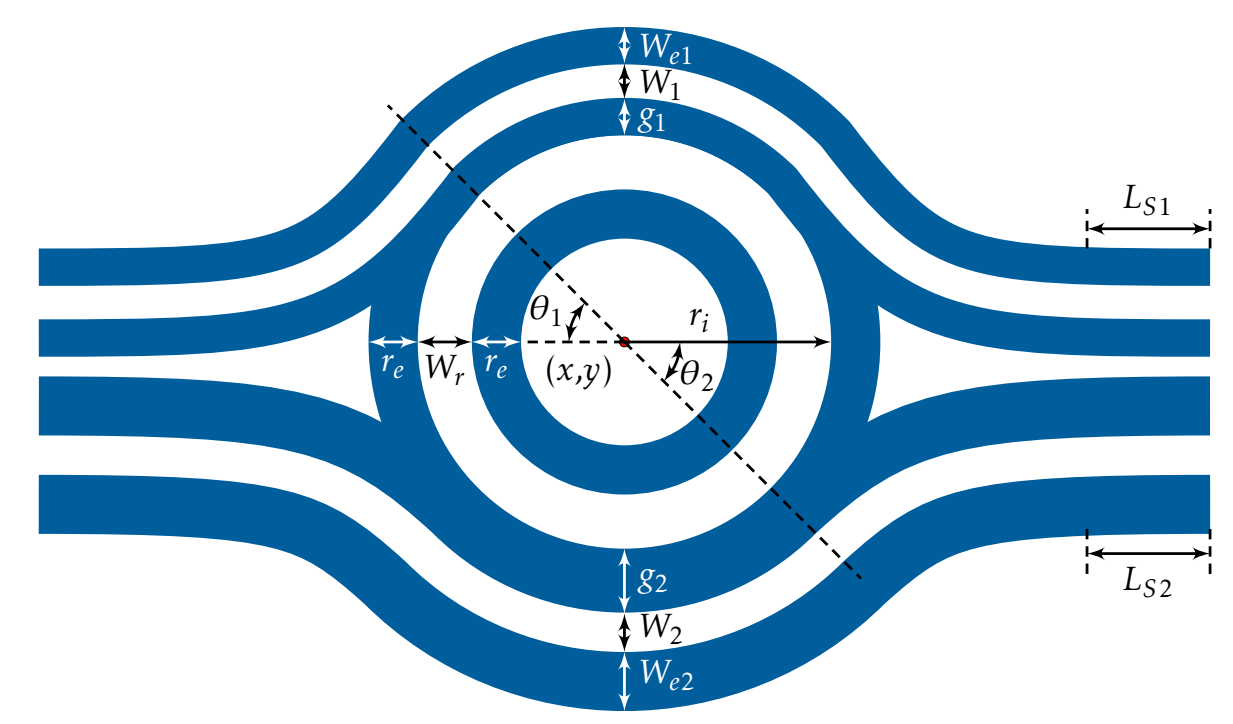

Figure 2.264: Example shape illustrating various parameters of the ringPullnvPAPul constructor. 
The following disc-ring waveguide pulley inverse positive tone structures are defined by the coupling length parameter $L_{c}$.
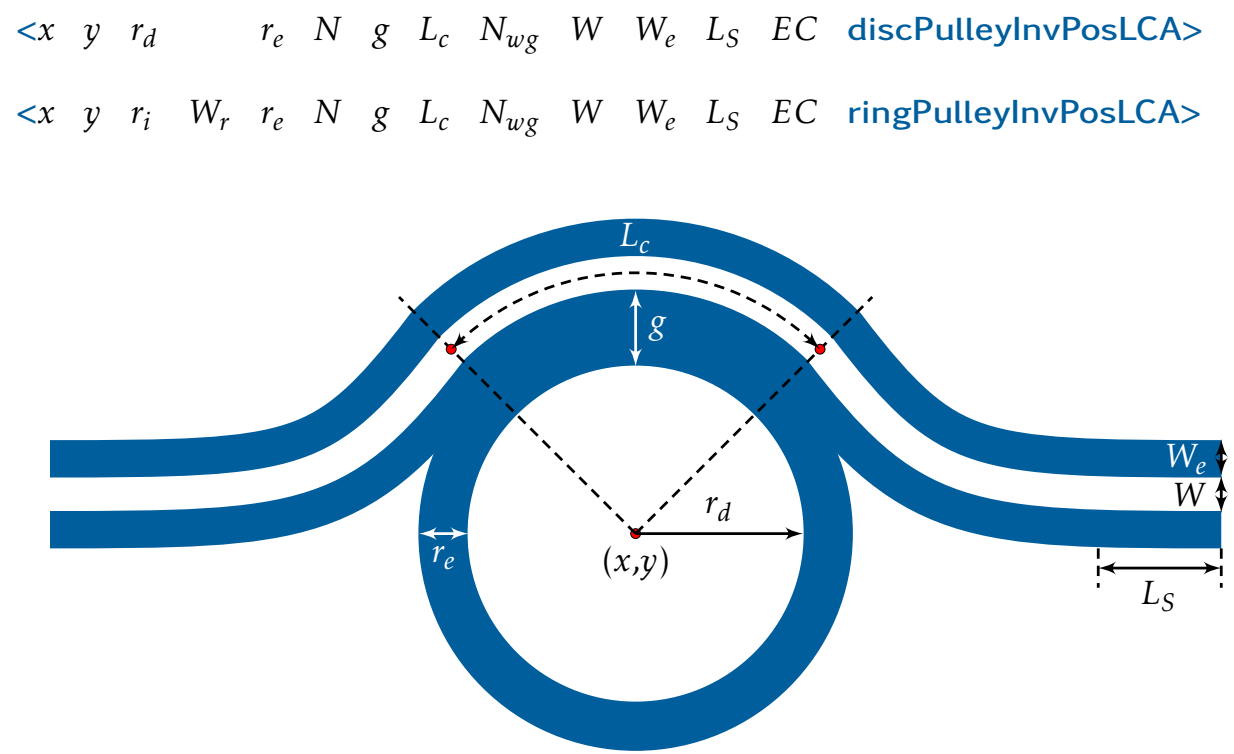

(a)

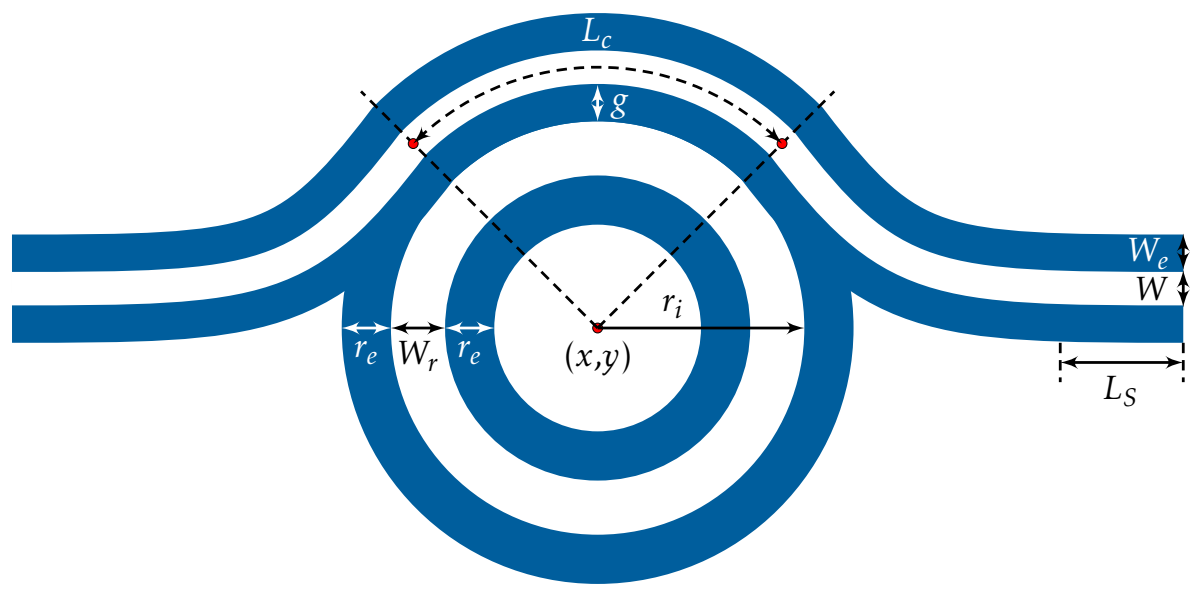

(b)

Figure 2.265: Example shapes illustrating various parameters of the (a) discPulleylnvPosLCA and (b) ringPulleylnvPosLCA constructors. 
Disc pulley inverse positive arc structure with an additional coupling waveguide.

$\begin{array}{lllllllllllllllllllllll}x & y & r_{d} & r_{e} & N & g_{1} & L_{c} & N_{w g} & W_{1} & W_{e 1} & L_{S} & g_{2} & W_{2} & W_{e 2} & L_{2} & E C & \text { discPullnvPLCADS }\end{array}$

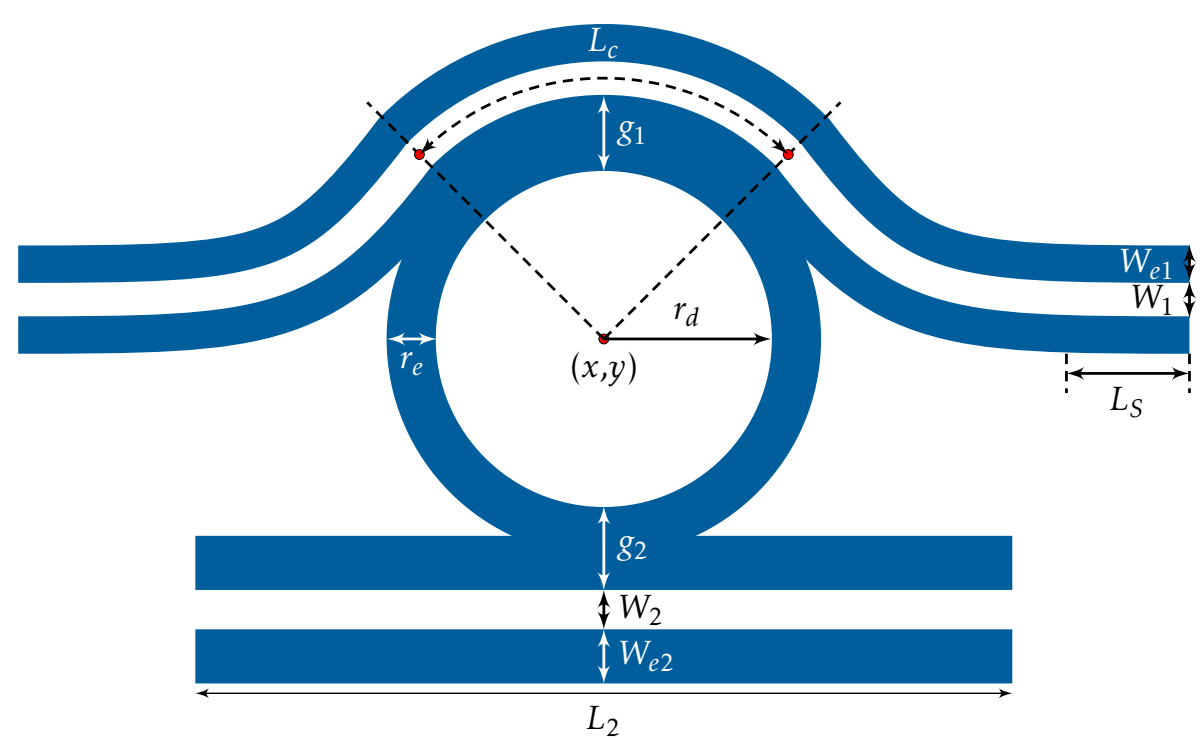

Figure 2.266: Example shape illustrating various parameters of the discPullnvPLCADS constructor. 
Disc pulley inverse positive arc structure with an additional coupling pulley.

\section{$<x y r_{d} r_{e} N g_{1} L_{c 1} N_{w g} W_{1} W_{e 1} L_{S 1} g_{2} L_{c 2} W_{2} W_{e 2} L_{2} E C$ discPullnvPLCAPul $>$}

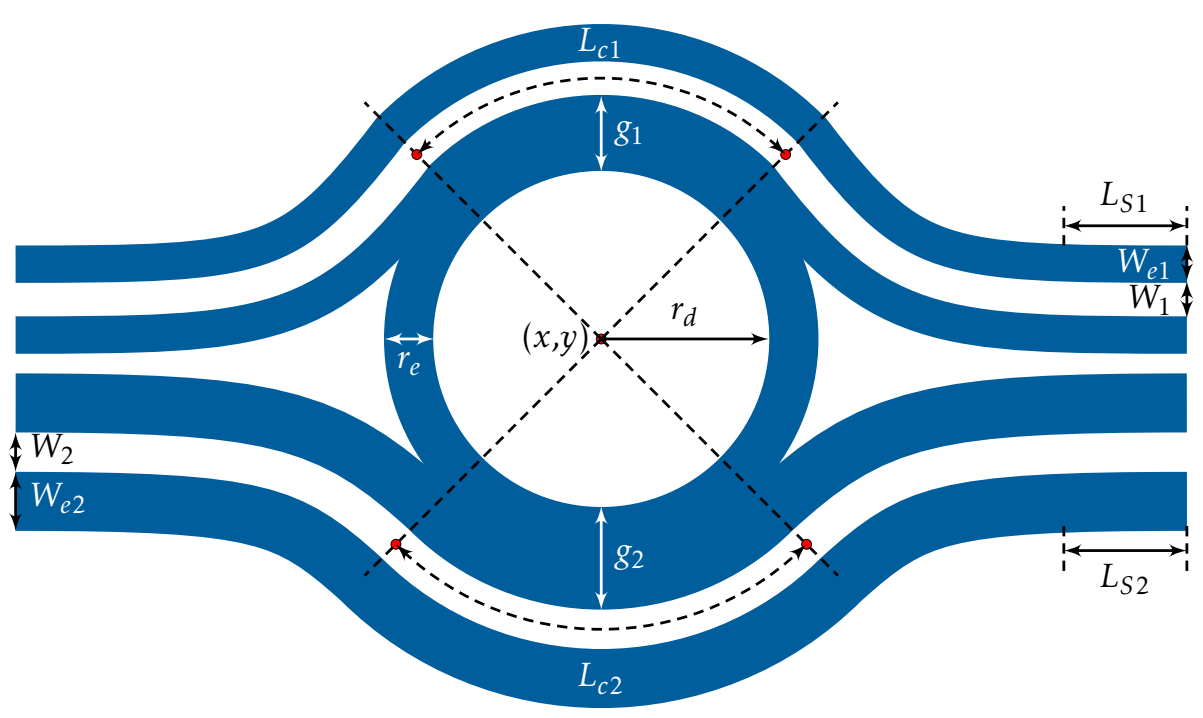

Figure 2.267: Example shape illustrating various parameters of the discPullnvPLCAPul constructor. 
Ring pulley inverse positive arc structure with an additional coupling waveguide.

$\begin{array}{lllllllllllllllllllllll}<x & y & r_{i} & W_{r} & r_{e} & N & g_{1} & L_{c} & N_{w g} & W_{1} & W_{e 1} & L_{S} & g_{2} & W_{2} & W_{e 2} & L_{2} & E C & \text { ringPullnvPLCADS }>\end{array}$

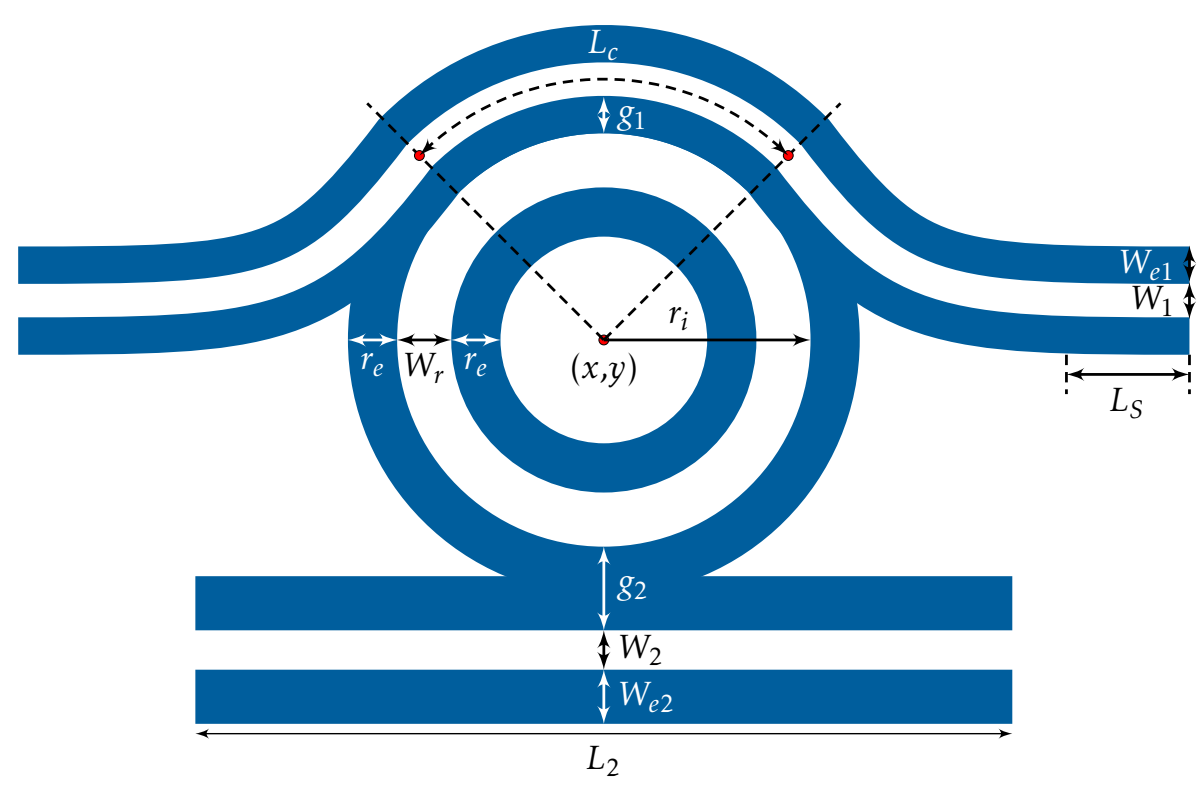

Figure 2.268: Example shape illustrating various parameters of the ringPullnvPLCADS constructor.

NIST • CNST Nanolithography Toolbox v2016.09.01 • http://www.nist.gov/cnst/ page 283 of 488 
Ring pulley inverse positive arc structure with an additional coupling pulley.

$<x y r_{i} W_{r} r_{e} N g_{1} L_{c 1} N_{w g} W_{1} W_{e 1} L_{S 1} g_{2} L_{c 2} W_{2} W_{e 2} L_{S 2} E C$ ringPullnvPLCAPul $>$

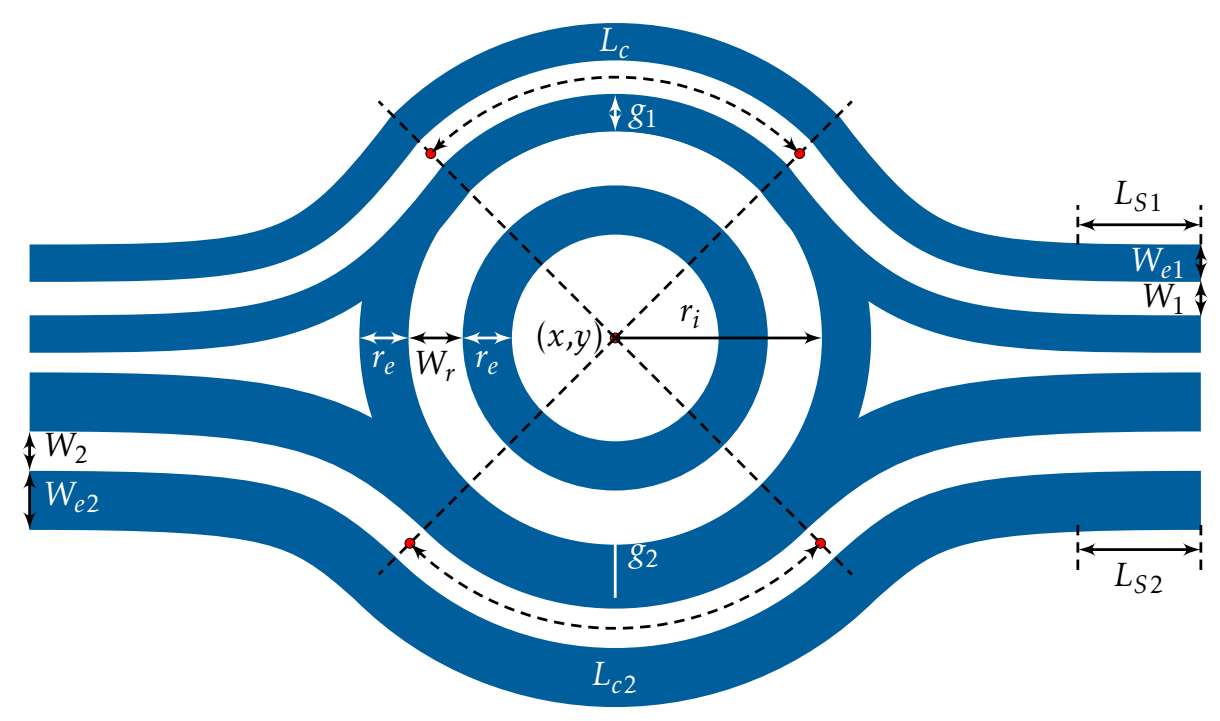

Figure 2.269: Example shape illustrating various parameters of the ringPullnvPLCAPul constructor. 


\subsubsection{Waveguide Inverse Photonic Crystals (Ellipse)}

Waveguide shapes characterized by elliptical perforations on either a periodic $(a=$ constant $)$ or varying grid $\left(a_{1}, a_{2}, \ldots a_{n}\right)$. Elliptical structures are defined by the two radii $\left(r_{x}\right.$ and $\left.r_{y}\right)$, the number of shape sides $(N)$ and the waveguide width $(W)$. The center waveguide position is $(x, y)$. Indexed numbers $(1-7$ and $1-5)$ correspond to the ellipse directly below.

$$
\begin{array}{llllllllllllllll}
r_{x_{1}} & r_{y_{1}} & N_{1} & r_{x_{2}} & r_{y_{2}} & N_{2} & \ldots & r_{x_{n}} & r_{y_{n}} & N_{n} x y \text { a } \mathrm{W} \text { waveGuidelnvPhC } \\
r_{x_{1}} & r_{y_{1}} & a_{1} & N_{1} & r_{x_{2}} & r_{y_{2}} & a_{2} & N_{2} & \ldots & r_{x_{n}} & r_{y_{n}} & a_{n} & N_{n} x y & W \text { waveGuidelnvPhCvary }
\end{array}
$$
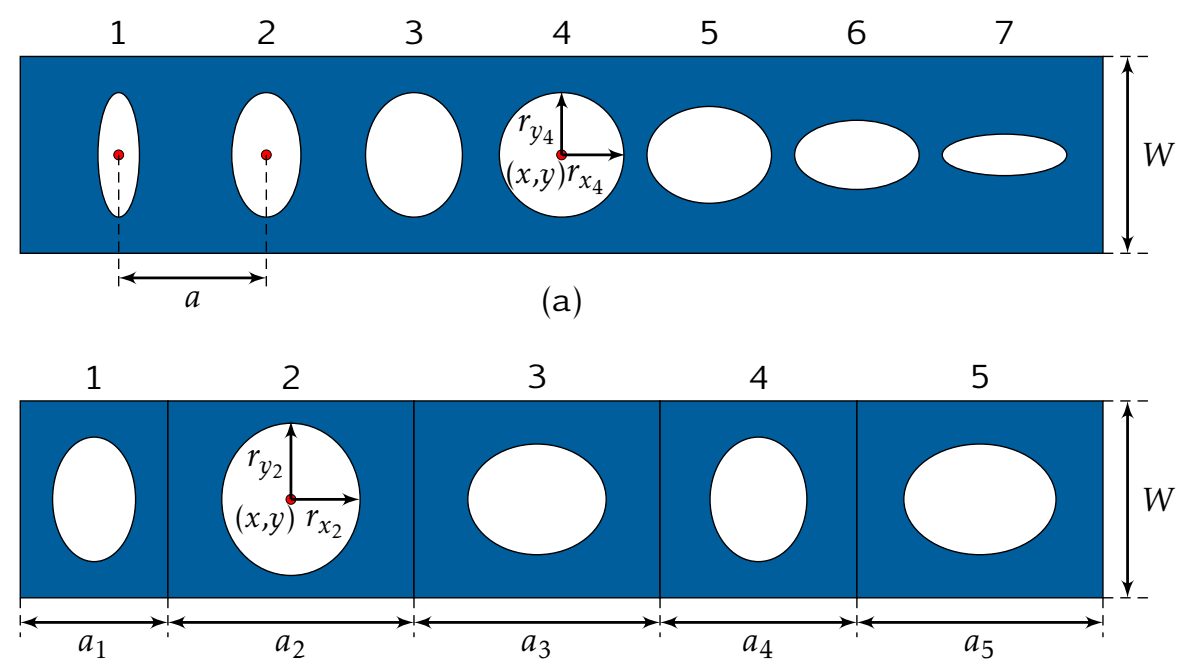

(b)

Figure 2.270: Example shapes illustrating various parameters from the (a) waveGuidelnvPhC and (b) waveGuidelnvPhCvary constructor. 


\subsubsection{Waveguide Photonic Crystals (Ellipse)}

Waveguide shapes characterized by elliptical structures on either a periodic ( $a=$ constant $)$ or varying grid $\left(a_{1}, a_{2}, \ldots a_{n}\right)$. Elliptical structures are defined by the two radii $\left(r_{x}\right.$ and $\left.r_{y}\right)$, the number of shape sides $(N)$ and the waveguide width $(W)$. The center waveguide position is $(x, y)$. The box defining the waveguide extends beyond the elliptical structures by the overhang $\left(S_{o}\right)$ and is of height $\left(S_{h}\right)$. Indexed numbers $(1-7$ and $1-5)$ correspond to the ellipse directly below.

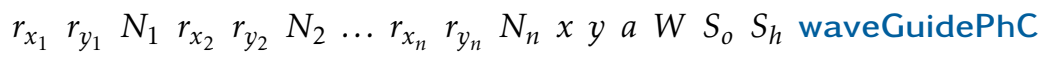

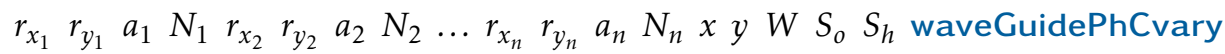

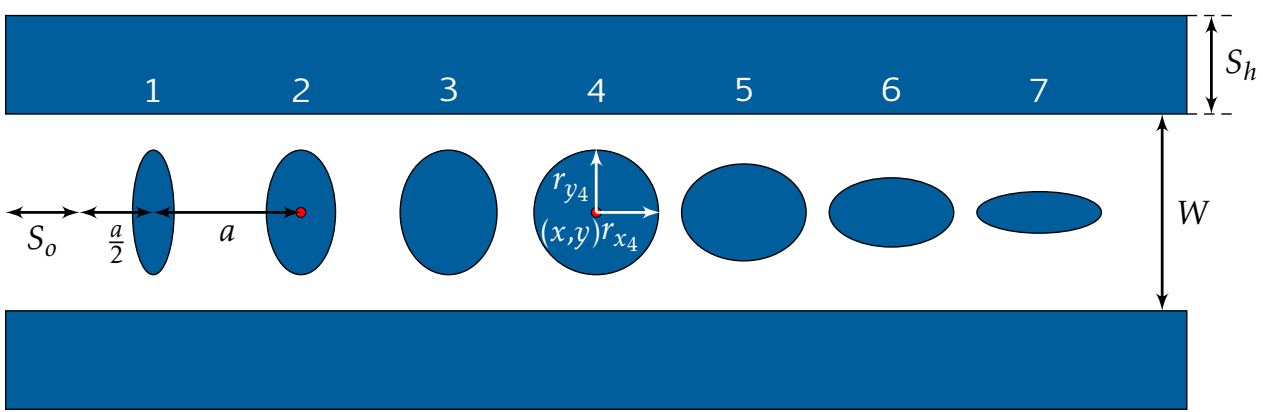

(a)

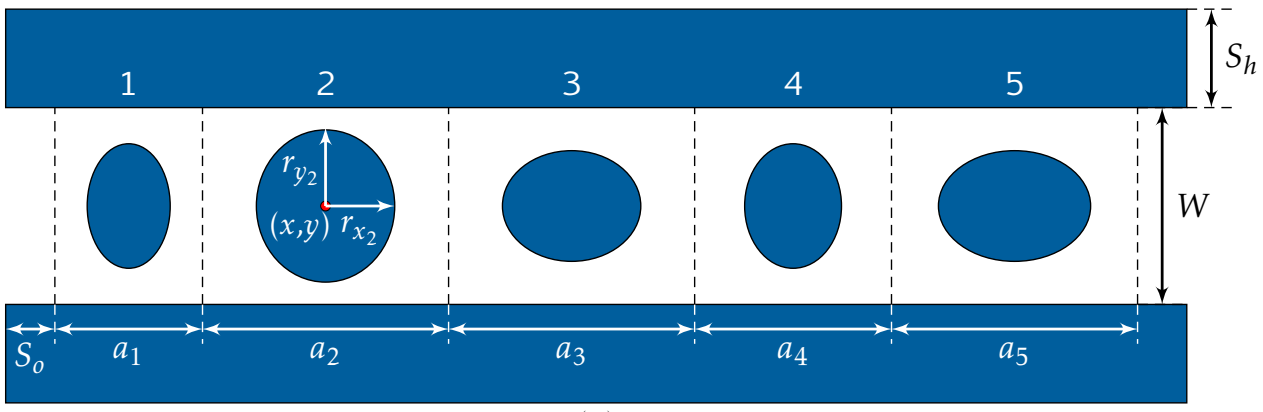

(b)

Figure 2.271: Example shapes illustrating various parameters from the (a) waveGuide$P h C$ and (b) waveGuidePhCvary constructor. 


\subsubsection{Waveguide Inverse Photonic Crystals (Rectangle)}

Waveguide shapes characterized by rectangular perforations on either a periodic ( $a=$ constant) or varying grid $\left(a_{1}, a_{2}, \ldots a_{n}\right)$. Rectangular structures are defined by the $L_{x}$ and $L_{y}$, and the waveguide width $(W)$. The center waveguide position is $(x, y)$. Indexed numbers $(1-7$ and $1-5)$ correspond to the rectangles directly below.

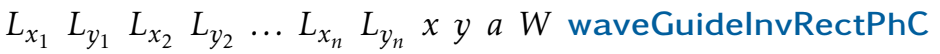

$$
\begin{aligned}
& \begin{array}{llllllllllllllllll}
L_{x_{1}} & L_{y_{1}} & a_{1} & L_{x_{2}} & L_{y_{2}} & a_{2} & \ldots & L_{x_{n}} & L_{y_{n}} & a_{n} & x & y & W & W \text { waveGuidelnvRectPhCvary }
\end{array}
\end{aligned}
$$
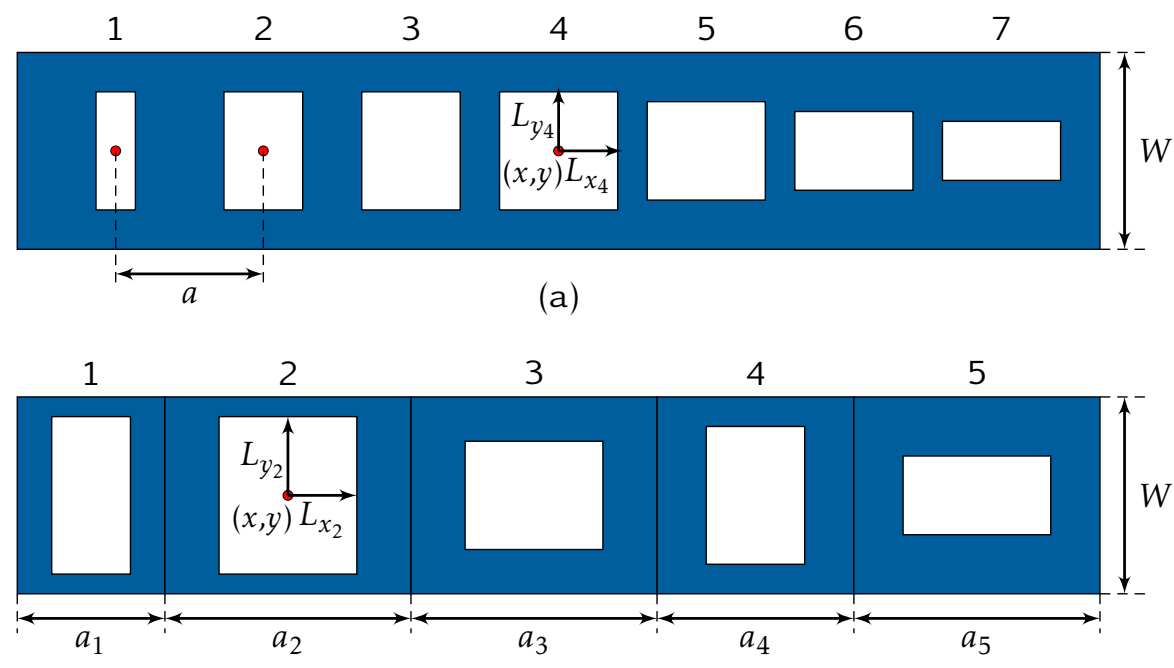

(b)

Figure 2.272: Example shapes illustrating various parameters from the (a) waveGuidelnvRectPhC and (b) waveGuidelnvRectPhCvary constructor. 


\subsubsection{Waveguide Photonic Crystals (Rectangle)}

Waveguide shapes characterized by rectangular structures on either a periodic $(a=$ constant $)$ or varying grid $\left(a_{1}, a_{2}, \ldots a_{n}\right)$. Rectangular structures are defined by the $L_{x}$ and $L_{y}$, and the waveguide width $(W)$. The center waveguide position is $(x, y)$. The box defining the waveguide extends beyond the rectangular structures by the overhang $\left(S_{o}\right)$ and is of height $\left(S_{h}\right)$. Indexed numbers $(1-7$ and $1-5)$ correspond to the rectangles directly below.

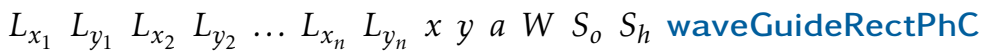

$$
\begin{aligned}
& \begin{array}{lllllllllllllll}
L_{x_{1}} & L_{y_{1}} & a_{1} & L_{x_{2}} & L_{y_{2}} & a_{2} & \ldots & L_{x_{n}} & L_{y_{n}} & a_{n} & x & y & W & S_{o} & S_{h} \text { waveGuideRectPhCvary }
\end{array}
\end{aligned}
$$

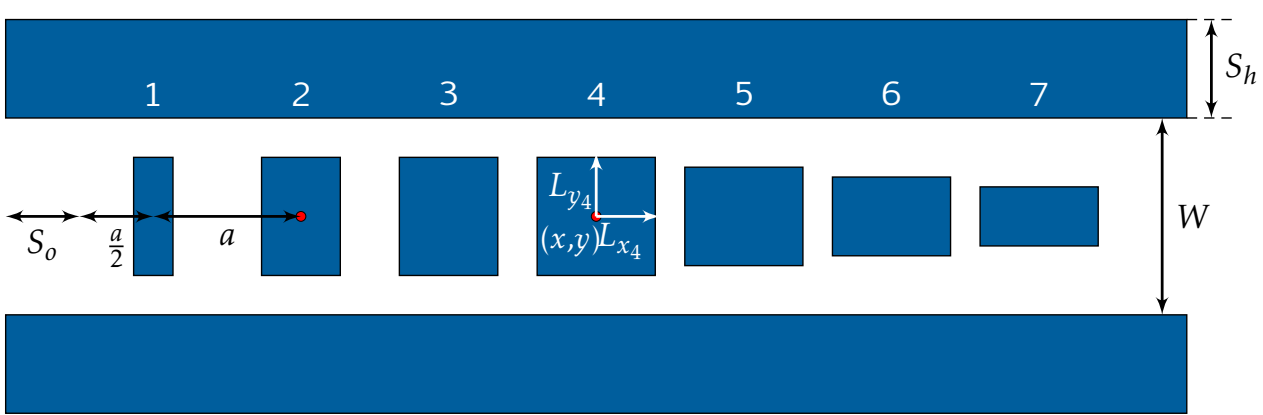

(a)

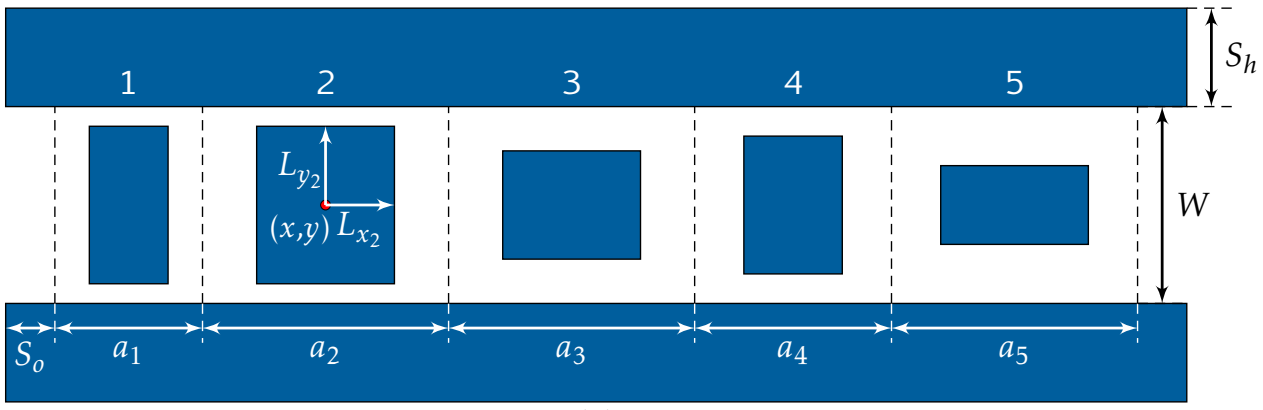

(b)

Figure 2.273: Example shapes illustrating various parameters from the (a) waveGuideRectPhC and (b) waveGuideRectPhCvary constructor. 


\subsubsection{Waveguide Photonic Crystals (Flush Rectangle)}

Waveguide shapes characterized by rectangular structures on a varying grid $\left(a_{1}, a_{2}, \ldots a_{n}\right)$. Rectangular structures are defined by the $L_{x}$ and $L_{y}$ positioned flush at a distance $d$ away from top of waveguide, and the waveguide width $(W)$. The center waveguide position is $(x, y)$. The box defining the waveguide (Figure 2.9.14) extends beyond the rectangular structures by the overhang $\left(S_{o}\right)$ and is of height $\left(S_{h}\right)$. Indexed numbers $(1-5)$ correspond to the rectangles directly below.

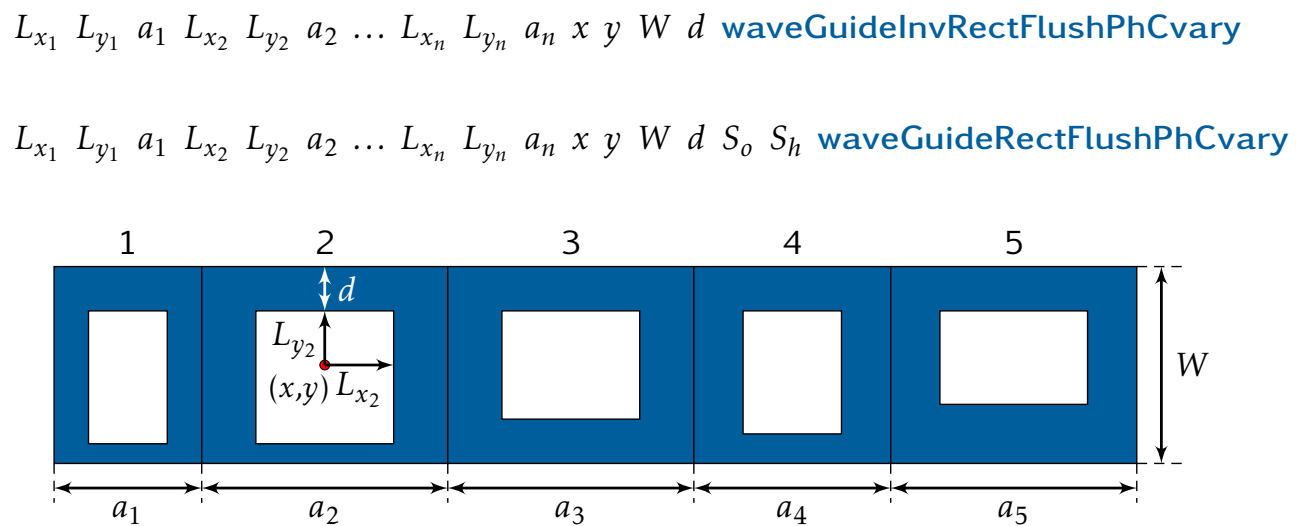

(a)

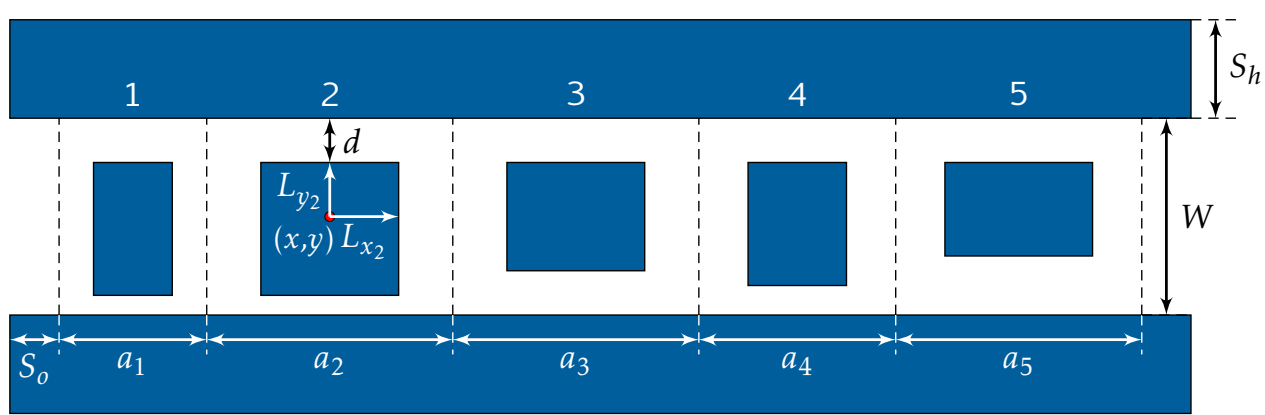

(b)

Figure 2.274: Example shapes illustrating various parameters from the (a) waveGuidelnvRectFlushPhCvary and (b) waveGuideRectFlushPhCvary constructor. 


\subsubsection{Various Waveguide-Disc-Tip Coupled Structures}

$\begin{array}{lllllllllllllllll}x & y & r & N_{\text {sides }} & d_{x} & d_{y} & w & L_{w g} & r_{w g} & s & g & r_{e} & d_{e} & c & d_{c} & \theta_{(x, y)} & \text { wgdcdV1 }>\end{array}$

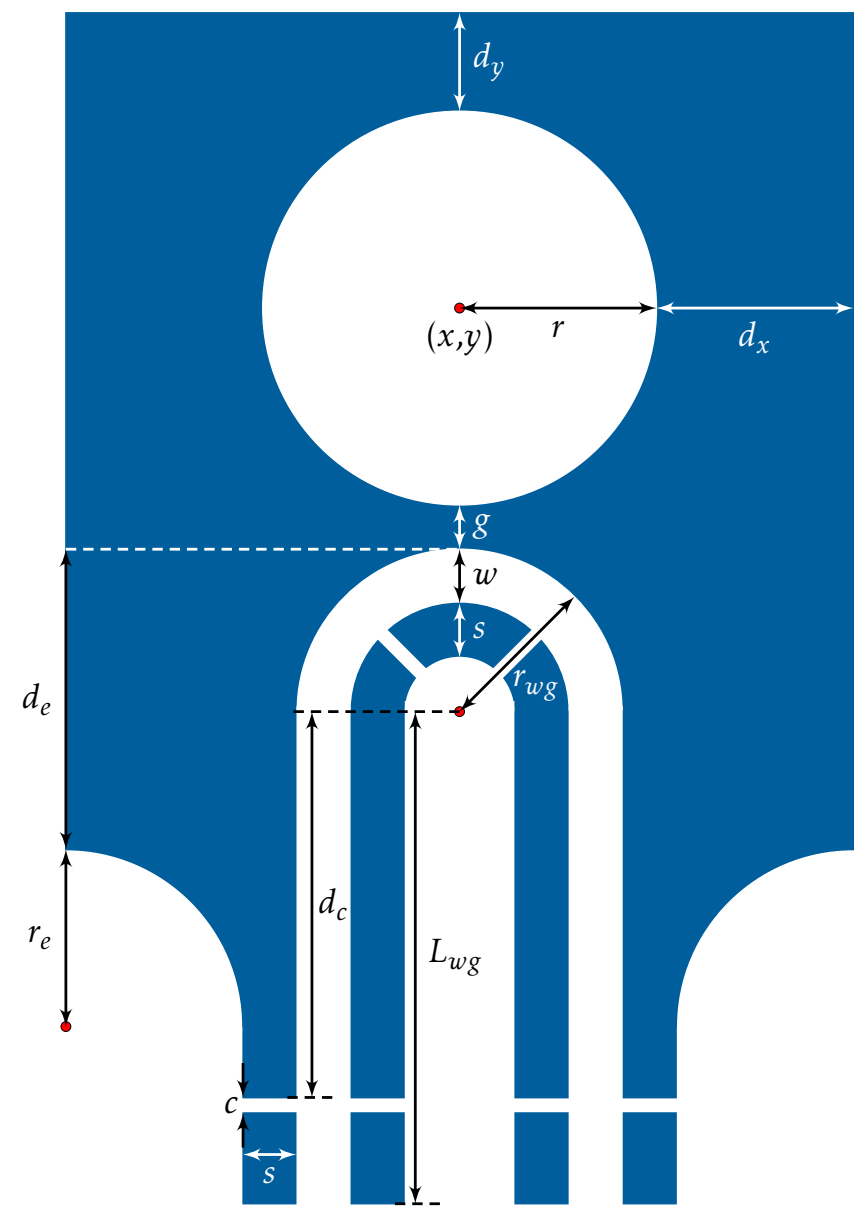

Figure 2.275: wgdcdV1 constructor. 
$<\begin{array}{lllllllllllllllllllll} & y & r & N_{\text {sides }} & d_{x} & d_{y} & H_{1} & W_{1} & t_{H} & t_{W} & t_{S} & t_{Y} & w & L_{w g} & r_{w g} & s & g & c & d_{c} & \theta_{(x, y)} & \text { wgdcdV2> }\end{array}$

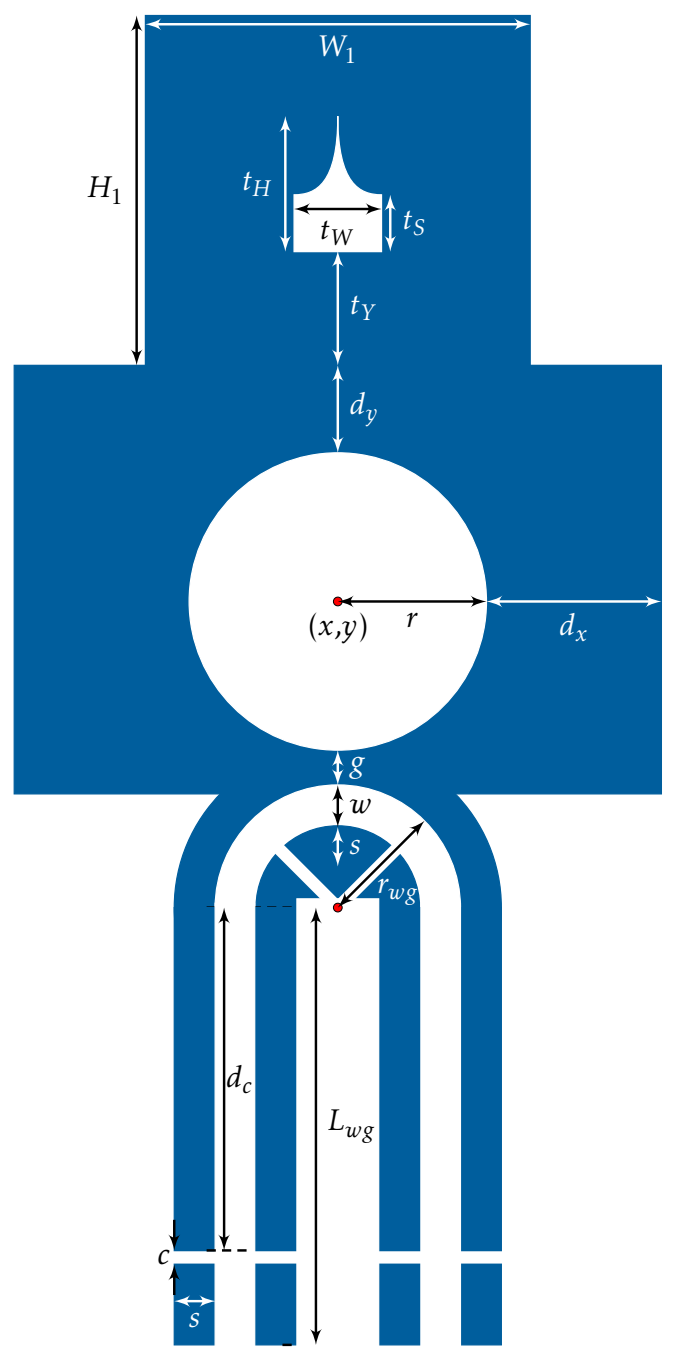

Figure 2.276: wgdcdV2 constructor. 
$\begin{array}{llllllllllllllllllll}<x & y & r & N_{\text {sides }} & d_{x} & d_{y} & d_{g} & H_{1} & W_{1} & w & L_{w g} & r_{w g} & s & g & c & d_{c} & D & \theta_{(x, y)} & \text { wgdcdV3 }\end{array}$

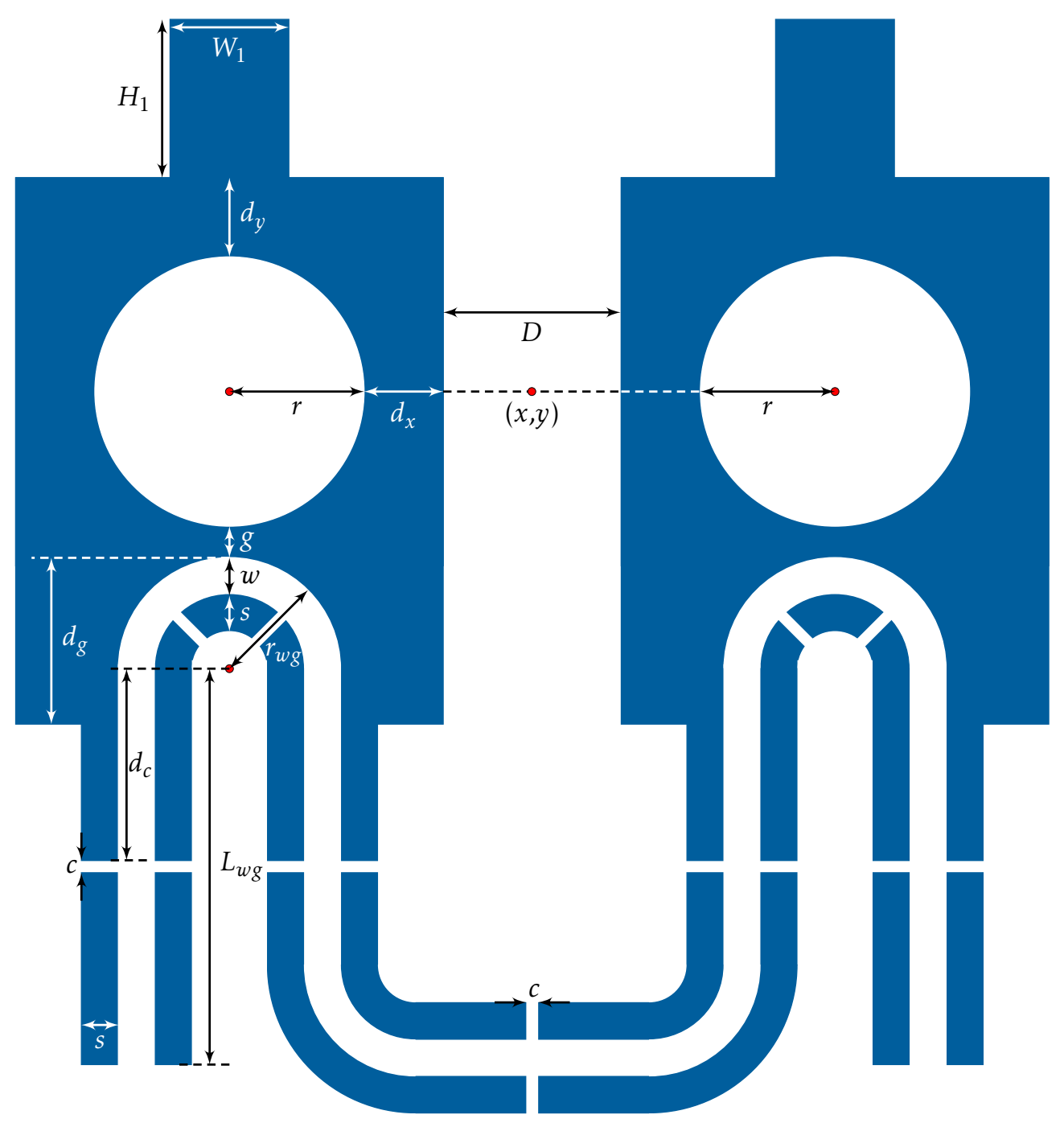

Figure 2.277: wgdcdV3 constructor. 
$\begin{array}{lllllllllllllllllll}x & y & r & N_{\text {sides }} & d_{x} & d_{y} & d_{g} & H_{1} & t_{H} & w & L_{w g} & r_{w g} & s & g & c & d_{c} & D & \theta_{(x, y)} & \text { wgdcdV4> }\end{array}$

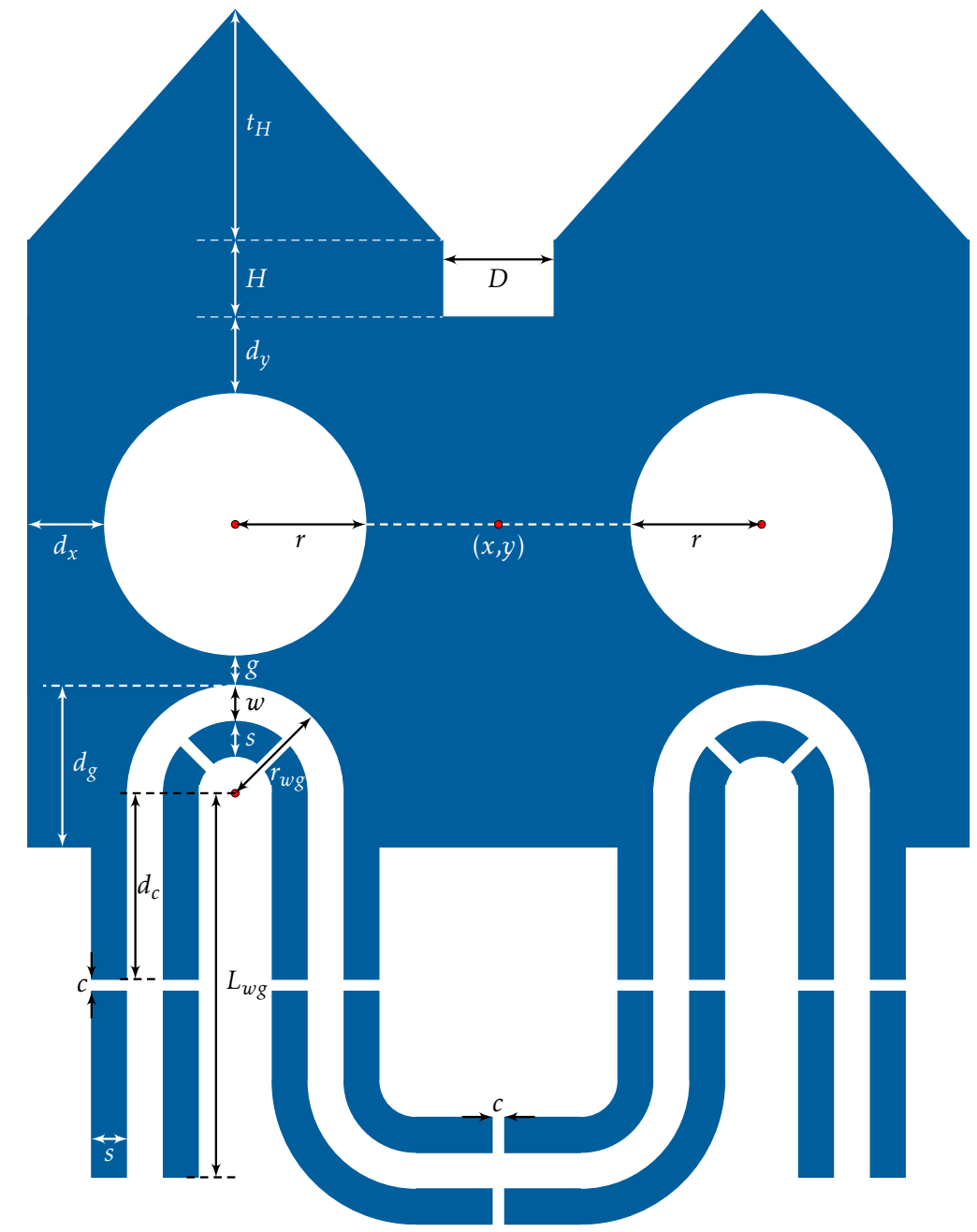

Figure 2.278: wgdcdV4 constructor. 
$\begin{array}{lllllllllllllllllllll}x & y & r & N_{\text {sides }} & d_{x} & d_{y} & d_{g} & t_{H} & w & L_{w g} & r_{w g} & s & g & c & d_{c 1} & d_{c 2} & \theta_{(x, y)} & \text { wgdcdV5 }>\end{array}$

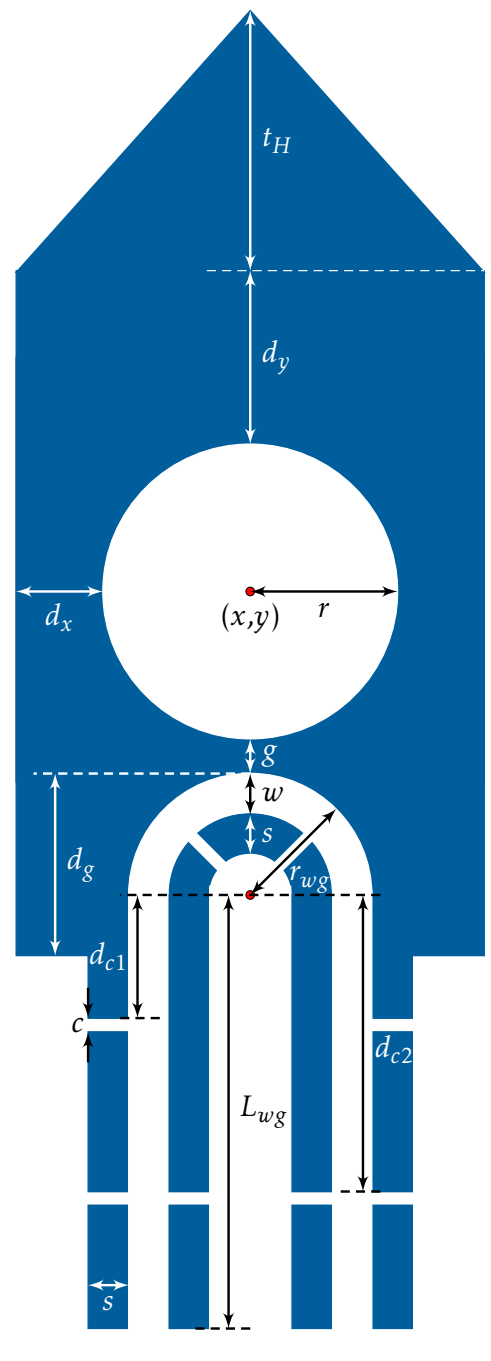

Figure 2.279: wgdcdV5 constructor. 
$\begin{array}{llllllllllllllllllll}< & y & r & N_{\text {sides }} & d_{x} & d_{y} & d_{g} & H & t_{H} & w & L_{w g} & r_{w g} & s & g & c & d_{c 1} & d_{c 2} & \theta_{(x, y)} & \text { wgdcdV6 }\end{array}$

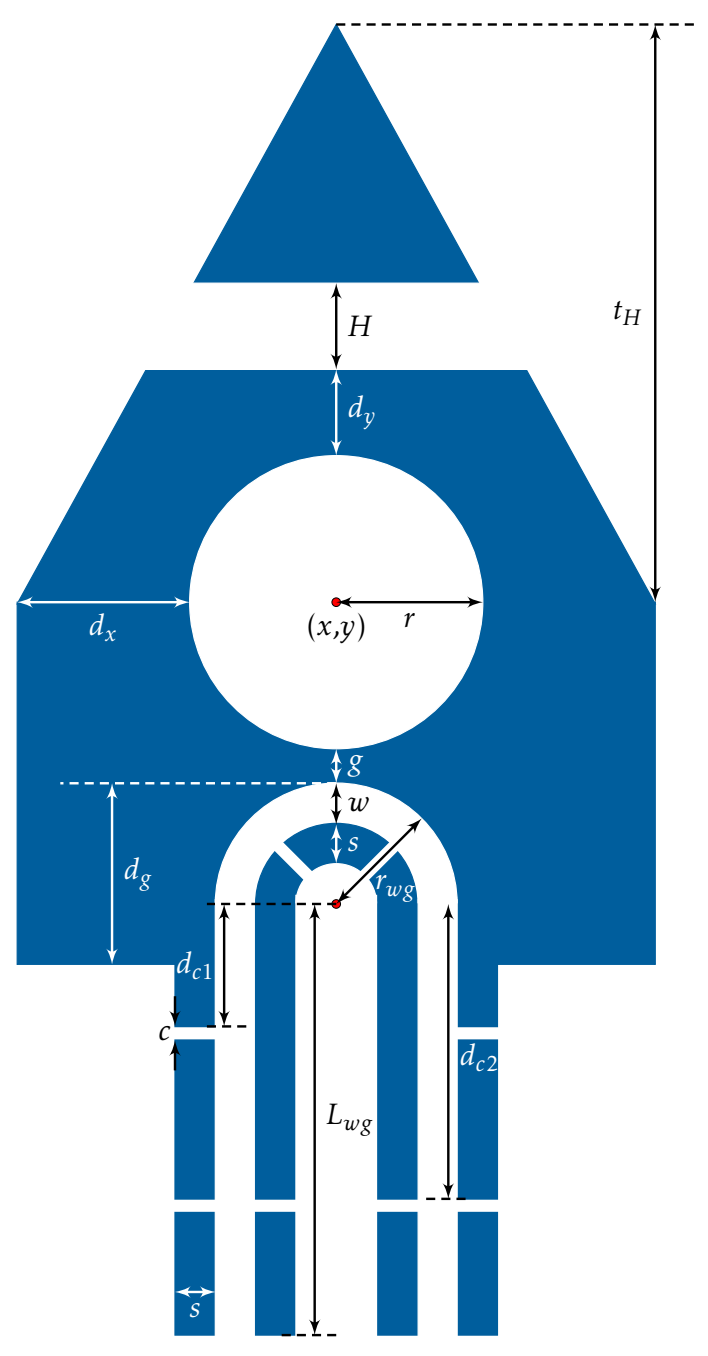

Figure 2.280: wgdcdV6 constructor. 
$\begin{array}{llllllllllllllll}< & y & r & r_{2} & N_{\text {sides }} & d_{y} & d_{x} & w & L_{w g} & r_{w g} & s & g & c & d_{c} & \theta_{(x, y)} & \text { wgdcdV7 }>\end{array}$

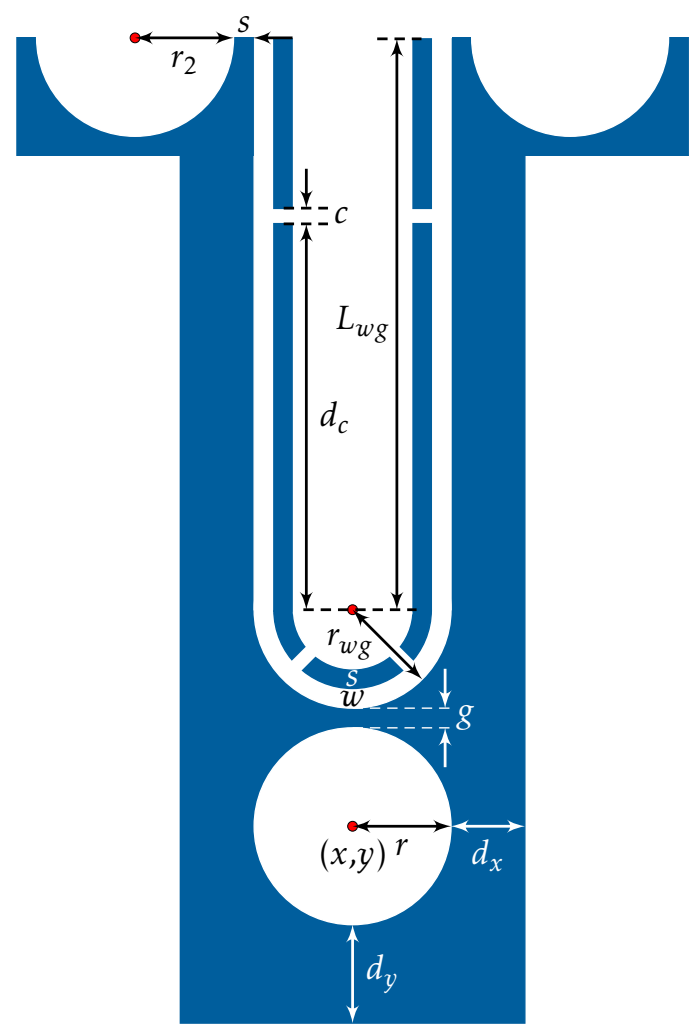

Figure 2.281: wgdcdV7 constructor. 
$\begin{array}{lllllllllllllllllllllll}<x & y & t & b & f & s & w_{1} & w_{2} & L_{s} & L_{t} & g & c & d_{c} & W & H & L_{1} & L_{2} & L_{3} & \theta_{(x, y)} & \text { wgdcdV8 } & \end{array}$

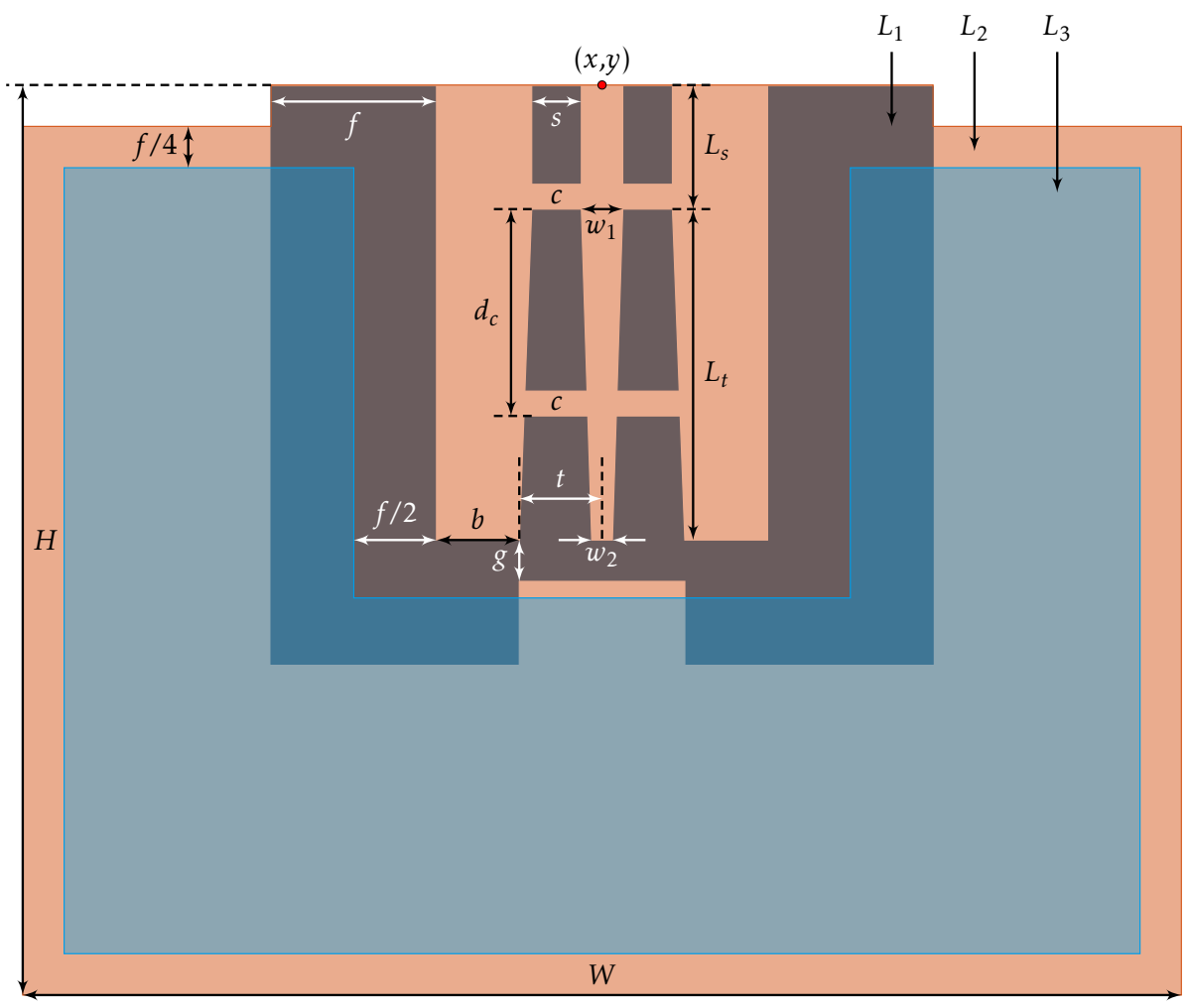

Figure 2.282: wgdcdV8 constructor. 


\subsection{MEMS - NEMS Library}

\subsubsection{Actuators}

\subsubsection{Bent Beams}

Bent beams form a class of linear displacement actuators. In this case, motion occurs via thermal expansion of the beam.

$$
\begin{array}{lllllllllllll}
x & y & w & l_{1} & l_{2} & l_{3} & b_{H} & b_{W} & a & L_{a} & \theta_{(x, y)} & \text { bentBeam }
\end{array}
$$

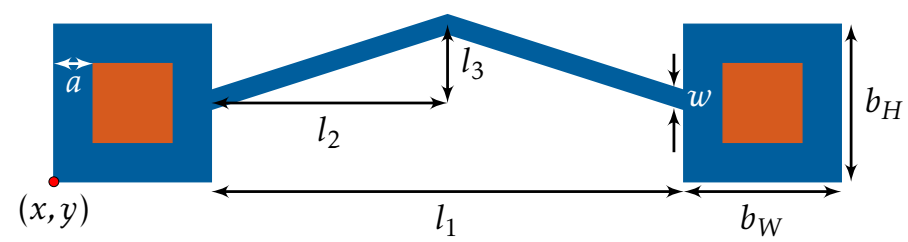

Figure 2.283: Bent beam of width $w$ suspended between two anchored electrodes. The anchor overlap and GDS layer parameters are defined by $a$ and $L_{a}$.

$\begin{array}{llllllllllllllllllll}x & y & w & l_{1} & l_{2} & l_{3} & l_{4} & h & p & N & c_{W} & d_{H} & d_{W} & L_{d} & b_{W} & a & L_{a} & \theta_{(x, y)} & \text { bentBeamArray }\end{array}$

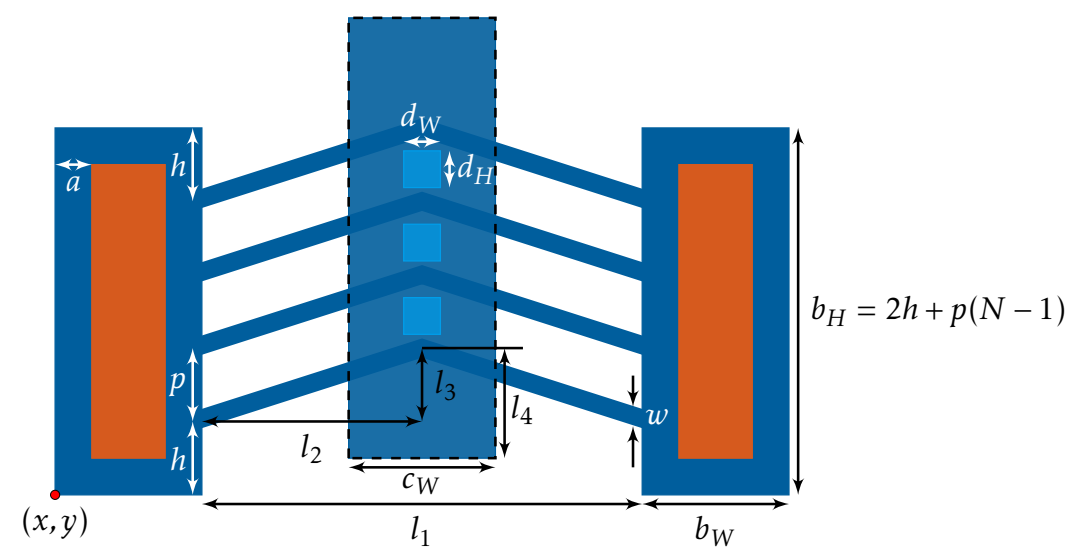

Figure 2.284: Array of $N$ bent beam elements with a central rectangular structure. The $d_{H}$ and $d_{W}$ parameter defines an extra GDS layer $\left(L_{d}\right)$ that could be used to perforate the central structure. 


\subsubsection{Bi-Morph Thermal Actuator}

Differential thermal expansion of the bimorph actuator allows for in-plane motion. This occurs when a voltage is applied, wherein current induced Joule heating causes uneven thermal expansion within the two beams of differing widths.

$\begin{array}{lllllllllllllllllll}x & y & w_{1} & w_{2} & w_{3} & w_{4} & l_{1} & l_{2} & l_{3} & p & s_{H} & s_{W} & L_{s} & b_{H} & b_{W} & a & L_{a} & \theta_{(x, y)} & \text { biMorph }\end{array}$

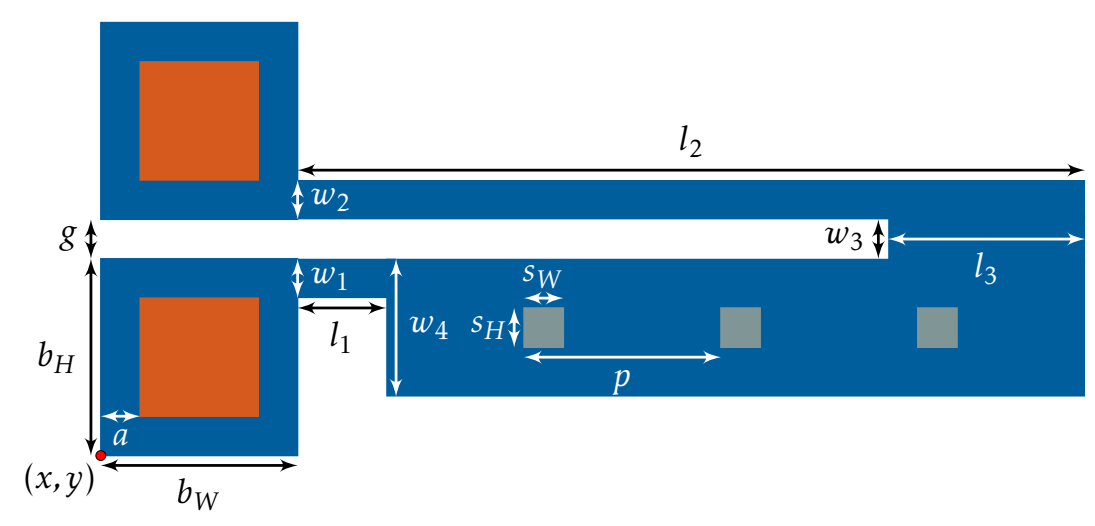

Figure 2.285: Bimorph thermal actuator. 


\subsubsection{Combs and Drive Elements}

Linear comb drive created using three GDS layers. Lower and upper base electrodes are created using the initialized and $L_{b}$ GDS layers respectively. The anchor overlap and GDS layers are correspondingly $a$ and $L_{a}$.

$$
\begin{array}{llllllllllllll}
x & y & w_{1} & w_{2} & l_{1} & l_{2} & N & p & b_{H} & L_{b} & a & L_{a} & \theta_{(x, y)} & \text { combDriveV1 }
\end{array}
$$

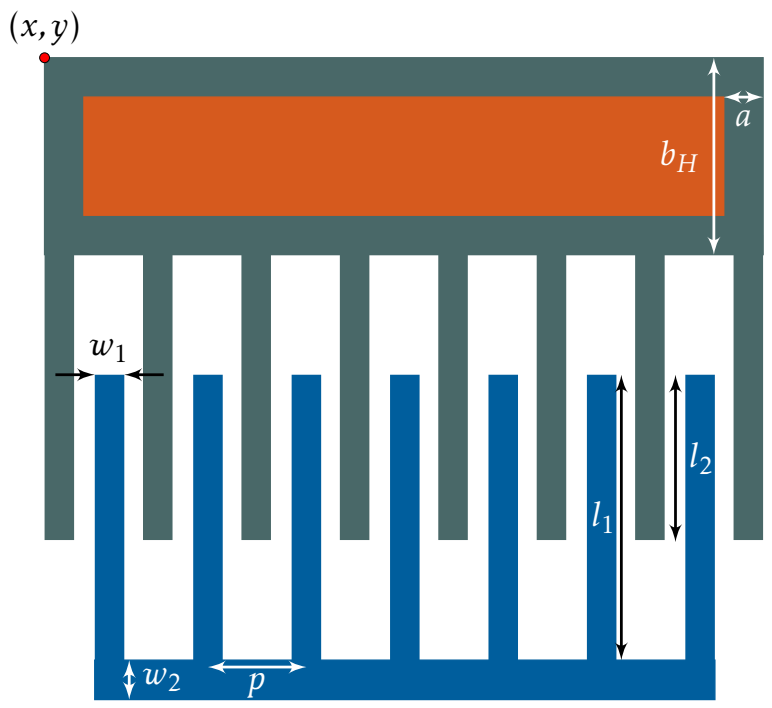

Figure 2.286: Linear comb drive. 
Number of electrode elements $(N)$ is used to calculate the number of rectangular rotor elements $\left(l_{1} \times w_{1}\right)$. The structure is composed from the initialized GDS layer (top and bottom electrodes), $L_{a}$ (anchor) and $L_{b}$ (rotor).

$\begin{array}{lllllllllllllllll}x & y & w_{1} & l_{1} & l_{2} & l_{3} & g & N & p & b_{H} & b_{W} & p_{b} & L_{b} & a & L_{a} & \theta_{(x, y)} & \text { linearDriveL1 }\end{array}$

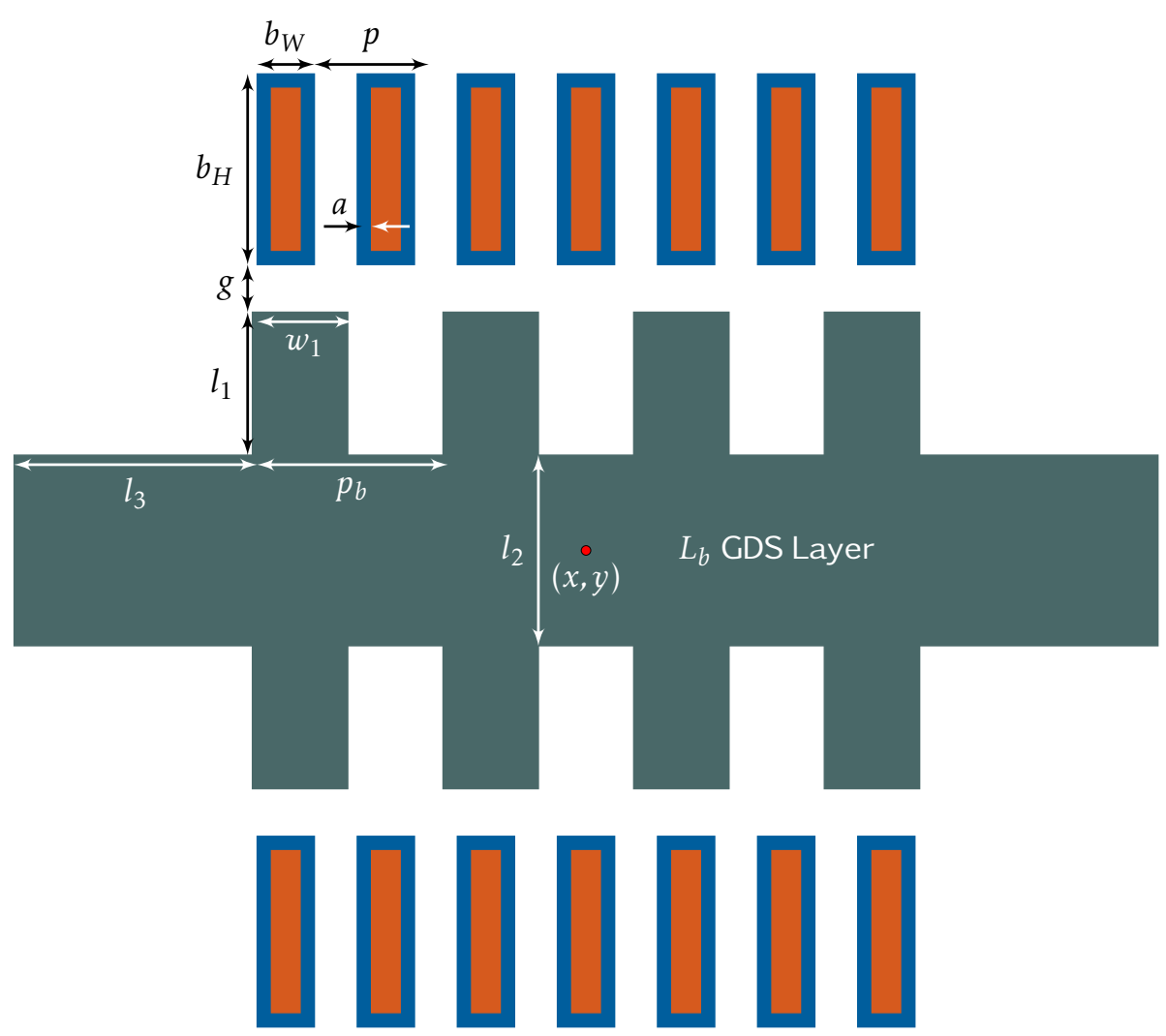

Figure 2.287: Linear drive composed of anchored electrodes and a central rotor. 


\subsubsection{Folded Springs}

$\begin{array}{llllllllllllll}x & y & w & l_{1} & l_{2} & p & A & N & b_{H} & b_{W} & a & L_{a} & \theta_{(x, y)} & \text { foldedSpring1A }\end{array}$

$\begin{array}{llllllllllllll}x & y & w & l_{1} & l_{2} & p & A & N & b_{H} & b_{W} & a & L_{a} & \theta_{(x, y)} & \text { foldedSpring1B }\end{array}$

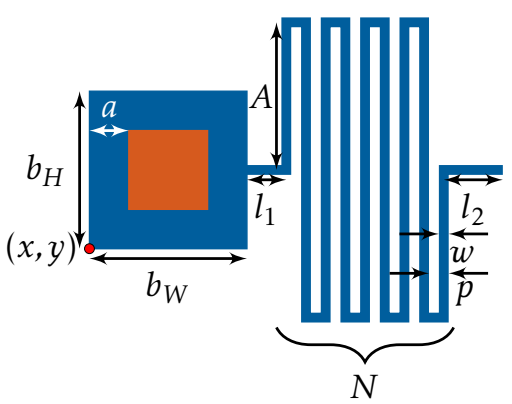

(a)

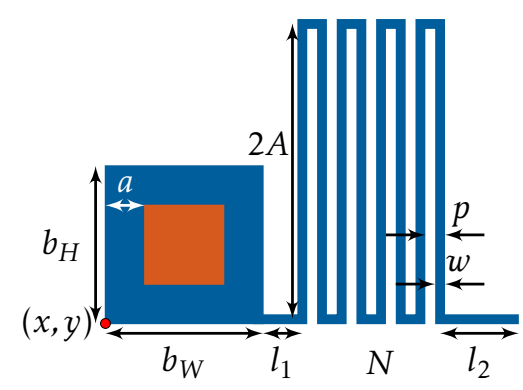

(b)

Figure 2.288: Folded springs with a single anchored pad. The spring meander initiates at the (a) midpoint and (b) bottom of pads. 


$$
\begin{array}{llllllllllllll}
x & y & w & l_{1} & l_{2} & p & A & N & b_{H} & b_{W} & a & L_{a} & \theta_{(x, y)} & \text { foldedSpring2A } \\
x & y & w & l_{1} & l_{2} & p & A & N & b_{H} & b_{W} & a & L_{a} & \theta_{(x, y)} & \text { foldedSpring2B }
\end{array}
$$

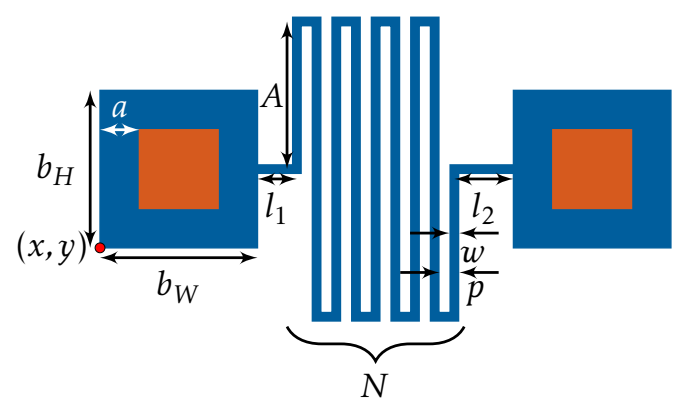

(a)

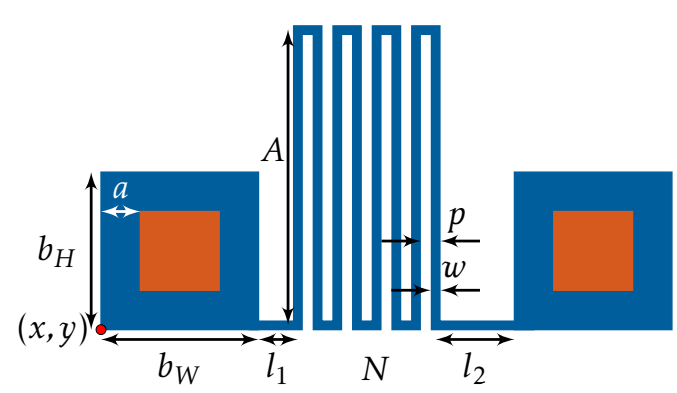

(b)

Figure 2.289: Folded springs with two anchored pads. The spring meander initiates at the (a) midpoint and (b) bottom of pads. 
$\begin{array}{llllllllllllllll}x & y & w & l_{1} & l_{2} & p & A & N & b_{H} & b_{W} & a & L_{a} & \theta_{(x, y)} & \text { foldedSpring2C }\end{array}$

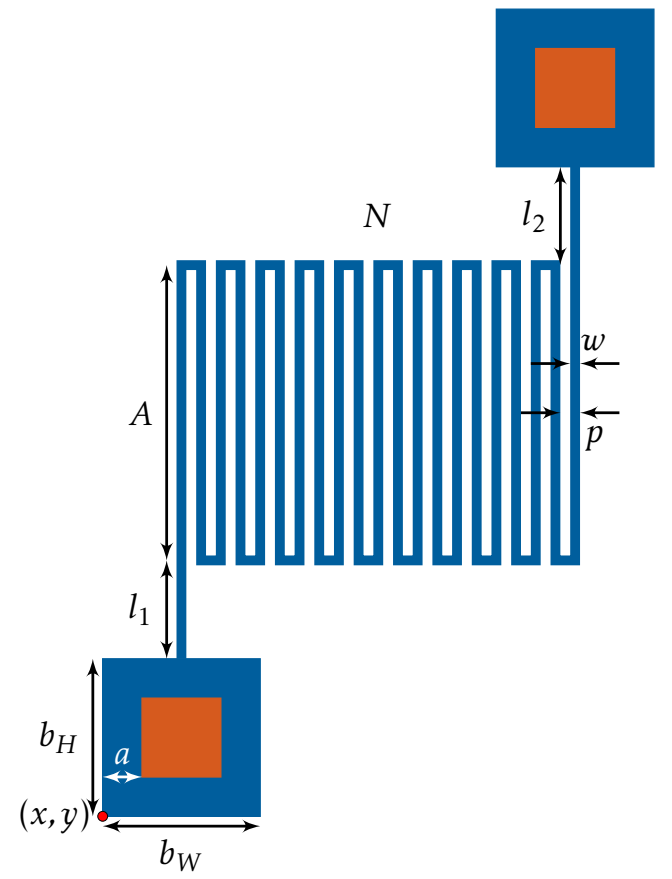

Figure 2.290: Folded springs with two anchored pads V2C. 
$\begin{array}{llllllllllllll}x & y & w & l_{1} & l_{2} & p & A & N & b_{H} & b_{W} & a & L_{a} & \theta_{(x, y)} & \text { foldedSpring2D }\end{array}$

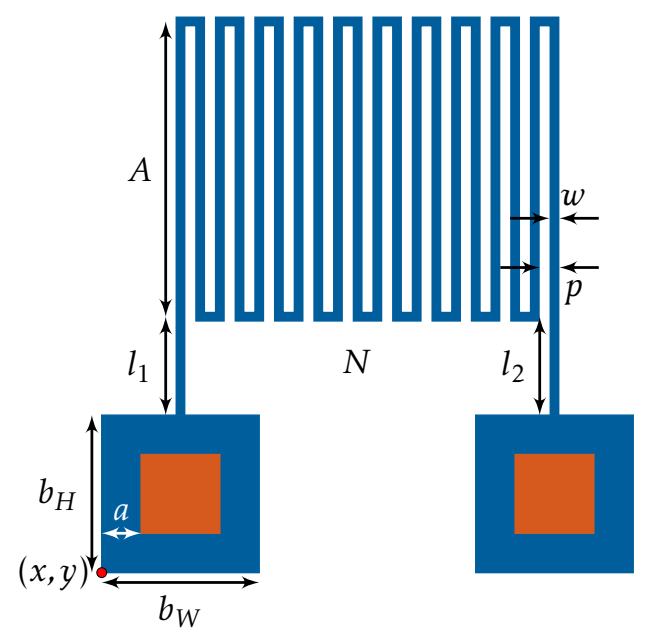

Figure 2.291: Folded springs with two anchored pads V2D. 

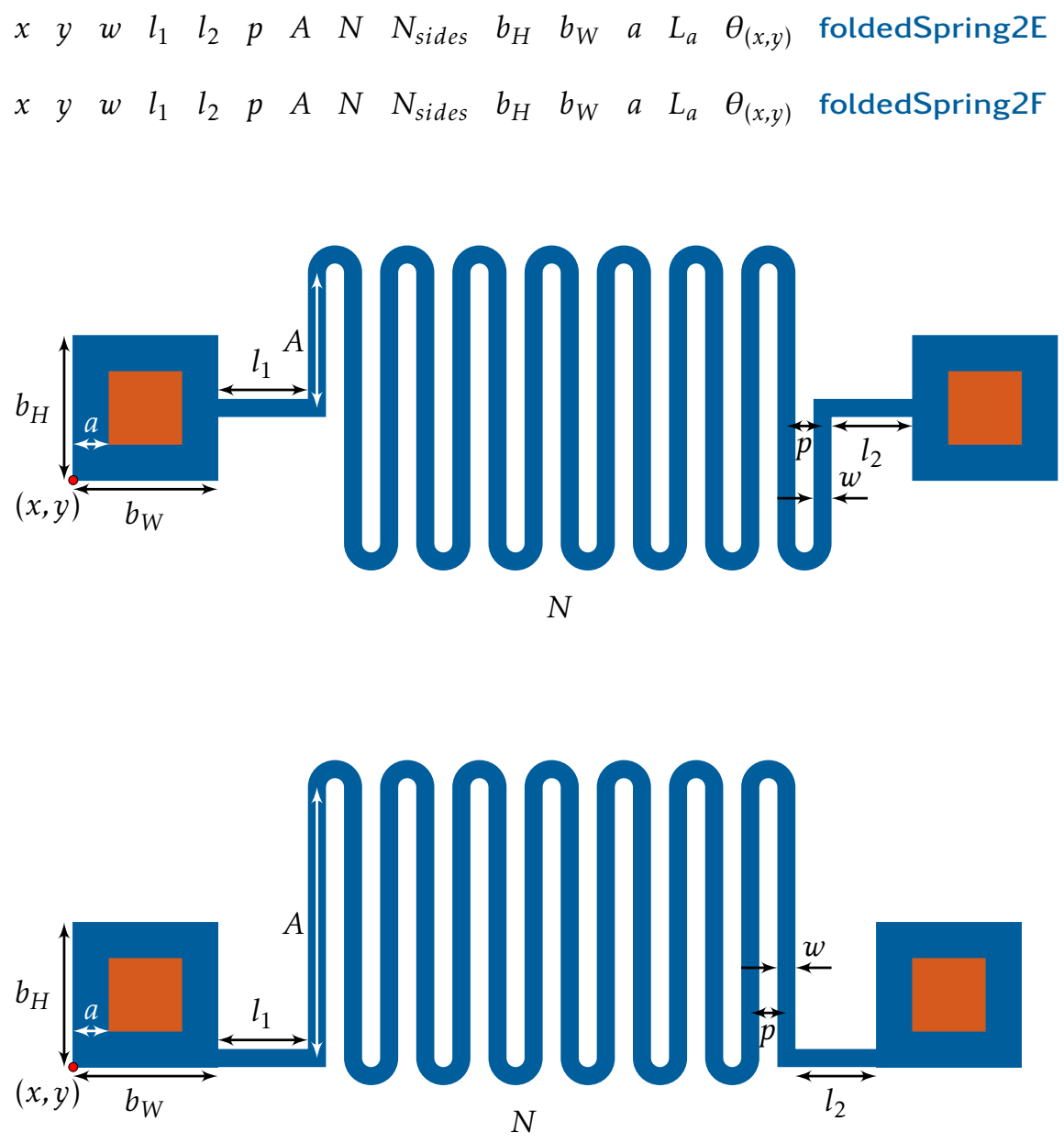

Figure 2.292: Folded springs with two anchored pads V2E and V2F. 
$\begin{array}{llllllllllllllllll}x & y & w & l_{1} & l_{2} & p & A & N & N_{\text {sides }} & b_{H} & b_{W} & a & L_{a} & \theta_{(x, y)} & \text { foldedSpring2G }\end{array}$

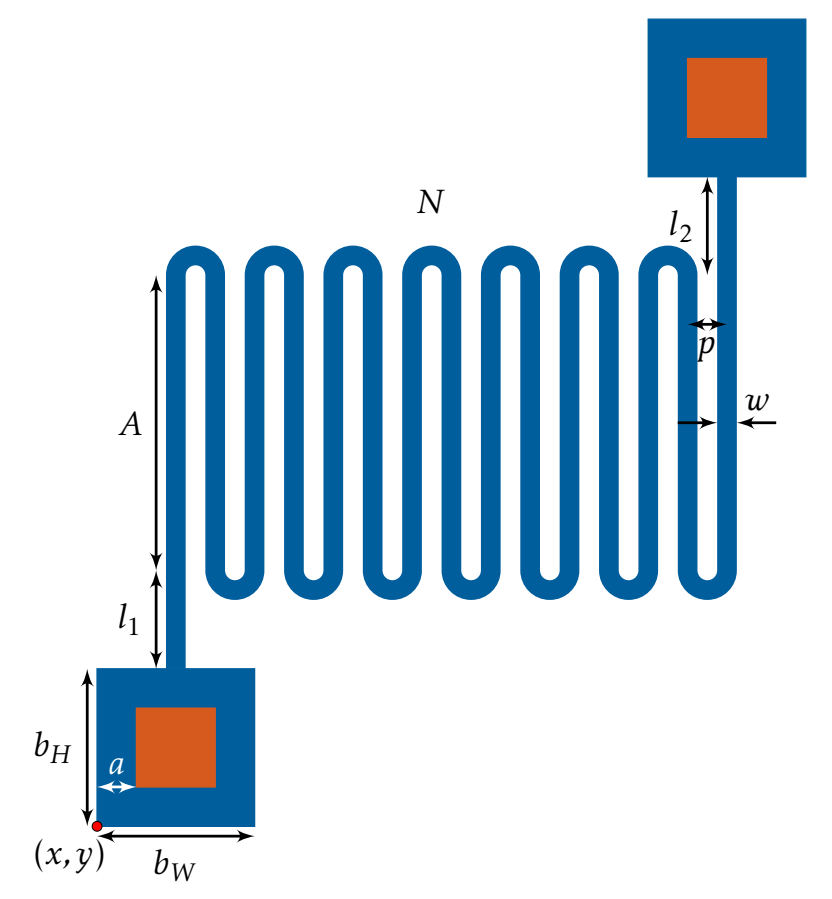

Figure 2.293: Folded springs with two anchored pads V2G. 
$\begin{array}{llllllllllllllllll}x & y & w & l_{1} & l_{2} & p & A & N & N_{\text {sides }} & b_{H} & b_{W} & a & L_{a} & \theta_{(x, y)} & \text { foldedSpring } 2 \mathrm{H}\end{array}$

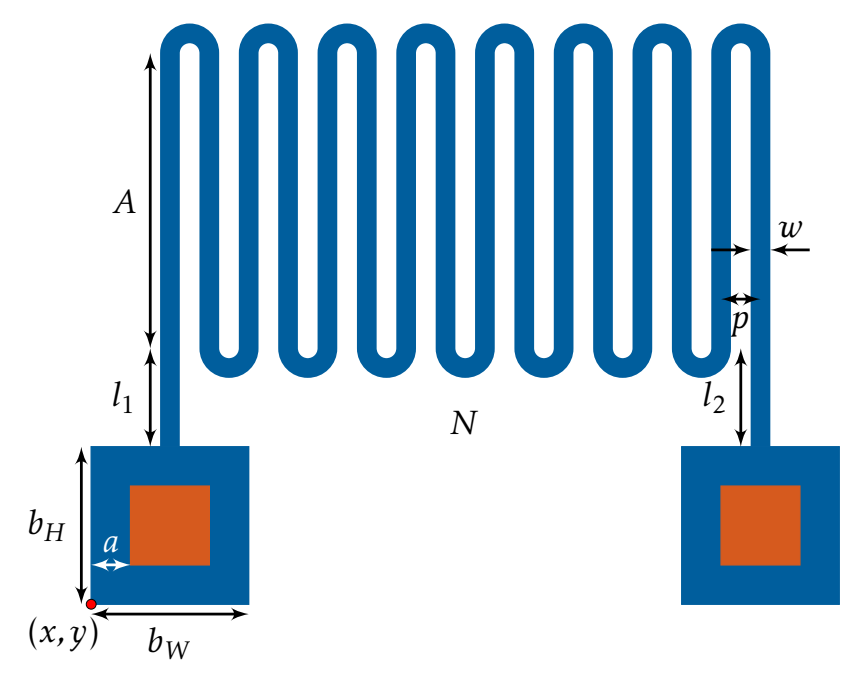

Figure 2.294: Folded springs with two anchored pads V2H. 
$\begin{array}{llllllllllllll}x & y & w & l_{1} & l_{2} & p & A & N & N_{\text {sides }} & r & a & L_{a} & \theta_{(x, y)} & \text { foldedSpring2l }\end{array}$

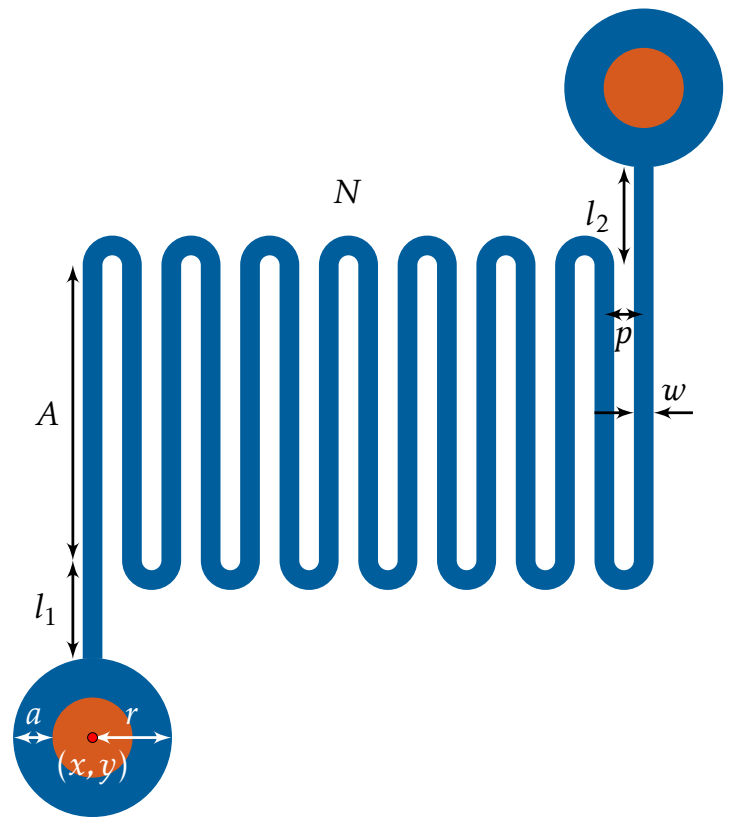

Figure 2.295: Folded springs with two anchored pads V2I. 
$\begin{array}{lllllllllllllll}x & y & w & l_{1} & l_{2} & p & A & N & N_{\text {sides }} & r & a & L_{a} & \theta_{(x, y)} & \text { foldedSpring2J }\end{array}$

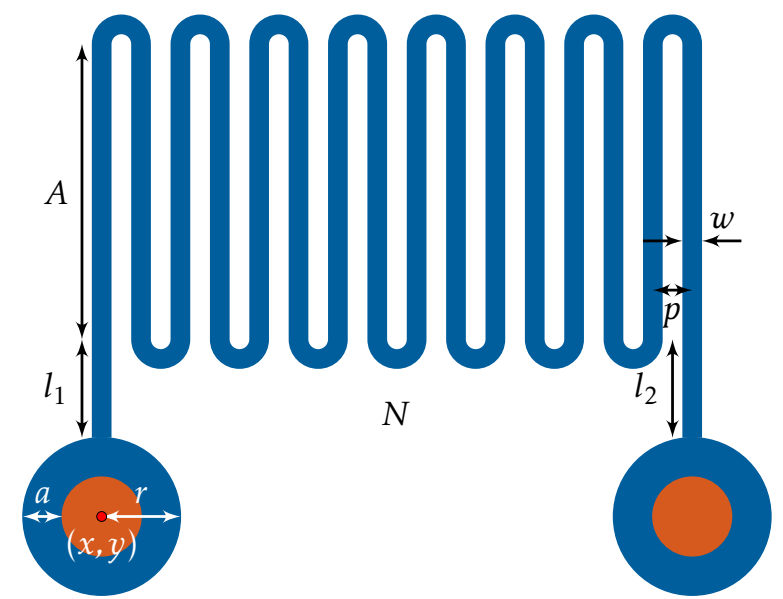

Figure 2.296: Folded springs with two anchored pads V2J. 


\subsubsection{Bolometers}

Below L-Shaped bolometer is constructed from GDS layers $L_{a}, L_{b}, L_{c}, L_{d}, L_{e}$, and $L_{f}$ using the below specified parameters. Rounded rectangles (layers $L_{d}$ and $L_{e}$ ) are defined by a radius $r_{i}$. The number of points defining the curved region of the rounded rectangle is defined by the shapeReso parameter. Meandering curved region defined by radius $r$ is constructed using $N_{\text {sides }}$ number of points.

$x y w_{1} w_{2} w_{3} g_{1} g_{2} g_{3} L_{1} L_{2} r r_{i} N_{\text {sides }} a b c d$ e $f L_{a} L_{b} L_{c} L_{d} L_{e} L_{f} \theta_{(x, y)}$ bolometerL

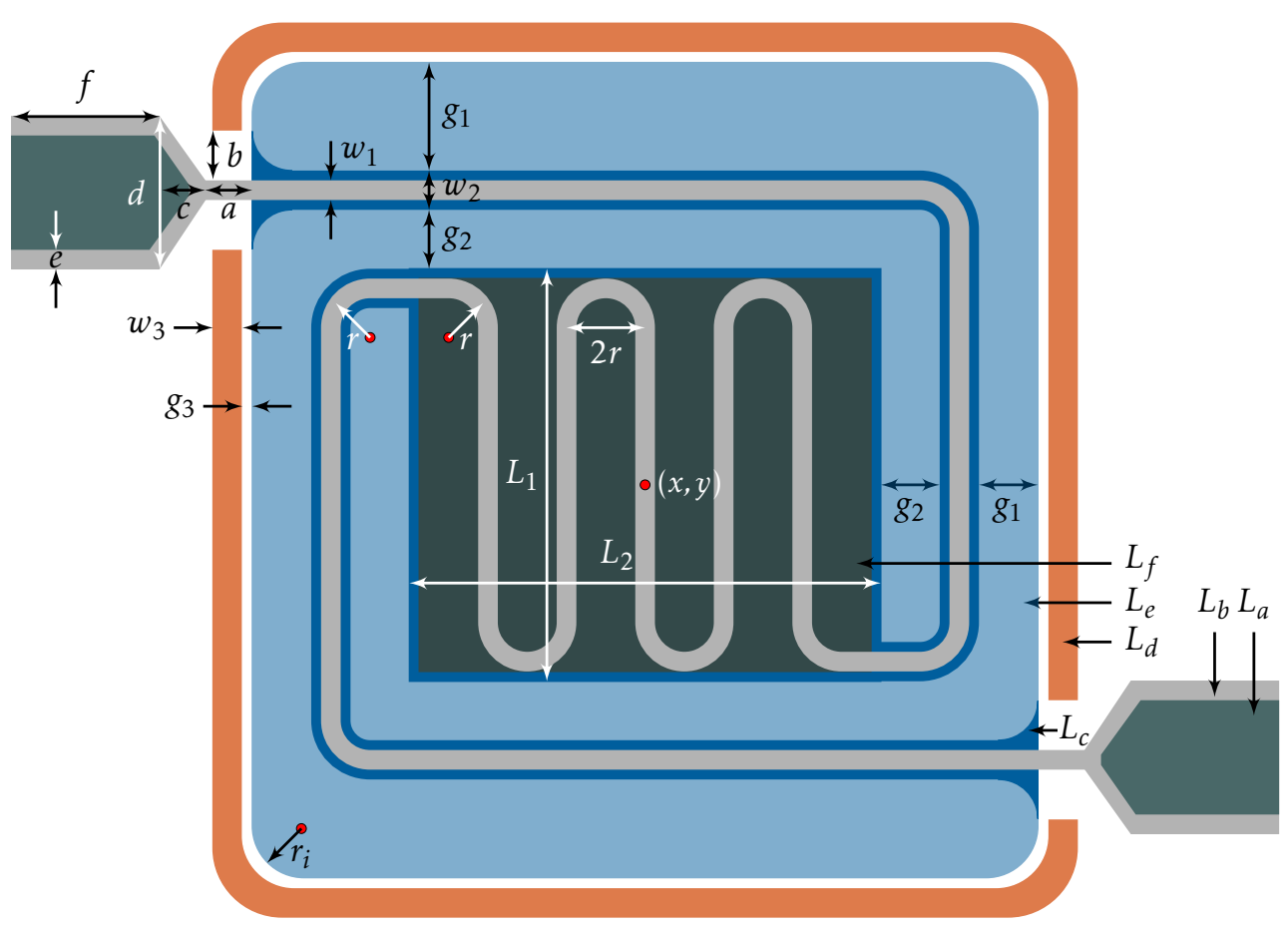

Figure 2.297: L-shaped bolometer structure. 
$x y w_{1} w_{2} w_{3} g_{1} g_{2} g_{3} L_{1} L_{2} r r_{i} N_{\text {sides }} a b c d$ e $f L_{a} L_{b} L_{c} L_{d} L_{e} L_{f} \theta_{(x, y)}$ bolometerU

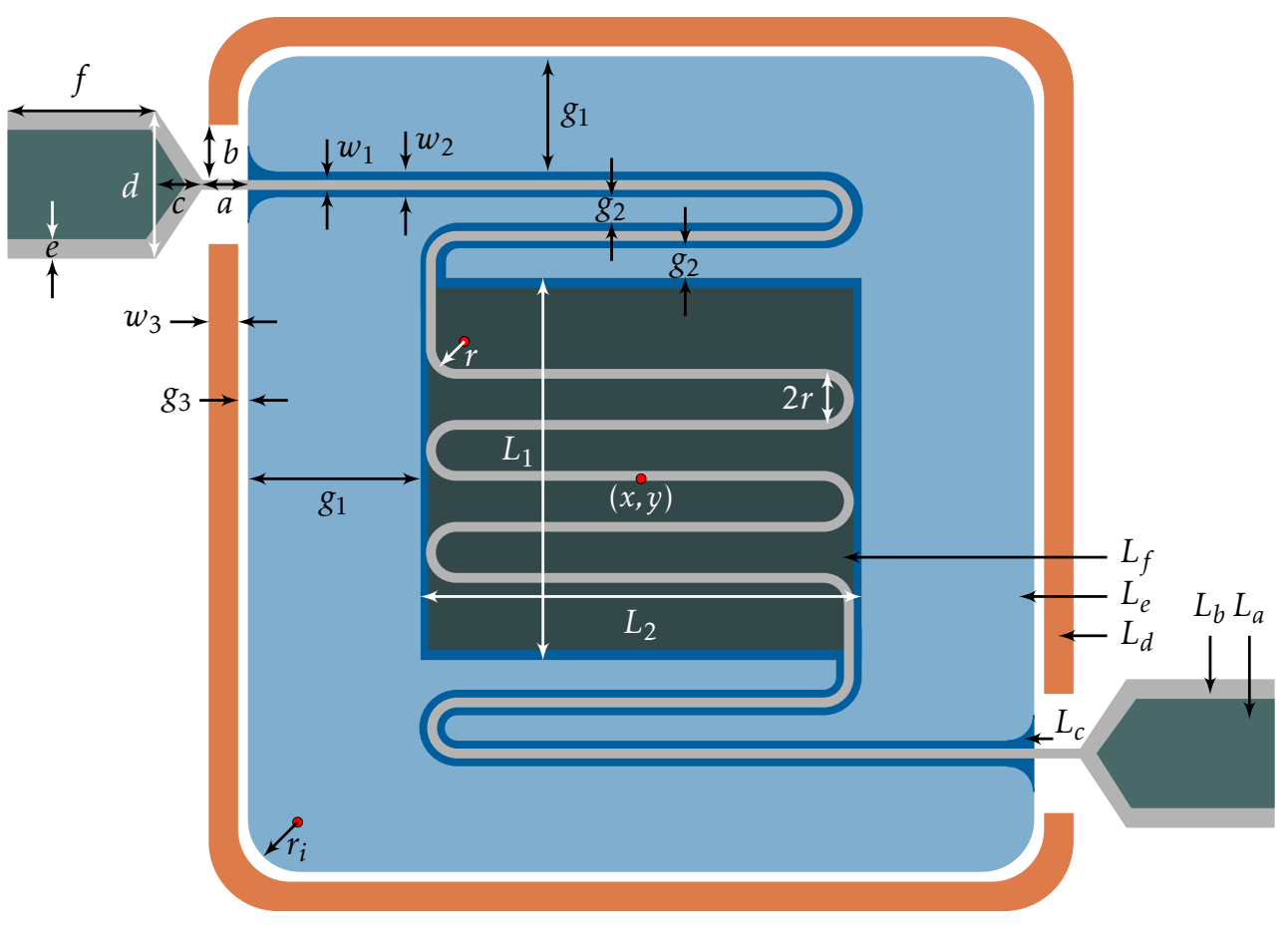

Figure 2.298: U-shaped bolometer structure. 


\subsubsection{Gears}

MEMS gear elements defined by the $(x, y)$ position, radius $r$ with $N$ sides, gear width and height, $w$ and $h$, respectively. Number of gear elements $N_{G}$ are evenly distributed along the disc circumference.
$\begin{array}{lllllllll}x & y & r & w & h & N_{G}\end{array}$
$N \theta_{(x, y)}$ gear
$\begin{array}{lllllllllllll}x & y & r & w & h & N_{G} & w_{t} & N & \theta_{(x, y)} & \text { gearT }\end{array}$

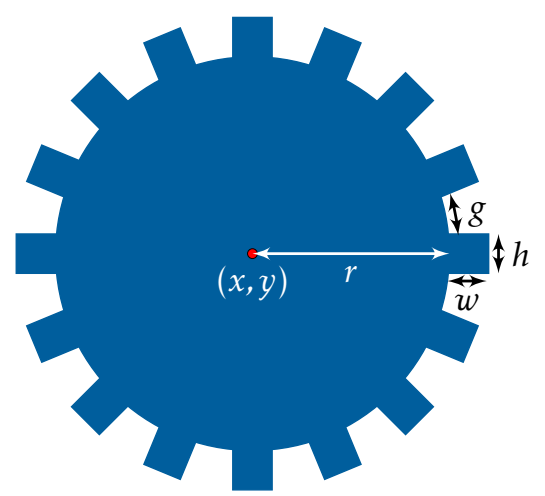

(a)

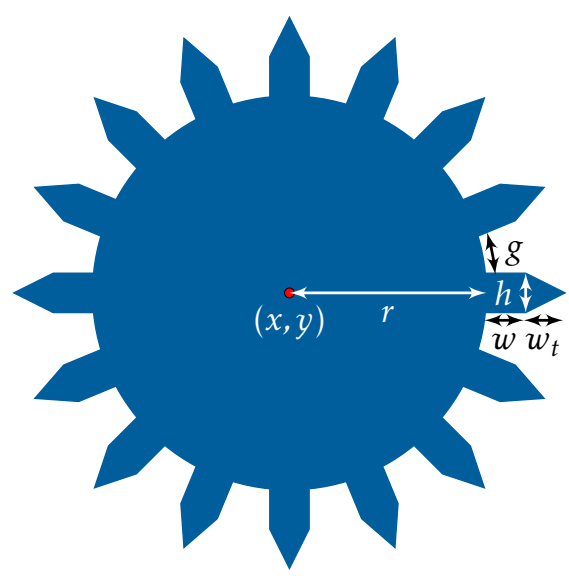

(b)

Figure 2.299: MEMS gear elements created using (a) gear and (b) gearT constructors. 


\subsubsection{Hub With Straight and Circular Springs}

$\begin{array}{lllllllllllll}x & y & w & r_{i} & w_{r} & R & N_{\text {sides }} & N_{\text {beams }} & a & L_{a} & \theta_{(x, y)} & \text { straightSpring }\end{array}$

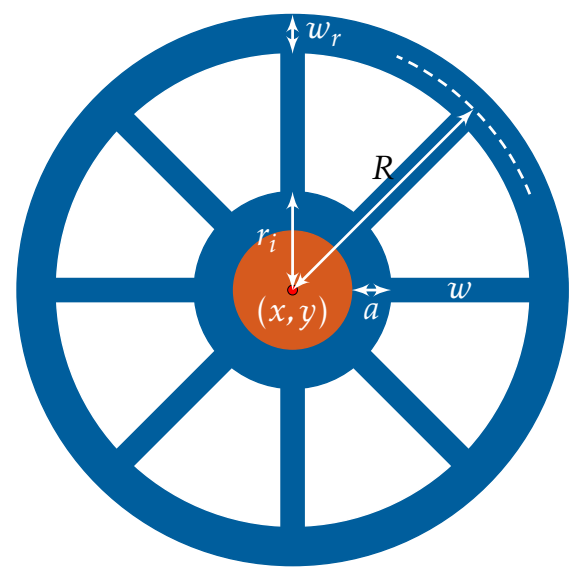

Figure 2.300: Concentric hub with straight springs.

$\begin{array}{llllllllllll}x & y & w & r_{i} & w_{r} & R & N_{\text {sides }} & N_{\text {beams }} & a & L_{a} & \theta_{(x, y)} & \text { circularSpring }\end{array}$

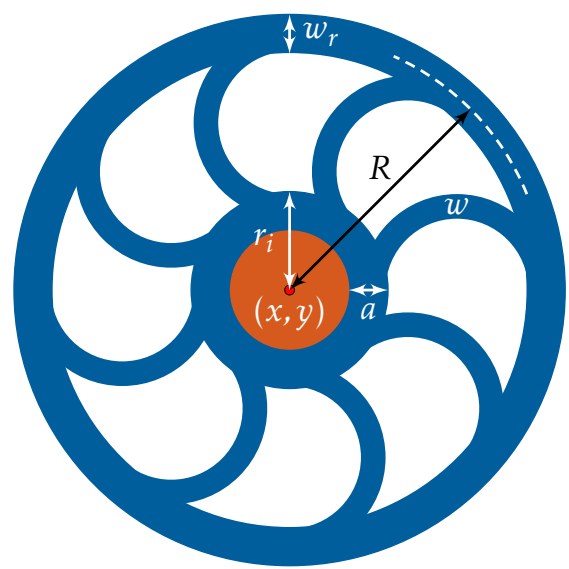

Figure 2.301: Concentric hub with circular springs. 
In figure 2.302, size of the surrounding electrodes depends on the number of electrodes $\left(N_{e}\right)$ and the dimensionless parameter $\beta$, where $0<\beta<1$. The gap between the electrodes is defined as $\beta L_{e s}$, where the electrode length segment is defined as $L_{e s}=C / N_{e} . C=2 \pi r^{\star}$ is the circumference of the innermost electrode, where $r^{\star}=R+w_{r} / 2+g$. Therefore, $\beta$ defines the electrode gap as the fraction of the electrode segment. Number of sides $N_{s}$ parameter is used to construct all circular objects.

$\begin{array}{lllllllllllllllll}x & y & w & r_{i} & w_{r} & R & N_{\text {sides }} & N_{\text {beams }} & g & W_{e} & N_{e} & \beta & e_{a} & a & L_{a} & \theta_{(x, y)} & \text { straightSpringE }\end{array}$

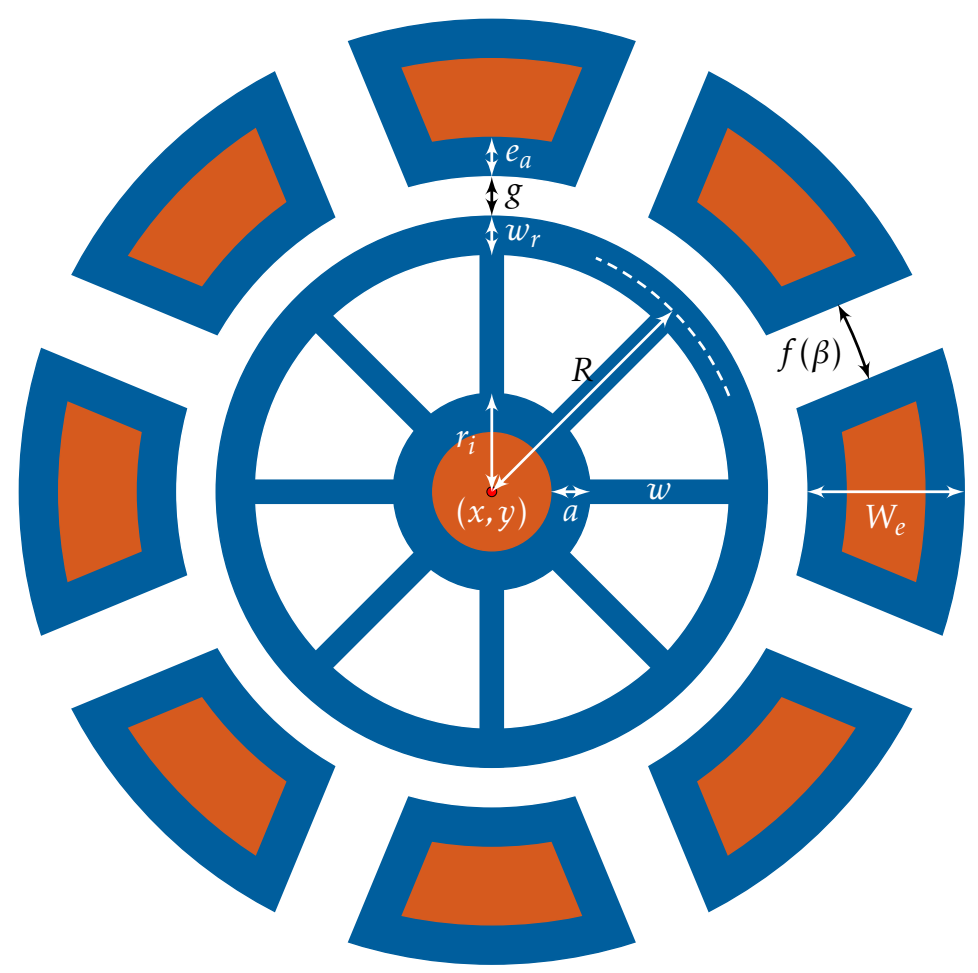

Figure 2.302: Concentric hub with straight springs and surrounding electrodes. 
$\begin{array}{lllllllllllllllll}x & y & w & r_{i} & w_{r} & R & N_{\text {sides }} & N_{\text {beams }} & g & W_{e} & N_{e} & \beta & e_{a} & a & L_{a} & \theta_{(x, y)} & \text { circularSpringE }\end{array}$

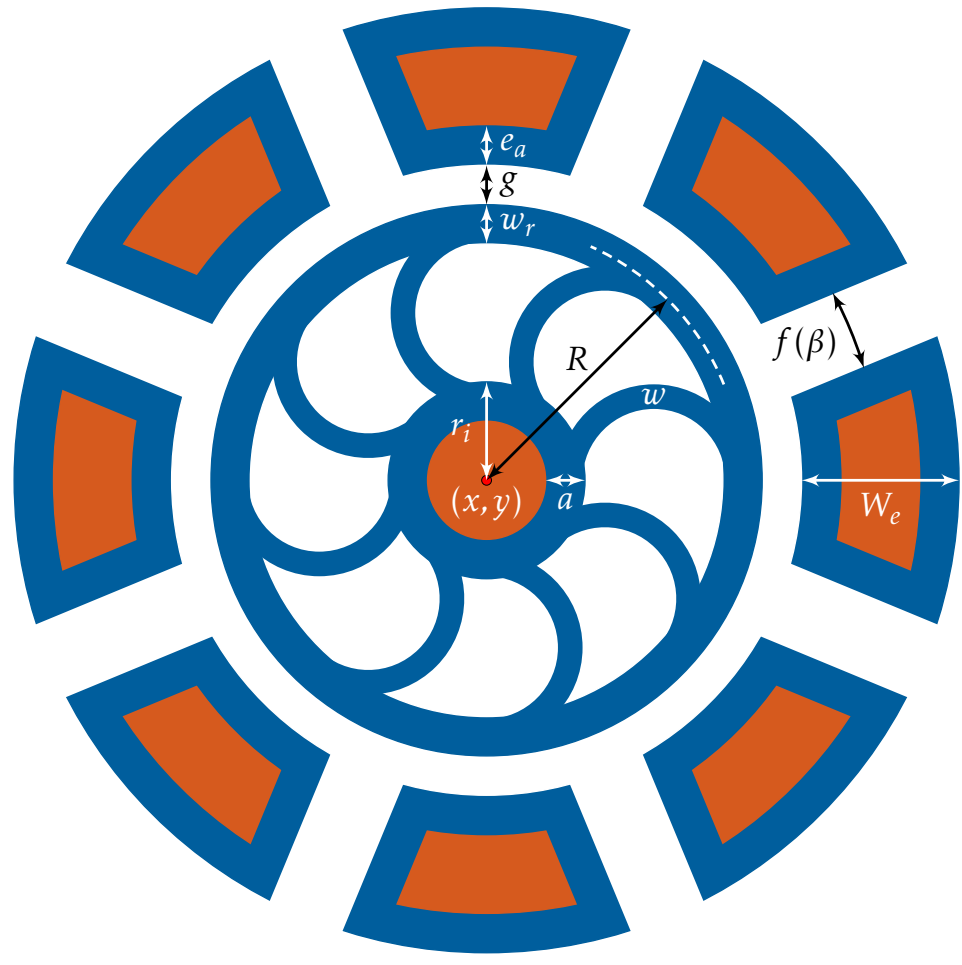

Figure 2.303: Concentric hub with circular springs and surrounding electrodes. 


\subsubsection{Radial Comb Drive}

Below constructors create radial comb-drives defined by an opening angle $(\theta)$, overlap angle $\left(\theta_{\circ}\right)$, electrode widths $\left(w_{1}, w_{2}\right)$, comb width $\left(w_{c}\right)$ and gap $(g)$, number of combs $\left(N_{\text {combs }}\right)$, number of sides for each comb arc segment $\left(N_{\text {sides }}\right)$, anchor overlap $(a)$ and anchor $\operatorname{GDS}$ layer $\left(L_{a}\right)$.

$\begin{array}{llllllllllllllll}x & y & w_{1} & r_{1} & w_{2} & r_{2} & w_{c} & g & N_{\text {combs }} & N_{\text {sides }} & \theta & \theta_{\circ} & a & L_{a} & \theta_{(x, y)} & \text { combRadialV1 }\end{array}$

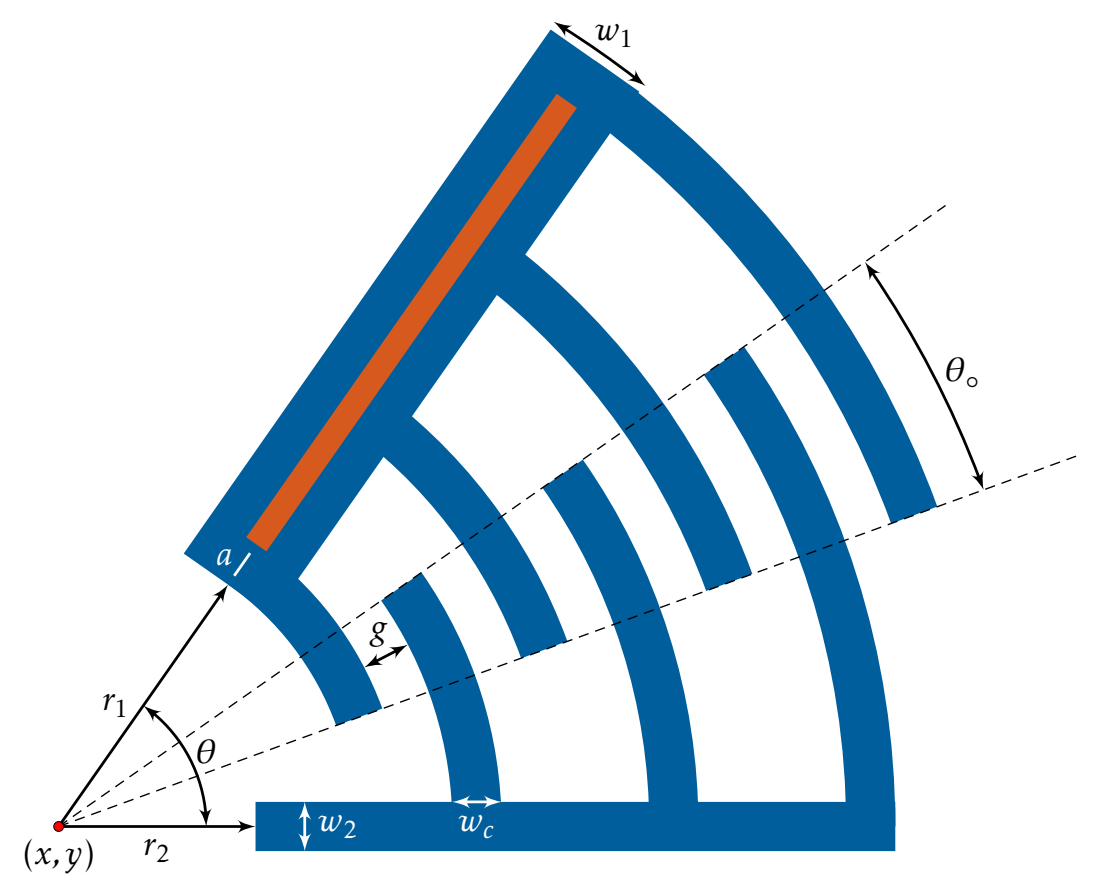

Figure 2.304: Radial comb drive V1. 
$\begin{array}{llllllllllllllll}x & y & w_{1} & r_{1} & w_{2} & r_{2} & w_{c} & g & N_{\text {combs }} & N_{\text {sides }} & \theta & \theta_{\circ} & a & L_{a} & \theta_{(x, y)} & \text { combRadialV2 }\end{array}$

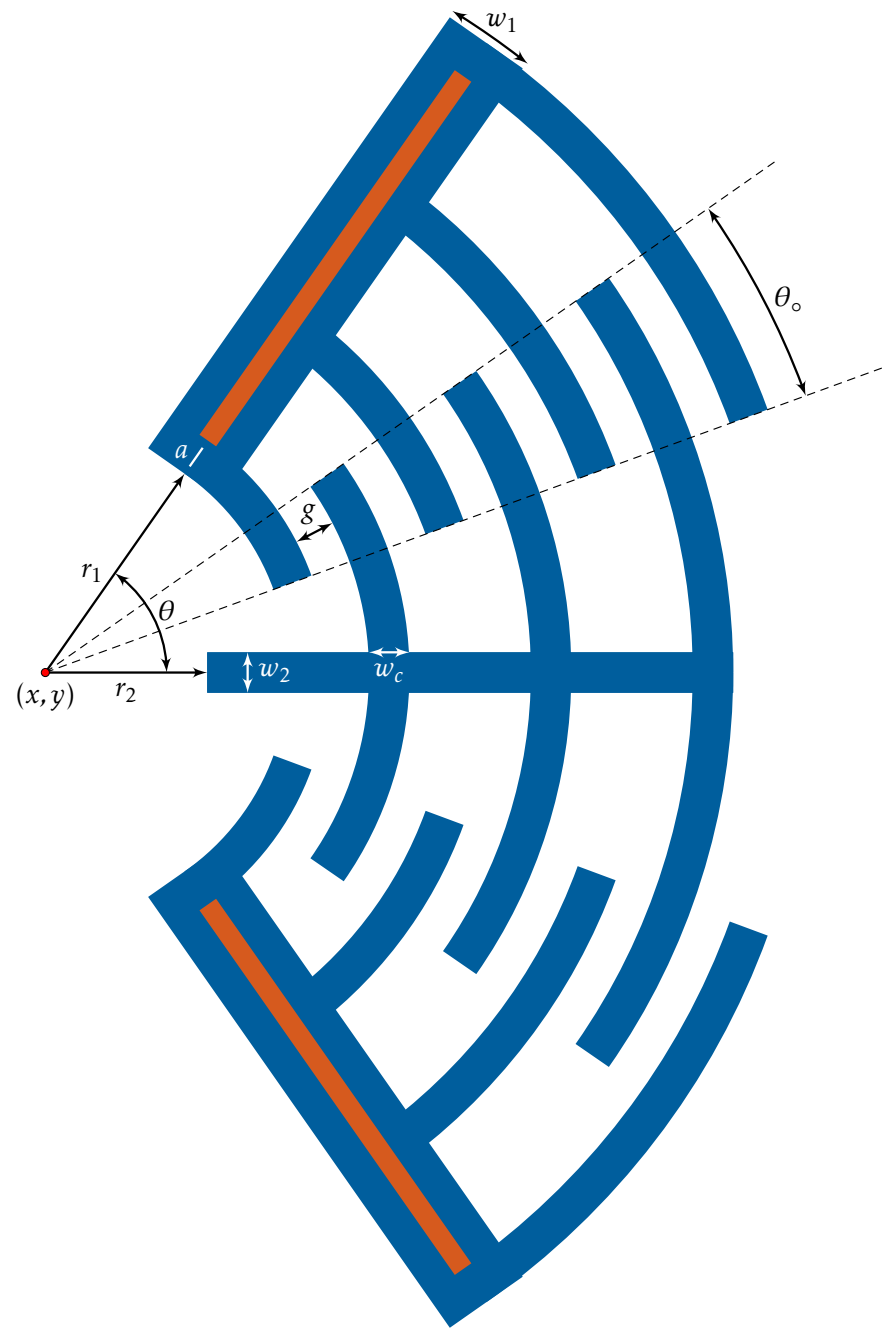

Figure 2.305: Radial comb drive V2. 


\subsubsection{Anchored Flexures}

The following constructors create accelerometer type anchored flexures with a proof mass. In all of the cases, the rectangular base support anchor is defined by the anchor support width $a$ and GDS layer $L_{a}$. Structural layer is defined by the active layer.

Anchored Flexure V2A

$\begin{array}{lllllllllllll}x & y & w & l_{1} & l_{2} & w_{m} & l_{m} & b_{H} & b_{W} & a & L_{a} & \theta_{(x, y)} & \text { flexure } 2 \mathrm{~A}\end{array}$

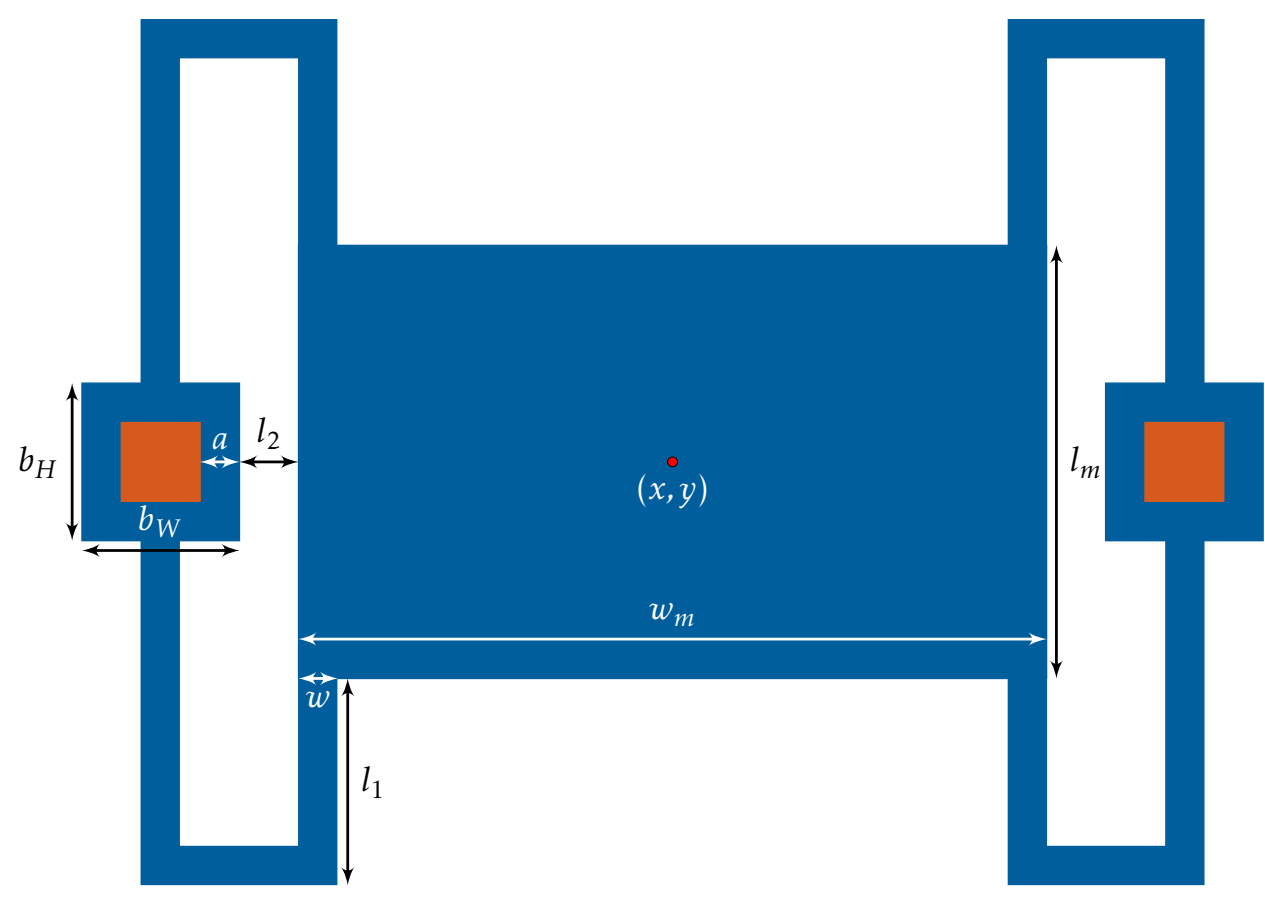

Figure 2.306: Anchored Flexure V2A with two hinges supporting a proof mass. 
Anchored Flexure V2B

$\begin{array}{lllllllllllllllll}x & y & w & l_{1} & l_{2} & w_{m} & l_{m} & c_{H} & c_{W} & s_{H} & s_{W} & b_{H} & b_{W} & a & L_{a} & \theta_{(x, y)} & \text { flexure2B }\end{array}$

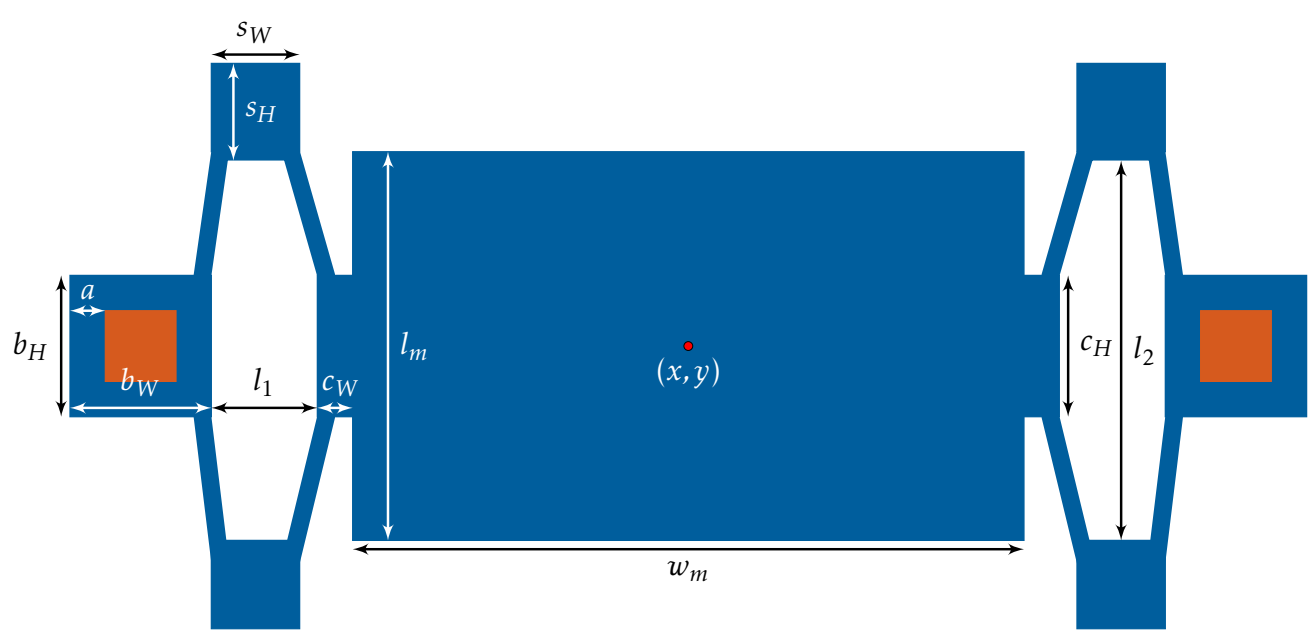

Figure 2.307: Anchored Flexure V2B with two hinges supporting a proof mass. 
Anchored Flexure V2C

$\begin{array}{lllllllllllllllllll}x & y & w & l_{1} & l_{2} & l_{3} & w_{m} & l_{m} & A & N_{p} & b_{H} & b_{W} & a & L_{a} & \theta_{(x, y)} & \text { flexure } 2 \mathrm{C}\end{array}$

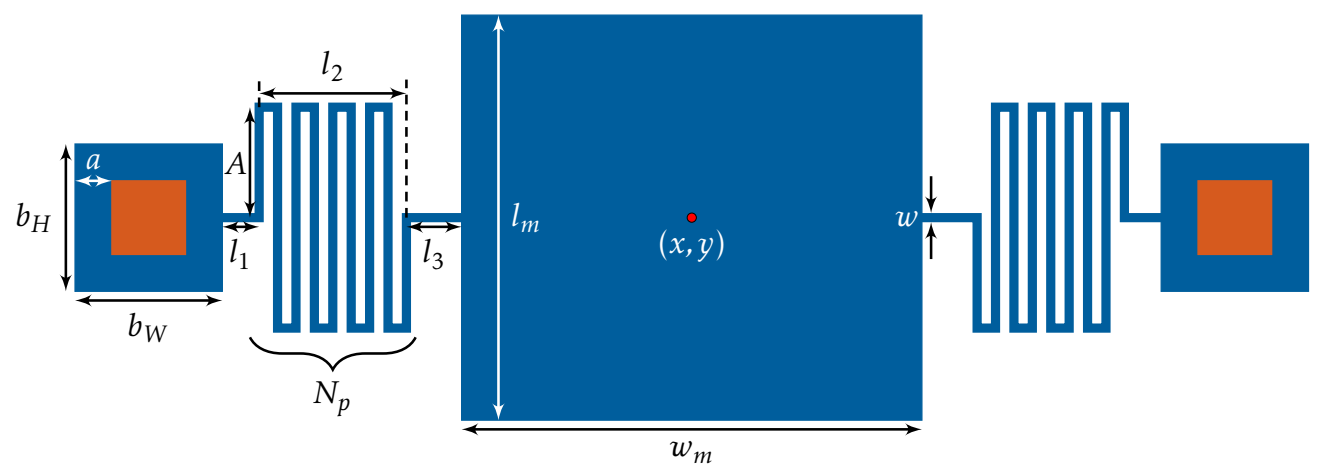

Figure 2.308: Anchored Flexure V2C with a meander supporting a proof mass. 
$\begin{array}{llllllllllllllllll}x & y & w_{1} & w_{2} & w_{3} & w_{4} & l_{1} & l_{2} & l_{3} & l_{4} & l_{5} & l_{6} & b_{W} & a & L_{a} & \theta_{(x, y)} & \text { flexure2D }\end{array}$

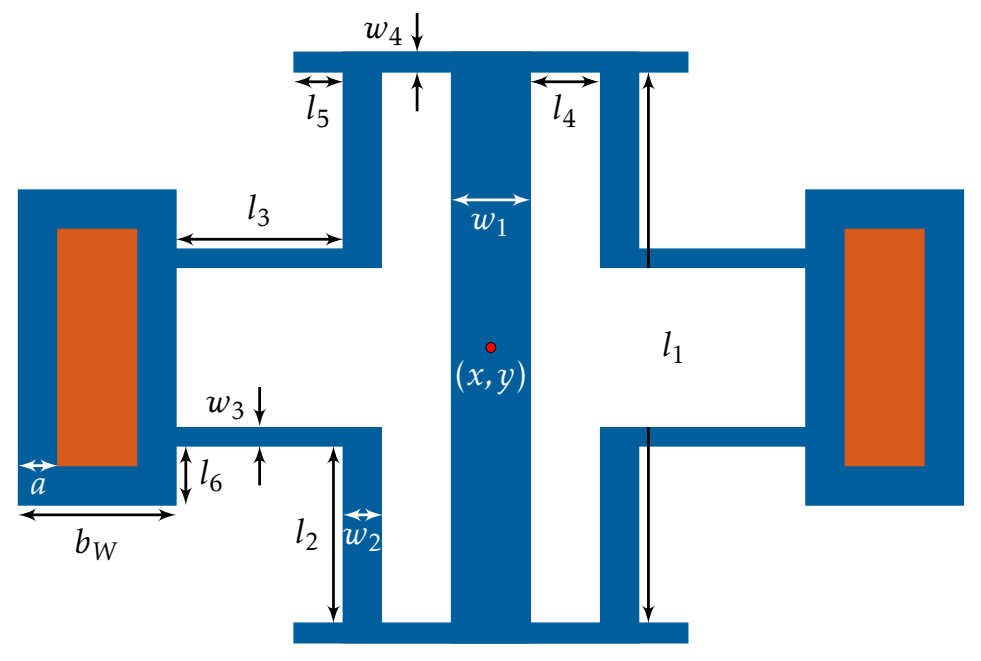

Figure 2.309: Anchored Flexure V2D. 
Anchored Flexure V2E

$\begin{array}{llllllllllllllllllll}x & y & w_{1} & w_{2} & w_{3} & w_{4} & w_{5} & l_{1} & l_{2} & l_{3} & l_{4} & g & b & b_{H} & b_{W} & a & L_{a} & \theta_{(x, y)} & \text { flexure } 2 \mathrm{E}\end{array}$

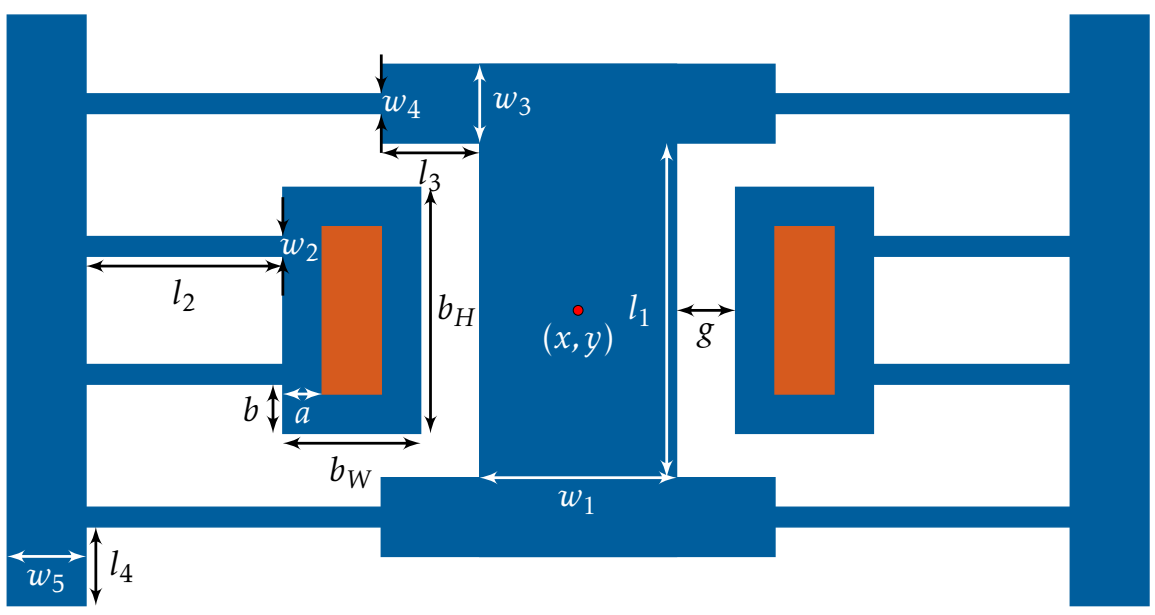

Figure 2.310: Anchored Flexure V2E. 
Anchored Flexure V4A

$\begin{array}{lllllllllllll}x & y & w & l_{1} & l_{2} & w_{m} & l_{m} & b_{H} & b_{e} & a & L_{a} & \theta_{(x, y)} & \text { flexure4A }\end{array}$

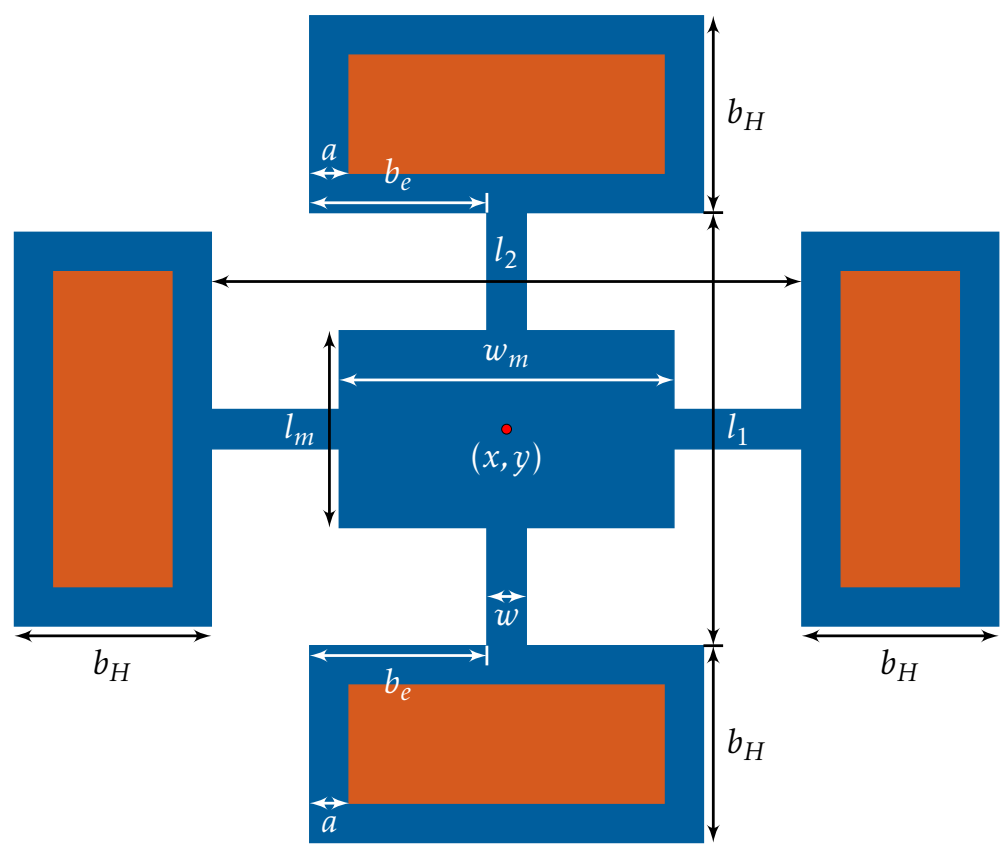

Figure 2.311: Anchored Flexure V4A with four hinges supporting a proof mass. 
Anchored Flexure V4B

$\begin{array}{lllllllllllll}x & y & w & l_{1} & l_{2} & w_{m} & g & b_{H} & b_{W} & a & L_{a} & \theta_{(x, y)} & \text { flexure4B }\end{array}$

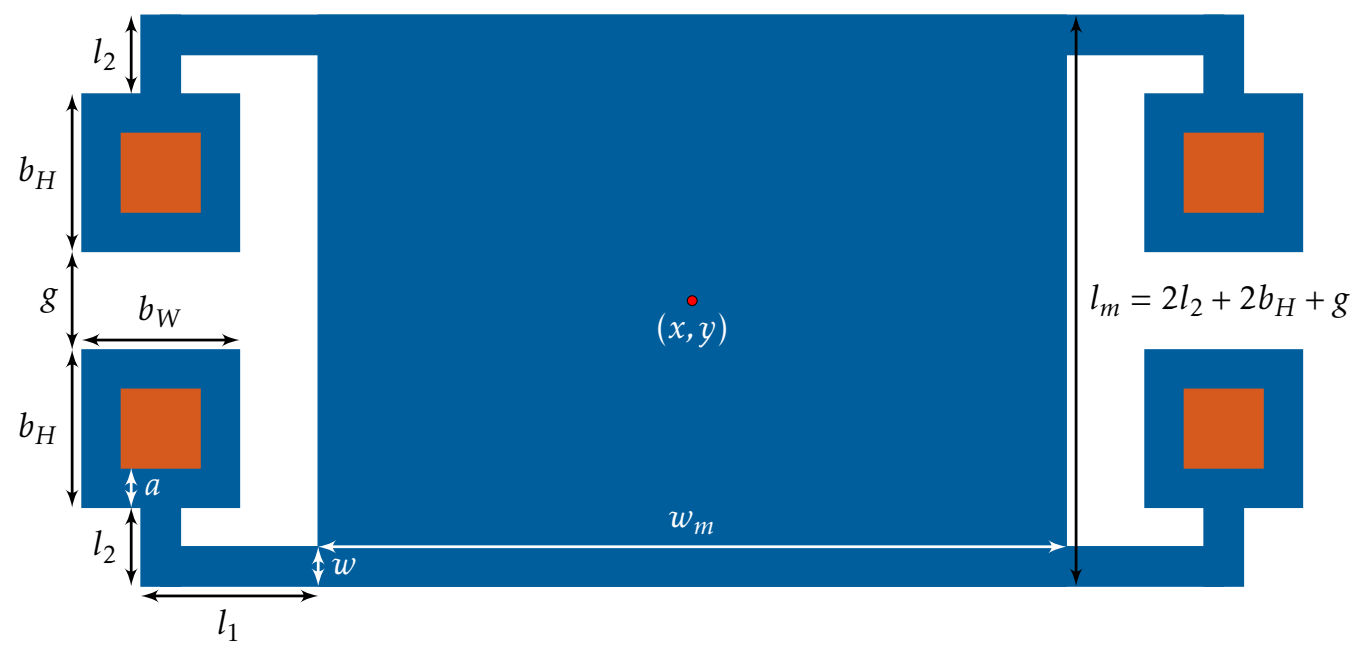

Figure 2.312: Anchored Flexure V4B with four hinges supporting a proof mass. 
Anchored Flexure V4C

$\begin{array}{lllllllllllllll}x & y & w & l_{1} & l_{2} & l_{3} & l_{4} & w_{m} & g & b_{H} & b_{W} & a & L_{a} & \theta_{(x, y)} & \text { flexure } 4 C\end{array}$

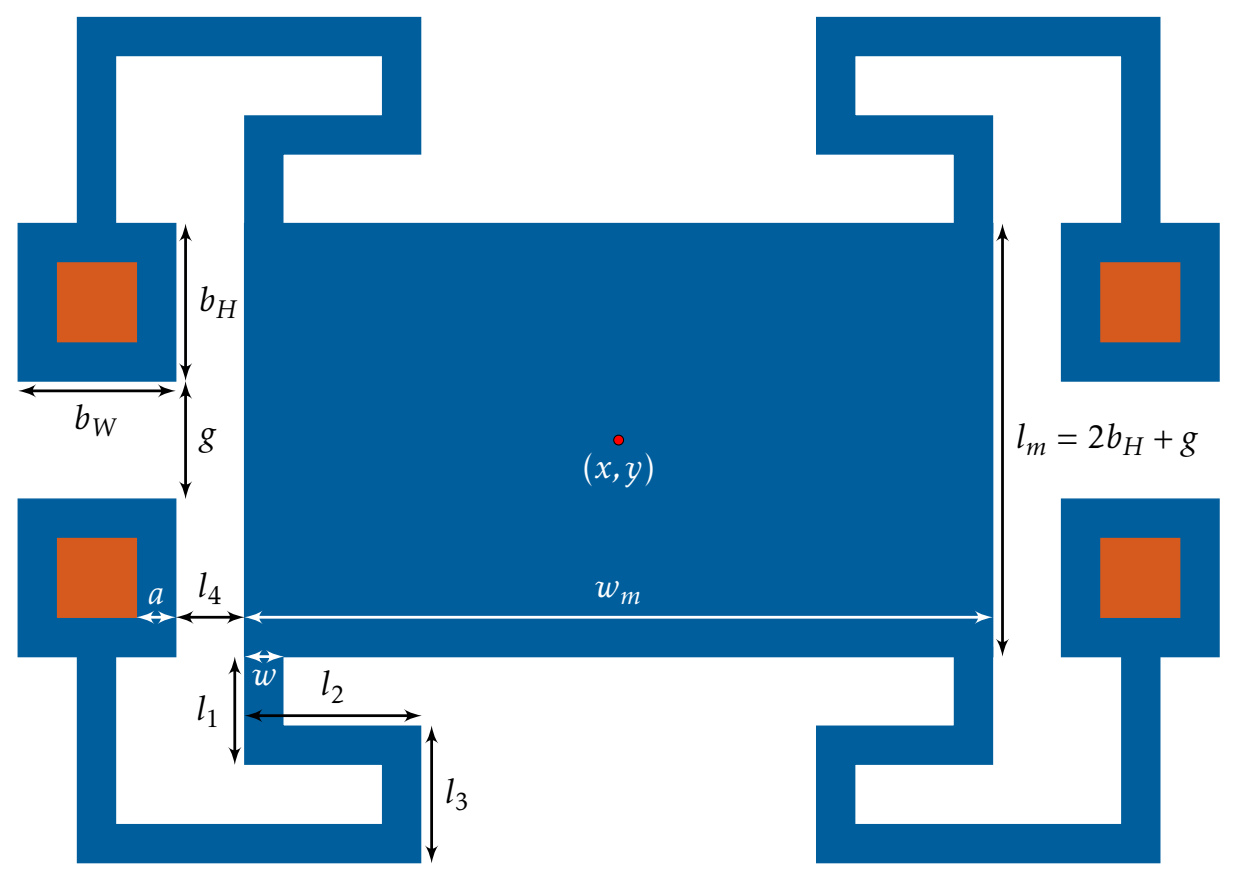

Figure 2.313: Anchored Flexure V4C with four hinges supporting a proof mass. 
Anchored Flexure V4D

$\begin{array}{lllllllllllllll}x & y & w_{1} & w_{2} & l_{1} & l_{2} & w_{m} & l_{m} & g & b_{H} & b_{W} & a & L_{a} & \theta_{(x, y)} & \text { flexure4D }\end{array}$

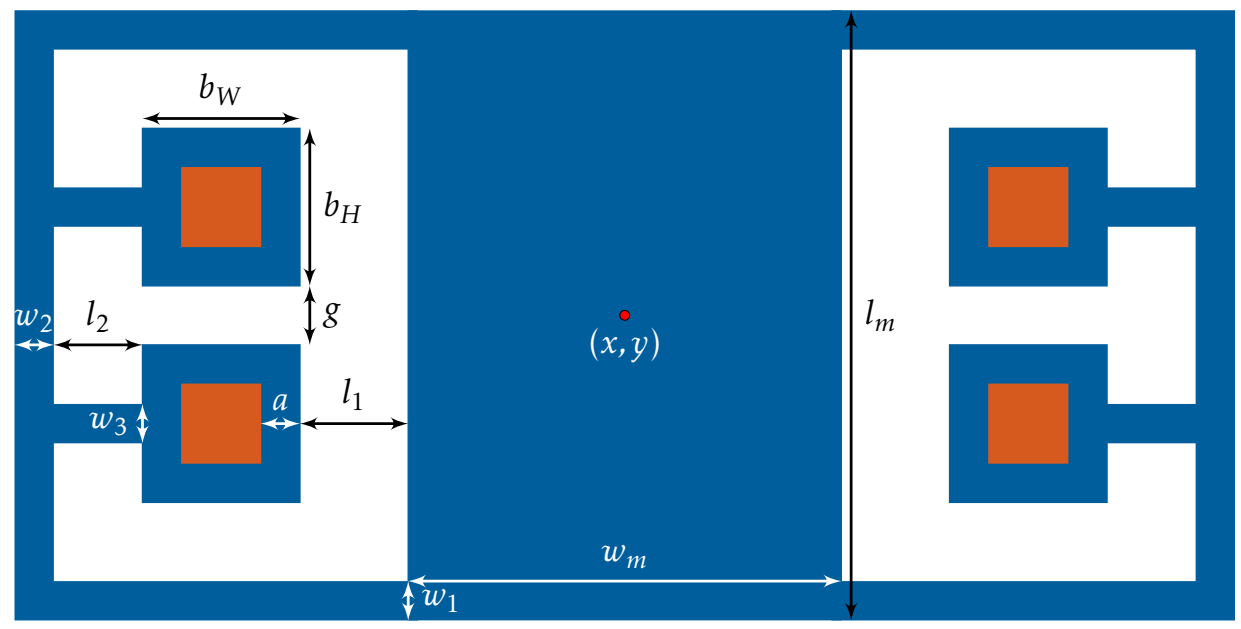

Figure 2.314: Anchored Flexure V4D with four hinges supporting a proof mass. 
Anchored Flexure V4E

$\begin{array}{llllllllllllllllll}x & y & w_{1} & w_{2} & w_{3} & w_{4} & l_{1} & l_{2} & l_{3} & l_{4} & l_{5} & b_{H} & b_{W} & a & L_{a} & \theta_{(x, y)} & \text { flexure4E }\end{array}$

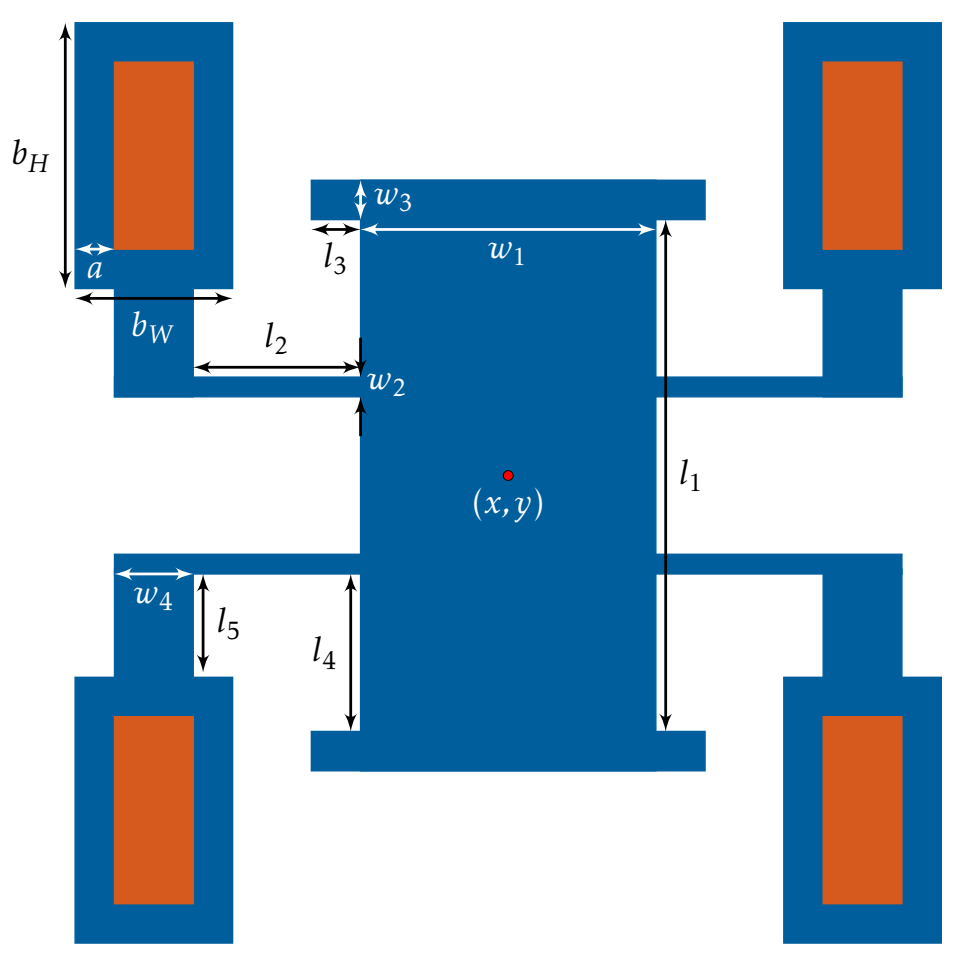

Figure 2.315: Anchored Flexure V4E. 


\subsubsection{Cantilevers}

The following cantilever beam structures have a linear, percentage or sinusoidal length variation with respect to the starting length $\left(s_{L}\right)$. The beams are periodically arranged on a base of height $b_{H}$ and of width that is a function of the number of elements $(n)$, beam width $(w)$, pitch $(p)$ and base extent $\left(b_{e}\right)$.
$\begin{array}{llllllllll}x & y & w & s_{L} & p & n & b_{H} & b_{e} & \Delta L\end{array}$
$\theta_{(x, y)} \quad$ cantileverL
$\begin{array}{lllllllllll}x & y & w & s_{L} & p & n & b_{H} & b_{e} & \text { Percent } \quad \theta_{(x, y)} & \text { cantileverP }\end{array}$
$\begin{array}{lllllllllll}x & y & w & s_{L} & p & n & b_{H} & b_{e} & \text { Amplitude } & \theta_{(x, y)} & \text { cantileverSine }\end{array}$
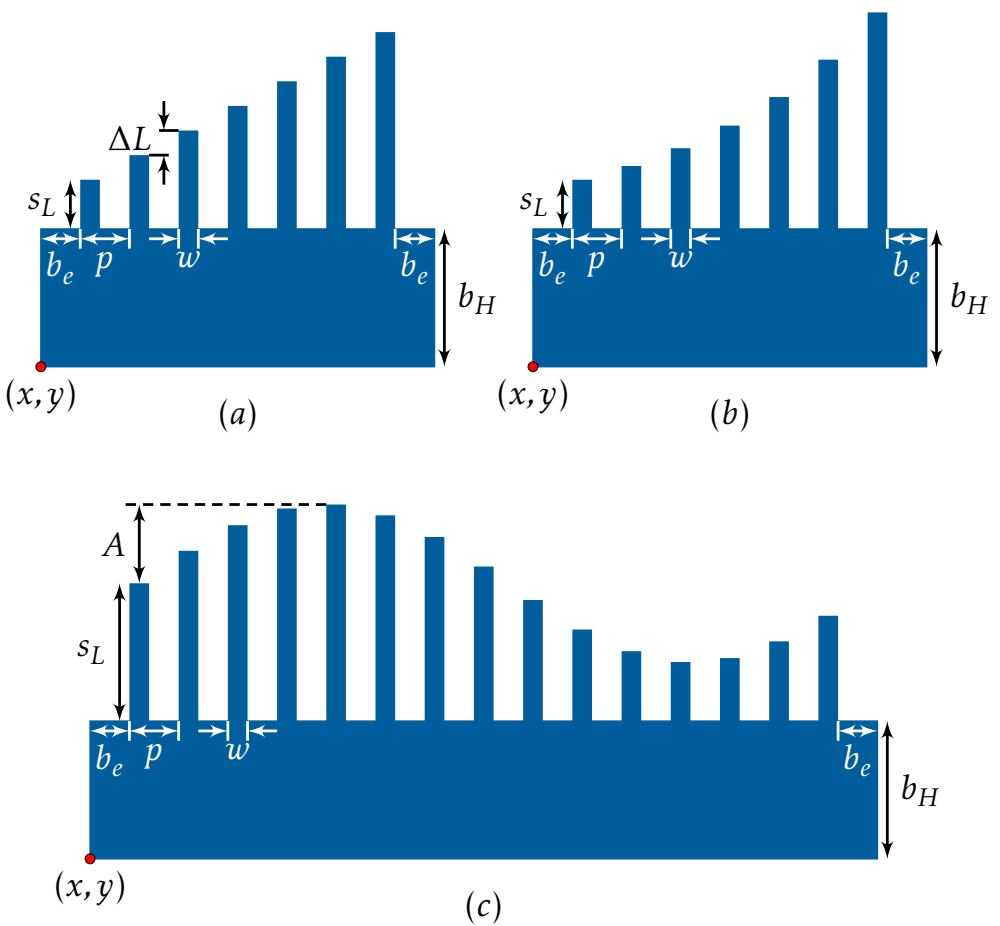

Figure 2.316: Cantilever arrays of varying length. (a) Linear (cantileverL) variation from $s_{L}$ with $\Delta L$ increments (b) Percentage (cantileverP) variation starting from $s_{L}$ and (c) sinusoidal (cantileverSine) variation over one period with amplitude $A$. 
Similar to figure 2.316, the cantilever variation for the below constructors is bound between the starting length $\left(s_{L}\right)$ and the end length $\left(e_{L}\right)$. Here, the origin $(x, y)$ is positioned at the lower left corner of the base, $w, s_{L}, e_{L}$ and $p$ are the cantilever width, start length, end lengths, and pitch, respectively. $n$ is the number of cantilever beams, $b_{e}$ and $b_{H}$ are the base extent and height. $\theta_{(x, y)}$ is the rotation about the origin $(x, y)$.

$$
\begin{array}{lllllllllll}
x & y & w & s_{L} & e_{L} & p & n & b_{H} & b_{e} & \theta_{(x, y)} & \text { cantileverLSE } \\
x & y & w & s_{L} & e_{L} & p & n & b_{H} & b_{e} & \theta_{(x, y)} & \text { cantileverNLSE }
\end{array}
$$

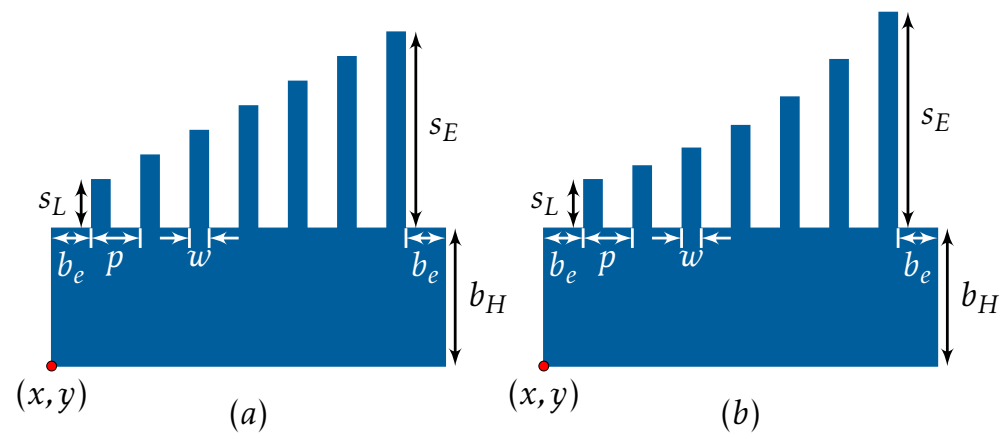

Figure 2.317: Cantilever arrays of varying length. (a) Linear (cantileverLSE) and (b) nonlinear (cantileverNLSE) length variation from $s_{L}$ to $e_{L}$. 
The following constructor creates cantilevers with custom parameters. Structural origin is defined by the lower left corner at $(x, y)$. Cantilever length and widths are defined by the $w_{i}$ and $l_{i}$ values. Space between the adjacent cantilevers is defined by $s_{1}, s_{2}, \ldots, s_{n}$. The base rectangle extends beyond the $n^{\text {th }}$ cantilever's right edge by the amount $s_{\text {end }}$.

$\begin{array}{llllllllllllllllll}x & y & s_{1} & w_{1} & l_{1} & s_{2} & w_{2} & l_{2} & \ldots & s_{n} & w_{n} & l_{n} & s_{\text {end }} & b_{H} & \theta_{(x, y)} & \text { cantileverCustom }\end{array}$

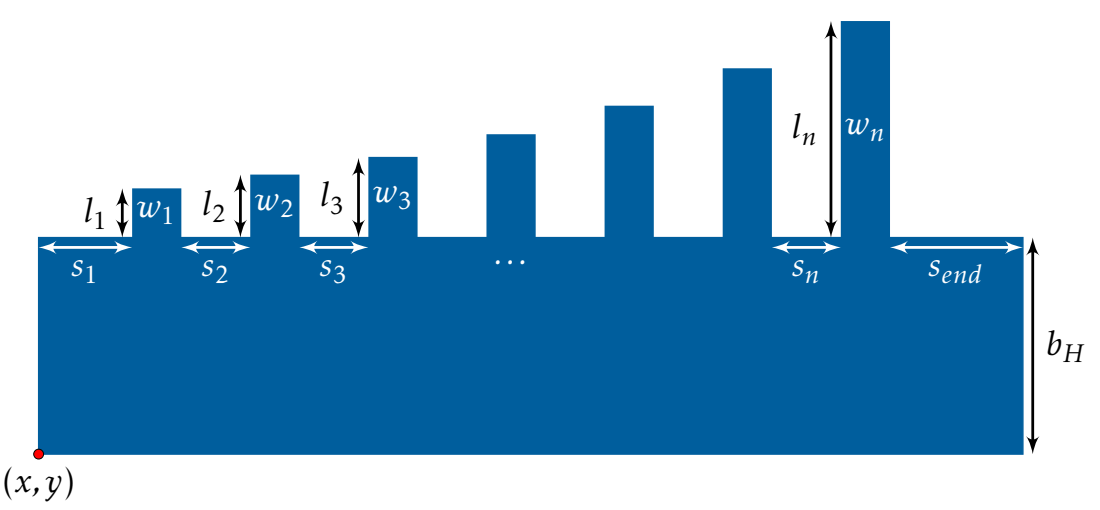

Figure 2.318: Cantilever array constructed with custom, user defined beam dimensions. 
Below constructors create rectangular (with and without a triangular tip), trapezoidal and paddle cantilever structures. The rectangular base support anchor is defined by the anchor support width $a$ and GDS layer $L_{a}$. Structural layer is defined by the active layer.

$$
\begin{aligned}
& \begin{array}{llllllllll}
x & y & w & l & b_{H} & b_{e} & a & L_{a} & \theta_{(x, y)} & \text { cantileverSR }
\end{array} \\
& \begin{array}{lllllllllll}
x & y & w & l & t_{H} & b_{H} & b_{e} & a & L_{a} & \theta_{(x, y)} & \text { cantileverSTri }
\end{array} \\
& \begin{array}{lllllllllll}
x & y & w_{a} & w_{b} & l & b_{H} & b_{e} & a & L_{a} & \theta_{(x, y)} & \text { cantileverSTrap }
\end{array} \\
& \begin{array}{llllllllllll}
x & y & w_{a} & w_{b} & l_{a} & l_{b} & b_{H} & b_{e} & a & L_{a} & \theta_{(x, y)} & \text { cantileverSPaddle }
\end{array}
\end{aligned}
$$

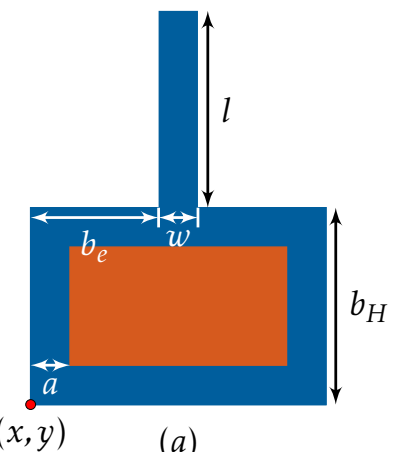

(a)

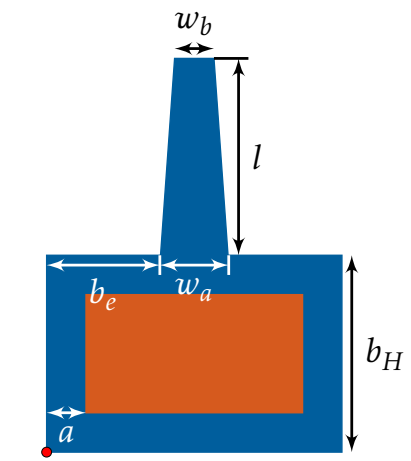

(c)

$(x, y)$

$(c)$

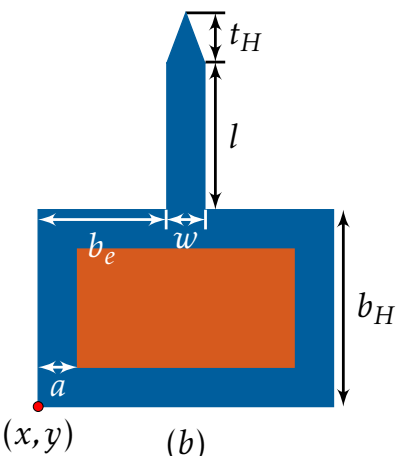

(b)

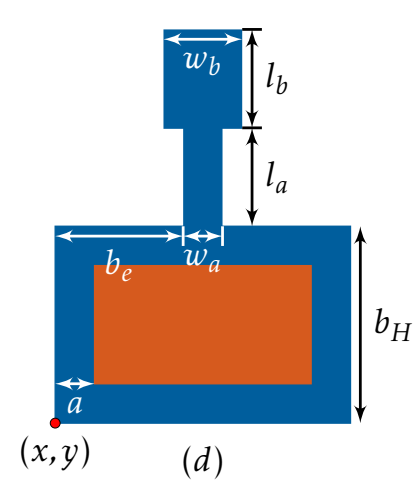

Figure 2.319: (a) Rectangular, (b) rectangular with a triangular tip, (c) trapezoidal and (d) paddle cantilever structures stemming from a rectangularly anchored base. 
Curved and straight-circular beams where the curved segment is defined by the number of vertices $N_{\text {sides }}$ and radius $R$.

$$
\begin{array}{llllllllllll}
x & y & w & R & N_{\text {sides }} & & b_{H} & b_{e} & a & L_{a} & \theta_{(x, y)} & \text { cantileverSCH } \\
x & y & w & R & N_{\text {sides }} & l & b_{H} & b_{e} & a & L_{a} & \theta_{(x, y)} & \text { cantileverSCF }
\end{array}
$$
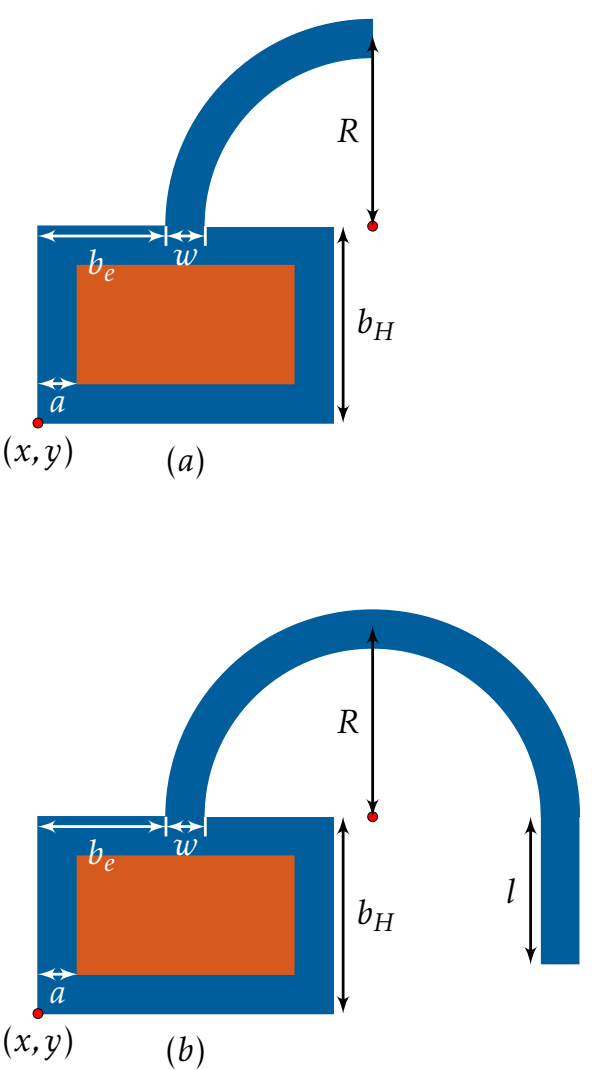

Figure 2.320: (a) Curved and (b) straight-circular beams stemming from a rectangularly anchored base. 
Below constructors create rectangular (with and without a triangular tip), trapezoidal and paddle cantilever structures. Constraints on parameter $h$ are $h<w / 2$ and $h<w_{a} / 2$.

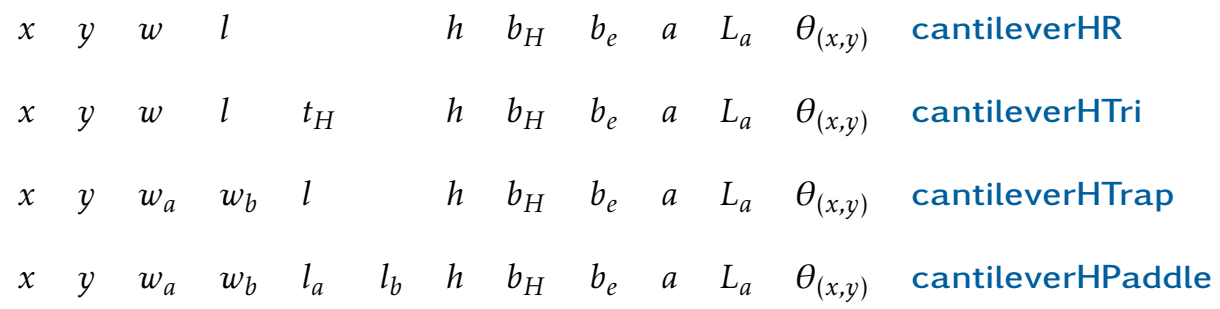
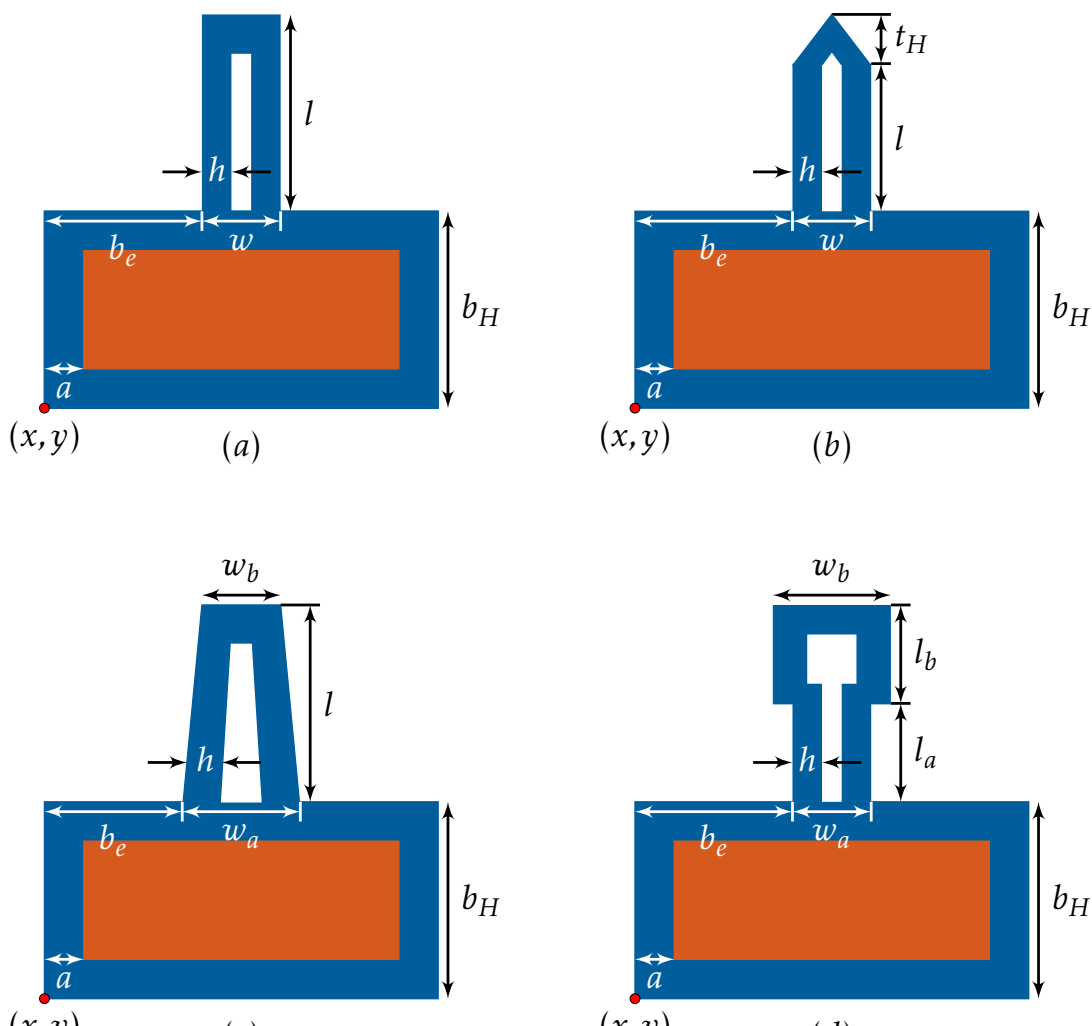

(c)

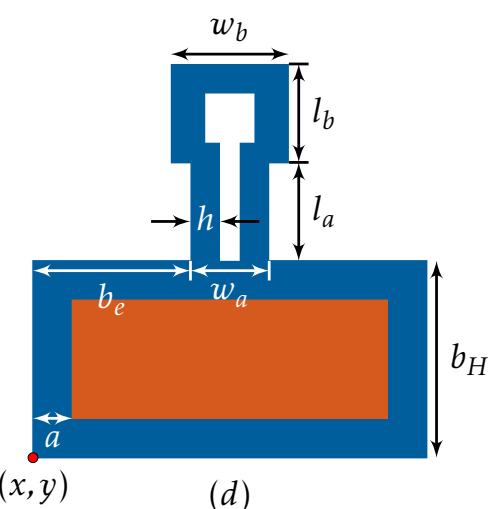

Figure 2.321: Hollow (a) rectangular, (b) rectangular with a triangular tip, (c) trapezoidal and (d) paddle cantilever structures stemming from a rectangularly anchored base.

NIST • CNST Nanolithography Toolbox v2016.09.01・http://www.nist.gov/cnst/ page 334 of 488 
The following two constructors create hollow curved and straight-circular beams. In both cases $h<w / 2$
$\begin{array}{lllll}x & y & w & R & N_{\text {sides }}\end{array}$
$h \begin{array}{llllll}h & b_{H} & b_{e} & a & L_{a} & \theta_{(x, y)}\end{array}$
cantileverHCH
$\begin{array}{llllllll}N_{\text {sides }} & l & h & b_{H} & b_{e} & a & L_{a} & \theta_{(x, y)}\end{array}$
cantileverHCF
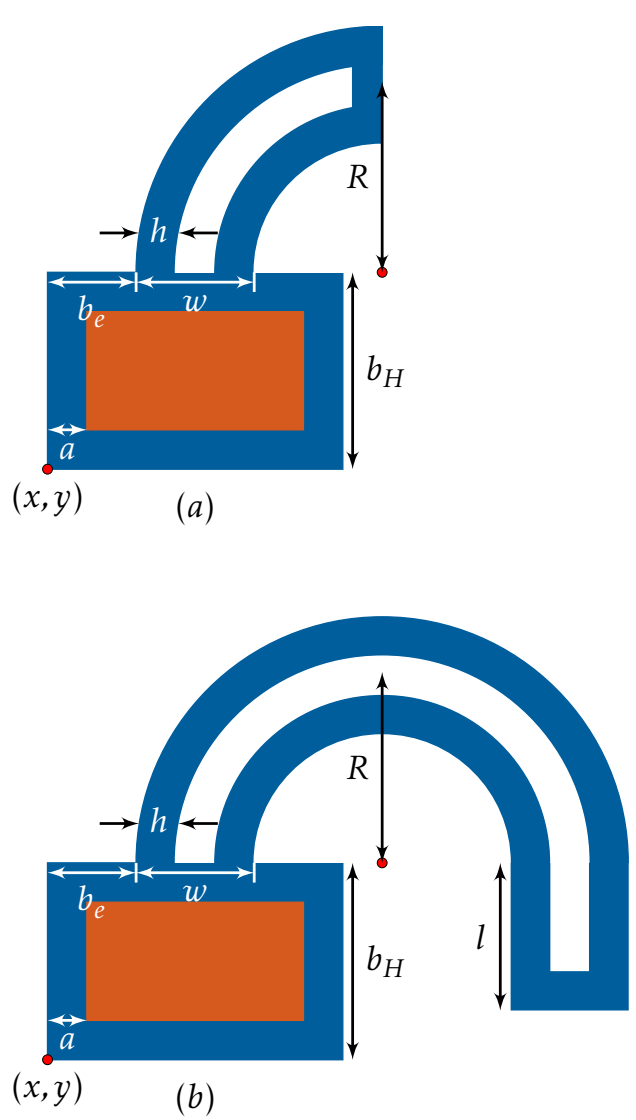

Figure 2.322: Hollow (a) curved and (b) straight-circular beams stemming from a rectangularly anchored base. 
Below constructors create connected parallel beams. The gap $(g)$ between the electrodes has the following constraint:

$$
\begin{aligned}
& g \leq \frac{l_{B}}{2}-2 w \quad 2 \text { parallel beams } \\
& g \leq l_{B}-2 w \quad 3 \text { parallel beams }
\end{aligned}
$$

$$
\begin{array}{llllllllllllll}
x & y & w & l_{1} & l_{2} & & l_{B} & g & b_{H} & b_{e} & a & L_{a} & \theta_{(x, y)} & \text { cantileverPB2 } \\
x & y & w & l_{1} & l_{2} & l_{3} & l_{B} & g & b_{H} & b_{e} & a & L_{a} & \theta_{(x, y)} & \text { cantileverPB3 }
\end{array}
$$
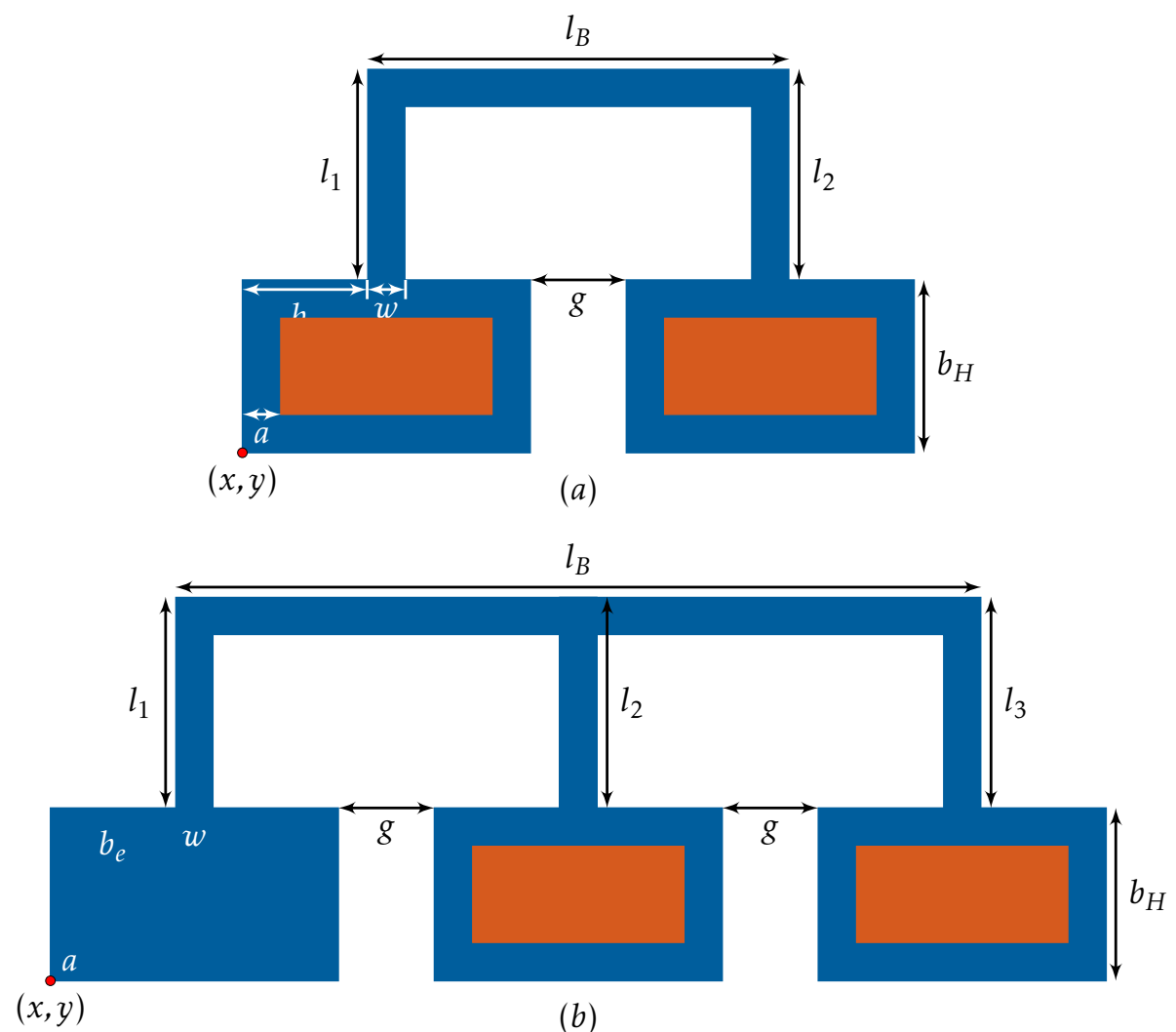

Figure 2.323: (a) Two and (b) three parallel, interacting beams. 
U-Shaped spring designs with sharp rectangular, circularly filleted and circular links. Parameters $a$ and $L_{a}$ are anchor overlap and anchor GDS layer respectively.

$$
\begin{array}{llllllllllllll}
x & y & w & l_{1} & l_{2} & l_{B} & & & b_{H} & b_{e} & a & L_{a} & \theta_{(x, y)} & \text { cantileverUR } \\
x & y & w & l_{1} & l_{2} & l_{B} & r & N_{\text {sides }} & b_{H} & b_{e} & a & L_{a} & \theta_{(x, y)} & \text { cantileverUCF } \\
x & y & w & l_{1} & l_{2} & D & N_{\text {sides }} & & b_{H} & b_{e} & a & L_{a} & \theta_{(x, y)} & \text { cantileverUC }
\end{array}
$$

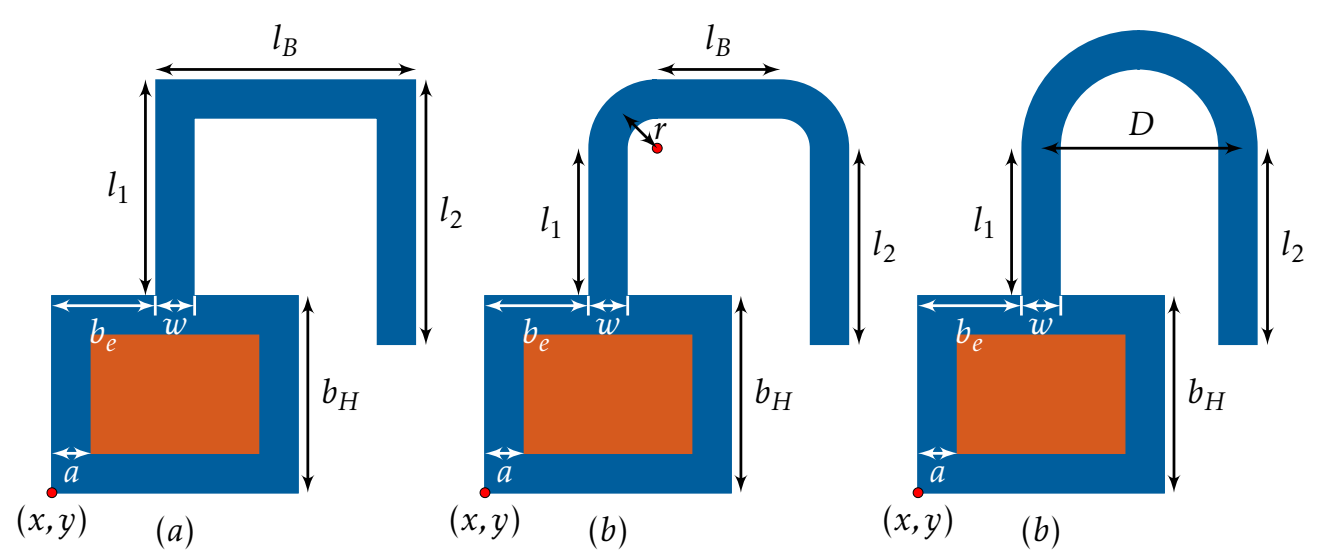

Figure 2.324: U-shaped (a) rectangular, (b) circularly filleted corners and (c) circular cantilever structures. 
U-shaped structures with a central cantilever and a central paddle. Parameters $a$ and $L_{a}$ are anchor overlap and anchor GDS layer, respectively. Central cantilever length $l_{2}$ can be either positive of negative, respectively yielding a cantilever of width $w_{2}$ below or above the top link.

$$
\begin{array}{lllllllllllllll}
x & y & w_{1} & w_{2} & l_{1} & l_{2} & & l_{3} & l_{B} & b_{H} & b_{e} & a & L_{a} & \theta_{(x, y)} & \text { cantileverUCC } \\
x & y & w_{1} & w_{2} & l_{1} & l_{2 a} & l_{2 b} & l_{3} & l_{B} & b_{H} & b_{e} & a & L_{a} & \theta_{(x, y)} & \text { cantileverUCP }
\end{array}
$$

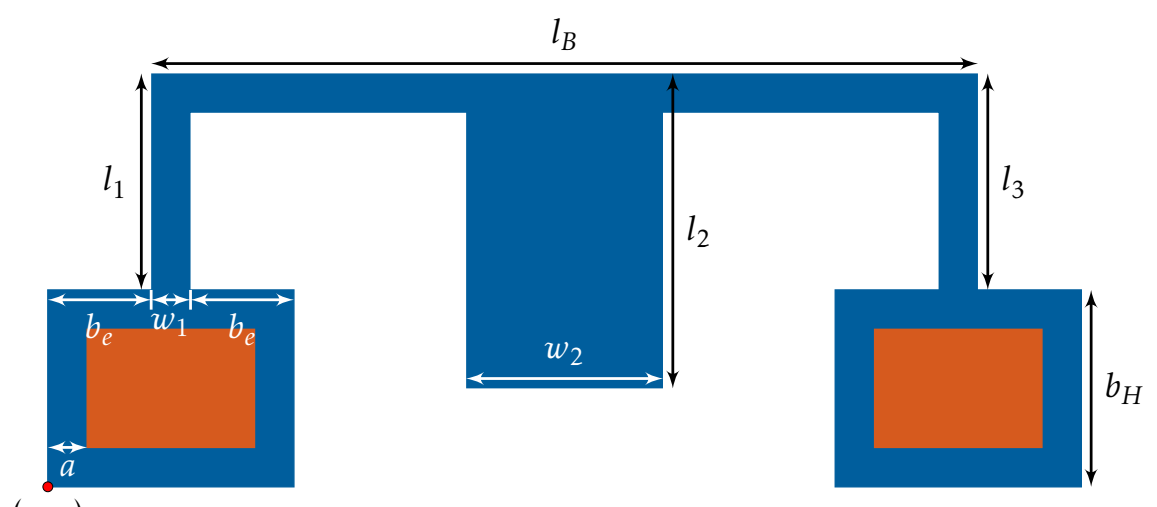

$(x, y)$

(a)

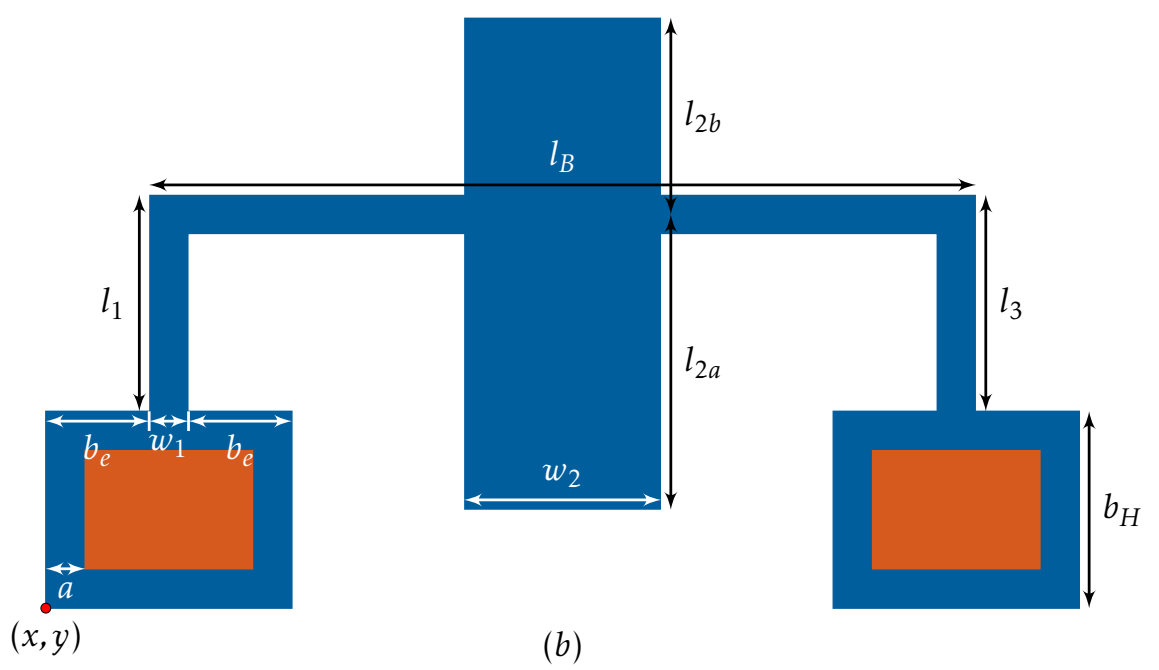

Figure 2.325: U-shaped structure with a center (a) cantilever and (b) paddle. 
Below constructors create curved cantilever structures. The rectangular base support anchor is defined by the anchor support width $a$ and GDS layer $L_{a}$. Structural layer is defined by the active layer. Curved segments of the upper and lower paddle oscillators are defined by ellipses with radii $\left(r_{x_{1}}, r_{y_{1}}\right)$ and $\left(r_{x_{2}}, r_{y_{2}}\right)$, respectively.

$$
\begin{array}{llllllllllllllllll}
x & y & w & l & r_{x} & r_{y} & & N_{\text {sides }} & & b_{H} & b_{e} & a & L_{a} & \theta_{(x, y)} & \text { cantileverCE } \\
x & y & w & l & r_{x_{1}} & r_{y_{1}} & r_{x_{2}} & r_{y_{2}} & N_{\text {sides }} & w_{p} & l_{p} & b_{H} & b_{e} & a & L_{a} & \theta_{(x, y)} & \text { cantileverCEPaddle }
\end{array}
$$

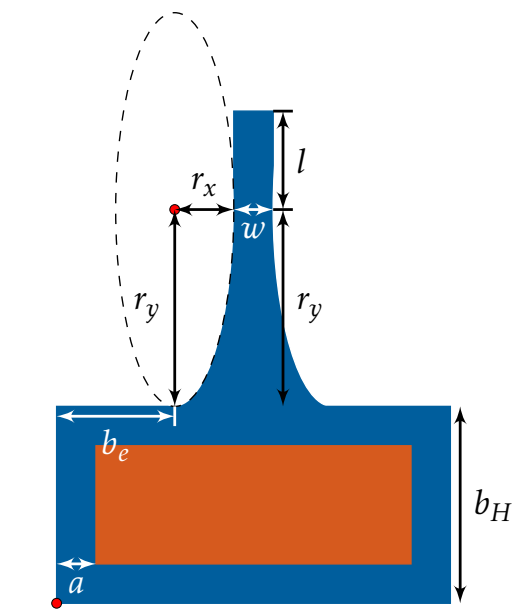

$(x, y)$ (a)

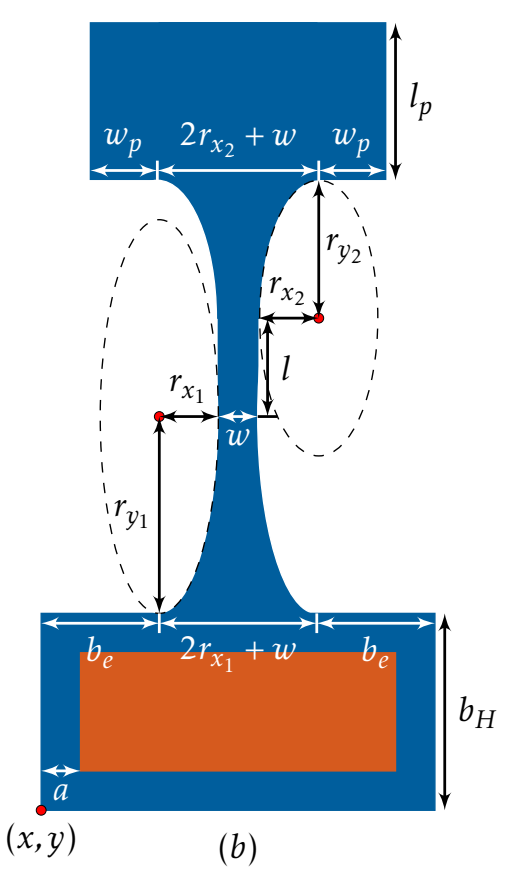

Figure 2.326: (a) Curved cantilever and (b) curved cantilever with a paddle. 


\subsubsection{Doubly Clamped Beams}

Similar to the cantilever structures in figures $2.316 \mathrm{a}$ and $2.316 \mathrm{~b}$, these are doubly clamped beams with a linear and percentage variation with respect to the starting length $\left(s_{L}\right)$.

$$
\begin{array}{lllllllllll}
x & y & w & s_{L} & p & n & b_{H} & b_{e} & \Delta L & \theta_{(x, y)} & \text { dcBeamL } \\
x & y & w & s_{L} & p & n & b_{H} & b_{e} & \text { Percent } & \theta_{(x, y)} & \text { dcBeamP }
\end{array}
$$
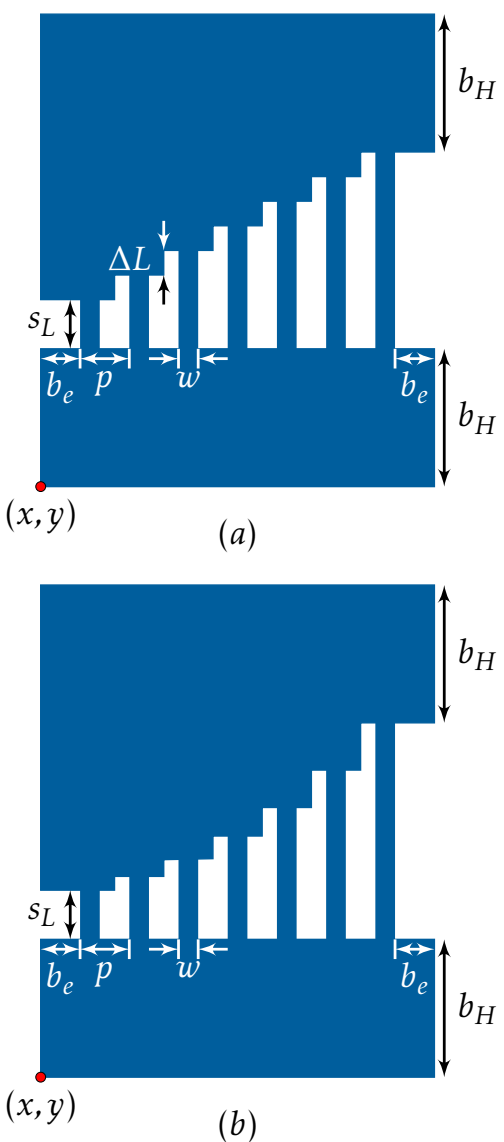

Figure 2.327: Doubly clamped beam arrays of varying length. (a) Linear (dcBeamL) variation from $s_{L}$ with $\Delta L$ increments and (b) Percentage (dcBeamP) length variation starting from $s_{L}$. 
Similar to the cantilever arrays in figure 2.317 , these are doubly clamped beams with length variation bound between the starting $\left(s_{L}\right)$ and the end length $\left(e_{L}\right)$ values.

$$
\begin{array}{lllllllllll}
x & y & w & s_{L} & e_{L} & p & n & b_{H} & b_{e} & \theta_{(x, y)} & \mathrm{dcBeamLSE} \\
x & y & w & s_{L} & e_{L} & p & n & b_{H} & b_{e} & \theta_{(x, y)} & \mathrm{dcBeamNLSE}
\end{array}
$$

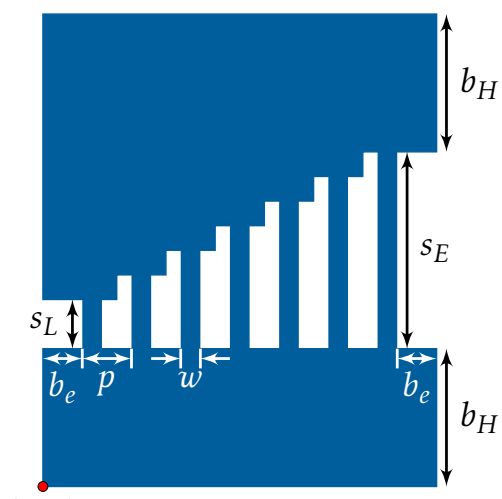

$(x, y)$

(a)

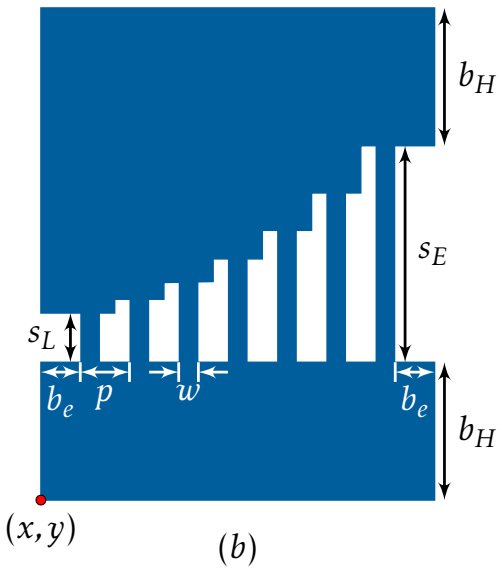

Figure 2.328: Doubly clamped beam arrays of length varying between $s_{L}$ and $s_{E}$. (a) Linear (dcBeamLSE) and (b) nonlinear (dcBeamNLSE) length variation from $s_{L}$ to $e_{L}$. 
The following constructor creates doubly clamped beams with used defined parameters. The structure is similar to custom cantilevers in Figure 2.318.

$\begin{array}{lllllllllllllllllll}x & y & s_{1} & w_{1} & l_{1} & s_{2} & w_{2} & l_{2} & \ldots & s_{n} & w_{n} & l_{n} & s_{\text {end }} & b_{H} & \theta_{(x, y)} & \text { dcBeamCustom }\end{array}$

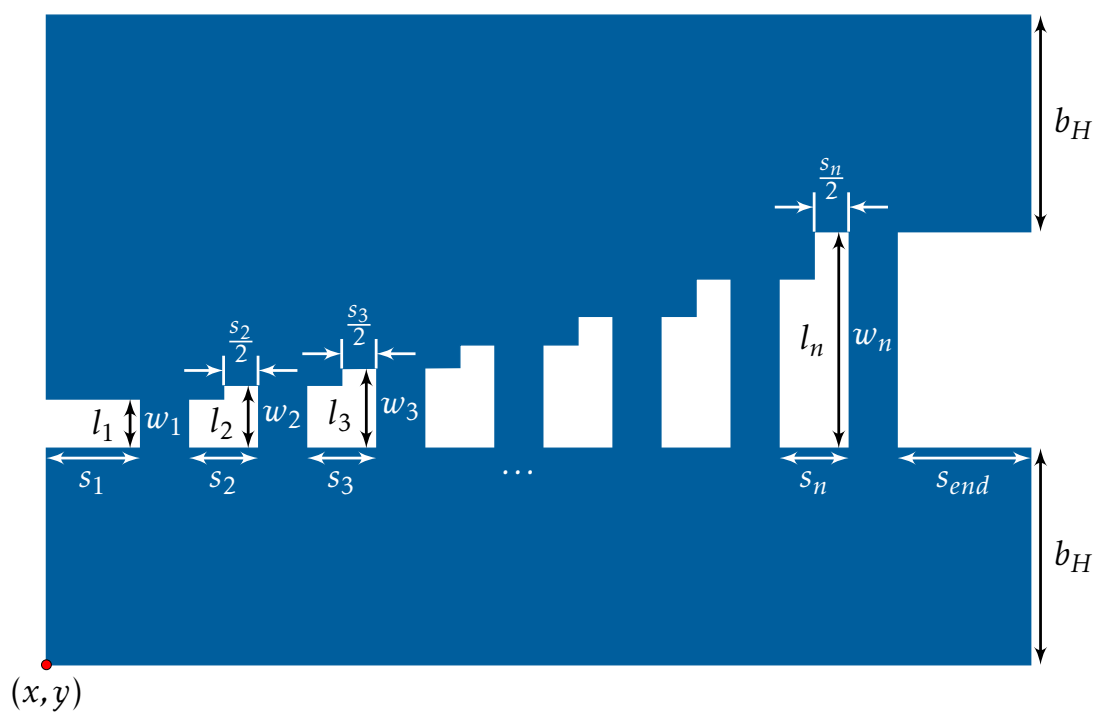

Figure 2.329: Doubly clamped array of beams constructed with custom, user defined dimensions. 
Below constructors create rectangular and torsional doubly clamped beam structures. The rectangular base support anchor is defined by the anchor support width $a$ and GDS layer $L_{a}$. Structural layer is defined by the active layer.
$\begin{array}{llll}x & y & w & l\end{array}$
$\begin{array}{llllllll}b_{H} & b_{e} & a & L_{a} & \theta_{(x, y)} & \text { dcBeamR }\end{array}$
$\begin{array}{llllllllllll}x & y & w_{1} & l_{1} & w_{2} & l_{2} & b_{H} & b_{e} & a & L_{a} & \theta_{(x, y)} & \text { dcBeamT }\end{array}$

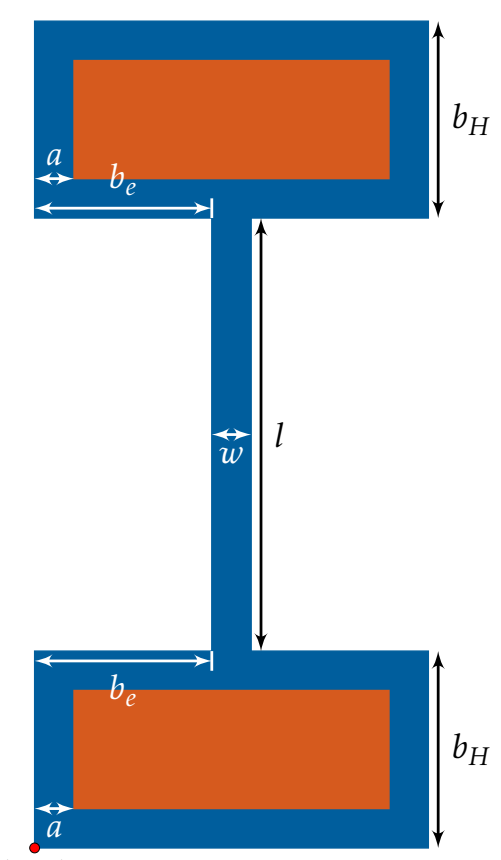

$(x, y)$

(a)

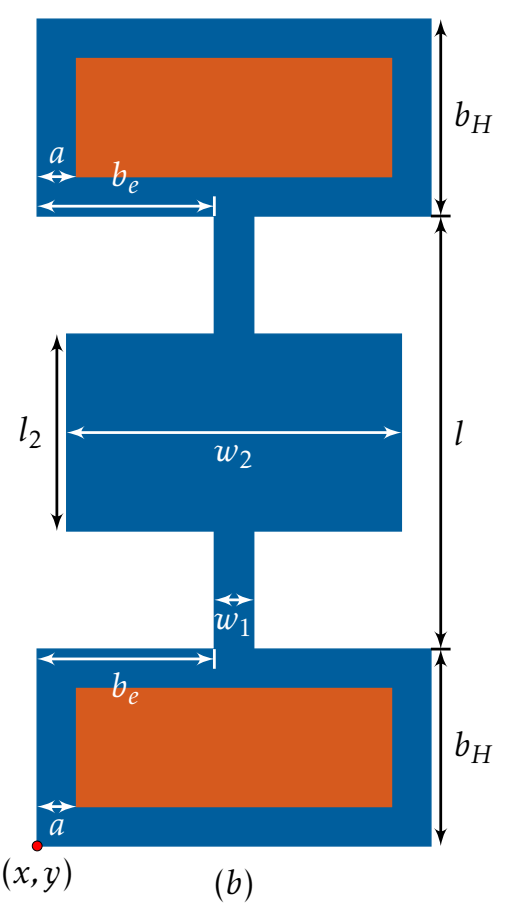

$(b)$

Figure 2.330: (a) Rectangular and (b) torsional doubly clamped beams. 
Doubly clamped torsional beam.

$\begin{array}{llllllllllllllll}x & y & w_{1} & w_{2} & w_{3} & w_{4} & l_{1} & l_{2} & l_{3} & g & b_{H} & b_{W} & a & L_{a} & \theta_{(x, y)} & \text { dcBeamT2 }\end{array}$

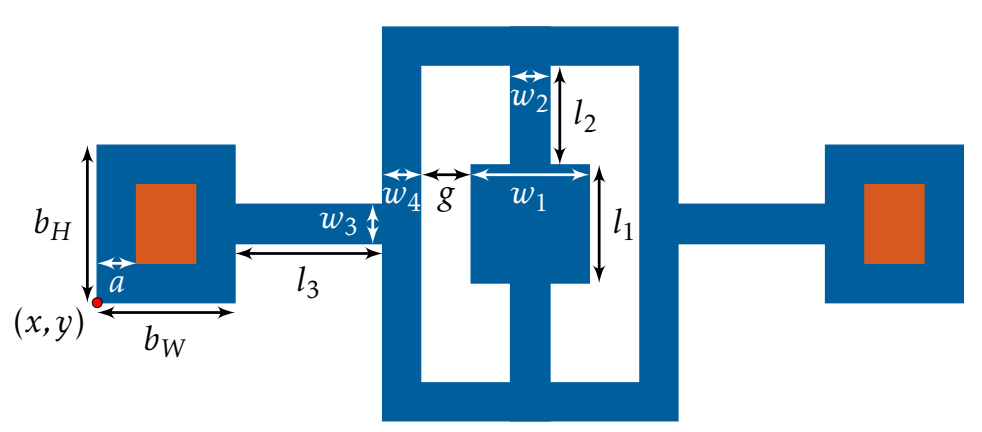

Figure 2.331: Doubly clamped torsional beam. 
Doubly clamped array of $N$ interacting beams with lengths ranging linearly from $L_{s}$ to $L_{e}$.

$\begin{array}{llllllllllll}x & y & w & L_{s} & L_{e} & N & b_{H} & b_{W} & a & L_{a} & \theta_{(x, y)} & \text { dcBeamCB }\end{array}$

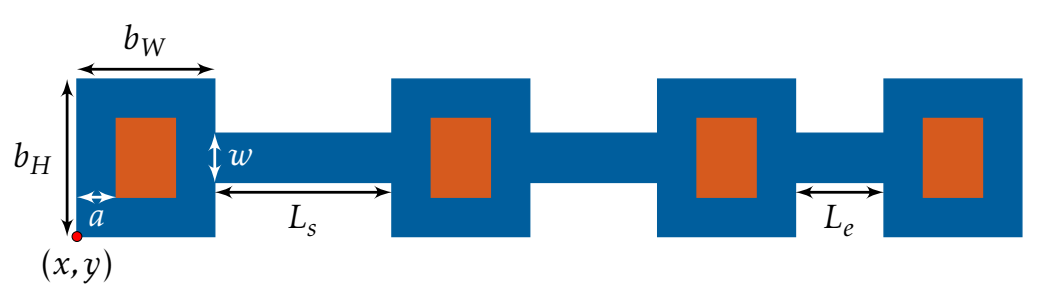

Figure 2.332: Doubly clamped coupled beams. 
Below constructor creates a curved doubly clamped beam structures. The rectangular base support anchor is defined by the anchor support width $a$ and GDS layer $L_{a}$. Structural layer is defined by the active layer. Curved segments of the upper and lower beams are defined by ellipses with radii $\left(r_{x_{1}}, r_{y_{1}}\right)$ and $\left(r_{x_{2}}, r_{y_{2}}\right)$, respectively.

$\begin{array}{lllllllllllllll}x & y & w & l & r_{x_{1}} & r_{y_{1}} & r_{x_{2}} & r_{y_{2}} & N_{\text {sides }} & b_{H} & b_{e} & a & L_{a} & \theta_{(x, y)} & \text { dcBeamC }\end{array}$

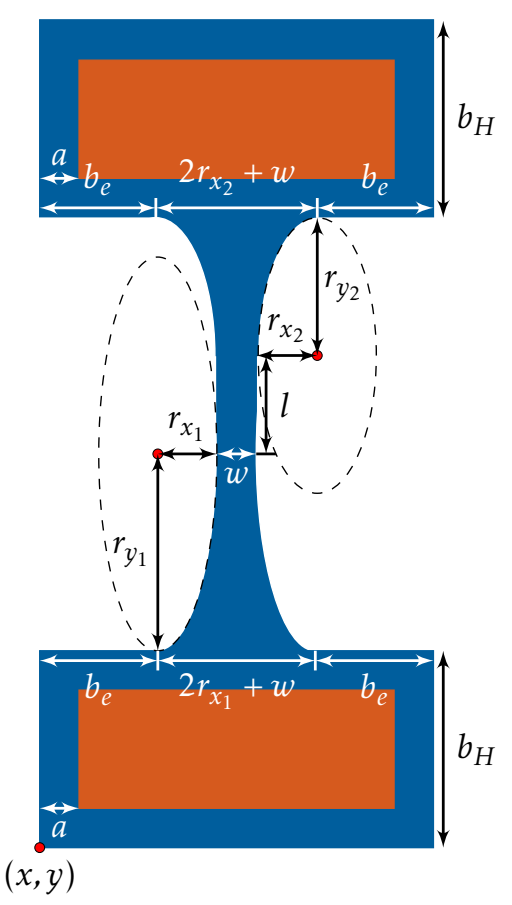

Figure 2.333: curved doubly clamped beams. 


\subsubsection{Interacting Arrays}

The following objects each have two constructors. The bracketed constructor ( $<i d$ parameters ... constructor $>$ ) creates hierarchical structures that are instantiated within the current structure. The $i d$ parameter is a string prefix all generated structures for the particular object. This parameter is a unique continuous string of characters (no spaces or tabs). To ensure compatibility with the GDSII standard, the allowed structure character names are $A-Z$, $a-z, 0-9$, underscore (_), question mark (?) and dollar sign (\$). Furthermore, the $i d$ parameter should be limited to no more than 15 characters, otherwise under certain circumstances, it's possible to overwriting existing structures. Two major benefits of this constructor are that processing is faster and smaller files are generated. Both are a consequence of GDS structural hierarchy.

The second constructor is nearly identical with the exception of not having brackets, the string $i d$ parameter and the constructor suffix s. Furthermore, the constructor does not create any additional structures, hence resulting structures entirely are generated within the initialized structure. Consequently, the resulting files are bigger and take longer to generate.

In both cases, structures are generated with the following 3 GDS layers:

$L y_{F} \quad$ - front side beam layer

$L y_{B} \quad$ - back side release layer

$L y_{M} \quad$ - front side circular metal dot layer

<id $x$ y $N L_{1} W_{1} L_{2} W_{2} s H_{\circ} L_{\circ} H_{e} L_{B} H_{B} d N_{s} L y_{F} L y_{B} L y_{M} \theta_{(x, y)}$ MARAs>

$x y N L_{1} W_{1} L_{2} W_{2} s H_{\circ} L_{\circ} H_{e} L_{B} H_{B} d N_{s} L y_{F} L y_{B} L y_{M} \theta_{(x, y)}$ MARA

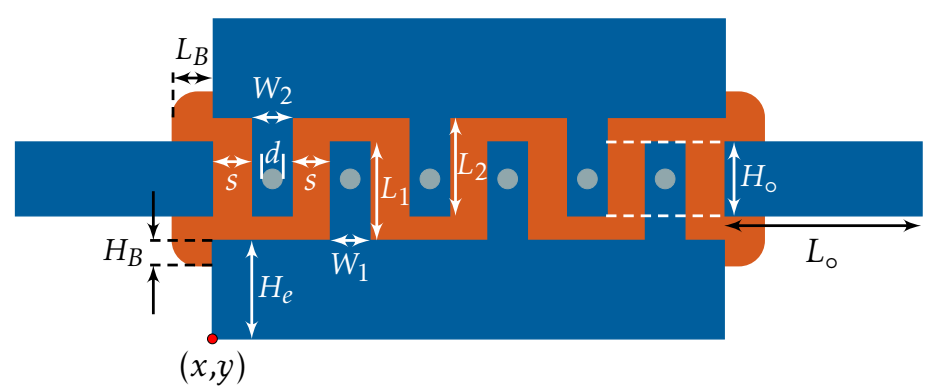

Figure 2.334: Two rectangular arrays of $N$ interacting beams created using MARAs and MARA constructors.

NIST • CNST Nanolithography Toolbox v2016.09.01 • http://www.nist.gov/cnst/

page 347 of 488 
Two trapezoidal array of $N$ interacting beams with identical parameters,

<id $x$ y N L W $W_{a} W_{b} s H_{\circ} L_{\circ} H_{e} L_{B} H_{B} d N_{s} L y_{F} L y_{B} L y_{M} \theta_{(x, y)}$ MATALWs $>$

$x y N L W_{a} W_{b} s H_{\circ} L_{\circ} H_{e} L_{B} H_{B} d N_{s} L y_{F} L y_{B} L y_{M} \theta_{(x, y)}$ MATALW

and with varying length $(L)$ and widths $\left(W_{a}\right.$ and $W b$ ) between the two arrays.

$<i d x$ y $L_{1} W_{1 a} W_{1 b} L_{2} W_{2 a} W_{2 b} s H_{\circ} L_{\circ} H_{e} L_{B} H_{B} d N_{s} L y_{F} L y_{B} L y_{M} \theta_{(x, y)}$ MATAs $>$

$x y N L_{1} W_{1 a} W_{1 b} L_{2} W_{2 a} W_{2 b} s H_{\circ} L_{\circ} H_{e} L_{B} H_{B} d N_{s} L y_{F} L y_{B} L y_{M} \theta_{(x, y)}$ MATA

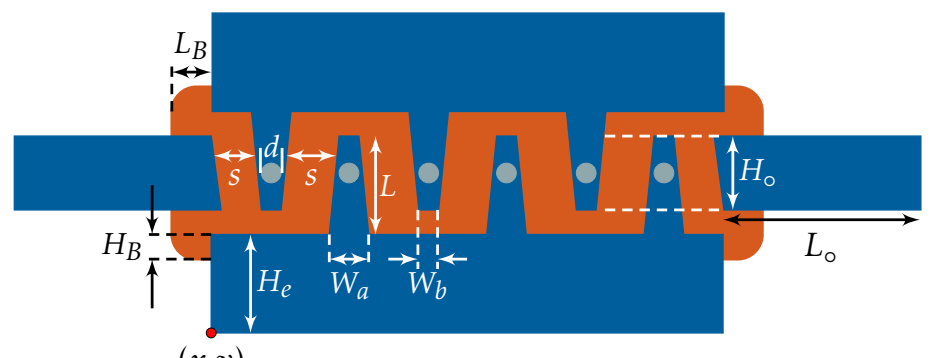

(a)

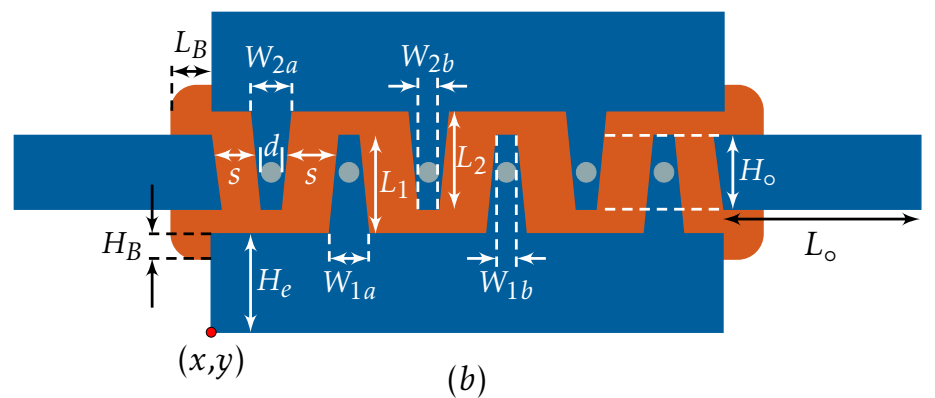

Figure 2.335: Two interacting trapezoidal beam arrays with (a) identical (MATALWs, MATALW) and (b) varying (MATAs, MATA) length and width parameters. 
Array of two interacting rectangular beams.

<id $x$ y $N L_{1} W_{1} L_{2} W_{2}$ s e $H_{\circ} H_{e} L_{s} L_{B} H_{B} d N_{s} L y_{F} L y_{B} L y_{M} \theta_{(x, y)}$ MAR2s $>$

$x$ y $N L_{1} W_{1} L_{2} W_{2}$ s e $H_{\circ} H_{e} L_{s} L_{B} H_{B} d N_{s} L y_{F} L y_{B} L y_{M} \theta_{(x, y)}$ MAR2

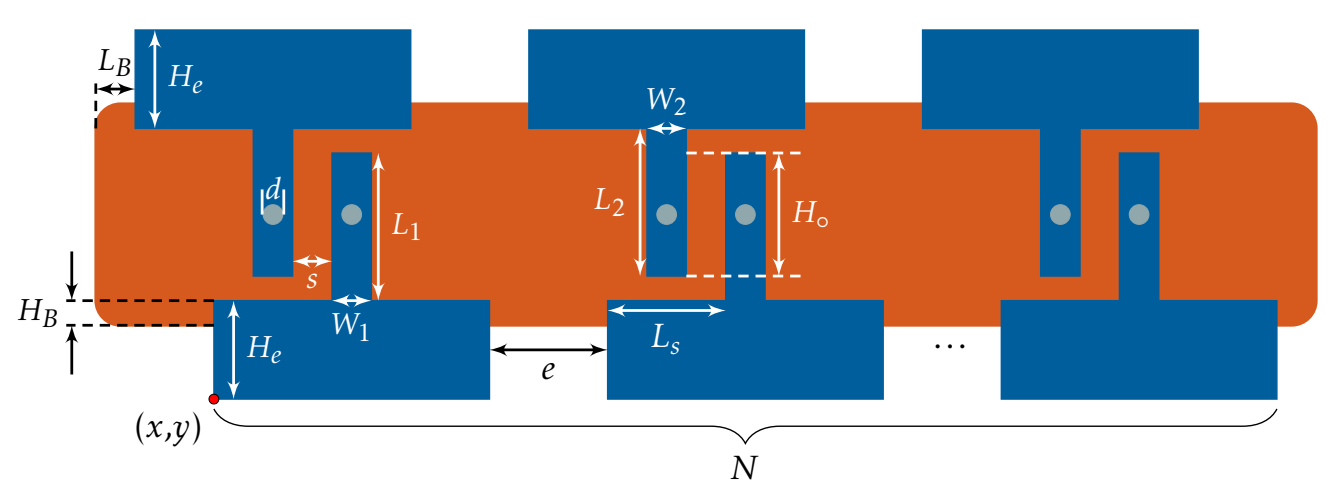

Figure 2.336: Array of two interacting rectangular beams created using the MAR2s and MAR2, constructors. 
Array of three interacting rectangular beams.

$<i d x y \quad N L_{1} W_{1} L_{2} W_{2}$ s e $H_{\circ} H_{e} L_{B} H_{B} d N_{s} L y_{F} L y_{B} L y_{M} \theta_{(x, y)}$ MAR3s $>$

$x$ y $N L_{1} W_{1} L_{2} W_{2}$ s e $H_{\circ} H_{e} L_{B} H_{B} d N_{s} L y_{F} L y_{B} L y_{M} \theta_{(x, y)}$ MAR3

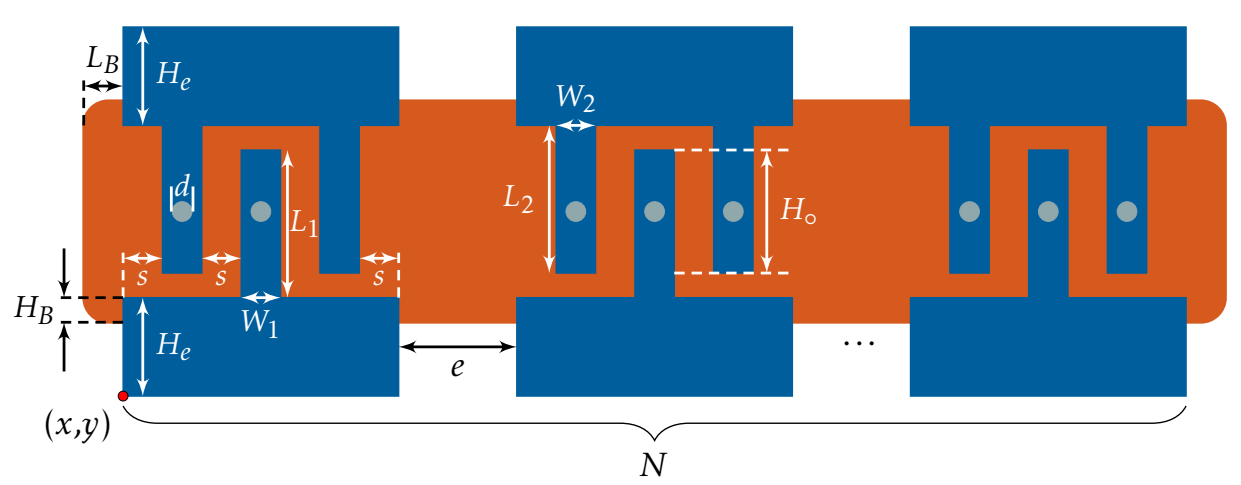

Figure 2.337: Array of three interacting rectangular beams created using the MAR3s and MAR3 constructors. 
Rectangular top array of elements interacting with single beams. The lower single beam elements are individually addressable via electrodes of width $H_{w}=W_{1}+2 W_{2}+4 s$ and height $H_{e}$.

<id $x$ y $N L_{1} W_{1} L_{2} W_{2}$ s e $H_{\circ} H_{e} L_{B} H_{B} d N_{s} L y_{F} L y_{B} L y_{M} \theta_{(x, y)}$ MARCs $>$

$x y N L_{1} W_{1} L_{2} W_{2}$ s e $H_{\circ} H_{e} L_{B} H_{B} d N_{s} L y_{F} L y_{B} L y_{M} \theta_{(x, y)}$ MARC

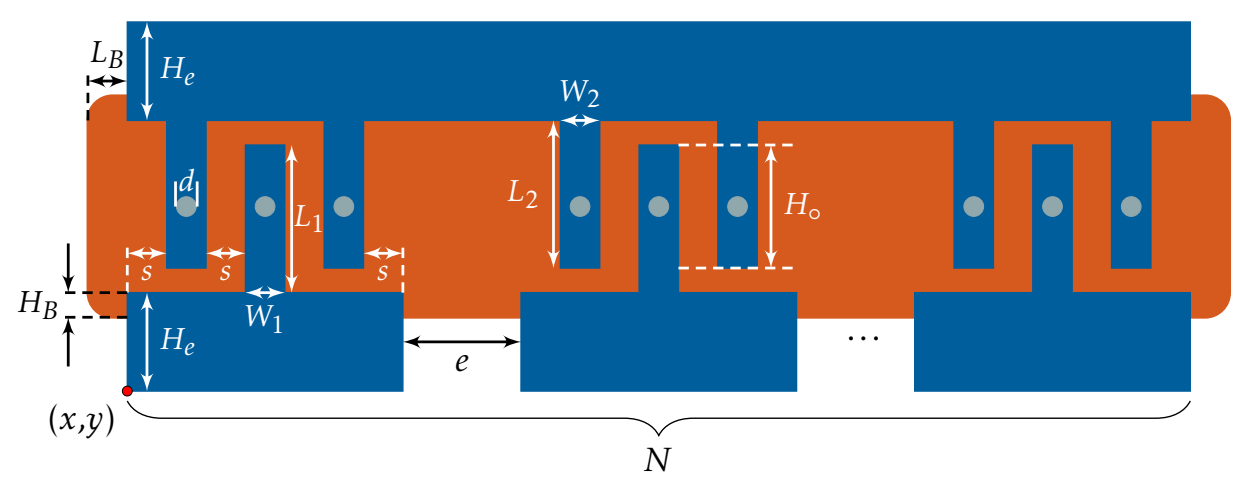

Figure 2.338: Array of $2 N$ rectangular beams interacting with $N$ single beams created using the MARCs and MARCconstructors. 
Array of two interacting trapezoidal beams.

<id $x$ y $N L_{1} W_{1 a} W_{1 b} L_{2} W_{2 a} W_{2 b}$ s e $H_{\circ} H_{e} L_{s} L_{B} H_{B} d N_{s} L y_{F} L y_{B} L y_{M} \theta_{(x, y)}$ MAT2s $>$

$x$ y $N L_{1} W_{1 a} W_{1 b} L_{2} W_{2 a} W_{2 b}$ s e $H_{\circ} H_{e} L_{s} L_{B} H_{B} d N_{s} L y_{F} L y_{B} L y_{M} \theta_{(x, y)}$ MAT2

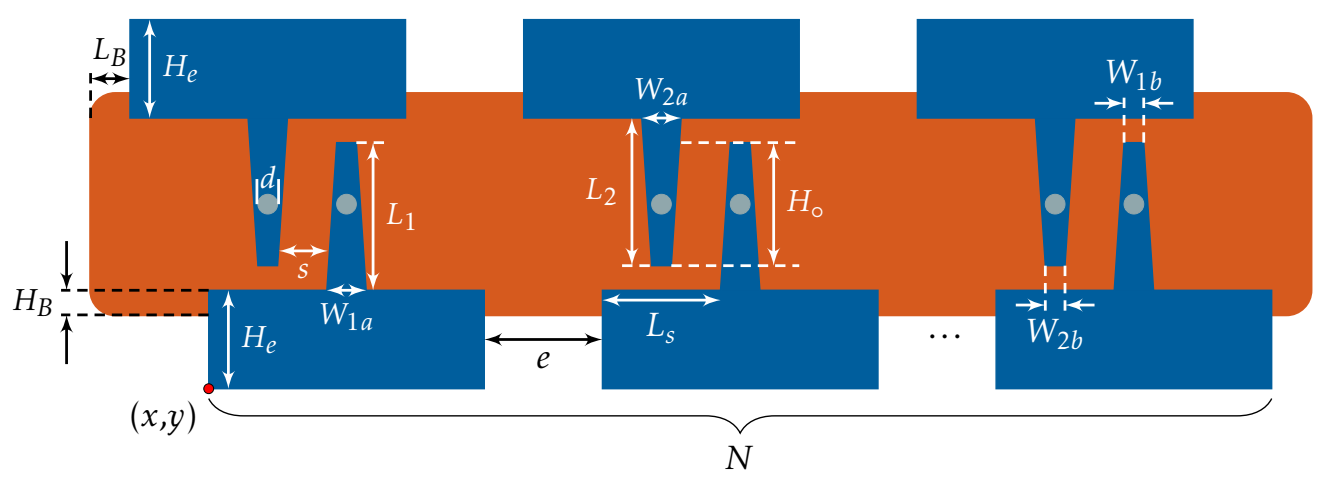

Figure 2.339: Array of two interacting trapezoidal beams created using the MAT2s and MAT2 constructors. 
Array of three interacting trapezoidal beams.

<id $x$ y $N L_{1} W_{1 a} W_{1 b} L_{2} W_{2 a} W_{2 b}$ s e $H_{\circ} H_{e} L_{B} H_{B} d N_{s} L y_{F} L y_{B} L y_{M} \theta_{(x, y)}$ MAT3s $>$

$x$ y $N L_{1} W_{1 a} W_{1 b} L_{2} W_{2 a} W_{2 b}$ s e $H_{\circ} H_{e} L_{B} H_{B} d N_{s} L y_{F} L y_{B} L y_{M} \theta_{(x, y)}$ MAT3

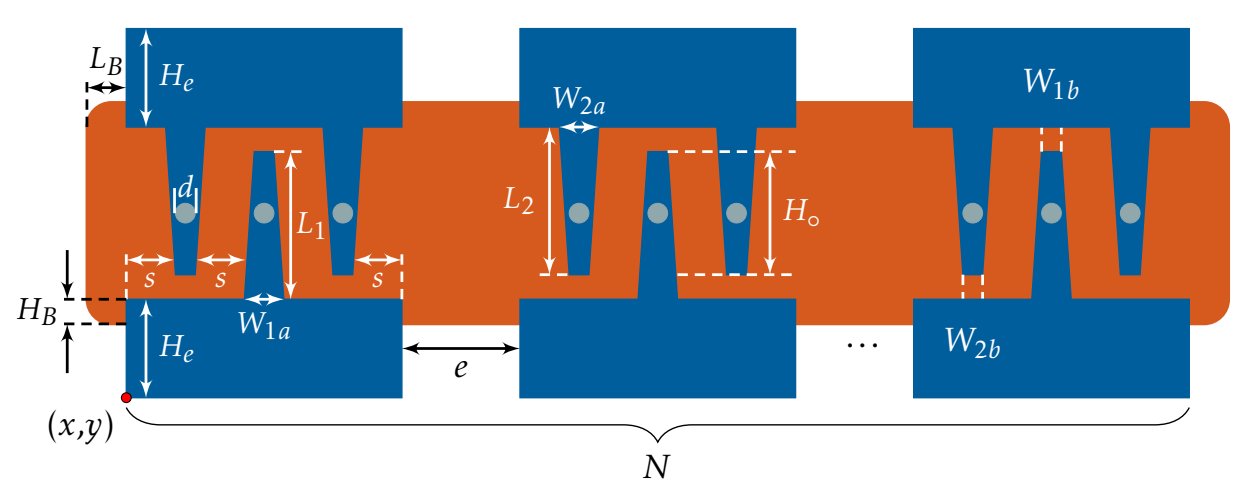

Figure 2.340: Array of three interacting trapezoidal beams created using the MAT3s and MAT3s constructors. 
Trapezoidal top array of elements interacting with single beams. The lower single beam elements are individually addressable via electrodes of width $H_{w}=W_{1 a}+2 W_{2 b}+4 s$ and height $H_{e}$.

<id $x$ y $N L_{1} W_{1 a} W_{1 b} L_{2} W_{2 a} W_{2 b}$ s e $H_{\circ} H_{e} L_{B} H_{B} d N_{s} L y_{F} L y_{B} L y_{M} \theta_{(x, y)}$ MATCs $>$

$x$ y $N L_{1} W_{1 a} W_{1 b} L_{2} W_{2 a} W_{2 b}$ s e $H_{\circ} H_{e} L_{B} H_{B} d N_{s} L y_{F} L y_{B} L y_{M} \theta_{(x, y)}$ MATC

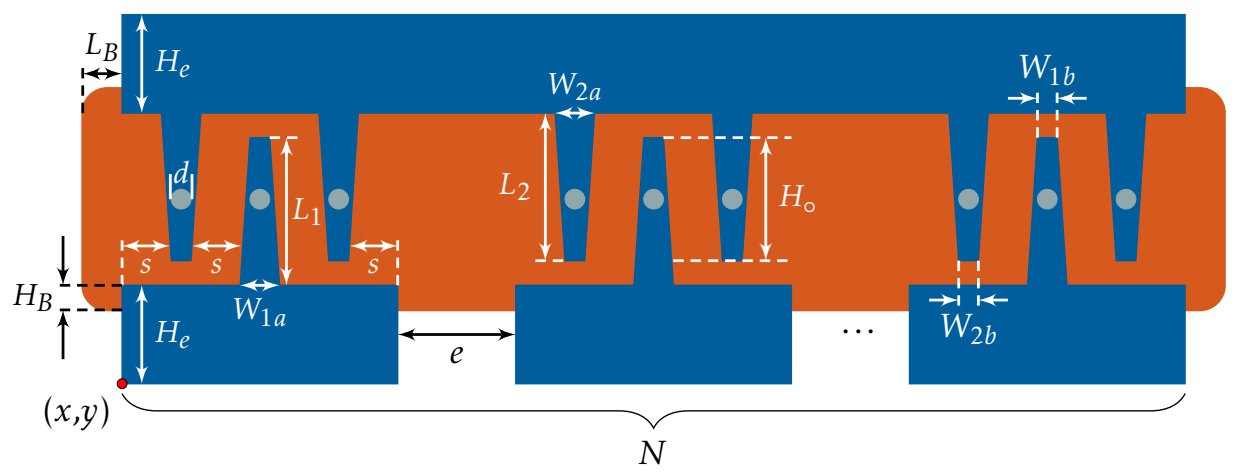

Figure 2.341: Array of $2 \mathrm{~N}$ trapezoidal beams interacting with a $N$ single beams created using the MATCs and MATC constructors. 
Two interacting rectangular arrays between elements of differing lengths. The electrode base varies linearly from the value of $H_{e}$ to $H e+\Delta$.

$<i d x$ y $N$ L $\Delta W$ s $H_{\circ} L_{\circ} H_{e} L_{B} H_{B} d N_{s} L_{1} L_{2} L_{3} \theta_{(x, y)}$ MARALINEARs $>$

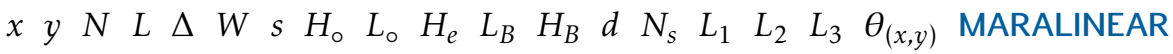

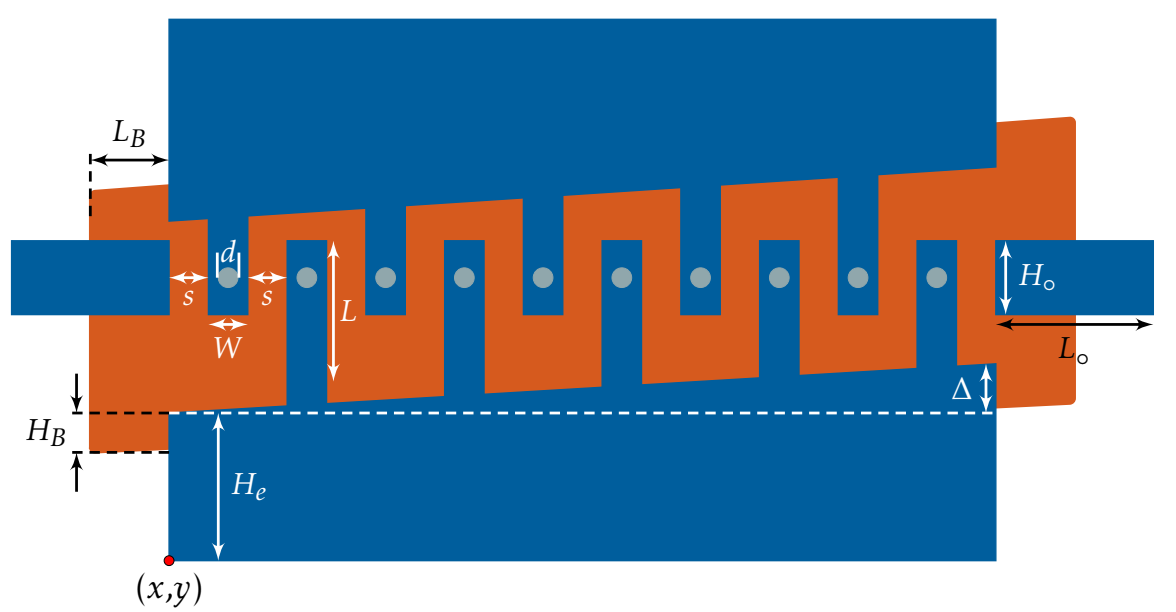

Figure 2.342: Two interacting rectangular arrays between elements of differing lengths. Beam lengths vary linearly across the electrode base. Structures are generated using the MARALINEARs and MARALINEAR constructors. 
Two rectangular arrays between elements of differing lengths. The electrode base has a slow non-linear variation from of $H_{e}$ to $H_{e}+\Delta$. The non-linear element was constructed using a Bezier curve.

<id $x$ y $N$ L $\Delta W$ s $H_{\circ} L_{\circ} H_{e} L_{B} H_{B} d N_{s} L_{1} L_{2} L_{3} \theta_{(x, y)}$ MARACURVEs >

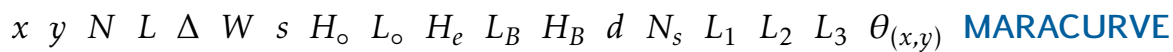

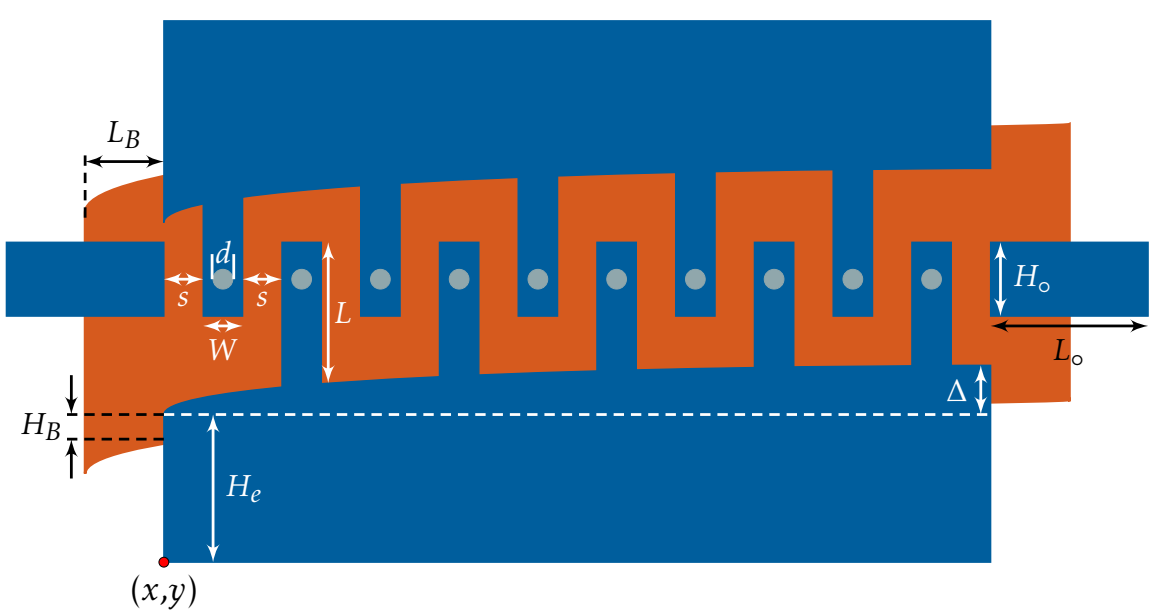

Figure 2.343: Two interacting rectangular arrays between elements of differing lengths. Beam lengths vary non-linearly across the electrode base. Structures are generated using the MARACURVEs and MARACURVE constructors. 


\subsubsection{Stress, Strain Measurement Structures}

\subsubsection{Guckel Rings}

Guckel ring structures are used to estimate residual stress in structural layers.

$\begin{array}{llllllllllllll}x & y & r_{W} & R & N_{\text {sides }} & b_{W} & b & L_{c} & W_{c} & a & L_{a} & \theta_{(x, y)} & \text { GuckelRing }\end{array}$

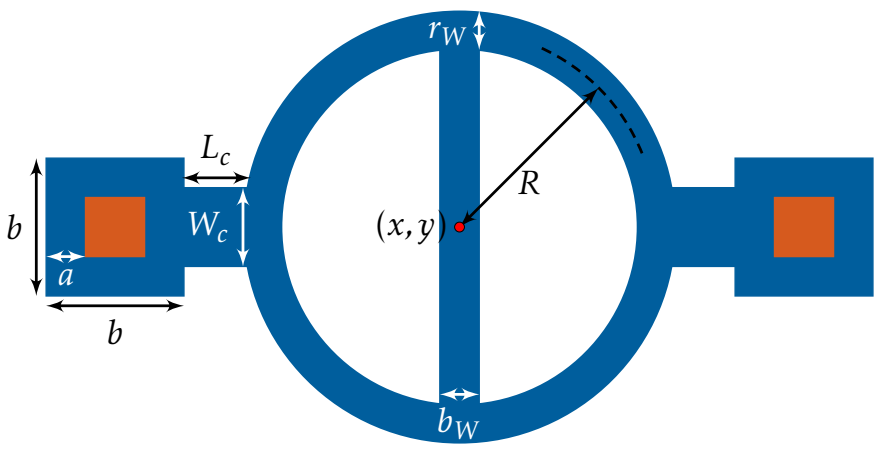

Figure 2.344: Schemaric of a Guckel ring structure used for measuring residual stress.

Below is a Guckel ring array structure. The varying radii range from $R_{s}$ to $R_{e}$ in increments of $\Delta R$.

$\begin{array}{llllllllllllllll}x & y & r_{W} & R_{s} & R_{e} & \Delta R & N & b_{W} & b & L_{c} & W_{c} & a & L_{a} & \theta_{(x, y)} & \text { GuckelRingArray }\end{array}$

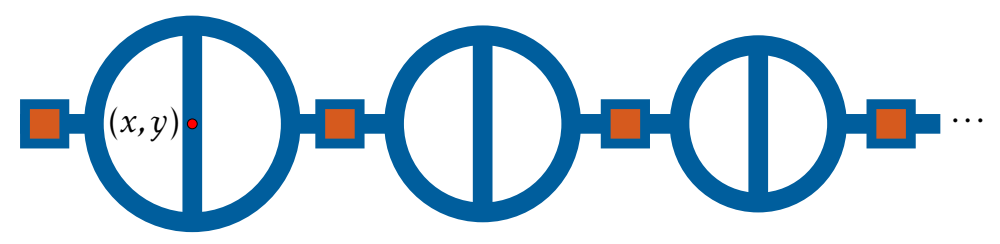

Figure 2.345: Schemaric of a Guckel ring array structure with varying radius.

NIST • CNST Nanolithography Toolbox v2016.09.01 • http://www.nist.gov/cnst/

page 357 of 488 


\subsubsection{Diamond Ring}

$\begin{array}{lllllllllllllll}x & y & w_{1} & w_{2} & w_{3} & l_{1} & l_{2} & l_{3} & b_{H} & b_{W} & a & L_{a} & \theta_{(x, y)} & \text { diamondRing }\end{array}$

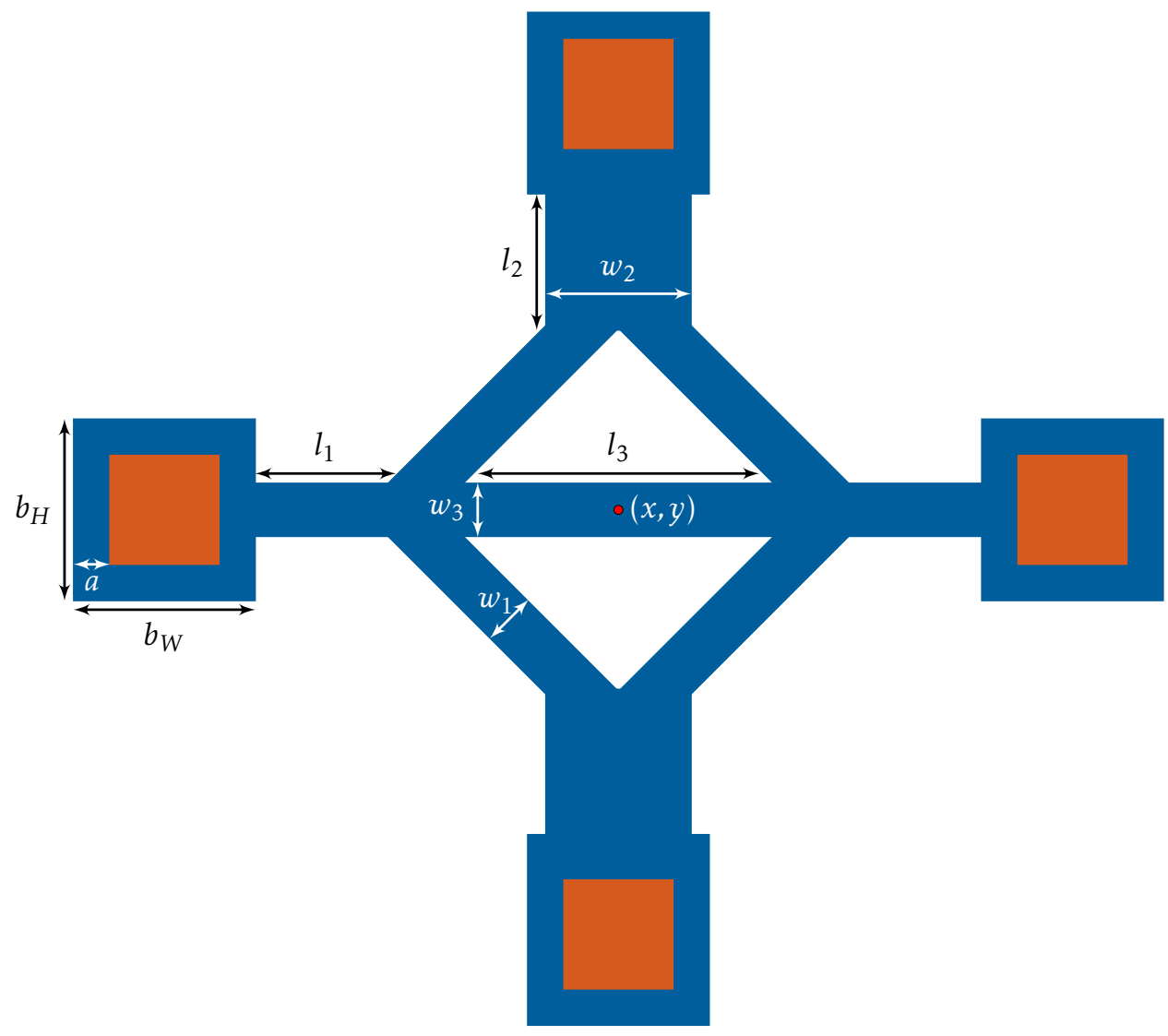

Figure 2.346: Stress measurement diamond ring. 


\subsubsection{Suspended Fluid Cell}

Fluid cell $\left(L_{c}\right)$ with a suspended region defined by radius $r_{3}$ and a spiral delay line heater $\left(L_{b}\right)$. Central red dot indicated coordinate $(x, y)$. The fluid input funnel shape are defined by the shapeReso parameter.

$x y w_{1} w_{2} w_{2 T} w_{3} w_{4} L_{1} L_{2} r r_{1} r_{2} r_{3} N_{\text {sides }} a b c d e f g h i L_{a} L_{b} L_{c} L_{d} L_{e} L_{f} \theta_{(x, y)}$ fluidCell

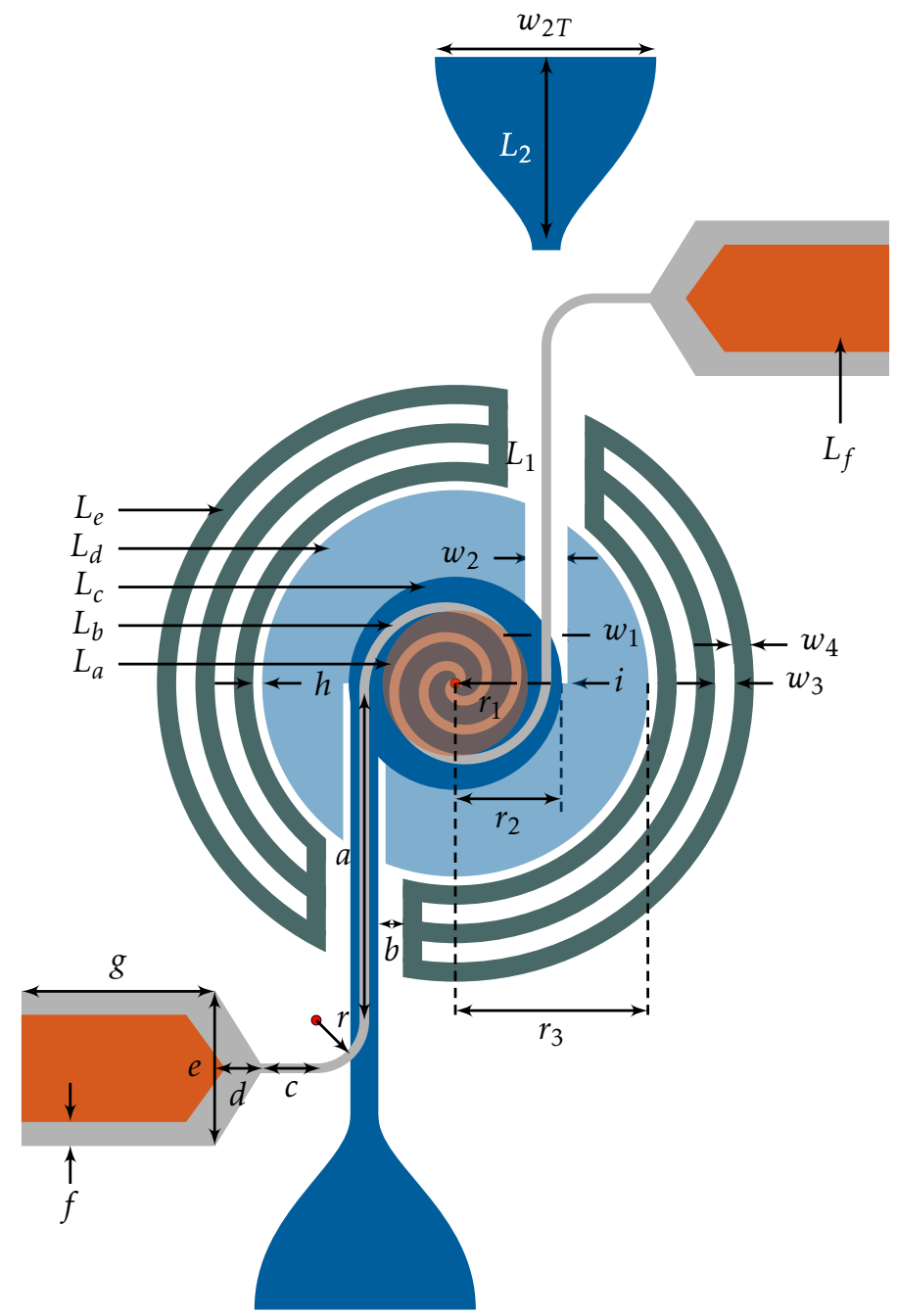

Figure 2.347: Fluid cell $\left(L_{c}\right)$ with an integrated spiral delay line heater $\left(L_{b}\right)$ and release trenches $\left(L_{e}\right)$. 


\section{Graphical User Interface}

\subsection{Basic Shapes}

\subsubsection{Pillar-Hole (Square/Hex) Array}

Within in this module, polygons are created and arrayed. User defined parameters consist of number of sides, size and rotation of individual elements, the GDS layer number, and lower left position, number of elements, and pitch between the arrayed structures. This module creates cells for individual elements used to create pillars and holes. Pillars are created by performing a boolean operation between a box of dimensions $\left(\right.$ Pitch $_{x} \times$ Pitch $\left._{y}\right)$ and the specified shape. The shapes are then instantiated within 4 arrayed cells consisting of square and hexagonal arrays of both polarities. Figures 3.348 $(a-h)$ show several examples of the pillar hole array.

\subsubsection{Torus}

This module creates arc shapes defined by the start $\left(\theta_{s}\right)$ and end $\left(\theta_{e}\right)$ sweep angles of the individual elements. Torus is created by sweeping an arc over $2 \pi$ radians, i.e. $\theta_{e}-\theta_{s}=360$ degrees. Default parameters for the torus module are $\theta_{s}=0$ degrees and $\theta_{e}=360$ degrees. Generated features are defined by the number of sides for the inner and outer arc segments, the GDS layer number, and rotation of individual shapes. Position, elements and pitch parameters define square and hexagonal array elements. Figures $3.348(i-j)$ show 4 examples of various arcs.

\subsubsection{Grating Coupler - Bulls Eye}

The module creates concentric arcs to form grating couplers or bulls-eye patterns. The arc section is defined by a sweep angle and arc width. The arrayed elements are located at the specified $(x, y)$ position. First element is placed at a distance defined by the radius midpoint. Parameter Rings defines the number of arrayed elements instantiated at the specified pitch. Figures $3.348(k-l)$ show several examples of grating coupler structures. 


\subsubsection{Spirals}

Spirals are defined by their center position, GDS layer number, shape width, and number of turns. Shape resolution is specified by the Increment parameter. Choosing many turns with a small increment (high resolution) will increase the GDS file generation time. Spiral structures are generated and placed into a GDS structure named top.

We offer three types of spirals, Archimedes, Fermat and Logarithmic (Figures 3.348 $(m-o))$. Archimedes spiral has a uniform spacing between turns $(s)$ and is defined as

$$
r=m \theta
$$

where

$$
m=\frac{s+w i d t h}{2 \pi}
$$

Fermat spiral is defined as

$$
r=\sqrt{a^{2} \theta}
$$

and Logarithmic spiral is defined as

$$
r=a e^{b \theta}
$$

\subsubsection{Gratings}

Gratings module generates user-defined lines arrayed at a predefined pitch. Each line of the table will create two cells, one for the line and the other for the grating array. Generated structure names will have a prefix $R X$ where $X$ represents the table row number. The load table button allows users to upload text files. Values from loaded text files will populate the table. Top cell will have an instance of each array separated by the TopCellSpacing value. Figures $3.348(p)$ shows an example of a grating structure. An example of a grating text file is located $\backslash$ loadFiles $\backslash$ gratingTable.

NIST • CNST Nanolithography Toolbox v2016.09.01 • http://www.nist.gov/cnst/

page 361 of 488 


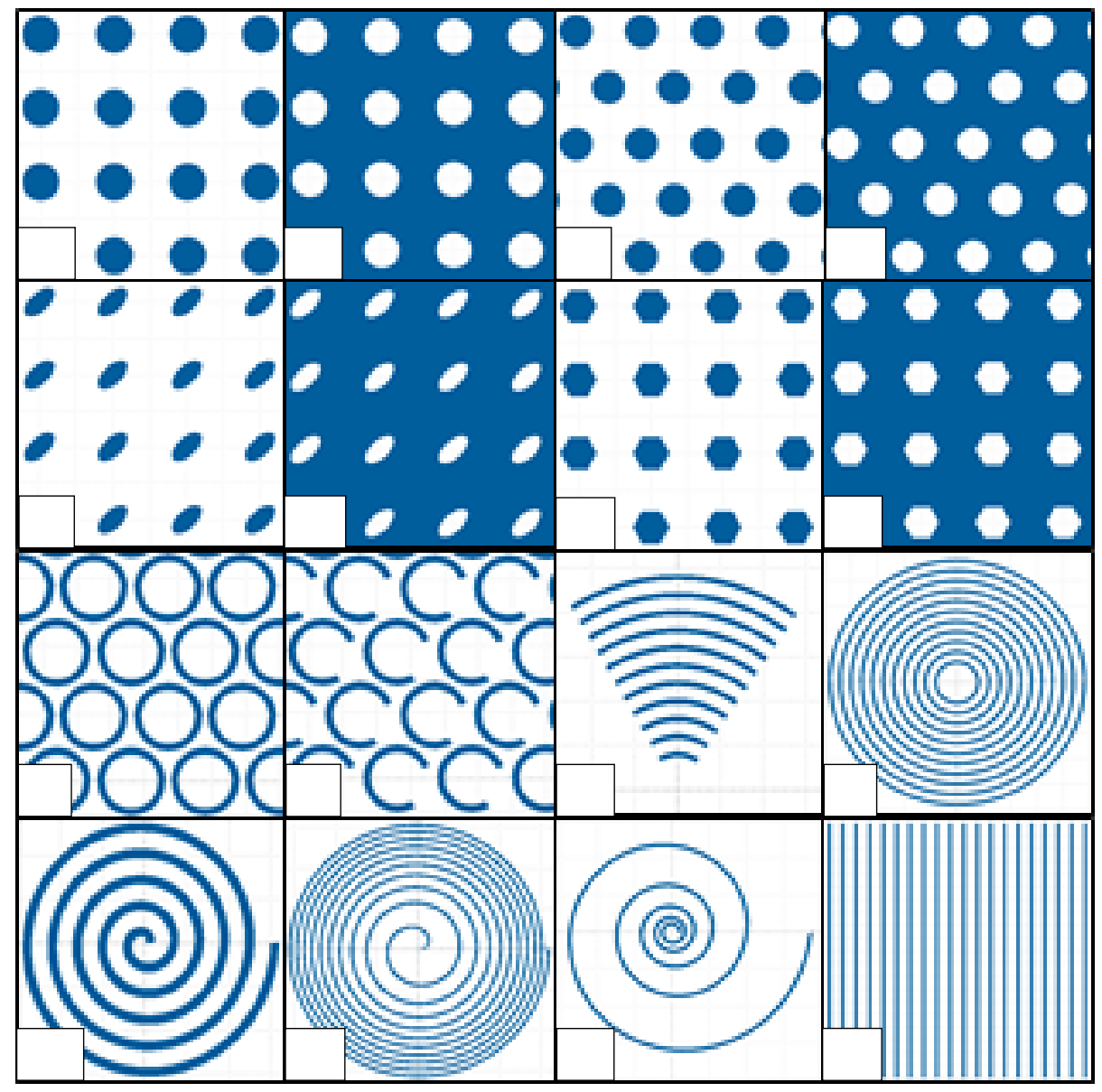

Figure 3.348: Various examples from the BasicShapes branch. Rectangular arrays of $(a, e, g)$ holes and $(b, f, h)$ pillars. Hexagonal array of (c) holes, (d) pillars, (i) torii and (j) arcs. (k) Grating coupler and (l) bulls-eye pattern. Spirals created using various parameters (m) Archimedes, (n) Fermat, (o) and Logarithmic. (p) Grating structure. 


\subsection{Lithography Machine Resources}

\subsubsection{CNST Reticle Frame Generator}

This module creates reticle frames for the CNST Nanofab ASML-PAS5500 i-line stepper and contact aligners. Default stepper option will print reticle marks, user defined label and barcode, NIST and CNST logos. ASML barcode and label are limited to 12 and 14 characters respectively. Two remaining options are for 5 inch and 7 inch contact masks. In both cases, label, along with NIST and CNST logos are printed near the periphery of the mask. Contact labels are limited to 22 characters.

\subsubsection{Generic Reticle Frames}

Within this module, reticle marks, label and barcode are generated for several steppers.

\subsubsection{Ebeam Lithography - Job and Schedule File Generator}

This module generates job and schedule files for the CNST JEOL 6300 electron beam lithography tool. The module offers a number of graphical features that help ensure proper pattern and alignment mark placement. Additionally, it features a wide variety of dose variation features including base dose matrices, single and multi-shot rank modulation, user defined dose table modulation, and several dose ramping functions. This node has 4 sections accessible using the tabs located at the top of the panel named Main, Align, Pattern and DoseMatrix.

\subsubsection{Default Values Initialization File}

Many features within the CNST JEOL 6300 JDF-SDF module are customizable using the provided CNST def aultValues. $x m l$ initialization file. For example, position size, auto-loader (ALD) position, paths, calibration parameters, alignment marks represent a few parameters that are stored within the initialization file. If this file is missing, hardcoded values will be displayed within the toolbox. Also, directly to the left of the About button within the Main tab, a string will indicate if values were loaded from the initialization file.

\subsubsection{Main}

Within the Main panel material parameters, jdf and sdf file names, alignment schemes, paths and calibration parameters are preset. The PATH and 
CALPRM variables are editable. Most of these parameters are predefined within the CNST defaultValues.xml initialization file. Figure 3.349 shows parameters under the Main panel.

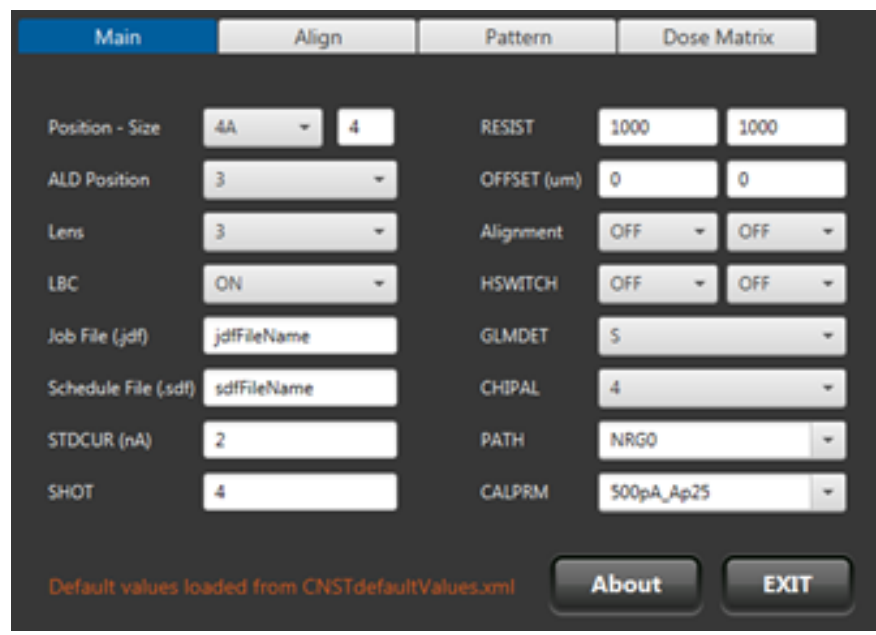

Figure 3.349: Main panel showing various available options under Main tab.

\subsubsection{Align}

The alignment panel allows users to define position and size of global $(P$ and $Q)$ and local $\left(M_{1}, M_{2}, M_{3}\right.$, and $\left.M_{4}\right)$ alignment marks. The positions are in wafer coordinates in units of micrometers. Under mark definition, mark width and length values are specified in micrometers for the the global $P(G L M P)$, global $Q(G L M Q R S)$ and chip (CHMARK) alignment marks. The Align section is only used if global and local marks are selected under the Alignment field within the Main tab. The left-most alignment option activates the global marks, while the right-most option controls local (chip) alignment. The options are by default inactive as indicated by the OFF state. Figure 3.350 shows parameters under the Align JEOL 6300 panel.

\subsubsection{Pattern}

This section defines pattern arrays within the job definition file. Pattern files specified, arrays are generated from those pattern files and stored into a vector array is accessed when job and schedule definition files are created. To add a pattern file to the database, type in a pattern file name then click the AddFile button. A string label will appear and indicate that the entered .v30 file was added to the pattern file list. More files are added by repeating the previous process. Entered pattern names will appear in the pull down selection box. 


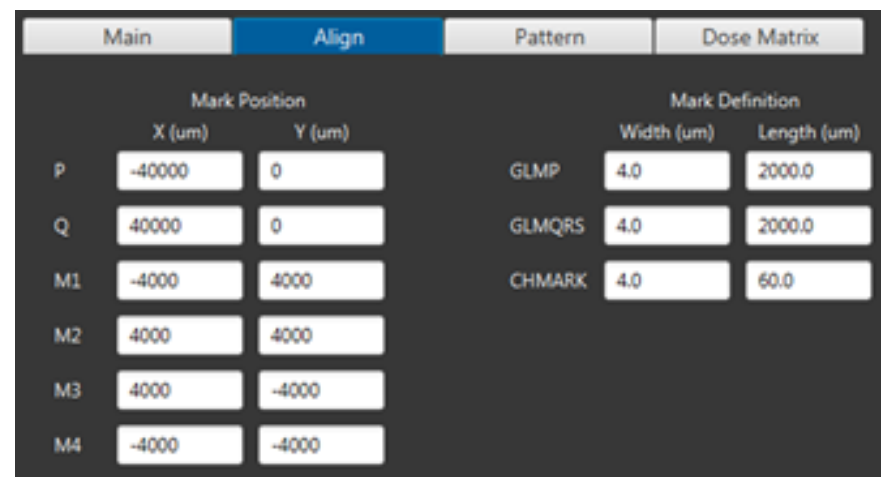

Figure 3.350: Alignment panel showing various alignment mark definitions under Align tab.

Select a pattern, then type underneath the ARRAY section, type in the starting coordinates of the first element of the array, then number of elements and the pitch between elements, then type in the dose modulation for the pattern file. For a single element leave both Element values at 1 . Click the Generate Array button. The value of the generated array will appear in a selection box directly to the left of the Generates Arrays field. Repeat the process, i.e. choose file name, then pattern position, number of array elements, pitch between elements, enter a dose modulation and click the GenerateArray button. Delete button to the right of the selection box within Generates Arrays field allows users to delete previously generated arrays stored within the selection box. Also, generated arrays can be modified by choosing an array from the saved list. Following parameter modifications, clicking the UpdateArray button overwrites the previous entry. Figure 3.351 shows parameters under the Pattern panel.

To view the arrays as well as the job and schedule files at any point during the array creation, click the Show SDF JDF button. A new frame will pop-up with a text area containing the contents of the jdf and sdf files. Another frame will pop-up and will show graphically where patterns are placed (Figure 3.352). The feature is similar to the JEOL Array Check program. Following any alteration of the generated array elements, clicking the Show SDF JDF button will update the job and schedule file panel as well as the array check panel.

\subsubsection{Dose Matrix}

Dose matrix module is a means to create complex dose and base dose matrices. The procedure initiates by choosing a pattern file name, the position, number of elements and pitch between the elements of the resulting dose array (Figure 3.353). There are various options under the DoseMethod tab. Standard DoseMatrix is the default method for executing a dose matrix on the chosen V30 file. Method BaseDose is used to determine a proper base dose for 


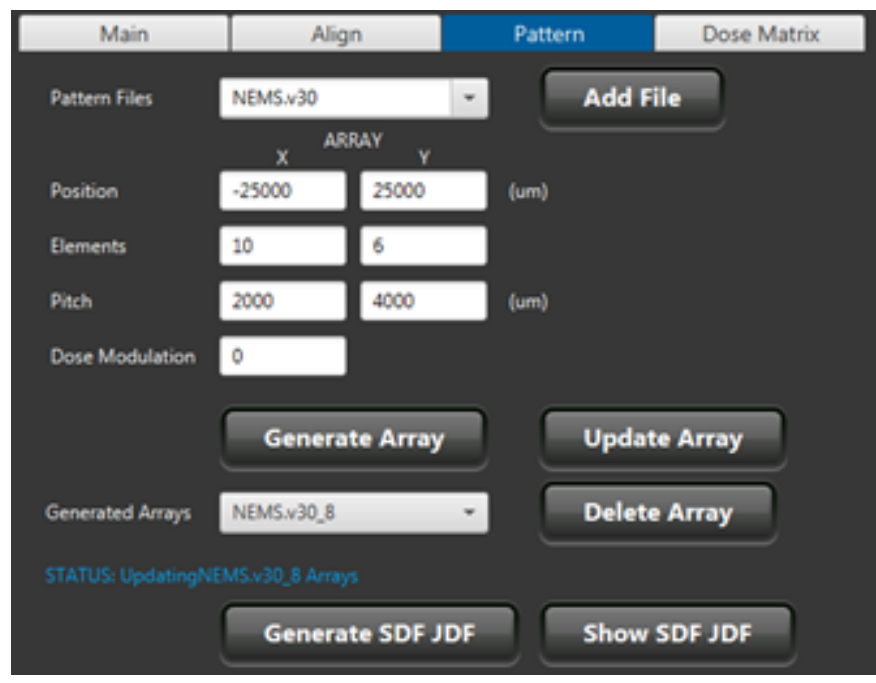

Figure 3.351: Pattern panel showing user-defined pattern files, array definitions, and generated arrays.

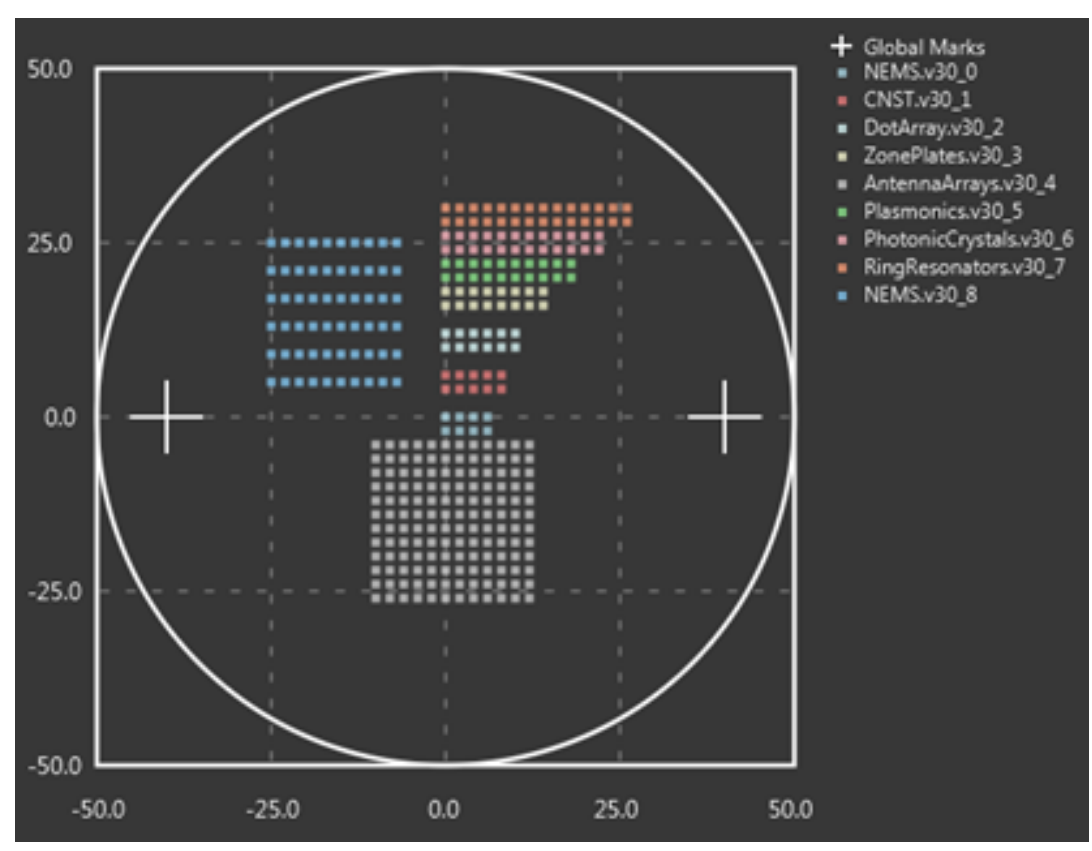

Figure 3.352: Display showing user generated pattern arrays and global mark positions (P and $Q)$. 
a proximity effect corrected pattern. Here base dose (RESIST parameter) is modified within the schedule file, the pitch in the two directions will represent the OFFSET parameter, with the RESIST value derived from the Start text field within this tabbed panel. There are 4 options within the Ramp selection menu. Linear and Percent options use respective right-most text-field values of Linear and Percentage to increment the dose array from the starting value represented by the Start text field. For instance, choosing Linear with a value of 10 within the Linear text field in conjunction with a Start value of 100 for an array of 4 elements, percentage modulation dose values will be 100, 110, 120 and 130. Analogously, with an active Percent ramp option and a value of 10 within the Percentage text field in conjunction with a Start value of 100 for an array of 8 elements,percentage modulation dose values will be 100, 110, $121,133.1,146.41,161.05,177.16$ and 194.87. LinearSE and NonlinearSE are two ramp methods that utilize number of arrayed elements, the Start and End text field values to determine the percentage dose modulation.

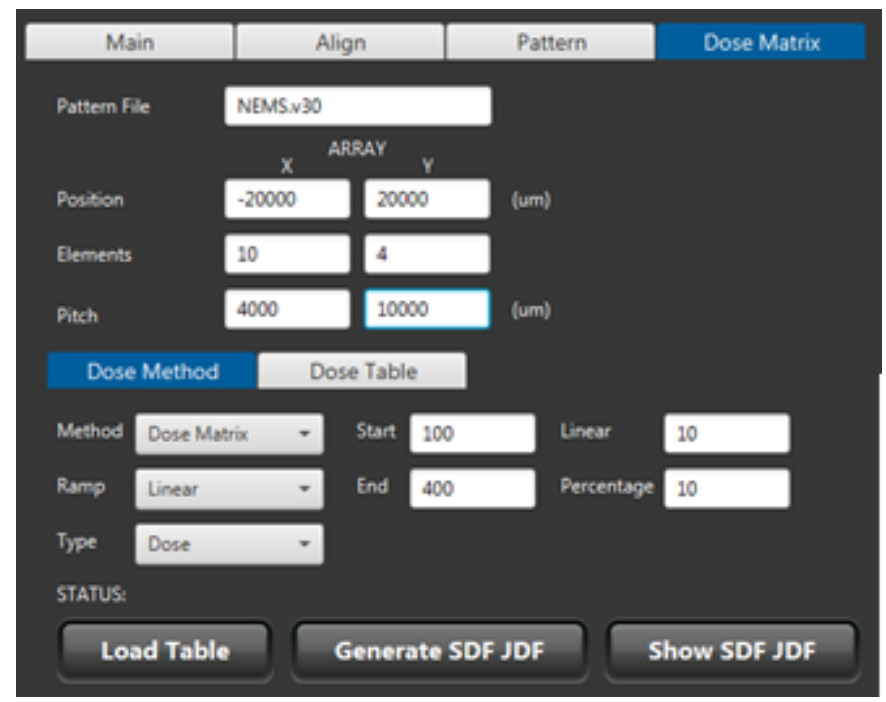

Figure 3.353: Dose matrix panel showing various user defined pattern, array and dose parameters.

Dose represents the default value of the Type selection. DoseTable option under Type uses values from the DoseTable tabbed panel. Within the Dose Table tabbed panel, values for the shot rank and modulation are either manually entered or uploaded from a text file (Figure 3.354). The method assumes the standard dose modulation table format, as seen in the layout beamer proximity effect corrected output file with extension JDI. An example dose matrix JDI file is located in the $\backslash$ loadFiles \jeol6300DoseTable directory. When using the table method, ramp can either be the default Linear or Percent option. Depending on which method is chosen, the respective Linear and Percentage text fields are used to modify the individual modulation values from the table. The sdf and jdf 
files could be shown or generated using the respective buttons at the bottom of the panel.

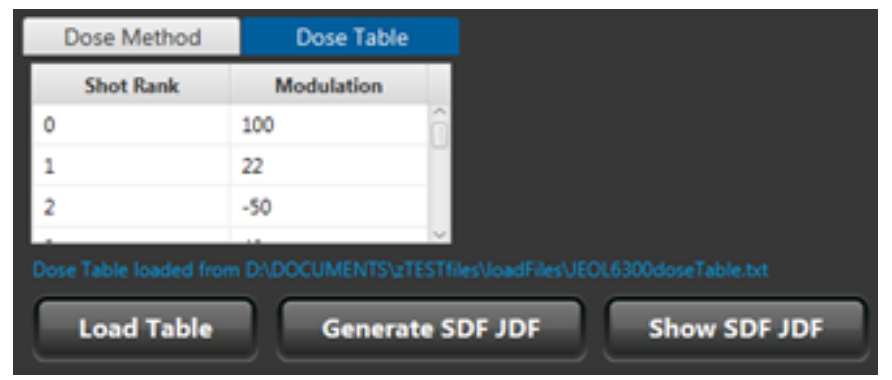

Figure 3.354: Dose matrix panel showing various user defined pattern, array and dose parameters.

\subsubsection{EBL Alignment Offset}

The JEOL alignment module calculates the offsets between the designed and observed wafer positions. Type in the designed mark positions of the $P$ (or $Q$ ) marks. This value is specified in material (or wafer) coordinates with micrometer units. Check the substrate holder center position and type in those values for the wafer center. Once the marks are located in the scanning electron microscope (SEM) of either of the CNST ebeam tools, type in the stage coordinates of this observed mark position, then press Enter or click the Calculate button. Within the OFFSET text field, calculated values will appear. These values should be used within the SETWFR routine to manually check global alignment. Furthermore, these values are placed within the schedule file (.sdf file) as:

$$
\text { OFFSET }\left(X_{\text {offset }}, Y_{\text {offset }}\right)
$$

\subsubsection{EBL Max Clock}

This module calculates the maximum writing frequency and the minimum shot time for the $4^{\text {th }}$ and $5^{\text {th }}$ lens electron beam lithography tools. Calculation is based on beam current, writing dose, shot pitch and tool type.

\subsubsection{EBL Write Time Estimation}

This module calculates an estimated electron beam lithography write time. Calculation is based on beam current, die area (written die area in micrometers squared - a value easily obtained from various $C A D$ and fracturing tools), writing 
dose, fields per die and the number of die. Stage time is 'estimated' at $1 \mathrm{sec}-$ ond per stage move. This is not quite correct ('estimate') since stage motion depends upon distance traveled and the EBL tool. The estimated calculation does not take into account tool calibration time. 


\subsection{Advanced CAD Resources}

Many of the modules in the advanced CAD resources are implemented within CNST scripting. Therefore, many modules refer to sections and figures within the scripting command reference chapter. Users should note that scripting offers more flexibility with many more features not implemented within its GUI counterpart.

\subsubsection{Label Maker}

Label Maker module is used to generate chip labels into a GDS file that could then be instanced into a user defined pattern. There are 4 label type options. The automated Outer and Row-Column will generate number arrays, whereas the custom variants are more versatile allowing imported tab delimited data to be cast as labels. Two test label files are included in \loadFiles\labels example directory. The files were generated in Excel and exported as plain text tab delimited.

Within the label maker module, first, number of elements, pitch between the elements, font specifications and label type are specified. Font resolution is the rendering resolution of the Bezier curves that construct font shapes. This parameter is defined as shapeReso within CNST scripting (see Section 2.2.6 and Figure 2.16). The four label types are described within the label maker scripting command reference guide. This module does not implement automatic letter labeling. Scripting section 2.6.3 further illustrates automatically generated Outer and Row-Column (Figure 2.45a,b) and the respective custom, user-defined labels (Figure 2.45c,d).

\subsubsection{Text To GDS}

Text to GDS module renders strings composed of vector fonts to GDS shapes. Font resolution parameter controls the rendered shape resolution. Example of font resolution and generated text are shown respectively in Figure 2.2.6 and 2.43 .

\subsubsection{Arbitrary Function Generator}

Arbitrary Function Generator module will create a GDS file with a shape defined by a mathematical equation. User will input a lower and upper bound, an increment and line width for the function. Within this module, users define a function $f(x)$ using the lower case variable $x$. Functional definitions follow the Java Math class. Scripting section 2.7.4 offers a more efficient function generator. This section also describes how functions are represented.

Functions constructed with a large number of points could take a consider-

NIST • CNST Nanolithography Toolbox v2016.09.01 • http://www.nist.gov/cnst/

page 370 of 488 
able amount of time to cast the resulting GDS file. Label indicator above the module buttons will display the path and file name once it's finished writing data to file. Also, casting a curved function defined by a large width could result in a shape with looped interiors.

\subsubsection{Binary Zone Plate}

In a binary zone plate, zones switch from opaque to transparent at radii,

$$
r_{n}=\sqrt{n \lambda f+\frac{n^{2} \lambda^{2}}{4}}
$$

where $n$ is an integer, $\lambda$ is the wavelength and $f$ is the focal distance measured from the center of the zone plate. Each shape is constructed with double the number of prescribed sides (interior and exterior) whereas the central element (circle) vertices are equal to the Sides module parameter. Figure 3.355 shows two examples of binary zone plates. Zones are either composed of a single user defined GDS layer, or cast into zone-respective GDS layers. This implies that zone at a radius $r_{n}$ is rendered to GDS layer number $n$ where $n=0,1,2, \ldots 255$. Due to 8-bit layer GDS standard restrictions, zone numbers above the upper bound (255) reset the counter back to GDS layer number 0 . Consequently, zones at radii $r_{256}, r_{257}, r_{258}, \ldots$ would respectively have GDS layer numbers $0,1,2, \ldots$.

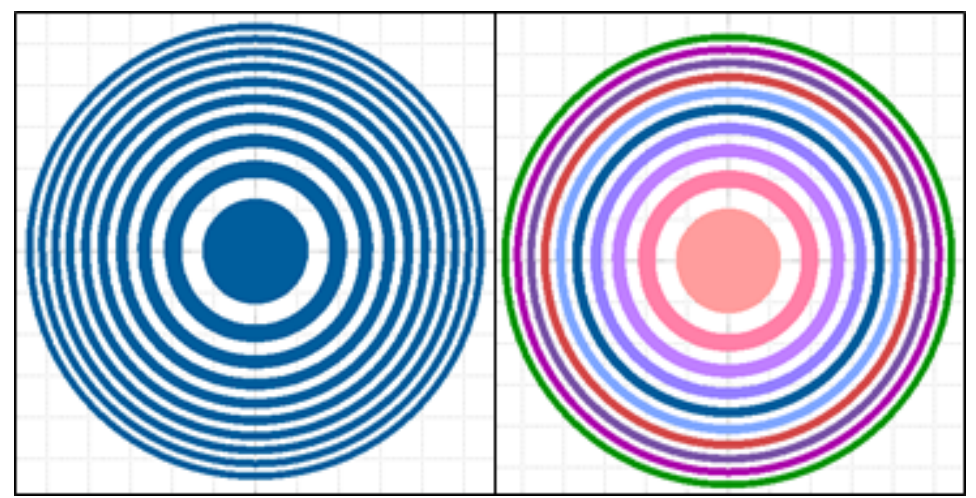

(a)

(b)

Figure 3.355: GDS output of a zone plate with 10 zones, 88 sides per zone, $\lambda=632.0 \mathrm{~nm}$, and $f=0.001 \mathrm{~m}$. (a) Single layer zone plate. (b) Enabling the different layers option casts each zone to a respective GDS layer number.

NIST • CNST Nanolithography Toolbox v2016.09.01 • http://www.nist.gov/cnst/ page 371 of 488 


\subsubsection{Photonic Crystals}

Photonic crystals module creates two hexagonal arrays of circles. Circles are defined by a diameter $(d)$, number of sides and GDS layer number parameters. Within the array circular elements are defined by the spacing proximity to the nearest neighbor $(h)$. Hexagonal arrays are defined by the separation between the two arrays $(s)$, and the array size $S_{x}$ and $S_{y}$ along the corresponding $x$ and $y$ directions (Figure 3.356). Table of values are either manually populated or data is loaded from a text file. Example photonic crystal table file is included in the $\backslash$ loadFiles\photonicCrystalTable directory. Each table value is tab separated, each table row starts on a new line. Figure 3.356 shows various parameters from the photonic crystal module.

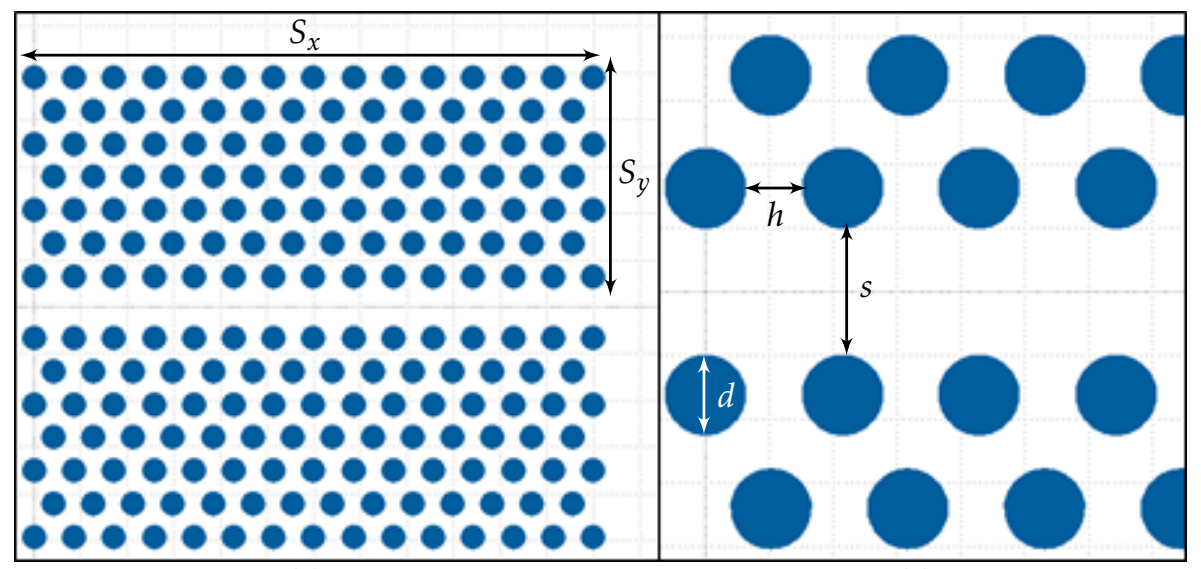

(a)

(b)

Figure 3.356: Photonic crystal example of a rendered GDS structure labeled with module parameters.

\subsubsection{Random Polygons}

Random Polygons module will randomly place polygons into a user defined area. Separation parameter defines the minimum separation between the outer radial perimeter of the objects. Polygons are defined by the outer radius and number of sides. The polygon placement area is defined by the Width and Length parameters. If the randomly generated coordinate violates the minimum separation distance, the module will keep generating random coordinates until the maximum number of failures (defined by the parameter Iteration) is reached.

By clicking the RandomRotation checkbox, each shape will be randomly rotated within the top cell. Enabling the option for features with many sides is 
not useful, however, with Sides $=3$ and random rotation produces randomly oriented triangles within the array (see Figure 2.78 in section 2.7.11).

This shape could be generated using the scripting constructor randomPolygons, as described in section 2.7.11.

\subsubsection{Random Rectangular Array}

Random rectangular array module will randomly place squares or rectangles into a user-defined area specified by the number of elements and pitch (in $x$ and $y$ directions). The shape size (square or rectangular) as well as the probability that a lattice point is occupied are user-defined parameters. Furthermore, user can load $x$ and $y$ coordinate pairs and cast them to lattice sites. This module was suggested by a researcher and used for generating disordered metamaterial structures.

This module allows users to load $x$ and $y$ coordinates where objects will be drawn. The ASCII file should be arranged with $x$ and $y$ pairs tab or space separated, with one pair per line. The first line of the data file is defined as a header and is consequently skipped. An example file is located under the $\backslash$ loadFiles \randomRectangularArray directory. To use the module, first choose the size of the square or rectangular object, then check the Load File check box, then click Load File, navigate to a directory with the text file, click on the file, then click OK. The chosen filename will be displayed in the label field directly below. Then click Create GDS to cast the shapes into a GDS file. NOTE: when loading from file, this module ignores the number of elements field, and will not display the total number of drawn shapes, skipped shapes, etc, within the results label field.

\subsubsection{Cantilever Arrays}

Cantilever Arrays module will create a GDS file with a modulated (linearly, nonlinearly, percentage, sinusoidally, etc) cantilever array structures. Number of resulting structures is defined by the Elements parameter. Levers are characterized by a width, pitch and variable length values. Cantilevers are connected to a rectangular base of a user specified height. The width of the base rectangle is defined by the number of elements, pitch and the base extent.

By choosing Linear or Percentage type variation, the generator will utilize the Length start value and will increment the length using the linear or percentage parameters respectively. Type Linear SE and non-linear SE use the Length start and Length end values and respectively vary the cantilever lengths linearly and non-linearly. Type Sinusoid will use the Length start value as the initial length value and will vary the cantilever lengths sinusoidally over a $2 \pi$ period with an amplitude defined by the sin Amplitude value.

The MEMS-NEMS library offers identical elements (section 2.10.5) as well as many other shapes with enhanced functionality. Figure 2.316 illustrates can-

NIST • CNST Nanolithography Toolbox v2016.09.01 • http://www.nist.gov/cnst/ page 373 of 488 
tilevers with linear, percentage, and sinusoidal length modulation. Figure 2.317 shows examples of a linear and exponential length variation using start and end length values.

\subsubsection{Verniers}

This module generates verniers between two specified alignment layers. User defined parameters consist of GDS layer numbers for layer $A$ and $B$, vernier resolution, number of vernier tick lines, text labels for the two layers, vernier line width, length and pitch. Table of values are either manually populated or data is loaded from a text file. Example vernier table file is included in the $\backslash$ loadFiles\vernierTable directory. Each table value is tab separated, each table row starts on a new line. Vernier example is shown in the scripting command reference section 2.8 .5 and figure 2.94 .

\subsubsection{Fractals}

This module contains Sierpinski triangle and carpet, curved tree, and the Vicsek saltire and cross fractals. Each fractal iteration is stored into a GDS structure with a prefix cell name concatenated with the iteration number. The extent of the final structure is defined by the module length and width parameters. Fractal examples are defined in the scripting command reference section 2.7.3 (see also Figure 2.58).

\subsubsection{Grayscale Image To GDS}

Grayscale Image to GDS module will convert a 8-bit gray scale BMP (PNG or JPG) and cast it to a GDS file where each level of gray (values 0 to 255) will be mapped to corresponding GDS layer numbers (GDS layers 0 to 255). This mapping is important for grayscale electron beam lithography. In this case, the generated file can be used in conjunction with a resist contrast curve to print three dimensional structures [104].

Resulting data is stored into a GDS structure named top. Within the module, data is read and converted line by line. Adjacent pixels of same value are merged together in order to minimize the resulting GDS file size. Also note that data within the top cell is not centered around the origin. The lower left hand corner of the image is mapped to the origin of the GDS file (point $(0,0)$ ). The image is mapped within the first quadrant $(+x,+y)$ of the GDS file.

If the BW option is checked, then all color values other than white (pixel value 255) will be cast to GDS layer 1 . In this case, adjacent pixels are merged, however, if 2 pixels are of different value, they will be both cast to GDS layer 1 , as 2 different GDS layer 1 pixels. To have pixels merged, use a graphic editing software package to cast image to black and white BMP, then change the mode to RGB 8-bit gray scale. Figure 3.357 shows an image and the resulting GDS

NIST • CNST Nanolithography Toolbox v2016.09.01 • http://www.nist.gov/cnst/

page 374 of 488 
files.

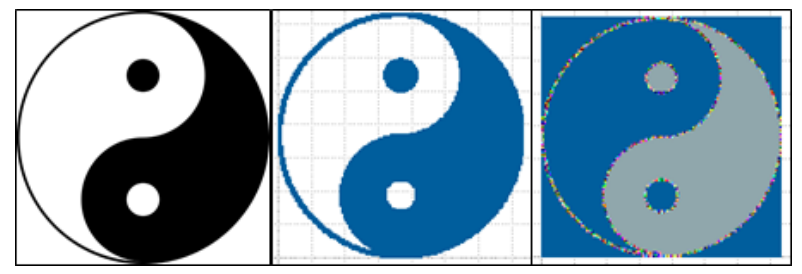

(a)

(b)

(c)

Figure 3.357: Grayscale to GDS. (a) 8-bit gray scale image (in this case black and white). The resulting GDS file (b) without and (c) with the BW option enabled. 


\section{Programming Reference}

\subsection{Programming Examples}

The nanolithography toolbox distribution includes a NetBeans 8.0.2 project entitled CNSTprogrammingExamples. The src directory contains a package with source .java example files. These files could be used with any integrated development environment (IDE) in conjunction with Java 8. Furthermore the nanolithography toolbox jar file must be included within the project compiletime libraries. The following examples illustrate access to existing scripting methods through programming. Furthermore, the project directory includes the output GDS example files.

The following is a procedure for setting up the included Netbeans example project:

1. Start NetBeans -> File -> Open Project ... -> navigate to \EXAMPLES \Programming and open the CNSTprogrammingExamples project

2. To fix the broken library link, right click on the project and choose Properties, click Libraries and remove the red-error highlighted compile-time library. Click the "Add JAR/Folder" button, then choose the CNST Nanolithography Toolbox JAR file and click OK.

\subsubsection{Template}

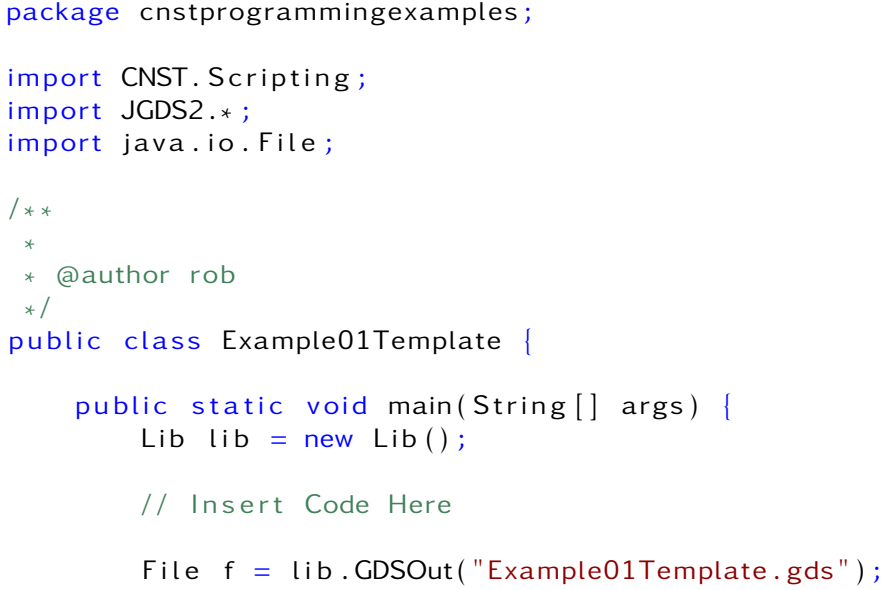




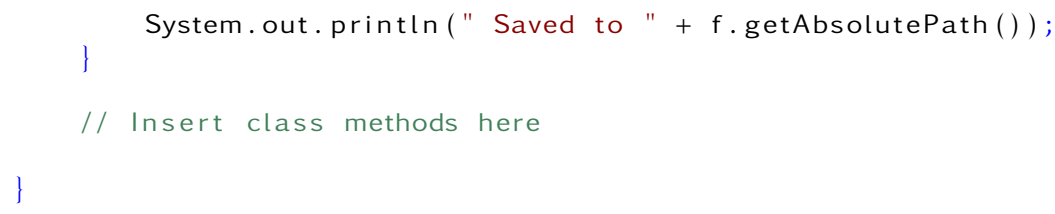

The above example is a template for java files accessing the CNST nanolithography toolbox. Lines 3 and 4 import all the objects needed to access scripting methods and create the GDS file. Line 5 import statement allows file input-output access. The example class contains a main method. Line 14 initializes the GDS library. Line 18 sets the GDS output file. The print statement (line 19) is optional but helpful since it displays the full path of the saved GDS file.

\subsubsection{Script Method Access}

The programming reference provides method headers for the available scripting methods within the toolbox. These methods either return a GDS Area object (GArea) and vector array of these objects (ArrayList $<$ GArea $>$ ), or are void in which case the the areas are placed within a particular GDS structure.

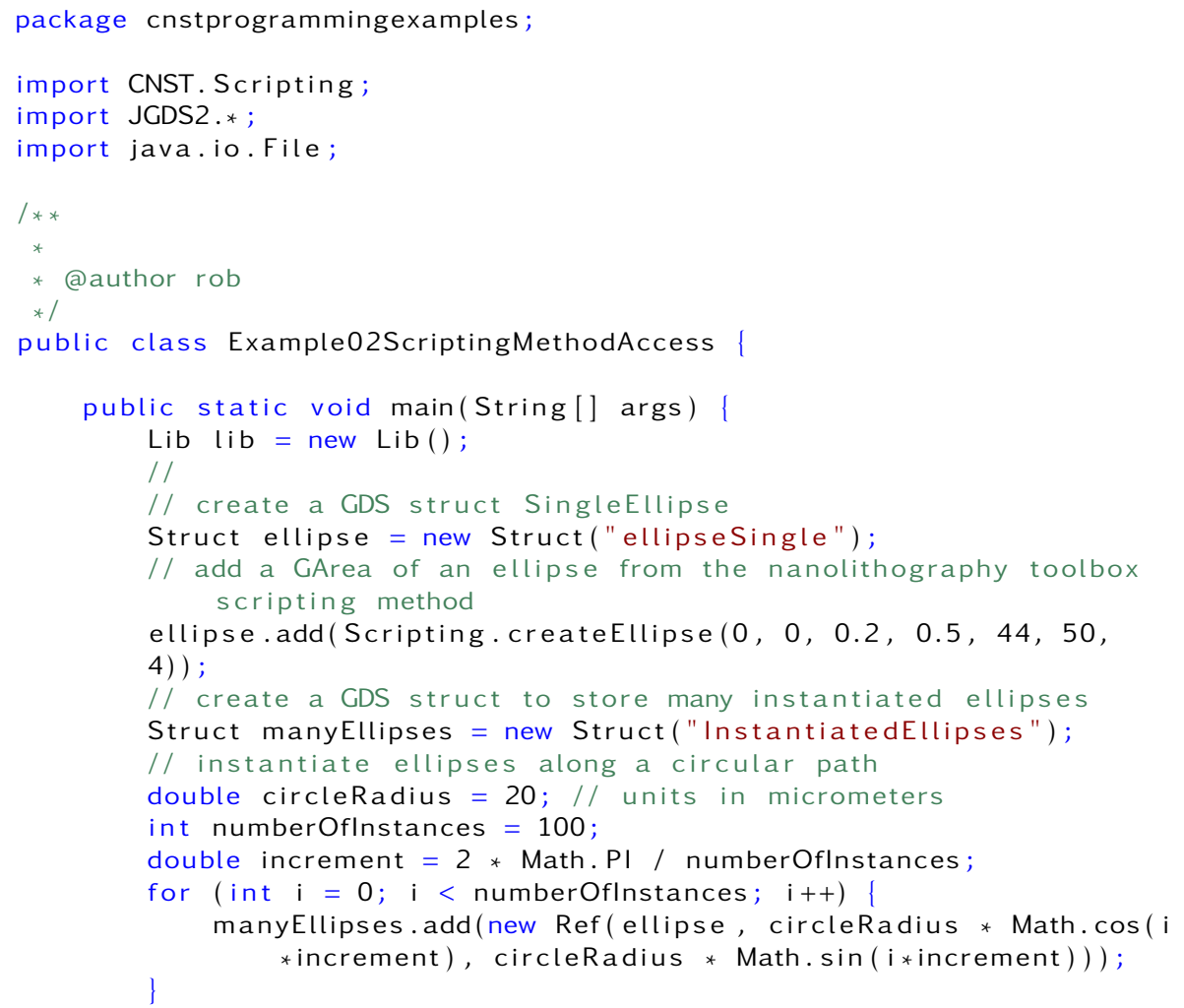




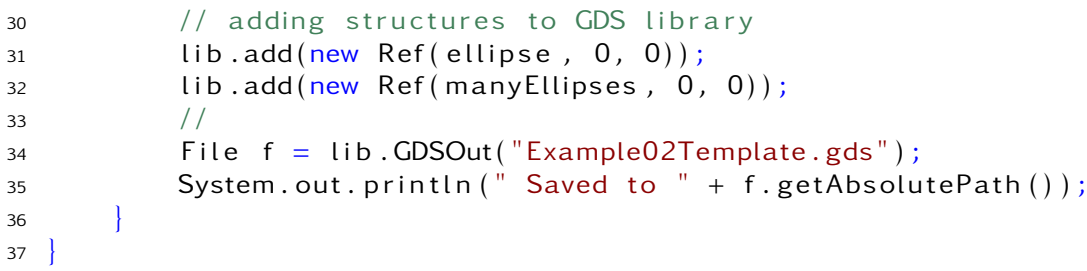

The above example creates a GDS structure object ellipse (line 17) with a structure name ellipseSingle. Line 19 adds a GDS area of an ellipse into the structure object. Here, the ellipse is created by one of the available nanolithography toolbox scripting methods using the following method header (Reference Methods section shows all the available methods)

public static GArea createEllipse (double $x$, double $y$, double radius , double radiusY, int numSides, double THETA, int gdsLayer)

Line 22 creates a GDS structure object manyEllipses. Using a for loop the ellipse object is instantiated along a circular path using the Ref constructor (line line 27). Figure 4.358 shows the output of the instantiated ellipse object. Lines 31 and 32 add the two structures to the GDS library. The following are Ref constructors

Ref(Struct structure, double $x$, double $y$ )

Ref(Struct structure, double $x$, double $y$, int mirror)

Ref(Struct structure, double $x$, double $y$, int mirror, double angle)

Ref(Struct structure, double $x$, double $y$, int mirror, double mag, double angle)

To use mirroring within Ref the existing class must implement an interface Const, then set int mirror to MIRROR. Please consult the JGDS user manual for examples and more information on GDS structure instantiation [76].

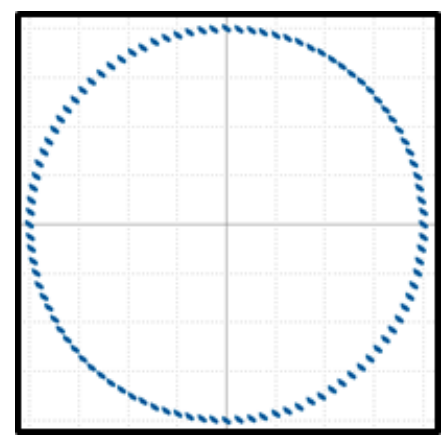

Figure 4.358: Programming example illustrating instantiation of objects created using the nanolithography scripting methods.

NIST • CNST Nanolithography Toolbox v2016.09.01 • http://www.nist.gov/cnst/

page 378 of 488 


\subsubsection{Boolean Operations and Affine Transformations}

The following example illustrates affine transformations of GDS areas and boolean operation between GDS areas. The code first generates a GDS structure (line 18) to store boolean substraction between a circle and a circle wave object. Lines 19 and 20 show instantiation of GDS areas using the available nanolithography toolbox methods. Line 21 subtracts the circle wave from the circle object. Subsequently the result (circle) is stored in the booleanCCW GDS structure. Figure 4.359 shows the rendered GDS shapes.

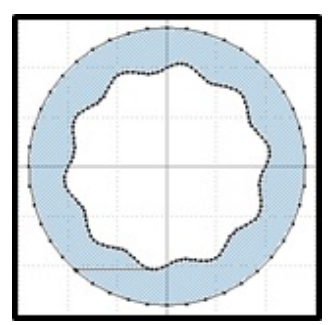

Figure 4.359: Rendered GDS output shape resulting from a subtraction between a circle and a circle wave object. The black dots represent shape vertices.

Lines 25-30 create a GDS structure and two circular areas, then add the areas to the structure. A copy of circle 1 into a temp area object occurs in line 34. Directly following, in line 35 a boolean OR operation with circle 2 occurs. Line 37 shows a GDS area translation using built in Java affine transformations. In a similar fashion, the areas could be scaled, rotated and sheared. Lines 40 to 59 repeat the process for a boolean AND, XOR, and SUBTRACT operations. Figure 4.360 shows the output of the rendered structures.

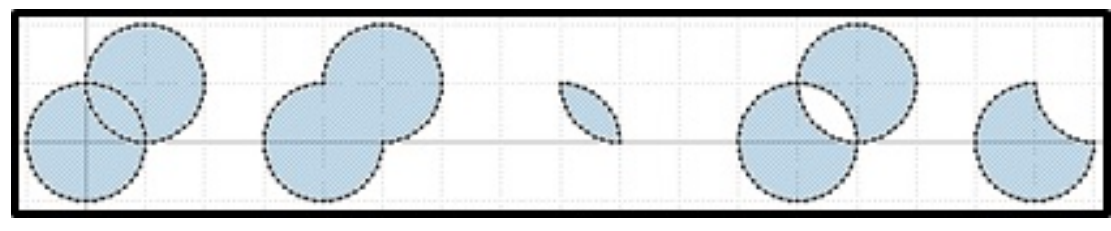

(a)

(b)

(c)

(d)

(e)

Figure 4.360: Rendered GDS output shape of the (a) original two circles and various boolean operations between the areas (b) OR, (c) AND, (d) XOR and (e) SUBTRACT.

1 package cnstprogrammingexamples;

2

NIST • CNST Nanolithography Toolbox v2016.09.01 • http://www.nist.gov/cnst/ page 379 of 488 


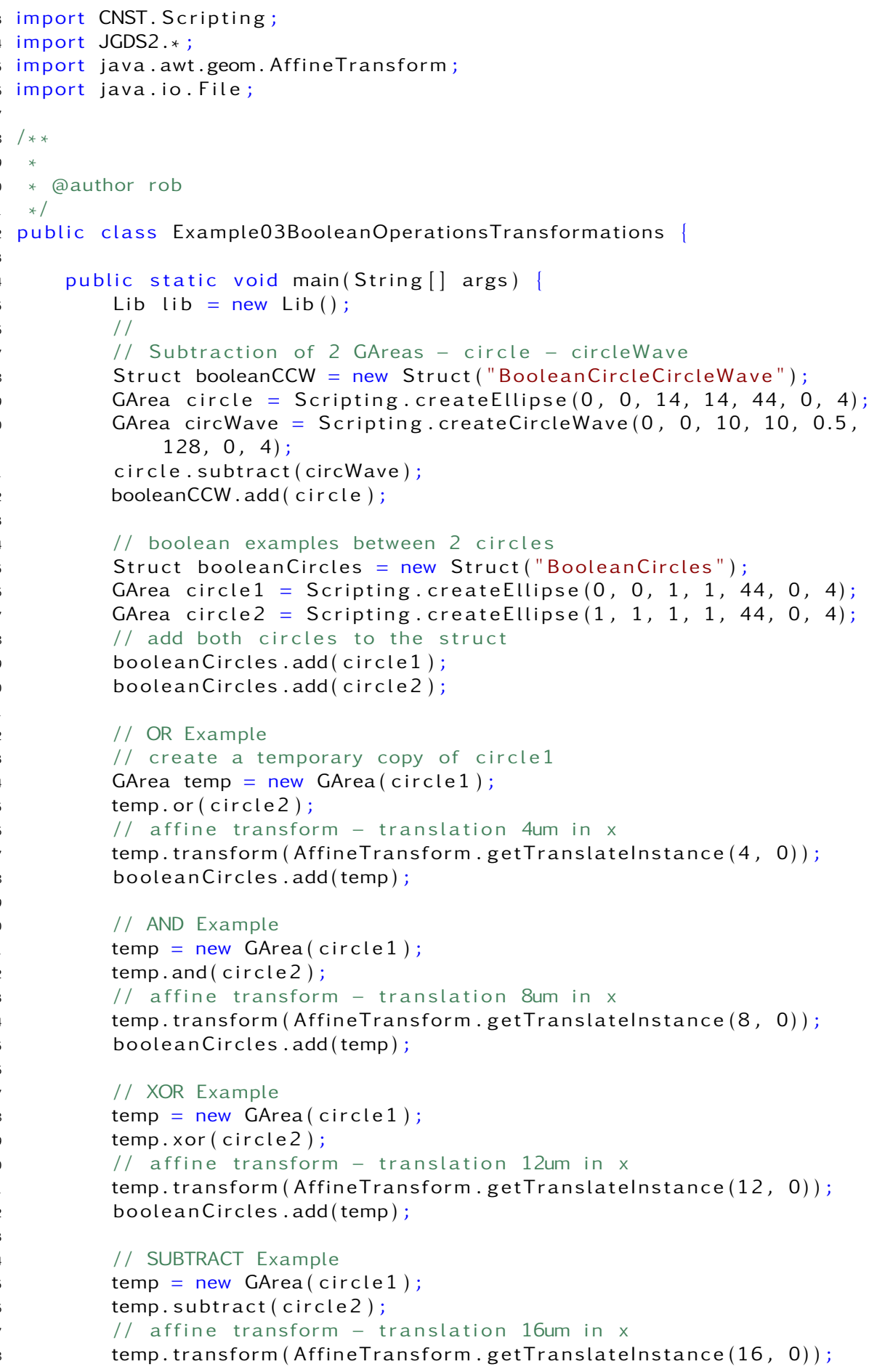

NIST • CNST Nanolithography Toolbox v2016.09.01 • http://www.nist.gov/cnst/

page 380 of 488 


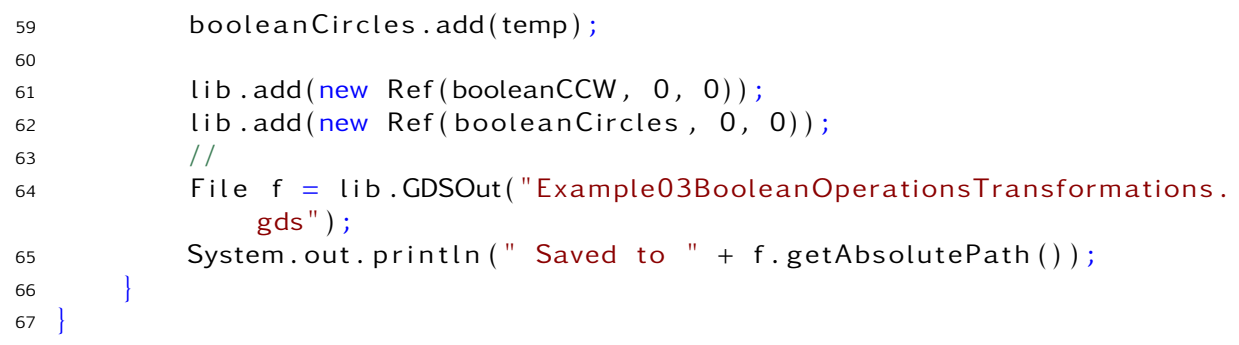

\subsubsection{Labeled Arrays}

Labeled arrays could be created in a variety of ways using the nanolithography toolbox scripting methods. For instance, general areas could be instantiated using any of the available array methods. The following example uses a for loop to iterate through various diameters and separations with

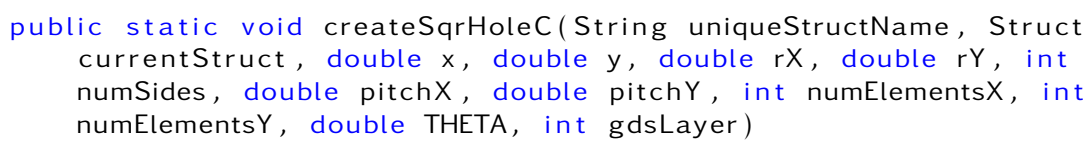

to create an array of circular objects, centered at position $(x, y)$. Within the loop, labels for each array are created using the centered text method

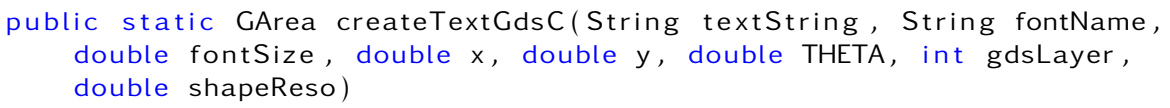

The program starts by initializing a GDS structure top (line 16). This structure will be used to hold instantiated arrays of various circular apertures. Lines 18-29 initialize a variety of variables used to create the arrayed structures. The nested for loop (lines 31-40) iterates through diameters and separations between circular array elements. In the inner loop, a pitch is calculated in line 33. Directly following, a string defining the GDS structure name of individual arrays is established (line 34). Number of array elements, based on the array extent and pitch, are calculated (line 35). Then createSqrHoleC method is used to create and instantiate the hole array patterns within the GDS structure top (line 36). A string label is defined using the diameter and pitch values (line 37) and a GDS area containing the text label is added to the GDS structure top (line 38). Figure 4.361 shows the rendered GDS output.

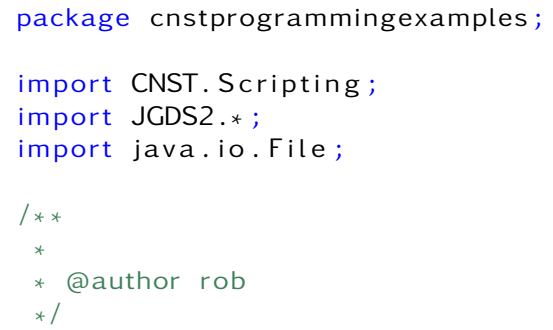

NIST • CNST Nanolithography Toolbox v2016.09.01 • http://www.nist.gov/cnst/ 


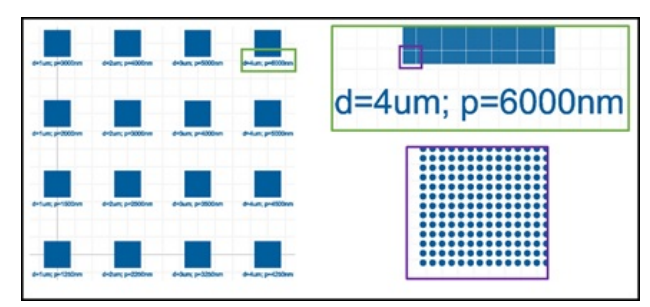

Figure 4.361: Programming example of labeled arrays.

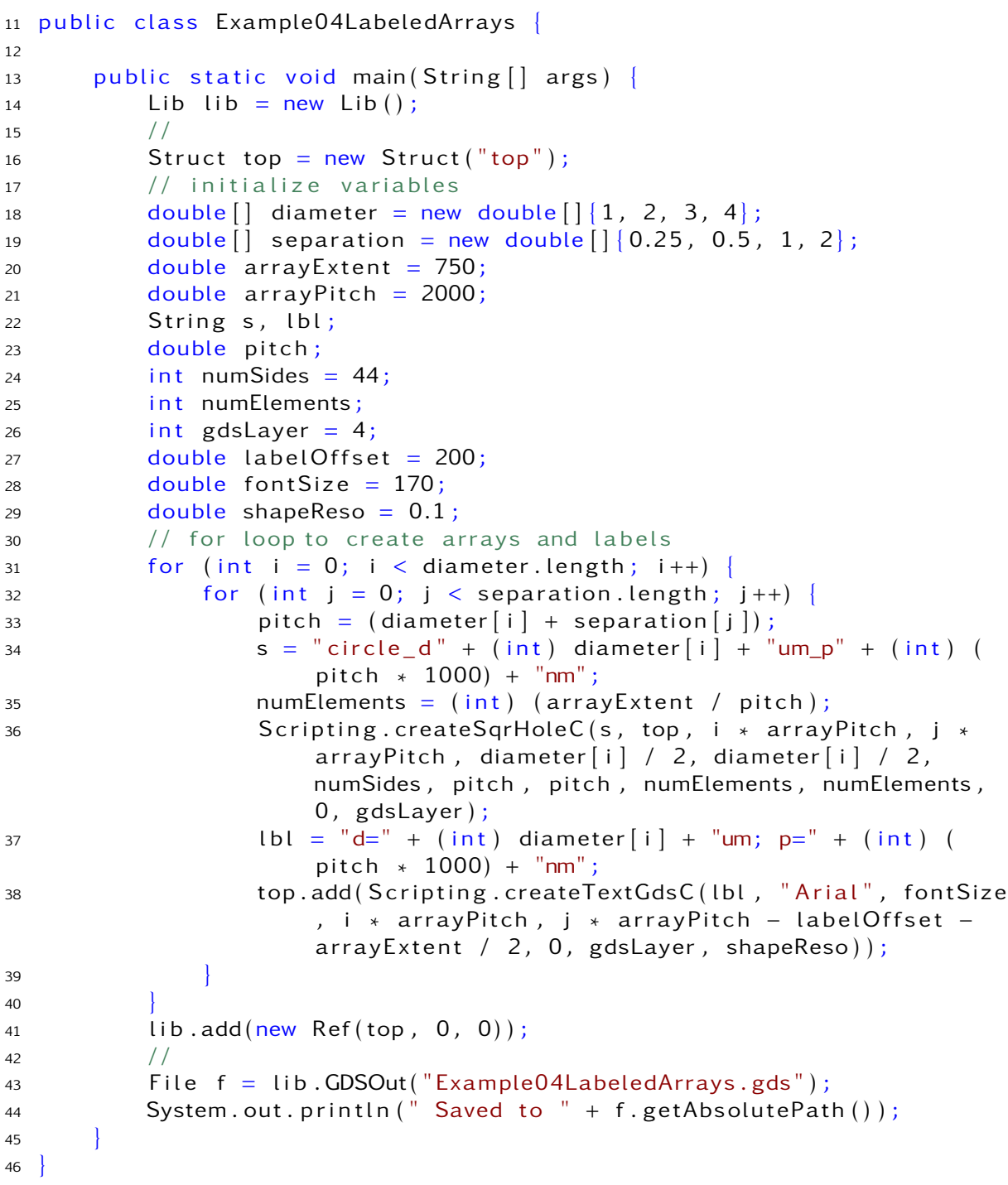




\subsubsection{MEMS Perforated Flexures}

The following code creates a perforated MEMS flexure. The scripting element is called flexure2C and described in subsection 2.10.4. The code describes storing vector array elements into a GDS structure, then extracting various layers from a Struct and storing them into GArea objects. The stored flexure and circle array GDS areas are then used to construct a perforated structure via boolean subtraction.

Lines 17 and 19 create two GDS structures. Line 21 stores the flexure2C element into an ArrayList<GArea>. Using the createStruct method, the vector array al is stored into a GDS structure (line 24). Line 27 stores an array of circles with GDS layer 7 into a GDS structure. Figure 4.362 a shows the GDS rendered output.

Lines 30 and 32 extract GDS areas with particular GDS layer numbers and stores the shapes into the instantiated GArea objects. Line 34 performs a boolean subtraction between the flexure and the circle array. The resulting perforated area is stored into the top structure (line 37). Line 40 extracts the anchors and stores them into the top structure. Figure $4.362 \mathrm{~b}$ shows the GDS rendered output.

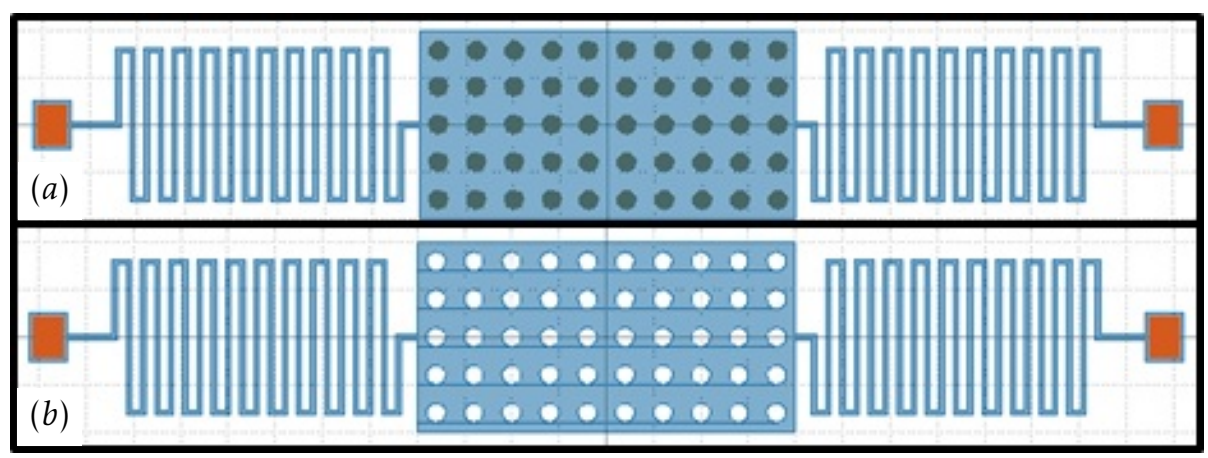

Figure 4.362: Perforated flexures example showing GDS structures (a) flexureWithCircles and (b) top.

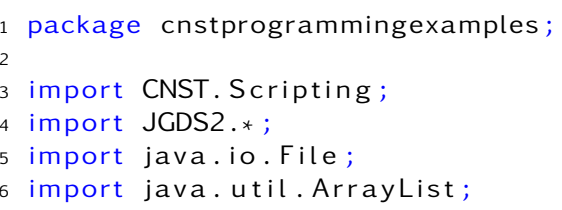

NIST • CNST Nanolithography Toolbox v2016.09.01 • http://www.nist.gov/cnst/ page 383 of 488 


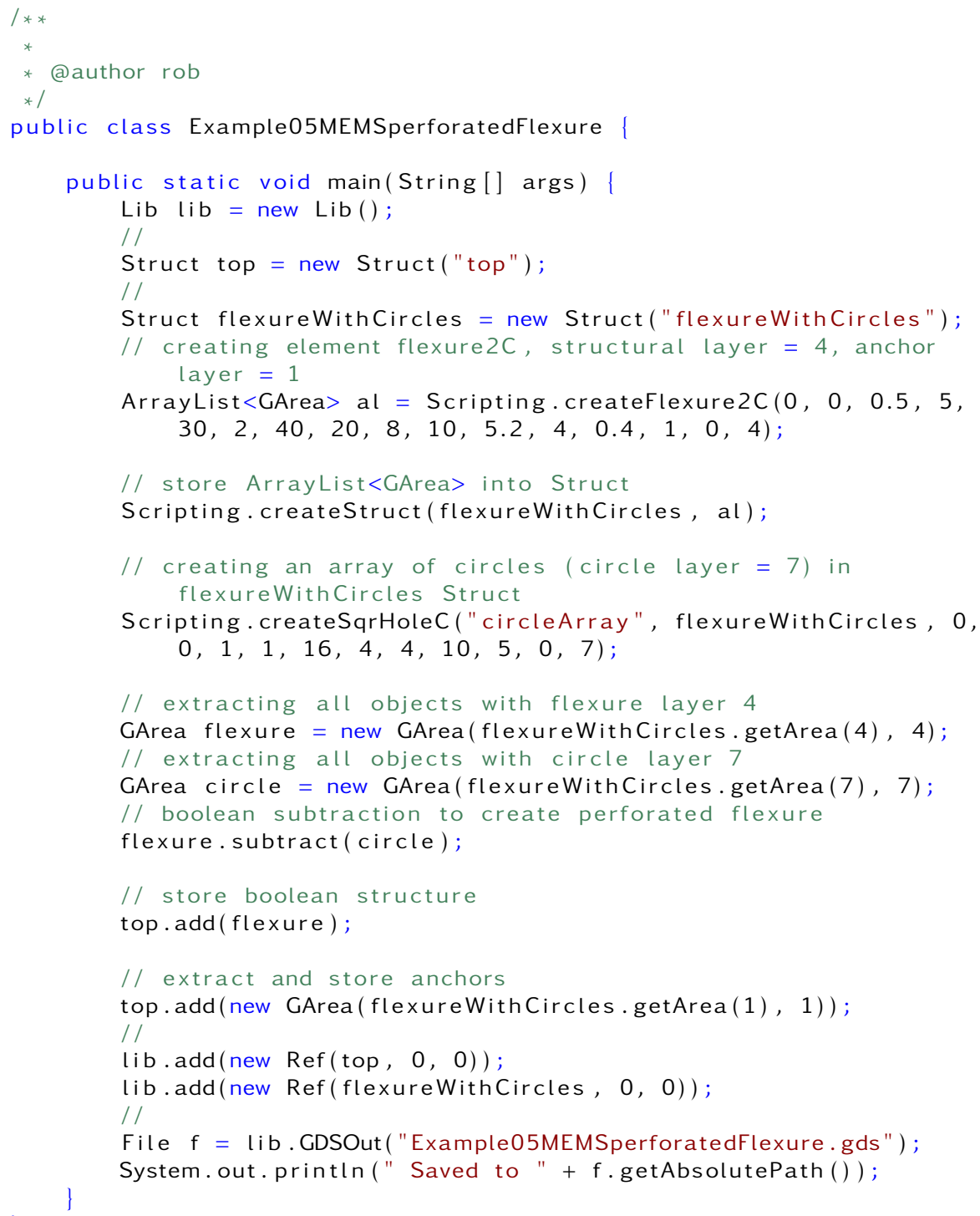




\subsubsection{Custom Class Methods}

The following example creates three methods identical to ones that generate yBend, yBendlnv and yBendlnvSlot structures described in corresponding sections 2.9.3.10, 2.9.3.11 and 2.9.3.12. Figure 4.363 shows the three 90 degree $y$-bend structures.

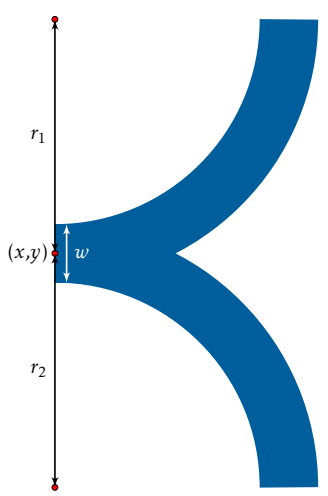

(a)

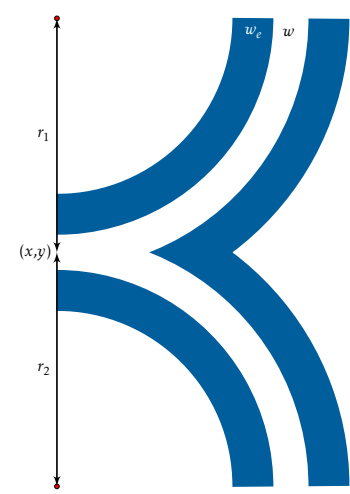

(b)

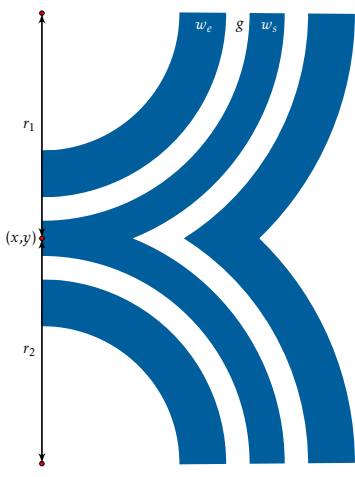

(c)

Figure 4.363: Programming example of creating 90 degree (a) $y$-bend, (b) $y$-bend inverse and (c) $y$-bend inverse slot structures.

The following code has four methods, main, createYBend90, createYBendInv90 and createYBendInvSlot90. Within the main method, line 14 initializes the GDS library and lines 16 to 20 initialize variables used to define the 90 degree $y$-bend structures. Lines 22 to 24 create GDS structures to store shapes created by the respective methods. Here we use the following constructor

Struct (String structName, GDS2Element shape)

Line 22 creates a 90 degree $y$-bend (figure 4.363a) by implementing the createYBend90 method defined by lines 37-41. The method simply creates upper arc using the Scripting . createTorusW method (line 38). The GDS area ga undergoes a Boolean OR with the lower arc. The arc shape is defined by the constructor torusW in section 2.3.15.

Similarly, line 23 and 24 creates the remaining two 90 degree y-bend structures (figure 4.363b and 4.363c). The methods createYBendInv90 and createYBendInvSlot90 reuse the defined createYBend90 method to create the resulting shapes. For instance, createYBendInv90 first creates a 90 degree $\mathrm{y}$-bend of width $w+2 w_{e}$ (line 47) and then subtracts a similar structure of width $w$ (line 48) to create a 90 degree $y$-bend inverse GDS area. Analogously, lines 54-58 define a method that creates a 90 degree $y$-bend inverse slot structure. 


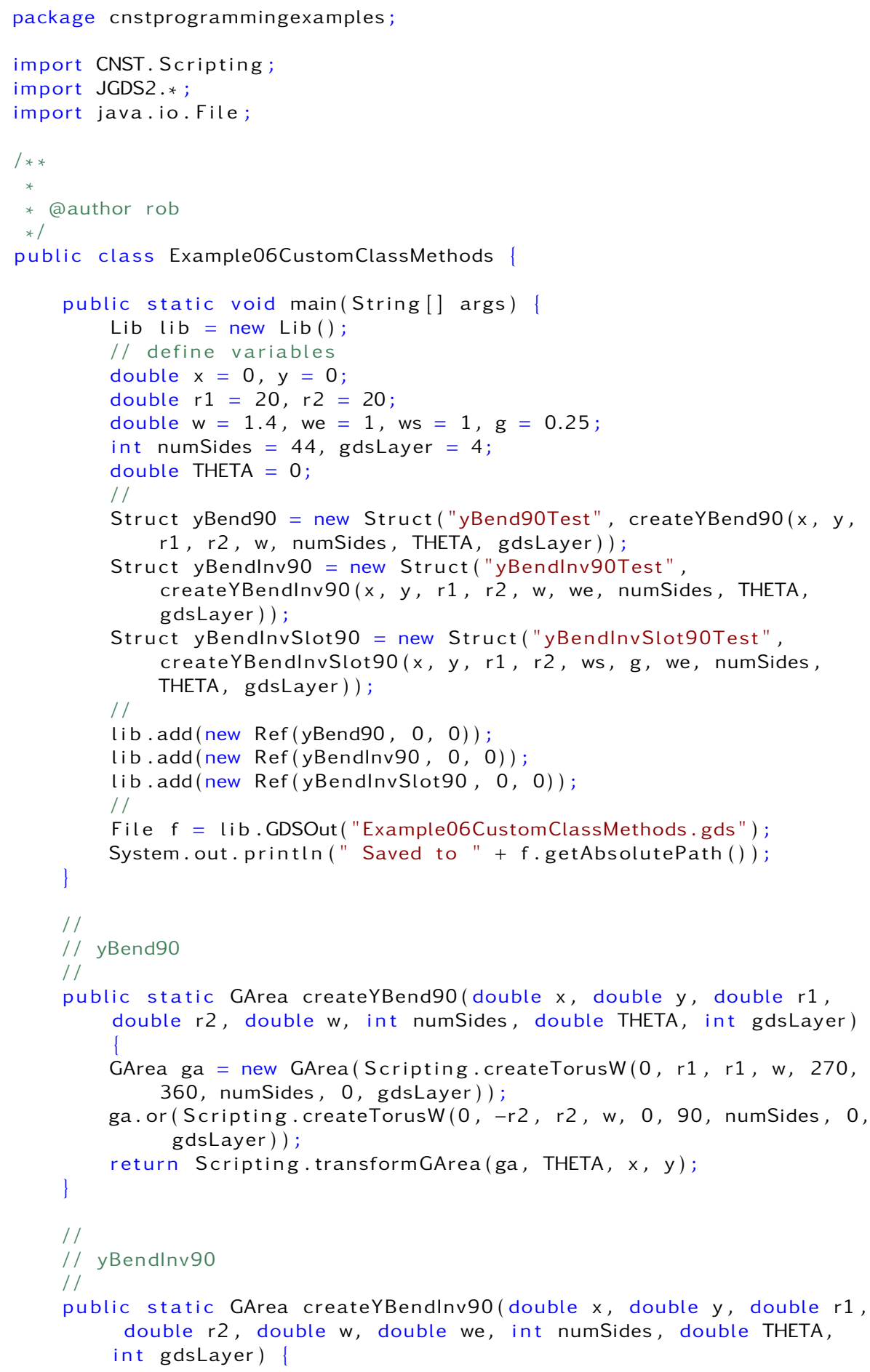




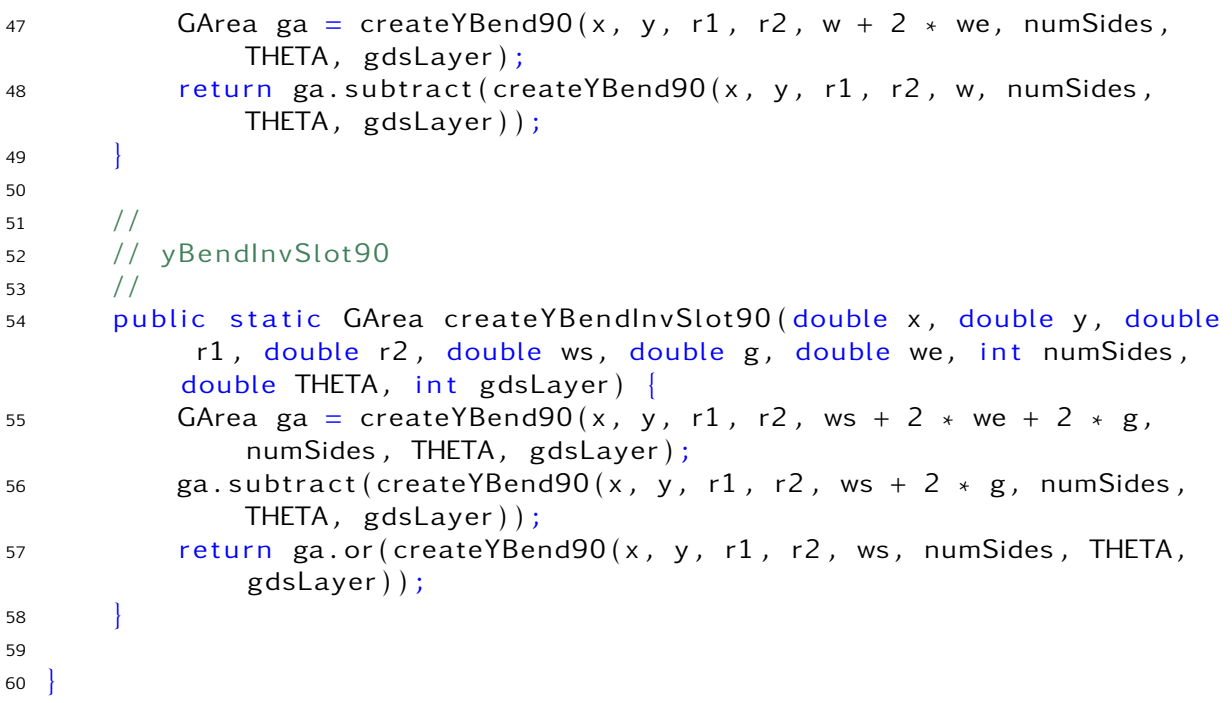

\subsubsection{GDS Area Objects - Layers and Data-types}

GDS layer definitions are typically defined within the method constructor. Layer data-types are either defined globally for subsequent GDS objects or GDS area objects are assigned to a data-type. For instance, within the following 4 lines, a structure is created, layer data-type 44 is set for subsequent shapes, the GDS area ellipse acquires the data-type value, and is added to the Struct.

1 Struct top = new Struct ("top");

2 Scripting.setDataType (44);

3 GArea ellipse $=$ Scripting. createEllipse $(0,0,0.4,0.8,44,0,4)$;

4 top.add(singleEllipse);

The following is an equivalent strategy that casts the GDS area object onto a particular datatype.

1 Struct top = new Struct ("top");

2 GArea ellipse = Scripting.createEllipse $(0,0,0.4,0.8,44,0,4)$;

3 singleEllipse. setDataType (44);

4 top.add(singleEllipse); 


\subsubsection{Centering GDS Area Objects}

The following code will find a centroid of a GDS shape. This procedure is useful for centering features around a specified origin or to create symmetric pattern extents. Lines 18 and 19 define a GDS structure and area, respectively. The GDS area is then added to the structure (line 20). In line 22, a new structure is formed that will store the resulting centered GDS area. The GDS area is extracted and stored into a Java Area (line23). Using the built-in Java Rectangle2D class, the area boundary is extracted in line 24 . The GDS structure containing the GDS area is then instantiated with offsets determined by the getCenterX() and getCenterY( ) methods from the Rectangle2D class. Instead of instantiating, the GDS area object could be added directly to a GDS structure using affine translational transforms with (-rec.getCenterX(), -rec.getCenterY()).

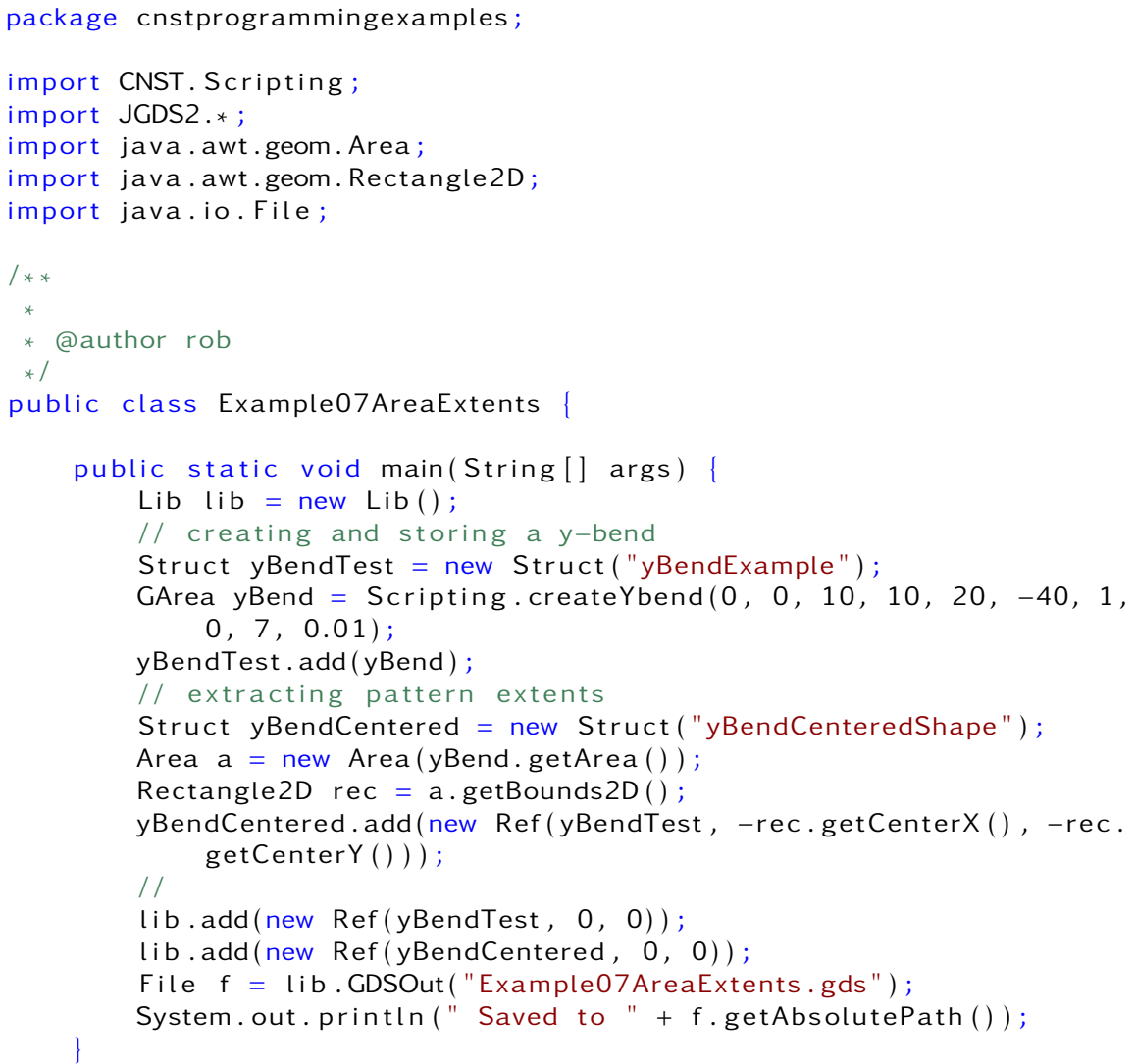

NIST • CNST Nanolithography Toolbox v2016.09.01 • http://www.nist.gov/cnst/ 


\subsubsection{PostScript to GDS}

The below code creates a GDS area using a string containing postscript commands. Unlike scripting, where the the number of postscript commands is practically unlimited, with the programming interface it is limited. This limitation stems from the Java String length limit. As defined by the Java specifications, a String has a maximum character length of Integer. MAX_VALUE $=\left(2^{31}-1\right)$. A host of memory dilemmas will be encountered prior reaching this limit, hence instead of holding all postscript commands in one String, the commands could be distributed over multiple Strings.

Example 8 implements an interface (Example08PSinterface.java) that contains a string $s$ with postscript paths (line 11). Variables are defined and a GDS structure is created (lines 16 to 21 ) in a similar manner to previous programming examples. Line 22 uses a method createPostScript in conjunction with a String psString to generate output GDS shapes. String variable psString is defined in the Example08PSinterface.java interface file. The following two methods are available for creating GDS shapes from vectorized objects:

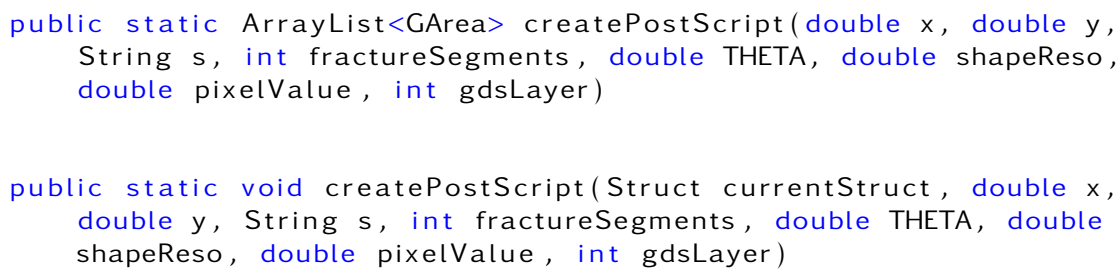

In our example we used the void method (line 22), that directly stores generated shapes into a specified (currentStruct) GDS structure. The lower left corner of the structure is positioned at $(x, y)$. The resulting structure is fractured into a number of equal segments defined by the integer value of fractureSegments. Parameters THETA, shapeReso, pixelValue and gdsLayer are rotation about $(x, y)$, rendering resolution of the vectorized shape(section 2.2.6), pixel scaling value of the postscript coordinates (section 2.6.5.1) and GDS layer number, respectively.

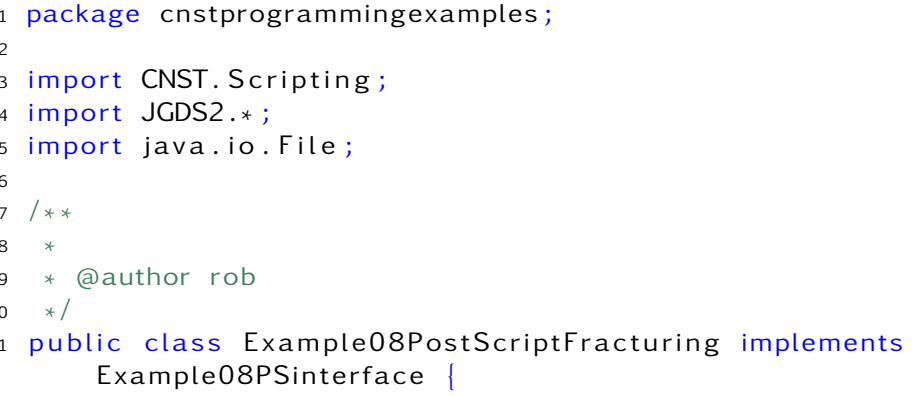

NIST • CNST Nanolithography Toolbox v2016.09.01 • http://www.nist.gov/cnst/ 


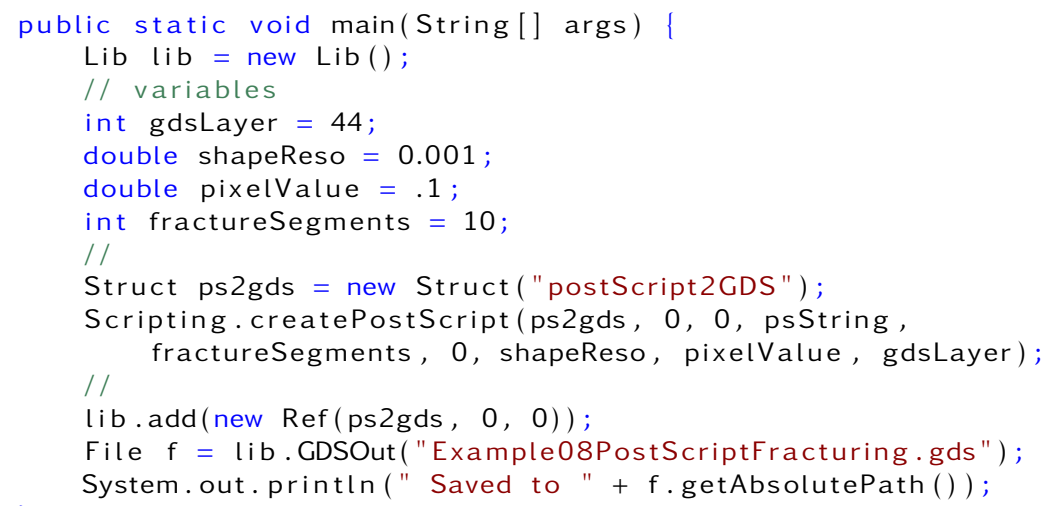




\subsubsection{Curved Fluidic Channels}

The following example creates two sets of labeled fluidic channels using predefined scripting methods. Straight segments were constructed using the waveguide method that defines a rectangular shape between points $\left(x_{1}, y_{1}\right)$ and $\left(x_{2}, y_{2}\right)$. The curved segments were created using either bezier curves or 90 degree torus bends.

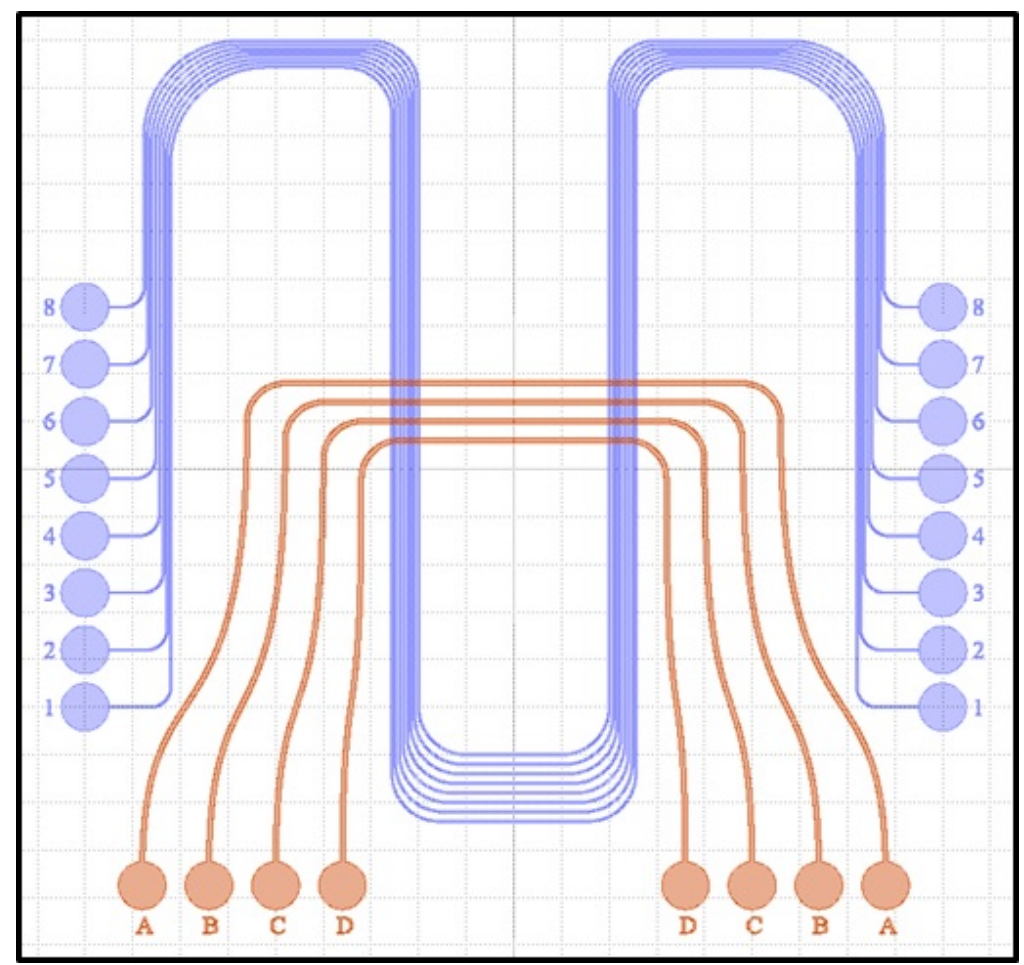

Figure 4.364: Programming example of labeled fluidic channels.

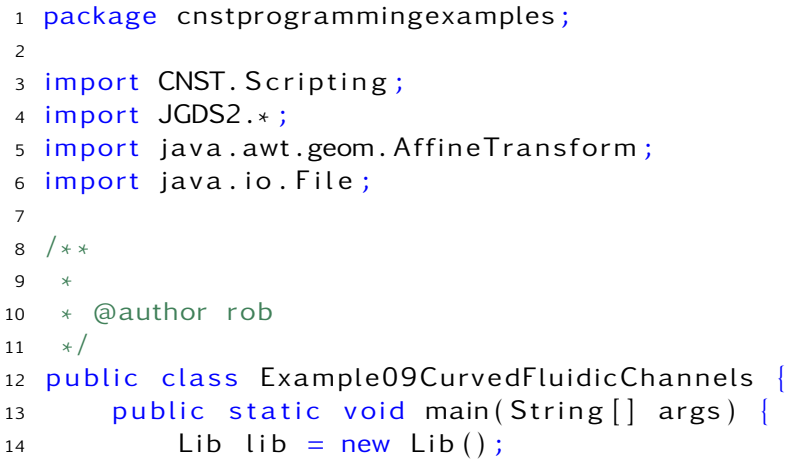




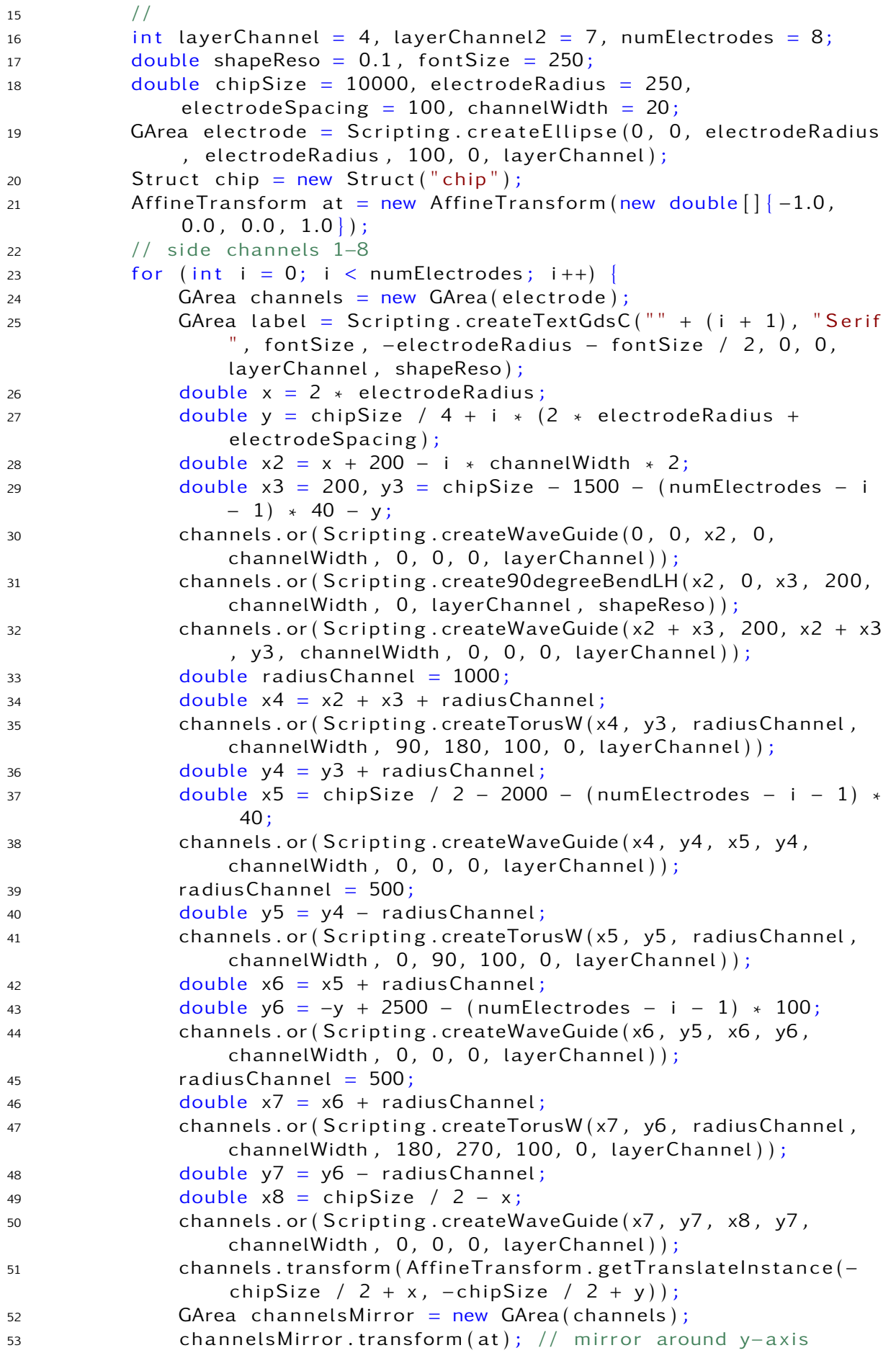

NIST • CNST Nanolithography Toolbox v2016.09.01 • http://www.nist.gov/cnst/

page 392 of 488 


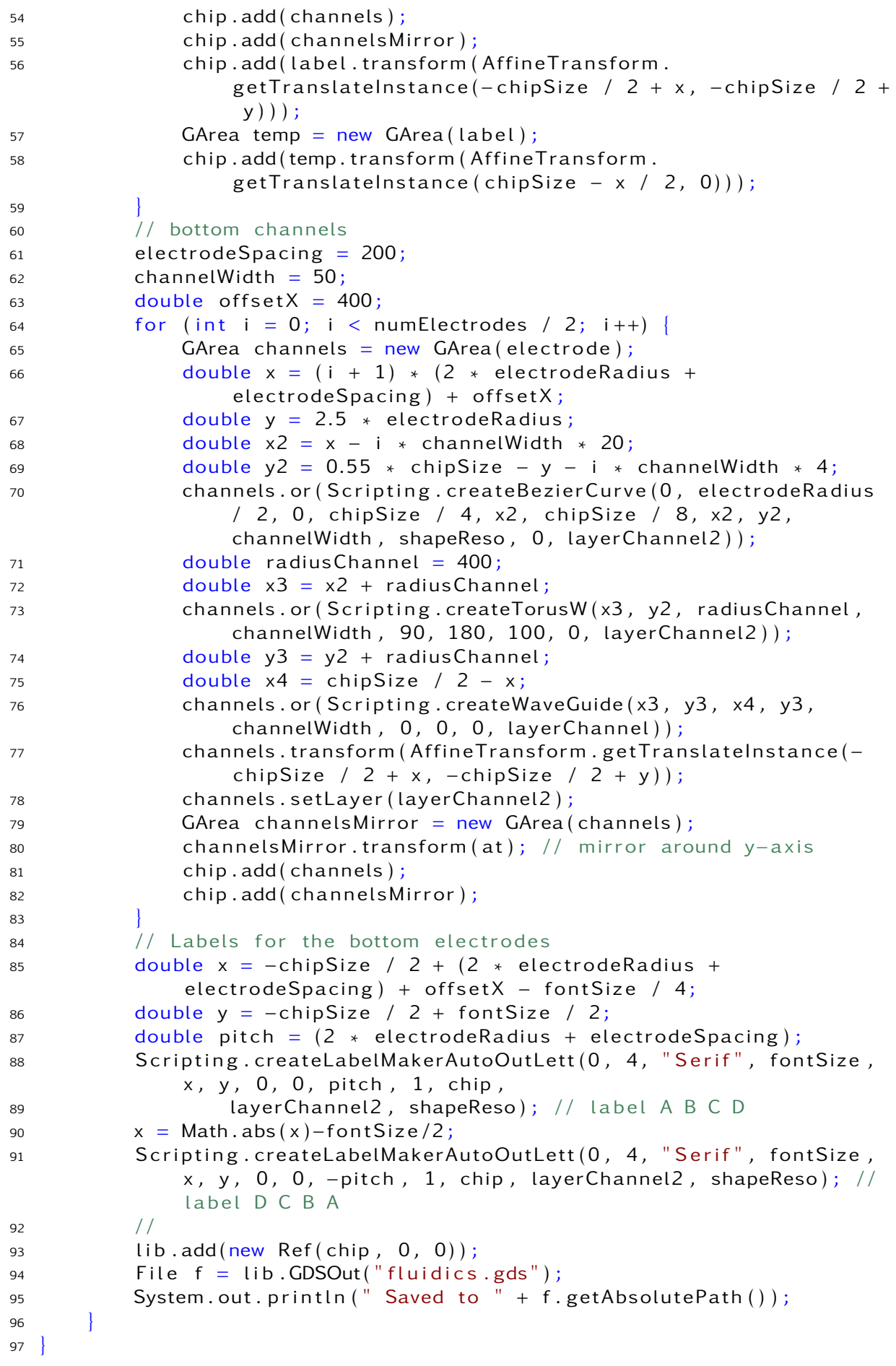




\subsection{Reference Methods}

The following subsection contain method headers that create complex GDS objects. These shapes are fully described in the GDS scripting chapter. Excluding access, mutator and miscellaneous methods, the shape methods names have the create followed by the script constructor name with the first letter capitalized. For instance, the ellipse scripting constructor (also shown in the previous comment line) would have a method entitled createEllipse

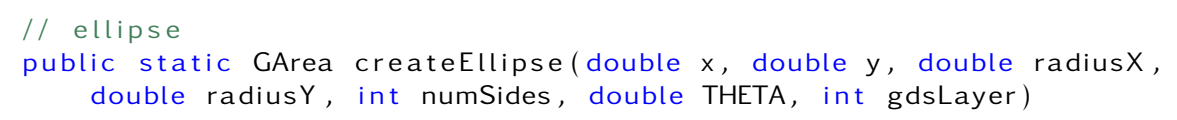

Directly following the method name are a set of parameters. All methods have a parameter list with the exception of accessor methods that have empty parameter lists. Methods return types are either void, GArea or ArrayList $<$ GArea $>$. Since Struct objects add GArea objects but not array lists of these objects, each vector array return types also have a void method. The void method stores the ArrayList $<$ GArea $>$ into the passed Struct currentStruct. Section 4.2.2 contains methods for dealing with GArea or ArrayList<GArea> objects. These are useful for controlling GDS area objects in custom user defined methods.

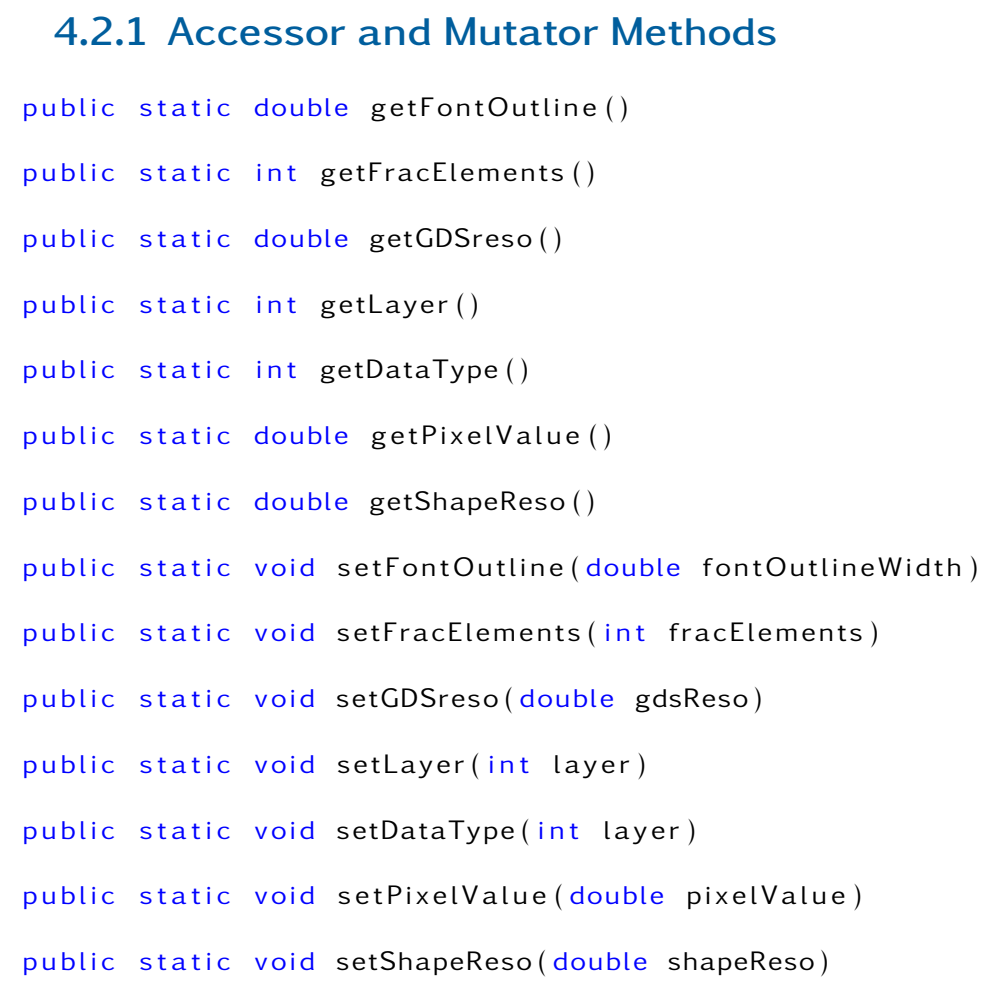

NIST • CNST Nanolithography Toolbox v2016.09.01 • http://www.nist.gov/cnst/

page 394 of 488 


\subsubsection{Miscellaneous GDS Area and Struct Methods}

These methods are used for affine transformations of single and vector array GDS area objects. Furthermore, the void methods place constructed vector arrays of GArea objects into a GDS structure.

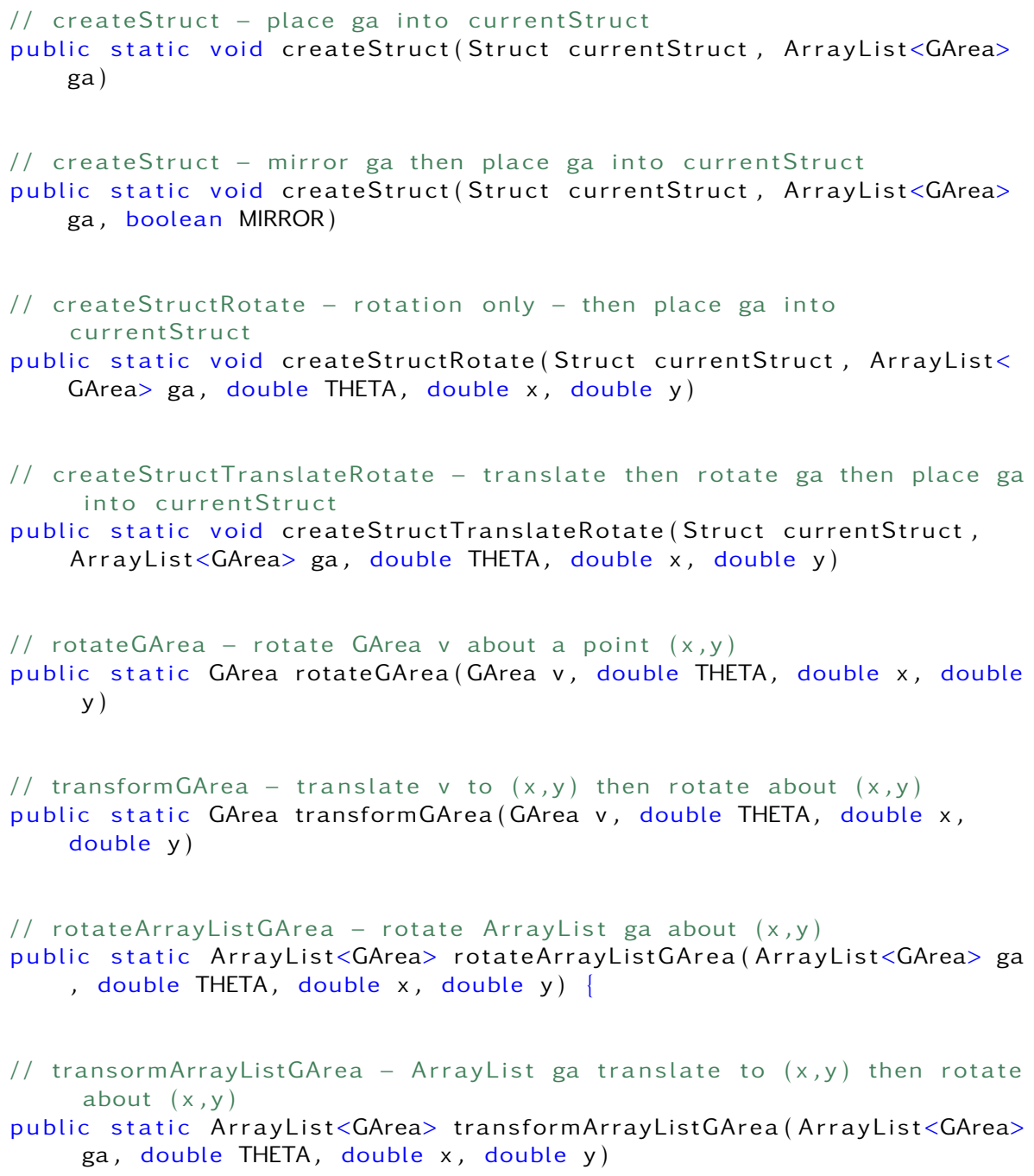

public static ArrayList $<$ GArea $>$ transformArrayListGArea (ArrayList $<$ GArea $>$ ga, double THETA, double $x$, double $y$ ) 


\subsubsection{Shape Methods}

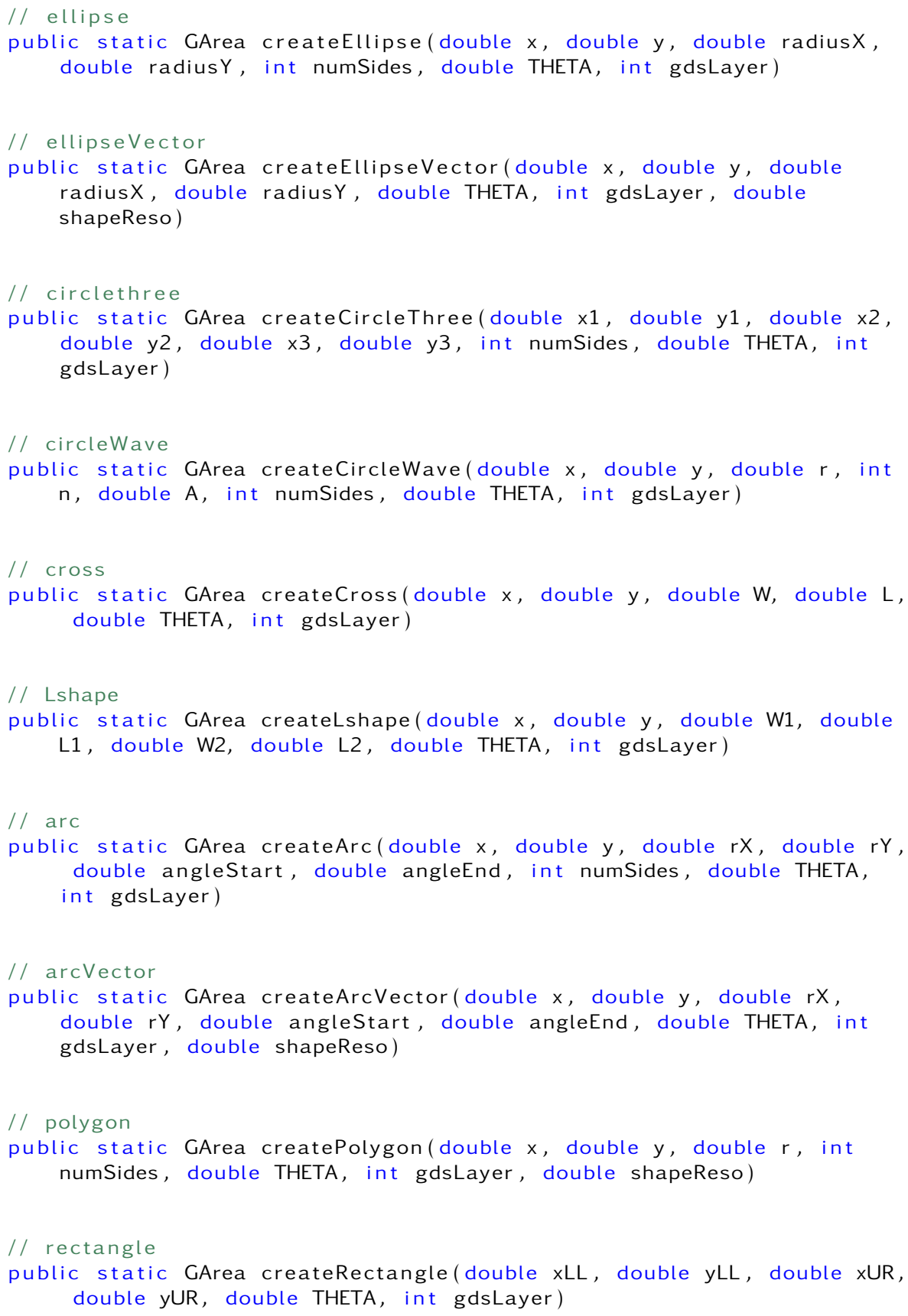




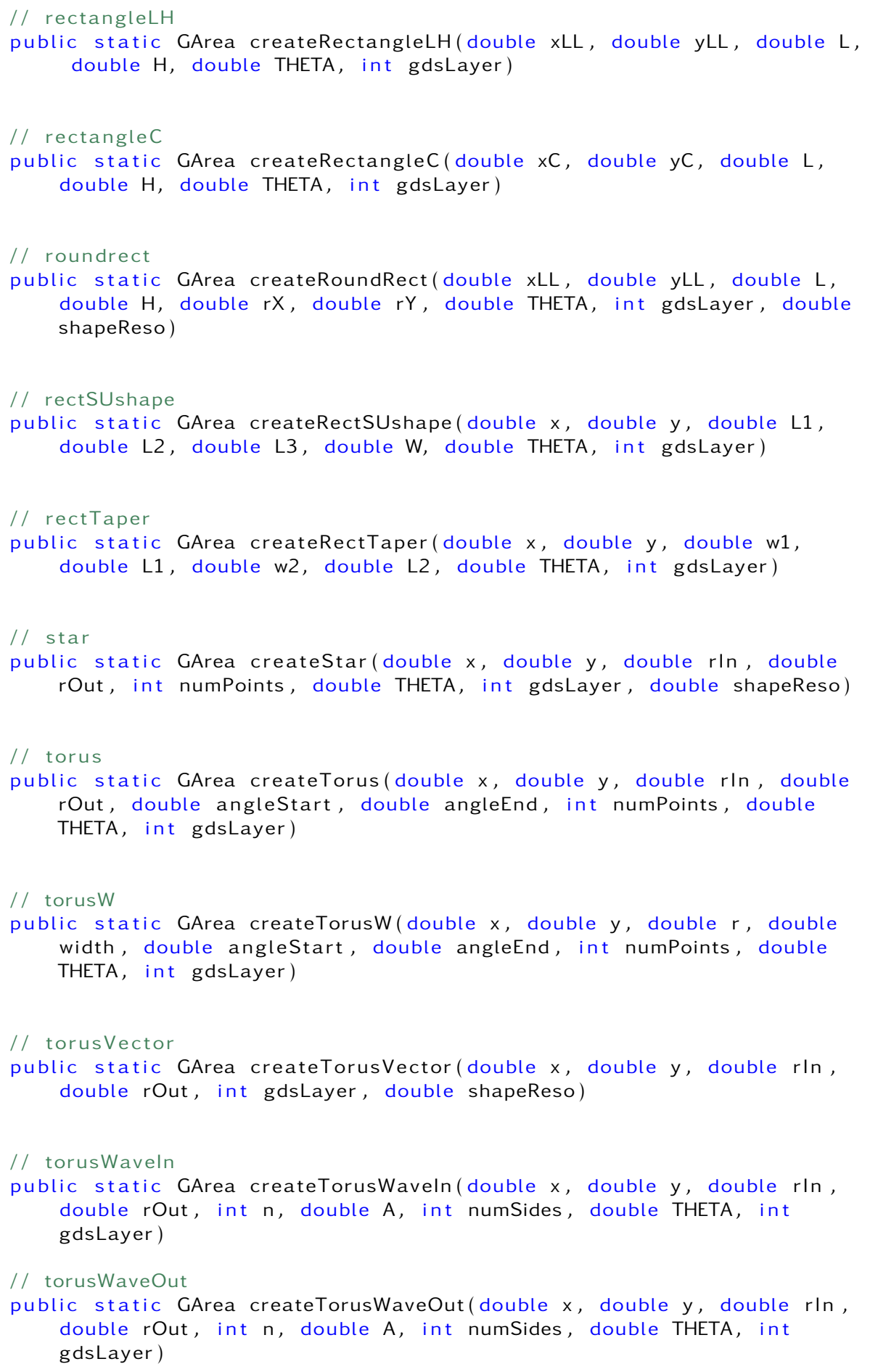

NIST • CNST Nanolithography Toolbox v2016.09.01 • http://www.nist.gov/cnst/ 


\subsubsection{Array Methods}

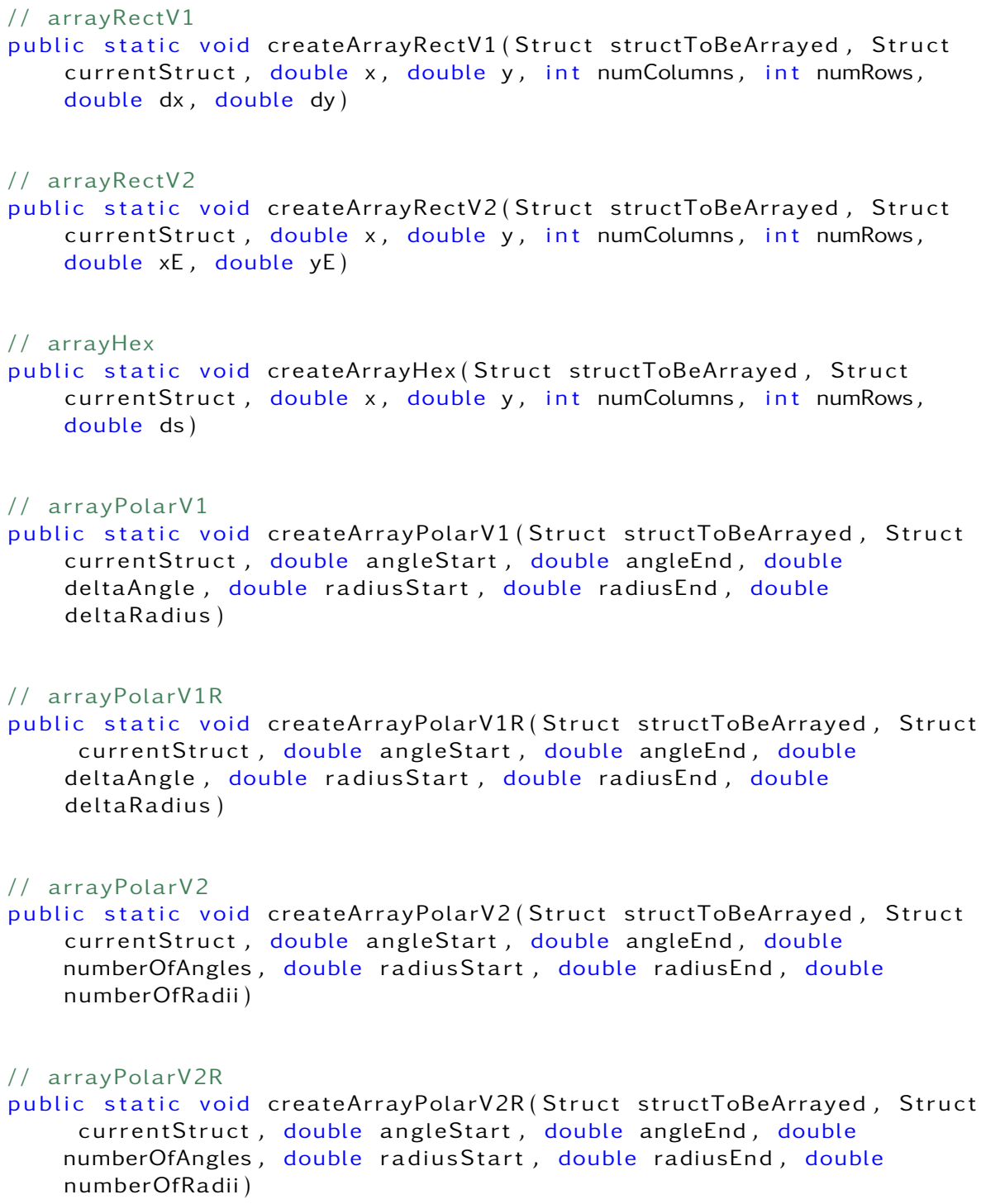

Instantiation using instance, instanceSym and points2instance can be accomplished using the JGDS reference (Ref) constructor. Refer to the JGDS manual for information on Ref. 


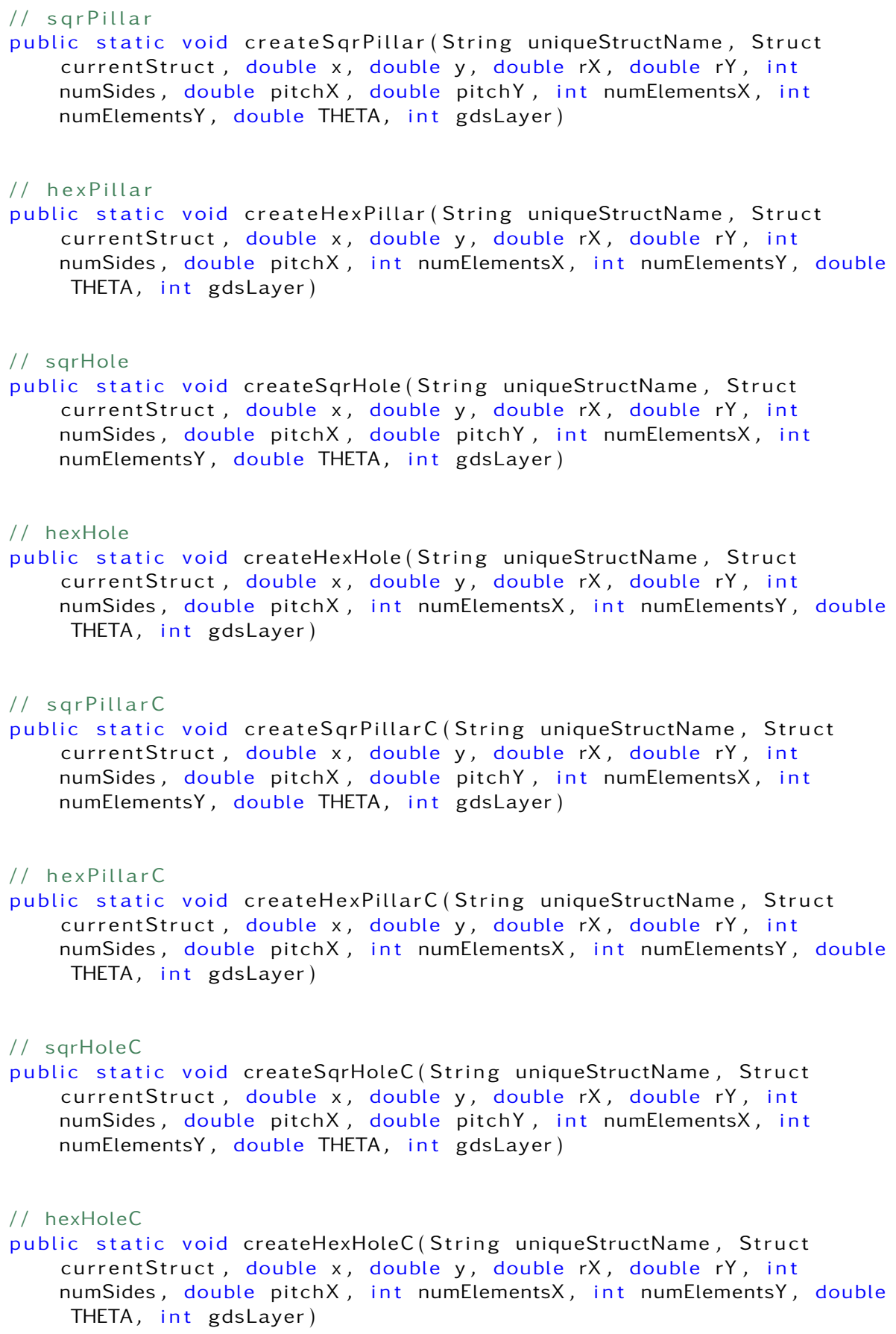




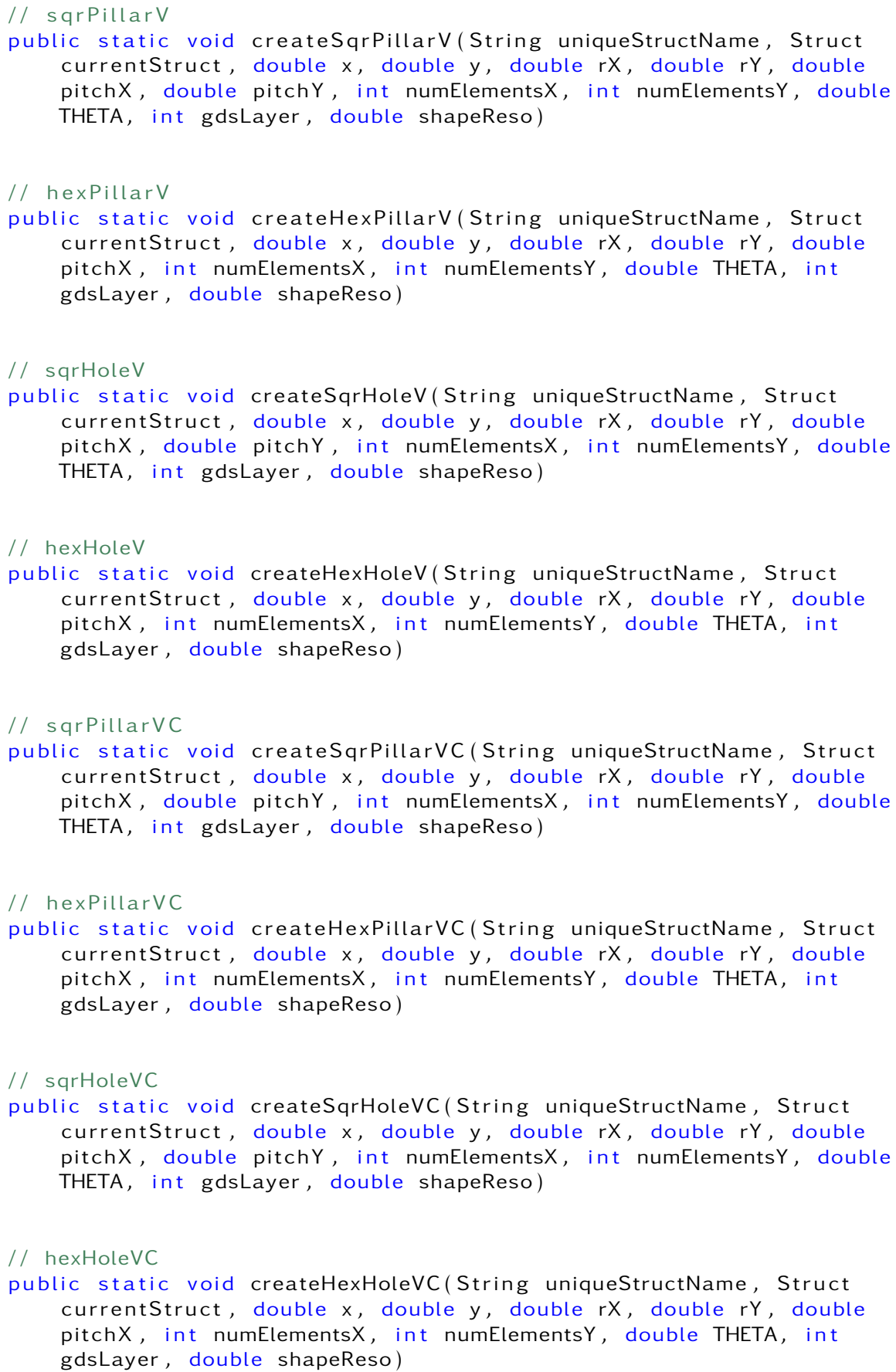




\subsubsection{Text, Labels, PostScript and Logo Methods}

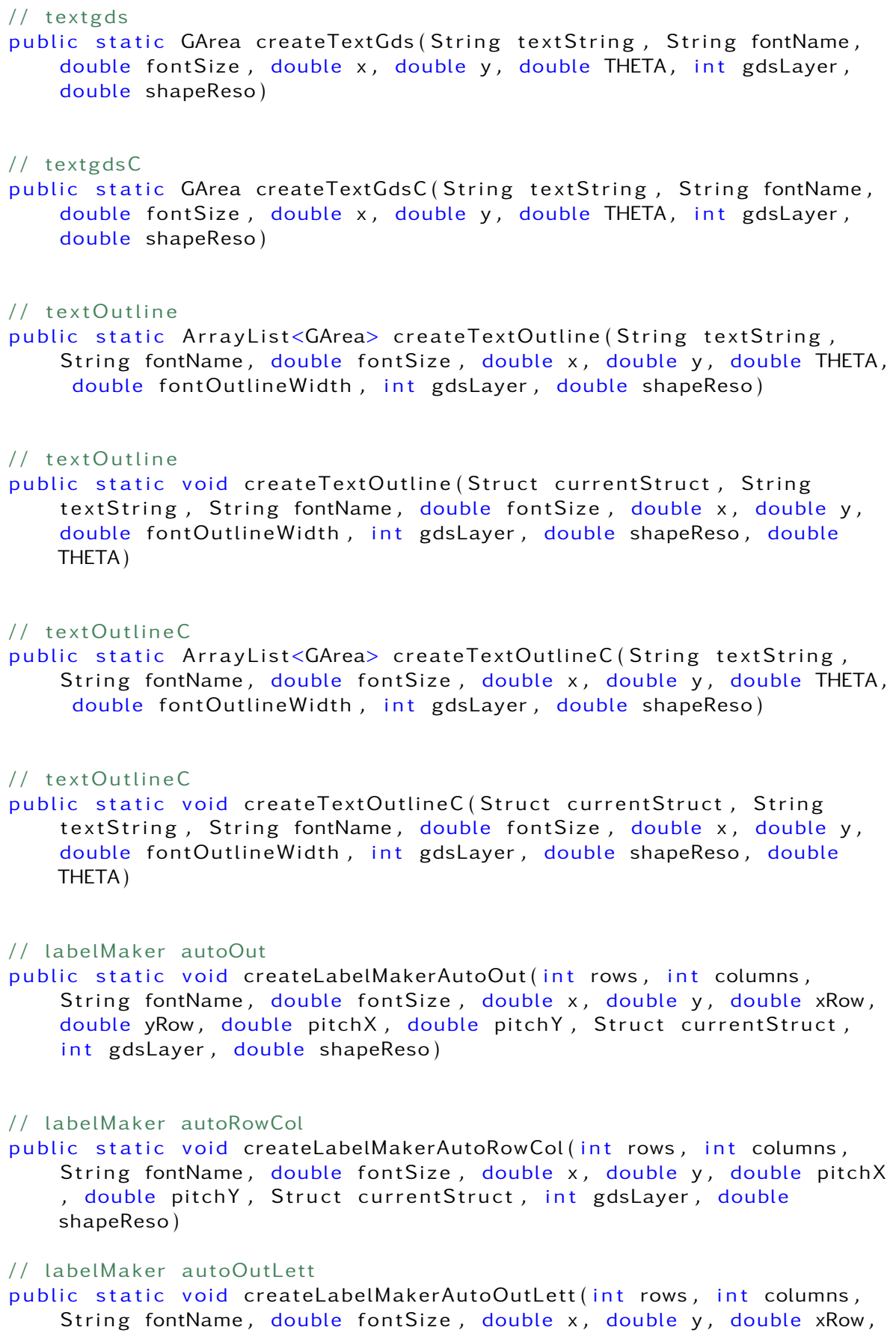


double yRow, double pitchX, double pitchY, Struct currentStruct, int gdsLayer, double shapeReso)

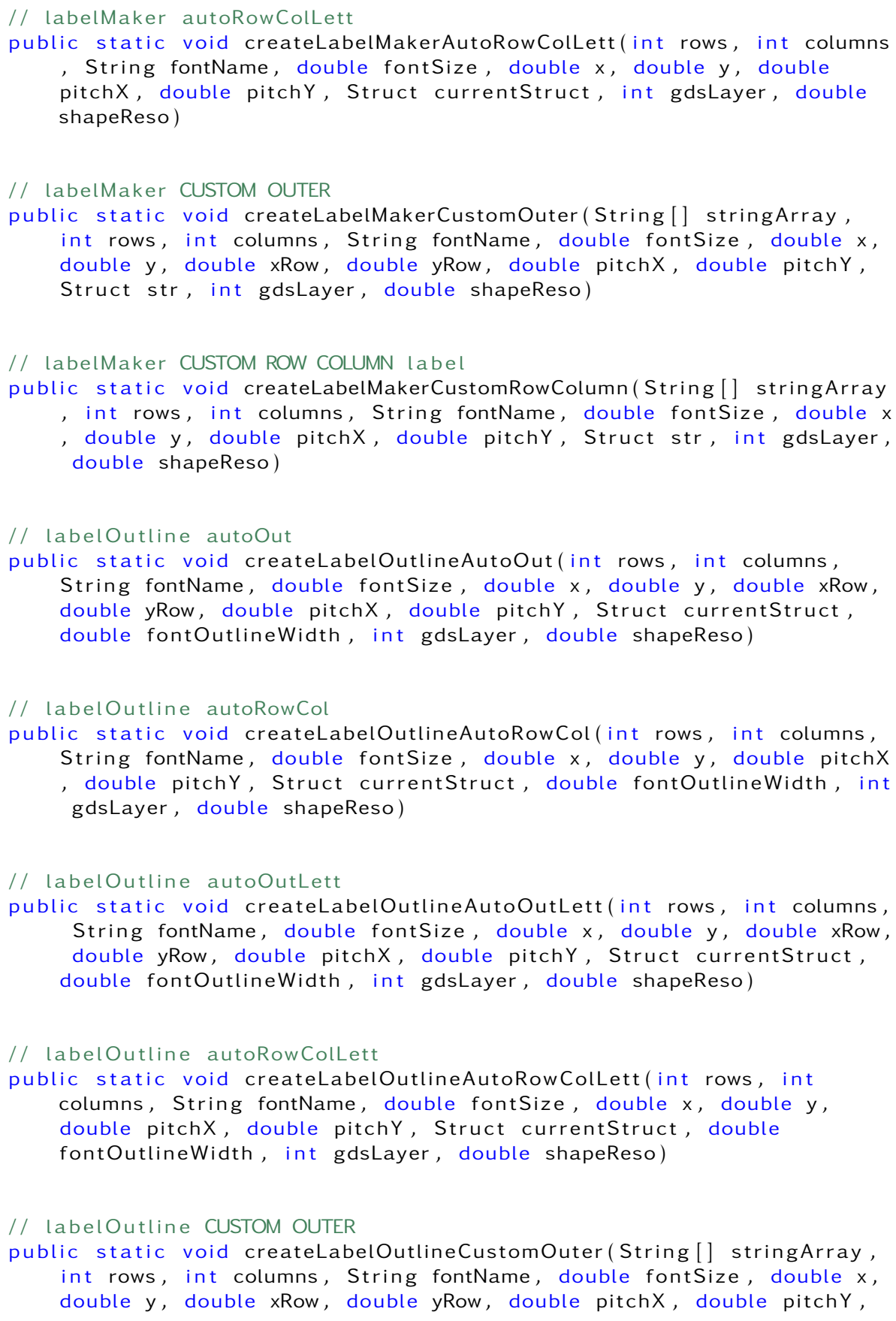




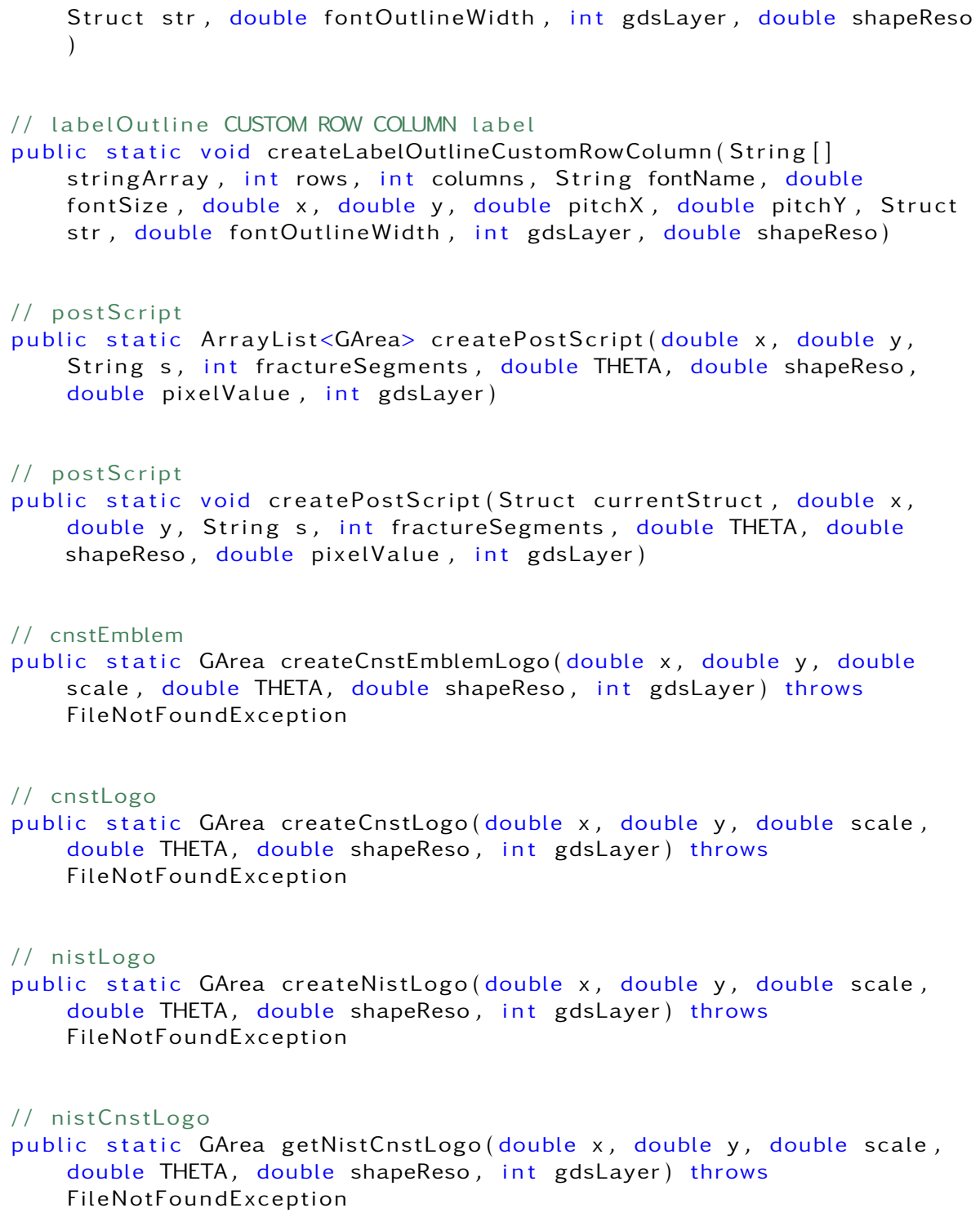




\subsubsection{Miscellaneous Object Methods}

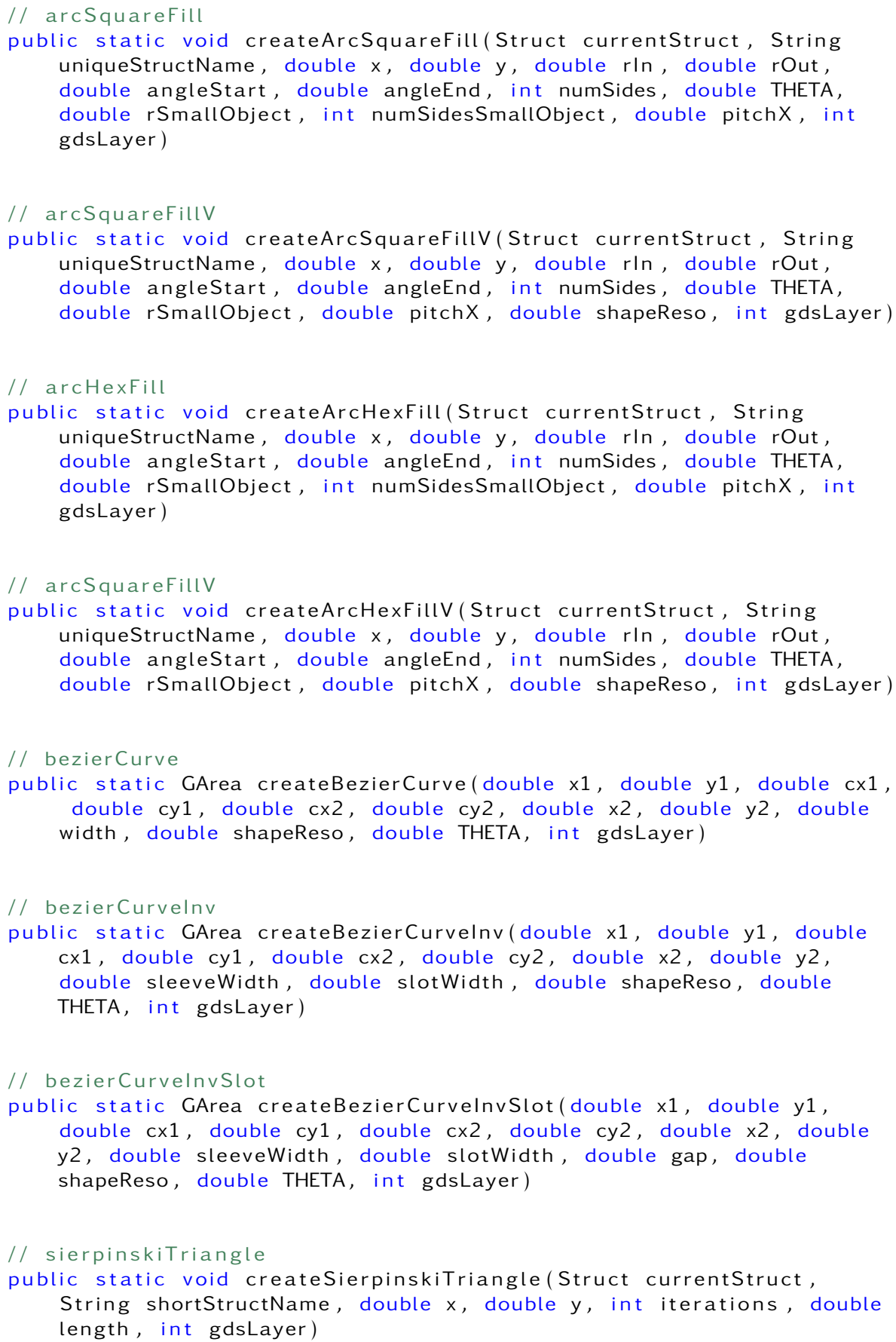




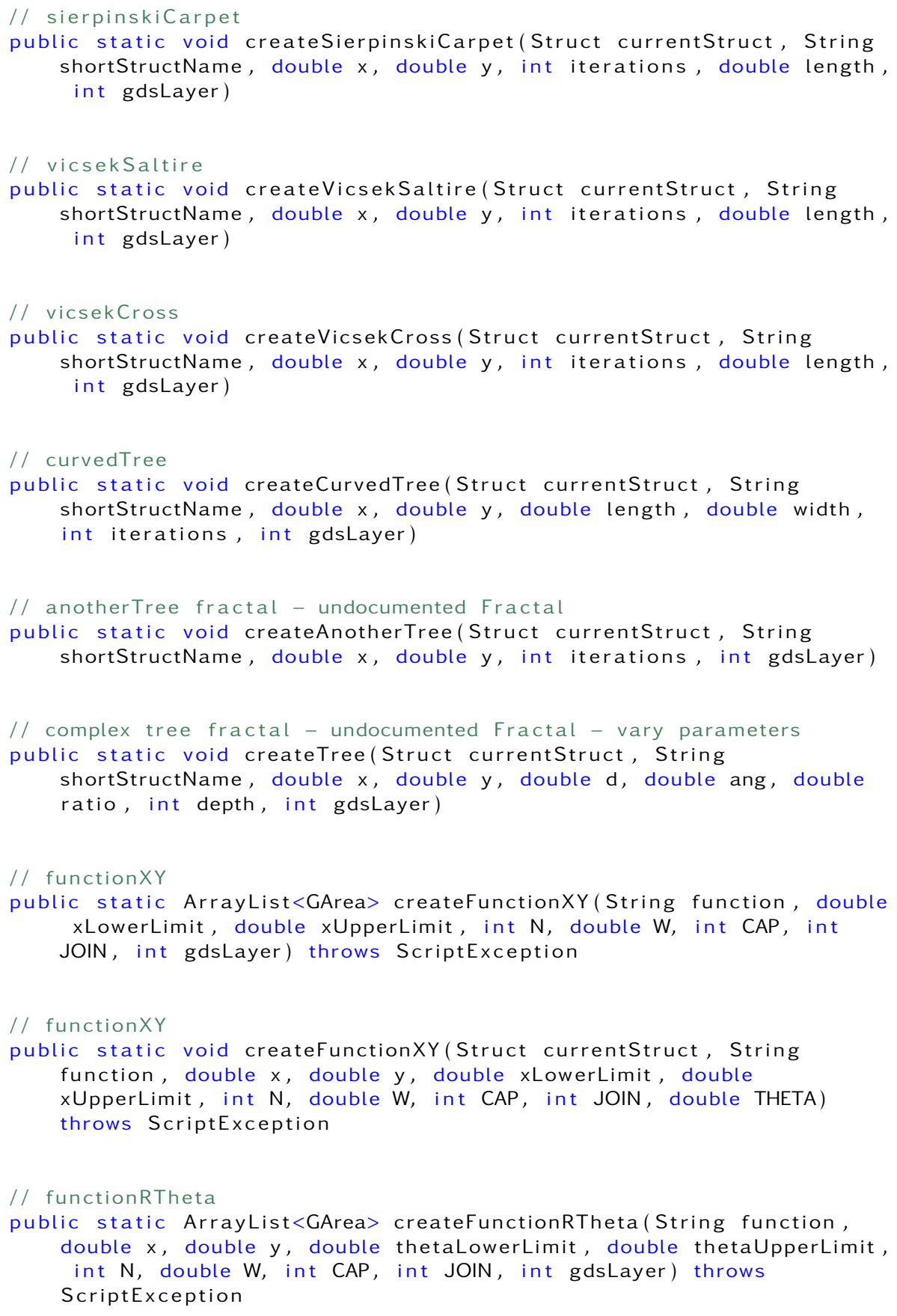

NIST • CNST Nanolithography Toolbox v2016.09.01 • http://www.nist.gov/cnst/ 


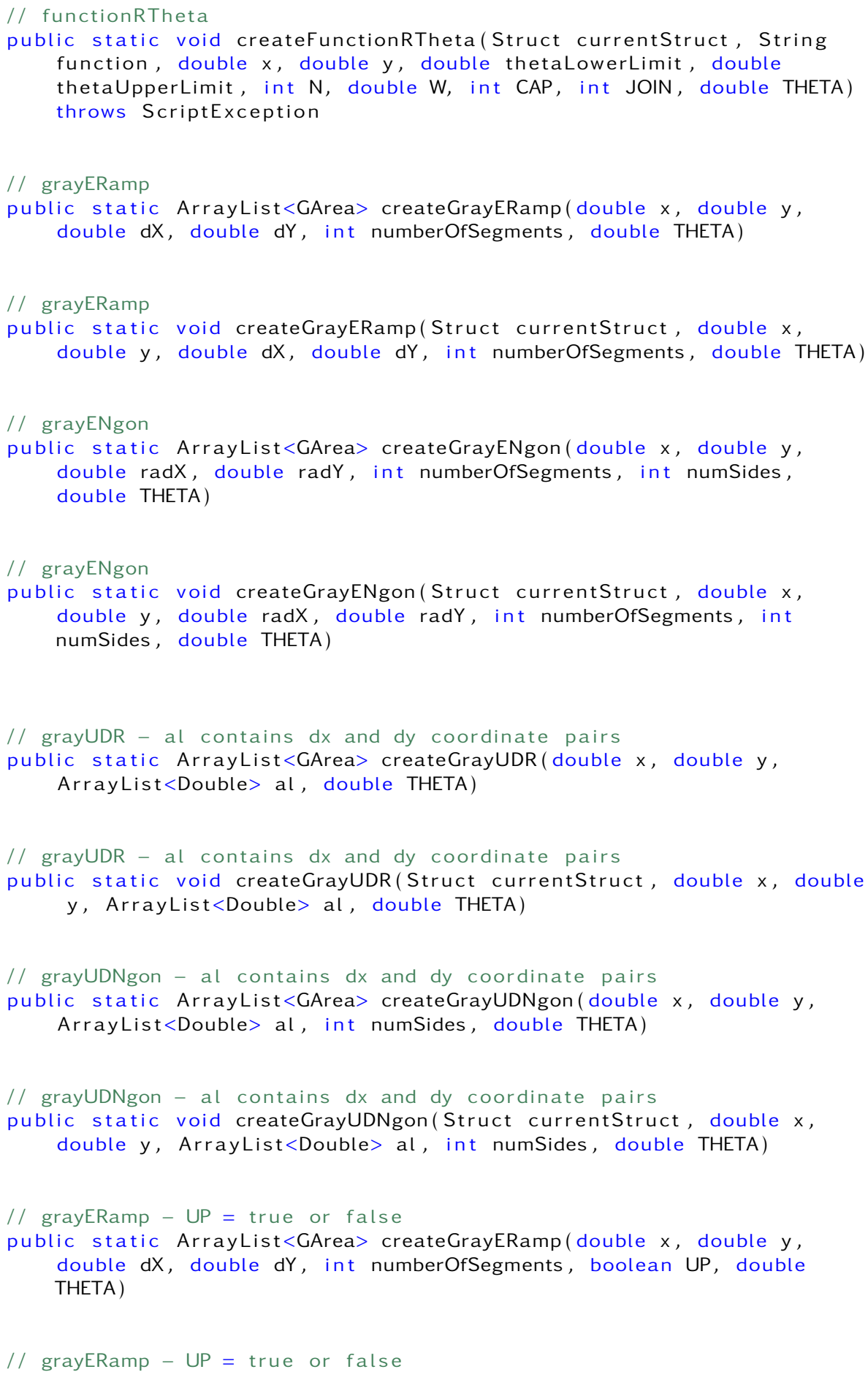


public static void createGrayERamp(Struct currentStruct, double $x$, double $y$, double $d X$, double $d Y$, int numberOfSegments, boolean UP, double THETA)

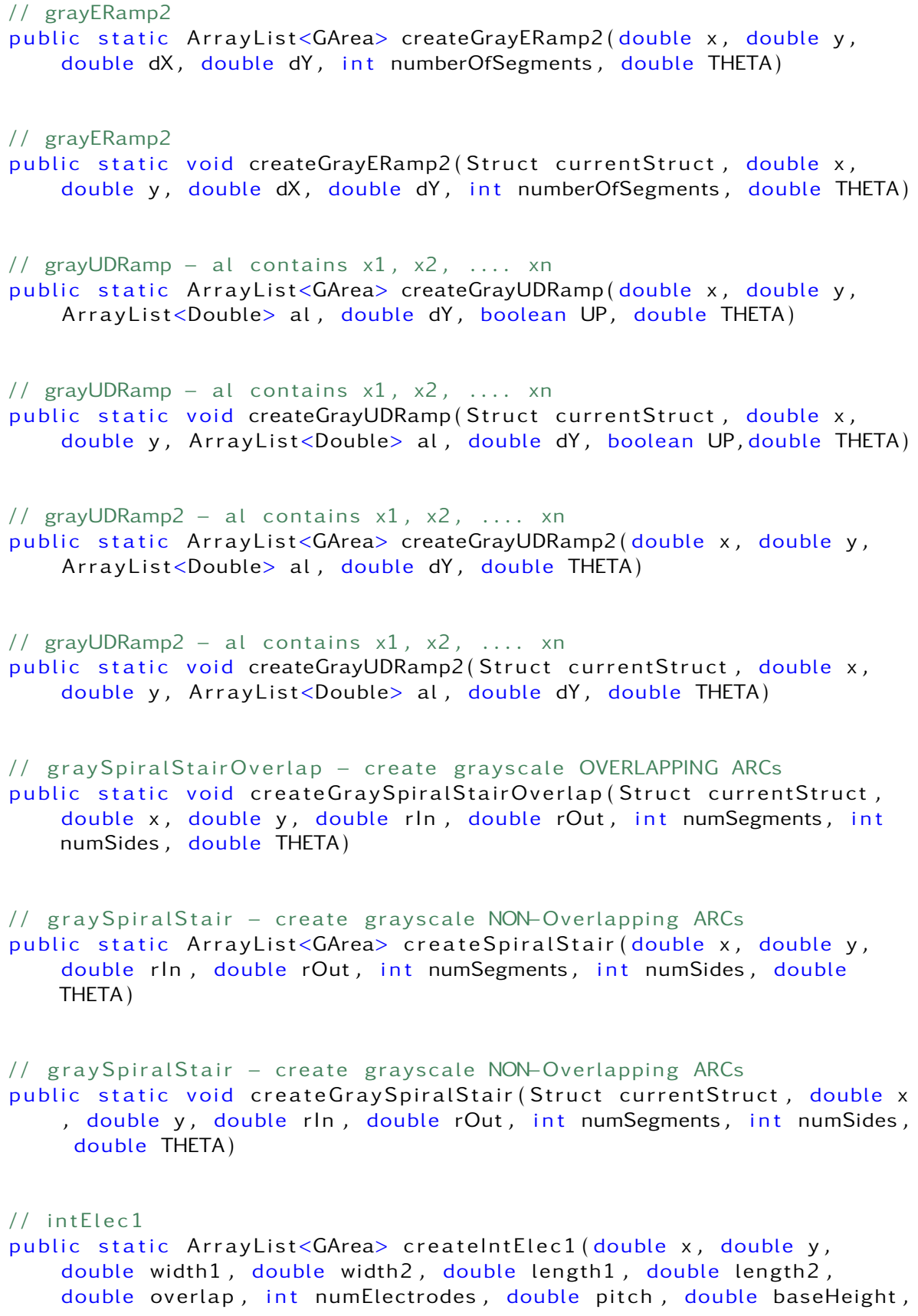


double baseWidth, double THETA, int gdsLayer)

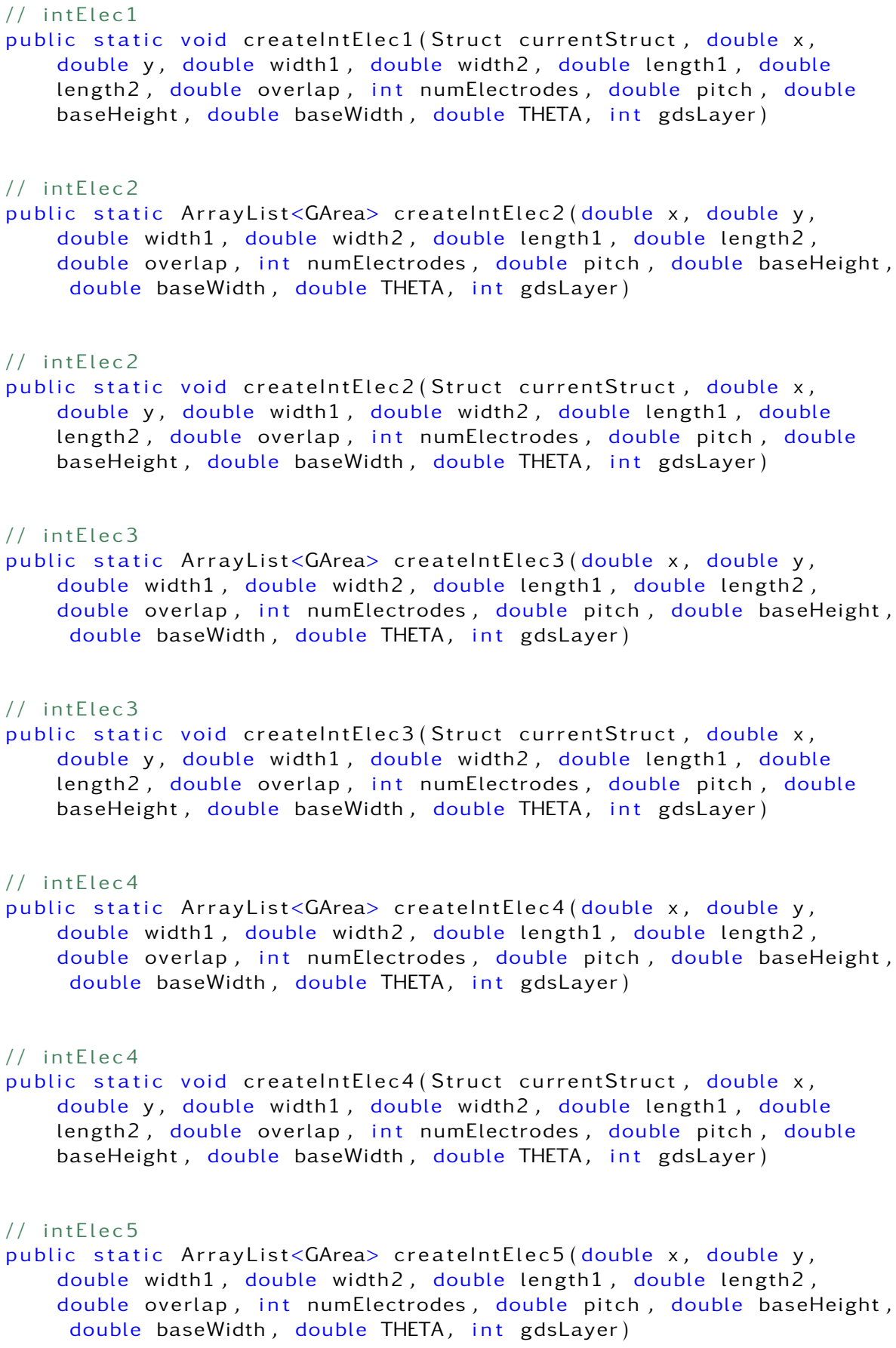

NIST • CNST Nanolithography Toolbox v2016.09.01・http://www.nist.gov/cnst/ 


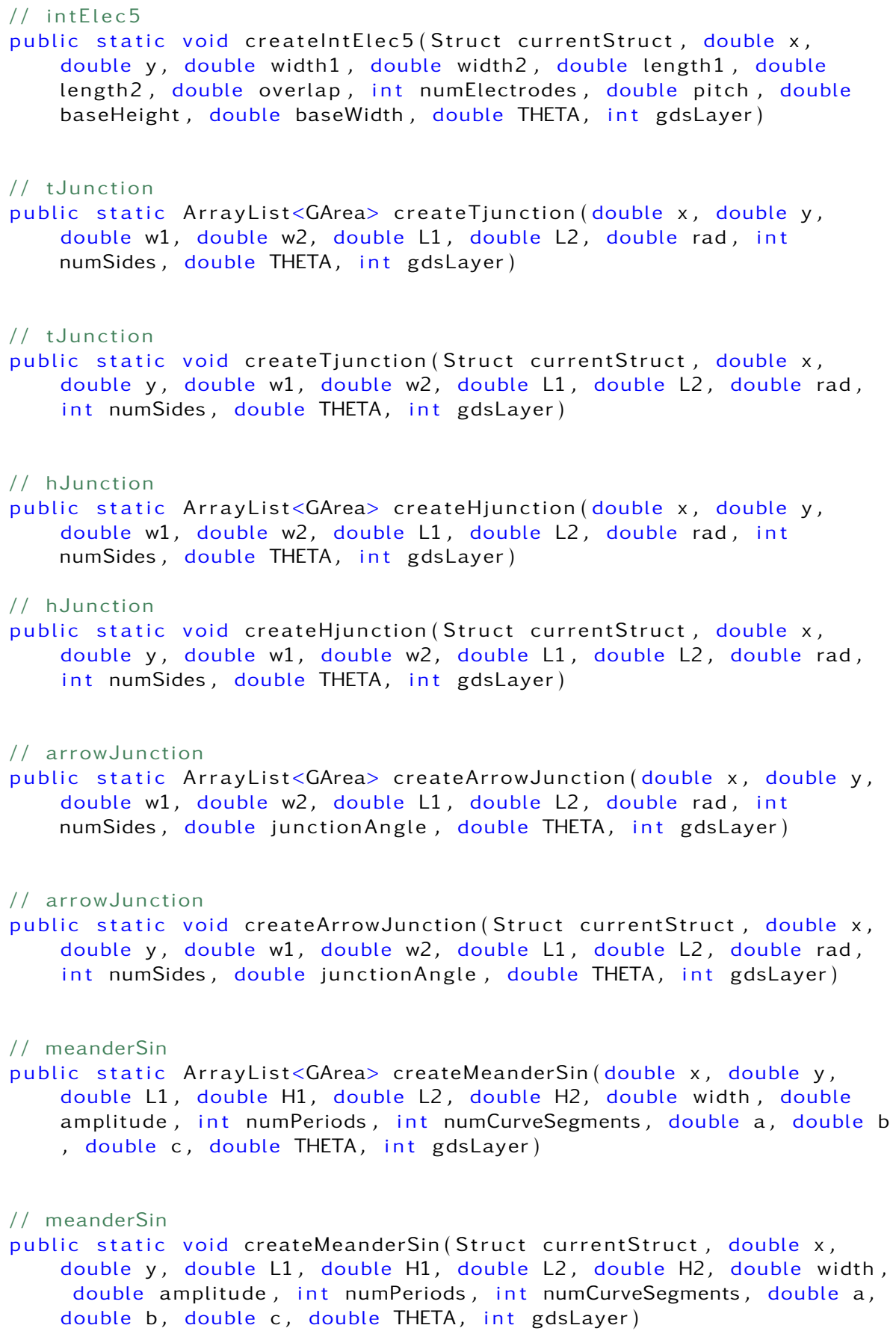




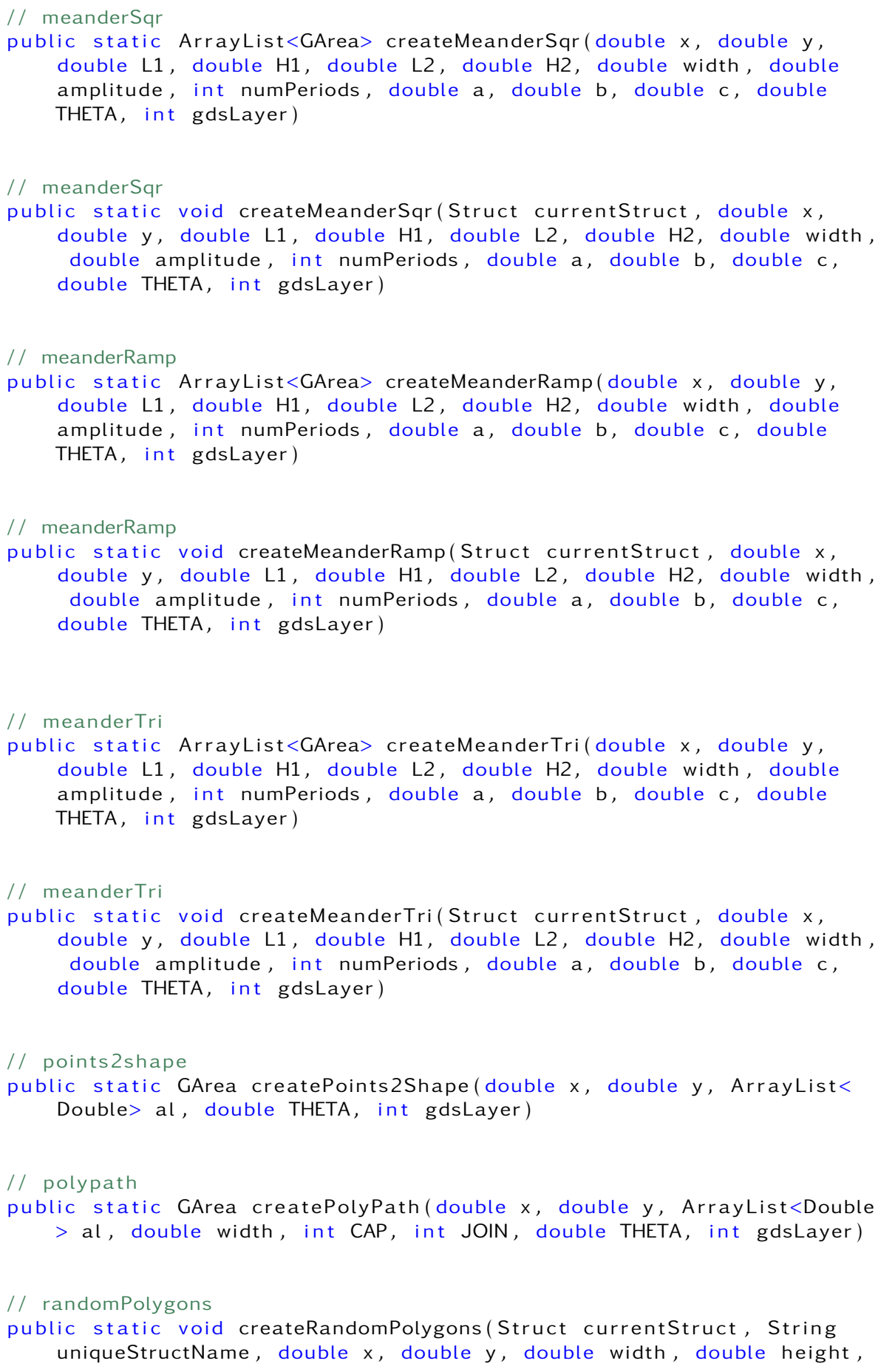


double radius, int numSides, double separation, int numElements, int iterations, boolean RANDOMROTATION, int gdsLayer)

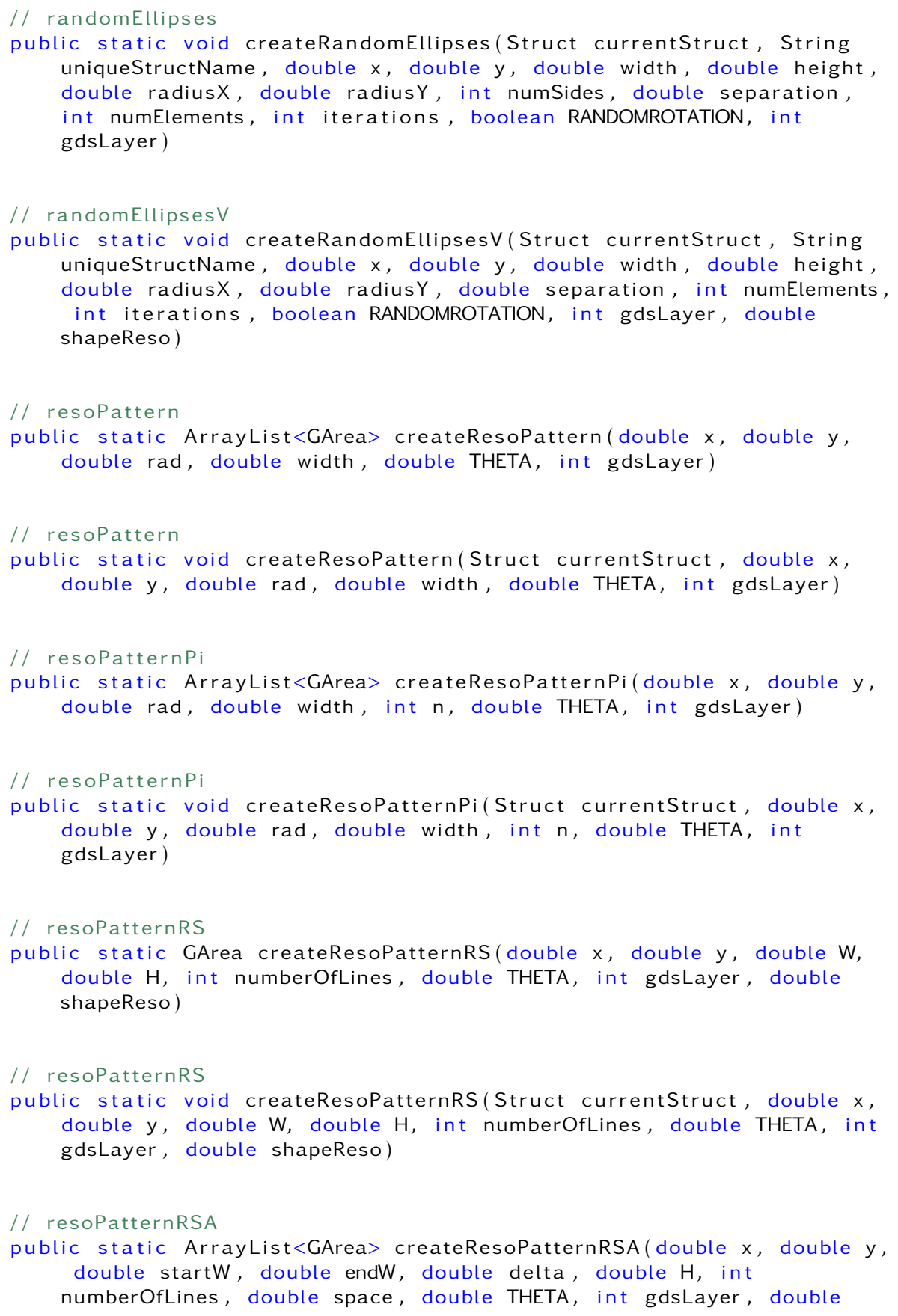

NIST • CNST Nanolithography Toolbox v2016.09.01 • http://www.nist.gov/cnst/ 
shapeReso )

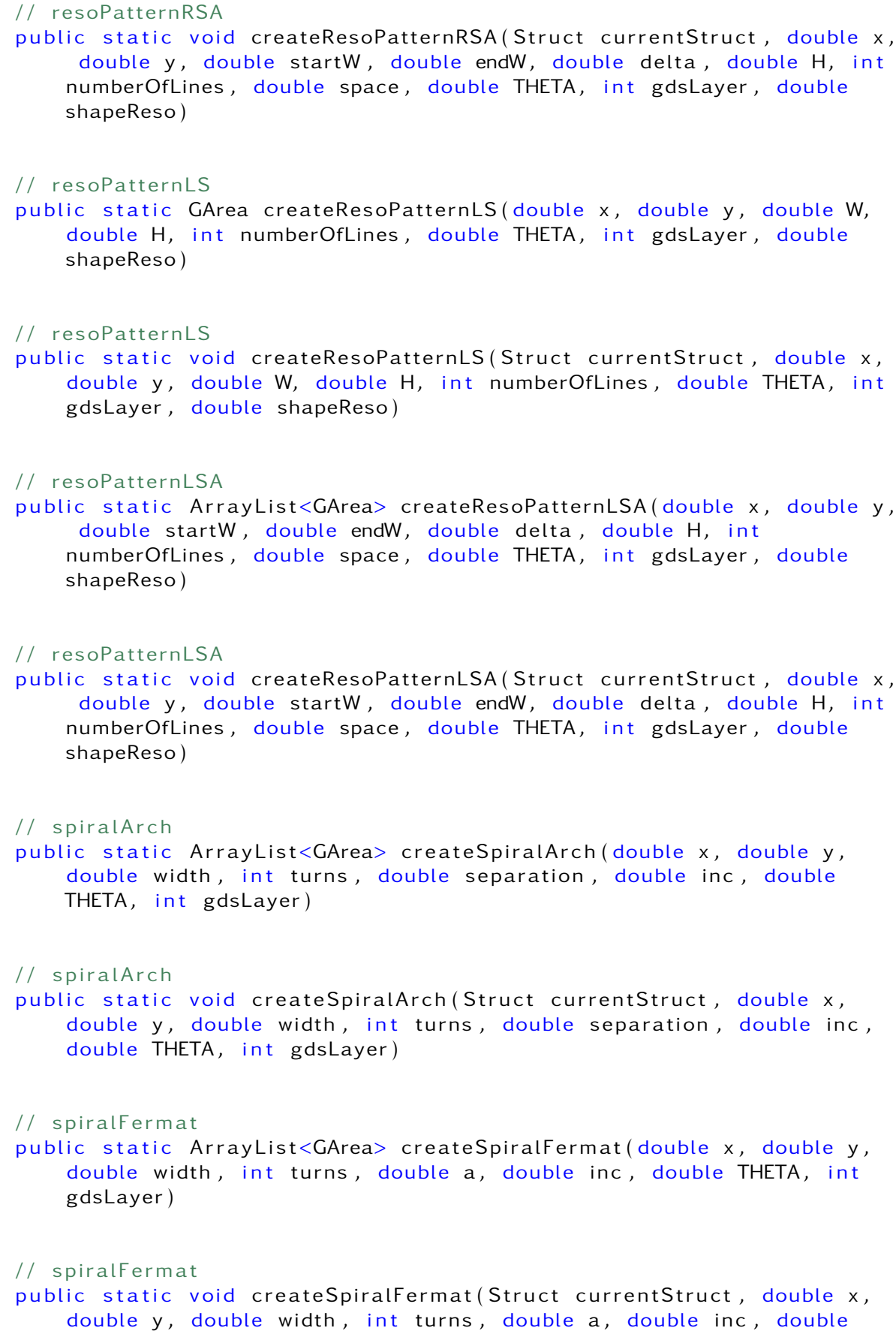


THETA, int gdsLayer)

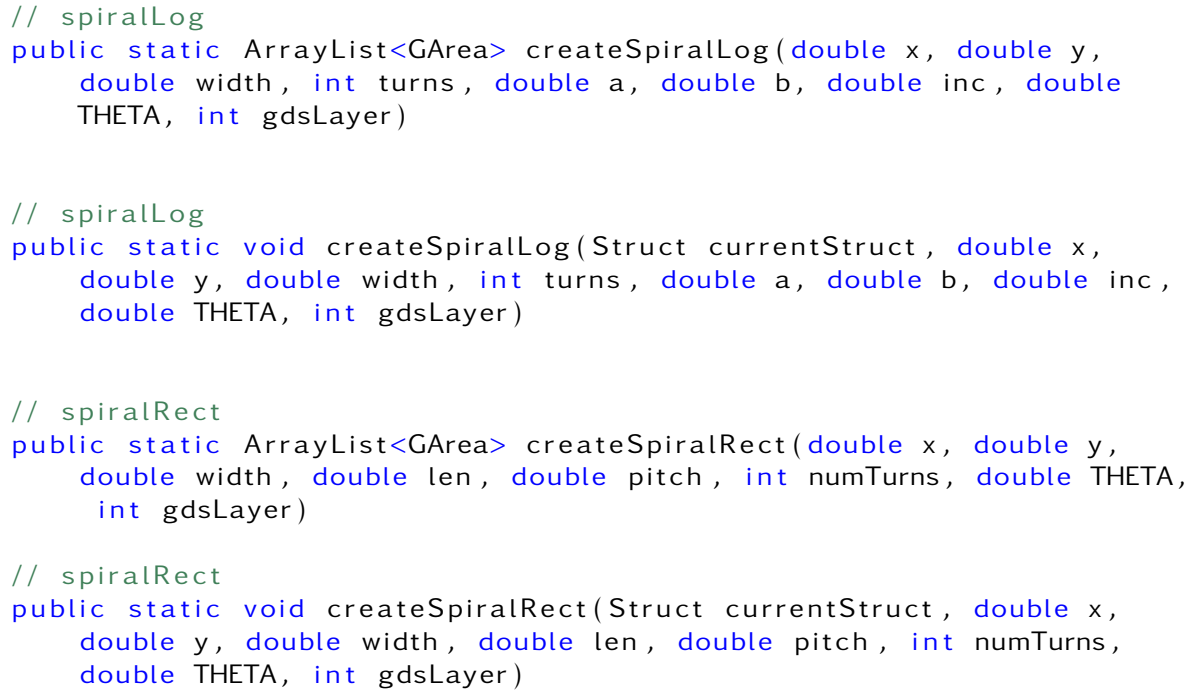




\subsubsection{Alignment and Reticle Element Methods}

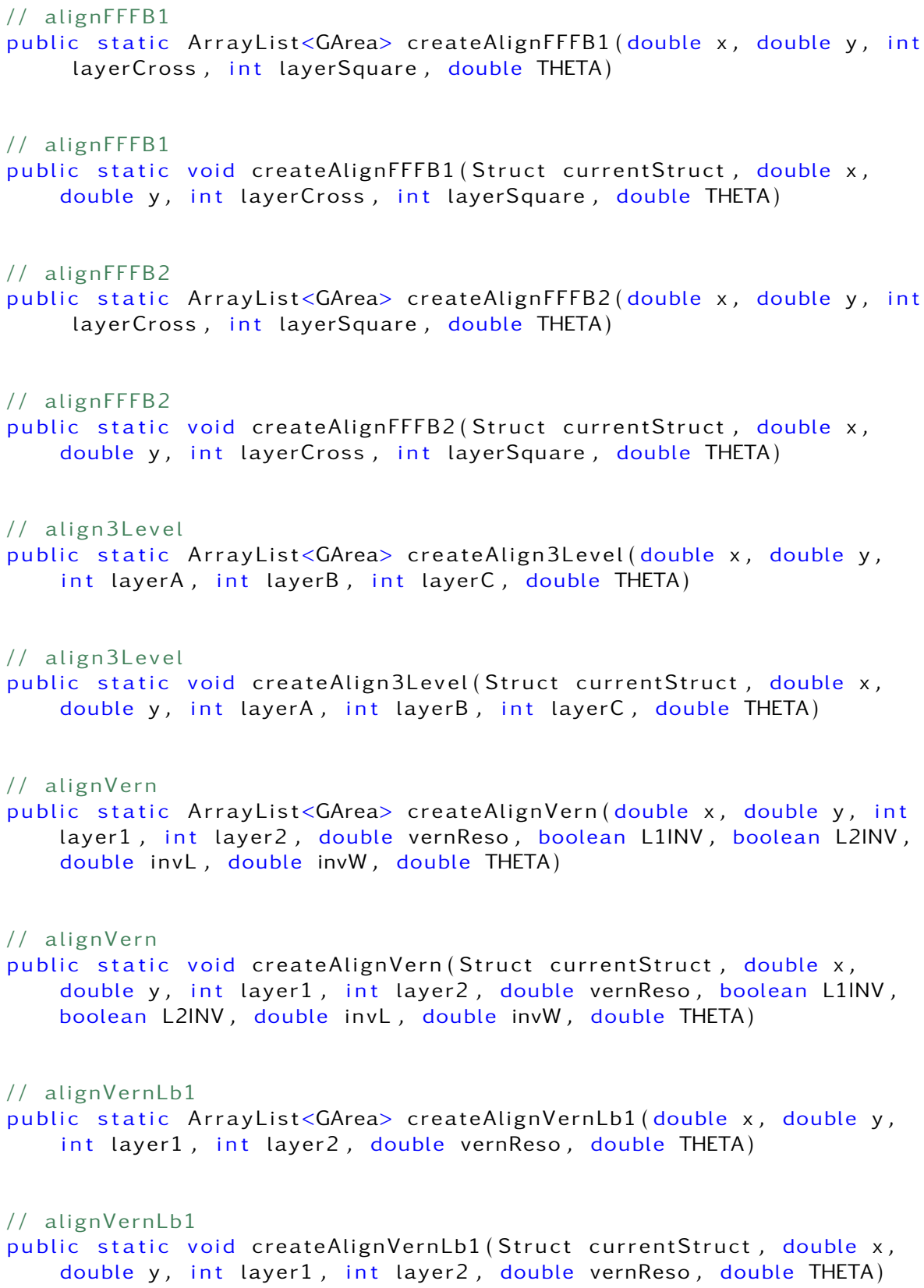




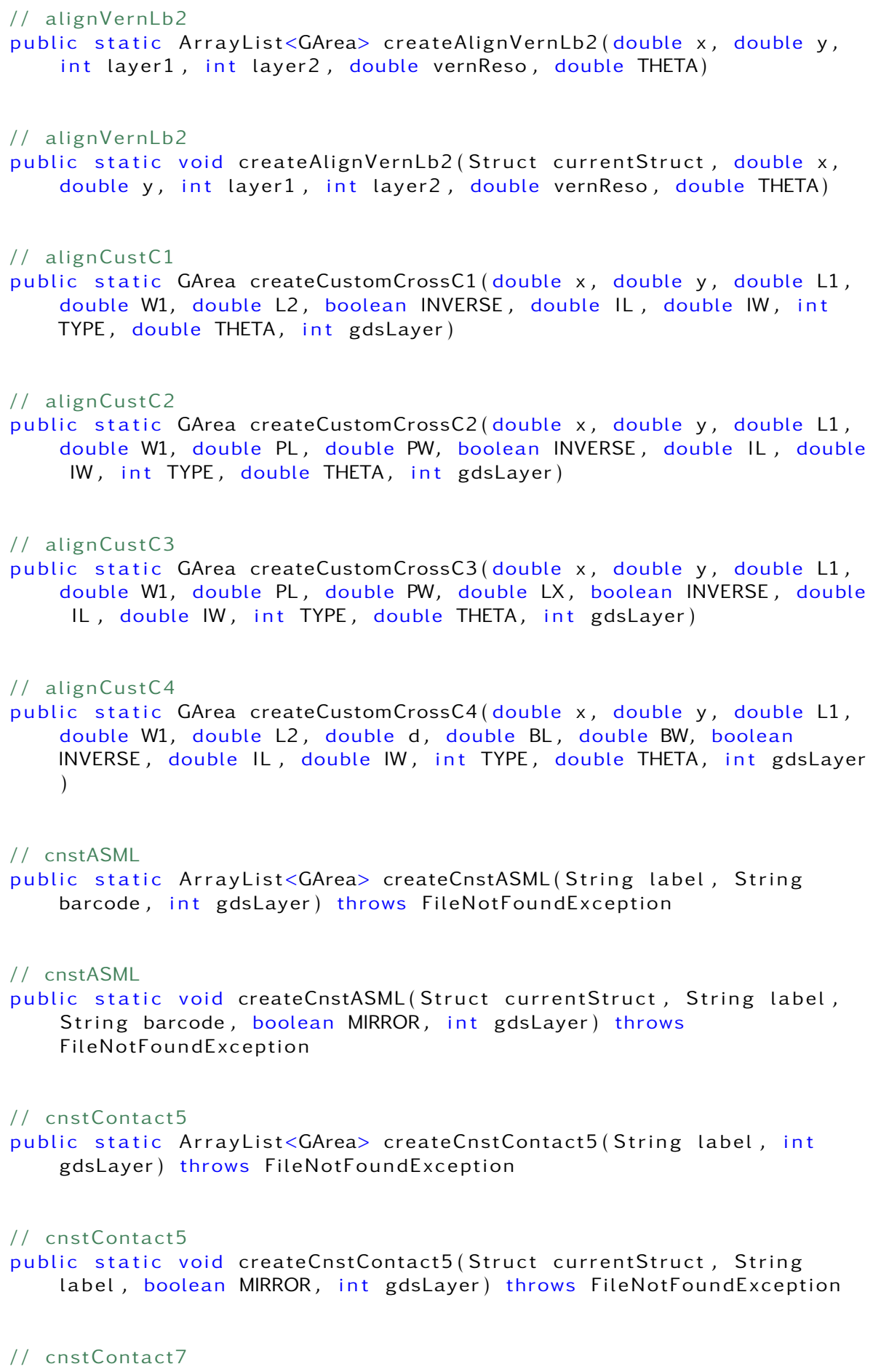




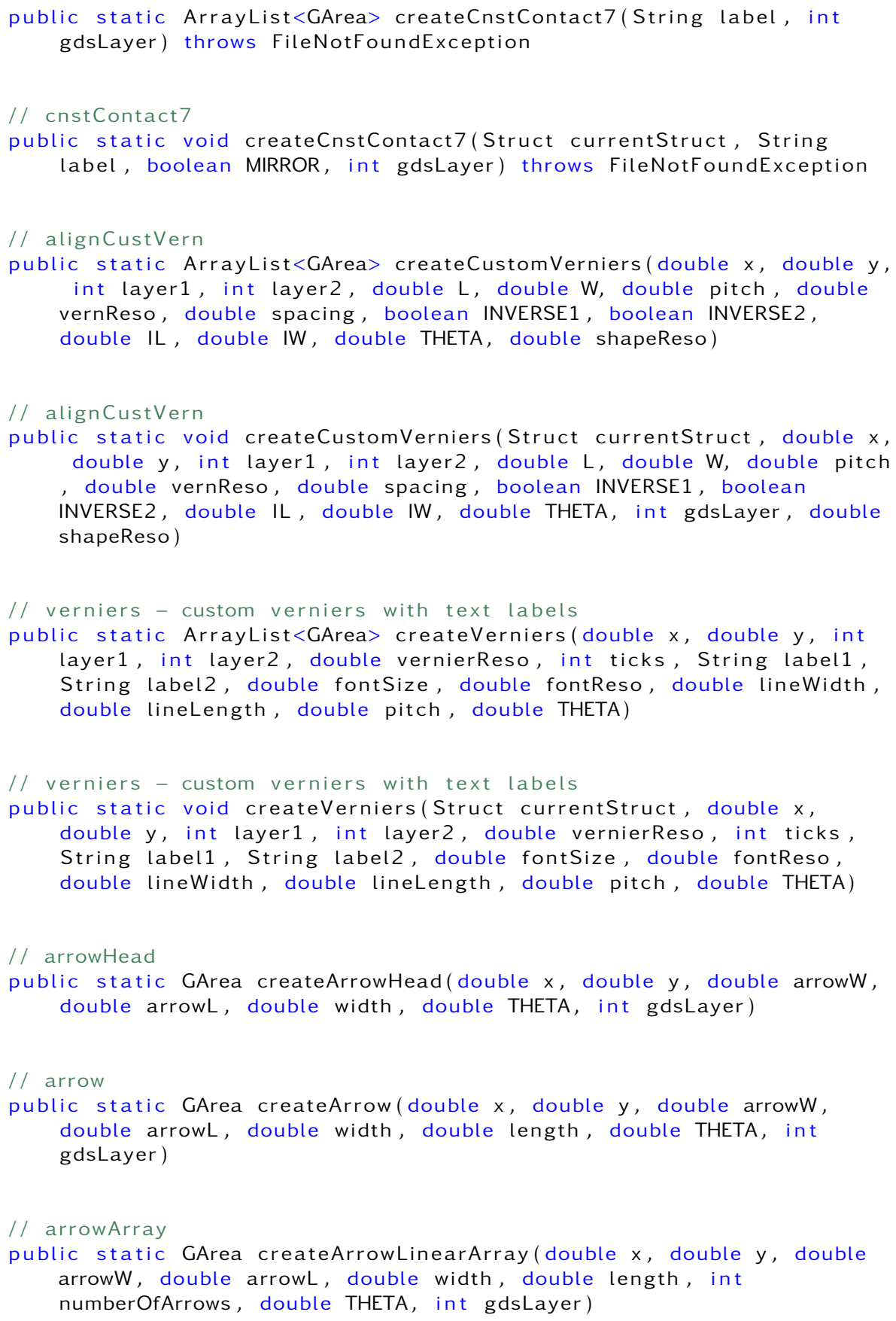




\subsubsection{Nanophotonics Library Methods}

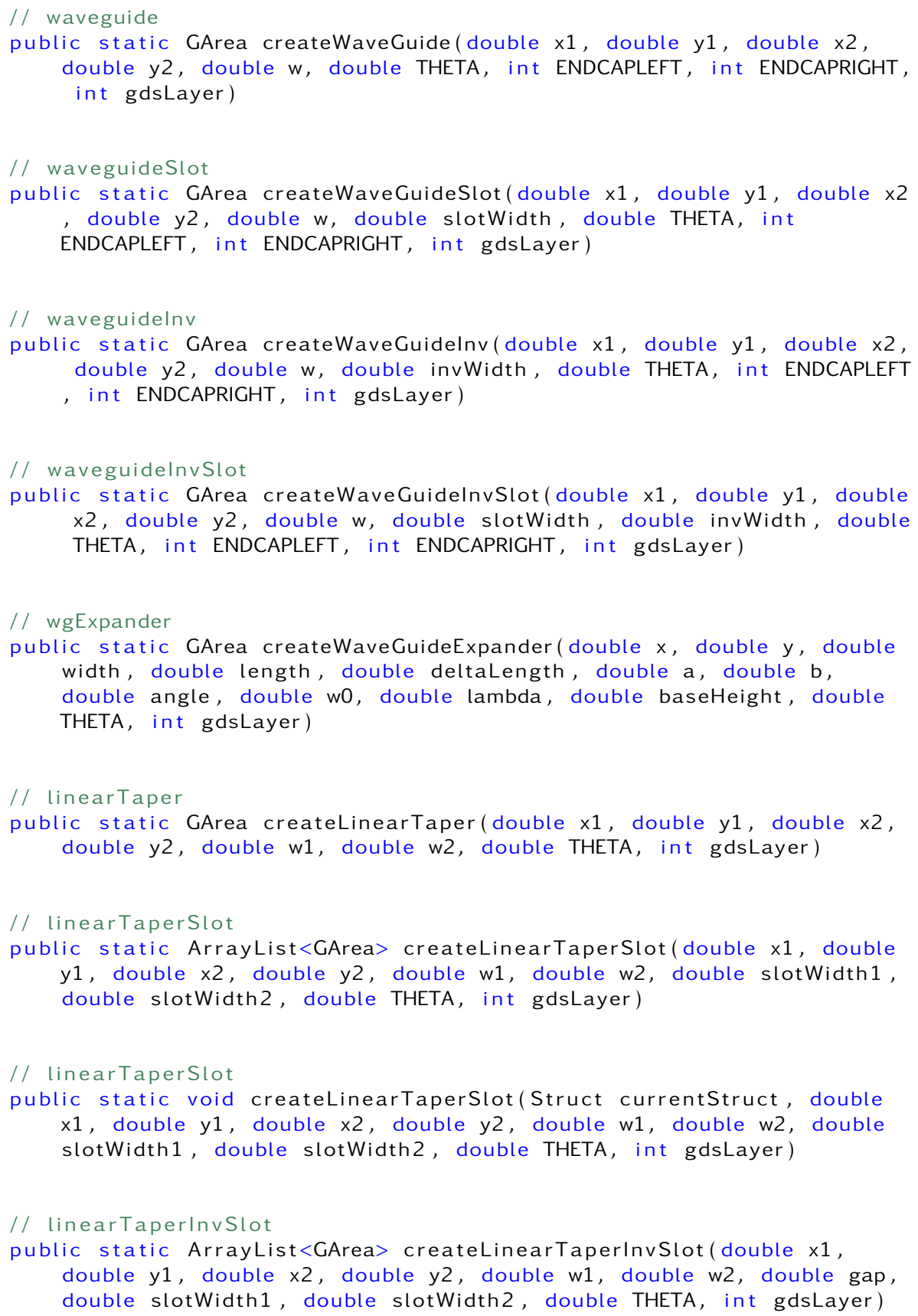

NIST • CNST Nanolithography Toolbox v2016.09.01 • http://www.nist.gov/cnst/

page 417 of 488 


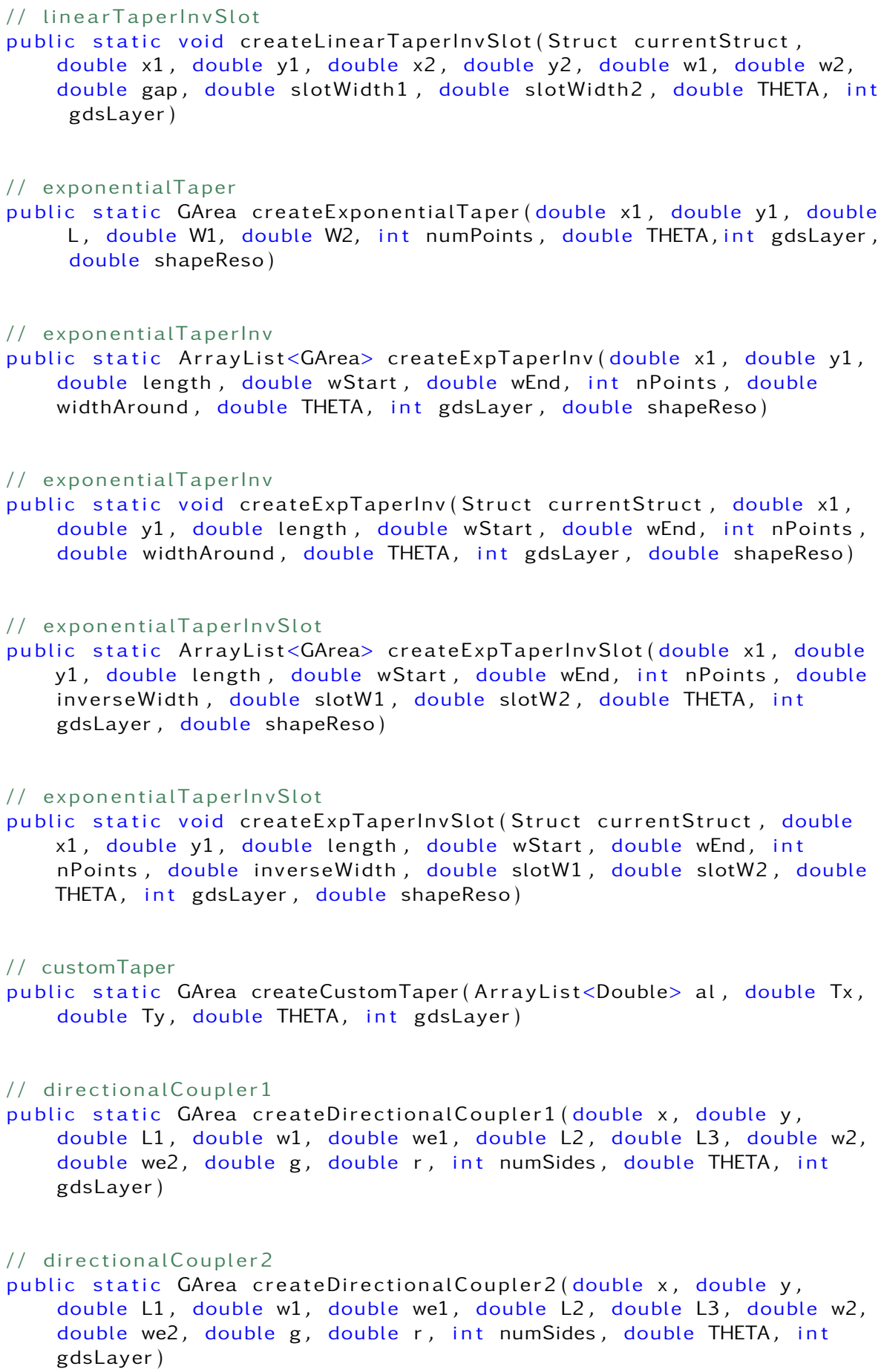




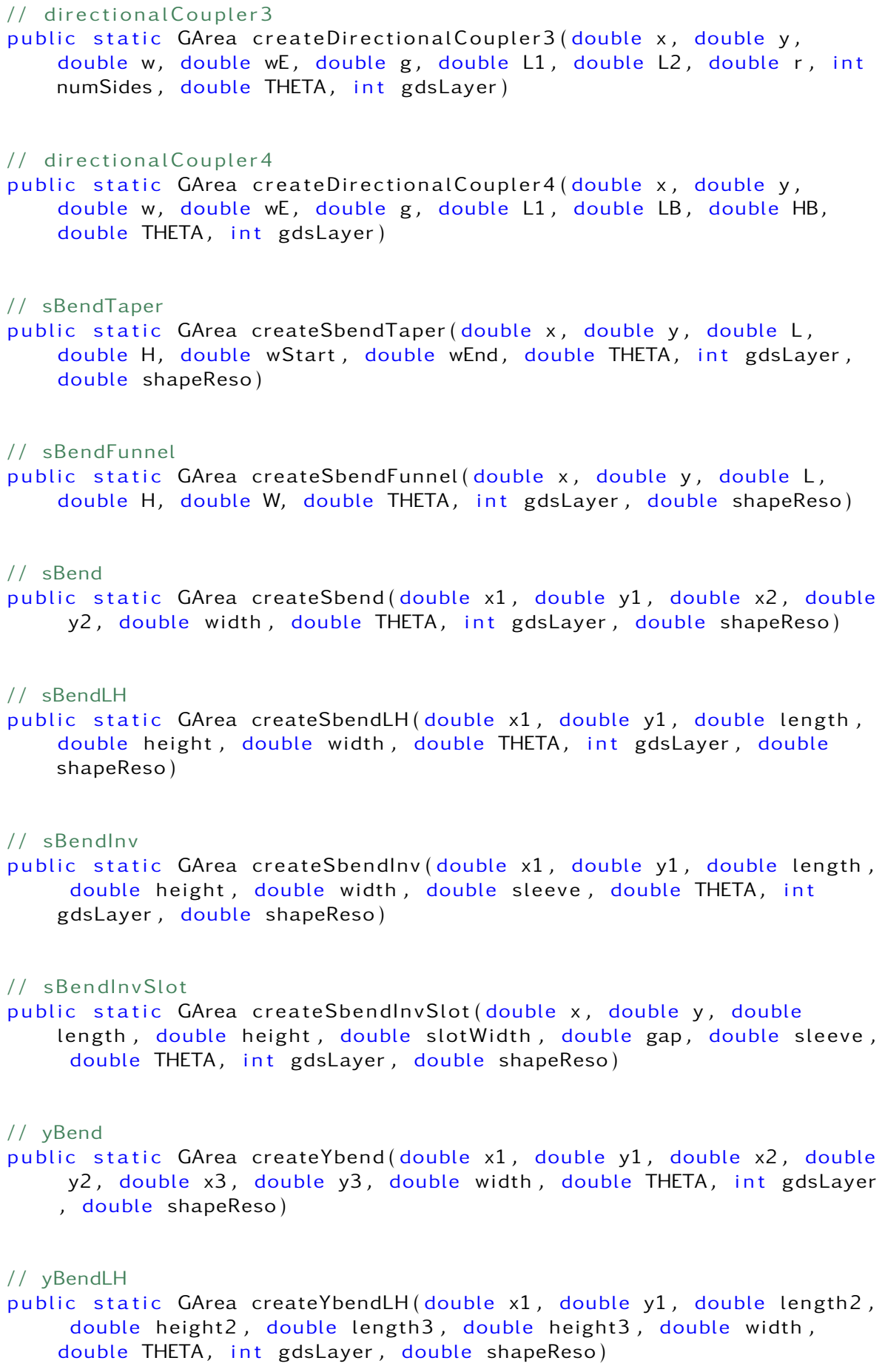

NIST • CNST Nanolithography Toolbox v2016.09.01 • http://www.nist.gov/cnst/

page 419 of 488 


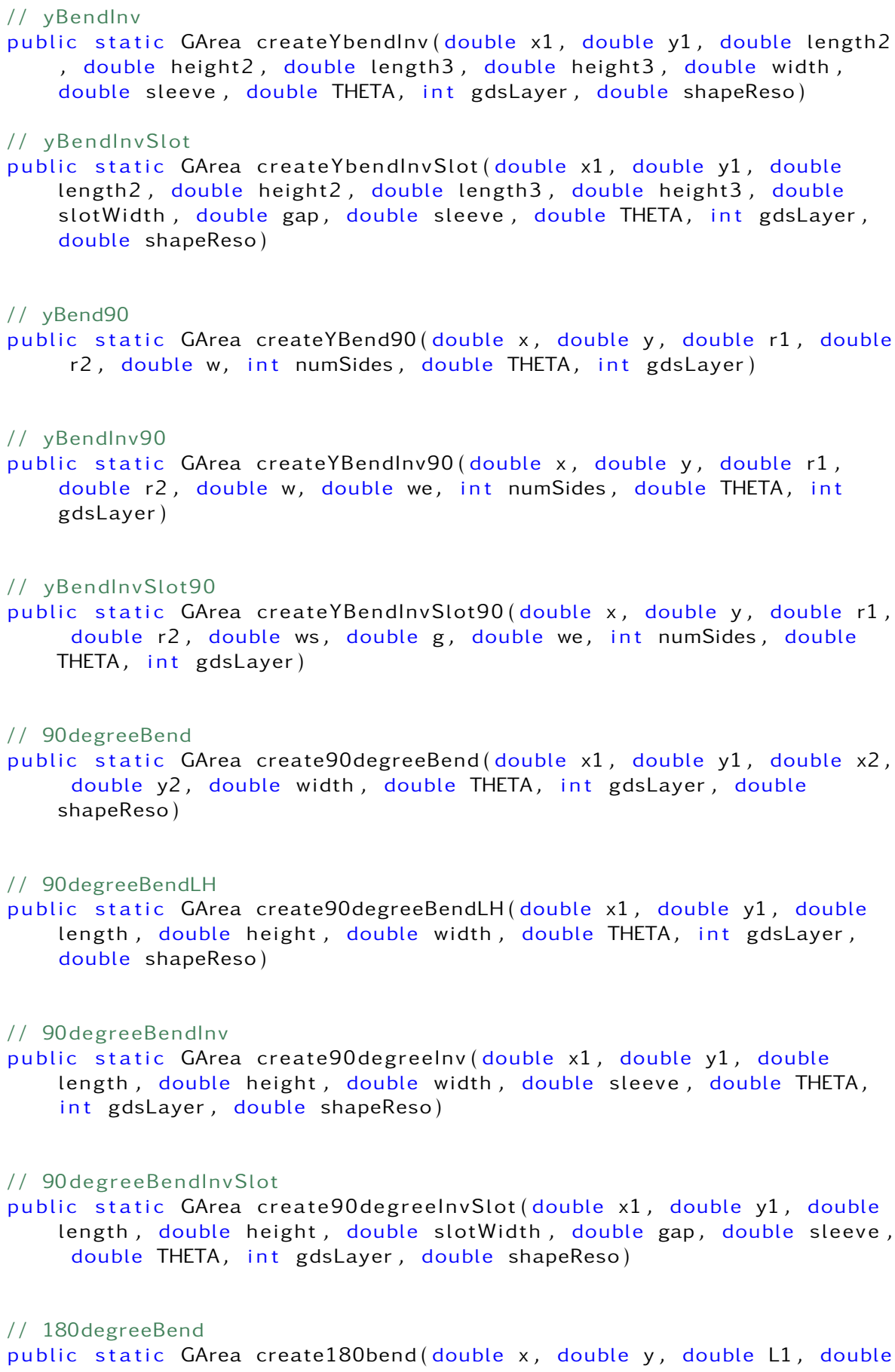


L2, double D, double W, int numSides, double THETA, int gdsLayer)

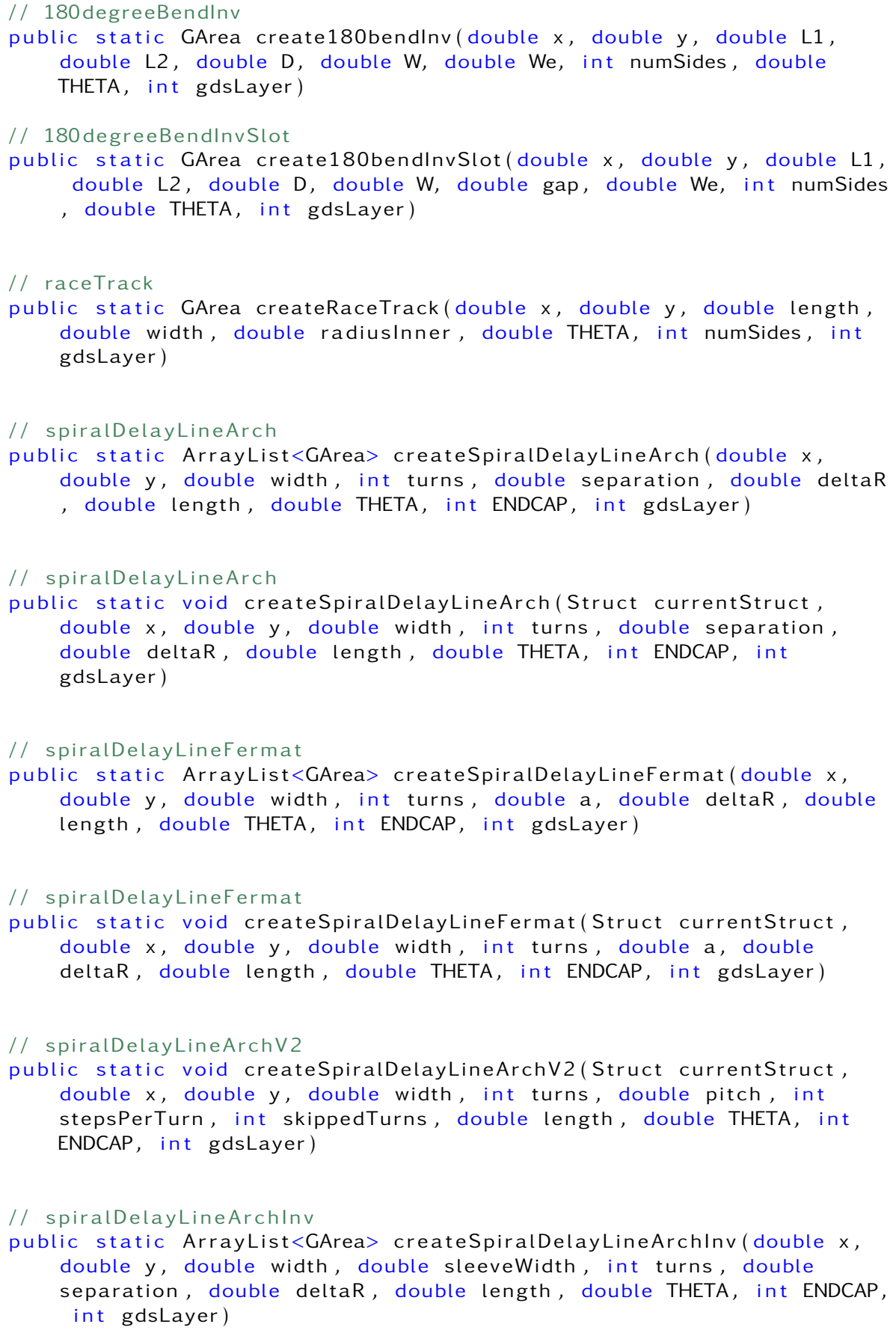

NIST • CNST Nanolithography Toolbox v2016.09.01 • http://www.nist.gov/cnst/

page 421 of 488 


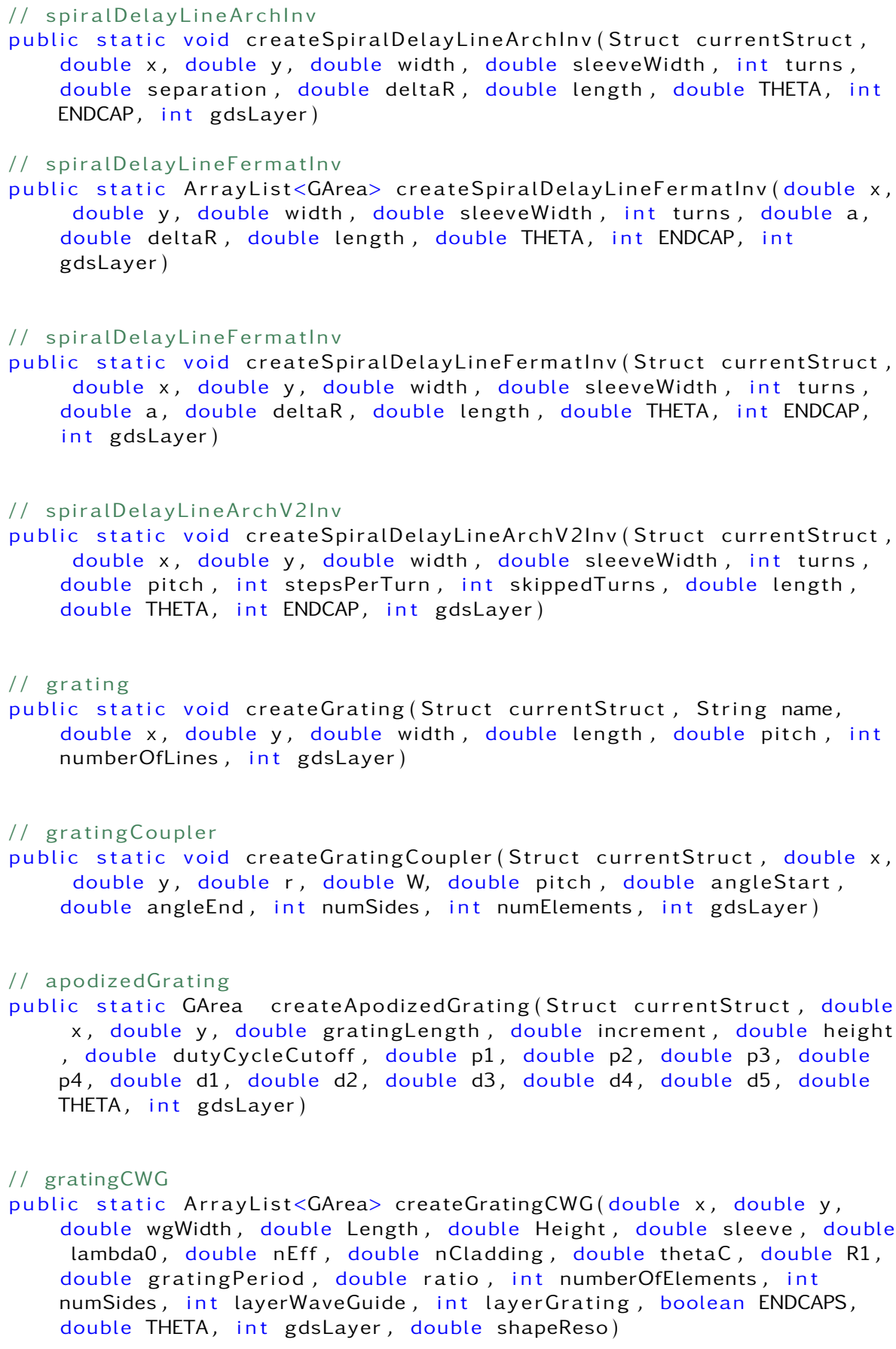

NIST • CNST Nanolithography Toolbox v2016.09.01・http://www.nist.gov/cnst/ 


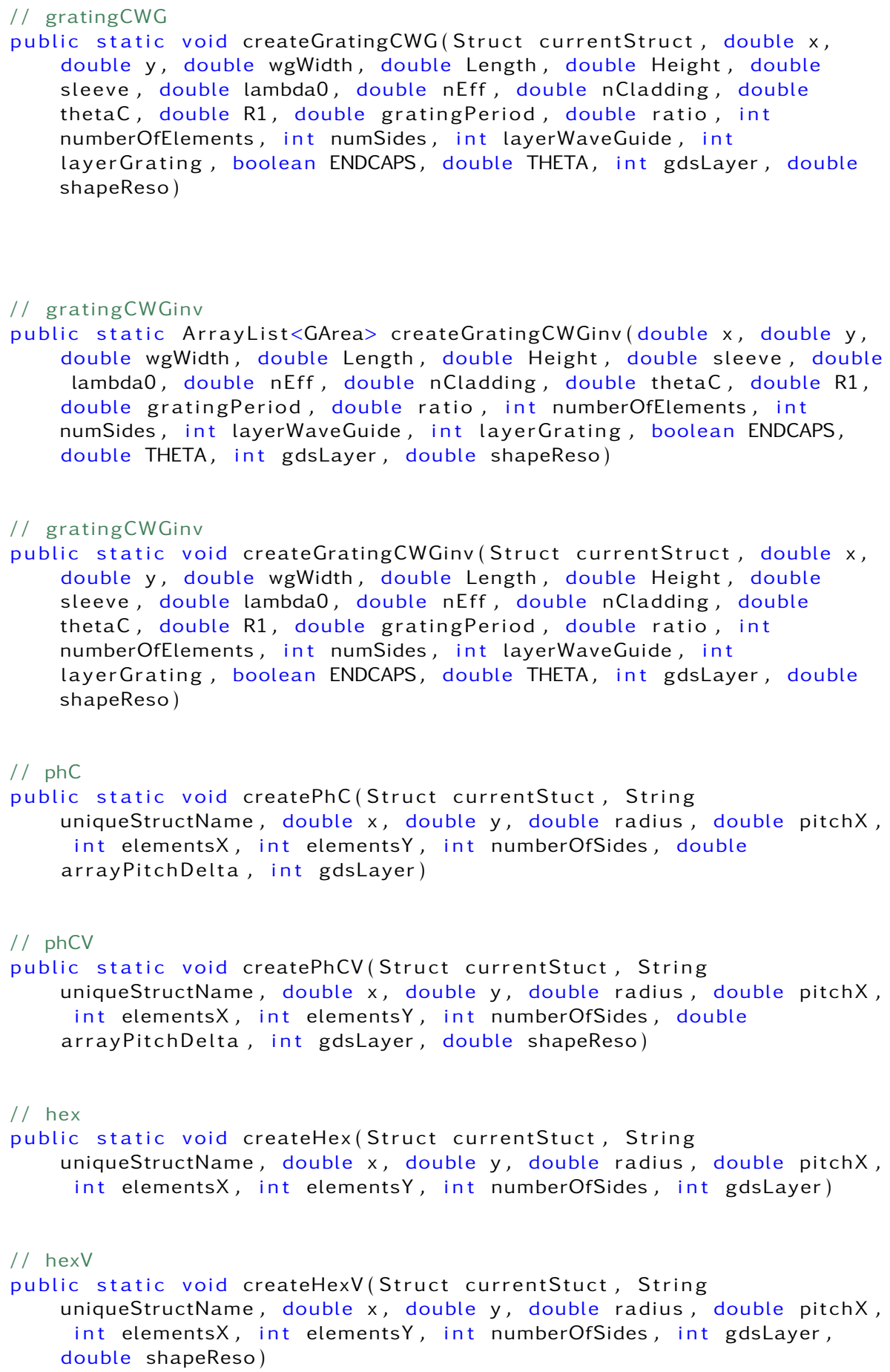




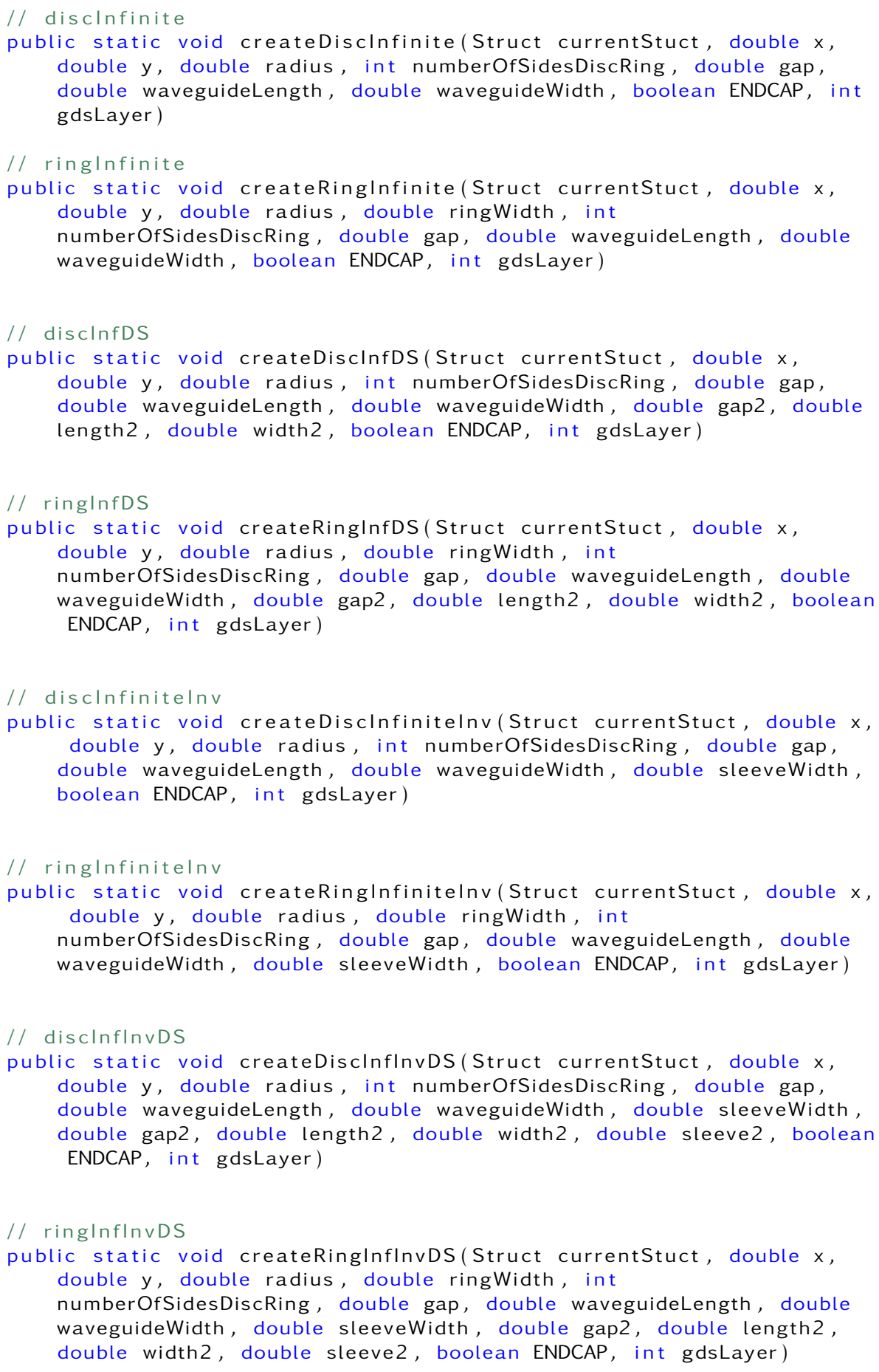

NIST • CNST Nanolithography Toolbox v2016.09.01・http://www.nist.gov/cnst/ 


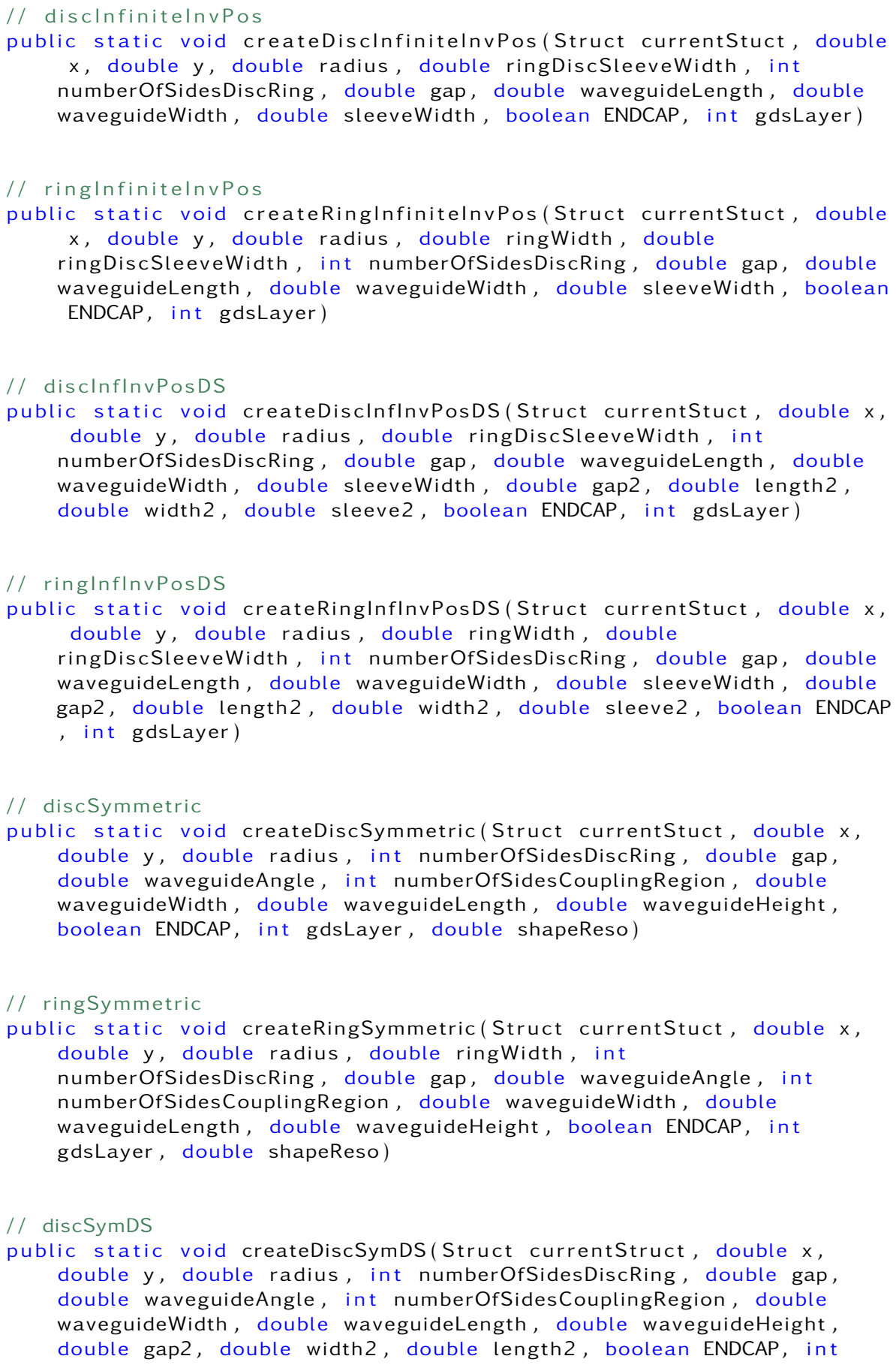


gdsLayer, double shapeReso)

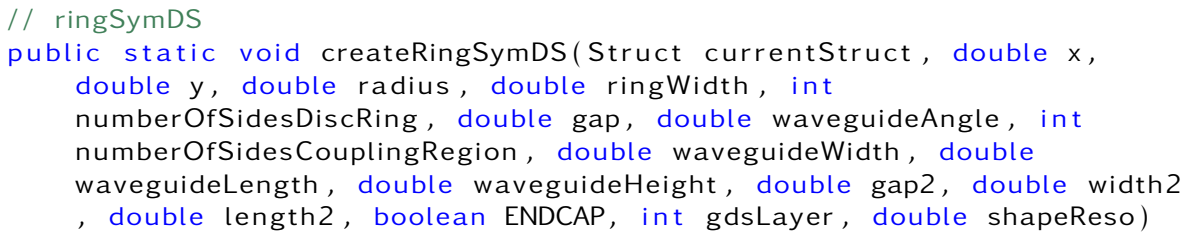

NIST • CNST Nanolithography Toolbox v2016.09.01・http://www.nist.gov/cnst/ 


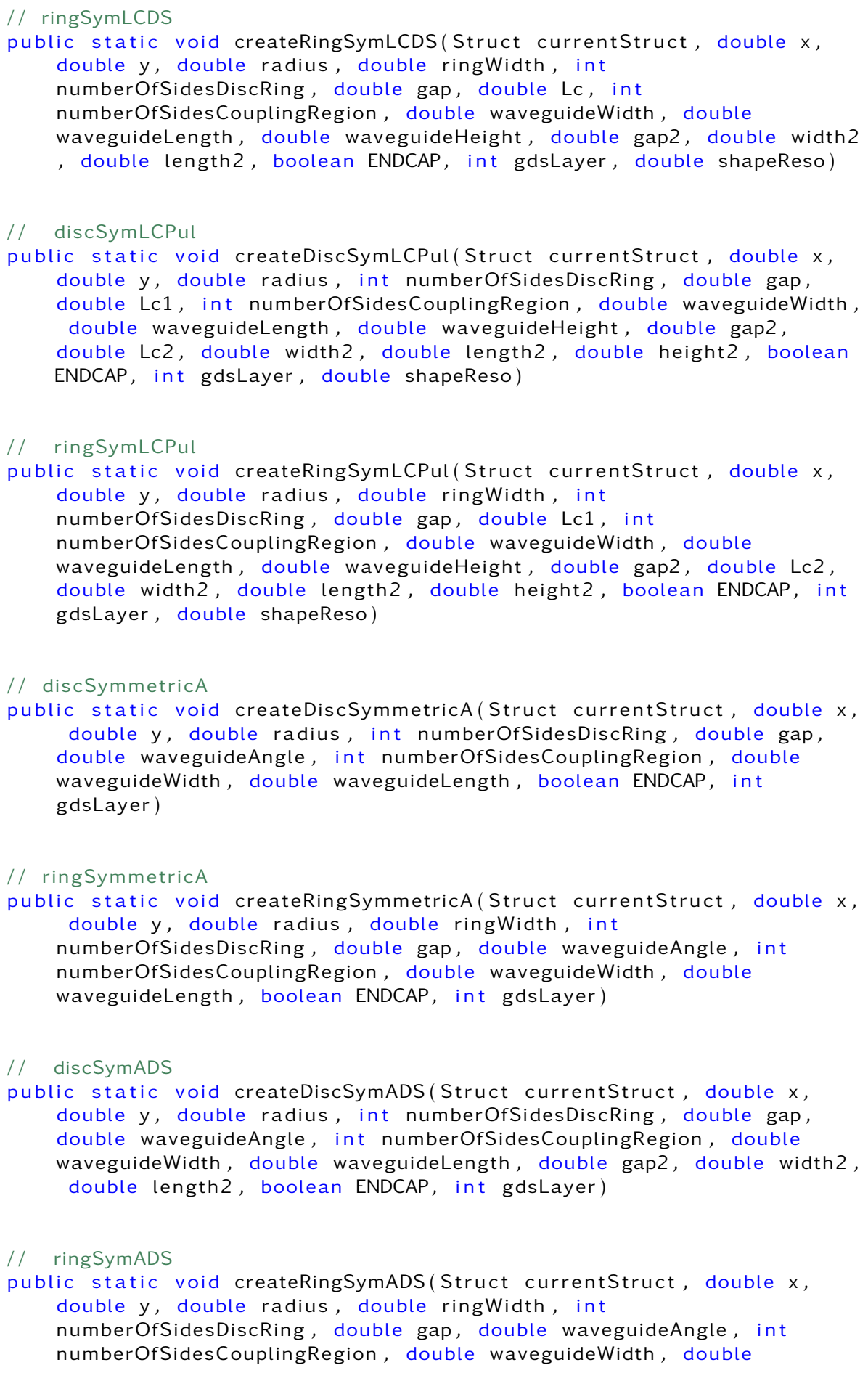

NIST • CNST Nanolithography Toolbox v2016.09.01 • http://www.nist.gov/cnst/ 
waveguidelength, double gap2, double width2, double length2, boolean ENDCAP, int gdsLayer)

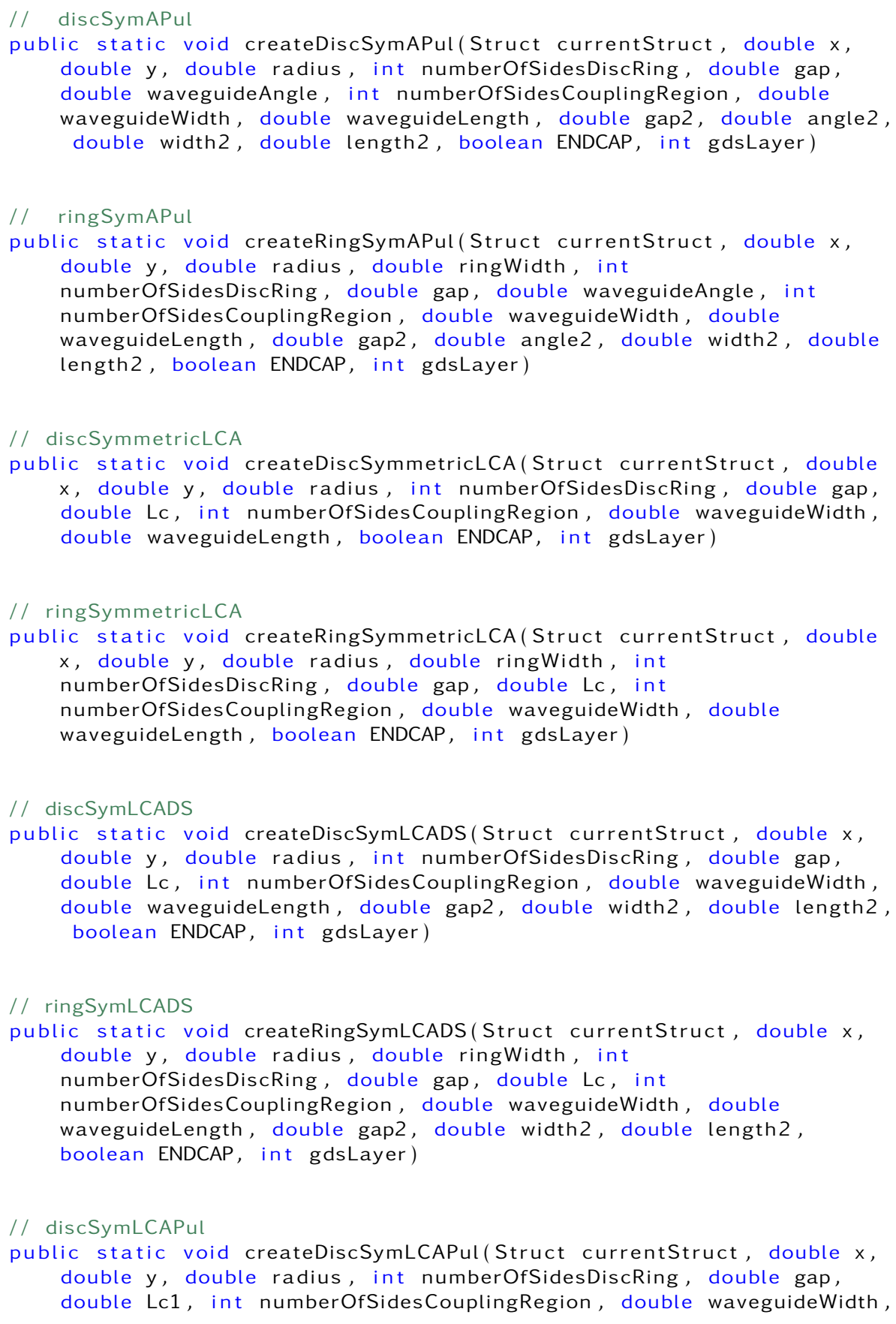


double waveguidelength, double gap2, double Lc2, double width2, double length2, boolean ENDCAP, int gdsLayer)

// ringSymLCAPul

public static void createRingSymLCAPul(Struct currentStruct, double $x$, double $y$, double radius, double ringWidth, int numberOfSidesDiscRing, double gap, double Lc1, int numberOfSidesCouplingRegion, double waveguideWidth, double waveguidelength, double gap2, double Lc2, double width2, double length2, boolean ENDCAP, int gdsLayer)

// discSymmetriclnv

public static void createDiscSymmetriclnv(Struct currentStruct, double $x$, double $y$, double radius, int numberOfSidesDiscRing, double gap, double waveguideAngle, int numberOfSidesCouplingRegion, double waveguideWidth, double sleeveWidth, double waveguideLength, double waveguideHeight, boolean ENDCAP, int gdsLayer, double shapeReso)

// ringSymmetriclnv

public static void createRingSymmetriclnv(Struct currentStruct, double $x$, double $y$, double radius, double ringWidth, int numberOfSidesDiscRing, double gap, double waveguideAngle, int numberOfSidesCouplingRegion, double waveguideWidth, double sleeveWidth, double waveguideLength, double waveguideHeight, boolean ENDCAP, int gdsLayer, double shapeReso)

// discSymlnvDS

public static void createDiscSyminvDS(Struct currentStruct, double $x$, double $y$, double radius, int numberOfSidesDiscRing, double gap, double waveguideAngle, int numberOfSidesCouplingRegion, double waveguideWidth, double sleeveWidth, double waveguideLength, double waveguideHeight, double gap2, double width2, double sleeve2, double length2, boolean ENDCAP, int gdsLayer, double shapeReso)

// ringSymInvDS

public static void createRingSymlnvDS(Struct currentStruct, double $x$, double $y$, double radius, double ringWidth, int numberOfSidesDiscRing, double gap, double waveguideAngle, int numberOfSidesCouplingRegion, double waveguideWidth, double sleeveWidth, double waveguideLength, double waveguideHeight, double gap2, double width2, double sleeve2, double length2, boolean ENDCAP, int gdsLayer, double shapeReso)

// discSyminvPul

public static void createDiscSyminvPul(Struct currentStruct, double $x$, double $y$, double radius, int numberOfSidesDiscRing, double gap, double waveguideAngle, int numberOfSidesCouplingRegion, double waveguideWidth, double sleeveWidth, double waveguidelength, double waveguideHeight, double gap2, double angle2, double width2, double sleeve2, double length2, double height2, boolean ENDCAP, int gdsLayer, double shapeReso) 


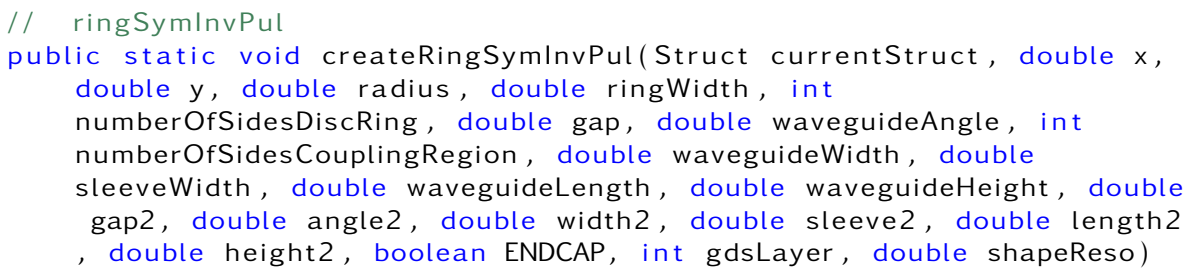

NIST • CNST Nanolithography Toolbox v2016.09.01・http://www.nist.gov/cnst/ 


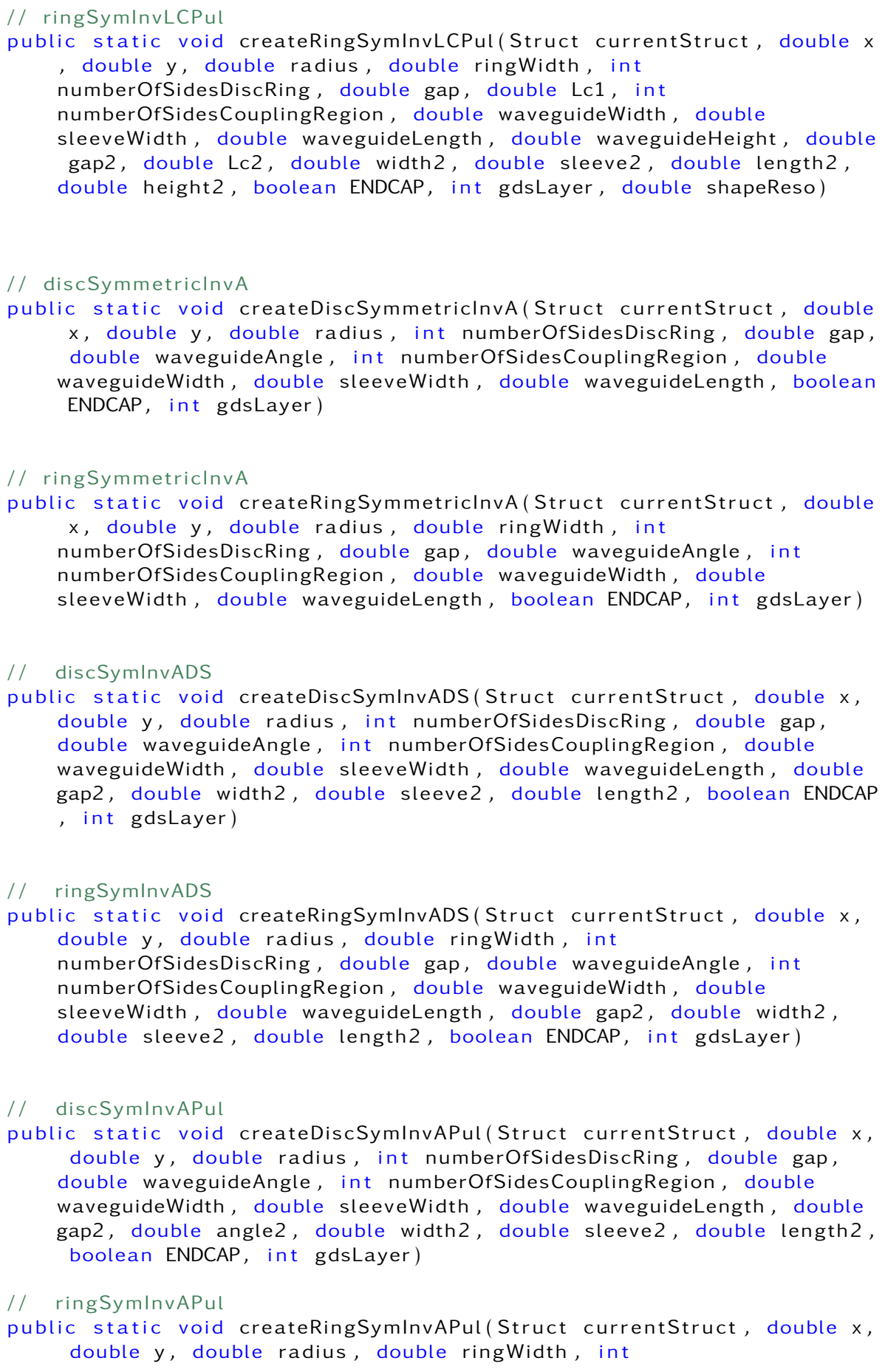

NIST • CNST Nanolithography Toolbox v2016.09.01 • http://www.nist.gov/cnst/

page 431 of 488 
numberOfSidesDiscRing, double gap, double waveguideAngle, int numberOfSidesCouplingRegion, double waveguideWidth, double sleeveWidth, double waveguidelength, double gap2, double angle2, double width2, double sleeve2, double length2, boolean ENDCAP, int gdsLayer )

// discSymmetriclnvLCA

public static void createDiscSymmetriclnvLCA(Struct currentStruct, double $x$, double $y$, double radius, int numberOfSidesDiscRing, double gap, double Lc, int numberOfSidesCouplingRegion, double waveguideWidth, double sleeveWidth, double waveguideLength, boolean ENDCAP, int gdsLayer)

// ringSymmetricInvLCA

public static void createRingSymmetriclnvLCA(Struct currentStruct, double $x$, double $y$, double radius, double ringWidth, int numberOfSidesDiscRing, double gap, double Lc, int numberOfSidesCouplingRegion, double waveguideWidth, double sleeveWidth, double waveguideLength, boolean ENDCAP, int gdsLayer)

\section{// discSymlnvLCADS}

public static void createDiscSyminvLCADS(Struct currentStruct, double $x$ , double $y$, double radius, int numberOfSidesDiscRing, double gap, double Lc, int numberOfSidesCouplingRegion, double waveguideWidth, double sleeveWidth, double waveguidelength, double gap2, double width2, double sleeve2, double length2, boolean ENDCAP, int gdsLayer )

\section{// ringSymInvLCADS}

public static void createRingSymlnvLCADS(Struct currentStruct, double $x$ , double y, double radius, double ringWidth, int numberOfSidesDiscRing, double gap, double Lc, int numberOfSidesCouplingRegion, double waveguideWidth, double sleeveWidth, double waveguidelength, double gap2, double width2, double sleeve2, double length2, boolean ENDCAP, int gdsLayer)

// discSymlnvLCAPul

public static void createDiscSymlnvLCAPul(Struct currentStruct, double $x$, double $y$, double radius, int numberOfSidesDiscRing, double gap, double Lc1, int numberOfSidesCouplingRegion, double waveguideWidth, double sleeveWidth, double waveguidelength, double gap2, double Lc2, double width2, double sleeve2, double length2, boolean ENDCAP, int gdsLayer)

// ringSymlnvLCAPul

public static void createRingSymlnvLCAPul(Struct currentStruct, double $x$, double $y$, double radius, double ringWidth, int numberOfSidesDiscRing, double gap, double Lc1, int numberOfSidesCouplingRegion, double waveguideWidth, double sleeveWidth, double waveguidelength, double gap2, double Lc2, double width2, double sleeve2, double length2, boolean ENDCAP, int gdsLayer ) 
// discSymmetriclnvPos

public static void createDiscSymmetriclnvPos(Struct currentStruct, double $x$, double $y$, double radius, double ringDiscSleeveWidth, int numberOfSidesDiscRing, double gap, double waveguideAngle, int numberOfSidesCouplingRegion, double waveguideWidth, double sleeveWidth, double waveguideLength, double waveguideHeight, boolean ENDCAP, int gdsLayer, double shapeReso)

// ringSymmetriclnvPos

public static void createRingSymmetriclnvPos(Struct currentStruct, double $x$, double $y$, double radius, double ringWidth, double ringDiscSleeveWidth, int numberOfSidesDiscRing, double gap, double waveguideAngle, int numberOfSidesCouplingRegion, double waveguideWidth, double sleeveWidth, double waveguideLength, double waveguideHeight, boolean ENDCAP, int gdsLayer, double shapeReso)

// discSymInvPosDS

public static void createDiscSymInvPosDS(Struct currentStruct, double $x$ , double $y$, double radius, double ringDiscSleeveWidth, int numberOfSidesDiscRing, double gap, double waveguideAngle, int numberOfSidesCouplingRegion, double waveguideWidth, double sleeveWidth, double waveguideLength, double waveguideHeight, double gap2, double width2, double sleeve2, double length2, boolean ENDCAP, int gdsLayer, double shapeReso)

// ringSymInvPosDS

public static void createRingSymInvPosDS(Struct currentStruct, double $x$ , double y, double radius, double ringWidth, double ringDiscSleeveWidth, int numberOfSidesDiscRing, double gap, double waveguideAngle, int numberOfSidesCouplingRegion, double waveguideWidth, double sleeveWidth, double waveguideLength, double waveguideHeight, double gap2, double width2, double sleeve2, double length2, boolean ENDCAP, int gdsLayer, double shapeReso)

// discSymInvPosPul

public static void createDiscSymlnvPosPul(Struct currentStruct, double

$x$, double $y$, double radius, double ringDiscSleeveWidth, int numberOfSidesDiscRing, double gap, double waveguideAngle, int numberOfSidesCouplingRegion, double waveguideWidth, double sleeveWidth, double waveguideLength, double waveguideHeight, double gap2, double angle2, double width2, double sleeve2, double length2 , double height2, boolean ENDCAP, int gdsLayer, double shapeReso)

// ringSyminvPosPul

public static void createRingSymInvPosPul(Struct currentStruct, double $x$, double $y$, double radius, double ringWidth, double

ringDiscSleeveWidth, int numberOfSidesDiscRing, double gap, double waveguideAngle, int numberOfSidesCouplingRegion, double

waveguideWidth, double sleeveWidth, double waveguideLength, double

NIST • CNST Nanolithography Toolbox v2016.09.01 • http://www.nist.gov/cnst/ page 433 of 488 
waveguideHeight, double gap2, double angle2, double width2, double sleeve2, double length2, double height2, boolean ENDCAP, int gdshayer, double shapeReso)

\section{// discSymmetriclnvPosLC}

public static void createDiscSymmetriclnversePosLC(Struct currentStruct , double $x$, double $y$, double radius, double ringDiscSleeveWidth, int numberOfSidesDiscRing, double gap, double Lc, int numberOfSidesCouplingRegion, double waveguideWidth, double sleeveWidth, double waveguidelength, double waveguideHeight, boolean ENDCAP, int gdsLayer, double shapeReso)

// ringSymmetricInvPosLC

public static void createRingSymmetriclnversePosLC(Struct currentStruct , double $x$, double $y$, double radius, double ringWidth, double ringDiscSleeveWidth, int numberOfSidesDiscRing, double gap, double Lc, int numberOfSidesCouplingRegion, double waveguideWidth, double sleeveWidth, double waveguidelength, double waveguideHeight, boolean ENDCAP, int gdsLayer, double shapeReso)

// discSymlnvPosLCDS

public static void createDiscSymlnvPosLCDS(Struct currentStruct, double $x$, double $y$, double radius, double ringDiscSleeveWidth, int numberOfSidesDiscRing, double gap, double Lc, int numberOfSidesCouplingRegion, double waveguideWidth, double sleeveWidth, double waveguidelength, double waveguideHeight, double gap2, double width2, double sleeve2, double length2, boolean ENDCAP, int gdsLayer, double shapeReso)

// ringSymInvPosLCDS

public static void createRingSymInvPosLCDS(Struct currentStruct, double $x$, double $y$, double radius, double ringWidth, double ringDiscSleeveWidth, int numberOfSidesDiscRing, double gap, double Lc, int numberOfSidesCouplingRegion, double waveguideWidth, double sleeveWidth, double waveguideLength, double waveguideHeight, double gap2, double width2, double sleeve2, double length2, boolean ENDCAP, int gdsLayer, double shapeReso)

// discSymlnvPosLCPul

public static void createDiscSymlnvPosLCPul(Struct currentStruct, double $x$, double $y$, double radius, double ringDiscSleeveWidth, int numberOfSidesDiscRing, double gap, double Lc1, int numberOfSidesCouplingRegion, double waveguideWidth, double sleeveWidth, double waveguideLength, double waveguideHeight, double gap2, double Lc2, double width2, double sleeve2, double length2, double height2, boolean ENDCAP, int gdsLayer, double shapeReso)

// ringSyminvPosLCPul

public static void createRingSymlnvPosLCPul(Struct currentStruct, double $x$, double $y$, double radius, double ringWidth, double ringDiscSleeveWidth, int numberOfSidesDiscRing, double gap, double 
Lc1, int numberOfSidesCouplingRegion, double waveguideWidth, double sleeveWidth, double waveguideLength, double waveguideHeight, double gap2, double Lc2, double width2, double sleeve2, double length2, double height2, boolean ENDCAP, int gdsLayer, double shapeReso)

// discSymmetriclnvPosA

public static void createDiscSymmetriclnvPosA(Struct currentStruct, double $x$, double $y$, double radius, double ringDiscSleeveWidth, int numberOfSidesDiscRing, double gap, double waveguideAngle, int numberOfSidesCouplingRegion, double waveguideWidth, double sleeveWidth, double waveguideLength, boolean ENDCAP, int gdsLayer)

// ringSymmetriclnvPosA

public static void createRingSymmetriclnvPosA(Struct currentStruct, double $x$, double $y$, double radius, double ringWidth, double ringDiscSleeveWidth, int numberOfSidesDiscRing, double gap, double waveguideAngle, int numberOfSidesCouplingRegion, double waveguideWidth, double sleeveWidth, double waveguideLength, boolean ENDCAP, int gdsLayer)

// discSymlnvPosADS

public static void createDiscSymInvPosADS(Struct currentStruct, double $x$, double $y$, double radius, double ringDiscSleeveWidth, int numberOfSidesDiscRing, double gap, double waveguideAngle, int numberOfSidesCouplingRegion, double waveguideWidth, double sleeveWidth, double waveguideLength, double gap2, double width2, double sleeve2, double length2, boolean ENDCAP, int gdsLayer)

// ringSymlnvPosADS

public static void createRingSymInvPosADS(Struct currentStruct, double $x$, double $y$, double radius, double ringWidth, double ringDiscSleeveWidth, int numberOfSidesDiscRing, double gap, double waveguideAngle, int numberOfSidesCouplingRegion, double waveguideWidth, double sleeveWidth, double waveguidelength, double gap2, double width2, double sleeve2, double length2, boolean ENDCAP , int gdsLayer)

// discSymlnvPosAPul

public static void createDiscSymlnvPosAPul(Struct currentStruct, double $x$, double $y$, double radius, double ringDiscSleeveWidth, int numberOfSidesDiscRing, double gap, double waveguideAngle, int numberOfSidesCouplingRegion, double waveguideWidth, double sleeveWidth, double waveguidelength, double gap2, double angle2, double width2, double sleeve2, double length2, boolean ENDCAP, int gdsLayer )

// ringSymInvPosAPul

public static void createRingSymlnvPosAPul(Struct currentStruct, double $x$, double $y$, double radius, double ringWidth, double ringDiscSleeveWidth, int numberOfSidesDiscRing, double gap, double waveguideAngle, int numberOfSidesCouplingRegion, double 
waveguideWidth, double sleeveWidth, double waveguideLength, double gap2, double angle2, double width2, double sleeve2, double length2, boolean ENDCAP, int gdsLayer)

// discSymmetriclnvPosLCA

public static void createDiscSymmetriclnvPosLCA(Struct currentStruct, double $x$, double $y$, double radius, double ringDiscSleeveWidth, int numberOfSidesDiscRing, double gap, double Lc, int numberOfSidesCouplingRegion, double waveguideWidth, double sleeveWidth, double waveguideLength, boolean ENDCAP, int gdsLayer)

// ringSymmetriclnvPosLCA

public static void createRingSymmetriclnvPosLCA(Struct currentStruct, double $x$, double $y$, double radius, double ringWidth, double ringDiscSleeveWidth, int numberOfSidesDiscRing, double gap, double Lc, int numberOfSidesCouplingRegion, double waveguideWidth, double sleeveWidth, double waveguideLength, boolean ENDCAP, int gdsLayer)

// discSymlnvPosLCADS

public static void createDiscSymlnvPosLCADS(Struct currentStruct, double $x$, double $y$, double radius, double ringDiscSleeveWidth, int numberOfSidesDiscRing, double gap, double Lc, int numberOfSidesCouplingRegion, double waveguideWidth, double sleeveWidth, double waveguideLength, double gap2, double width2, double sleeve2, double length2, boolean ENDCAP, int gdsLayer)

// ringSymlnvPosLCADS

public static void createRingSymlnvPosLCADS(Struct currentStruct, double $x$, double $y$, double radius, double ringWidth, double ringDiscSleeveWidth, int numberOfSidesDiscRing, double gap, double Lc, int numberOfSidesCouplingRegion, double waveguideWidth, double sleeveWidth, double waveguidelength, double gap2, double width2, double sleeve2, double length2, boolean ENDCAP, int gdsLayer)

// discSymlnvPosLCAPul

public static void createDiscSymlnvPosLCAPul(Struct currentStruct, double $x$, double $y$, double radius, double ringDiscSleeveWidth, int numberOfSidesDiscRing, double gap, double Lc1, int numberOfSidesCouplingRegion, double waveguideWidth, double sleeveWidth, double waveguidelength, double gap2, double Lc2, double width2, double sleeve2, double length2, boolean ENDCAP, int gdsLayer )

// ringSymInvPosLCAPul

public static void createRingSymInvPosLCAPul(Struct currentStruct, double $x$, double $y$, double radius, double ringWidth, double ringDiscSleeveWidth, int numberOfSidesDiscRing, double gap, double Lc1, int numberOfSidesCouplingRegion, double waveguideWidth, double sleeveWidth, double waveguidelength, double gap2, double Lc2, double width2, double sleeve2, double length2, boolean ENDCAP, int gdsLayer )

NIST • CNST Nanolithography Toolbox v2016.09.01 • http://www.nist.gov/cnst/ 


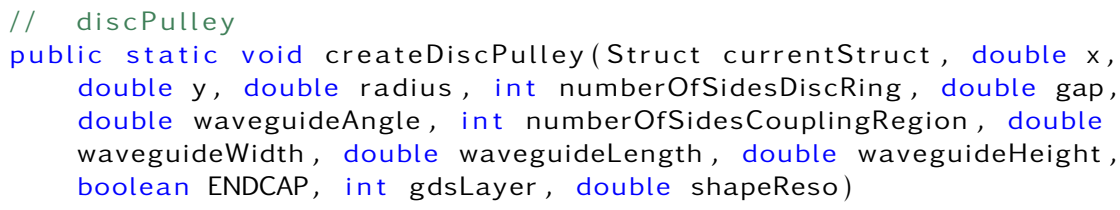




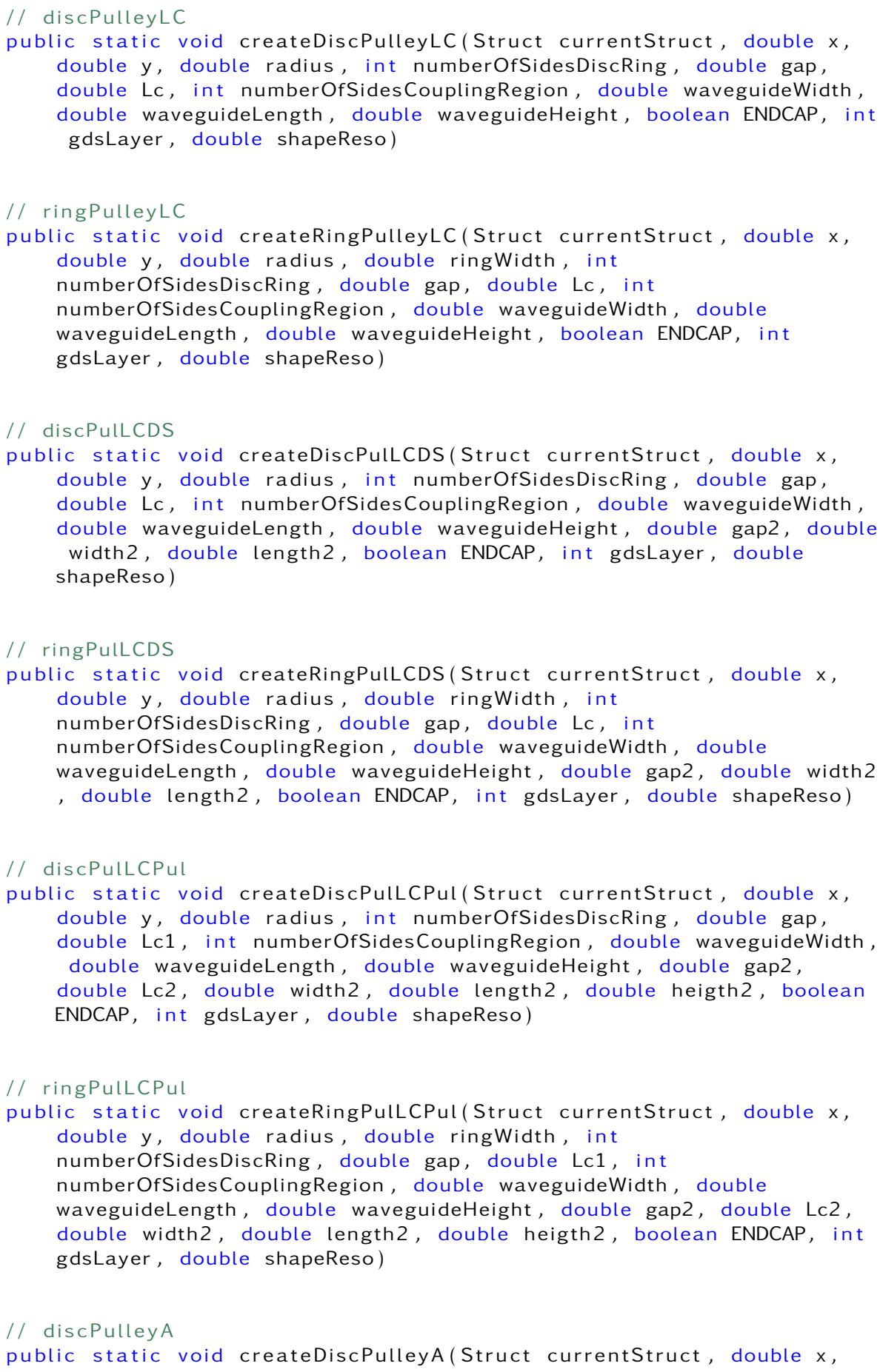

NIST • CNST Nanolithography Toolbox v2016.09.01・http://www.nist.gov/cnst/ 
double y, double radius, int numberOfSidesDiscRing, double gap, double waveguideAngle, int numberOfSidesCouplingRegion, double waveguideWidth, double waveguideLength, boolean ENDCAP, int gdsLayer )

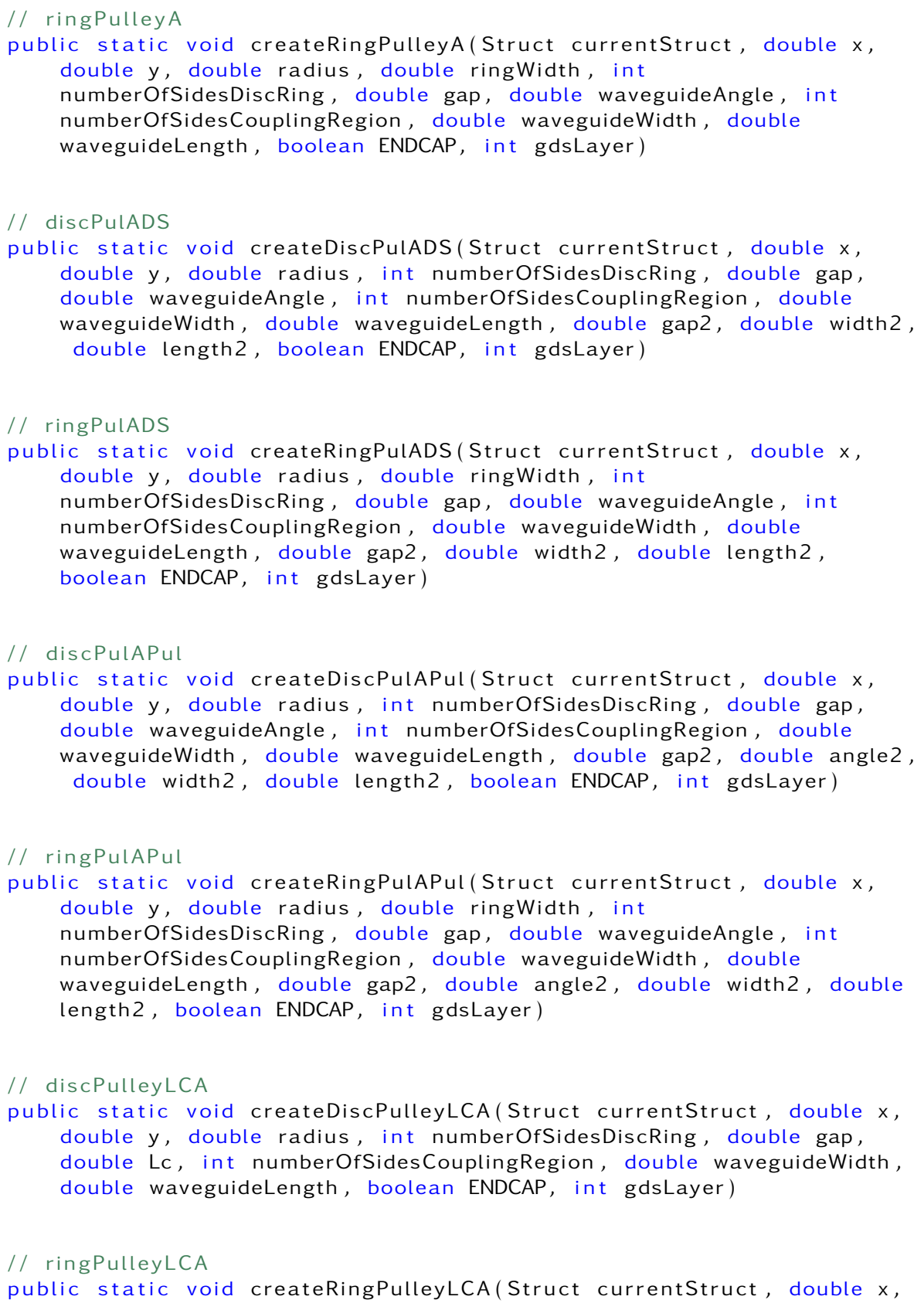


double $y$, double radius, double ringWidth, int numberOfSidesDiscRing, double gap, double Lc, int numberOfSidesCouplingRegion, double waveguideWidth, double waveguideLength, boolean ENDCAP, int gdsLayer)

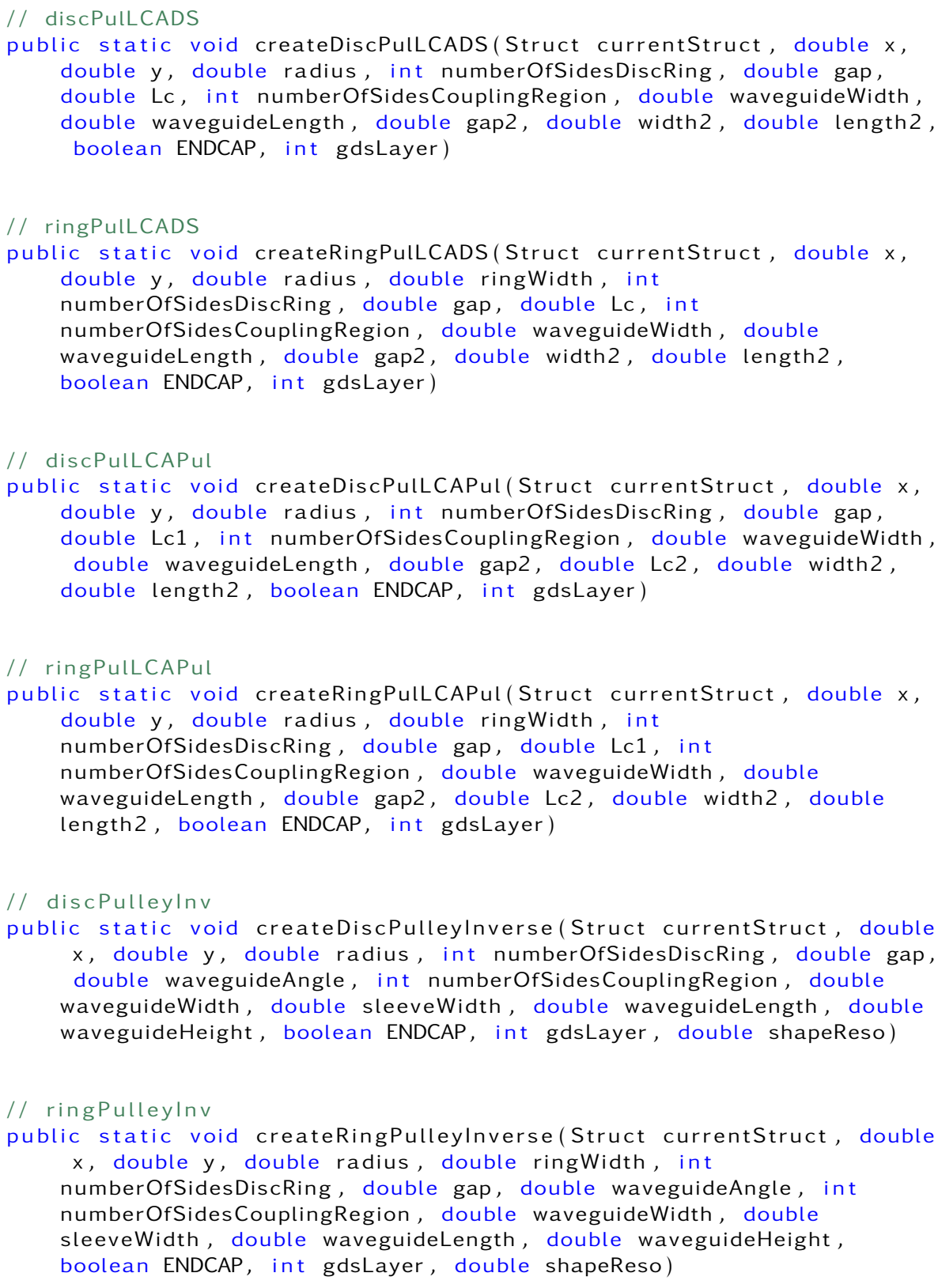

NIST • CNST Nanolithography Toolbox v2016.09.01 • http://www.nist.gov/cnst/ 


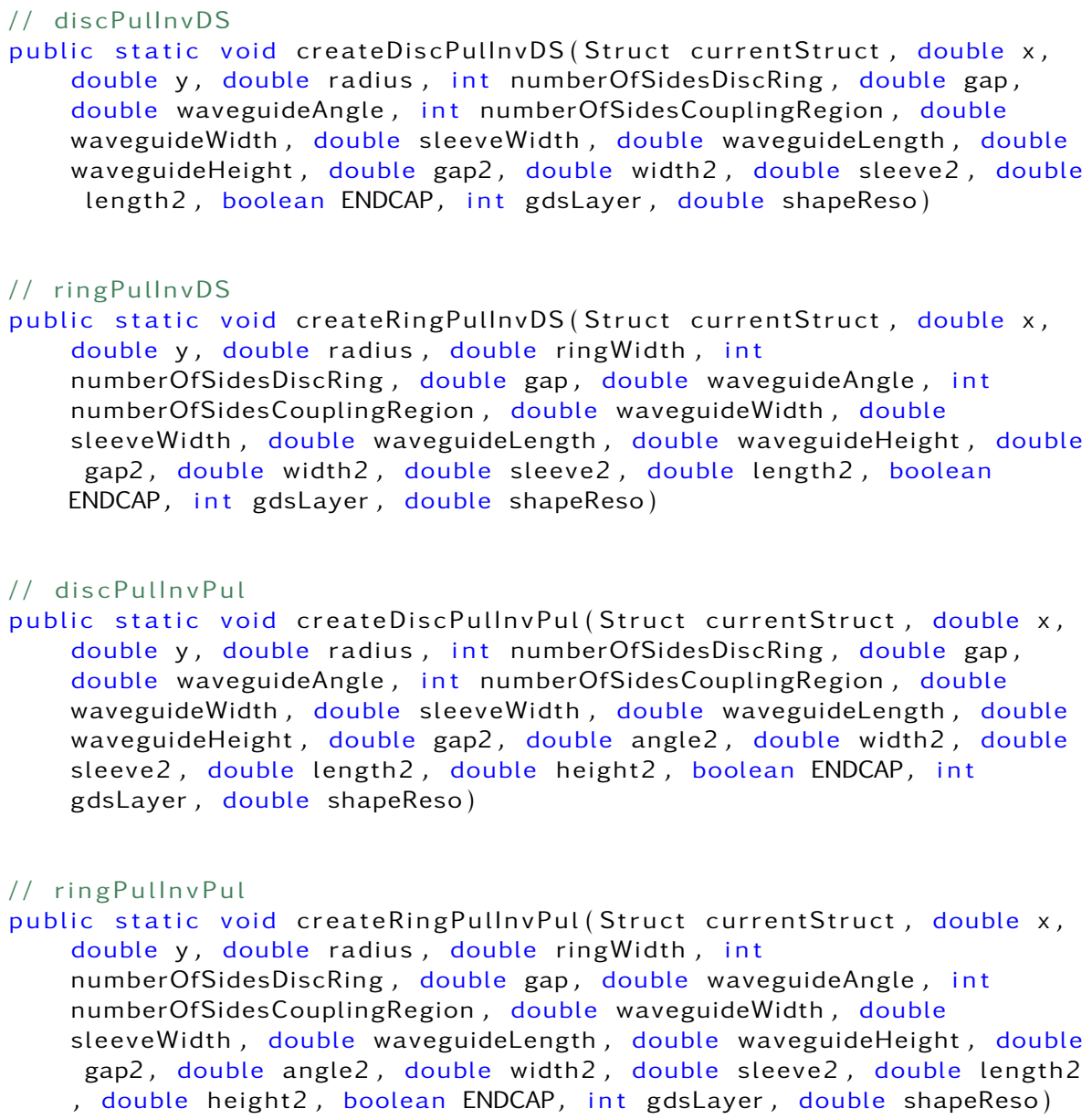


double $y$, double radius, int numberOfSidesDiscRing, double gap, double Lc, int numberOfSidesCouplingRegion, double waveguideWidth, double sleeveWidth, double waveguideLength, double waveguideHeight, double gap2, double width2, double sleeve2, double length2, boolean ENDCAP, int gdsLayer, double shapeReso)

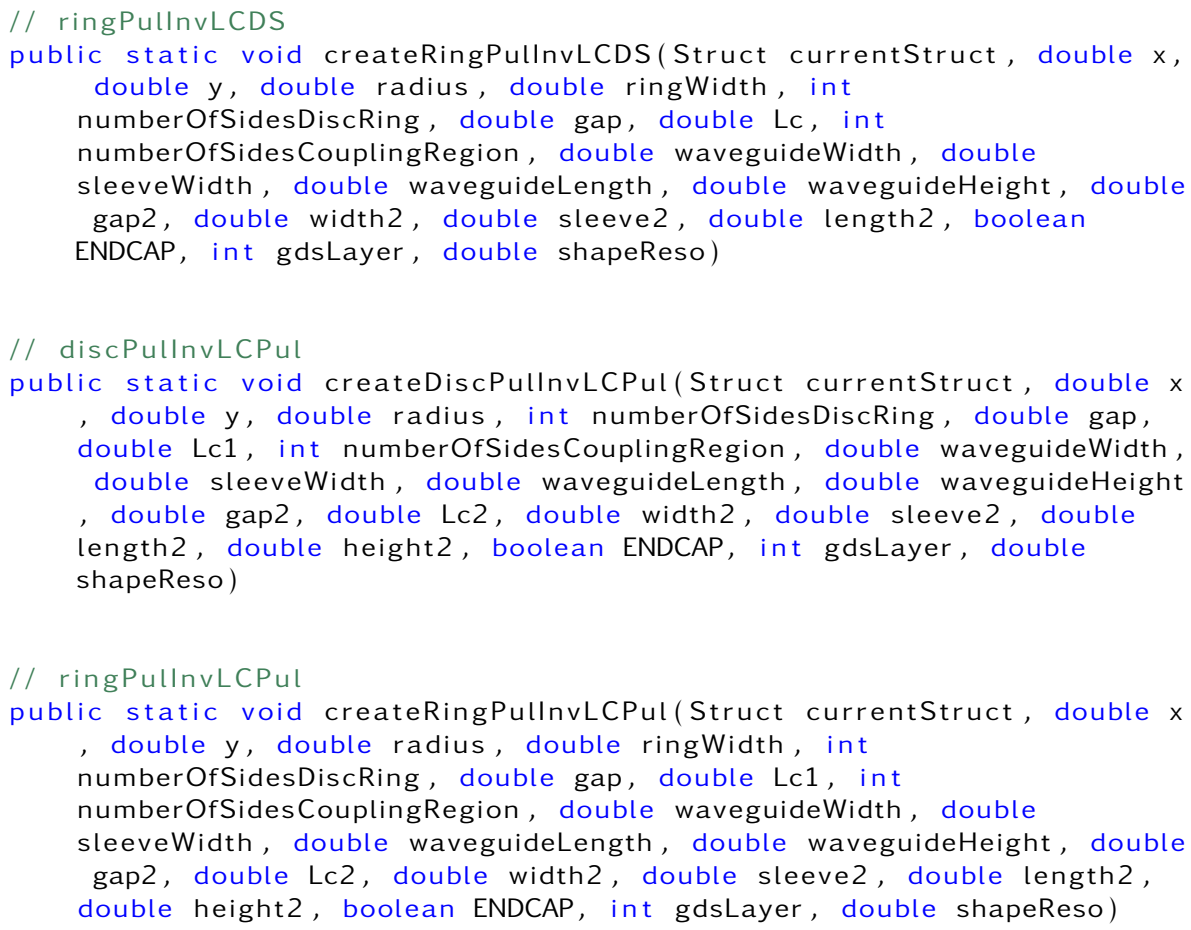


waveguideWidth, double sleeveWidth, double waveguideLength, double gap2, double width2, double sleeve2, double length2, boolean ENDCAP , int gdsLayer)

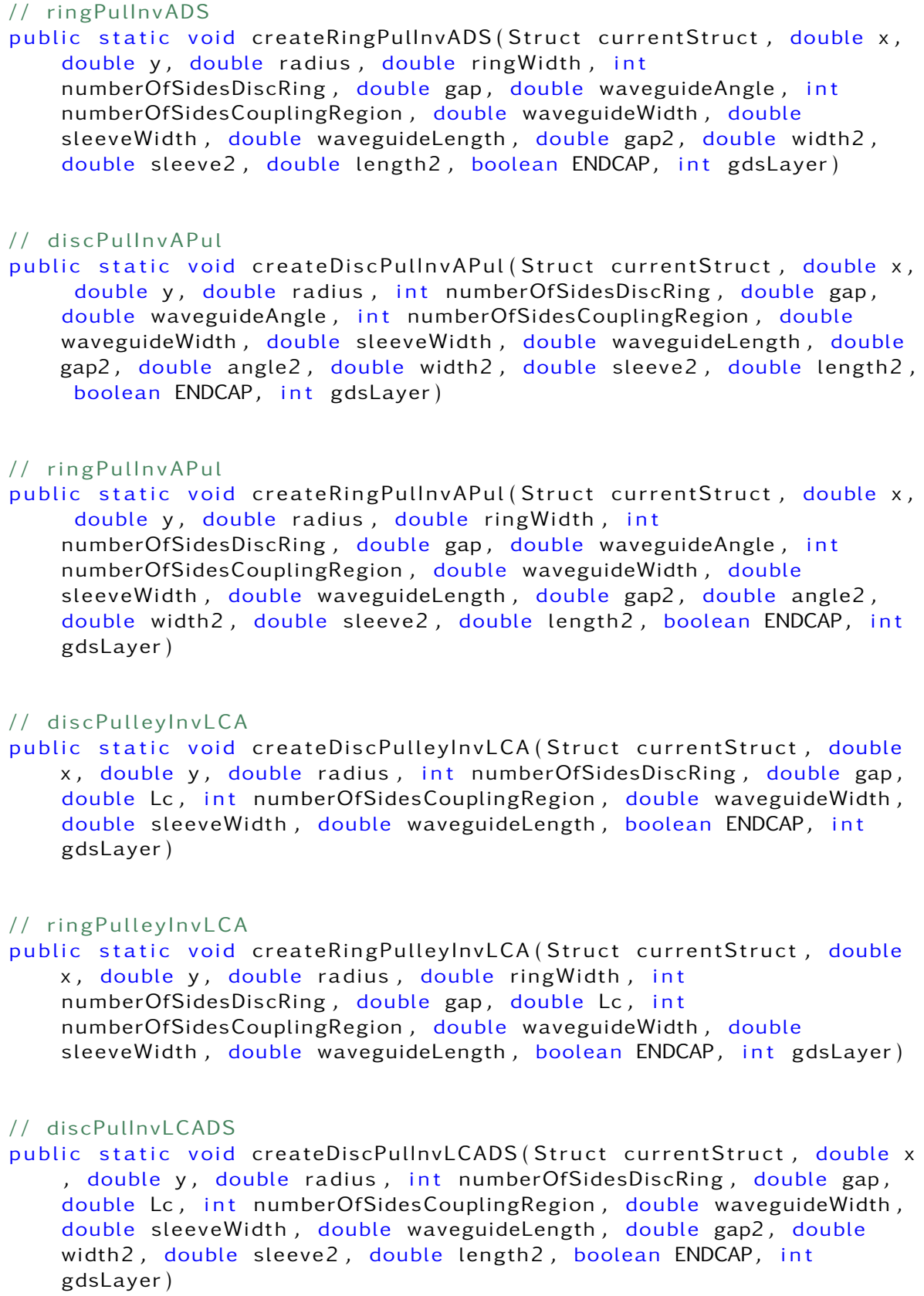




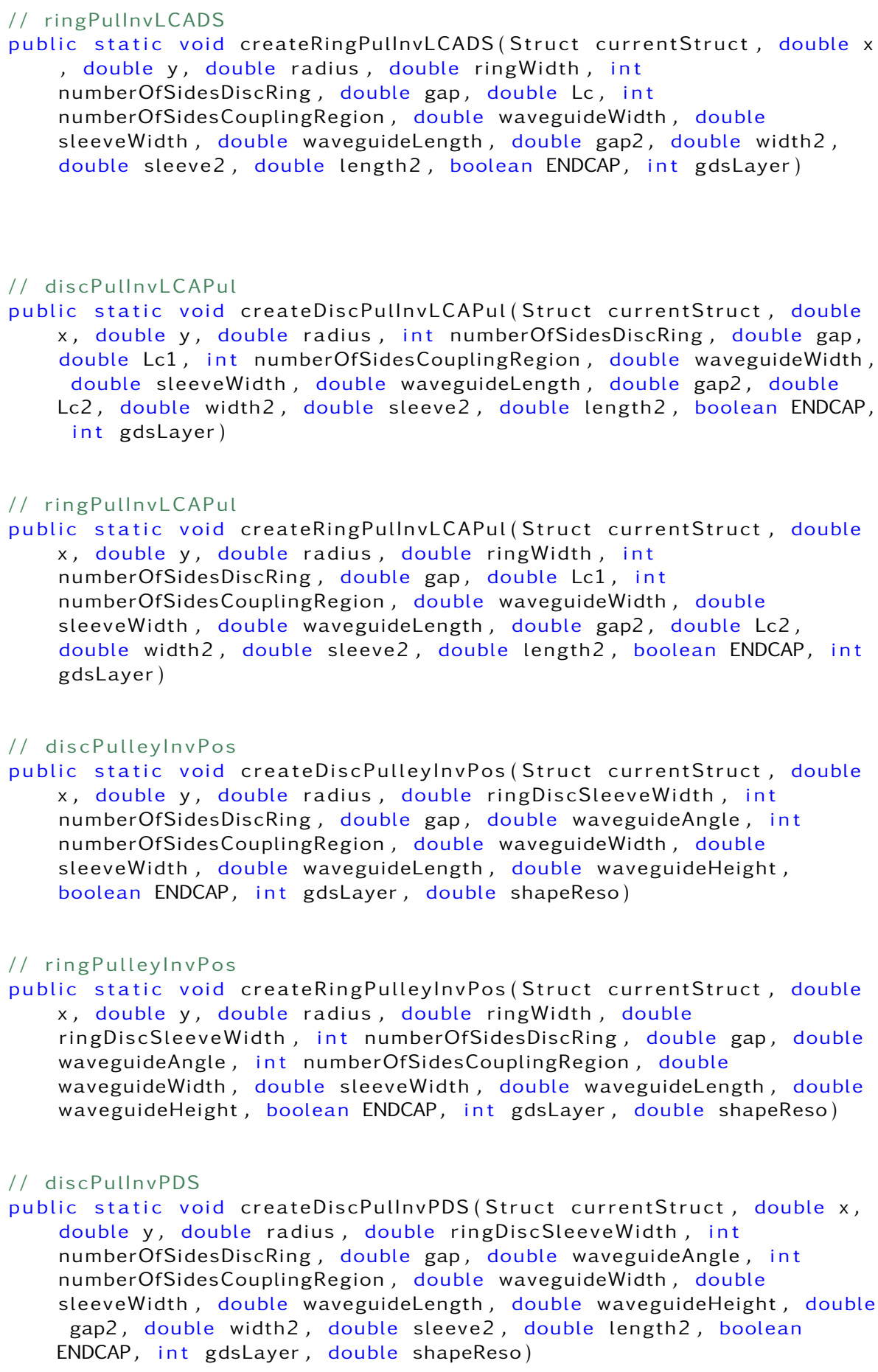




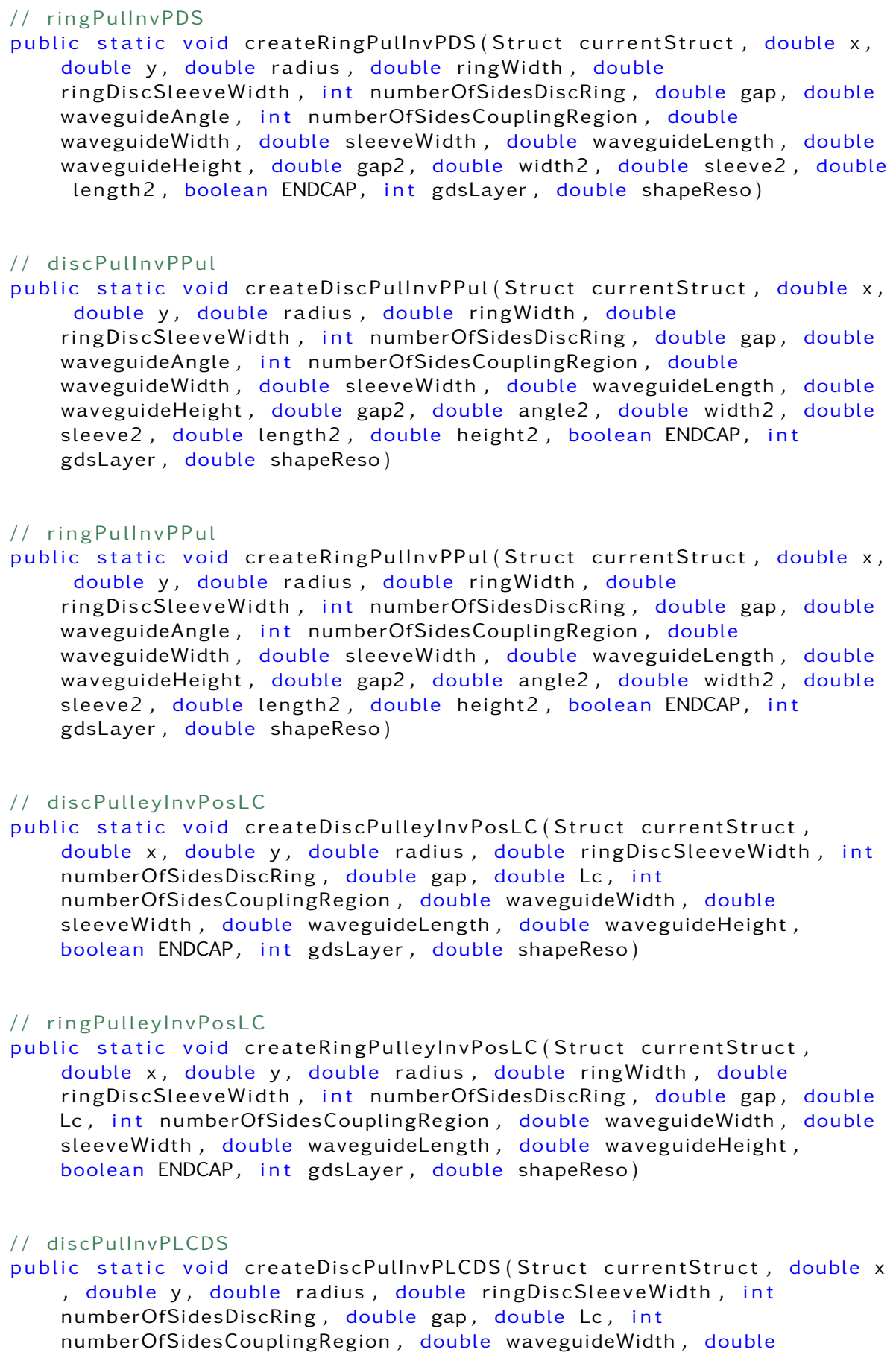

NIST • CNST Nanolithography Toolbox v2016.09.01 • http://www.nist.gov/cnst/ 
sleeveWidth, double waveguideLength, double waveguideHeight, double gap2, double width2, double sleeve2, double length2, boolean

ENDCAP, int gdsLayer, double shapeReso)

\section{// ringPullnvPLCDS}

public static void createRingPullnvPLCDS(Struct currentStruct, double $x$ , double y, double radius, double ringWidth, double

ringDiscSleeveWidth, int numberOfSidesDiscRing, double gap, double Lc, int numberOfSidesCouplingRegion, double waveguideWidth, double sleeveWidth, double waveguideLength, double waveguideHeight, double gap2, double width2, double sleeve2, double length2, boolean ENDCAP, int gdsLayer, double shapeReso)

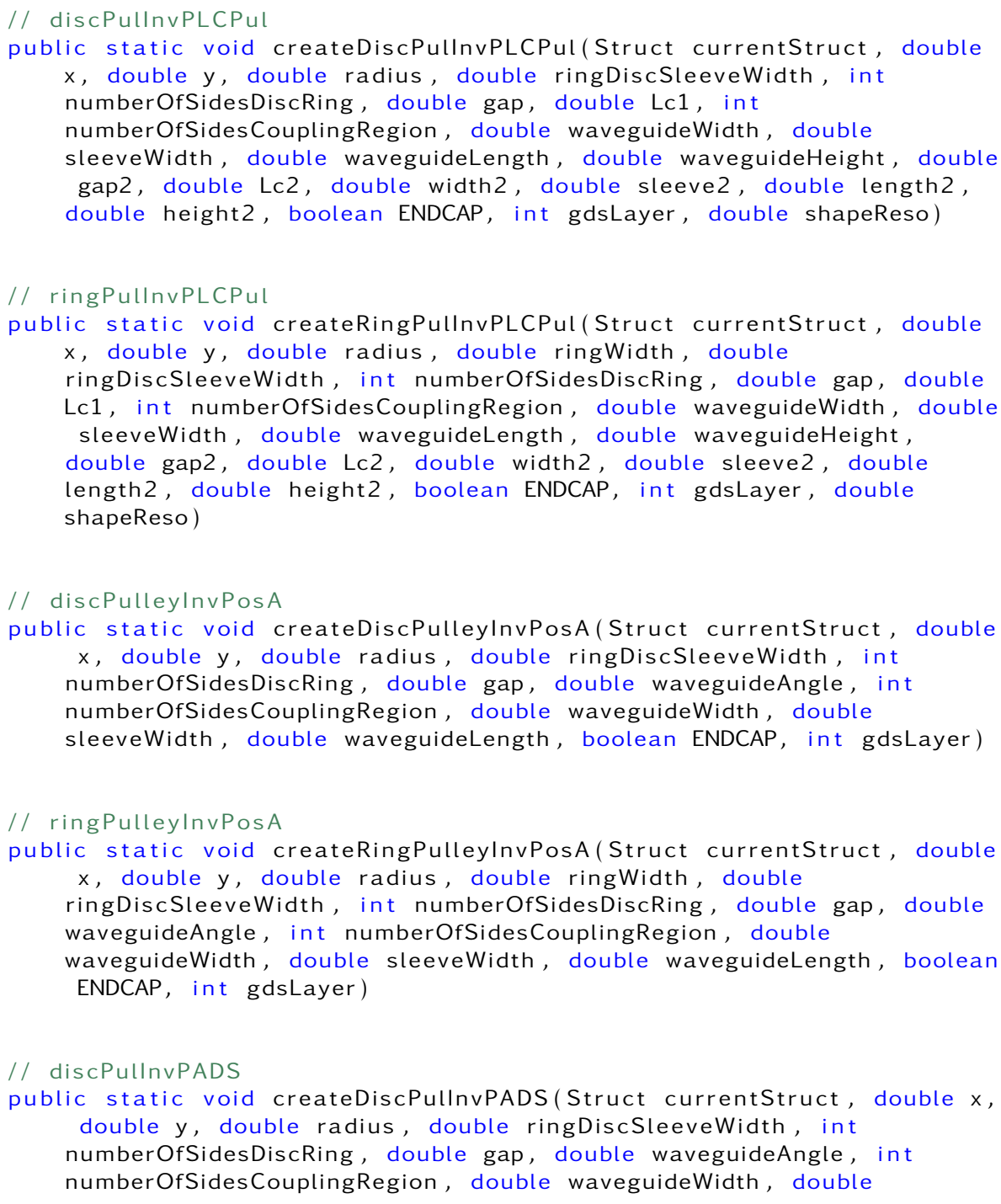


sleeveWidth, double waveguidelength, double gap2, double width2, double sleeve2, double length2, boolean ENDCAP, int gdsLayer)

// ringPullnvPADS

public static void createRingPullnvPADS(Struct currentStruct, double $x$, double y, double radius, double ringWidth, double

ringDiscSleeveWidth, int numberOfSidesDiscRing, double gap, double waveguideAngle, int numberOfSidesCouplingRegion, double

waveguideWidth, double sleeveWidth, double waveguideLength, double gap2, double width2, double sleeve2, double length2, boolean ENDCAP , int gdsLayer)

// discPullnvPAPul

public static void createDiscPullnvPAPul(Struct currentStruct, double $x$ , double y, double radius, double ringDiscSleeveWidth, int numberOfSidesDiscRing, double gap, double waveguideAngle, int numberOfSidesCouplingRegion, double waveguideWidth, double sleeveWidth, double waveguidelength, double gap2, double angle2, double width2, double sleeve2, double length2, boolean ENDCAP, int gdsLayer )

// ringPullnvPAPul

public static void createRingPullnvPAPul(Struct currentStruct, double $x$ , double $y$, double radius, double ringWidth, double

ringDiscSleeveWidth, int numberOfSidesDiscRing, double gap, double waveguideAngle, int numberOfSidesCouplingRegion, double waveguideWidth, double sleeveWidth, double waveguideLength, double gap2, double angle2, double width2, double sleeve2, double length2, boolean ENDCAP, int gdsLayer)

// discPulleylnvPosLCA

public static void createDiscPulleylnvPosLCA(Struct currentStruct, double $x$, double $y$, double radius, double ringDiscSleeveWidth, int numberOfSidesDiscRing, double gap, double Lc, int numberOfSidesCouplingRegion, double waveguideWidth, double sleeveWidth, double waveguideLength, boolean ENDCAP, int gdsLayer)

// ringPulleylnvPosLCA

public static void createRingPulleylnvPosLCA(Struct currentStruct, double $x$, double $y$, double radius, double ringWidth, double ringDiscSleeveWidth, int numberOfSidesDiscRing, double gap, double Lc, int numberOfSidesCouplingRegion, double waveguideWidth, double sleeveWidth, double waveguideLength, boolean ENDCAP, int gdsLayer)

// discPullnvPLCADS

public static void createDiscPullnvPLCADS(Struct currentStruct, double $x$, double $y$, double radius, double ringDiscSleeveWidth, int numberOfSidesDiscRing, double gap, double Lc, int numberOfSidesCouplingRegion, double waveguideWidth, double sleeveWidth, double waveguidelength, double gap2, double width2, double sleeve2, double length2, boolean ENDCAP, int gdsLayer) 


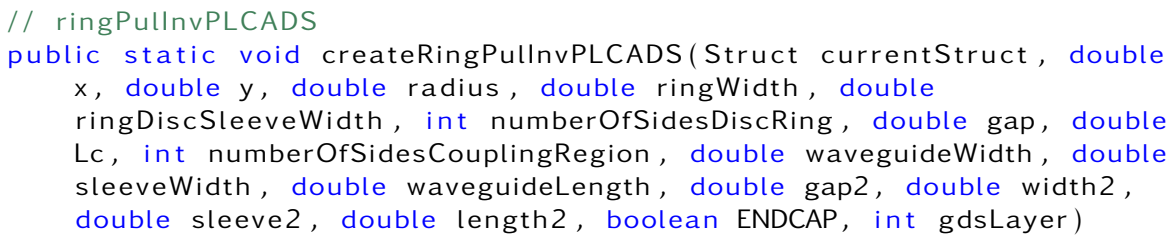




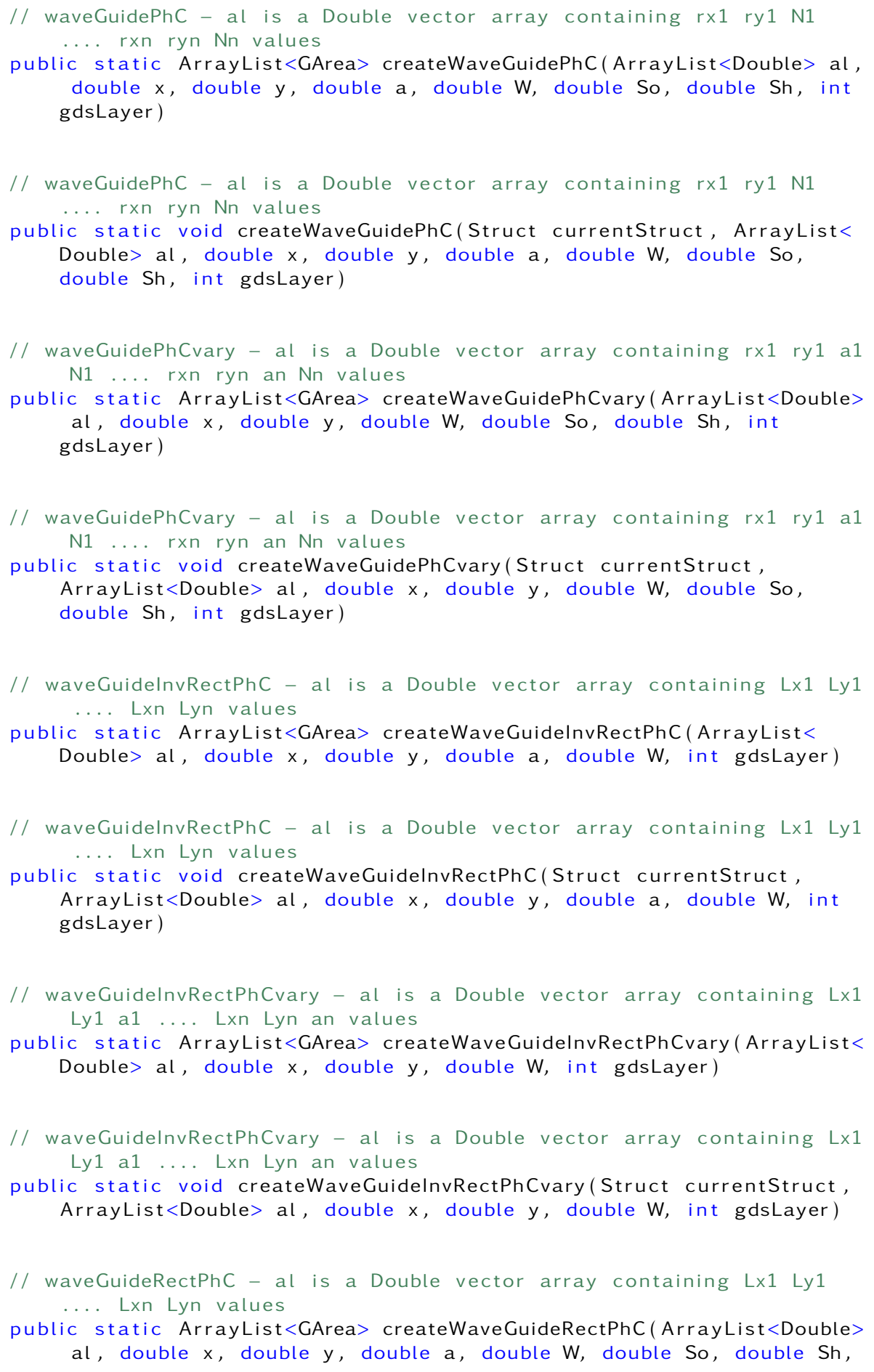




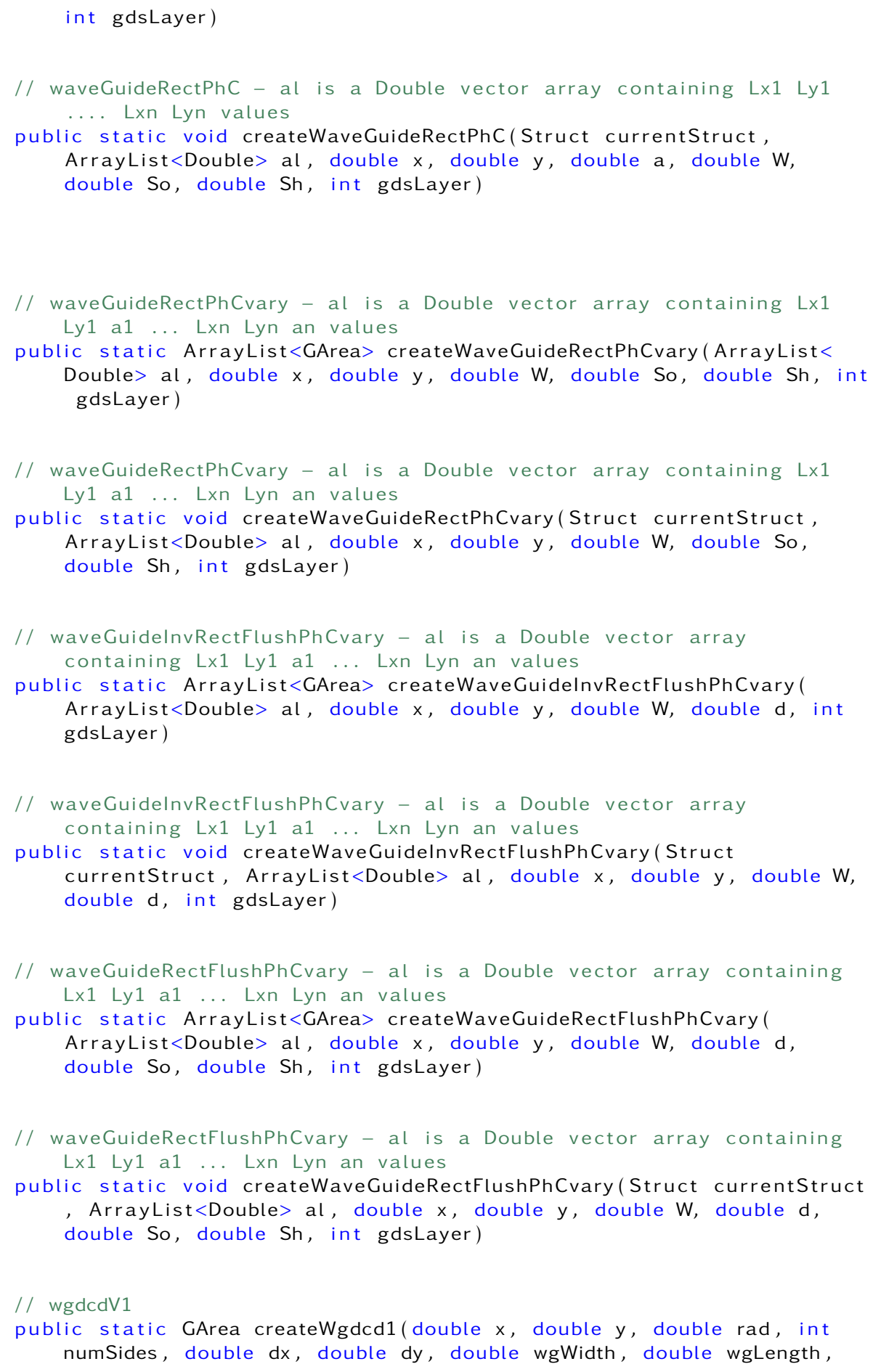


double wgRad, double sleeve, double gap, double ry, double dEllipse, double cutWidth, double cutPosition, double THETA, int gdsLayer)

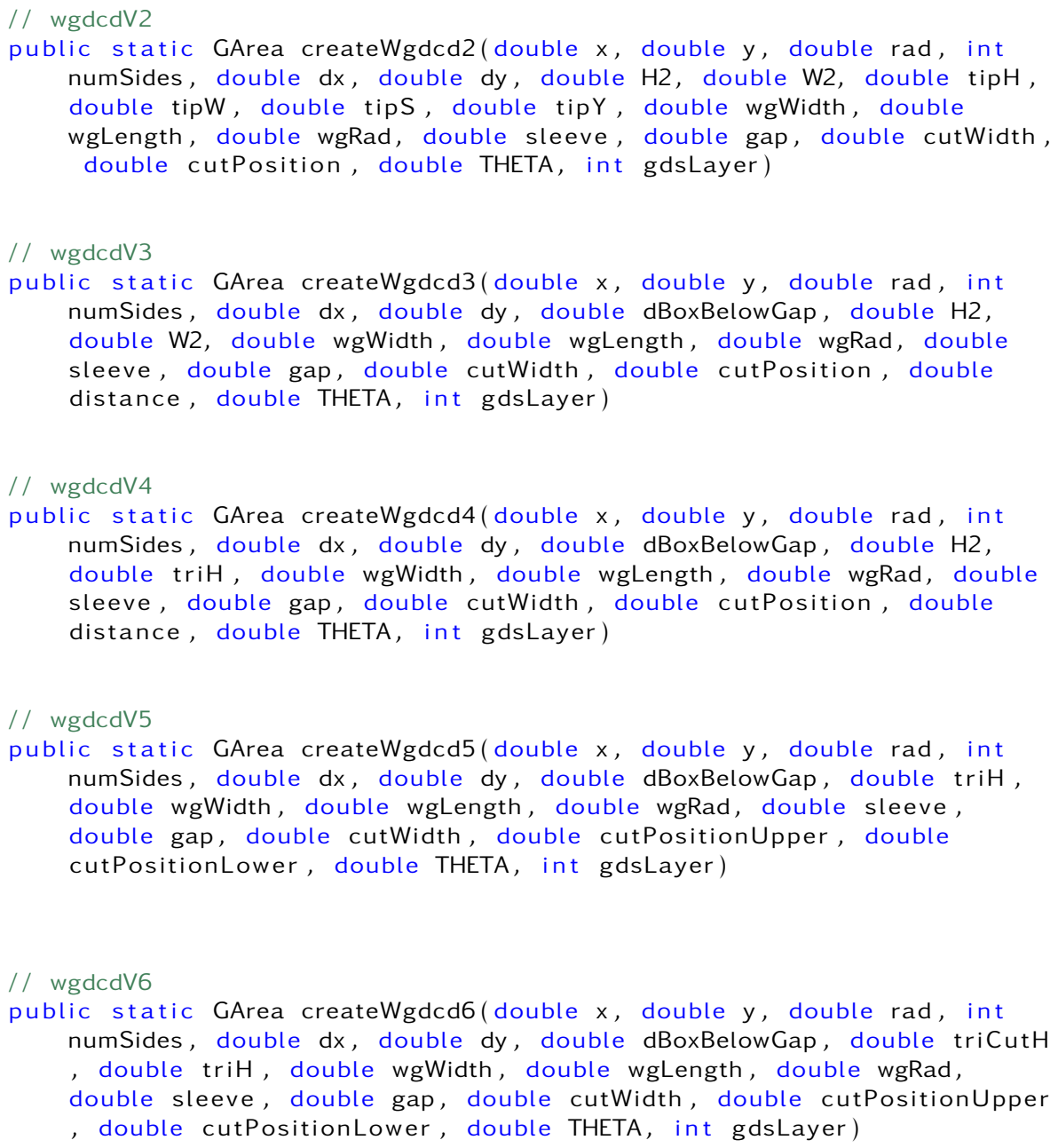

NIST • CNST Nanolithography Toolbox v2016.09.01 • http://www.nist.gov/cnst/

page 451 of 488 
cutWidth, double dCut, double largeBoxW, double largeBoxH, int L1, int L2, int L3, double THETA, int gdsLayer) 


\subsubsection{MEMS NEMS Library Methods}

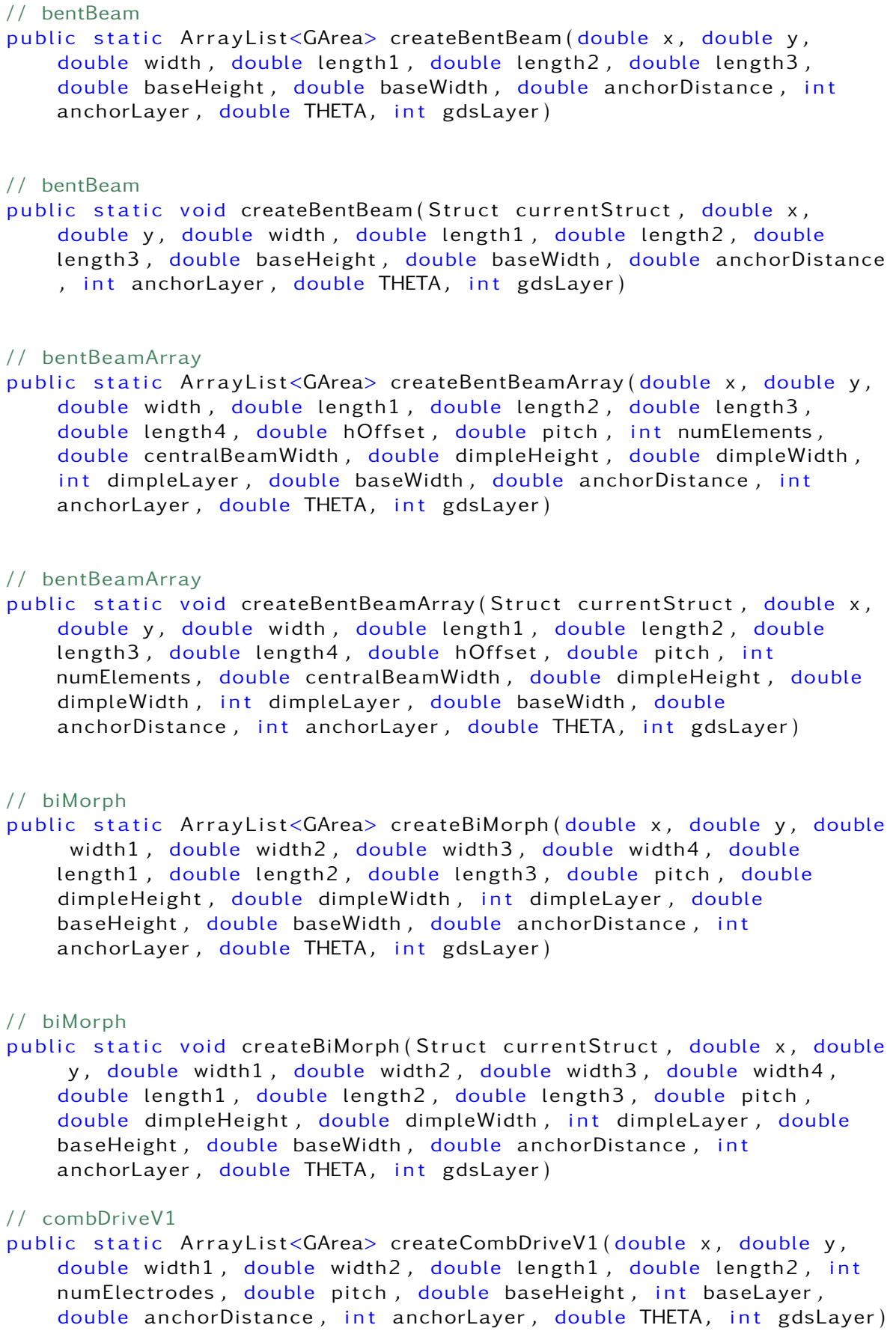




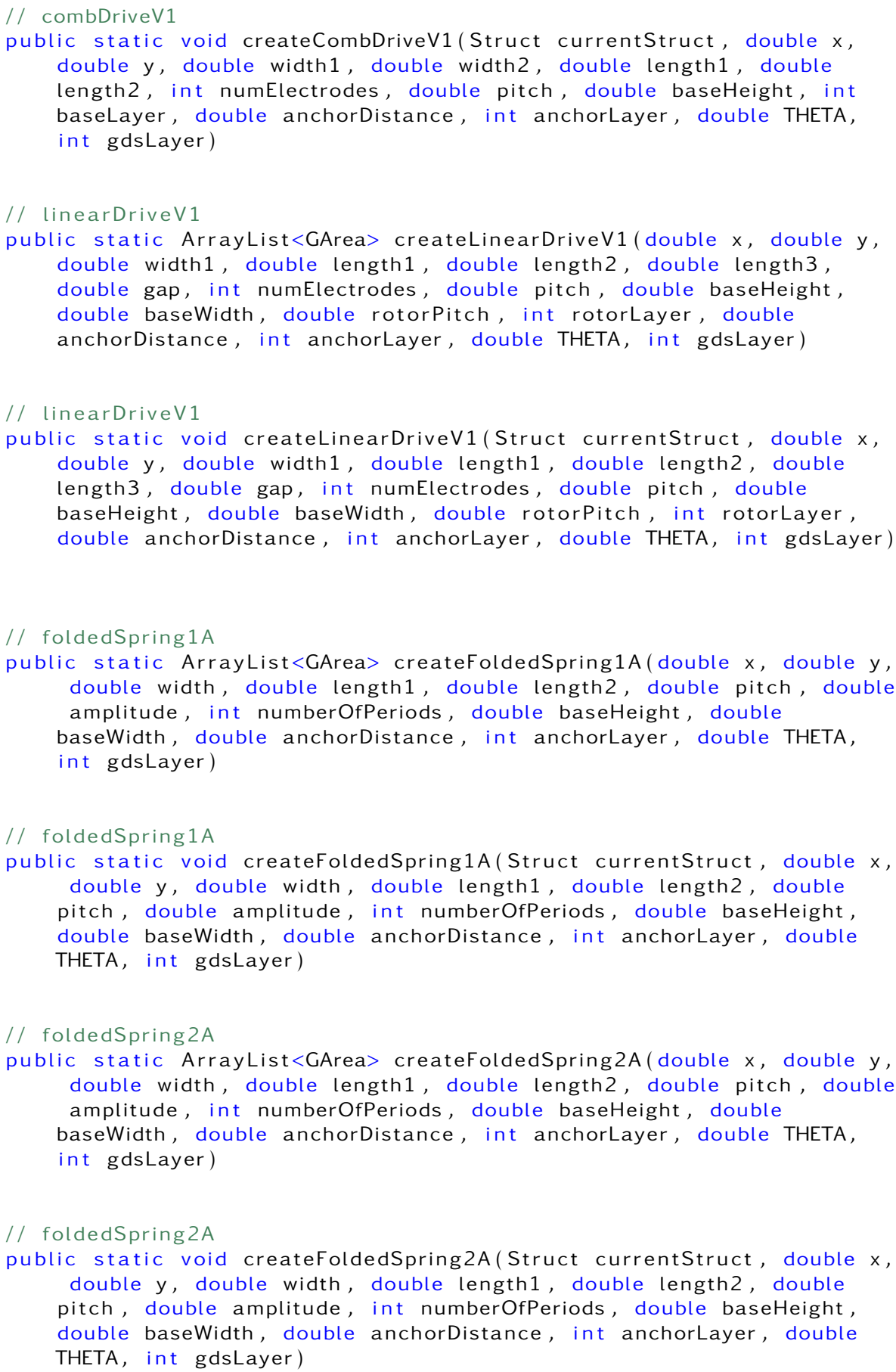

NIST • CNST Nanolithography Toolbox v2016.09.01 • http://www.nist.gov/cnst/ 


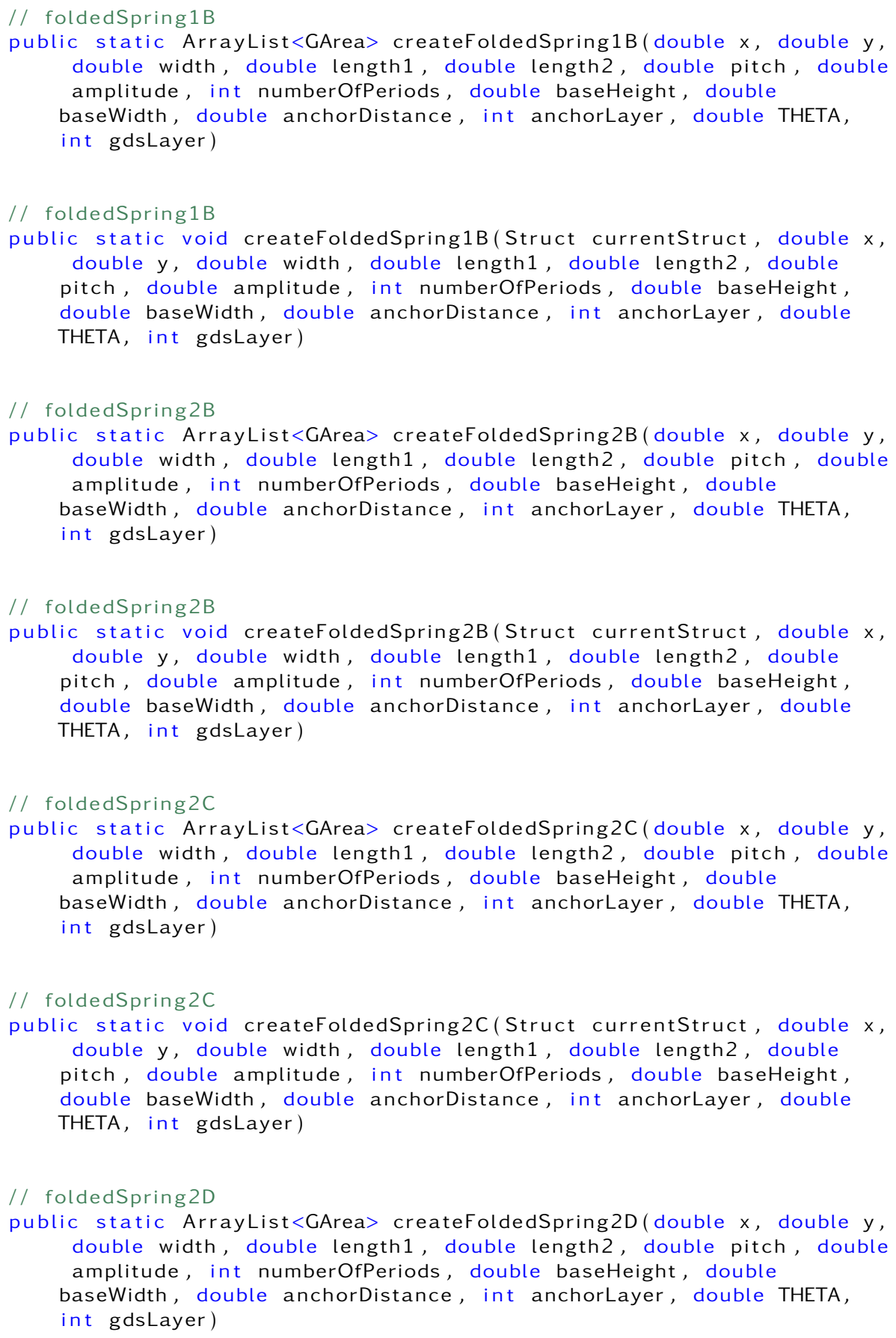

NIST • CNST Nanolithography Toolbox v2016.09.01・http://www.nist.gov/cnst/ 
// foldedSpring2D

public static void createFoldedSpring2D(Struct currentStruct, double $x$, double $y$, double width, double length1, double length2, double pitch, double amplitude, int numberOfPeriods, double baseHeight, double baseWidth, double anchorDistance, int anchorLayer, double THETA, int gdsLayer)

// foldedSpring2E

public static ArrayList<GArea> createFoldedSpring2E(double $x$, double $y$, double width, double length1, double length2, double pitch, double amplitude, int numberOfPeriods, int numSides, double baseHeight, double baseWidth, double anchorDistance, int anchorLayer, double THETA, int gdsLayer)

// foldedSpring2E

public static void createfoldedSpring2E(Struct currentStruct, double $x$, double y, double width, double length1, double length2, double pitch, double amplitude, int numberOfPeriods, int numSides, double baseHeight, double baseWidth, double anchorDistance, int anchorLayer, double THETA, int gdsLayer)

// foldedSpring2F

public static ArrayList<GArea> createFoldedSpring $2 \mathrm{~F}$ (double $\mathrm{x}$, double $\mathrm{y}$, double width, double length1, double length2, double pitch, double amplitude, int numberOfPeriods, int numSides, double baseHeight, double baseWidth, double anchorDistance, int anchorLayer, double THETA, int gdsLayer)

// foldedSpring2F

public static void createFoldedSpring $2 F$ (Struct currentStruct, double $x$, double $y$, double width, double length1, double length2, double pitch, double amplitude, int numberOfPeriods, int numSides, double baseHeight, double baseWidth, double anchorDistance, int anchorlayer, double THETA, int gdsLayer)

// foldedSpring2G

public static ArrayList<GArea> createFoldedSpring2G(double $x$, double $y$, double width, double length1, double length2, double pitch, double amplitude, int numberOfPeriods, int numSides, double baseHeight, double baseWidth, double anchorDistance, int anchorLayer, double THETA, int gdsLayer)

// foldedSpring2G

public static void createFoldedSpring2G(Struct currentStruct, double $x$, double $y$, double width, double length1, double length2, double pitch, double amplitude, int numberOfPeriods, int numSides, double baseHeight, double baseWidth, double anchorDistance, int anchorLayer, double THETA, int gdsLayer)

NIST • CNST Nanolithography Toolbox v2016.09.01 • http://www.nist.gov/cnst/ page 456 of 488 


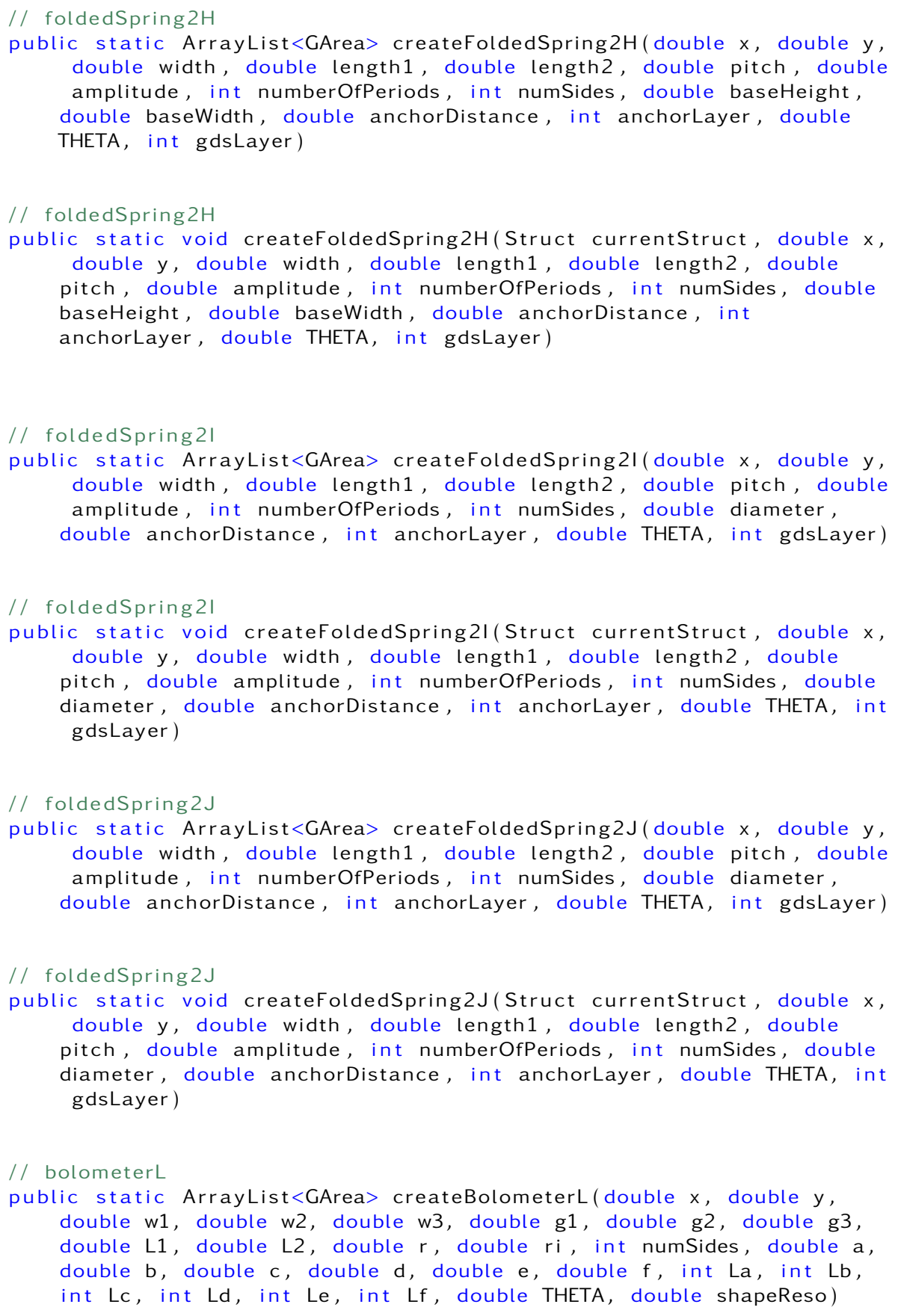




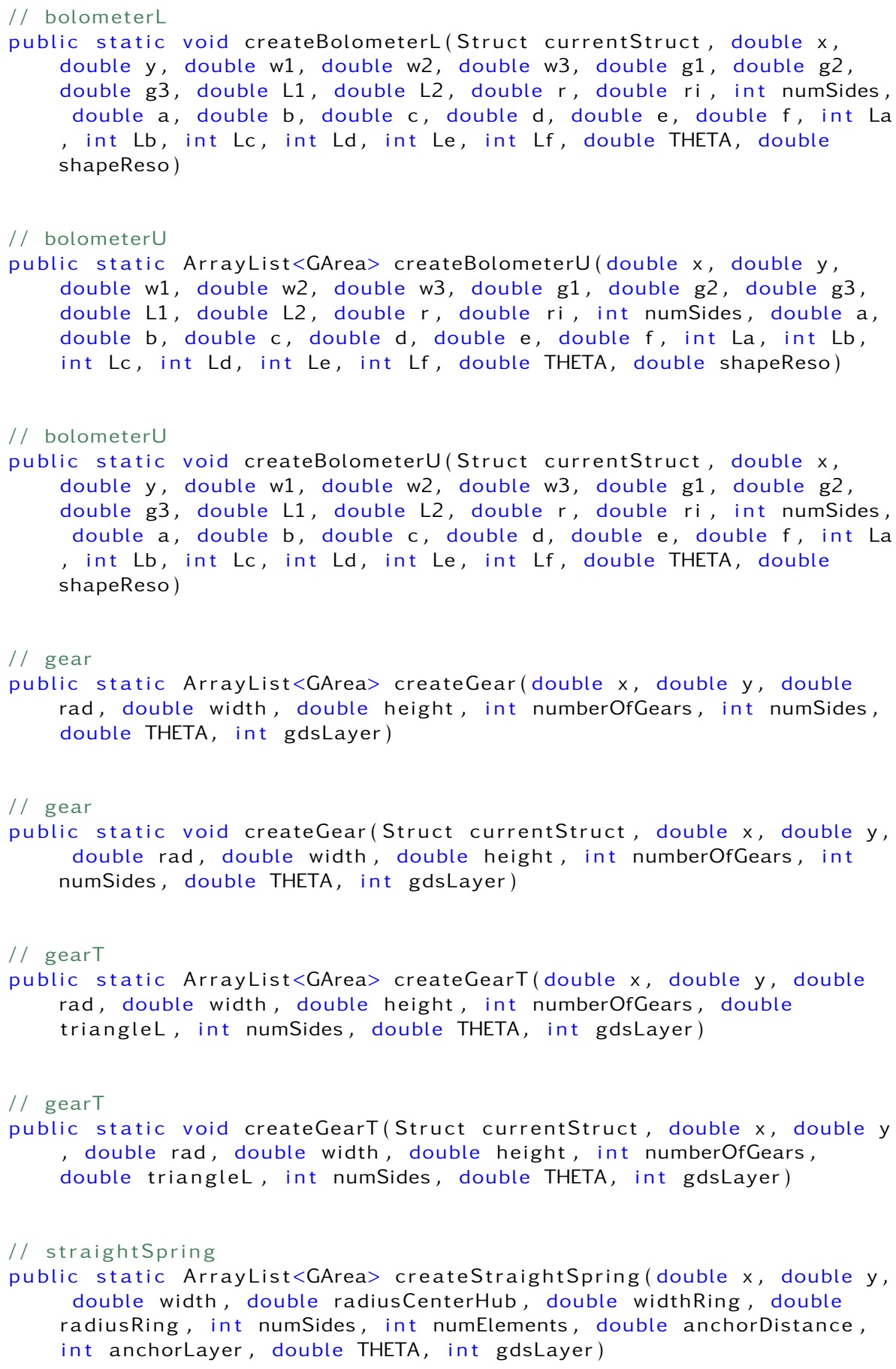

NIST • CNST Nanolithography Toolbox v2016.09.01・http://www.nist.gov/cnst/ 


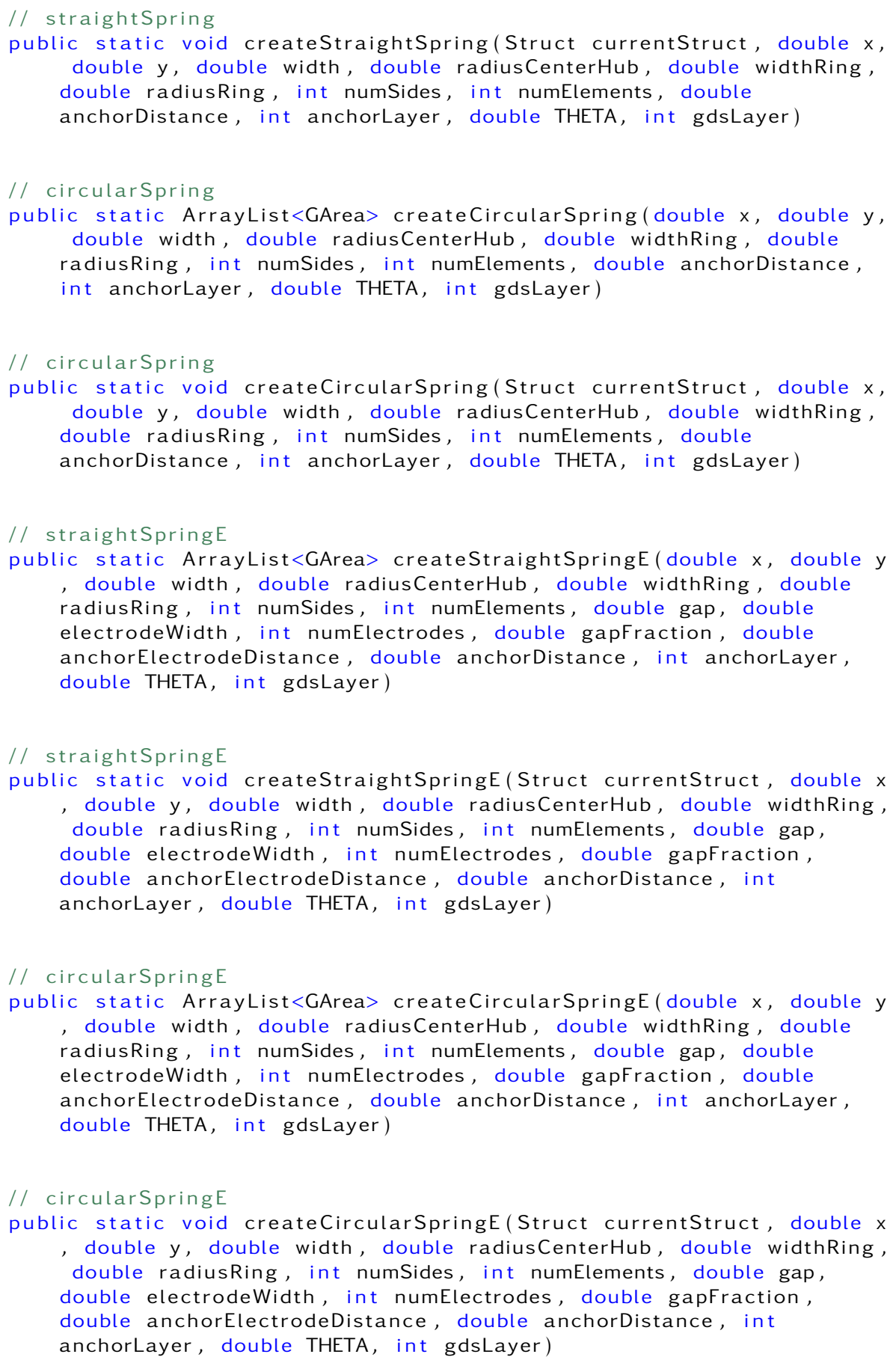

NIST • CNST Nanolithography Toolbox v2016.09.01・http://www.nist.gov/cnst/ 
// combRadialv1

public static ArrayList $<$ GArea $>$ createCombRadialV1 (double $x$, double $y$, double w1, double $r 1$, double w2, double $r 2$, double wc, double gap, int numElements, int numSides, double thetaComb, double thetaOverlap, double anchorDistance, int anchorlayer, double THETA, int gdsLayer)

// combRadialv1

public static void createCombRadialV1(Struct currentStruct, double $x$, double $y$, double $w 1$, double $r 1$, double $w 2$, double $r 2$, double wc, double gap, int numElements, int numSides, double thetaComb, double thetaOverlap, double anchorDistance, int anchorlayer, double THETA , int gdsLayer)

// combRadialv2

public static Arraylist<GArea $>$ createCombRadialV2(double $x$, double $y$, double w1, double r1, double w2, double r2, double wc, double gap, int numElements, int numSides, double thetaComb, double thetaOverlap, double anchorDistance, int anchorLayer, double THETA, int gdsLayer)

// combRadialv2

public static void createCombRadialv2(Struct currentStruct, double $x$, double y, double w1, double r1, double w2, double r2, double wc, double gap, int numElements, int numSides, double thetaComb, double thetaOverlap, double anchorDistance, int anchorLayer, double THETA , int gdsLayer)

\section{// flexure2A}

public static ArrayList $<$ GArea $>$ createFlexure2A(double $x$, double $y$, double width, double length1, double length2, double widthMass, double lengthMass, double baseHeight, double baseWidth, double anchorDistance, int anchorLayer, double THETA, int gdsLayer)

\section{// flexure2A}

public static void createflexure2A(Struct currentStruct, double $x$, double $y$, double width, double length1, double length2, double widthMass, double lengthMass, double baseHeight, double baseWidth, double anchorDistance, int anchorLayer, double THETA, int gdsLayer)

\section{// flexure2B}

public static ArrayList $<$ GArea $>$ createFlexure2B(double $x$, double $y$, double width, double length1, double length2, double widthMass, double lengthMass, double connectorHeight, double connectorWidth, double squareHeight, double squareWidth, double baseHeight, double baseWidth, double anchorDistance, int anchorLayer, double THETA, int gdsLayer) 


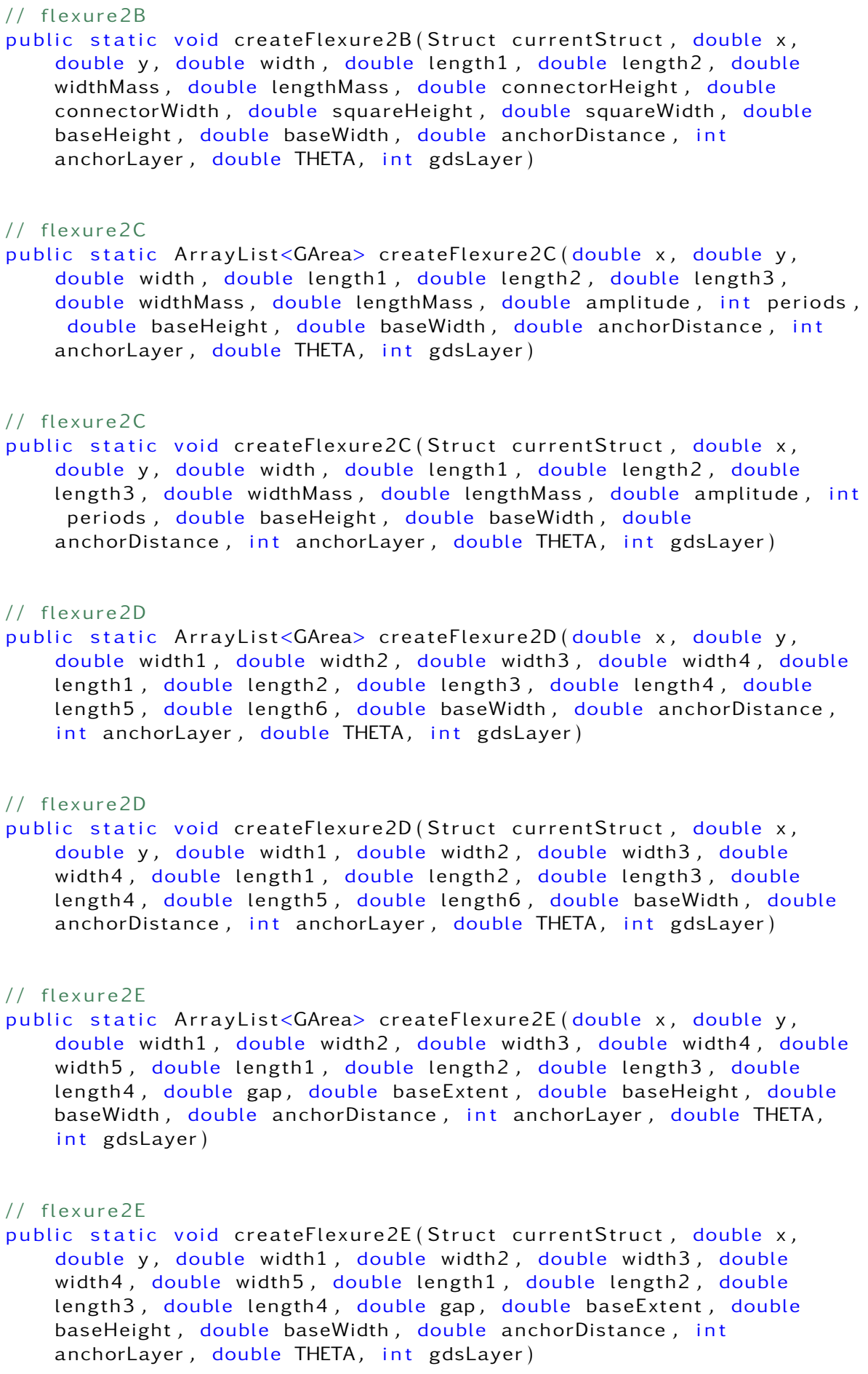

NIST • CNST Nanolithography Toolbox v2016.09.01 • http://www.nist.gov/cnst/ 


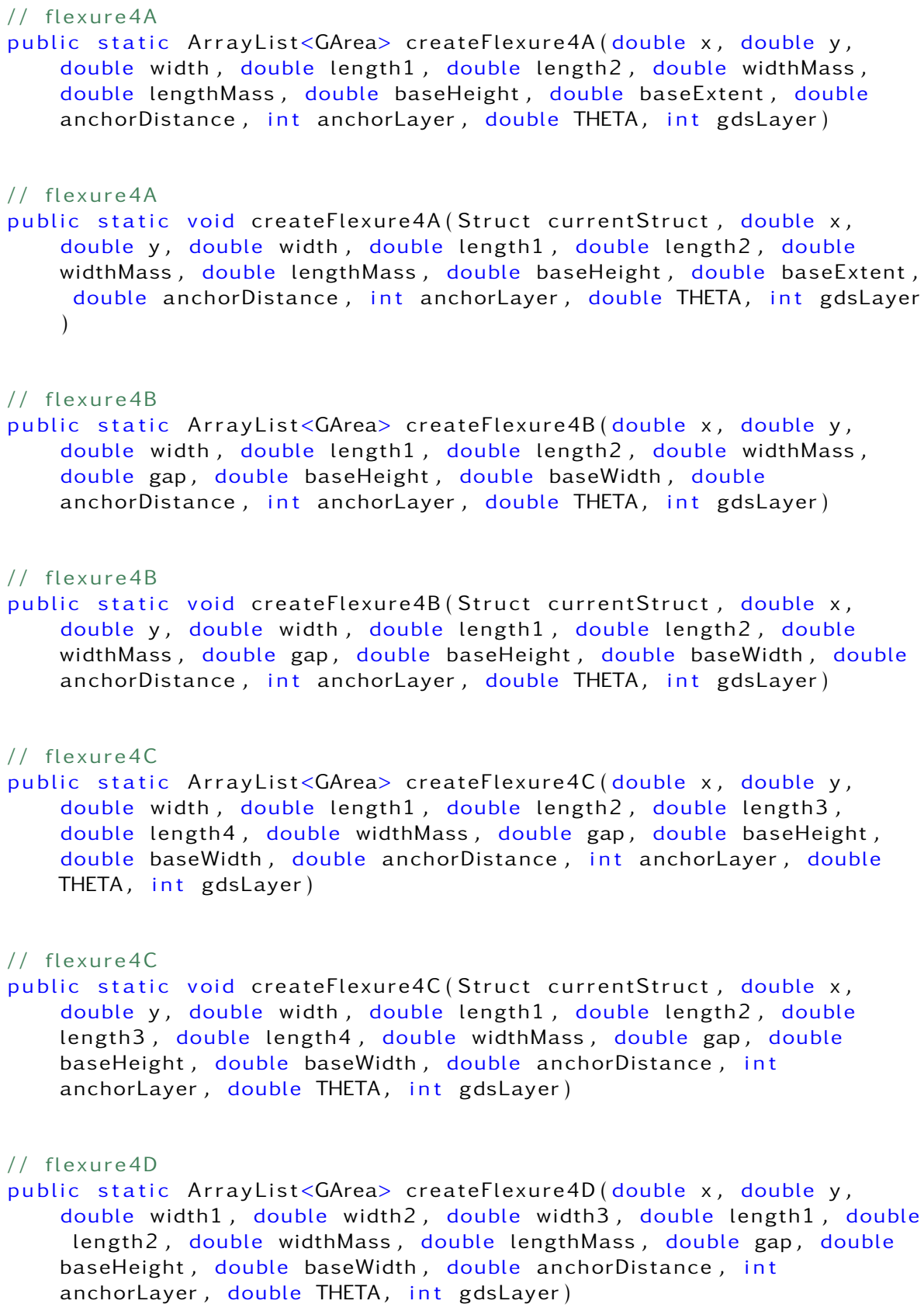




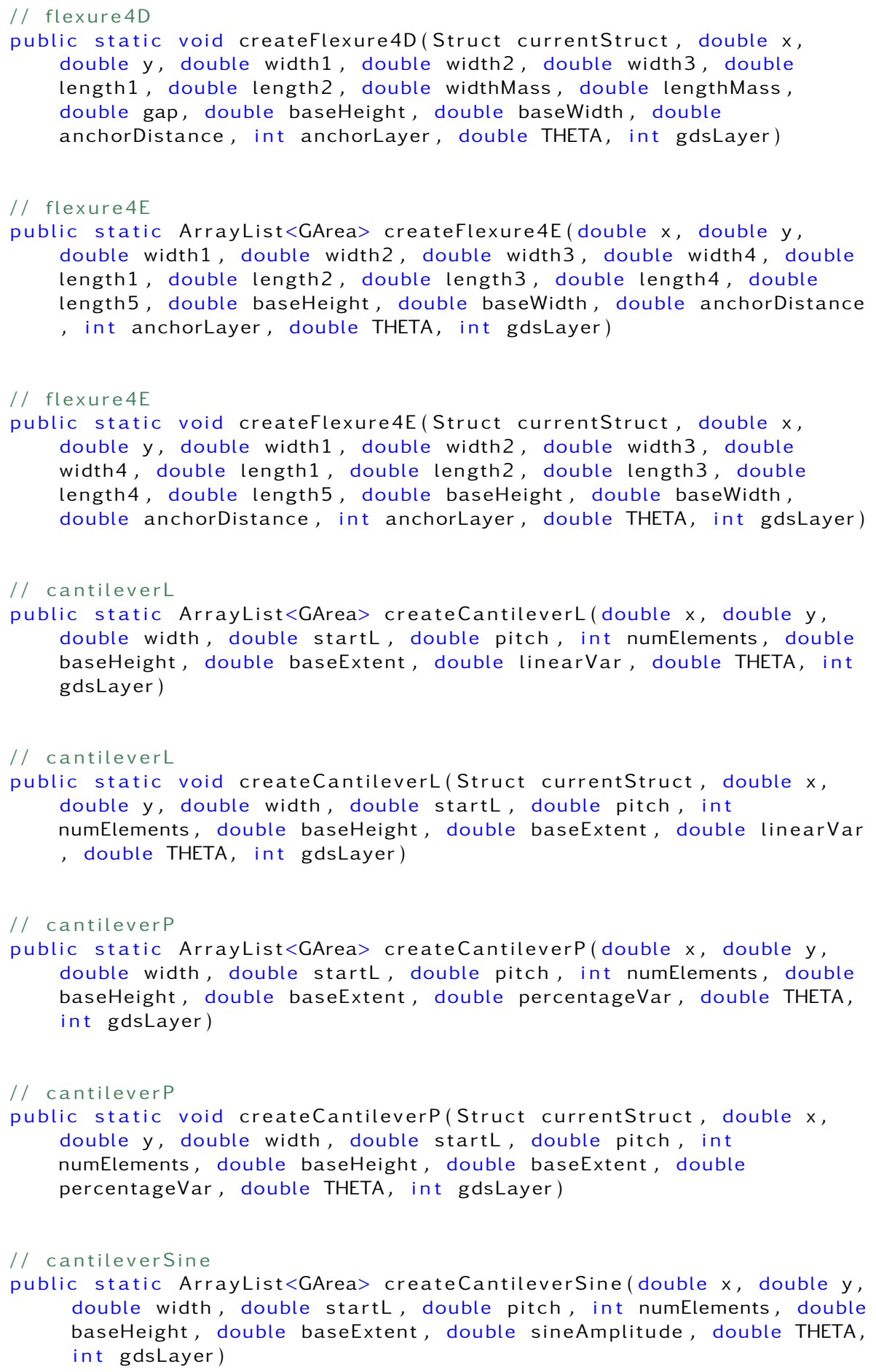

NIST • CNST Nanolithography Toolbox v2016.09.01・http://www.nist.gov/cnst/

page 463 of 488 


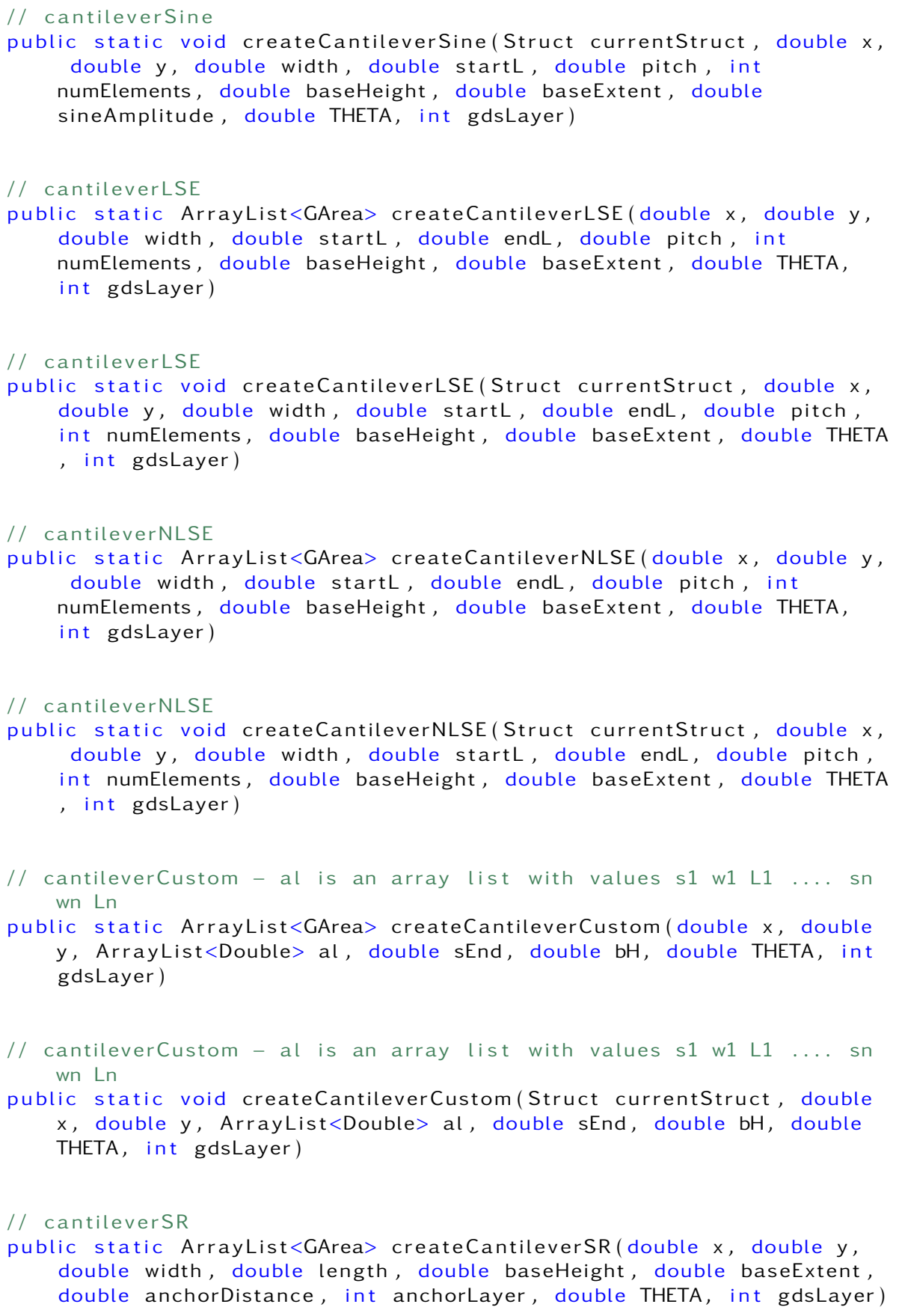




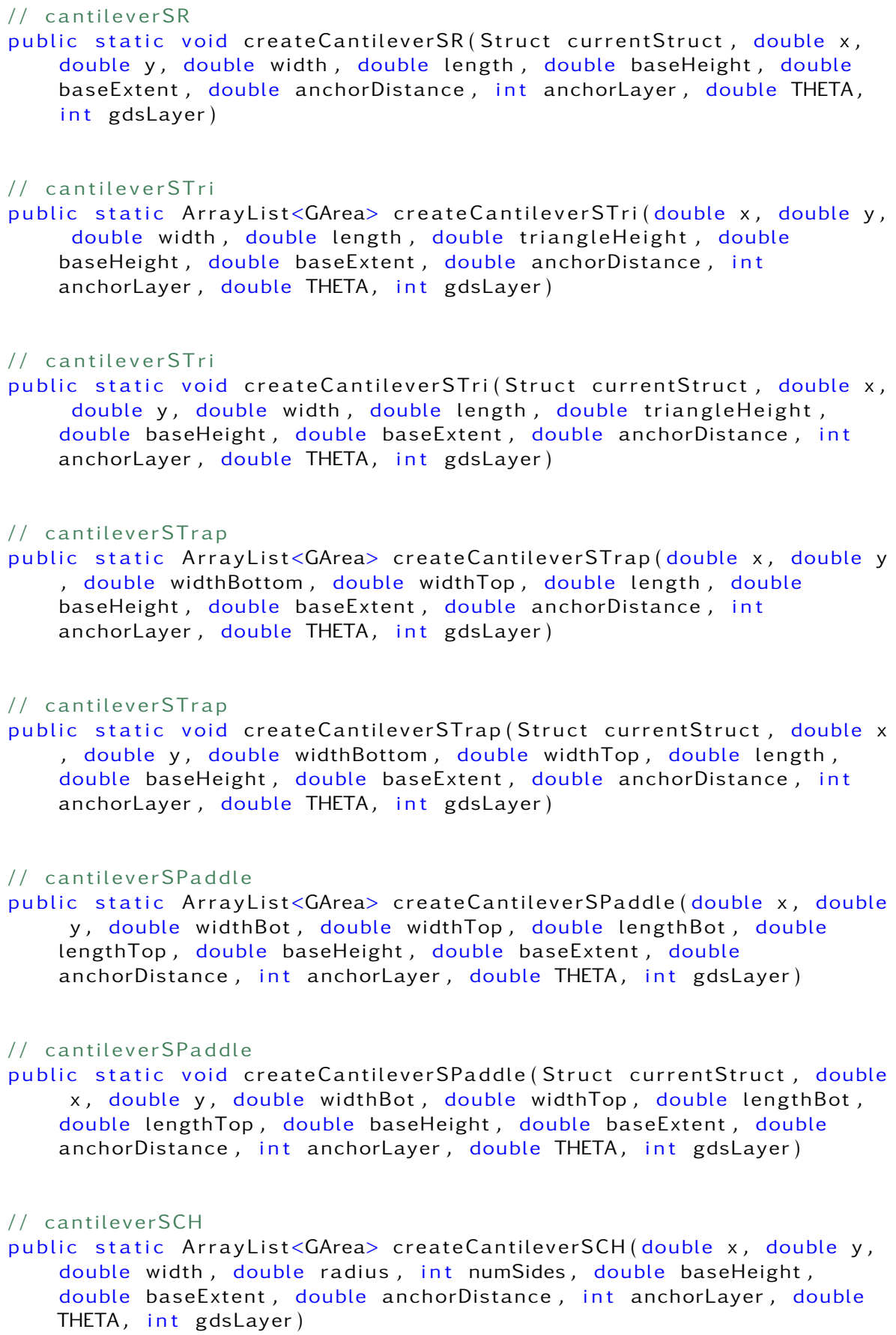




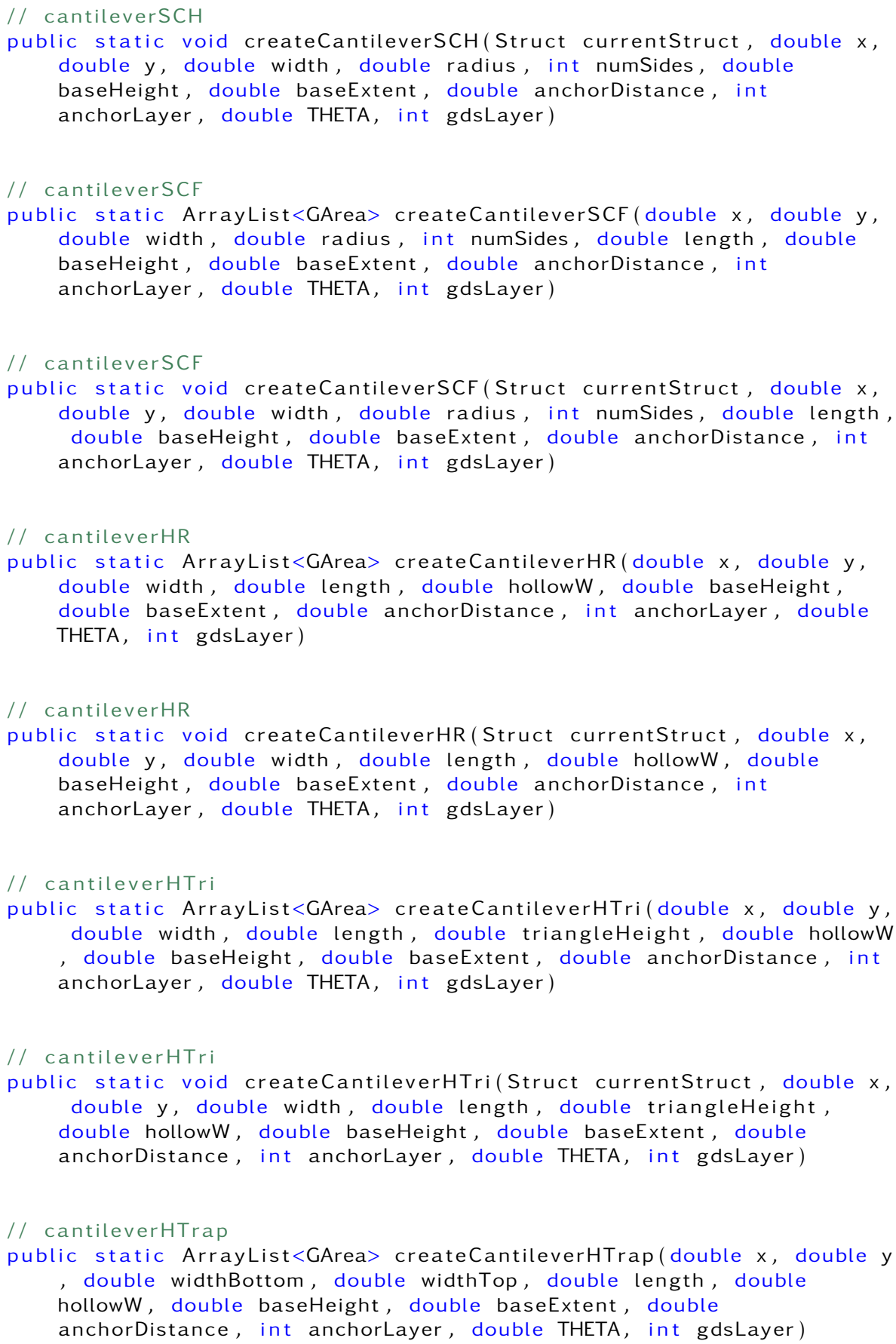

NIST • CNST Nanolithography Toolbox v2016.09.01・http://www.nist.gov/cnst/ 


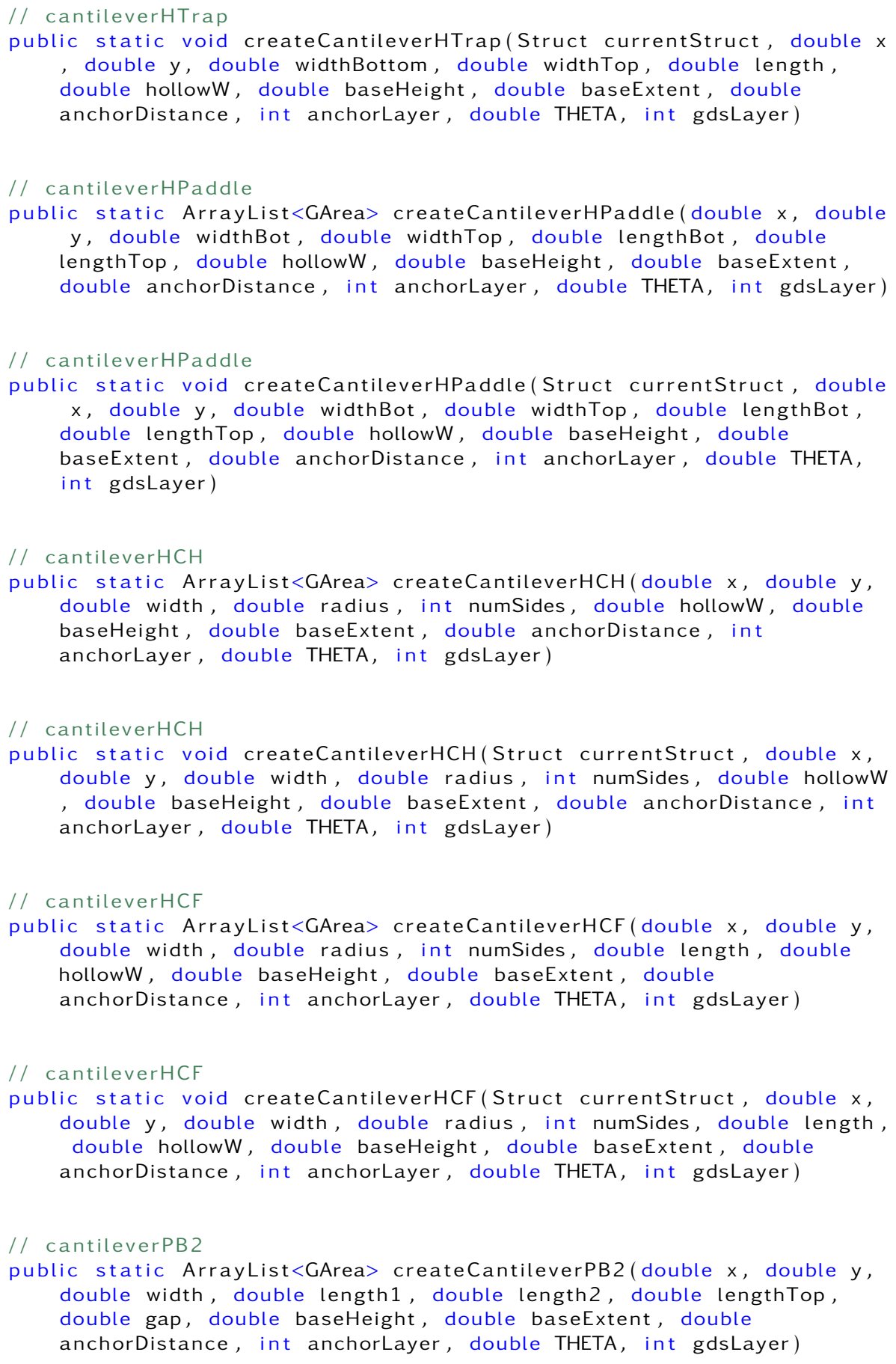

NIST • CNST Nanolithography Toolbox v2016.09.01 • http://www.nist.gov/cnst/ 


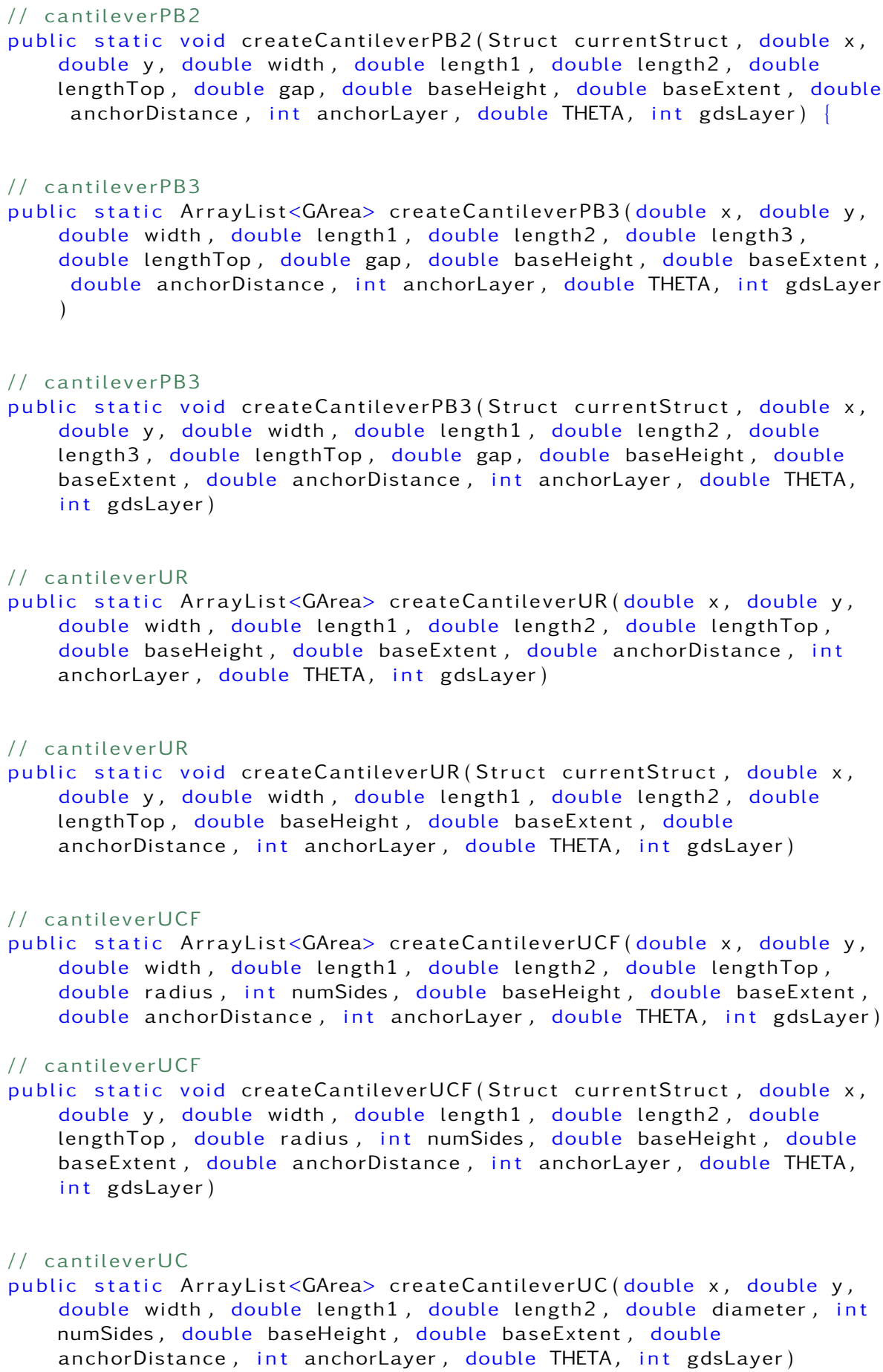

NIST • CNST Nanolithography Toolbox v2016.09.01・http://www.nist.gov/cnst/ 


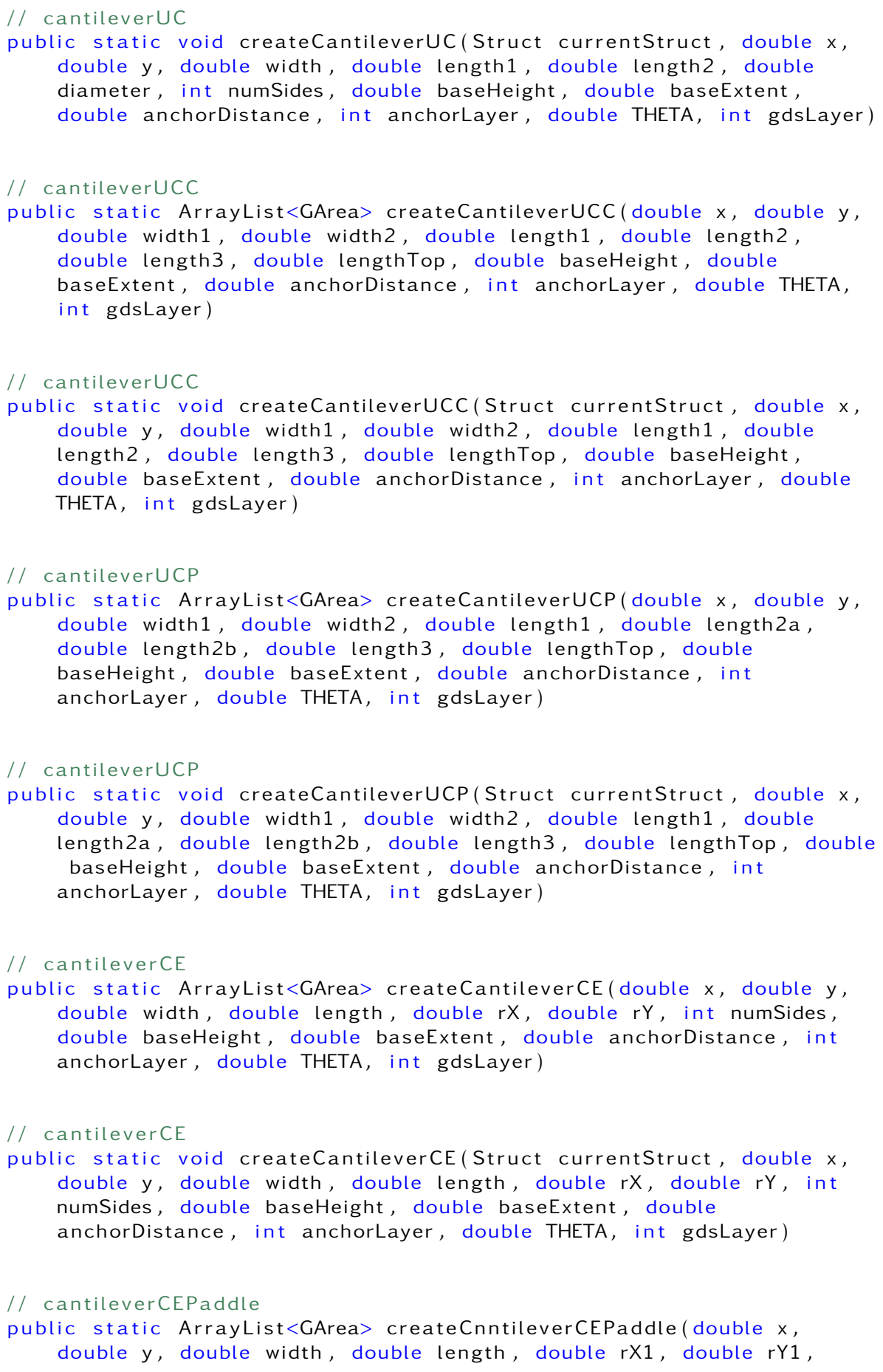

NIST • CNST Nanolithography Toolbox v2016.09.01 • http://www.nist.gov/cnst/ 
double $r X 2$, double rY2, int numSides, double paddleW, double paddleL, double baseHeight, double baseExtent, double anchorDistance, int anchorLayer, double THETA, int gdsLayer)

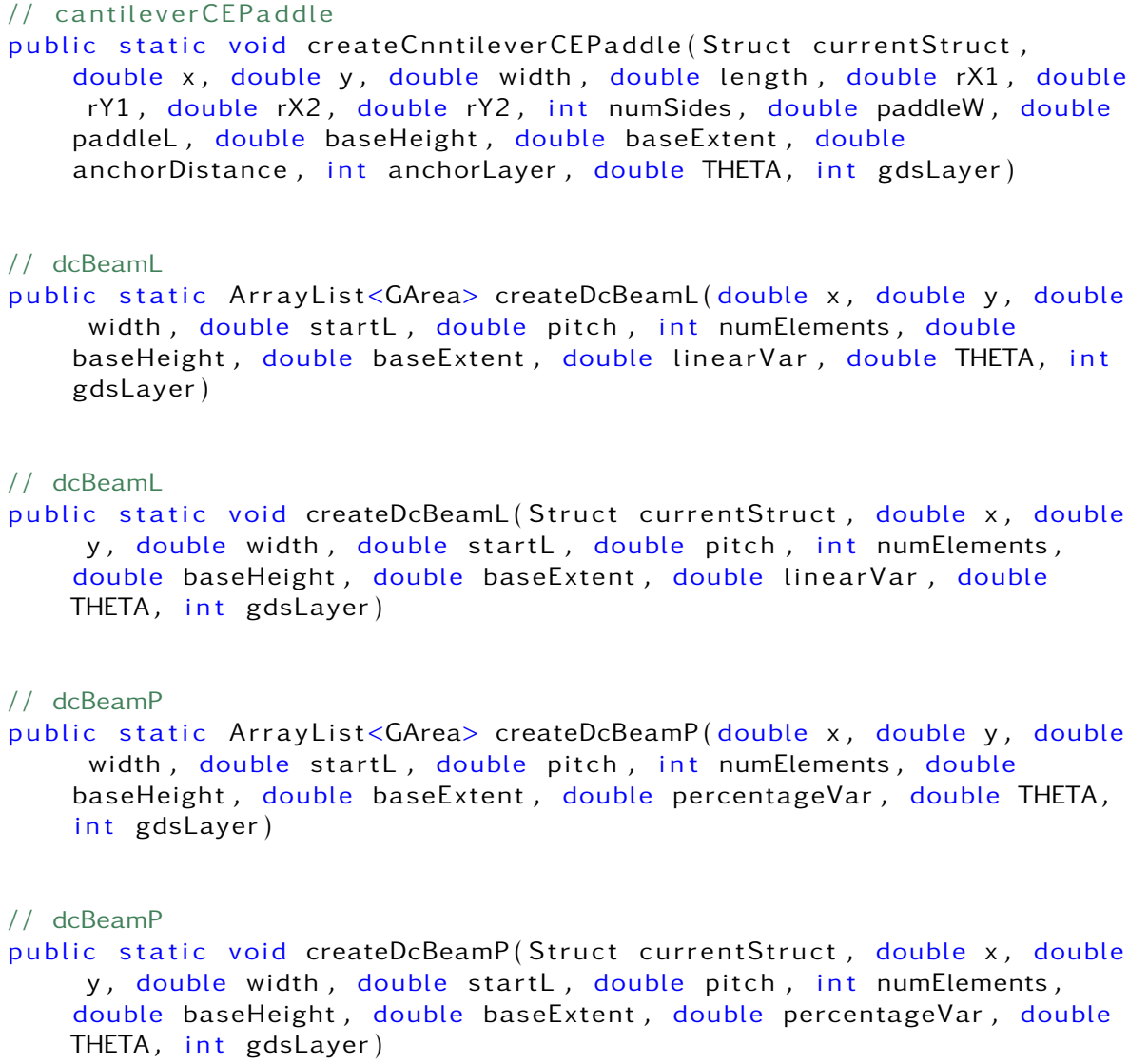




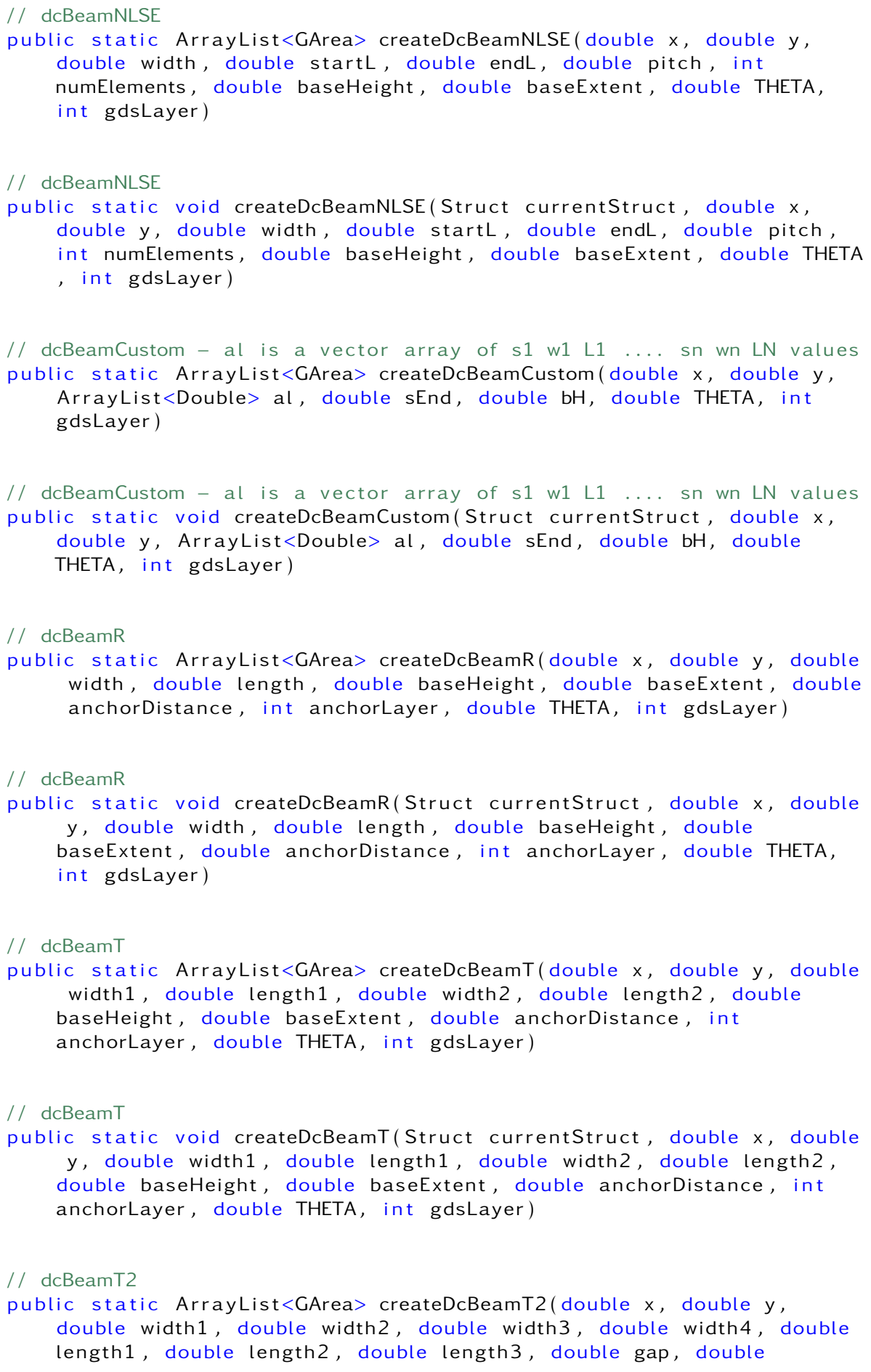


baseHeight, double baseWidth, double anchorDistance, int anchorlayer, double THETA, int gdsLayer)

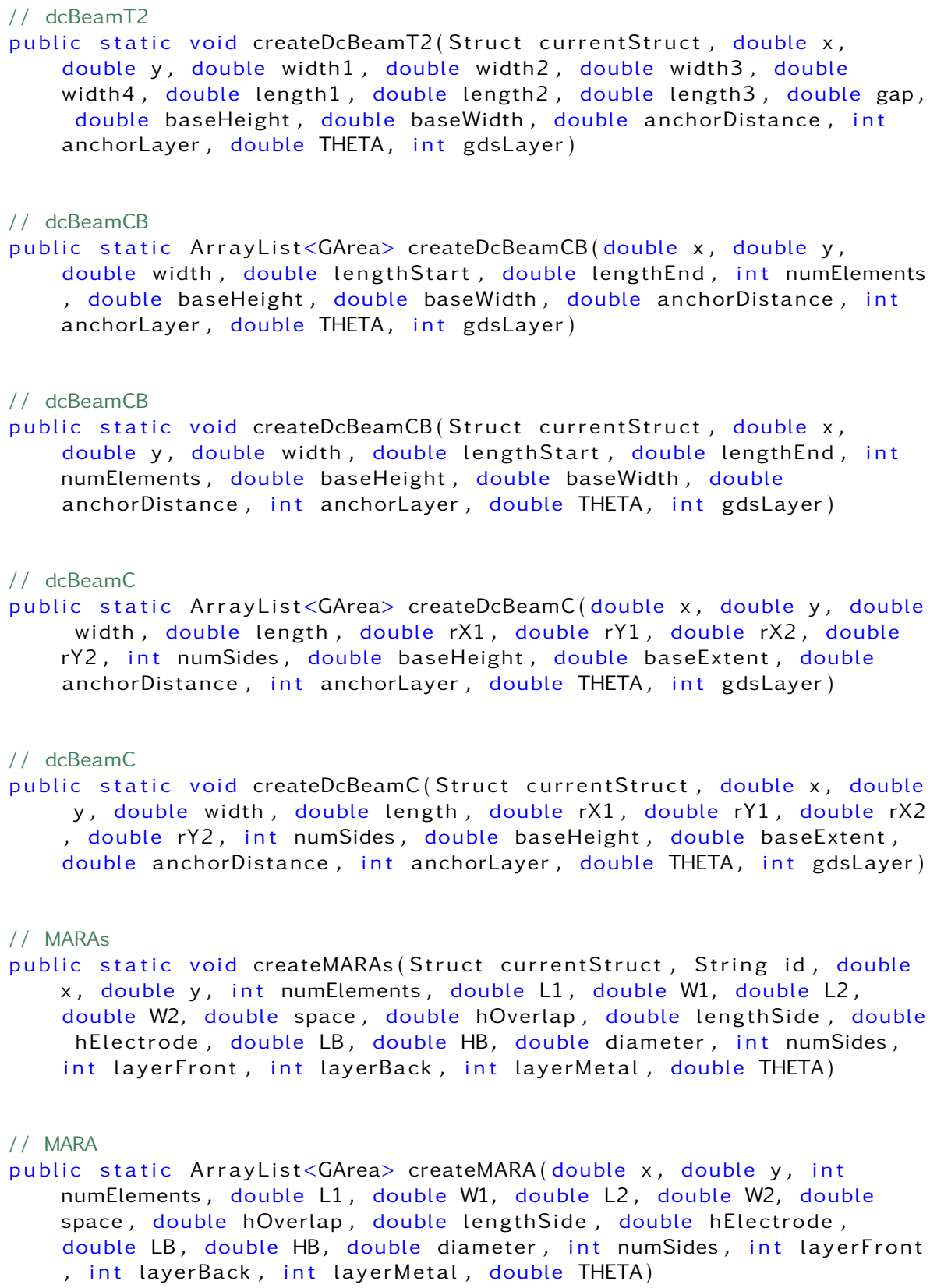




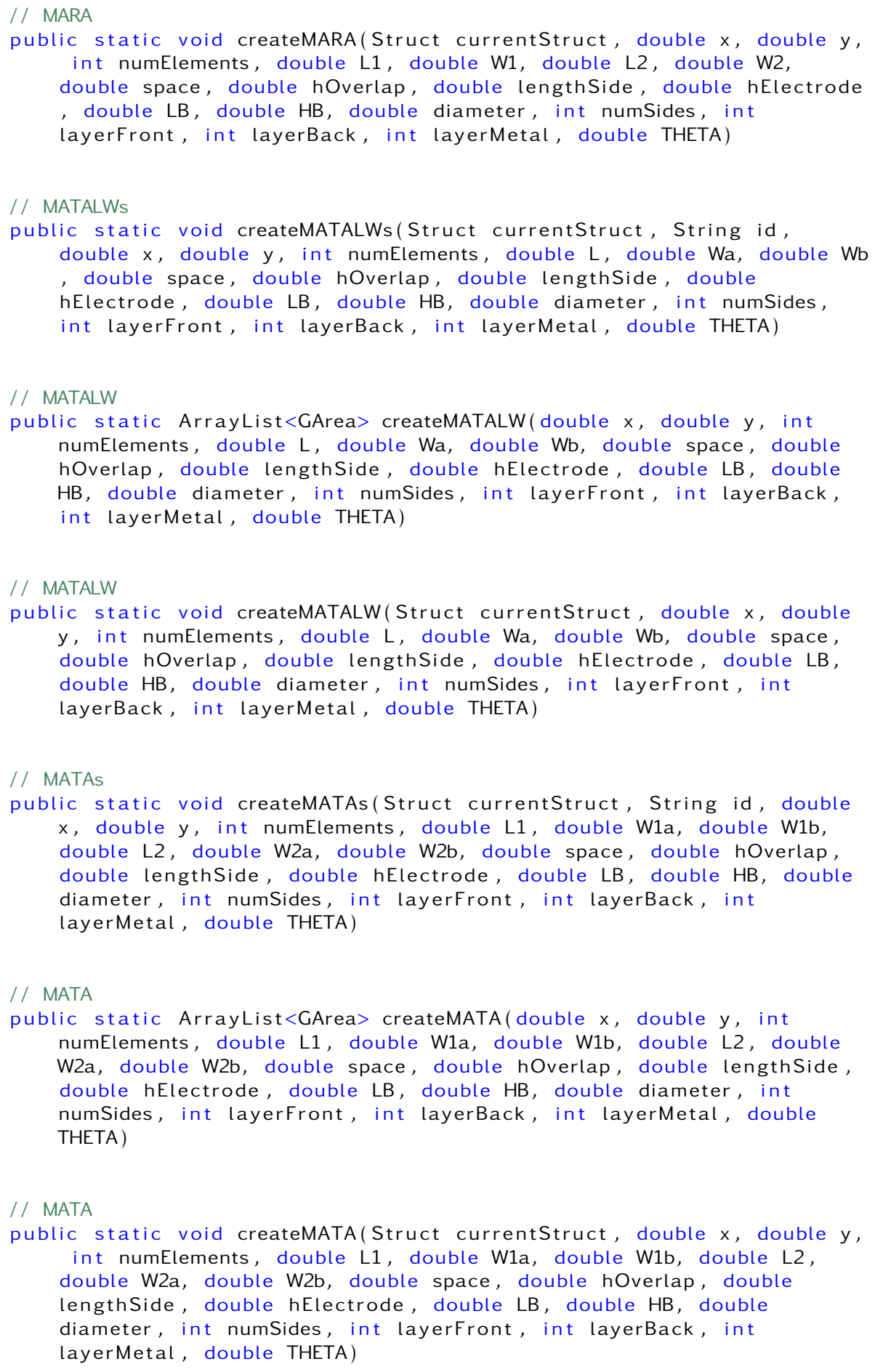




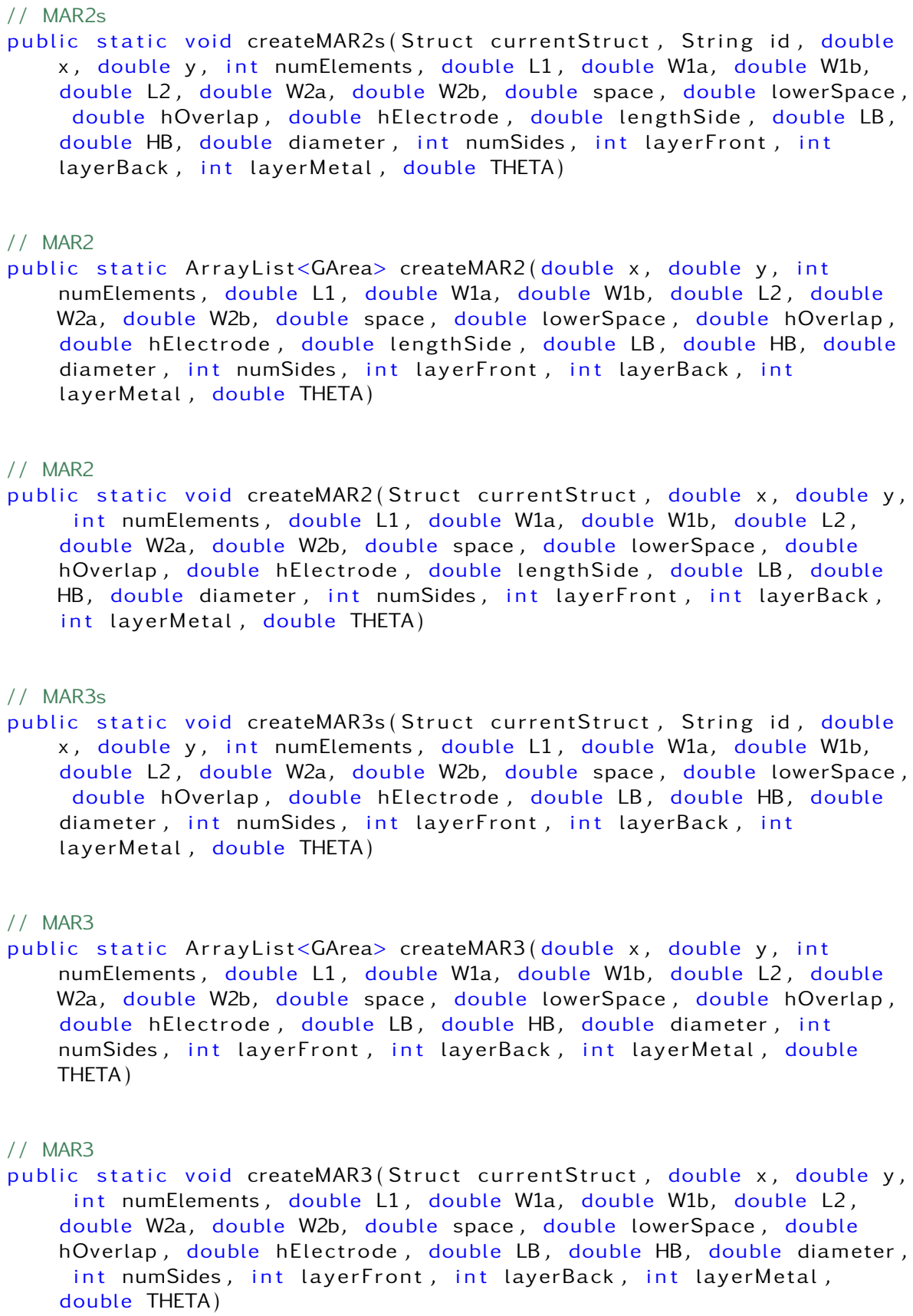




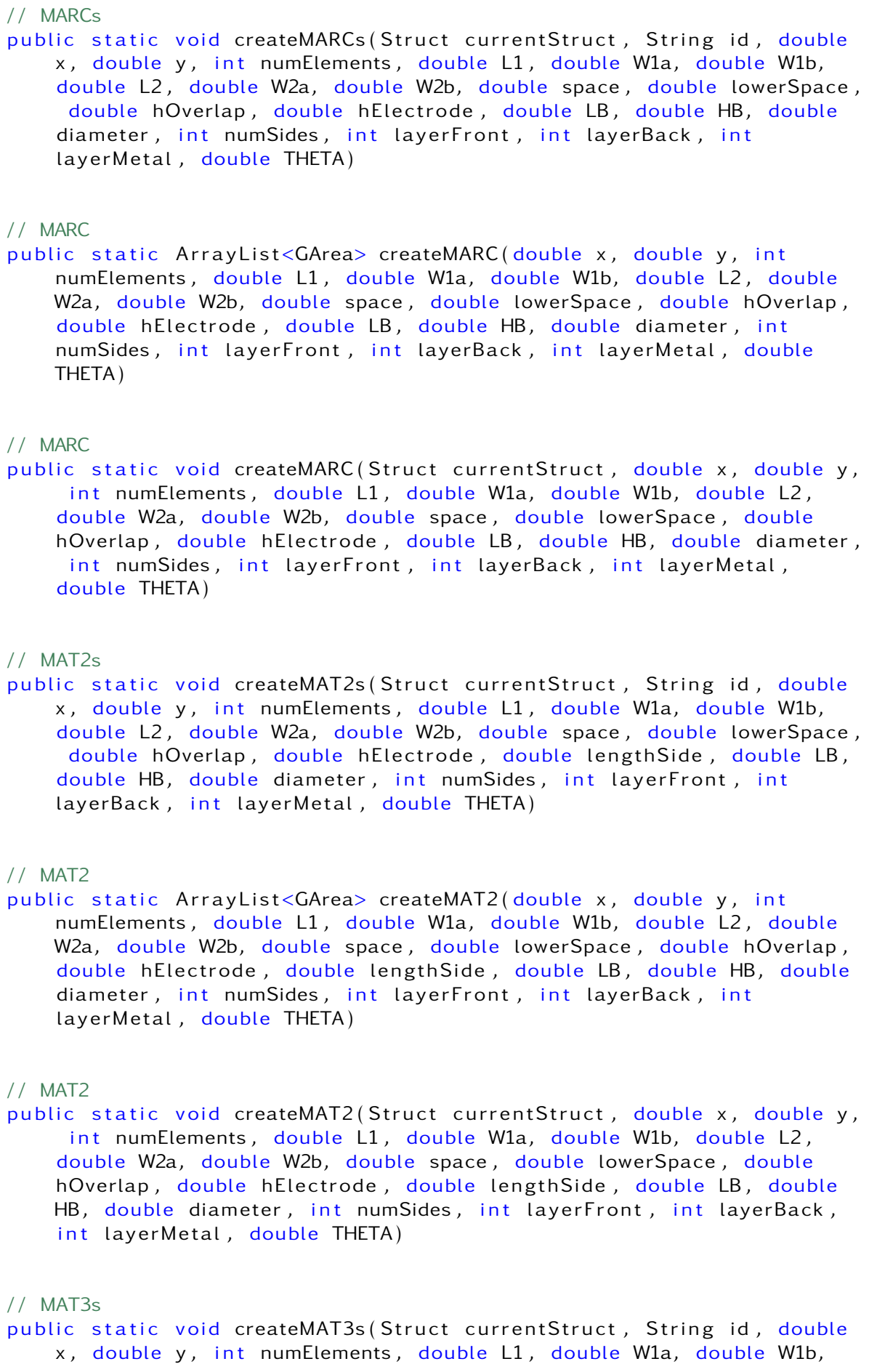

NIST • CNST Nanolithography Toolbox v2016.09.01 • http://www.nist.gov/cnst/ 
double L2, double W2a, double W2b, double space, double lowerSpace, double hOverlap, double hElectrode, double LB, double HB, double diameter, int numSides, int layerFront, int layerBack, int layerMetal, double THETA)

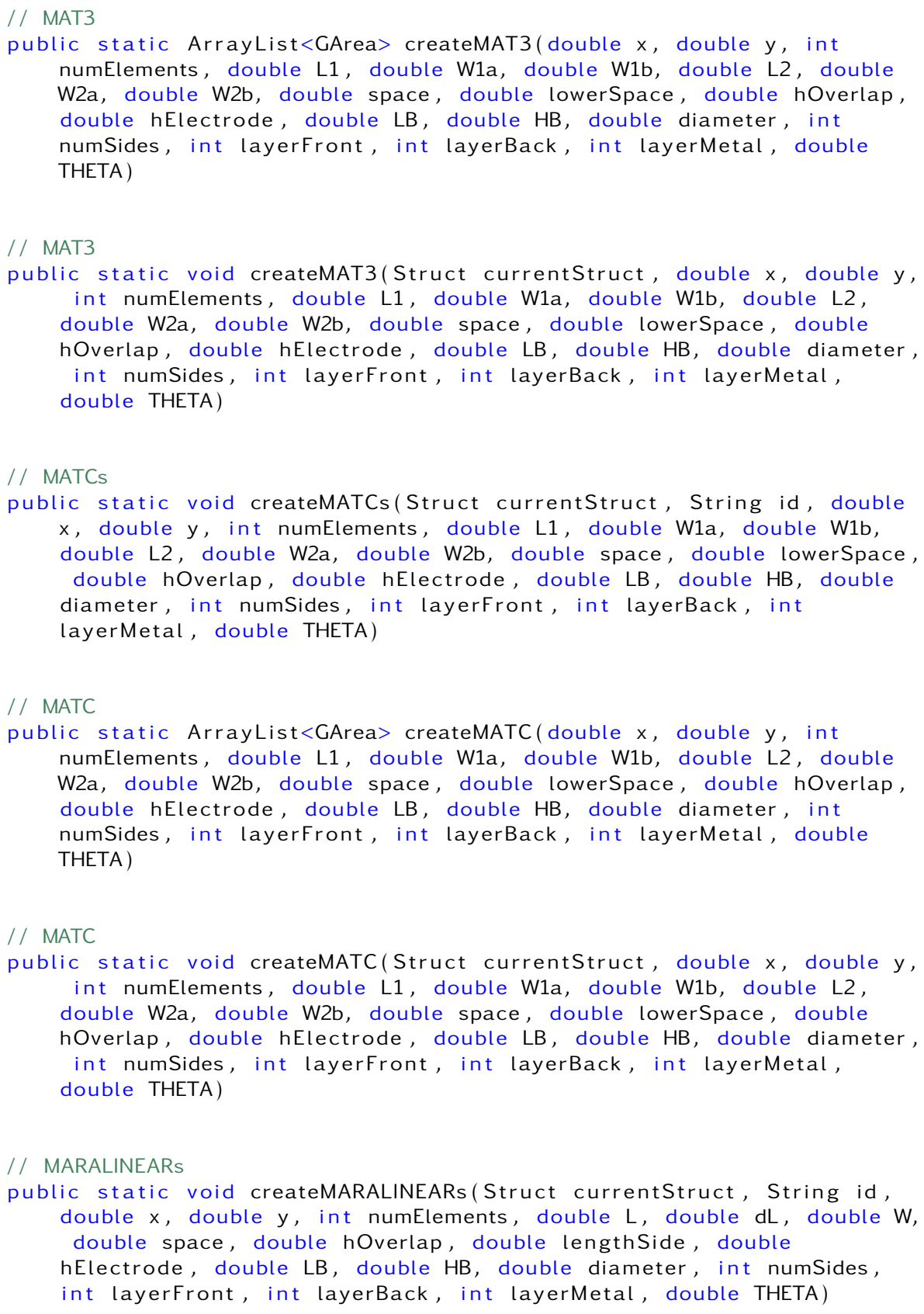

NIST • CNST Nanolithography Toolbox v2016.09.01・http://www.nist.gov/cnst/ 


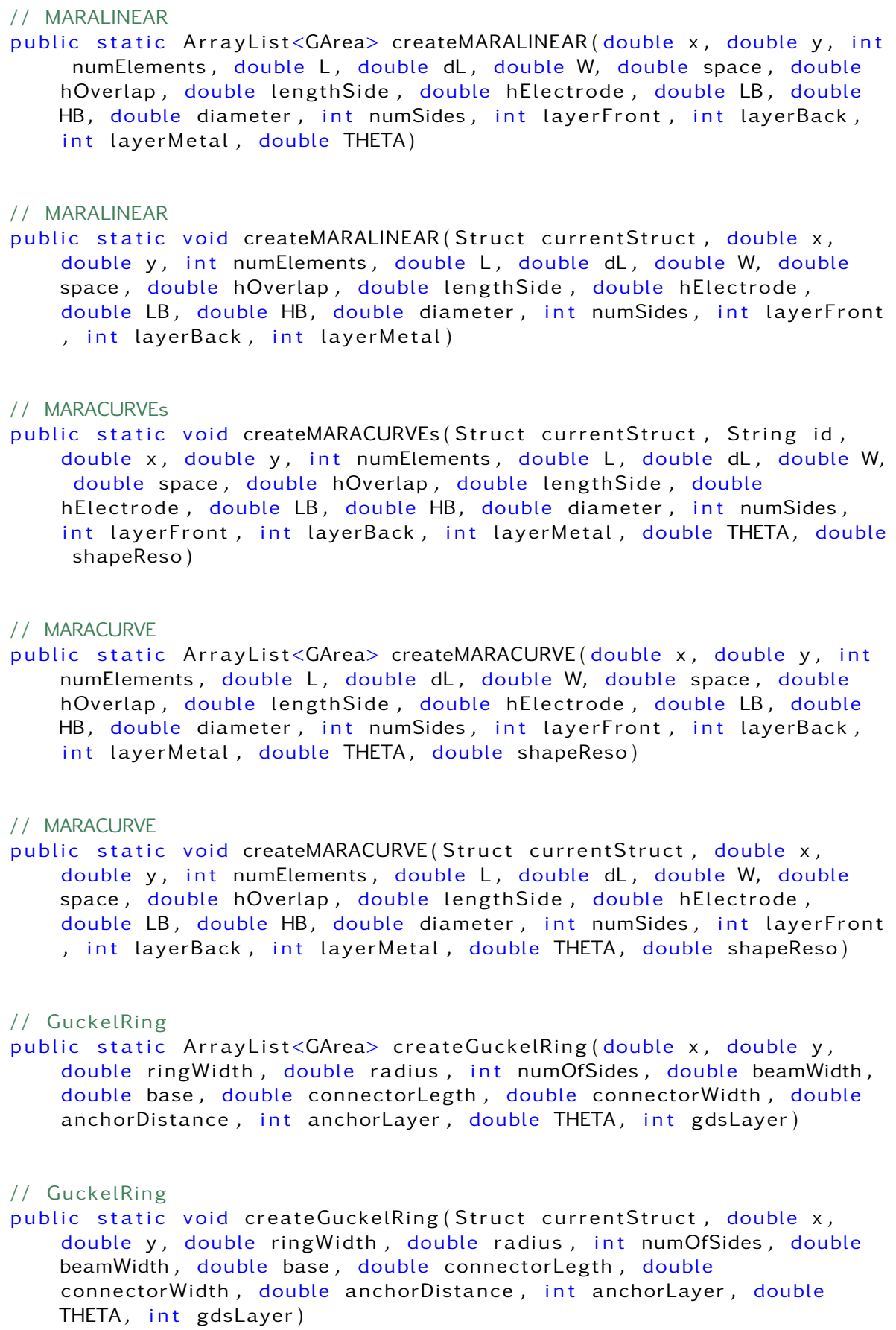

NIST • CNST Nanolithography Toolbox v2016.09.01 • http://www.nist.gov/cnst/ 


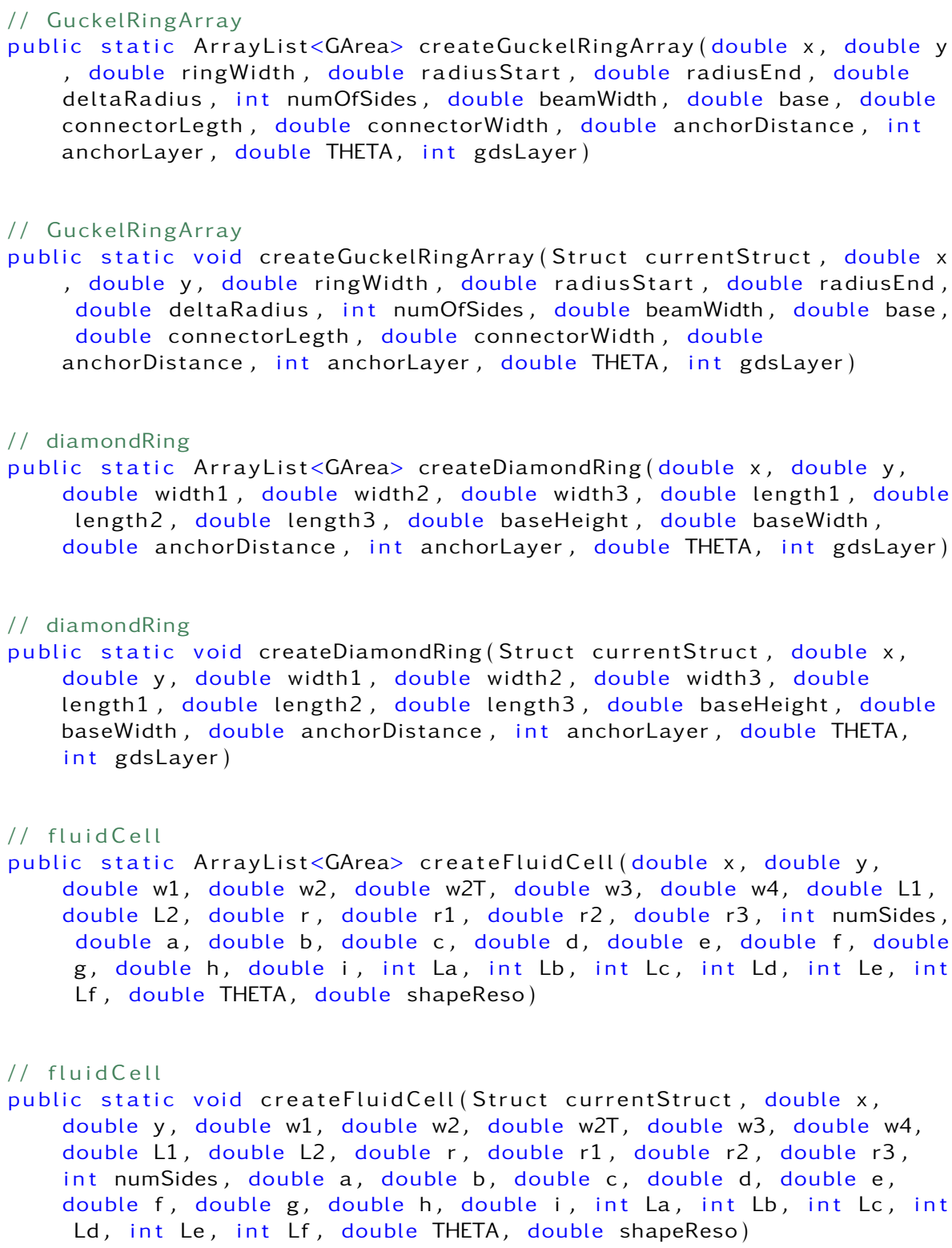


[1] A. J. Speth, A. D. Wilson, A. Kern, and T. H. P. Chang, "Electron beam lithography using vector scan techniques," Journal of Vacuum Science \& Technology, vol. 12, pp. 1235-1239, Nov. 1975.

[2] W. D. Grobman and T. W. Studwell, "Data compaction and vector scan e-beam system performance improvement using a novel algorithm for recognition of pattern step and repeats," Journal of Vacuum Science \& Technology, vol. 16, pp. 1803-1808, Nov. 1979.

[3] W. D. Grobman, "An overview of pattern data preparation for vector scan electron beam lithography," Journal of Vacuum Science \& Technology, vol. 17, pp. 1156-1163, Sept. 1980.

[4] S. M. Arnold, "Electron beam fabrication of computer-generated holograms," Optical engineering, vol. 24, no. 5, pp. 245803-245803, 1985.

[5] T. Shiono, K. Setsune, O. Yamazaki, and K. Wasa, "Computer-controlled electron beam writing system for thin film micro-optics," Journal of Vacuum Science \& Technology B, vol. 5, pp. 33-36, Jan. 1987.

[6] V. Bogli, P. Unger, H. Beneking, B. Niemann, P. Guttmann, and W. MeyerIlse, "Electron Beam Lithography And Nanometer Structures: Fabrication Of Microzone Plates," Optical Engineering, vol. 27, no. 2, pp. $272143-$ 272143-, 1988.

[7] M. A. Gesley, F. J. Hohn, R. G. Viswanathan, and A. D. Wilson, "A vector scan thermal field emission nanolithography system," Journal of Vacuum Science \& Technology B, vol. 6, pp. 2014-2018, Nov. 1988.

[8] U. Klein and F. Gotz, "Definition of geometries with complicated, curved boundaries for electron beam pattern generation," Microelectronic Engineering, vol. 9, no. 1, pp. 495-497, 1989.

[9] K. S. Urquhart, S. H. Lee, C. C. Guest, M. R. Feldman, and H. Farhoosh, "Computer aided design of computer generated holograms for electron beam fabrication," Applied Optics, vol. 28, pp. 3387-3396, Aug. 1989.

[10] R. W. Hawley and N. C. Gallagher Jr, "Efficient electron-beam pattern data format for the production of binary computer-generated holograms," in OE/LASE'90, 14-19 Jan., Los Angeles, CA, pp. 11-23, International Society for Optics and Photonics, 1990. 
[11] T. Shiono and H. Ogawa, "Diffraction-limited blazed reflection diffractive microlenses for oblique incidence fabricated by electron-beam lithography," Applied optics, vol. 30, no. 25, pp. 3643-3649, 1991.

[12] T. Erdogan, O. King, G. W. Wicks, D. G. Hall, E. H. Anderson, and M. J. Rooks, "Circularly symmetric operation of a concentric-circle-grating, surface- emitting, AlGaAs/GaAs quantum-well semiconductor laser," Applied Physics Letters, vol. 60, no. 16, p. 1921, 1992.

[13] O. King, "Curved grating fabrication techniques for surface-emitting distributed feedback lasers," Journal of Vacuum Science \& Technology B: Microelectronics and Nanometer Structures, vol. 10, p. 2974, Nov. 1992.

[14] D. M. Newman, R. W. Hawley, D. L. Goeckel, R. D. Crawford, S. Abraham, and N. C. Gallagher, "Efficient storage, computation, and exposure of computer-generated holograms by electron-beam lithography," Applied optics, vol. 32, no. 14, pp. 2555-2565, 1993.

[15] T. Shiono and H. Ogawa, "Planar-optic-disk pickup with diffractive microoptics," Applied optics, vol. 33, no. 31, pp. 7350-7355, 1994.

[16] F. Vasey, "Electron-beam lithography of curved structures with an enhanced vector-scan pattern generator supporting conic-based primitives," Journal of Vacuum Science \& Technology B: Microelectronics and Nanometer Structures, vol. 12, p. 3460, Nov. 1994.

[17] E. H. Anderson, V. Boegli, and L. P. Muray, "Electron beam lithography digital pattern generator and electronics for generalized curvilinear structures," Journal of Vacuum Science \& Technology B, vol. 13, pp. 25292534, Nov. 1995.

[18] J. Fan, D. Zaleta, K. S. Urquhart, and S. H. Lee, "Efficient encoding algorithms for computer-aided design of diffractive optical elements by the use of electron-beam fabrication," Applied optics, vol. 34, no. 14, pp. 2522-2533, 1995.

[19] K. A. Goldberg, R. Beguiristain, J. Bokor, H. Medecki, D. T. Attwood, K. Jackson, E. Tejnil, and G. E. Sommargren, "Progress towards $\lambda / 20$ extreme ultraviolet interferometry," Journal of Vacuum Science \& Technology $B$, vol. 13, pp. 2923-2927, Nov. 1995.

[20] D. Prongue, H. Rothuizen, F. Vasey, and P. Vettiger, "Enhanced e-beam system for the fabrication of optical elements," Microelectronic engineering, vol. 27, no. 1, pp. 163-166, 1995.

[21] S. J. Spector, C. J. Jacobsen, and D. M. Tennant, "Process optimization for production of sub-20 nm soft x-ray zone plates," Journal of Vacuum Science \& Technology B, vol. 15, pp. 2872-2876, Nov. 1997. 
[22] H. Rothuizen, D. Prongue, F. Vasey, and P. Vettiger, "A conic primitivebased pattern generator for electron-beam lithography of diffractive optical elements," Microelectronic Engineering, vol. 34, pp. 243-260, Dec. 1997.

[23] E. Di Fabrizio, F. Romanato, M. Gentili, S. Cabrini, B. Kaulich, J. Susini, and R. Barrett, "High-efficiency multilevel zone plates for keV X-rays," Nature, vol. 401, pp. 895-898, Oct. 1999.

[24] E. H. Anderson, D. L. Olynick, B. Harteneck, E. Veklerov, G. Denbeaux, W. Chao, A. Lucero, L. Johnson, and D. Attwood, "Nanofabrication and diffractive optics for high-resolution x-ray applications," Journal of Vacuum Science \& Technology B, vol. 18, pp. 2970-2975, Nov. 2000.

[25] K. K. Lee, D. R. Lim, H.-C. Luan, A. Agarwal, J. Foresi, and L. C. Kimerling, "Effect of size and roughness on light transmission in a $\mathrm{Si} / \mathrm{SiO}_{2}$ waveguide: Experiments and model," Applied Physics Letters, vol. 77, no. 11, p. 1617, 2000.

[26] W. Chao, B. D. Harteneck, J. A. Liddle, E. H. Anderson, and D. T. Attwood, "Soft X-ray microscopy at a spatial resolution better than $15 \mathrm{~nm}$," Nature, vol. 435, pp. 1210-1213, June 2005.

[27] http://www.genisysgmbh.de/web/applications.html, "Mixed Exposure Options, HighResolution and Fast Writing, Multi-Pass Exposure, Fracture Optimization, Application Notes."

[28] E. Gogolides, V. Constantoudis, G. P. Patsis, and A. Tserepi, "A review of line edge roughness and surface nanotexture resulting from patterning processes," Microelectronic Engineering, vol. 83, pp. 1067-1072, Apr. 2006.

[29] M. Lu, D. M. Tennant, and C. J. Jacobsen, "Orientation dependence of linewidth variation in sub-50-nm Gaussian e-beam lithography and its correction," Journal of Vacuum Science \& Technology B, vol. 24, pp. 2881-2885, Nov. 2006.

[30] K. Evans-Lutterodt, A. Stein, J. M. Ablett, N. Bozovic, A. Taylor, and D. M. Tennant, "Using Compound Kinoform Hard-X-Ray Lenses to Exceed the Critical Angle Limit," Physical Review Letters, vol. 99, p. 134801, Sept. 2007.

[31] M. Ubaldi, V. Stasi, D. Piccinin, and M. Martinelli, "Molecular roughness analysis of developed resist by LER method," Microelectronic Engineering, vol. 84, pp. 1088-1091, May 2007.

[32] M. Lu, L. E. Ocola, S. K. Gray, and G. P. Wiederrecht, "Fabrication of metallic nanoslit waveguides with sharp bends," Journal of Vacuum Science \& Technology B, vol. 26, pp. 2151-2155, Nov. 2008. 
[33] S. Sardo, F. Giacometti, S. Doneda, U. Colombo, M. D. Muri, A. Donghi, R. Morson, G. Mutinati, A. Nottola, M. Gentili, and M. C. Ubaldi, "Line edge roughness (LER) reduction strategy for SOI waveguides fabrication," Microelectronic Engineering, vol. 85, pp. 1210-1213, May 2008.

[34] A. Stein, K. Evans-Lutterodt, N. Bozovic, and A. Taylor, "Fabrication of silicon kinoform lenses for hard $\mathrm{x}$-ray focusing by electron beam lithography and deep reactive ion etching," Journal of Vacuum Science \& Technology B, vol. 26, pp. 122-127, Jan. 2008.

[35] L. E. Ocola, "Nanoscale geometry assisted proximity effect correction for electron beam direct write nanolithography," Journal of Vacuum Science \& Technology B, vol. 27, pp. 2569-2571, Nov. 2009.

[36] R. J. Bojko, J. Li, L. He, T. Baehr-Jones, M. Hochberg, and Y. Aida, “Electron beam lithography writing strategies for low loss, high confinement silicon optical waveguides," Journal of Vacuum Science \& Technology B, vol. 29, no. 6, p. 06F309, 2011.

[37] C. Browning, T. Quaglio, T. Figueiro, S. Pauliac, J. Belledent, A. Fay, J. Bustos, J.-C. Marusic, and P. Schiavone, "Photonic curvilinear data processing," p. 92350V, Oct. 2014.

[38] M. I. Davanco and K. Srinivasan, "Efficient spectroscopy of single embedded emitters using optical fiber taper waveguides," Optics Express, vol. 17, pp. 10542-10563, June 2009.

[39] M. Davanco and K. Srinivasan, "Fiber-coupled semiconductor waveguides as an efficient optical interface to a single quantum dipole," Optics Letters, vol. 34, pp. 2542-2544, Aug. 2009.

[40] M. T. Rakher, L. Ma, O. Slattery, X. Tang, and K. Srinivasan, "Quantum transduction of telecommunications-band single photons from a quantum dot by frequency upconversion," Nature Photonics, vol. 4, pp. 786791, Nov. 2010.

[41] M. Davanco and K. Srinivasan, "Hybrid gap modes induced by fiber taper waveguides: Application in spectroscopy of single solid-state emitters deposited on thin films," Optics Express, vol. 18, pp. 10995-11007, May 2010.

[42] M. Davanco, M. T. Rakher, W. Wegscheider, D. Schuh, A. Badolato, and K. Srinivasan, "Efficient quantum dot single photon extraction into an optical fiber using a nanophotonic directional coupler," Applied Physics Letters, vol. 99, p. 121101, Sept. 2011.

[43] M. Davanco, M. T. Rakher, D. Schuh, A. Badolato, and K. Srinivasan, "A circular dielectric grating for vertical extraction of single quantum dot emission," Applied Physics Letters, vol. 99, p. 041102, July 2011. 
[44] M. T. Rakher and K. Srinivasan, "Subnanosecond electro-optic modulation of triggered single photons from a quantum dot," Applied Physics Letters, vol. 98, p. 211103, May 2011.

[45] F. Ferdous, H. Miao, D. E. Leaird, K. Srinivasan, J. Wang, L. Chen, L. T. Varghese, and A. M. Weiner, "Spectral line-by-line pulse shaping of onchip microresonator frequency combs," Nature Photonics, vol. 5, pp. 770 776, Dec. 2011.

[46] K. Srinivasan, H. Miao, M. T. Rakher, M. Davanco, and V. Aksyuk, "Optomechanical Transduction of an Integrated Silicon Cantilever Probe Using a Microdisk Resonator," Nano Letters, vol. 11, pp. 791-797, Feb. 2011.

[47] M. T. Rakher, L. Ma, M. Davanco, O. Slattery, X. Tang, and K. Srinivasan, "Simultaneous Wavelength Translation and Amplitude Modulation of Single Photons from a Quantum Dot," Physical Review Letters, vol. 107, p. 083602, Aug. 2011.

[48] S. Ates, I. Agha, A. Gulinatti, I. Rech, M. T. Rakher, A. Badolato, and K. Srinivasan, "Two-Photon Interference Using Background-Free Quantum Frequency Conversion of Single Photons Emitted by an InAs Quantum Dot," Physical Review Letters, vol. 109, p. 147405, Oct. 2012.

[49] M. Davanco, J. Chan, A. H. Safavi-Naeini, O. Painter, and K. Srinivasan, "Slot-mode-coupled optomechanical crystals," Optics Express, vol. 20, pp. 24394-24410, Oct. 2012.

[50] M. DavanÃ ğo, J. R. Ong, A. B. Shehata, A. Tosi, I. Agha, S. Assefa, F. Xia, W. M. J. Green, S. Mookherjea, and K. Srinivasan, "Telecommunicationsband heralded single photons from a silicon nanophotonic chip," Applied Physics Letters, vol. 100, p. 261104, June 2012.

[51] F. Ferdous, H. Miao, P.-H. Wang, D. E. Leaird, K. Srinivasan, L. Chen, V. Aksyuk, and A. M. Weiner, "Probing coherence in microcavity frequency combs via optical pulse shaping," Optics Express, vol. 20, pp. 2103321043, Sept. 2012.

[52] I. Agha, M. Davanco, B. Thurston, and K. Srinivasan, "Low-noise chipbased frequency conversion by four-wave-mixing Bragg scattering in $\mathrm{SiN}_{\mathrm{x}}$ waveguides," Optics Letters, vol. 37, pp. 2997-2999, July 2012.

[53] H. Miao, K. Srinivasan, and V. Aksyuk, "A microelectromechanically controlled cavity optomechanical sensing system," New Journal of Physics, vol. 14, p. 075015, July 2012.

[54] Y. Liu, H. Miao, V. Aksyuk, and K. Srinivasan, "Wide cantilever stiffness range cavity optomechanical sensors for atomic force microscopy," Optics Express, vol. 20, pp. 18268-18280, July 2012. 
[55] S. Ates, L. Sapienza, M. Davanco, A. Badolato, and K. Srinivasan, "Bright Single-Photon Emission From a Quantum Dot in a Circular Bragg Grating Microcavity," IEEE Journal of Selected Topics in Quantum Electronics, vol. 18, pp. 1711-1721, Nov. 2012.

[56] F. Intravaia, P. S. Davids, R. S. Decca, V. A. Aksyuk, D. Lopez, and D. A. R. Dalvit, "Quasianalytical modal approach for computing Casimir interactions in periodic nanostructures," Physical Review A, vol. 86, p. 042101, Oct. 2012.

[57] K. J. Chau and H. J. Lezec, "Revisiting the Balazs thought experiment in the case of a left-handed material: electromagnetic-pulse-induced displacement of a dispersive, dissipative negative-index slab," Optics Express, vol. 20, pp. 10138-10162, Apr. 2012.

[58] P.-H. Wang, F. Ferdous, H. Miao, J. Wang, D. E. Leaird, K. Srinivasan, L. Chen, V. Aksyuk, and A. M. Weiner, "Observation of correlation between route to formation, coherence, noise, and communication performance of Kerr combs," Optics Express, vol. 20, pp. 29284-29295, Dec. 2012.

[59] R. Kumar, J. R. Ong, J. Recchio, K. Srinivasan, and S. Mookherjea, "Spectrally multiplexed and tunable-wavelength photon pairs at 1.55 Îjm from a silicon coupled-resonator optical waveguide," Optics Letters, vol. 38, pp. 2969-2971, Aug. 2013.

[60] Y. Liu, M. Davanco, V. Aksyuk, and K. Srinivasan, "Electromagnetically Induced Transparency and Wideband Wavelength Conversion in Silicon Nitride Microdisk Optomechanical Resonators," Physical Review Letters, vol. 110, p. 223603, May 2013.

[61] I. Agha, S. Ates, M. Davanco, and K. Srinivasan, "A chip-scale, telecommunications-band frequency conversion interface for quantum emitters," Optics Express, vol. 21, pp. 21628-21638, Sept. 2013.

[62] K. C. Balram, M. Davanco, J. Y. Lim, J. D. Song, and K. Srinivasan, "Moving boundary and photoelastic coupling in GaAs optomechanical resonators," Optica, vol. 1, pp. 414-420, Dec. 2014.

[63] M. Davanco, S. Ates, Y. Liu, and K. Srinivasan, " $\mathrm{Si}_{3} \mathrm{~N}_{4}$ optomechanical crystals in the resolved-sideband regime," Applied Physics Letters, vol. 104, p. 041101, Jan. 2014.

[64] M. Davanco, C. S. Hellberg, S. Ates, A. Badolato, and K. Srinivasan, "Multiple time scale blinking in InAs quantum dot single-photon sources," Physical Review B, vol. 89, p. 161303, Apr. 2014.

[65] I. Agha, S. Ates, L. Sapienza, and K. Srinivasan, "Spectral broadening and shaping of nanosecond pulses: toward shaping of single photons from quantum emitters," Optics Letters, vol. 39, pp. 5677-5680, Oct. 2014. 
[66] R. Zhang, C. Ti, M. I. Davanco, Y. Ren, V. Aksyuk, Y. Liu, and K. Srinivasan, "Integrated tuning fork nanocavity optomechanical transducers with high fMQM product and stress-engineered frequency tuning," Applied Physics Letters, vol. 107, p. 131110, Sept. 2015.

[67] K. Grutter, M. Davanco, and K. Srinivasan, "SiN Nanobeam Optomechanical Crystals," IEEE Journal of Selected Topics in Quantum Electronics, vol. 21, pp. 1-11, July 2015.

[68] K. E. Grutter, M. I. Davanco, and K. Srinivasan, "Slot-mode optomechanical crystals: a versatile platform for multimode optomechanics," Optica, vol. 2, p. 994, Nov. 2015.

[69] K. A. Twedt, J. Zou, M. Davanco, K. Srinivasan, J. J. McClelland, and V. A. Aksyuk, "Imaging nanophotonic modes of microresonators using a focused ion beam," Nature Photonics, vol. 10, pp. 35-39, Jan. 2016.

[70] T. Tamir and S. T. Peng, "Analysis and design of grating couplers," Applied physics, vol. 14, pp. 235-254, Nov. 1977.

[71] K. A. Bates, L. Li, R. L. Roncone, and J. J. Burke, "Gaussian beams from variable groove depth grating couplers in planar waveguides," Applied Optics, vol. 32, p. 2112, Apr. 1993.

[72] R. Waldhausl, B. Schnabel, P. Dannberg, E.-B. Kley, A. Brauer, and K. Wolfgang, "Efficient Coupling into Polymer Waveguides by Gratings," Applied Optics, vol. 36, pp. 9383-9390, Dec. 1997.

[73] D. Taillaert, F. Van Laere, M. Ayre, W. Bogaerts, D. Van Thourhout, P. Bienstman, and R. Baets, "Grating Couplers for Coupling between Optical Fibers and Nanophotonic Waveguides," Japanese Journal of Applied Physics, vol. 45, pp. 6071-6077, Aug. 2006.

[74] F. Van Laere, G. Roelkens, M. Ayre, J. Schrauwen, D. Taillaert, D. Van Thourhout, T. Krauss, and R. Baets, "Compact and Highly Efficient Grating Couplers Between Optical Fiber and Nanophotonic Waveguides," Journal of Lightwave Technology, vol. 25, pp. 151-156, Jan. 2007.

[75] F. Van Laere, T. Claes, J. Schrauwen, S. Scheerlinck, W. Bogaerts, D. Taillaert, L. O'Faolain, D. Van Thourhout, and R. Baets, "Compact Focusing Grating Couplers for Silicon-on-Insulator Integrated Circuits," IEEE Photonics Technology Letters, vol. 19, pp. 1919-1921, Dec. 2007.

[76] http://www.skinni.com, "JGDS - Java GDS Library."

[77] http://www.genisysgmbh.de/web/applications.html, "GenlSys - 3-D eBeam Lithography Application Note,"

NIST • CNST Nanolithography Toolbox v2016.09.01 • http://www.nist.gov/cnst/

page 485 of 488 
[78] A. Schleunitz and H. Schift, "Fabrication of 3d nanoimprint stamps with continuous reliefs using dose-modulated electron beam lithography and thermal reflow," Journal of Micromechanics and Microengineering, vol. 20, no. 9, p. 095002, 2010.

[79] A. Schleunitz, C. Spreu, M. Vogler, H. Atasoy, and H. Schift, "Combining nanoimprint lithography and a molecular weight selective thermal reflow for the generation of mixed 3d structures," Journal of Vacuum Science \& Technology B, vol. 29, p. 06FC01, Nov. 2011.

[80] A. Schleunitz, V. A. Guzenko, A. Schander, M. Vogler, and H. Schift, "Selective profile transformation of electron-beam exposed multilevel resist structures based on a molecular weight dependent thermal reflow," Journal of Vacuum Science \& Technology B, vol. 29, p. 06F302, Nov. 2011.

[81] H. Schift, M. Altana, and A. Schleunitz, "Sidewall-angle dependent mold filling of three-dimensional microcavities in thermal nanoimprint lithography," Journal of Vacuum Science \& Technology B, vol. 30, p. 06FB09, Nov. 2012.

[82] N. D. Bassim, A. Giles, J. D. Caldwell, and L. E. Ocola, "Focused lon Beam Direct Write Nanofabrication of Surface Phonon Polariton Metamaterial Nanostructures," Microscopy and Microanalysis, vol. 20, pp. 358-359, Aug. 2014.

[83] E. I. Bromley, J. N. Randall, D. C. Flanders, and R. W. Mountain, "A technique for the determination of stress in thin films," Journal of Vacuum Science \& Technology B, vol. 1, pp. 1364-1366, Oct. 1983.

[84] H. Guckel, T. Randazzo, and D. W. Burns, "A simple technique for the determination of mechanical strain in thin films with applications to polysilicon," Journal of Applied Physics, vol. 57, pp. 1671-1675, Mar. 1985.

[85] M. G. Allen, M. Mehregany, R. T. Howe, and S. D. Senturia, "Microfabricated structures for the insitu measurement of residual stress, Young's modulus, and ultimate strain of thin films," Applied Physics Letters, vol. 51, pp. 241-243, July 1987.

[86] M. Mehregany, R. T. Howe, and S. D. Senturia, "Novel microstructures for the insitu measurement of mechanical properties of thin films," Journal of Applied Physics, vol. 62, pp. 3579-3584, Nov. 1987.

[87] H. Guckel, D. Burns, C. Visser, H. Tilmans, and D. Deroo, "Fine-grained polysilicon films with built-in tensile strain," IEEE Transactions on Electron Devices, vol. 35, pp. 800-801, June 1988.

[88] K. Najafi and K. Suzuki, "A novel technique and structure for the measurement of intrinsic stress and Young's modulus of thin films," in IEEE 
Micro Electro Mechanical Systems, 1989, Proceedings, An Investigation of Micro Structures, Sensors, Actuators, Machines and Robots, pp. 9697, Feb. 1989.

[89] L.-S. Fan, R. Muller, W. Yun, R. Howe, and J. Huang, "Spiral microstructures for the measurement of average strain gradients in thin films," in IEEE Micro Electro Mechanical Systems, 1990. Proceedings, An Investigation of Micro Structures, Sensors, Actuators, Machines and Robots, pp. 177181, Feb. 1990.

[90] R. Pratt, G. Johnson, R. Howe, and J. Chang, "Micromechanical structures for thin film characterization," in , 1991 International Conference on Solid-State Sensors and Actuators, 1991. Digest of Technical Papers, TRANSDUCERS '91, pp. 205-208, June 1991.

[91] H. Guckel, D. Burns, C. Rutigliano, E. Lovell, and B. Choi, "Diagnostic microstructures for the measurement of intrinsic strain in thin films," Journal of Micromechanics and Microengineering, vol. 2, no. 2, p. 86, 1992.

[92] Y. Gianchandani, K. Najafi, and B. Orr, "Silicon micromachined thermal profilers," in Electron Devices Meeting, 1993. IEDM '93. Technical Digest., International, pp. 191-194, Dec. 1993.

[93] L. Lin, R. Howe, and A. Pisano, "A passive, in situ micro strain gauge," in Micro Electro Mechanical Systems, 1993, MEMS '93, Proceedings An Investigation of Micro Structures, Sensors, Actuators, Machines and Systems. IEEE., pp. 201-206, Feb. 1993.

[94] F. Ericson, S. Greek, J. Soderkvist, and J. Schweitz, "High Sensitive Internal Film Stress Measurement By An Improved Micromachined Indicator Structure," in The 8th International Conference on Solid-State Sensors and Actuators, 1995 and Eurosensors IX.. Transducers '95, vol. 2, pp. 8487, June 1995.

[95] Y. Gianchandani and K. Najafi, "Bent-beam strain sensors," Journal of Microelectromechanical Systems, vol. 5, pp. 52-58, Mar. 1996.

[96] L. Lin, A. Pisano, and R. Howe, "A micro strain gauge with mechanical amplifier," Journal of Microelectromechanical Systems, vol. 6, pp. 313321, Dec. 1997.

[97] G. T. A. Kovacs, Micromachined transducers sourcebook. Boston, Ma: WCB, 1998.

[98] S. D. Senturia, Microsystem design. Boston: Kluwer Academic Publishers, 2001.

[99] M. Gad-el Hak, The MEMS handbook. Boca Raton, FL: CRC Press, 2002.

NIST • CNST Nanolithography Toolbox v2016.09.01 • http://www.nist.gov/cnst/ page 487 of 488 
[100] N. Lobontiu and E. Garcia, Mechanics of microelectromechanical systems. New York: Kluwer Academic, 2005.

[101] N. Lobontiu, Mechanical design of microresonators: modeling and applications. McGraw-Hill nanoscience and technology series, New York: McGraw-Hill, 2006.

[102] J. A. Kubby, A guide to hands-on MEMS design and prototyping. Cambridge, UK ; New York: Cambridge University Press, 2011.

[103] V. Kempe, Inertial MEMS: principles and practice. Cambridge ; New York: Cambridge University Press, 2011.

[104] N. Unal, D. Mahalu, O. Raslin, D. Ritter, C. Sambale, and U. Hofmann, "Third dimension of proximity effect correction (PEC)," Microelectronic Engineering, vol. 87, pp. 940-942, May 2010. 